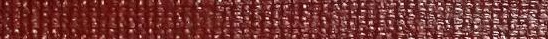

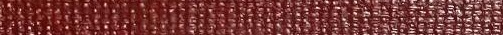




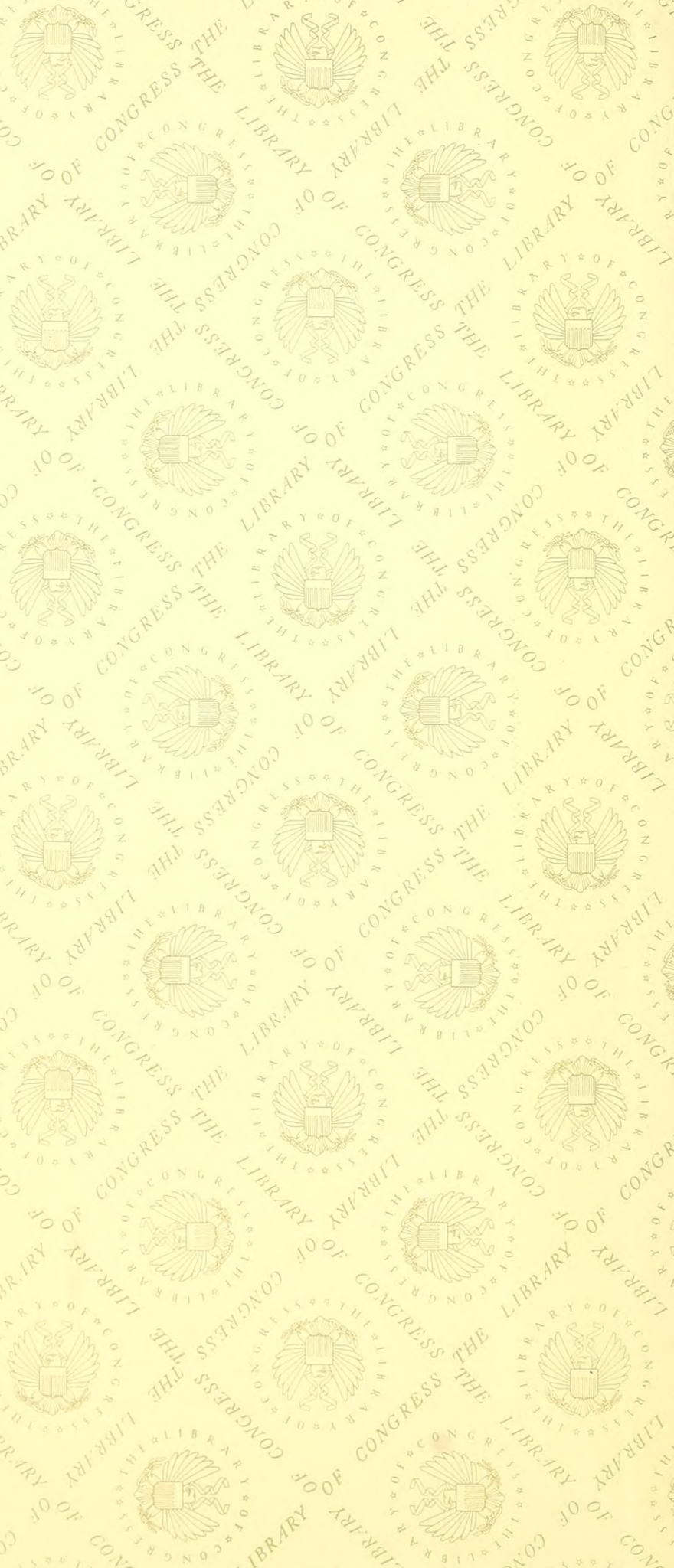




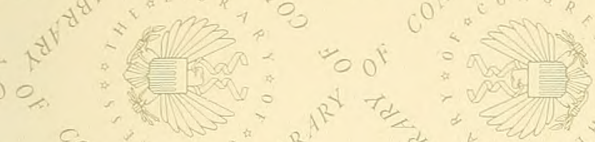

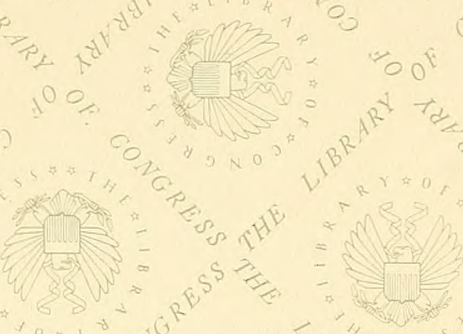

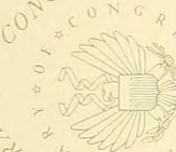
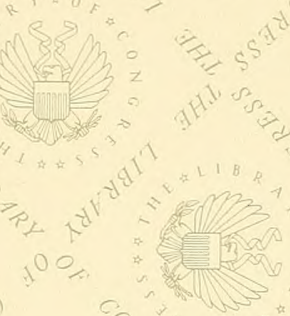

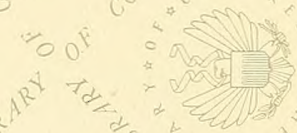

.00
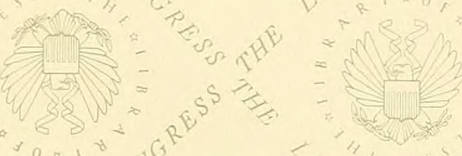

$\lim _{3 \rightarrow 1}$
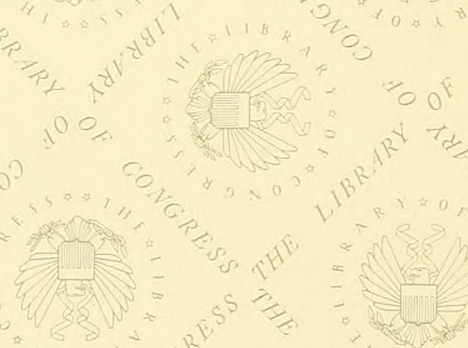

$0^{0}$
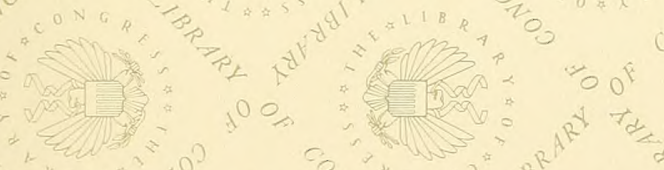

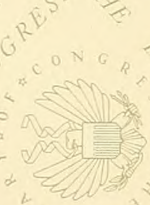

$0^{0}$ B 





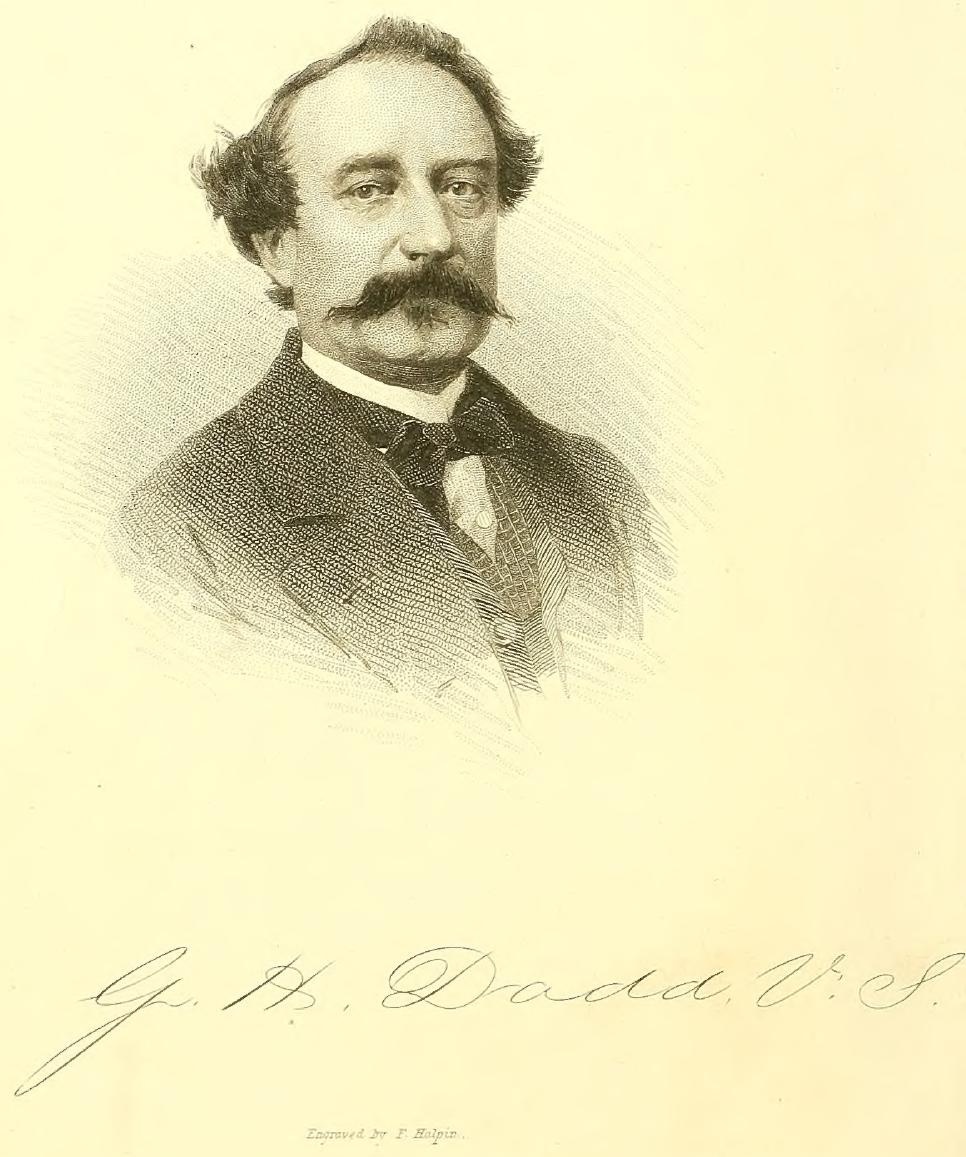




\section{A D D'S}

\section{THEORY AND PRACTICE}

of

VETERINARY

MEDICINE AND SURGERY,

CONTAINING

THE CURATIVE TREATMENT OF DISEASES OF

\section{HORSES AND CATTLE,}

SHEEP AND SWINE,

AND EMBRACING ALL THE LATEST INFORMATION ON THE RINDERPEST AND TRICHINA.

By GEORGE H. DADD, Veterinary Surgeon, Author of "Anatomy and Physiology of the Horse," "Modern Horse Doctor," "Cattle Doctor," etc., Professor of Anatomy and Physiology in the Veterinary Institute of Chicago.

\section{ELEGANTLY ILLUSTRATED。}

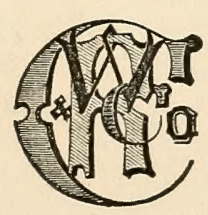

C CINCINNATI:

R. W. CARROLL \& CO., PUBLISHERS, 117 WEST FOURTH STREET. 
Entered according to Act of Congress, in the year 1867, by R. W. CARROLL \& CO.,

In the Clerk's Office of the District Court of the United States for the Southern District of Ohio. 


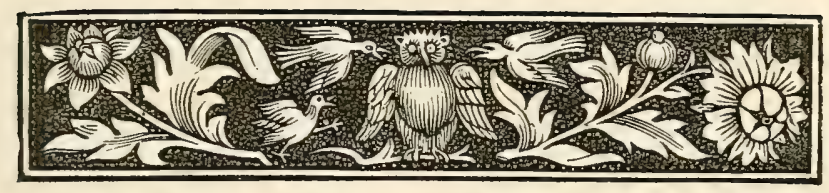

\section{P R E F A C E.}

-

A MERICANS rank high in the estimation of the scientific Al world for their genius and industry in the great work of intellectual, moral, and scientific improvements. See what has occurred in the arts of printing, electrotyping, etc. The eloquence of the great literary magicians of the New World, the honored graduates of free institutions and free schools, no sooner falls from their lips than the phonographist transfers it to paper, the nimble fingers of the compositor puts it into "form ;" next the "battery" deposits on the face of the type a more durable coating of copper, and "Hoe's press" sends off " impressions" with almost magical rapidity." No less surprising are the wonderful feats performed by American mechanics, in the construction of fast yachts and steamers, which often pluck a laurel from the so-called "Mistress of the Sea ;" and old "Uncle John" gives us due credit for perfecting a race of fast trotters that can beat the world of horseflesh.

Improvements in every department of Science and Art are constantly treading on the heels of improvement; yet, as regards the science of Veterinary Medicine and Surgery, America seems almost the last among civilized nations to put her shoulder to the wheel. What can be the reason? Her youth may be offered as a plea for excuses. The daughter of the Old World has not yet, in this department, arrived at years of discretion; she is yet in her teens. Her sons have borne the heat and burden of the day in establishing their liberty, in founding cities, extending commerce, and in taming the wild face of soil, by uprooting the giant oak, and in clearing away the forest, so that the husbandman, with plow and harrow in hand, might follow on, and next impregnate mother 
earth with germs for fructification, which, in the day of the harvest thereof, might be gathered and garnered for the benefit of man and the inferior orders of creation.

And long ere the woodman's ax ceases to vibrate its clear stroke-notes in the ears of the new settlers, a giant enterprise is in contemplation. Space and distance must be comparatively annihilated; the iron horse, with his lungs of steel and breath of steam, must have his highway and byway, and the result is, the choice spirits of progression have been earnestly engaged in dotting a vast surface of United States Territory with a magnificent net-work of railway and telegraphic wire; and various other enterprises, with which the reader is familiar, have so occupied the American mind, that the Science of Life, as it applies to animals, has been almost at a stand-still.

In the prosecution of these objects, so essential as they have proved in the economy of an infant republic, it is not surprising that our scicnce should fail to receive attention, and that America should be in the rear of the veterinary squadrons of the Old World; yet, notwithstanding this seeming indifference, veterinary science does, in some parts of this country, occupy as high a platform as that which obtained in England, about seventy years ago, when the Apostle of Mercy, ST. BEL, first landed on British soil.

The science which we here advocate is as valuable and charitable to nature's menials, for the purpose of ministering to their physical wants, relieving their aches and pains, and of prolonging their lives, as that practiced on the more noble of created life; and, at the present day, testimony can be produced going to show that among us can be found "good Samaritans" who are always ready to minister to the wants of the inferior orders of creationa work as acceptable to the God of Nature, and as creditable to manhood, as when the welfare of one of us is concerned. And should we estimate the science in exact ratio to the value and usefulness of the legion host of domestic animals that have been reared in this country, and those which, without regard to cost, have been imported, to whose welfare it directly contributes, the reader will readily perceive that it is entitled to the consideration and support of a nation of husbandmen.

It must be borne in mind that a knowledge of the principles of veterinary medicine can not be acquired without diligent study 
and close application. It is only the property of the industrious and devoted student.

In order to acquire the necessary tact, skill, and experience for the practice of our art, some of the shining lights-the early disciples-have spent the May-day of their existence in solving its problems, and, when near its close, have declared, with a modesty indicative of true genius, that their education was yet incomplete.

Such testimony goes to show that there is no republican nor royal road to veterinary knowledge. In fact, after a man has perseveringly pursued the routine studies of the most popular collegiate institutions, he may still be found "wanting."

Let us contemplate for a moment the superior advantages which human practitioners have over our craftsmen, both as regards their scientific qualifications and the superior means at their command for ascertaining the character, location, and intensity of the various maladies peculiar to the human race. For example, the practitioner of human medicine, if he has been a faithful and industrious student, enters upon the active duties of his profession, having acquired a valuable fund of knowledge of anatomy, physjology, pathology, and other necessary branches of study; and, having disciplined his mind in the regular school, and in that of experience, he is expected to be able to rejoice in the possession of a well-trained mind, which enables him to understand and interpret the physiological laws which preserve health and life in the constitution of organized beings; also to comprehend the why and wherefore of disease; to be able to institute sanitary and other regnlations, and to select suitable medicinal preparations, in view of meeting the various indications of each and every form of disease. And when a man becomes the subject of sickness, and the doctor is called upon to exercise his skill, the latter receives valuable aid, in view of making a correct diagnosis, from the patient and his friends. Each can be questioned, and their responses throw considerable light on the history and nature of the malady. Now, as regards the latter advantages, they have no parallel with the men of our craft. Our patients are deprived of the power of speech, and we can only judge of the state of their health, and the nature of their maladies, by signs revealed or elicited through physical exploration. We have other difficulties to encounter of no less magnitude. For example, our patients are often located in situations unfit for a well animal to reside in, and they do not 
always receive that attention from their owners which the urgency of the case demands. In fact, the good nursing, kind attention, etc., which often proves of so much value in the restoration from sickness of one of our race, is often denied the inferior ereature; and other matters, too numerous to mention at the present time, tend to show the advantages which our brethren of a sister science have over us, and how necessary and important it is that a practitioncr of our art should be thorough master of his profession. It is a lamentable fact that the great majority of our husbandmen have not hitherto realized that the superstructure of veterinary science rests on the same intelligent basis as that of human medicine; but such is really the case. In the language of the lamented Percivalu, we are assured that " whether we prescribe for a man, horse, or any animal, the laws of the animal economy are the same; all require the same treatment," that is to say, the propositions for restoration are all founded on the same intelligent basis.

The importance, therefore, of educating men for the profession, and of furnishing reliable works for study, is very evident; and the necessity is further illustrated in the daily experience of those persons who, as a matter of necessity, are obliged to do the best they ean for the sick and dying of their flocks and herds, having no knowledge of those advantages which a thorough course of training affords. Such persons must, necessarily, feel that they are groping in darkness, and when a ray of light does flit across their medical path, it only serves to make them better acquainted with their own want of skill. Persons thus circumstanced, unable to procure the services of a good veterinary surgeon, in consequence of a searcity of the "genuine material," are often compelled to assume the responsibility and risk of medical attendant; and, no doubt, they prescribe with good intention and honesty of purpose, but disease and death has the vantage of them, for they know not the modes of pathological warfare, nor are they acquainted with means best calculated to insure a favorable issue ; and they will certainly hail, as a great blessing, any and every effort to improve our veterinary literature, and thus diffuse knowledge so sadly needed.

It is well known that prior to the introduction of a rational system of veterinary medicine in the mother country, millions of domestic animals were annually sacrificed at the shrine of igno- 
rance; but science came to the rescue, and now some of the disciples of St. BEL, Coleman, their co-workers and kindred spirits, can, by the aid of their vast materia medica, their anæsthetic agents, scalpel, etc., accomplish unheard of wonders. We are now working to dispel the awful cloud of ignorance and superstition which has too long lingered around the stable and barn. We aim to illuminate the dark spots that have existed for many hundreds of years, and to obliterate the false theories that have been handed down to us by the Egyptians and Arabians, and the modern jugglers of this science.

There never was a period in the history of the United States when the services of educated veterinarians were so much needed as at the present time; for the live stock of this country do not enjoy immunity from those pestiferous epizoötic maladies which have formerly operated as a withering simoom on the enterprise of British husbandmen in the breeding and raising of live stock. For example: the people of the Western States complain that a disease occasionally makes its appearance among cattle, to which they have applied the name of "trembles," or " milk sickness," and it has so scourged both the superior and inferior orders of creation, that the former have often abandoned the old homestead, in view of seeking a location where there seemed to be some prospect of enjoying immunity from the pestilential scourge. A contagious and infectious disease often prevails among swine, carrying them off by hundreds and by thousands, yet many of us are in the dark regarding its cause, nature, and treatment. Typhoid affections, puerperal fever, apoplexy, and dropsy of the brain are just as prevalent here as in England. Miscarriage or abortion is fearfully on the increase. Diseases of climatic origin are more rife in this country than in England. This is, perhaps, owing to the diversity which exists in the climatic temperature of our vast territory, and to our various faulty modes of management. In fact, there is searcely any disease known to veterinarians of the Old World but that has prevailed in the New.

We must have reliable text-books and educated surgeons, in order to understand the nature and treatment of the diseases incidental to domestic animals. It is not only a matter of national, but of individual, policy and interest; and should we view the matter with the eyes of business men, we shall see that such enterprise must surely pay. 
Persons who have paid any attention to the rise and progress of veterinary science in this country, are painfully aware of its great imperfections, and the author's object in preparing this work is to endeavor to lessen and remove them, by giving the reader the benefit of an experience which extends through a period of twenty-five years.

In view of furnishing reliable information, and of showing how little of medicine is required for the treatment of various forms of disease, a number of cases, recorded in the author's note-book, are introduced in various parts of the work. These cases may, perhaps, also give the unprejudiced reader juster views of the relations of nature and art to diseases; for it is a notorious fact that very many well-instructed persons of all classes have hitherto exercised a blind faith in the medical art for the cure of disease, when it is a fact well known to those who practice rational medicine that Nature possesses vastly greater powers than Art in curing diseases. What is here meant by nature, is the conservative power inherent in the living body. For a better understanding of this subject, the reader is referred to the works of Sir JoHN Forbes, Oliver W. Holmes, and Professor Bigelow.

In view of aiding nature in the cure of disease, the author has introduced to the reader's attention a new class of remedies, viz.: Fluid Extracts of a sanative character. They have proved more safe and efficacious in the practice of rational medicine than all the other heroic arms of physic.

These remedies have been carefully tested for several years by the author, and those students who have, from time to time, placed themselves under his instruction, and the result has been very satisfactory; otherwise, they would not be recommended in this work.

Finally, the author feels it due to himself to state that the almost constant occupation of his time, professionally, has given him less opportunity than he desired for the production of this work, yet he entertains a hope that he has not labored in vain; and thus this mite is cast into the common treasury of Veterinary Science.

G. H. D.

Chicago, Nov. 3, 1866. 


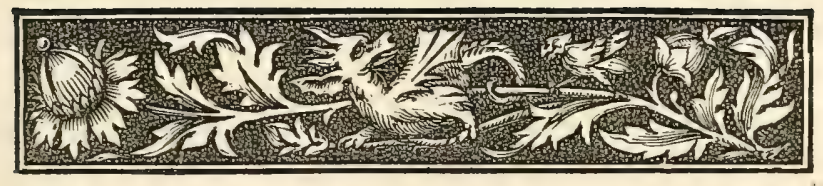

\section{CONTENTS.}

\section{SECTION I.-The Causes of Disease.}

(Page 19-39.)

Remarks on Veterinary Science, and the Relation it bears to Social ScienceVeterinary Science; how to inaugurate it-What Benefits have we derived from its Study-Inflammation; its Nature and Treatment

\section{SECTION II.-Diseases of THE Ere.}

(Page 40-57.)

Amaurosis, or Glass Eye (Gutta Serena) - Foreign bodies within the EyelidsSpecks, or film on the eye, known as Opacity of the Cornea-Cataract-Worm in the Eye-Ophthalmia-Treatment of Inflammation-Purulent OphthalmiaSpecific Ophthalmia.

\section{SECTION III.-Diseases of the Brain and Nervous System.}

(Page 58-85.)

Abscess within the Substance of the Brain-Hydrocephalus, or Dropsy of the Brain-Stomach Staggers-Apoplexy, or Cerebral Hemorrhage-Apoplexy of Spine, or Spinal Hemorrhage, producing Paraphlegia-Epilepsy, or Fits-Tetanus-Vertigo-Acute or General Disease of the Brain, known as EncephalitisSleepy Staggers-Cerebral Meningitis-Chorea, or Stringhalt.

\section{SECTION IV.-Diseases of the Respiratory Passages and Organs.}

(Page 86-186.)

Importance of Ventilating Stables-Brief Exposition and Description of the Function of the Lungs-Spasm of the muscles of the Glottis and Epiglotis-Laryngytis, (suppurative and inflammatory)-Croup-Chronic Cough-RoaringPolypus-Bronchocele-Influenza, or epizoütic catarrh-Pneumonia, or Inflammation of the Lungs, including Typhoid Affections, Pleurisy, and Dropsy of the Chest. 


\section{SECTION V.-Dentition and Diet.}

(Page 127-146.)

The Horse's Age as shown by his Teeth-On the Diet of Horses-The effects of various kinds of Food-As regards the quantity of Food required-As regards changes in Diet-Conclusion.

\section{SECTION VI:-GLANDERS AND FAROY.}

(Page 147-159.)

Glanders-Glanders can be communicated to Man-Contagiousness of Glanders-Suspected Glanders-How came the Disease to be called Glanders?-Diagnostic Symptom of Glanders-Treatment of Glanders--Farcy (Disease of the Absorbents).

\section{SECTION VII.-Diseases of the Digestive Organs.}

(Page 160-222.)

Lampas-Spontaneous Salivation-Bots-Inflammation of Stomach-Rupture of Stomach-Gorged, or over-distended Stomach-Meteorization-Flatulent Colic-Spasmodic Colic-Inflammation of the Peritoneum-Ascitis-Inflammation of the Intestines-Strangulation of Intestines-Wounds penetrating the Abdominal Carity-Splenic Apoplexy-Functional Disease of the Liver-Pancreas; its Function-Parasites which affect the Intestines.

\section{SECTION VIII.-Diseases of the URinary Organs.}

$$
\text { (Page 223-237.) }
$$

Inflammation of the Kidneys-Inflammation of the Bladder-Stone in the Bladder-Suppression of the Urine-Retention of the Urine-Diabetes, or Profuse Stalling-Rupture of the Bladder-Albuminous Urine-Hæmaturia, or Bloody Urine.

\section{SECTION IX.-Strgical Operations.}

(Page 239-273.)

Of Surgical Operations and the various Restraints it is sometimes necessary to place the Horse under for their performance-Casting-Slinging-Castration-French method of Castration - Castration by Ligature - Lithotomy Traceotomy-Esophagotomy--Neurotomy-Mode of Performing NeurotomyPeriosteotomy-Division of the Flexor Tendons-Amputations-Amputation of the Penis-Amputation of the Tail-Nicking-Firing-Blistering-Ammoniacal Blister-Rowelling-Setons-Abstraction of Blood, or Bleeding-Division of the Temporal Artery-Bleeding by the Palate-Bleeding by the Toe-PhlebotomyBleeding by the Jugular Vein. 


\section{SECTION X.-Diseases of the Skin and Sub-Tissues,}

(Page 274-301.)

Iteh and Mange-Lice on Animals-Grease and Scratches-Surfeit-Dropsical Limbs-Scarletina-Ringworm-Prurigo--Poll-evil-Fistulous Withers-Warts on the Skin-Purpura Hemorrhagica-Out of condition-Hide-bound-Herpes.

\section{SeCtion XI.-Breeding, and the Principles of the same.}

$$
\text { (Page 302-318.) }
$$

Breeding-The Principles of Breeding-In-and-in Breeding-Permanent Varieties-Animals become Parents too early-Objections to In-and-in Breeding answered-The Advantage of In-and-in Breeding-The Importance of Studying Anatomy and Physiology.

\section{SECTION XII.-Lameness, From Various Causes.}

$$
\text { (Page 319-385.) }
$$

Curbs-Elbow Tumor-Capped Hock-Varix, or Bog Spavin-Atrophy, or Wasting of Muscles-Thorough-pin-Navicular Disease, or Lameness-Pleuro-dynia-Acute Rheumatisn-Chronic Rheumatism-Ring-bone-Splint-SpavinOsteo Porosis-Curvature of the Spine-Hoof-bound-Laminitis-Sand-crackQuarter Crack-Navicular Thritis-Stifle Out-Contraction of the Hoof-Bowed Legs-Corns-Lameness.

\section{SECTION XIII.-Horse Management, eto.}

$$
\text { (Page 387-415.) }
$$

Prolapsus Ani-Hemorrhoids, or Piles-Pricking and Nicking Horses' TailsDocking Horses-Anæmia-The Use and Abuse of the Curry-comb-Horses and Cattle should have a supply of Pure Water-Galled Back-The Heart-The quantity of Blood contained in a Horse's Body-Rapidity of the CirculationThe transfusion of Blood-Management of the Horses put in the Stable-Strangles. 


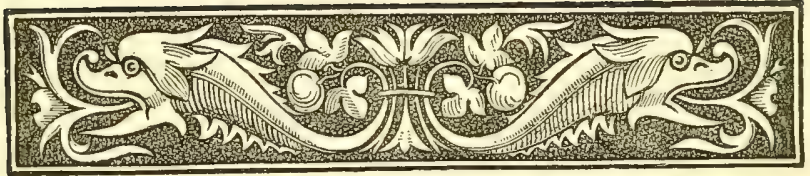

\section{CONTENTS OF BOVINE DEPARTMENT.}

\section{SECTION XIV.-The Rinderpest, or Contagiods Pleuro- Pneumonia.}

(Page 419-438.)

Origin of the Disease in Massachusetts-Spread of the Disease-The Cattle Plague in Kentucky.

\section{SECTION XV.-Diseases of the Organs of Respiration.}

$$
\text { (Page 439-456.) }
$$

Croup-Laryngitis-Bronchitis-Inflammation of the Lungs-Hoose or Common Catarrh-Epizoötic Catarrh-Consumption-Pharyngitis (Sore Throat)Pulmonary Apoplexy-Pleurisy, and Description of the Pleura.

\section{SECTION XVI.-Diseases of the Digestive Organs.}

$$
\text { (Page 457-491.) }
$$

Description of the Esophagus-Foreign Bodies in the Gullet-Esophagotomy-Laceration of the Esophagus-Aphthæ-Deseription of Stomach-Rumination-Bloat-Distension of the Rumen with Food-Gastro-intestinal Inflammation-Dentition of Cattle-Synopsis of Dentition-Suspended Rumination, or Loss of Cud-Inflammation of the Bowels-Invagination of Intestine-Diarrhea-Chronic Diarrhea-Gut Tic-Internal Rupture of Oxen-Flatulent ColicSpasm of the Bowels-Constipation of Bowels.

\section{SECTION XVII.-PARTURITION.}

(Page 492-502.)

Signs of Labor, or Parturition-Natural Labor-Unnatural Labor-Fore-legs Presenting-One Fore-leg Presenting--IIead Presenting without the Legs-Extraction of a Calf on its Back-Hind Legs Presenting-Breech PresentationCleansing, or Removal of the After-birth-Back of the Calf Presenting at the Brim of the Pelvis-Uterine Hemorrhage-Birth of Twins-Triplets. 


\section{SECTION XVIII.-Diseases of the Generative Organs.}

$$
\text { (Page 503-516.) }
$$

Constriction of the Neck of the Uterus-Embryotomy-Treatment of Cows during Pregnancy - Symptoms of Pregnancy-Dropsy of the Womb-Puerperal Fever-Falling of the Womb.

\section{SECTION XIX.-Diseases of the Udder, Teats, etc.}

(Page 517-528.)

Mammitis-Stricture in Cows' Teats-Obstruction at the end of the TeatsObstruction in the Teats-Tumors in the Teats-Injuries to the Teats-Sore Teats-Chapped Teats and Chafed Udder-Inversion of the Vagina-Laceration of the Vagina-Abortion-Inflammatory Affections of the Hind Limbs after Calving.

\section{SECTION XX.-Diseases of the Urinary Organs.}

$$
\text { (Page 529-539.) }
$$

Description of the Urinary System-The Ureters-The Bladder-The Urethra-Hæmaturia-Inflammation of the Kidneys-Urinary Calculi-Calculi in the Bladder-Black Water--Red Water.

\section{SECTION XXI.-The Heart-Its Function and Diseases.}

$$
\text { (Page 540-552.) }
$$

Description of the Heart-The Heart's Function-Diseases of the Heart-Dilatation of the Heart-Pericarditis-Hypertrophy-Endocarditis.

\section{SECtion XXII,-Diseases of the Ere and its Membranes.}

$$
\text { (Page 553-561.) }
$$

Description of the Eye-Humors of the Eye-Coats of the Eye-Muscles of the Eye and Eyelids-Ophthalmia-Foreign Bodies in the Eyes-Specific or Periodical Ophthalmia-Cancer in Cattle.

\section{SECTION XXIII.-HERNIA, OR RUPTURES.}

$$
\text { (Page 562-567.) }
$$

Forms of Rupture-Inguinal Hernea-Strangulated Hernia-Ventral Hernia.

\section{SECTION XXIV.-Diseases of the Bones.}

(Page 568-599.)

Cachexia Ossafraga-Bone Disorders-Mechenism and Structure of BonesComposition of Bones-Ultimate Constituents of Bones-Dilitation of the Jaw 
Bones-Abscess beneath the Periosteum, at the Angle of the Jaw-Exostosis, or Deposit of the Calcareous Matter on the Surface of Bones-Caries, or Ulceration of Bones-Preliminary to Horn-Ail-On Sympathy and the Sympathetic Relations which exist in the Animal Economy-Horn-Ail-Tail-Aịl.

\section{SECTION XXV.-Diseases of the Brain.}

(Page 600-610.)

Inflammation of the Brain and its Membranes-Sturdy in Cattle-Shaking Palsy-Hydrophobia.

\section{SECTION XXVI.-The Liver and its Diseases.}

$$
\text { (Page 611-619.) }
$$

Description of the Liver-Secretion of Bile; its Uses, etc.-Gall-bladder-Inflammation of the Liver-Hydatids-Jaundice, or Yellows.

\section{SECTION XXVII.-Diseases of the Skin. (Page 620-630.)}

Exanthemata, or Eruptive Fever-Mange-Foul in the Foot-Warts-Gadfies-Yoke Galls-The Hair of Cattle an Epidermic Appendage-Pemphygis or Vesicular Eruption-Cracks in the Heels of Cattle.

\section{SECTION XXVIII.-Varioůs Operations and Diseases.}

$$
\text { (Page 631-667.) }
$$

Castration-Enzoötic Milk Sickness, or Trembles-Improved Method of Milking-Diseased Thymus Gland--Cords in Young Calves-Steamed and Cooked Food for Stock-Cheap Fodder for Cows-Black Tongue-Fractured BonesFractured Radius of a Calf-Light in Barns-Diarrhea in Calves-Sprains or Strains-Spaying Cows.

\section{Section XXiX.-Remarks on the Cattle Plague.}

(Page 668-705.)

\section{SECTION XXX.-Diseases of Sheep.}

$$
\text { (Page 706-716.) }
$$

Improvement in Sheep-Rot, or Hydatids in the Liver-Yellows-Vertigo, or Giddiness-Jackets or Blankets for Sheep-Foot-rot-Grubs in the NostrilsTicks-Inflammation of the Eye-Inflammation of the Lungs-Common CatarrhDiarrhea and Dysentery-Constipation of the Bowels-Tympanites, or Windy Distension of the Intestines and Abdomen. 


\section{SECTION XXXI.-Diseases of Swine.}

\section{(Page 717-746.)}

Effects of Impure Air on Swine-Measles in Pork-Quinsy-Hernia, or Rupture-Intestinal Worms-Hog Cholera-Trichina Spiralis.

GuIDE to Diagnosis...................................................... 755

TABLE OF WEIGHTS......................................................... 768

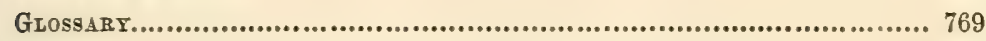

IVDEX....................................................................... 773 


\section{LIST OF ILLUSTRATIONS.}

\section{EQUINE DEPARTMENT.}

Skeleton of the horse.

A horse bent on mischief-the subject of phrenitis.

The test for tetanus or locked-jaw.....

Contents of the thorax.

Representation of the insertion of the tracheotomy tube.

Nasal polypus.

Appearance of the tumor of bronchocele.

Plan of a vapor bath

Main arteries on the inside of one of the fore legs.

The horse's age, as shown by the teeth, twelve illustrations......127-132

Section of a horse's head showing glanders

Section of a horse's head in a healthy condition.

Burning a horse's mouth for lampas.. 161

Estrus equi, (bots). 172

Estrus hemorrhoidalis.

Appearance of the intestines on opening the abdominal eavity. 202

Surgieal instruments used in veterinary practice.

238

Acarus, or itch insect......................... 274

Curbs and elbow tumor....................... 320

A foundered, or dead lame horse....... 324

View of some of the deep-seated muscles in the region of the hock and stifle 328

External muscles and tendons of the off hind leg.
Extremity of one of the fore legs...... 351 Section of a diseased spine................ 361

The patella or stifle bone diseased..... 363

Articulating surface of the lower end of the thigh bone........................... 365

Spavined in off hind leg-ring bones on all pasterns.............................. 371

A perfect fore 1 leg ................................ 379

Mode of operating for thoroughpin, splint and spavin......................... 379

Bottom of the hoof represented........ 379

Anatomy of one of the fore legs........ 386

\section{BOVINE DEPARTMENT}

Representation of the geneology of pleuro-pneumonia as it occurred in Massachusetts........................... 418

Section of a cow's stomach................. 459

Imported Dutch cow "Purmer"........ 474

Dentition of cattle, fourteen illustrations. $475-477$

Falling of the womb......................... 513

The heart, its circulation................... 541

Celebrated bull "Dutchman "........... 552

View of some of the superficial muscles ............................................. 567

Pelvis and ribs diseased and distorted 578 Specimen of an Alderney bull........... 610

Trichina spiralis and ova, as seen in the muscles of the human subject, magnified

Trichina spiralis in pork, magnified 737 Trichina in various stages of development 


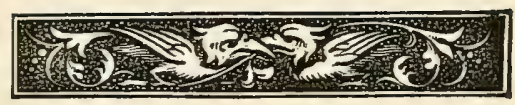

\section{EQUINE DEPARTMENT.}

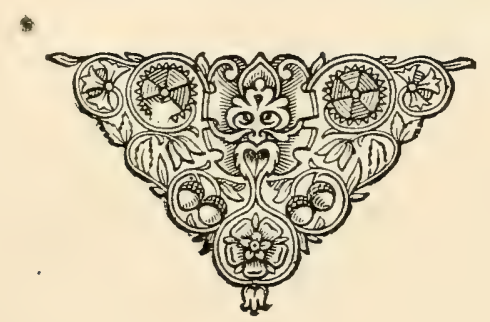

2 


\section{SKELETON OF THE HORSE.}

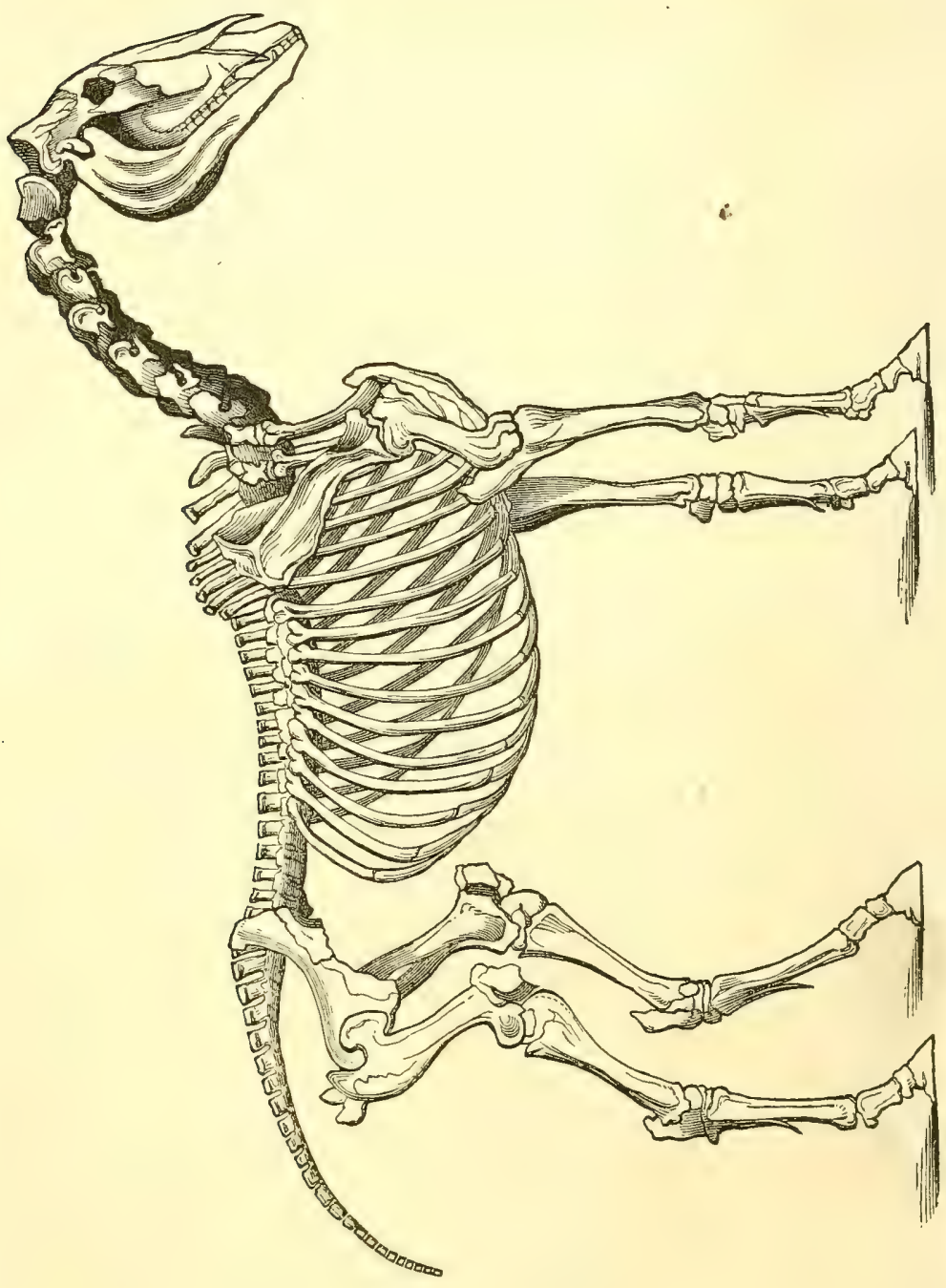

FROM A CELEBRATED ANATOMICAL MODEL. (Engraved expressly for this work.) 


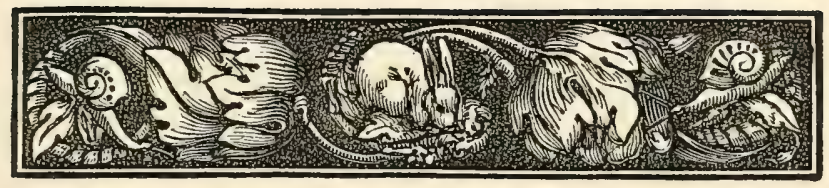

\title{
THEORY AND PRACTICE
}

OF

\section{VETERINARY MEDICINE AND SURGERY.}

\section{SECTION I.}

\author{
ON THE CAUSES OF DISEASE.
}

Remarks on Veterinary Science, and the Relation it bears to Soctat Science-Veterinary Scrence; how to Inadgurate it-What Benefits have We derived from its Study-Inflamation; its Nature and TreatMENT.

TTO explain the cause or causes of disease occurring among 1 domestic animals is an herculean undertaking, when expounded by the theory and faulty logic as applied to the ancient method of reasoning on "false facts ;" but, thanks to the genial spirit of progression and improvement, we are enabled to throw some light on the subject. We have been taught to believe that many of the causes of disease are invisible and mysterious; but this is not the fact. We are in possession of evidence going to show, very conclusively, that the causes of disease may be classified very distinctly and intelligently under two heads, viz.: PREDISPOSING and Exciting; yet this classification is not offered by the author as absolute, but is a more convenient arrangement than any other to study the multifarious conditions which people believe are remotely or immediately concerned in the production of disease. For example, many interesting and valuable facts are on record, going to show that the peculiar properties, characters, and qualities of the parent-whether good or bad, healthy or dis- 
eased, external or internal-are transmitted to the offspring; or, in common phraseology, are predisposing and hereditary.

"Among horses and cattle, we find, as in the human subject, ample illustration of the hereditary tendency of external form, disposition, habit, and disease. The parent transfers to its offspring size, shape, and general conformation similar to its own; and the aphorism, 'like produces like,' is as applicable to faulty and disproportioned as to beautiful and symmetrical form, to diseased and debilitated as to healthy and vigorous constitution, to gentle and tractable as to fiery and indomitable disposition. The size, weight, general appearance, expression of countenance, fleetness, and temper of the horse are all hereditary. Many illustrations might be given of particular families being remarkable, during several generations, for good or bad points, as for well or ill-formed head; for high and well-developed or for low and weak withers; for fine, strong, and well-turned, or for coarse, weak, and ill-formed limbs. Peculiarities of color often extend through many generations, and are so constant in their transmission as sometimes to form one of the distinctive characteristics of a race. Indeed, most breeds of horses have a prevailing color, to which there are few exceptions. The heavy horses of Lincolnshire, for example, are generally of black; the Cleveland, bay; and the wild horses of the plains of Eastern Siberia, dun. Particular markings, also-as white spots on various parts of the body, stars and blazes on the face, one or more white feet or legs-often continue for many generations, and are peculiar to certain families.

"There are some maladies in which it is comparatively easy to trace the connection between conformation and disease. In the horse, certain sorts of limbs notoriously predispose to certain diseases. Thus, bone spavins are most usually seen where there is a disproportion in the size of the limb above and below the hock; curbs, where the os calcis is small and the hock straight; strains of the tendons of the fore-leg, where the limb is round and the tendons and ligaments confined at the knee; and navicular disease, where the chest is narrow and the toes turned out. Among horses so formed, these diseases are unusually common, and are developed by causes which would be quite inadequate to produce them in animals of more perfect conformation. But it appears to us that internal and constitutional hereditary diseases also depend upon the altered conformation or texture of the parts specially 
affected, or of some disturbance of the relation which should subsist between the different constituents of these parts. This abnormal state of the internal parts is seldom within the limits of our means of observation or investigation; but its existing in animals having an hereditary predisposition to disease can not, we think, be doubted, as we shall now endeavor to show. The ground of our reasoning rests chiefly on the analogy which subsists, in all respects, between external and internal parts. The same law which regulates the hereditary transmission of form, texture, and relation of external and visible parts, also operates with equal force in regard to the form, texture, and relations betwixt the component parts of internal, and, it may be, inaccessible to ordinary powers of investigation. Then if, as we have shown, external hereditary diseases, such as lamenesses, are traceable to external hereditary peculiarities of conformation, we do not think it pushing our analogy too far in asserting that, in like manner, internal hereditary diseases must, in great part at least, depend upon some inherent hereditary peculiarity of the internal parts affected."

The common disease known as bone spavin is inherent, or dependent on predisposition, as related above; for the author never knew a horse to be the subject of this malady unless he had faulty hocks; or, in other words, in so far as the conformation of the parts were concerned, predisposition was evident. The same remarks apply to ringbone. Either the pasterns are too upright, thereby causing jar and concussion, or they slant unnaturally, and the consequence is, strain of the ligamentary structures, ending often in ringbone and other osseous diseases.

Ample evidence can be adduced to show that various diseases of the eye are hereditary. The tendency to ophthalmia shows itself in a peculiar conformation of the eye, and parts in the vicinity of it. Periodical ophthalmia is notoriously hereditary, and usually ends in cataract.

Colic, also, can be traced to inherent tendencies; for animals subject to flatulent colic are known to have a morbid appetite; they have, also, a large, roomy abdomen, which gives them an ungainly appearance. Yet it is true that diseases may occur accidentally, without the intervention of predisposition; but predisposition exists in almost all cases, and it only requires some anatomical and physiological knowledge on the part of husbandmen to detect it. 
F. Dus, a lecturer in the Edinburgh Veterinary College, tells us that diarrhea and colic are, to a certain extent, hereditary, "inasmuch as they are very prone to attack horses of particular form and constitution, as those with narrow loins, large flat sides, and of what is generally termed a washy appearance. If such animals be overworked, (especially soon after being fed,) if their food be suddenly changed, or if they be allowed an unusual quantity of fluid, they are almost certain to be attacked either by purging or colic. The tendency to these diseases appears, in such cases, to depend on a want of adjustment among the different organs of the body; a want of balance among the different functions of digestion, circulation, and respiration.

"Many farm-horses, as well as others without much breeding, are remarkable for consuming large quantities of food, for soft and flabby muscular systems, and for round limbs containing an unusual proportion of cellular tissue. These characters are notoriously hereditary, of which indubitable evidence is afforded by their existence in many different individuals of the same stock, and their long continuance, even under the best management and most efficient systems of breeding. Such characters indicate proclivity to certain. diseases, as swelled legs, weed, and grease. If horses of this description stand long, the circulation of the blood through the limbs is retarded; for, as the contraction of the muscles which materially aid circulation are wanting, the blood in the veins rises with difficulty against its own gravity, while the soft and lax condition of the venous coats, and of the muscles in contact with them, permits the passage of the fluid parts of the blood, giving rise to a serous effusion, which is soft, and pits on pressure. This anasarcous condition, although troublesome, and frequently recurring, is easily removed by friction, exercise, or a little physic, and does not unfit the animal for ordinary work.

"But the same conformation and constitution which induce simple swelled legs also give rise to the more serious affection known as weed, or a shot of grease. This consists in a disturbance of the balance which naturally subsists between the waste of the system and the supply of new material to repair that waste. Food is assimilated in larger quantity than the wants of the system require; the chyle so formed accumulates in the absorbent vessels and glands, which become, in consequence, irritated and inflamed. That part of the absorbent system situated in the hinder extremi- 
ties is usually the principal seat of the disease. The animal suddenly becomes lame; the inguinal and other glands in the groin become enlarged and very painful, and the swelling and pain gradually extend downward along the course of the absorbents, while the limb becomes a great deal larger than its natural size. There is, at the same time, a good deal of constitutional fever, with a full and bounding pulse. The swelling of the leg is, in the first instance, inflammatory, being hot and tender, and the skin, over the part affected, hard and tense. Such swellings may, by judicious treatment, be removed; but, in cases of a chronic character, or where the same limb has been previously affected, lymph is effused, forming hard and nodulous, and even diffuse swellings, which often cause lameness, by interfering with the motions of the joints or tendons. These indurated swellings must be carefully distinguished from the serous effusions above noticed, which, although giving the animal an unsightly appearance, do not materially impair his usefulness.

"Grease consists in a morbid condition of the sebaceous glands of the horse's heels and fetlocks. It occurs in various degrees of intensity; sometimes as a mere scurfy itchiness of the skin about the fetlocks, more commonly of the hind extremities; sometimes attended with much inflammation, causing great heat, pain, and swelling, and an ichorous fetid discharge; sometimesscausing falling off of the hair about the heels, and the formation of deep cracks and fissures; and sometimes becoming so violent and inveterate as to cause eversion of the sebaceous glands, formation of granulations, and secretion of pus, constituting the loathsome complaint termed the grapes. There are few diseases better deserving the epithet of hereditary than grease, and few in which the hereditary nature can be more easily discovered and traced. Almost every practitioner can bring to his recollection cases showing the tendency of this disease to descend from parent to offspring. A friend of mine, some years ago, purchased a valuable four-year old entire horse, adapted for agricultural purposes. When bought, he appeared perfectly sound, and his limbs were nearly black, well-formed, and fine; within a short time, however, they became thick and greasy. And, although the mares to which he was put were perfectly free from such faults, the progeny have shown, in every case where they can be traced, unmistakable evidence of their inheriting the greasy diathesis of their 
sire. They have all been found liable to swelled legs when they stand idle for a few days; most of them have been the subjects of repeated attacks of weed; all are affected, particularly in spring, with scurfiness of the skin of the hind extremities and excessive itchiness, and lose, at a very early age, their flatness and smoothness of limb. The faults occur, to a greater or less degree, in all the stock of this horse, by many different mares, and are distinctly traceable to the third generation. But, although grease is undoubtedly hereditary, and is, therefore, readily induced by comparatively simple causes, still it is frequently caused, and is always aggravated, by neglect of cleanliness; and of this there is ample evidence in the fact that it is most common in foul and badly-managed stables, and where no pains are taken to keep the horses' feet and legs elean and dry."

The scrofulous predisposition is very marked in certain breeds of horses; it occasions rickets, softening, deformity, and various forms of disease in the bones, as, for example, big head, big jaw, etc. The same author, just quoted, says in reference to scrofula:

"From their weak and unsound constitution, horses of a scrofulous diathesis are unusually prone to glanders and farcy-two forms of a disease peculiar (at least as an original disease) to the equine species. As has been already remarked, it is characterized by a specific unhealthy inflammation, identical in all important characteristics with the syphilitic inflammation in man. From the dire and loathsome nature of glanders, and the terror in which it is held, animals affected by it are never used for breeding, so that we have little opportunity of judging of its hereditary nature. There is no evidence (so far as I know) which proves it to be directly hereditary, $*$ but there is no doubt that the progeny of a glanderous horse would exhibit an unusually strong tendency to the disease. Its ordinary predisposing causes are, many of them, hereditary; it is very prone to attack animals of a weak or vitiated constitution. It is emphatically the disease which cuts off all horses that have had their vital energies reduced below the healthy standard, either by inherent or acquired causes. Glan-

* "Though I am not aware of any facts proving glanders to be congenital, yet I think there is every probability that such is the case; for it is notorious that syphilis, the analagous disease in the human subject, is congenital, and often appears at birth in the children of women affected by that disease." 
ders is, also, sometimes caused by inoculation ; is frequently produced in healthy subjects by mismanagement, as by insufficient food, want of shelter, and overwork; and often supervenes on bad attacks of influenza, strangles, diabetes, and other diseases which debilitate the system, or impair the integrity of any of its more important parts. These causes appear to possess the power of engendering in the constitution of a horse a peculiar poison, .which, as it reproduces itself, and spreads to all parts of the body, gives rise to the characteristic symptoms of glanders, causing, sooner or later, a breaking up of the system, and a fatal prostration of the vital powers. This poison produces in the blood abnormal changes, which vitiate that fluid, and unfit it for healthy nutrition.* From the irritant action of the morbid fluids passing through them, the lymphatic glands and vessels become inflamed, and lymph is deposited. This, however, being of an unhealthy nature, soon runs on to softening, which extends to the skin overlying the part, and ulcerating farcy-buds are formed. On the surface of the more vascular mucous membranes effusions of tubercular matter are also poured out; these take on an unhealthy inflammation, and degenerate into chancrous ulcers, which may generally be seen on the mucous membrane of the nostrils in most bad cases of glanders.

"These are the most common scrofulous diseases of horses; but an animal of the scrofulous diathesis, besides being specially subject to these, is little able to withstand ordinary morbific causes, and hence is also unusually liable to many ordinary diseases; in such a subject, too, disease is very apt to be severe and complicated, and to be acted on tardily and imperfectly by all remedies."

Having now furnished the reader with a few valuable facts bearing on hereditary predisposition, we shall briefly discourse

* "A comparison of the two subjoined analyses will show the great difference in composition between the blood of healthy and of glanderous horses-a difference consisting chiefly in a diminution of the red corpuscules, and a proportional increase of the fibrine and albumen.

Blood of Healthy Horse.

\begin{tabular}{|c|c|c|c|}
\hline \multirow{6}{*}{$\begin{array}{l}\text { Water............................. } \\
\text { Fibrine ............................ } \\
\text { Blood corpuscules......... } \\
\text { Fat .................................. } \\
\text { Albumen ...................... } \\
\text { Soluble salts................ }\end{array}$} & of Healthy Horse. & Blood of Gla & $\begin{array}{c}\text { nderou } \\
\text { B. }\end{array}$ \\
\hline & $804.75 \ldots \ldots \ldots . . . . . . . . . . . . . .$. & 842. & \\
\hline & $2.41 \ldots \ldots \ldots \ldots \ldots \ldots$ & $6.60 \ldots . . . . . . . . . . . . . . .$. & 8.7 \\
\hline & $117.13 \ldots \ldots \ldots \ldots . . . . . . . . . . . .$. & $68.20 \ldots \ldots \ldots \ldots \ldots \ldots . . . . . . . . . . .$. & 44.20 \\
\hline & $\left.\begin{array}{r}1.13 \\
67.85\end{array}\right\} \ldots .$. & $76.80 \ldots . . . . . . . . . . . . . . . .$. & 82.27 \\
\hline & $6.82 \ldots \ldots \ldots \ldots \ldots \ldots \ldots$ & $6.50 \ldots \ldots \ldots \ldots \ldots \ldots \ldots$ & 5.38 \\
\hline
\end{tabular}


in reference to some of the exciting causes which in general produce those diseases to which the horse, by virtue of his constitution, is liable. The following quotation, brief and pointed, will be found pertinent:

"By far the easiest and best mode of curing diseases of animals is to kcep the diseases and the cattle separate. With cattle, as with ourselves, disease is caused, in most instances, by an excess of carefulness or of carelessness. Too much and too rich food is as injurious as too little and too poor. Perhaps fewer diseases, in proportion to the numbers treated in each way, spring from privation than from surfeit. Too little as well as too great exposure to extremes of heat and cold has a tendency to create disease; in the one case it causes disease, in the other it renders man or animal more liable to it. Let a wealthy and a warmhearted man own and take charge of a favorite animal, and the chances are that it will be 'killed with kindness;' if it is tended, in part, by a hireling, the equilibrium may be restored. In our experience-no very limited one, by the way-care is more needed among cattle than cures; and all the diseases which came under our treatment sprang from the want of judicious care. Has a cow a cough, has she the hoven, has she the red-water, or the puerperal fever? it is from overcare-too much warmth or too much feed. Is she mangy, lousy, hide-bound, or affected with horn-ail? the chances are that she has been neglected. Of course there are very many exceptions to this and to every other general rule. But this is our experience. Let few farmers, however, feed their animals less than they now do, or give them more airy lodgings than they now do, because the greater number of the cattle in this country suffer from privation and exposure to the inclemencies of the weather rather than from pampering. But animals, as well as men, will, with the best care we can bestow on them, become sick at times. Our judgment is often at fault when our intentions are the best. Sometimes our back is turned, or our eyes are not opened sufficiently wide. At all events, our stock will become sick, some of them; and recourse must be had to medical remedies."

It is usual to classify causes; yet, for all practical purposes, it is only necessary for us to know that exciting causes are those from which disease most directly springs. For example, suppose that in the act of shoeing a horse a nail is driven in a wrong direction, 
enters the sensitive tissues of the foot, and lameness occurs; the nail, in this event, may be classed as a special or mechanical exciting cause; and, under the same head, we class poisons and injuries of all sorts.

When horses become the subjects of glanders, or farcy, by being exposed to the maladies, they are then said to have taken it either by infection or contagion, and the cause is said to be specific. The terms infection and contagion are now used as synonymous, and, undoubtedly, if reference be had to the etymology of the words, the arrangement is correct. Coming now to plain matter of fact, the exciting cause of disease is that which, when taken into the body, or applied outwardly, is followed by disease or derangement.

The exciting causes of disease may, therefore, be summed up thus: Debility, induced by bad management, exposure, unwholesome food; plethora, brought on by over-feeding and want of exercise; overdosing animals with improper medicines and poisons; overtaxing the muscular powers of the animal, and the breathing of an impure atmosphere.

A great proportion of the diseases occurring among horses and cattle in the Western States is due to the fact that they are not properly housed at night, and the food that they often gather in their exposed situations lacks the nitrogenous and nutritious matter for the manufacture of good, healthy blood and strong muscles.

Disease sometimes, however, occurs when no exciting nor any other cause appears; yet, with the exception of those maladies supposed to be produced by miasmatic influences, it is probăble that the cause, in such cases, has not been discovered. Finally, it may be said that the causes of disease among animals are pithin their bodies as well as around and about them.

\section{Veterinary Science, and the relation it bears to Social Science.-} The necessity which exists for the services of educated Veterinary Surgeons throughout the United States, and in cavalry regiments of the United States army, is evident to every thinking man; yet, prior to the advent of our late national troubles, it was found almost impossible to interest the right kind of men, so that they might use their influence for the purpose of directing the attention of the General Government to the value and 
importance of veterinary science; but the probability is that Congress will soon legislate on the subject, and before long we may expect to hear of the appointment of a Veterinary Surgeon and Assistant Surgeon to each cavalry regiment.

There exists no earthly reason why our profession should not rank as high in America as in England and France. The great losses which are continually occuring among army horses is a monstrous and growing evil, and, in view of preventing unnecessary diseases and premature deaths, the Government must organize a veterinary corps.

It is very important that our science should attract the notice of legislators; for it bears a very intimate relation with social science, inasmuch as men and animals are subject to similiar diseases, which are communicable one to the other, and which often arise spontaneously, and are transmitted from first to second. For example, there is a disease which often occurs among cattle, known as "Anthrax." It is a malignant disease, and many persons have lost their lives by absorbing the virulent "anthrax poisons" from the carcasses of dead animals.

A cutaneous disease often occurs among the bovine species, known as "ring-worm," which affects the hands and arms of some persons who attend such animals.

It is asserted, also, that the vesicular murrain, which at times prevails among cows, gives rise to the development of a virus which is often squeezed into the milk-pail, and produces very bad effects on children when fed on the same.

It has been discovered that the little vesicles found in measled pork are tape-worms, in a certain stage of development, and if not destroyed by thoroughly cooking the infected meat, may develop the dangerous parasite in the human intestines.

That awful disease known as virulent "glanders" (spontaneous in some horses) has destroyed many of our race. A small portion of the glandered matter coming in contact with an abraded or absorbing spot on any part of a man's body, will surely cause him to die the most horrible of all deaths; and the same remarks apply to the disease known as " malignant farcy."

We might enumerate many other forms of disease which are communicable from the superior orders of animality to the inferior, and vice versî, but the above must suffice, in view of attracting attention to the value and importance of the subject. 
In regard to the origin of diseases, it is known to the profession that the diseases of men and animals are often due to similar causes; that the evils of domestication, which operate to develop disease in animals, are as notorious as the evils of civilization, which induces unnecessary diseases and premature death among members of the human family; therfore, our science does bear an intimate relation to social science, and it appears to us that no scientific mind can fail to appreciate the advantages to be derived from a more extended knowledge of the fundamental principles of veterinary science.

Let the husbandmen and stock-raisers of this country put their shoulders to the wheel, in view of establishing veterinary schools and colleges throughout the length and breadth of our immense agricultural domain, and soon we can boast of having a class of veterinary surgeons, educated on American soil, conversant with the diseases incidental to the live stock of America. In that event, we can do our own doctoring, without foreign interference.

We are not disposed to find fault, nor scold about the itinerant veterinary practice which prevails throughout the United States, for we are aware that all try to do the best they can for the relief of the inferior orders of ereation. The fault is not with the practitioners, but with the people, who have failed to furnish the means of education.

Resume.-Since writing the above, the "Trichina" disease has made its appearance in Berlin, creating a terrible consternation among the consumers of pork. The disease in its symptoms somewhat resembles cholera, but on making post-mortem examinations, there was soon discovered in the flesh of some persons an immense number of small microscopic parasites; these were traced back to the flesh of the hog, where they were discovered in the form of chrysali imbedded in a little shell of lime; these, on being subjected to a slight degree of heat, speedily brought forth myriads of small parasites, of various forms and sizes, a dozen of which were frequently found in a piece of tissue as large as a pin's head. The process of incubation was discovered to have been produced in the intestines of those who had eaten the pork, by the animal warmth of the stomach. Thus freed from their shells, the creatures make their way through the walls of the intestines in every direction, choosing the hard and fleshy tissues especially, and none of them have yet been found in the blood. Most remarkable to 
relate, the "Trichina" is said to have come from the "fieldmouse," which the hog, by eating, introduces into his system, from whence it is introduced into the system of man. A few years ago, a number of persons in London were very seriously affected with vomiting and dysentery after partaking of sausages, the meat of which was found to be in a putrid state. Liebig, the great chemist, informs us that "several hundred cases are known in which death has occurred from the use of bad sausages in a state of putrefaction." This kind of testimony might be multiplied to an indefinite length, but what has been written must suffice. The reader can not fail to perceive that, in the study of comparative anatomy and physiology, a wide field for investigation and research is before us, which will amply repay the devoted student, and be the means of accomplishing much good.

Veterinary Science-How to Inaugurate it-What Benefits have we derived from its Study? - Shortly after the commencement of our late national struggle, the author was consulted in reference to the most feasible plan of inaugurating Veterinary Science, so that it might be made available for the restoration of sick, lame, and otherwise disabled horses in the Government service, which drew forth the following reply: Up to the present period there exists no law authorizing the employment of regularly-educated veterinary surgeons; therefore any rational plan calculated to ameliorate the condition of army horses, or to prevent the many unnecessary cases of disease and premature deaths which are now constantly occurring, or to guard against the great pecuniary losses which the Government and people of this country have been compelled to submit to in the condemnation and forced sales of deteriorated horses, can not, as I understand the matter, be considered by the powers that be until Congress shall legislate upon the subject. I now propose to inform the reader how to render the knowledge we possess available.

In the first place, I should follow the systems of instruction and the organizations of the veterinary schools of London, Edinburgh, Alfort, and Saumur, and prepare suitable text-books adapted to the wants of a nation of people who have never given the subject that attention which its importance demands.

In the selection of pupils, I favor the plan of General MeClellan, which is as follows: "The pupils for the veterinary school might 
be selected from among the best recruits; indeed, it is not improbable that the advantages of such an institution would induce excellent men to enlist for the purpose of availing themselves of its benefits. Should such be found to be the results, it would be well to require them to enlist for longer than the usual time, as a compensation for the time spent at school." Cavalry officers, farriers, or blacksmiths should be permitted to attend lectures on anatomy, physiology, and the obvious diseases and lameness of horses.

In cases of emergency, a competent veterinarian should be employed in each regiment, whose duty it should be to select an intelligent farrier from each company, and instruct such person, in a brief and practical manner, on the management of cavalry horses in camps, the prevention of diseases, and the most rational method of treating diseases incidental to camp life.

Should it be found impossible to secure the services of a sufficient number of competent veterinarians for the above purpose, let those who have shown themselves qualifier, by an examination before a medical board, march from camp to camp, give instructions, see that the sick and disabled are separated from healthy animals, and that the former be placed in a covered hospital, and then issue special regulations for the use of mounted troops in garrison and in the field, for the purpose of guarding against the consequences of ignorance and abuse of the animal machine.

It would be advisable to establish a veterinary professorship at West Point, and there locate the National School. The Government has there a very fine French model of the horse, a skeleton, and various preparations of morbid anatomy, etc., which, together with other material available, will furnish all that is necessary for the purposes of veterinary tuition. It would also be advisable for the Chief Veterinary Surgeon to issue to his subordinates such orders as shall secure proper sanitary regulations in camp. The horses should be as well cared for as the troops, for in cavalry and artillery service, the success of a campaign, or attack and defense, depends much on the health and efficiency of horses. It should be the business of some members of the veterinary corps to station themselves at accessible points, and there erect temporary hospitals for the reception of sick and lame horses, where the latter shall receive the benefits of a rational system of medication and nursing, whereby, in their restoration to useful- 
ness, many millions of dollars might be saved to the Government in a short time. I contend that the condemnation and ruinous sale of sick and lame horses is a wanton waste of property, and it would be just as rational, if rational at all, to sacrifice, neglect, or abandon sick or disabled soldiers simply because they are not in fit condition for present duty.

Importance of Veterinary Education.-The importance of educating men for the scientific practice of Veterinary Medicine and Surgery is self-evident, and is further illustrated by the daily experience of those persons whom, as a matter of necessity, have been obliged to prescribe for the sick and dying of their flocks and herds, at the same time, having no knowledge of those advantages which a thorough course of training affords, must necessarily feel that they are groping in comparative darkness, and when a ray of medical light does flit across their path, it often serves to make them better acquainted with their own want of skill. Persons thus circumstanced, unable to procure the services of veter1nary surgeons, in consequence of a scarcity of the "genuine material," are often called upon to assume the responsibilities of medical practitioners; and they, no doubt, prescribe with good intention and honesty of purpose, but disease and death have the vantage of them, for the disease is not often rightly diagnosed, nor are they acquainted with the means of securing a favorable issue; and they will hail, as a great blessing, every attempt made for the purpose of diffusing a knowledge of Veterinary Science.

We all know that there is greater need for scientific qualification on the part of those who propose to practice on brutes than in the case of a practitioner of human medicine; and if a special course of study and qualification, obtained under the guidance of competent teachers at college or elsewhere, be essentially necessary in the one case, surely they can not be dispensed with in the other. Now, it is well known that prior to the introduction of a rational system of veterinary medicine in the mother country, millions of domestic animals were annually sacrificed at the shrine of ignorance; but science came to the rescue, and now the disciples of St. Bex, Coleman, their co-workers (the founders of the science in England), and kindred spirits, can, by aid of their vast materia medica-their anesthetic agents, their scalpel, and other appliances-accomplish unheard of wonders. 
The Samaritans of our craft have solved the problems on which the fundamental principles of physiology and pathology are founded; they have, to a certain extent, dispelled the awful cloud of ignorance and empiricism which had reigned in the British Isles. They have succeeded in illuminating the dense and dark spots that have existed in horse and cattle practice since the advent of the ancient Egyptians, up to that period which introduced a new era in our art. They have done more than this: our craftsmen have often robbed death of some of its victims, and have succeeded in blunting the keen edge of many an epizoötic sword; and thus they have succeded in stealing a march on the grim monarch of epizoötic destiny. Their weapons were often such as were wielded by JENNER, the benefactor of our race. Our efforts may not always be successful, but as our veterinary fathers have accomplished much, we ought not to despair.

The field of enterprise in the United States is composed of virgin soil, and the "bridegroom of promise" hath not yet put on the complete mantle of fertility; so that there is a splendid chance for the teachers and graduates of American schools to distinguish themselves and earn the laurel of fame.

The husbandmen of this country have also suffered immense losses by the death of valuable animals, and since the landing of the Pilgrims on Plymouth Rock, up to this very moment, death hath run riot in the camping-ground of horses and cattle, and people have scarcely been informed of the "why or wherefore." The legion host of "itinerant" practitioners which necessity and circumstances have created, remain remarkably silent and noncommittal; and probably this is the best course they can pursue, for, if "ignorance is bliss," and that pays well, it would be decidedly unbusiness-like in them to assume wisdom which might not pay.

There never was a period in the history of this country when the services of educated veterinary surgeons were so much needed as at the present time. The live stock of "Young America" do not enjoy immunity from those pestiferous epizoötic maladies which have in former years operated as a "withering simoom" on British husbandry. For example, a dreadful malady known as pleuro-pneumonia has made sad havoc among live stock in Massachusetts, and the terrible alarm there ereated in consequence, is keenly felt, not only in that State, but in many others. In some 
of the Western States, a malady known as "milk-sickness," or trembles, afflicting both man and beast, sometimes prevails, attended with such disastrous results that many farmers have often been compelled to abandon the old homestead in view of seeking a location where there was some prospect of enjoying immunity from the pest.

Hog cholera, or pleuro-pneumonia, (among swine) has often slain its millions and tens of millions. Contagious typhus prevails, and puerperal fever has carried off hundreds of thousands of our best stock. Apoplexy, softening of the brain, dropsy of the brain, are just as prevalent here as in England. Abortion, or miscarriage, is fearfully on the increase. Diseases of climate and spontaneous origin are constantly occurring. In fact, to shorten a lengthy story, there is scarcely any disease known to the veterinarians of the Old World that has not prevailed in the New. The mere mention of these matters should at once arouse stock-owners to a sense of duty to themselves and their domestic :animals.

We must have educated veterinary surgeons for the treatment of diseases peculiar to the live stock of this country. It is a matter of national policy, a work of benevolence; and should we take a business view of the affair, it is a cent and dollar enterprise that will surely pay.

We want schools and colleges in this country, where the young men of America can acquire a republican veterinary education, so that in the era before us, the people in this country can employ the graduates of their own schools, endowed and fostered by their own enterprise and industry. Some persons have an idea that veterinary science can only be acquired under the banner of royalty, but this is a great mistake. I am aware that the people of this country have been so absorbed in promoting its growth and developing its resources, that there has been but little chance for great scientific achievement in our calling, yet within the past few years some of the most learned of our profession, educated under the auspices of royalty and aristocracy, have been amazed at the contributions to science furnished by a few devoted men in this country that never saw the inside of an European college.

As a national affair, therefore, it should be the pride of every American citizen to put his shoulder to the wheel for the purpose just indicated, for the enterprise will surely redound to the honor 
and glory of the nation. The science which we are laboring to render popular is vast and boundless as the ocean ; and although it has been explored by many distinguished scholars, it is capable of unfolding new truths, and when the united intellect of intelligent Americans shall be brought to bear upon it, will advance with a rapidity truly astonishing. What the American people have accomplished, during the past few years, in rendering other sciences popular, is proof positive of what shall occur when the current of investigation is directed to the science of veterinary medicine and physiology.

In the absence of educated veterinary surgeons throughout the country, it is of vast importance that stock-owners generally should study, as far as they can, the diseases to which their property is subject, and acquire a knowledge of, at least, the ordinary remedies. By doing so, thousands of dollars' worth of valuable horses, cattle, and swine may be annually saved to the country, and the intense misery which these poor brutes suffer frequently be relieved. It is our object in this work to simplify the science so that it may be acquired, to a very useful extent, by any intelligent man. If we shall succeed in enabling stock-owners to practice the healing art on their dumb but faithful companions, we shall feel that we have done a good work.

Inflammation; Its Nature and Treatment.-Inflammation is said to consist of pain, heat, and redness. It is usually considered as an enemy to the patient; and we have been taught to subdue it by means of bleeding and purging, at all hazards; and some practitioners, when they undertake the job, generally succeed, yet they lose the patient for their pains.

Some writers have made inflammation cut up all manner of pranks. It is now sanative in healing a wound or injury, in repairing damages which occur in various parts of the animal economy; and in the same paragraph the writers declare that inflammation is the cause of nearly all the deaths that occur. This is sheer nonsense, and will not stand the test of logic, nor the daily experience of that practitioner who deals in fact, and does his own thinking, independently of the dictum of some of the schools, and the ancient method of reasoning on false facts. A distinguished medical reformer, writing on this subject, says: "Numerous hypotheses or opinions respecting the true nature of 
inflammation have for ages been advanced, and, for a time, sustained; but even at the present day the various doctrines appear to be considered altogether problematical."

We often hear, in common parlance, that, "if inflammation sets in, the horse will die." Now, the very reverse of this is the case ; for, while inflammation lasts, there is strength, vitality, and angmented action, though there may be much pain. This should be interpreted as the warning voice of Nature, to apprise us of a loss of equilibrium in the circulation of the blood, or some deranged condition of the system; and we should heed the warning, and array ourselves on the side of Nature, in view of promoting a fuli and free circulation of the blood over the system, so that there shall be no excess anywhere; and the derangement, wherever it exists, must next command our attention.

While inflammation and pain exists, there is hope. Take, for example, inflammation of the intestines. While that stage lasts, the animal is not in danger; but so soon as inflammation and pain ceases, (often accomplished through the means of bleeding and narcotism, we then open up a new page of pathology. In the course of a few hours the animal dies. Of what-inflammation? No. He dies of mortification, or gangrene of the bowels. Therefore death was not caused by inflammation.

So in regard to phrenitis (inflammation of the brain). A certain amount of pain and activity in the parts are observable; the inflammation may now be attacked after the true Sangrado fashion ; the inflammation is subdued, but still the nimal is no better. He dies. Of what does he die? Not of inflammation, for the autopsy reveals, very often, softening of the brain, and fluid is found within the lateral ventricles. Softening of the brain is equivalent to gangrene (death of a part). Then, agaiu, horses can not be said to die of inflammation when the practitioner declares that he heroically subdued it some time previous to the death of the animal.

There is no proof that bleeding has any directly beneficial influence over the course of inflammations, either external or internal. Surgeons never bleed now in external inflammation; and educated and liberal physicians have given up all arguments in favor of the practice for internal inflammation. Good, in his "Study of Medicine," scems to have given us some definite information regarding the effect of blood-letting in inflammation, 
which should agree with the sentiments of all men desiring to see veterinary science joined in the march of improvement which is now progressing in almost every other department of science and industry. "The immediate effect of profuse and repeated bleeding is exhaustion. While this exhaustion continues there is a diminution of action of every kind, and hence an imposing appearance of relief to the symptoms of disease; but it no sooner takes place than an instinctive effort is made by the vis medicatrix natura to remedy the evil hereby produced, and to restore the system to its former balance of power. This balance is called a rallying, or reaction of the living principle. The arteries contract to adapt themselves to the measure of blood that remains; the sensorial organ is roused to the secretion of a large proportion of nervous power to supply the inordinate drain that takes place. During the general commotion, all is in a state of temporary hurry and urgency, and, for the most part, irregularity of action, while the instinctive effort is proceeding. And hence, no sooner is the immediate effect of prostration and exhaustion overcome than the heart palpitates, the pulse beats forcibly with a jerking bound, the head throbs, and the eyes flash fire. Now, it often happens that these concurrent signs are mistaken for proofs of latent or increased vigor, instead of being proofs of accelerated action; and action, too, that adds as largely to the exhaustion as the depletion that produced it; and the unhappy patient is bled a second, a third, and even a fourth time, * till no reaction follows, at which time it is strangely supposed that the plethora or inflammatory diatheses is subdued and lulled into a calm, because the patient has been so far and fatally drained of the living principle that there is no longer any rallying or reactive power remaining, and gives up the ghost, in a few hours, to the treatment instead of the disease." This is the case with thousands and tens of thousands of valuable animals that annually die in this country, in

* My attention was recently called to a valuable stallion, which had lately been brought from Nova Scotia. He was attacked with what the attending would-be physician called "founder," but which was a pure case of acute rheumatism. In three different bleedings, forty-two pounds of blood had been abstracted! and I found the patient so weak and prostrated that he was scarcely able to stand. It seems to me that every man having the least spark of charity for so noble an animal as a horse, should discountenance such outrageous treatment as this. 
consequence of our misguided notions of inflammation, and uur want of knowledge of a suitable remedy to treat it. But a brighter day is dawning, and the antiquated notions of disease and its treatment are fast giving way to a more rational and successful system; and the day is not far distant when blood-letting for the cure of inflammation will be entirely abandoned.

It is very gratifying to the author to be able to record that many of the progressive medical writers of the present day are on the right side of Nature, teaching us that "she is ever busy, by the silent operation of her own forces, in the cure of disease;" and they are inaugurating a very great and desirable revolution in the theory and practice of human as well as veterinary medicine.

Nature of Inflammation.-The physical characteristics of inflammation are, as I have just written, redness, heat, pain, and sometimes swelling. It is, and always was physiologically, operating for the good of humanity and the inferior orders of creation. Its curative power none can dispute. We see it, in the form of a blush, on the cheek of offended humanity. Friction, injuries, poisons and disease, etc., excite Nature to hoist the symbol of distress-inflammation. She calls loudly for help, but she does not always get it; and instead of acknowledging her autocracy, and furnishing what she wants to use in her own way, viz.: the water, oil, and wine of the ancient Samaritans, we offer fire, knife, and poison.

Inflammation being an exalted condition of local arterial circulation, it can only be excited by some mental emotion, injury, loss of function, or by what is known as disease, in parts adjacent or remote from its seat. Hence, all diseases of an acute character are preceded and accompanied, to a certain stage or period, by inflammation. Hence, also, according to ancient usage and the dictum of alma mater, we are constrained to talk and write as though inflammation was the great evil or disease which required our services; and thus we coquette with Nature by means of sharp-edged tools, while the actual disease steals a march on us, and we lose the patient in consequence of our want of knowledge.

Treatment of Inflammation.-Inflammation being more or less active, according to the intensity of the disease of which it is a 
forerunner, or rather an indication, and being accompanied by pain, it becomes our duty merely to restore the equilibrium of the circulation. This removes the inflammation, and promotes a free and full circulation of the blood all over the system, so that there shall be no excess (inflammation) anywhere. This is what we call subduing the inflammation, with this difference, however, that we resort to natural means and sanative medication instead of using the fleam and eathartics. The means are warmth, moisture, stimulants, and friction; and clothing to the extremities and to parts of the body that are cold; and cold applications to parts that are unnaturally hot. One of the best equalizers of the circulation is the "Roman bath," the vapor bath, and wet packing. Should the inflammation exist internally, then give plenty of warm water, emolient drinks, and emolient enemas, and keep up a physiological action and heat on the surface of the body by means of friction and warm clothing. In this simple way the circulation of the blood is equalized, or, in other words, the inflammation is "cured;" and, at the same time, this treatment may cure the disease as well as the inflammation.

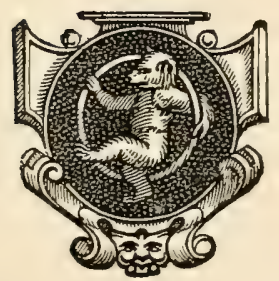




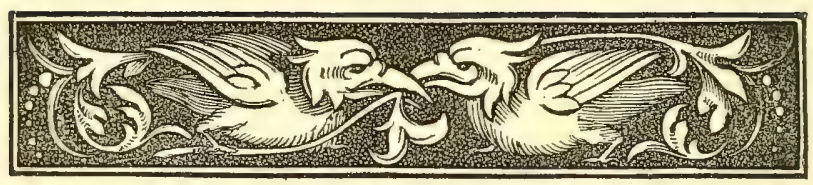

\title{
SECTION II.
}

\author{
DISEASES OF THE EYE.
}

Amaurosis, or Glass Ere (Gutta Serena)-Foreign Bodies within the Eyelids-Specks, or Film on the Eye, known as Opacity of the CorneaCataracr-Worm in the Eye-Ophthalmia-Treatment of InflammationPurulent Ophthalmia-Specific Ophthalmia.

\section{Amaurosis ("Grass Eye"-Gutta Serena.)}

MAUROSIS is known to most men as "glass eye." Physi1 cians, however, have named the disease gutta serena. The abnormal condition, which consists in dilatation of the pupil, uninfluenced by light or darkness, is occasioned by paralysis of the optic nerve and its ultimate expansion.

Causes.-Some horses, of an excitable, nervous temperament, often become the subjects of dilated pupil, without any assignable cause; while that form of amaurosis occurring among plethoric subjects, or those whose digestive organs are deranged or occupied by a large quantity of undigested food, (they, the subjects bordering on that state known as stomach staggers,) can easily be explained on the well-known law of sympathy.

We may, however, with propriety, assign a cause for its occurrence in the eyes of nervous, excitable horses; for this very condition of the nervous system, which gives rise to the excitability, perhaps goes to show that the brain is actually diseased, either in function or structure. Animals subject to this affection are gencrally of a "bony," spare, muscular development, and have wiry sinews, thin tapering ears, delicate lips and nostrils, diminution in the quantity of the hair in the mane and tail, but remarkable for compactness of texture. We generally find, under ordinary cireumstances, the black color preponderates in nervous horses over the various shades of equine coloring-matter found in the 
hair, eyes, etc. I had a case of this kind, and the owner s statement was as follows: The animal (mare) had been in his possession about five years. She had four successive attacks of blindness, which had never been treated by a certified surgeon, and it was concluded that she must have a periodical attack in spite of all efforts to prevent it. Now, this is what we call a case of idiopathic periodical amaurosis, and if it does not owe its origin to some hereditary idiosyncracy, it is the result of an abnormal condition of the brain.

When our attention shall be called to a case of this character, we must bear in mind the fact that this animal survived four periodical attacks of the same malady, and performed the ordinary duties of a horse, in the intervals of health, with far-seeing eyes, and without the aid of science; and this goes to show that this condition of the nervous structure of the eyes of some horses, as well as many other diseases incidental to them, are often "selflimited," and the subjects will recover, if carefully nursed and left unembarrassed by "poisonous drugs." This opinion is indorsed by some of the most distinguished surgeons of the present day. I have no desire to try to saddle the reader with any peculiar notions of medication emanating from my own fancy or brains. I am not offering sensible people a mirror of my own reflection, but I wish to present the truth in that mirror, so that they may see it and judge for themselves. Excessive medication is a quicksand which we must try to avoid. Thousands of wellmeaning medical aspirants have actually committed professional suicide by clinging to the absurd theory of medication as practiced in bygone days. The lancet, and many of the heroic medicines which our predecessors placed great confidence in, and in their writings have urged us to use, are now almost discarded. It is not because an essential change has taken place in the nature of equine maladies, but because we have greater faith in Nature as our curative agent, and have become better acquainted with anatomy, pathology, and physiology.

But to resume the subject of amaurosis. If from the history of the case, we have reason to believe that it is of a periodical character, and if it be the sequel to, or an accompaniment of, some hereditary affection, we may be sure that the malady will exist for a certain length of time in spite of all we may do. In such cases, all that is necessary is to have the animal comfortably 
housed, fed on a light, laxative diet, and pay careful attention to the condition of the secretory and excretory functions of the body.

That form of amaurosis occasioned by a deranged condition of the digestive organs, plethora, or over-distension of the same, will cease so soon as the cause is removed, and may be considered as a sympathetic affection.

What that cause is we must endeavor to decide on by careful examination, and then frame our plan of treatment in accordance with the indications to be fulfilled.

Should we consult authorities on amaurosis, we shall find that much diversity of opinion exists. YoUATT, who is quoted in this country by some persons as high authority, has mistaken the disease, or else has strangely distorted facts. He contends that "the treatment of amaurosis is quite as difficult as that of cataract." Now, every one knows that cataract occurring among horses is notoriously incurable; and Percrvall writes, "A man must be able to perform miracles ere he cures cataract."

Now, I contend that amaurosis, or dilatation of the pupil, is not of itself a disease, but the symptom of one; hence, between cataract-a disease resulting from altered structure of the eye-and amaurosis there exists no analogy. Whenever altered structure exists in the mechanism of the eye, then we have an incurable case.

Mr. Spooner, the reviser of YouatT's work on the horse, assures us that amaurosis is often connected with diseased liver, thus controverting YouATT's theory of incurability; for animals often recover from very severe attacks of liver disease.

I have called the attention of our readers to the opinions of Youatt, Percivald, and Spooner merely to show the importance of exercising our own reasoning faculties. We must not place too much confidence in mere book authority, no matter how high the source, unless experience and intelligence indorses the theory or facts in the case.

Speculative and false theories are only surface deep. They need not the eye of the philosopher, nor the mental genius of a Webster, in order to discover their flimsy texture and develop the truth. Those who propose to do their own thinking have the ability to judge between right and wrong. Let us commit to memory all matters which appear as facts, and forget the rest.

Amaurosis can be artificially produced by administering a few 
doses of extract of belladonna, and this amaurotic state of the pupil is also observed during etherization, and disappears so soon as the animal is restored to consciousness.

Percivall informs us that amaurosis may proceed from some abnormal condition of the optic nerve or brain, which may prove, as he says, the last link of a series of marked phenomena, originating in some remote part of the body, operating sympathetically on the nervous system, and, through it, extending to the eye. Cases are occasionally met with which may be termed symptomatic amaurosis, occasioned by congestion, tumors, or hydrocephalus.

Symtomatic amaurosis may follow cranial fracture, and, consequently, depression. In such cases, the skill of the surgeon is called into requisition to trephine the uninjured region of the skull, in order to elevate the depressed and fractured parts. The potent remedy is the removal of the pressure. Amaurosis is occasionally associated with anæmia. Now, anæmia is a condition of the body which indicates a loss of the circulating fluid, either of quantity or quality. The remedy for this condition is carbonaceous food, mineral and vegetable tonics. Preparations of iron and golden seal are the best remedial agents.

Symptoms.-I shall now turn the reader's attention, for a moment, to some of the symptoms attending the amaurotic condition. The head of the patient is elevated, and he is known, in popular language, as a "star-gazer." His ears are kept in constant motion, one turned backward and the other forward, in quick succession, to catch the sound of approaching vehicles, so that he may have timely notice of their whereabouts. When started, and while walking, the fore-feet are raised from the ground to an unnecessary height. This is probably done for the purpose of sounding the ground, and, at the same time, of avoiding obstacles which may lie in the road; for the sight is defective, and the animal must depend more on the senses of hearing and feeling.

Should such an animal be attached to a vehicle and left alone in the street, we shall observe that when an omnibus or heavyloaded wagon approaches him he becomes restless, and exhibits signs of fear. Such are the principal symptoms which usually accompany defective sight, or total paralysis of the optic nerve, producing amaurosis.

Treatment.-Sympathetic amaurosis should be treated by means of antispasmodics and alteratives. One ounce of tincture of assa- 
fetida may be given every morning, and in the evening, one ounce of the fluid extract of sassafras. So soon as the animal regains his natural vision, the medicine may be discontinued.

\section{Foreign Bodies withix the Exelids.}

When foreign hodies, such as small particles of hay or dirt, get within the eyclids, they create great pain and uneasiness, and if allowed to remain there, produce a very grave form of disease, often ending in disorganization and total blindness. Should any thing of the kind be discovered, it may be removed by raising the upper or depressing the lower lids; then introduce and explore the eyeball by means of a camel's hair pencil, to which the foreign body will usually adhere, when it is easily brought away. The parts should then be sponged with lukewarm water. After the lapse of a few hours, should the membranes of the eye and lids appear much reddened, use the following:

No. 1. Rose water .................... 4 oz.

Fluid extract of gelseminum....... $2 \mathrm{dr}$.

Put the patient on a diet of sloppy bran mash, and place him where the rays of sunlight shall not affect the eye.

\section{Films or Specks on the Eye, known as Opacity OF THE CORNEA.}

Opacity of the cornea sometimes results from injuries; at others they are symptomatic, and accompany other diseases. They are of very common occurrence in the various stages of influenza and other catarrhal affections, and sometimes a distinct speck will remain long after the disease which gave rise to it has disappeared. The author remembers many cases in which the sight has been completely destroyed by using substances of a corrosive or stimulating character. The proper mode of treatment is to bathe the eye occasionally with a portion of the following lotion:

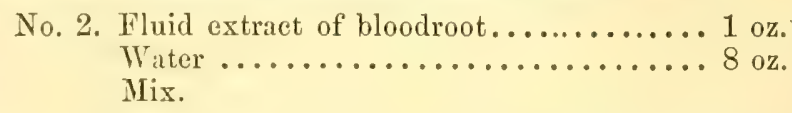

Give the patient one ounce, morning and evening, of fluid extract of phytolacea decandra (poke-root). This acts as an alterative, and will expedite the cure. 


\section{Cataract.}

Cataract in the horse is usually the result of previous attacks of disease. It consists of opacity of the crystalline lens, or the capsule of the same. The disease is incurable, because if an operation was performed for the breaking-up or depression of the cataract, such as is practiced in human medicine, vision would still be as imperfect as ever without the aid of magnifying glasses, which would be a great inconvenience to the horse, and scarcely of any practical service.

\section{WORM IN THE EYE.}

Small parasites are occasionally observed in the eyes of horses. They float about in the watery humor, often for a great length of time, without appearing to do any mischief; yet, being a foreign body, they are, to say the least, injurious. The author has never seen but one case of worm in the eye, and that occurred in a horse, the property of a resident of Chicago. The animal was kept on exhibition for the inspection of the curious, and was the scource of considerable profit to the owner. It is possible that such worm might be extracted by a surgical operation. A writer in the "Veterinary Review" thus alludes to this affection:

"It may also be here noticed that there is a disease which frequently affects the eyes of horses in India, but which, as far as I know, has never been observed in Europe. A worm, which, from the description I have received from different people, may be compared to the common ascaris, is generated in the anterior chamber, and can at times be distinguished swimming about, with apparently great vigor, in the aqueous humor. It produces a great deal of irritation and inflammation, the effects of which ultimately destroy the organ. The natives of India cure the disease by making an incision through the cornea, and extracting the worm. Though I have never had an opportunity of examining an eye affected with this curious disease, the circumstantial accounts, from several accurate observers, leave no doubt in my mind of its existence; and the fact accords with what is known to take place regarding the formation of worms, not only in the human body, but more particularly in the liver, lungs, brain, and other organs of the inferior animals." 
Ophthalmia, or Acute Disease of the Eye.

This is a very common affection, yet a most painful one, and can not well be mistaken. The animal keeps the eyelids closed. They are more or less swollen, and the internal surface of the same appears very red. There is an unnatural flow of tears running down the cheeks. The haw, or nictitating membrane, is swollen and very vascular. This condition of the haw is known to some persons as the hooks, and serves as an excuse for the performance of a barbarous operation, not sanctioned by science or common sense, viz.: amputation of the haw, which only aggravates the disease, and deprives the horse of a useful appendage to the organs of vision.

In the early stage of this disease, and when scalding tears run copiously over the cheeks, we denominate it catarrhal ophthalmia. The disease is not of a very serious nature, but often becomes so in consequence of the outrageous treatment practiced, such as bleeding, purging, and the local application of lunar caustic. The proper mode of treatment is to keep the patient on a light diet, and occasionally bathe the region of the eye with an infusion of poppies or hops; sometimes with tepid or cold water, as the case may seem to indicate, according to the rational judgment of the owner of the afflicted animal. It is the very best application that can be made, and is much more scientific, according to the dictum of the God of Nature, than many of the agents heretofore used. The fact is, many indications of disease designated as inflammation are curable only through the intervention of time and a rational expectancy. Don't do any violence to nature in the use of destructive or poisonous agents, and should the case be curable, it will terminate favorably under this treatment. According to the dictum of the schools of veterinary medicine, inflammation of the eye, as well as inflammatory action of other parts of the body, has to be treated heroically, which system of treatment kills more than it ever cures. As the subject of inflammation is a very important one, worthy the attention and consideration of American husbandmen, I here introduce an article from the pen of A. S. Copeman, who was formerly associated with me in conducting the "Veterinary Institute of Boston," Massachusetts : 


\section{Treatment of Inflammation.}

"It must be admitted by all who contemplate the actual state of medical practice at the present day, that the use of blood-letting and of other antiphlogistic remedies, has, within a recent period, greatly declined. According to YouAtT and Percivall, such remedies, and more especially blood-letting, were formerly highly successful in arresting diseases, in the treatment of which we now know they not only fail, but are even highly injurious. The inference has been drawn from these supposed facts, that inflammation itself is no longer the same; that its type has altered from an inflammatory to a typhoid character. In short, it seems to have been the opinion of certain writers that an advanced knowledge of physiology and pathology has had little influence in producing this great revolution in our treatment, but that the constitutions of animals are fundamentally altered, and that medical men were as right in bleeding thirty years ago as they are correct in now abstaining from it. In opposition to these views, it will be my endeavor to show, 1st, That little reliance can be placed on the experience of those who, like Blain, Percivald, and YouATt, were unacquainted with both histology and organic chemistry, and, per consequence, the nature of inflammations; $2 \mathrm{~d}$, That inflammation is the same now as it has ever been; $3 \mathrm{~d}$, That the principles on which blood-letting and antiphlogistic remedies have hitherto been practiced are fallacious, and opposed to pathology; 4th, That an inflammation once established can not be cut short, and that the object of judicious medical practice is to conduct it to a favorable termination; 5th, That all positive knowledge of the experience of the past, as well as the more exact observations of the present day, alike establish the truth of the preceding propositions as guides for the future.

1st Proposition.- - That little reliance can be placed on the experience of those who, like Blain, Percivall, and Youatt, were unacquainted with histology, and, per consequence, the nature of inflammations.

Inflammation, for many years, was generally recognized, especially in external parts, by the existence of pain, heat, redness, and swelling, and in internal parts by fever, accompanied by pain and impeded function of the organ affected. In fact, groups of symptoms, in accordance with the nosological systems of the day, 
constituted inflammation. But the school of morbid anatomy, by showing that inflammation was a diseased condition of a part, entirely overthrew the errors and confusion inherent in all such nosological systems; while more recent histological research, by exhibiting to us that inflammation is, in truth, a disease of nutrition, governed by the same laws that determine growth and waste of the tissues, has united physiology and pathology into our science, and has removed our present knowledge still further from the traditional errors of the past. Now, if it could be shown that the group of symptoms formerly called inflammaticn always induced the same morbid lesions, former experience might still be useful to us. But we contend that this is what clinical observation proves to be impossible. Such are the contradictory statements and the confusion resulting from the unacquaintance of the past race of practitioners with a correct diagnosis and pathology, that no confidence whatever can be placed in their impressions as to what cases were benefited by bleeding. Medicine is not a scientific art, which is dependent for its principles on the study of a commentary on the older writers. What they thought and what they said are not, and ought not, in a question of this kind, to be our guide as to what was or is. On the contrary, it is the book of Nature, which is open to all, that we ought to study; and why should we read it through the eyes of past sages, when the light of science was comparatively feeble and imperfect, instead of bringing all our improved modern appliances and advanced knowledge to elucidate her meaning?

2D Prop.-That inflammation is the same now as it has ever been.

The essential nature of inflammation has been already alluded to, viz. : a series of changes in the function of a part, terminating in exudation or effusion of lymph. Now, what proof is there that any of these necessary changes have, of late years, undergone any modification? If a healthy animal receives a blow, or any other injury, are the resulting phenomena, in these days, in any way different from those which took place in the days of Youstт and Percivall? Were the effects which followed wounds in 1830 different from those which resulted from similar injuries in 1860? This has not yet been shown. Again: if a healthy horse, nowadays, be exposed to wet and cold, and be seized with an inflammation of the lung or pleura, is not the lung hepatized in 
the one case? and does not effusion follow in the other, in exactly the same way as formerly? But what should this asserted change in the nature and character of inflammation lead us to infer? It is said that inflammation and its results are entirely changed within the last thirty years. It is, then, argued that horses, in all parts of the world, since the days of BLAIN, YouATt, and PERCIVALL, have become so debilitated and deteriorated; that their constitutions have been so altered for the worse; that, attacked by the same lesion, and to the same exfent, there is no longer the same reaction. If so, where is the evidence of this?

For my own part, I have earnestly sought for but can not discover a shadow of evidence for such a belief. Moreover, I have a most lively remembrance of all the facts and circumstances connected with the bleeding of hundreds of patients, thirty years ago, when I first commenced the study of veterinary medicine, and my impression is, that not the slightest difference exists between the character of inflammation now and what it was then.

3D Prop.-That the principles on which blood-letting and antiphlogistic remedies have hitherto been practiced are fallacious and opposed to pathology.

Large and early bleeding have been practiced, under the idea that, by diminishing the amount of circulating fluid, 1st, the materus morbi in the blood would be diminished; $2 d$, less blood would flow to the inflamed parts; $3 d$, the increased quantity of blood in the part would be lessened; 4 th, the character of the pulse was the proper index to the amount of blood that ought to be drawn. Let us examine a few of these principles of practice. The increased throbbing and circulation of blood in an inflamed part may be shown not to be the cause of inflammation, but the result of it, and that the idea of so-called determination of blood to inflamed parts is fallacious. Now, if we attend to what takes place in the finger from a thorn entering the skin and remaining unextracted, we find the irritating body first acts upon the cellular constituents-the nerves and blood-vessels of the part; then comes on the congestion and exudation, and, lastly, follows the throbbing, which is the evidence of so-called determination, and result of the inflammation, and not a cause of it. The blood, in this case, instead of being sent by a vis a tergo, is, in fact, drawn by a vis a fronte, and, as we shall endeavor to show, for the most important purposes. But why should Nature, in cases 
of inflammation, draw an increased amount of blood toward the part? She does so, it seems to us, in obedience to one of her wisest laws, but one which has been too much ignored by medical practitioners. It must be obvious, however, that an inflammation having occurred, the great work now to be accomplished is the removal of the exudation-to eliminate from the injured part either directly by discharge externally, or by passage into the blood, to be finally excreted through the emunctories. In all such cases, the blood is not sent or determined, but drawn to the part, in consequence of the increased actions going on in them; in short, it is absolutely imperative that the part in which these changes go on should receive more blood than in health. But, hitherto, medical practitioners have supposed that this phenomenon is injurious, and ought to be checked by blood-letting and. antiphlogistics. The rapid flow of blood, which is so necessary, they have sought to diminish, and the increased amount in the neighborhood of the part which is so essential for the restoration to health, it has been their object to destroy. In doing so, we argue they act in opposition to sound theory, and, as we shall afterward attempt to show, good practice, also.

The inconsistency of the theraupeutical rules on this head will become more manifest when we remember that it is necessary, in the opinion of many medical practitioners, not only to weaken the pulse when it is strong, but to strengthen it when it has been made weak. Now, although it is obviously good practice to support the strength when the calls upon the nutritive functions have exhausted the economy, it is injurious to diminish, by bloodletting, the nutritive processes themselves, when they are busily engaged in operating on the exudation and eliminating the morbid products. In short, the phenomena of fever and excitability accompanying inflammation have been wrongly interpreted, and danger is to be apprehended from them, not directly, but from the subsequent exhaustion which all great exertions of the animal economy produce. In themselves, these exertions are sanative, and indicate the struggle which the economy is engaged in when attempting to get rid of the diseased processes; and whenever we lessen the vital powers at such a critical juncture, we diminish the chances of that struggle terminating favorably. This proposition seems to be universally admitted in the case of essential fevers, and its truth ought to be accepted equally in inflammation. 
It has been argued, however, that the immediate effect of bloodletting justifies the practice. But, assuming it is granted that in some cases the pain is for a time relieved by bleeding, and that in pneumonia the respiration temporarily becomes more free, at what a cost are those advantages obtained, should the patient be so weakened as to be unable to rally! Even if he does rally, a large bleeding almost always prolongs the disease. Of course, we are now speaking of a true pneumonia, and not of that combination of symptoms commonly called a chill, or lung fever.

4TH PROP.-That an inflammation once established can not be cut short, and that the object of judicious medical practice is to conduct it to a favorable termination.

There was a time when it was supposed that the progress of influenza, distemper, and many other specific fevers which are now generally allowed to run their natural course, could be arrested by medical interference. But with regard to them has been established the principles, first, of prevention, and, second, when this fails, of simply conducting them to a favorable termination.

It appears to me that the same rule ought to hold with regard to internal inflammations, and that this will be admitted when it is made apparent, not only that every inflammation, once formed, runs through a definite course, but what that course is. This I now proceed shortly to consider. If a violent blow or injury has been received, a greater or less amount of exudation is infiltrated among the contused and torn tissues, which undergo disintegration, is absorbed and excreted from the economy; if not, it collects, in the form of a fluid, and constitutes an abscess. The series of changes here referred to have always been found to be best perfected in animals of vigorous constitutions, while in the weak, poor, and broken-down they proceed slowly, or not at all. Surely, it can not be maintained that in cases similar to the above, we can favor the reparative processess by blood-letting and lowering the strength of the economy?

But in internal inflammations, say of the lungs or intestines, are the processes different? Certainly not. But because the processes by which Nature relieves herself have been hid from vien, physicians have supposed that instead of treating the inflamed parts, as the surgeon does, he ought to attack the general symptoms which result from the lesion. In cases of fracture and contusion there are also febrile syraptoms, increased pulse, and so on; 
but does the surgeon imagine that callous will form better, or an abscess be resolved or reach maturity sooner, by general bloodletting and antiphlogistics? Experience teaches him otherwise; and in the same manner it may be most reasonably argued that such treatment can not favor the natural termination of internal inflammations.

5тн Prop.-That all positive knowledge of the experience of the past, as well as the more exact observation of the present day, alike establish the truth of the preceding propositions as guides for the future.

Before it is possible, however, to determine with exactitude the value of any practice, it is essential to ascertain the natural duration of the disease we propose to treat. Fortunately, we have now some data which will enable us to arrive at this information with regard to many diseases. We have seen many severe cases of pneumonia submitted to homepathic remedies-that no reasonable medical man can suppose to be any thing else than inertyet most of these cases got well, and, I think, may be considered as excellent studies of the disease left entirely to Nature. Many years' experience and close observation have convinced me that uncomplicated pneumonia, especially in young and vigorous constitutions, almost always gets well, if, instead of being lowered, the vital powers are supported, and the excretion of effete products assisted. It is in exactly these cases, however, that we were formerly enjoined to bleed most copiously, and that our systematic works even now direct us to draw blood largely, in consequence of the supposed imminent danger of suppuration destroying the texture of the lung. Such danger is altogether illusory, and the destruction to lung tissues, so far from being prevented, is far more likely to be produced by the practice. In fact, the only cures in which it occurs are in the aged or enfeebled constitutions, in which nutrients, and not antiphlogistics, are the remedies indicated. We can, however, readily understand how blood-letting, practiced early, and in young and vigorous constitutions, does less harm, or, to use a common expression, is "borne better," than when the disease is advanced, or the patient weak, and this because then the vital powers are less affected by it. But that it cures the greater number of animals attacked, or shortens the duration of the disease, is disproved by every fact with which we are acquainted. Before closing, we have a few words to offer on 
'mercurials.' The confident belief in their power of causing absorption of lymph, by operating on the blood, is not only opposed to sound theory, but, like blood-letting, is not supported by experience, which has been so confidently appealed to in their favor. I can not, therefore, resist the conclusion that the principles which led to an antiphlogistic practice in inflammation were erroneous, and are no longer in harmony with the existing state of pathology. I think it has been further shown, that in recent times our success in treatment has been great just in proportion as we have abandoned 'heroic remedies,' and directed our attention to furthering the natural progress of the disease.

Internal inflammations are cured, not by bleeding and drugs, but by a natural process as distinct and definite as the process of normal nutrition. What we may do by our interference, may either aid, promote, and even accelerate, this natural tendency to get well, or it may very seriously impair and retard, and even altogether stop, that salutary process. If, then, this view of the nature of the means by which inflammation is resolved in internal organs be correct, it is not unreasonable to assume that a very depressed state of vital power is unfavorable to the healing process.

Indeed, if you watch those cases in which nothing at all has been done, or in which nothing has been done to lower the vital powers, you will find that the mere inflammatory process itself, especially in an organ so important as the lung, depresses the strength of the patient each day more and more. You will perceive, then, that, according to these views, there are strong $\dot{a}$ priori reasons in favor of the policy of upholding our patients, even in the earliest stages of acute diseases, by such food as may be best suited to their digestive organs, such as is most readily assimilated, and calls for the least effort, the smallest expenditure of vital force for its primary digestion-nutritive matters, tea, sweetened milk, etc., and also alcohol, which is directly absorbed, and tends to keep up the heat of the body.

If, then, it has been satisfactorily shown, in consequence of our advanced knowledge of diagnosis and pathology, that an antiphlogistic practice is opposed to the cure of diseases, it follows that many of the principles which have hitherto guided us in their treatment must be considerably modified. That medical practice has undergone a great revolution during the last fifteen years, is a fact already so well established that it can be no longer denied. 
In my discourse on inflammation, reference was made to the views held by the 'neuro-pathologists.' 'Now, nervous pathology has been in medicine the 'great scape-goat' upon which more professional sins have been heaped than any other. 'Nervous exhaustion,' nervous irritation, etc., are a few of the many terms with which we seek to cloak our ignorance of the real nature of many disorders, the intimate nature of which is beyond our ken. Many accomplished practitioner's still maintain that abnormal, vital phenomena may be, and are likely to be, occasioned by dynamic aberrations alone, and that such phenomena are correctly designated as functional disease. We can not concur in this opinion. What is called force of every description is connected with, if not dependent on, changes in the atoms of matter. Force is the hypothetic agent which underlies the phenomena of material change; and to affirm that dynamic modifications of vital function may exist without alteration of material organization, is to ignore the fundamental principles of philosophic physiology. All diseases, therefore, in our opinion, is organic, even mental and nervous diseases of every kind and form. Not a thrill of sensation can occur, not a flashing thought or a passing feeling can take place, without changes in the living organism; much less can diseased sensation, thought, or feeling occur without such changes-changes which we are not able to detect, and which we may never be able to demonstrate, but which we are, nevertheless, certain of. For, whether we adopt the theory that the states and things which we call heat, electricity, vitality, etc., are distinct entities of what is called 'imponderable' matter, or the far more probable theory that they are only phenomena belonging to ordinary ponderable matter, an atom or a cell, charged with electricity or heat, or in a state of chemical activity, is essentially in a different condition to a cell or an atom in chemical or electrical equilibrium with surrounding substances. Organic actions can not exist without corresponding changes' in material condition. The only force capable of explaining any of the phenomena of life is the chemical one, and this only in a state of constant activity and interminable change. In disease, the chemical composition of the cells, or general matter, is altered from the standard of health, and this alteration of chemical composition is the real groundwork of organic disease. Those abnormal states which depend upion an altered condition of the blood, are not less 
strictly organic than all other diseases; for not only can no change take place in the composition of the blood without in some degree affecting all parts which are nourished by it, but that this fluid is, strictly speaking, itself a living organism, and every change which takes place therein is organic. Life can not be arrested without material change in the organs necessary to life. Every autopsy in which we fail to discover these changes, proves merely that our knowledge of life and death is defective, not that the changes do not exist."

\section{Purdlent Ophthalmia.}

Purulent ophthalmia takes its name from the profuse discharge of pus, or matter, which escapes from the eyelids. In regard to the human subject, the authorities contend that there are several varieties of this malady prevalent in man, one of which, the Egyptian ophthalmia, is contagious; but no such disease was ever known to occur among horses.

Causes.-The various maladies which affect the eyes sometimes owe their origin to accidents; at other times they occur in badly ventilated stables, where a large number of horses are congregated together, and then they are supposed to be contagious. But it appears that there is no necessity, in such ease, to advance the theory of contagion, by way of explanation of the outbreak; for the same cause which produced the malady in the first horse would be more or less operative in all the rest. A hot, foul atmosphere has a very bad effect on the eyes of both men and horses. The membrane lining the eyelids, which also covers the eyeball, is very vascular and sensitive, and is extremely susceptible to irritation from the action of the ammoniacal gases which prevail in foul stables; hence, in order to prevent the disease, proper attention must be paid to ventilation and cleanliness.

Treatment.-For the treatment of purulent ophthalmia the author recommends the following lotion:

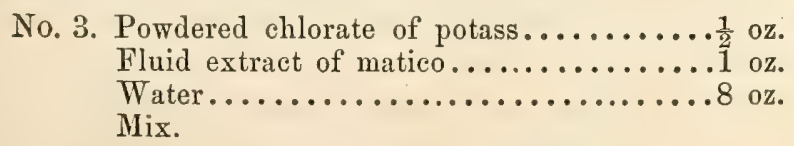

A portion of this lotion should be applied to the eyes, by means of a soft sponge, two or three times a day; the eyes as well as the 
lids must, however, be previously cleansed with lukewarm water. The patient should have a few doses of fluid extract of poke-root. About half an ounce of the extract, night and morning, will suffice, which will act as an alterative.

\section{Specific Ophthalmia.}

This form of disease affects animals periodically, and is dependent on some peculiar predisposition; hence it may be termed hereditary.

Symptoms.-There usually comes on very suddenly, perhaps in a single night, a great tenderness in one eye, commonly marked by the eyelids being shut, a copious secretion of tears, the white of the eye appearing slightly red, and the whole anterior chamber of the eye dim and clouded, there being no distinct speck on the cornea, as takes place in the common inflammation of the eye. The redness of the eyeball is never very remarkable, even though the disease assumes its most aggravated form; but the dimness of the anterior chamber increases rapidly, and in two or three days, or even a shorter period, a yellow spot appears at the bottom of that carity, arising from the formation of pus. Sometimes the quantity of pus is very considerable, and I have seen it fill at least twothirds of the anterior chamber. After lasting one, two, or three weeks, the inflammation and watering usually begin gradually to subside. The pus, though in very large quantity, is sometimes almost entirely absorbed, so that scarcely any vestige is to be seen; and in other instances thin webs of opaque matter remain, which destroy the transparency and luster of the eye, and which, by their adhesion to the edges of the pupil, interfere with its motions and destroy its form.

It is astonishing how acute dealers in horses are in discovering an eye which has had an attack of this kind.

Sooner or later, while the horse appears in a state of perfect health, the eye is again attacked, the disease being accompanied by the same symptoms, making a similar progress, and having the same termination, while each new attack is accompanied with the deposition of more and more opaque matter. These attacks succeed each other at very different, and sometimes at very distant, intervals, until the whole pupil is filled with an opaque white matter, and the sight of the eye completely destroyed. 
During this progress, the disease is often confined to one eye; at least one eye is usually much more severely affected than the other. In some cases the two eyes are simultaneously affected, and, finally, by a succession of attacks, the horse becomes completely blind. The probability is, that after a horse has suffered several times from periodical attacks he will finally become blind.

Treatment.-The same treatment as recommended for common inflammation of the eye is applicable to this complaint.

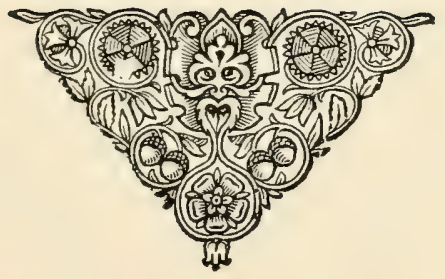




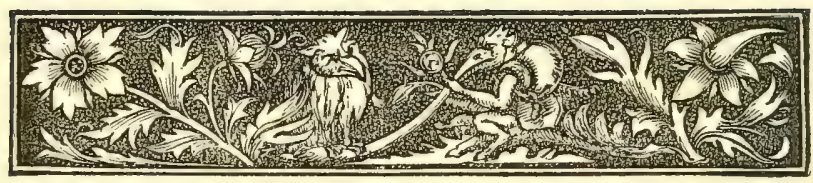

SECTION III.

DISEASES OF THE BRAIN AND NERVOUS SYSTEM.

Abscess within the Substance of the Bratn-Hydrocephalus, or Dropsy of the Brain-Stomach Staggers-Apoplexy, or Cerebral Hemorrhage- Apoplext of Spine, or Spinal Hemorrhage, producing ParaphlegiaEpilepsy, or Fits-Tetanus-Vertigo-Acute or General Disease of the Brain, known as Encephalitis-Sleepy Staggers-Cerebrat MeningitisChorea, or Stringhalt.

\section{Abscess within the Substance of the Brain.}

7 THE author, having some doubts about the curability of abscess 1 within the brain, can not offer the reader much encouragement as regards the cure; yet, for the purposes of research, and in view of prosecuting our Samaritan-like calling on a noble and valuable animal, it is proper that the reader be put in possession of the facts in the case. The symptoms and morbid conditions connected with various forms of brain disease are, at the present state of our knowledge, very obscure. Even in human practice, the authorities contend that the subject of symptoms and conditions is full of uncertainty and apparent irregularity. Doubtless there is some constant and uniform connection of cause and effect between the altered physical states of the brain and the altered manifestations of its functions, but we have not yet been successful in our search after those settled relations, nor have we but partial and imperfect glimpses of them.

Causes.- It is probable that the same causes which produce abscess in other parts of the body, not clearly traceable to local injuries, are operative in regard to the brain. Abscess is, as a matter of course, always preceded by an active stage, known as inflammatory, and when not called into existence by local injury, must depend on both predisposing and exciting causes. The predisposition lurks in breed, and the exciting causes may be among 


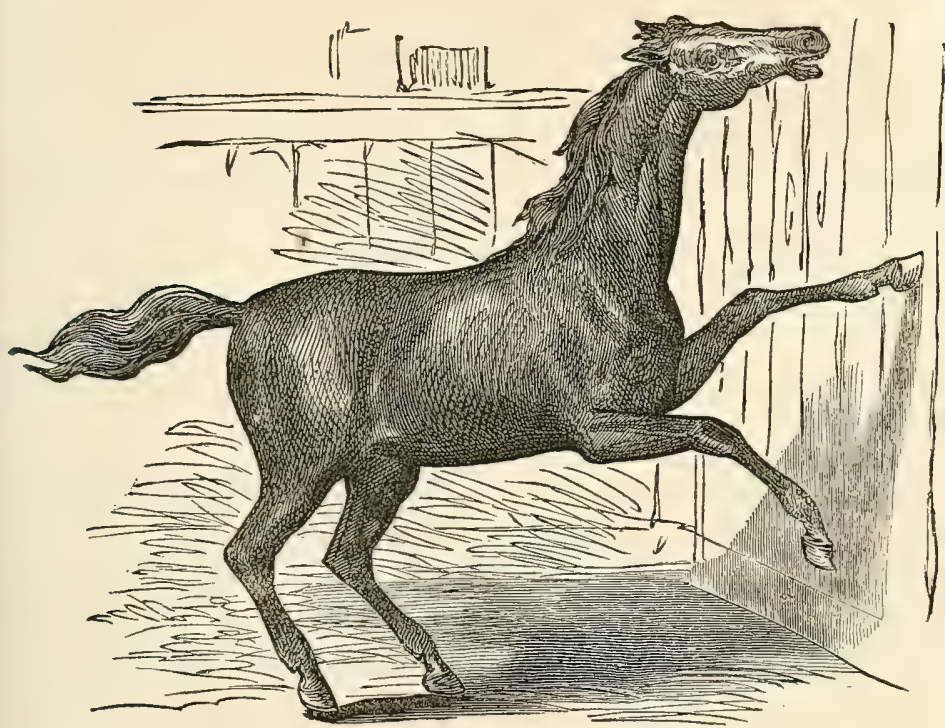

A HORSE BENT ON MISCHIEF-THE BUBJECT OF PHBENITIS.

those which produce disorder in the digestive function or organs. It is well known, however, that this, like some other diseases of the brain, is constantly occurring among members of the human family as well as the equine species, they being the subjects of constitutional defect in the form of scrofula. Derangement of the digestive organs almost always affect the brain, in consequence of sympathetic relations existing between the two. Hence, in view of preventing disease of the brain, we must keep the stomach in good working order, by means of an intelligent system of dieteties, and the exhibition of sanative medicines when they seem to be needed.

Symptoms.-The observable symptoms of abscess within the bran do not differ materially from those which are present in dropsy of the brain. In the early stage, the animal appears lethargic, sleepy, and, when urged to move, reels and comes near falling. The head is usually somewhat depressed, yet it is often inclined to one side: the pupil of the eye is dilated, and the membranes of the lids are congested and reddened. As the disease advances, a state of torpor sets in. Blindness, from pressure on the brain, ensues; the animal gets upon the floor, soon abrades the skin from the regions of the hips and shoulders, until, as a matter of charity, the owner puts an end to the sufferings of the patient. 
The lethargic condition, therefore-dilated pupils, torpor, and reeling of the animal-indicate this serious difficulty in the substance of the brain or its cavities.

Treatment.- Should the surgeon or the proprietor of the affected animal desire to attempt a cure, the author recommends the following:

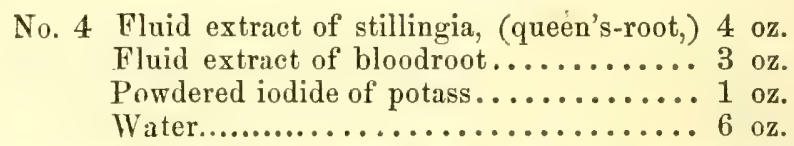

Dissolve the iodide in the water, and then add the stillingia and bloodroot.

Dose, two ounces, twice daily. Local treatment in this malady does not amount to much, if any thing at all, for the only way in which the matter within the brain can be got rid of, if got rid of at all, is by exciting the absorbents to action, so that the matter may be absorbed. At the same time, alteratives are to be used; and, for this purpose, the above prescription is recommended.

\section{Hydrocephalus (Dropsy of the Bratn).}

Hydrocephalus is the termination of some disorder in the brain itself, or the membranes surrounding it; yet some medical writers contend that dropsy is rather a symptom of disease than disease in itself. It may be so in some cases, which constitutes the exception to a general rule; but the author regards dropsy of the brain as the last stage of organic disease of that organ. On the dissection of the brain of some horses that have died of this malady, scrofulous tubercles are often found in the substance of the brain, and tubercular deposits also manifest themselves on the membranes covering the brain. In fact, the pathological appearance revealed on dissecting the brain of a horse which has died of hydrocephalus does not differ from that found in the human subject. CoNDIE says: "Tubercles, varying in size from that of a pin's head to that of a pea, are very generally found scattered irregularly over the surface of the pia mater, following it between the convolutions. Occasionally, however, they occur in distinct patches of an inch or more in extent. They are commonly hard and semi-transparent; sometimes opaque, and of a whitish, grayish, or yellowish color. They are found upon all parts of the surface, the convex and lateral portions as well as the base, in the infractuosities of the convolutions, 
and in the fissures. According to Rilliet and Barthez, they are more frequent upon the convex surface of the hemispheres than at the base. Dr. Hamernjh found them more frequently at or near the base of the brain. They are much more abundant upon the brain than upon the cerebellum. They are met with, also, imbedded in the gray matter of the brain, and are here often surrounded by a halo of redness, usually connected with an enlarged vessel, ramifying from the pia mater. More rarely, tubercles are detected in the medullary portion of the brain, where they are often overlooked, in consequence of their pale, semi-transparent, yellowish tint. The plexus choroides is, also, often covered with tubercles. They are very commonly met with, likewise, on the serous membranes of the thorax and abdomen, in the lungs, and occasionally in the substance of the liver. In twenty-seven out of thirty-three cases of hydrocephalus, Barthez and Rilliet found tubercles or granulations associated with inflammation of the pia mater; in four cases the meningitis was unattended by any trace of tubercular deposition in the encephalon; and in two cases the granulations, or meningial tubercles, were unattended with any traces of inflammation. In all the thirty-three cases the symptoms were nearly identical."

Causes.-Among the causes of this disease, therefore, we may consider the scrofulous diathesis as being the most potent; yet we can not always prove the fact until after the death of our patient. Yet an autopsy held on such a case is really valuable to the inquiring mind; for, in discovering the true pathology of the affection, we are not only enabled to comprehend its character, but also the modus operandi of treatment.

The exciting causes of the malady are not always so apparent. The author has known it to follow castration, puncture of the foot, staggers, and acute disease of the stomach.

In some cases, faulty nutrition is the exciting cause; in short, the symptoms sometimes supervene on the subsidence of some other malady.

Symptoms.-As regards the symptoms of hydrocephalus, they do not differ materially from those alluded to in the preceding article. At first, torpor, unconsciousness, unsteadiness on moving; dilation of the pupil; the animal gets upon the floor, in many cases never to rise again. Stertorous (grunting) breathing takes place; the animal tosses his head about in an unnatural manner, often throwing it backward-a very noticeable feature of this affection-oceasion- 
ally raising it from the ground. Finally, convulsions set in, and the subject dies in that state, perfectly unconscious.

When hydrocephalus occurs after phrensy, or what is known as "mad staggers," it always proves fatal.

Treatment. - For the treatment of hydrocephalus give the following:

No. 5. Fluid extract of buchu............ 4 oz.

Water.......................6 6 oz.

Iodide of potass................ 2 oz.

Mix.

Dose, two ounces, morning and evening.

The patient should have injections of soap-suds, once or twice daily; and should the disease have made its appearance very suddenly, or, in other words, be of an acute character, so that the parts in the region of the brain feel hot, they should be then sponged very frequently with cold water; then give two drachms of fluid extract of gelseminum twice daily, until the pulse feels soft, or until the acute stage subsides.

\section{Stomach Staggers.}

The cerebral disease usually denominated stomach staggers prevails among horses which are overfed, whereby the function of digestion becomes deranged ; and thus the food given accumulates, and finally gorges the stomach, producing cerebral derangement, which makes the horse reel and stagger like a drunken man. The horse may be said to be drunk from the effect of food. Sometimes the cause is accidental. A horse gets loose in the stable, and, finding a lot of meal or oats incautiously exposed, he devours voraciously a large quantity, and very soon after becomes the subject of stomach staggers.

Symptoms.-A stomach surcharged with food, without any accompanying distension, does not appear to occasion any local pain, but operates with that kind of influence upon the brain which gives rise to symptoms, not stomachic, but cerebral; hence the analogy between this disease and staggers, and the appellation for it of "stomach staggers." The unnaturally-filled stomach produces, for the first time, a sense of satiety; the horse grows heavy and drowsy, reposes his head upon the manger, falls asleep, and makes a stertorous noise. All at once he rouses from his lethargy, and violently thrusts his head against the rack or wall of the stable, or any thing, 
in fact, that happens to oppose him, and in this posture parss with his fore-feet, or performs the same action with them as he would were he trotting, evidently all the while unconscious of what he is about. His eye, which at first was full of drowsiness, has now acquired a wild, unmeaning stare, or has already become dilated and insensible to light. The respiration is tardy and oppressed; the pulse slow and sluggish; the excretions commonly diminished.

The late Professor Coleman used to relate a circumstance, in his lectures, connected with this disease, which throws considerable light on its origin. The artillery horses stationed in London during the winter of 1817 suffered very considerably from stomach staggers; so much so that it was considered to be endemical, and of an infectious character. With his usual penetration, he soon discovered the cause, and found that, from some new regulations about that time, the stablemen were not allowed any candles, and during the winter the horses were bedded up at five o'clock in the evening, and not fed again until eight o'clock on the following morning, when they consumed their breakfast voraciously, gorging their stomach, not to the degree likely to produce acute indigestion, but sufficiently distending them as to oppress the blood-vessels and the circulation through them. This practice, continued day after day, caused a specific inflammation of the stomach-an inflammation of a peculiar character, differing from gastritis or inflammation of the part. The symptoms produced were regarded as resulting from the sympathetic connection between the stomach and the brain, united to the effects that would arise from the daily distension, throwing a vast quantity of blood on the brain. An order was obtained for candles for the use of the stablemen, which enabled the horses to be fed at a later hour in the evening, and an earlier one in the morning, when the disease disappeared.

A common error still prevails, in many districts, that staggers is a contagious disease; but should the horses on a farm be attacked occasionally with slight fits of this kind, the farmer may rest assured that there is mismanagement somewhere in the feeding department.

From such evidence as this, it will be inferred that there exists no doubt regarding the cause of stomach staggers.

Treatment.-We now propose to show how this disease ought to be treated. The proposition of cure is, that the digestive function shall be aroused, and the only way to accomplish that is by admin- 
istering bitter tonics and stimulants. In this view, the following prescription is offered:

No. 6. Fluid extract of black pepper.......4 oz.

Fluid extract of ginger............ 6 oz.

Hyposulphite of soda............ 2 oz.

Water...................... 4 oz.

Dissolve the hyposulphite in the water, then add the pepper and ginger. Give the animal a wine-glassful every four hours. A stimulating injection may be thrown into the rectum occasionally, composed of a handful of fine salt to about four quarts of water.

The animal should be allowed to stand quietly in the stall, and the medicine must be given with care, for the least excitement may augment the cerebral difficulty. So soon as the medicine arouses the digestive function, and the food gradually passes the pylorus into the intestines, the animal will obtain relief. Both food and water should be withheld until there is some marked improvement; the patient has had enough of food for some time, and water only retards digestion.

\section{Apoplexy (Cerebral Hemorrhage).}

As regards the cause of apoplexy, the author has nothing to offer, except he has noticed that the subjects of this affection generally have short, thick necks, and, as the saying is, "chunky" heads. From this he infers that, in so far as conformation is concerned, there lurks in the system of such animals a peculiar predisposition.

Symptoms. - An animal may be on the road, trotting along as usual, without any apparent impairment of health, when suddenly he falls down; the pupils of the eyes become dilated; stertorous breathing sets in; a deprivation of the sense of feeling and of motion immediately occurs; a tremulous motion of various parts of the body is observed; the pulse beats with unnatural force, yet the animal appears to be in a deep, snoring sleep. It may be said that the functions of animal life are suspended, excepting those of respiration and pulsation. The animal is unable to swallow, and if fluids be put into the mouth, they appear to choke him, or they run out again at the corners of his mouth. The prognosis of apoplexy is very uncertain. Some horses die in a few hours, while others live for several days. This depends on the amount of blood 
extravasated on the surface of the brain; but, in most cases of cerebral hemorrhage, the horse falls never to rise again. He may be conveyed home on some vehicle constructed for the purpose, but the finger of Death is on him; his days are numbered, and the owner charitably puts him out of existence.

The reader must bear in mind that in apoplexy the horse falls, and is suddenly deprived of all voluntary motion; is insensible to the prick of a pin, and the breathing, so soon as he is on the ground, becomes stertorous. This disease, therefore, is not to be confounded with others of the brain and spinal marrow. For example, a horse may have an attack of simple apoplexy without hemorrhage, fall down, and, by judicious treatment, recover; or he may be the subject of epilepsy, which oceasions a temporary suspension of consciousness, with spasms recurring at intervals. Hence, in case of doubt or mistake, it will be advisable to treat the case in the following manner:

Treatment.-Procure a few ounces of spirits of ammonia, with which saturate a sponge, then apply it to the nostrils. In the mean time, sponge the head with cold water, and rub the body and limbs briskly with a brush or whisp of straw. If he revive under this treatment, there may be some hopes of recovery; and should it appear that the act of swallowing can be performed, give a drench composed of

No. 7. Powdered chlorate of potass........... 2 oz.
Boiling water...............

When cool, administer. The action of chlorate of potass on the blood is to oxygenize it, and thus liberate carbonic acid gas. With the same object in view, we apply ammonia to the nostrils, viz. : to decarbonize unpurified blood.

Blood-letting is inadmissible, for it can not accomplish any good; neither will it act as a purificator of the vital current which the lungs have failed to arterialize.

\section{Apoplexy of the Spine, (or Spinal Hemorrhage, producing Paraplegia.)}

Paraplegia signifies paralysis of the posterior half of the body. Spinal apoplexy may be classed in the same order of disease as cerebral apoplexy - that is to say, in so far as the pathology of the two forms is concerned-and is usually just as fatal. 
Canses.-These are very obscure, except in cases of fracture of the spine, or injury to the same, by falling. Then the disease is accounted for. It always ends in paraplegia-palsy of hind limbs.

Symptoms.-The disease is usually sudden in its attack. A profuse perspiration ushers it in; next, the hind limbs fail to support the body, and the animal makes desperate efforts to support himself, and gradually crouches and falls to the ground, unable, perhaps, ever to get up again.

It has been noticed that paraplegia may also be occeasioned by effusion of serum within the coverings of the spinal cord; yet we can not demonstrate the fact only by autopsy; and if we could, I fear that medicine would be of little value. However, if it is the owner's wish that the case should be treated, then the same course as recommended for cerebral apoplexy must be pursued.

\section{EPILEPSY-Fits.}

Epilepsy consists of a temporary suspension of consciousness. This disease, if properly managed, is not often fatal. It varies in duration. Sometimes a horse will suddenly fall, lose all sensibility and consciousness, exhibit spasmodic contraction of the voluntary muscles, go into convulsions, recover, and get up again in the course of ten minutes; or he may lie on the ground and have a succession of paroxysms, which may last for half an hour or more. If protracted beyond an hour or so, the patient is very apt to die. According to the best authority, "the functions that are affected in this disease are functions of the brain. Sensation, thought, and motion, regulated by the will, are the natural functions of that organ: The temporary abeyance of sensibility, thought, and volition, $*$ and violent and irregular action of the muscles, which are thus withdrawn from the government of the will, constitute a paroxysm of epilepsy."

The fit is generally brought on by a derangement in the relation between the arterial and venous circulation within the head and a temporary pressure on the brain; in other words, a determination

\footnotetext{
Horses think and reason just as man does. Their manifestations of mind do not.differ from ours in kind, but only in degree. "The noble and daring warhorse, when he sniffs the distant field of blood, neighing for joy, instils a desperate courage into the veteran trooper's quailing heart, gives evidence of a soul, the proper attribute of man."
} 
of blood to the head. After the horse has fallen, by his struggles and herculean efforts to battle with the malady, although unconciously, he soon breaks out into a profuse perspiration. This has the effect of relaxing the capillaries so that the blood circulates more freely and uniformly. An equilibrium of the circulation takes place, and this is the end of epilepsy for the time being. But a horse once having had a fit of this kind must be looked upon with suspicion; for he is liable, when under excitement from wanton punishment, or from exercising great feats of strength in drawing heavy loads, to have a re-attack.

Cause.-The predisposing cause of epilepsy has an hereditary origin. Horses subject to it have a misshapen head. It is not symmetrical-does not correspond with the conformation of the neck and body. In the language of the turf, "the head is too coarse." It has been found, also, among members of the human family, that epileptics have heads of an unnatural shape. WATson says: "There is no doubt that a tendency to epileptic disease is frequently hereditary. It may be bequeathed from parent to child, or it may skip over a generation or two, and appear in the grandchild or great-grandchild; or it may be traceable only in the collateral branches of the ancestry." Epilepsy, however, may not always arise as an hereditary affection; for a mere passive congestion of the brain, owing to a loss of equilibrium in the circulation of the blood, may produce it. In regard to the horse, it is very difficult for us to decide on the universal hereditariness of the malady, because we have no reliable history of the ancestry and idiosyncrasies of our equine patients; yet if we study carefully the external conformation of well-formed horses, and make ourselves conversant with anatomy, we shall be better able to judge whether or no such an animal carries about with him the inherent tendencies to particular diseases; and this knowledge will make up, to a certain extent, for the advantage which human medicine has over the veterinary in this department of knowledge. The principal symptoms of epilepsy are as follows:

Symptoms.- Suppose the horse attached to a vehicle, and traveling along at any given pace. He gives a sudden, snorting, loud noise, and falls to the ground instantly, as if felled by some unknown power. Here he lies, to all appearance, totally unconscious, violently convulsed in every limb, his eyes staring as though they would burst out of their sockets; the mouth foams 
with saliva, and violent convulsions will sometimes affect the whole frame. Such are the principal symptoms attending this formidable malady.

It will be quite a novelty to some of our readers to be informed that the horse is actually subject to the same diseases which afflict his master; and in view of showing that there is a reality in the author's views on the subject now under consideration, the following quotation from the "Veterinarian" is offered. It will show, by way of comparison, the features of the disease as it occurs in the horse and in man:

"In the first place, as to the exciting cause or causes. It is well known that among the exciting causes of epilepsy in the human subject, mental or moral emotions have long been considered as holding a foremost rank. Without going into detail on this subject, or offering illustrations of this statement, it will suffice to remark that the experience of all adequately acquainted with medical literature, or tolerably familiar with medical practice, can not fail to supply them with numerous instances wherein this class of causes has been in operation as concerned in the production of the disease in question. Over and over again I have myself witnessed cases of epilepsy, either during or after the paroxysm or fit, in which I have had good reason for entertaining the opinion that certain mental or moral emotions had largely, if not entirely, contributed to this result. Now, without denying that, in some instances, fright may so far affect the horse as to prove an exciting cause of epilepsy in that animal, still, I think that, almost as a general rule, the class of causes now under consideration may be excluded from further notice as tending toward the production of this disease in the horse.

Assuming this position for the sake of argument, we thus eliminate at once, so far as concerns this animal, most of such cases of epilepsy as, if speaking in reference to the human subject, would be regarded as cases of epilepsy of centrie origin. Without altogether denying that, in some instances, the presence of tumors, or morbid growths, or excrescences of any kind, or of spiculæ of bone in certain parts of the brain, or spinal cord, or their membranes, may occasionally operate as causes of epilepsy, yet I am by no means prepared to admit that their presence in such places, by irritation of these structures, is so frequent a cause of this disease as is affirmed by some medical authorities. 
Yet, whatever view of the question, whether affirmative or negative, may be taken, the same reasoning will apply equally to man and the horse in regard to the disease under consideration. We are told that, in some instances, such foreign bodies have been found in these situations on examination after death. This I do not deny; but, at the same time, the evidence that these had much, if any thing, to do in the production of epilepsy, still less that the disease took its origin from them, is, in my judgment, far from complete, and, in some cases that have been recorded, unsatisfactory to the last degree. Again, the circulation of blood in an unhealthy state, in its accustomed channels through the substance of the delicately-constructed brain or spinal cord, appears to me not unlikely to be a frequent cause of this disease in man. The comparatively recent physiological researches of Dr. BrownSequard have thrown much light upon the heretofore obsçure pathology of epilepsy in the human subject; and I conceive that his arguments, and the conclusions deduced therefrom, are, for the most part, perfectly applicable to an animal so high in the zoölogical series as the horse is well known to be."

Treatment.-So soon as the horse falls, some hay or straw should be placed under his head and around him. Bathe the region of the cranium with cold water, and carefully wash the foam from his mouth, taking care not to let any water, hay, or dirt enter the nostrils. Officious persons are very apt to attempt, by force of strength, to raise the horse on his legs; but this ought not to be done. Let him rest quietly until consciousness returns; then, should he attempt to get up, help from the bystanders may then be of some service. When on his legs, deal gently with him. Let the external surface of the body be rubbed until the skin is dry; then administer two ounces of fluid extract of valerian, and let the patient be provided with comfortable quarters. For a few days the patient should be excused from work, and be fed lightly. The only way to prevent a re-attack is to keep him at light work, and treat him in the most gentle manner, both in the stable and out of it.

In cases of this character, as well as many others, men are apt to place too much confidence in medicine, and dose the animal accordingly; but, as we understand the practice of medicine, it is the province of the good physician to know when to do nothing. 


\section{Tetands.}

We recognize tetanus by the unnatural condition of the muscles of various parts of the body, more particularly those of the face and neck. But the fault lies not in the muscles, but in the nervous system. This disease constitutes one of the most terrible and fatal to which the horse is subject. It is not only frightful to look upon, but is productive of the most distressing agony to the animal.

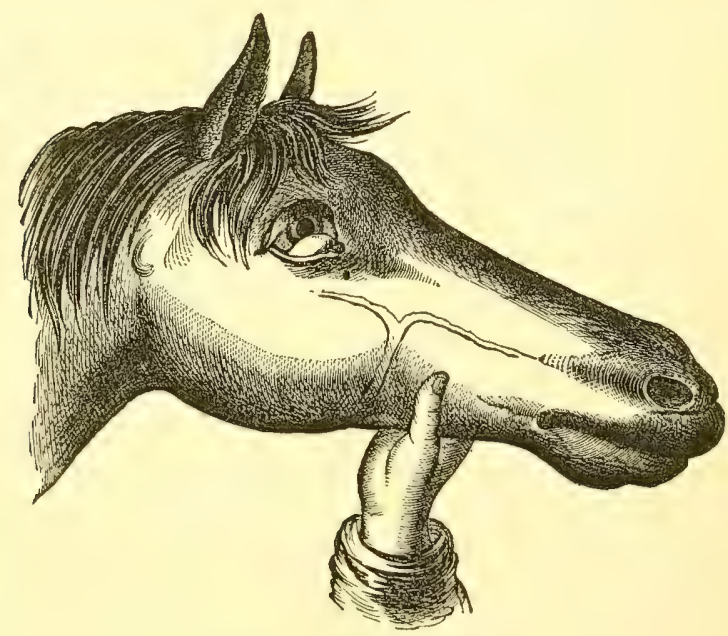

THE TEST FOR TETANUS OR LOCKED-JAW.

Explanation.-On placing a hand under the angle of the jaw, and pushing the head in an upward direction, the nictitating membrane (haw) will be suddenly thrown partly over the pupil, as seen in the above picture. This is the diagnostic symptom of tetanus.

There is no difficulty in recognizing this disease. It is characterized by involuntary and persistent cramps of the voluntary muscles. The muscles which seem first to be affected are those of the jaws, neck, and throat; and soon all the other muscles are involved. One of the principal tests for the discovery of the disease is to place a hand under the jaw and raise the head. Immediately the haw, or nictitating membrane, shoots over the eyeball. (See cut.) There is no other disease with which tetanus can be confounded; therefore, a minute description of the symptoms seems superfluous at this point of our argument.

When the disease affects the muscles of the jaw only, it is called trismus; but it is only a variety of the same disease, and, therefore, 
requires the same treatment. In former years the author met with but little success in the treatment of this malady, yet recently, by using more mild and sanative agents, he has been very fortunate. Bleeding, purging, and blistering has had its day, but that day has now past. We have learned that to do violence to the animal system is not to do good; and our aim now is to "pair off" with Nature, endeavor to sustain the vital powers, or, rather, by sanative medication and nutritious fluids, keep the animal alive, while the disease runs its course. Physicians have no power to arrest the disease, and those who think so only deceive themselves and their employers; and those who attempt the feat of cutting the disease short by heroic medicines, are arrayed in hostility to Nature, and an unnecessary death is often the consequence. Any of our readers who happen to have great faith in drugs will probably feel little comfort in the perusal of the following quotation, uttered by one of the most distinguished physicians of France. It was intended for the benefit of mankind, but it also applies to veterinary medicine, simply from the fact that the diseases of animals are to be treated on the same general principles which apply to man :

"The sick-room no longer resembles the sample department of of a drug warehouse. Our physicians have consciences and common sense. They recognize Nature as the great antagonist of disease, and endeavor to assist her in her struggle to expel it, instead of negatively helping disease by prostrating the physical strength of its victims with drastic cathartics, cantharides, and the lancet. In ailments for which gallons of medicine were given half a century ago, as many ounces are not administered at the present day, and bleeding and blistering have almost fallen into disuse. Not long before his death, the great French surgeon, physician, and medical chemist, Majendie, told his pupils, in the college of France, that the old hospital practice was mere humbug; that he himself had prescribed the drugs of the dispensary at the Hotel Dieu, in Paris, without having the least idea why or wherefore, and that on administering bread pills and colored water to one division of his patients, and the preparations of the pharmacopœia to another, he found that the mortality was least among those who took no medicine! 'You tell me,' said this extraordinary man, in one of the lectures of his final course, "that doctors cure people. I grant you people are cured. But how? Gentlemen, Nature does a good deal; imagination does a good deal. Doctors do very little, when 
they do n't do harm. You ask, then, what is the use of attending medical lectures? I'll tell you. We come here (to the college of France) to study Nature, to learn to reinforce and aid Nature, not to spin fine theories. I would not give a centime for all the theories in the world. Give me stubborn facts.'

"Such was the bold and candid language of one of the greatest anatomists and physicians of the age but a short time before his death. Our medical men do not take quite such strong ground as Majendic took, but they show their lack of faith in what was formerly called 'vigorous treatment' by sparing medication, and a still more limited use of the lancet and other mechanical means of depletion. Nature, after having been professionally misused for centuries, seems at last to have found a friend and ally in the medical faculty."

We now come more directly to the treatment of tetanus; and, in view of showing what unwarrantable outrages are perpetrated on the poor uncomplaining brute, the following article is here introduced:

"Wounds of tendinous and ligamentous parts are the common cause of tetanus, more generally known as locked-jarr, so-called because the first symptoms of the violent spasmodic affection are detected in the jaw. Castration, nicking, docking, lacerations, and punctures, particularly of the feet, are the kinds of wounds that end in tetanus. Even slight contusions will bring it on. It is a dreadful and, too often, a fatal disease. The animal is nearly paralyzed by the constant spasm of all the voluntary muscles. The symptoms are unmistakable: First, a certain stiffness about the throat, and difficulty in swallowing or turning the head. This gradually extends to the jaws, contracting the mouth. The horse possesses a desire to masticate, but, in the earliest stage of the disease, the work is imperfectly performed, with great pain. The eyes become vivid in appearance, and present a retroverted aspect. The discase continues to spread, and when it affects the voluntary muscles of the trunk and the extremicies, the animal becomes a living picture of agony and distress. The cocked yet quivering tail, the distended legs, the contraction of the abdominal museles, the tremulous and irregular pulse, the hurried respiration, the sweat-marks, and the fixed and sunken eyes, all speak unmistakably of the violent and painful nature of the disease. The animal sometimes suffers for six or seven days, when it falls, completely exhausted 
by continued irritation and inanimation; or it expires in turrible convulsions. Tetanus, indisputably, is a nervous affection. An injury to a small fiber of some nerve extends to the origin of the nerve, when the brain becomes affected, and a diseased action of the body ensues. When locked-jaw results fatally, it will be before the expiration of nine days, as the horse dies, without a remission of the spasms, from sheer exhaustion. The free use of the lancet is regarded as one of the surest means of curing tetanic cases, as by a flow of blood we open the bowels and tranquilize the system. Venesection is the most powerful sedative for muscular spasms. The nervous influence passes off with the blood; therefore the flow should be most copious. We may bleed the horse until he falls, before the triumph is complete. The permanent strength of the animal is not hazarded by the free use of the lancet, but we simply make an attack upon the seat of the disease. Physic, also, is a matter of much importance. Profuse bleeding will cause the muscles of the jaws to relax, when the dose may be introduced into the mouth. Clysters assist the action of the purgatives; therefore are useful. The application to the spine of green sheep-skins, warm from the slaughtered animal, will sometimes diminish the sufferings of the patient. By gently rubbing the spine with the hand, and then by using an opiate liniment, a relief may be afforded. From eight to ten drachms of aloes should be administered as a physic. The farina of croton-nut is also a powerful purgative. A solution of Epsom salts constitutes a safe injection as a clyster. Opium, when given in doses, varying from one half to two drachms, is a valuable medicine in cases of lockjaw. The disease is a violent one, and to check it prompt measures must be resorted to."

Contrast this with the following case: While in the city of St. Louis, a short time ago, I was requested to visit a bay gelding, aged eight years, the property of Captain SiLva. The messenger informed me, not knowing that it was a case of tetanus, that the horse was "all stiffened up." On an examination, the following symptoms were presented: The muscles in the region of the neck and back, rigid; the eyes had a sort of squinting appearance; the nose protruded; the ears were erect and stationary; the nostrils were expanded to their utmost capacity; the head, neck, and trunk seemed to be immovable, so that it was impossible to make him turn in any direction, or describe the least segment of a circle. 
The abdomen appeared "tucked up" as the saying is. This arose from the rigid state of muscles of the abdomen. The hind limbs presented a straddling appearance, and the fore ones were unnaturally advanced far beyond the axis of the shoulder-blade; the bowels were constipated; the pulse was wiry, and the respirations were accelerated and laborious. The case was diagnosed as tetanus from puncture of the off hind-foot. It appeared that the animal had picked up a nail, five days previous, which was withdrawn by a blacksmith, and the foot was dressed in the usual way. The treatment was as follows:

The rigid muscles were rubbed, twice daily, with a portion of the following antispasmodic liniment:

No. 8. Cod-liver oil.................. 12 oz.

Oil of cedar................. 4 oz.

Sulphuric ether................ 3 oz.

The application of the liniment was continued during a period of ten days, in which time four drachms of Indian hemp were daily placed on the tongue. The jaws now relaxed, so that the patient could eat bran mashes. At the expiration of two weeks all signs of tetanus had disappeared.

\section{Vertigo.}

The term vertigo signifies giddiness or dizziness. It is usually the result of some latent disease within the brain; and when that is present, any exciting cause, which we shall presently refer to, may bring on a fit of vertigo. The following article, by surgeon HAYcock, will, perhaps, prove interesting to the reader:

"By vertigo is meant a chronic disease of the horse, chiefly indicated by a disturbance of the sensitive faculties, ocasioning derangement in the ordinary functions of life. Much that is incorrect has been written regarding the seat, properly so-called, of the evil. At present, most veterinary surgeons are agreed in seeking the proximate cause, not as formerly, on the brain, but in the abdominal organs, and on considering the cercbral affection as purely secondary."

Vertigo often succeeds acute disease of the brain.

Causes.-Its chief exciting causes are confinement in hot and badly-aired stables, cold, extreme fatigue, blows and injuries on the head, indigestion, unwholesome or too much food in proportion 
to the exercise taken. The fear of punishment, especially if the whip, occasionally gives rise to it, in sensitive and irritable animals. Some horses have an hereditary predisposition to it, and mares are considered more subject to it than stallions. Further, it is scarcely ever observed except in hot weather; and as it is gencrally at the beginning of summer that it commences to appear, it goes away always in autumn, at least with respect to its chief symptoms.

Symptoms.-The horse having, previous to the attack, been lively and active, begins, all of a sudden, to appear heavy and indolent. He is dejected, and prefers to keep himself in the darkest corner of the stable. Eyes, dull; look, fixed and stupid; eyelids, half shut; inattention to every thing, forgetting even himself, and, as it were, asleep, his head hanging down, or resting on the manger. His gait is heavy, slow, and unsteady; he raises his feet very high, and puts the entire sole to the ground, raising and letting down the limbs in a manner purely mechanical, and, as it were, unconsciously. He exhibits much awkwardness in turning, and can not be pulled back except by depressing the head very much, and pushing it latterly. He also leans to one side in walking. To maintain his equilibrium the better, he places the fore-legs beneath the belly, and moves his ears backward in a peculiar manner. According as the disease progresses, he becomes less and less sensible to external impressions. Mastication is performed slowly. He takes, from time to time, a monthful of food, masticates it, swallows a portion of it, but keeps the remainder in his mouth. He prefers taking his food off the ground rather than in any other way, and when drinking, he plunges his head into the water, even above his nostrils. During and after some rather violent movements, his symptoms become much aggravated, and the signs of complete insensibility become more and more marked. The animal runs on quite blind till some obstacle stops him, or turns round, or remains tranquil, with his head depressed, and the legs crowded beneath the body, without being able to change this unusual attitude, unless assisted to do so. There is never any fever. The pulse is often from ten to twelve pulsations slower than in the normal state.

In the same way, also, the respiration is constantly slow, deep, and frequently of a sighing character. In almost all cases, the tongue is foul, and the mouth dry and elammy. With respect 
to treatment, the remedies which have succeeded best with me are, camomile (some doses), then sulphur and nux vomica. In a particular case, where, independently of the symptoms peculiar to vertigo, the conjunctiva, tongue, and mouth were more yellow, the horse frequently flexed his fore-legs, seldom lay down, the fieces were hard, and he passed but little urine.

The reader will perceive that many of the symptoms above alluded to are present in other diseases of the brain; therefore, some difficulty may be encountered in diagnosing the case. It is best for us, however, to diagnose the case on the symptoms of dizziness or giddiness; then a mistake is not likely to occur. Yet, after all, a mistake in the true nature of the malady will not prove disastrous, provided the patient be treated on the general principles laid down in this work, by means of sanative medicines and little good common sense.

Treatment.-For example, should a horse have an attack of vertigo on the road, the driver must immediately stop and loosen the throat-latch and check-rein. Then let the animal stand in quietude for a few minutes, during which time he may possibly recover, and soon be able to resume the journey. If not, he must be taken out of the harness, and carefully led to the nearest stable, or where he shall be free from annoyances of every kind. After the excitement is over, the animal may be led home, and put into a roomy stall, where he must be dieted according to his condition. If fat and plethoric, bran mashes are indicated, into which should be stirred a drachm or two of hyposulphite of soda. Should the patient be poor in flesh, a few good oats are indicated, to which add a small quantity of powdered ginger and balmony. The surface of the body being cold, it must be warmed, after the usual fashion, by means of clothing. But should the surface of the borly feel hot, more particularly in the region of the head, then tonics, stimulants, and food are to be withheld. A bucket of cold water is then to be placed before him, into which about four drachms of nitrate of potass may be stirred.

\section{Acute and General Disease of the Brain known as Encephaltits.}

The contents of the cranium are called, collectively, the encephalon; hence the term encephalitis, which signifies inflammation 
of any, or all portions of the contents of the cranial cavity. Various terms are applied to disease of this character, such as "sleepy staggers," "coma," "phrenitis," cerebritis," and “cerebral meningitis," the latter being formerly" recognized as blind or sleepy staggers. These several terms merely apply to the various stages of the acute disease as it gradually invades the membranes covering the brain, or the substance of the brain itself. It sometimes appears to invade at once the whole of the parts within the skull, or, beginning in one part, it extends rapidly to all the rest, so that the term encephalitis seems to be more applicable than those just enumerated. It is a matter of impossibility for us to tell precisely what are the pathological conditions of the parts affected. Nor are the symptoms always the same. They may range from a state of phrenzy to one of coma. Still, in our treatment, we shall not be led astray; for, being an acute affection, (or affections, as some persons may term it,) we have to treat it on the same general principles which obtain in many or all diseases of an acute character, viz.: by means of sedatives, laxatives, cold water, spare diet, and rest.

Should the patient die during the acute stage of disease of the brain, an autopsy will reveal great vascularity and softening of the cerebral mass, and thickening of its membranes; but should the disease run on unchecked to a fatal termination, pus and fluid may be found within the lateral ventricles of the brain. This enables us to explain the difference between the symptoms which prevail in the early and latter stages of the malady; for, at the commencement of the acute stage, the loss of equilibrium in the circulation sends the red arterial blood, in undue quantities, to the brain - the part, perhaps, most predisposed to diseased action, or, it may be, at the time actually in a pathological condition; hence the loss of equilibrium in the circulation-which, in consequence of accelerated respiration, becomes highly charged with oxygen, acts as a potent stimulus, not only to the nervous system, but to the muscular system also, producing those active and phrenitic symptoms which have led us to infer that the patient is going or is actually mad; hence the name which some persons have applied, "mad staggers." This activity can not last long; for it is potent to exhaust the vital forces. Organs and parts of the body become overworked; then comes organic changes-softening of the brain, effusion, formation of pus, which 
ends in coma, which is the latter stage just referred to, devoid of any mad or phrenitic symptoms, but marked by lethargy, or a prolonged comatose condition, from which no stimulus will arouse the patient. He is not only the subject of altered structure within and around the brain, but the latter is compressed by the serum, or pus, as the case may be, and thus sensibility is more or less destroyed. The degree of coma, however, will be directly proportioned to the degree of pressure. Now, the reader will perceive that the proposition of treatment, just referred to, for the acute stage will not apply to the comatose condition ; for here we have to stimulate and give tone to the system, so as to sustain the failing vital powers.

In regard to this subject, Professor Copeman observes: "In inflammation of the brain, phrenitis, mad staggers, I have told you that it may be said to be impossible to distinguish, with any thing like certainty, in individual cases, acute inflammation of the substance of the brain from those affecting the membranes or ventricles. The reason will be obvious when we reflect that the phenomena, in every instance, are, in fact, attributable to pressure on the entire contents of the skull, encephalon, viz.: cerebrum, cerebellum, medulla, oblongata, and membranes; and if this be rapid and general, it can matter little whether it originates from the membranes or the brain. Acute inflammation does, however, sometimes appear to invade at once the whole of the parts that are lodged within the skull; or, beginning in one part, it extends rapidly to all the rest. The symptoms which usually mark an attack of cerebritis are the following: The horse becomes sleepy, heavy, or more or less comatose, accompanied by general fever. After a time a convulsive attack supervenes. He becomes morose, and shows delirium; perhaps rears both forelegs into the manger, from which position he may reel around and fall, kicking and tearing every thing about him. During the fit his respiration is much excited, and he sweats profusely. If he gets upon his legs, he makes sudden and violent efforts, dashing against rack, manger, or stall, totally disregarding any thing that may be said or done to him. Thus he is continually struggling, panting, and perspiring, perhaps foaming at the mouth, leading the ordinary observer to believe he is not only delirious but actually ' mad.' But all these symptoms vary much in different cases. Coma occurs frequently, but often only tem- 
porarily. Great prostration and muscular debility are generally observed.

The seat of 'sleepy staggers,' or cerebral meningitis, is the socalled subarachnoid cavity, in which is a quantity of loose areola tissue, richly furnished with blood-vessels. It generally results that the exudation poured into this cavity is quickly accompanied or followed by pus. Hence, we find that what is generally called a recent layer of coagulable lymph, covering the convolutions in meningitis, is, in point of fact, a layer of pus, generally presenting a molecular character. As to the diagnosis, notwithstanding the efforts which have been made to distinguish meningitis of the convolutions from that of the base, or either of these from a simple effusion into the ventricles, I have in vain sought for any precise symptoms, which could be relied on, as indicative of the situation of the disease. Drowsiness and coma, causing slow and subsequently rapid pulse, succeeded by restlessness. The horse is excited; he flings himself about, frequently jerking his head up and down, sometimes rearing; perhaps, into the manger; tension of the limbs, thrusting the head into the rack. The faculties of the organs of sense are lost, for the horse neither hears nor sees. The state of excitement may terminate, more or less quickly, in convulsions and death, or the patient may relapse into a state of coma, and ultimately result in partial or complete recovery.

The gradual mode of invasion, and the succession of the symptoms to one another, are also characteristic, and differ markedly in degree from those which attend sudden attacks of apoplexy caused by hemorrhage. They are both the result of general pressure on the brain, and hence the reason why mere effusion can not be distinguished from hemorrhage."

Treatment.-The treatment of the preceding forms of acute disease of the brain was formerly, and is at present, to some extent, conducted on the absurd antiphlogistic plan, by blood-letting, purging, and blistering, which practice kills more than it ever cures. The plan now adopted by the author is to give drachm doses of gelseminum every four hours, until relief is apparent. The cranial region is kept constantly bathed with cold water; the rectum is kept free from excrement by means of injections of soapsuds; the bowels are kept in working order, by mixing Glauber salts with thin bran mashes. Half a pound of salts, dissolved in about four quarts of mash, will generally prove laxative. Should 
it fail to have this effect, after a lapse of about six hours, the dose may be repeated. This plan of treatment is more rational, and has proved more successful, than that just alluded to.

Should the disease progress so that the animal manifests symptons of coma, or lethargy, then chlorate of potass is the best agent. It should be given in half-ounce doses, every four or six lours, in the form of Irench, or it may be dissolved in the water which the animal is allowed to drink. A few doses of the following preparation must also be given:

\section{No. 9. Fluid extract of golden seal........ Mix.}

Dose, two ounces every morning.

Keep the rectum empty by injections, and, if the case be curable, such treatment as this, followed up by careful nursing, will accomplish the object. Copeman, who is authority in this disease, fully indorses this treatment, and says:

"Hitherto the treatment of meningitis (sleepy staggers), whether real or supposed, has been antiphlogistic, but it is impossible to say that any benefit has ever been effected by the practice. The early stages of the disease are probably generally overlooked. So long as the horse retains his appetite and his consciousness, no suspicion of disease arises. It is only when exudation or effusion has been poured out in such quantity as to cause drowsiness and stupor that our suspicions are awakened, and thus it is very difficult to understand how blood-letting or purging could facilitate its absorption. Besides, we have seen that the tendency of such effusion is to pass into the circulation. Hence, the treatment which favors the reabsorption of the exudation, as I have previously explained, must be most effectual. For this purpose time is required, and the vital strength, instead of being lowered, should be supported. In short, the duty of the practitioner is to support the economy as much as possible, to give nutrients with moderate stimulants, to unload the bowels, from time to time, artificially, by injections, etc., and in this way to gain time, which will enable the effused matters to pass through their natural transformations, to be absorbed and ultimately excreted. It has appeared to me that the collection of serous fluid, whether in the ventricles or over the surface of the brain, either with or without exudation, is consecutive on obstruc- 
tion of the vessels, and is, therefore, more allied to the dropsies than to inflammations. It is the collection of serum which does the mischief-presses on the brain, and causes the coma and stupor. If so, the occurrence of those symptoms should be regarded as secondary, instead of as primary, and as analogous to ascites, dropsy, or anasarca, following disease of the kidneys. These pathological considerations are, it appears to me, wholly opposed to the idea of blood-letting and antiphlogistics being beneficial after effusion has occurred."

\section{Equine Chorea, or Stringhait.}

Chorea, or stringhalt, consists of an irregular and involuntary spasmodic action of some of the muscles of the hind extremities. Equine chorea differs somewhat from human chorea. In the latter case, it usually begins with slight twitches in the muscles of the face, or in the upper extremities, and various parts of the body twitch and contort in such a singular and unnatural manner, that some persons have denominated the disease "insanity of the muscles."

In the case of horses, no such "insanity of muscles" has been observed. It is mainly confined to the posterior limbs. Mr. FEron, a distinguished V. S., contends that stringhalt bears some affinity to what is known in human medicine as chorea, or "St. Vitus' dance." He does not, however, wish to convey the idea that they are essentially the same disease, only they are both of a convulsive or spasmodic character, wherein the mind, will, or instinct has lost more or less of its control over the voluntary muscles of the hind extremities, and the peculiar feat is thus accomplished. When the animal has lifted his hind leg from the ground, which is always done with a convulsive twitch, the fetlock nearly approaches the belly, and, by some other remarkable irregularities in its action, before the foot can be replaced on the ground, displays such unnatural movements as to convince us that volition is impaired. Hence, we may infer that, in a majority of cases, stringhalt is the result of some abnormal condition of the nervous system. What occasions that condition is a matter of fact and argument, which remains as an open question for some future pathologist to decide. So far as the author's experience goes, he is satisfied from actual dissection of the parts, that some 
stringhalt horses are the subjects of ulcerative disease of the hockjoint, and they finally become sprained.

In such cases, it is possible that stringhalt may be the result of hock disease, which irritates the nerves in the vicinity of the part, and thus affects that portion of the nervous system which controls the muscles concerned. ANDral tells us that "chorea, like epilepsy, may be excited by irritation." Hence, in the commencement of spavin, or so soon as osseous incrustations are thrown out, they may, during the active motion of the joint, produce local irritation, of nervous filaments, in the vicinity, which irritation may be communicated to larger and more important nerves, and thus induce stringhalt.

We all know that local irritation produced by the prick of a nail, or sometimes docking and pricking, is often productive of locked-jaw, simply because some nervous or tendinous structure: is injured; and so the local irritation in the region of the hock may be productive of the malady now under consideration. The author has paid considerable attention to this subject, and is convinced that almost all horses of the nervous or excitable temperament, the subjects of inter-articular spavin, manifest more or less catching-up of the limb (stringhalt) at some period during the progress of spavin, and that when the bones of the hock are anchylosed (united), and the motion and irritation of the parts have ceased, the stringhalt becomes modified. The reader, however, must not receive this opinion as absolute; for at times, in consequence of some peculiar idiosyncrasy, the stringhalt gradually grows worse. Among some members of the human family, of the peculiar temperament to receive the impression, almost any thing which makes a forcible impression upon the nervous system may act as an exciting cause of chorea. The subject is a very important one, and most veterinary writers of the English school have rather mystified the subject, and at last have come to the conclusion that the disease is incurable. This was formerly the author's opinion, but a change has taken place in his sentiments, and he is unwilling to deprive the afflicted animal of the benefits of progressive science. More light on the subject may enable us to effect many cases of cure; at least it will enable us better to understand the pathology of the case, which is an important step in the right direction. In view, therefore, of furnishing the reader with some useful information, whether he be a veterinary 
practitioner or not, we shall borrow from analogy, in the introduction of the following article from "Watson's Practice" :

"In certain of M. Magendie's experiments on animals, the following curious facts were ascertained: When a vertical section of the cerebellum of a rabbit was made, leaving one-fourth of the whole adhering to the crest of the right side of the cranium, and three-fourths to that of the left, the animal rolled over and over incessantly, turning itself toward the injured side. The same phenomenon occurred upon the division of the crus cerebelli. The animal lived for eight days, and continued, during the whole of that time, to revolve upon its long axis, unless stopped by coming in contact with some obstacle. How like is this to the symptoms exhibited at one period in the girl whose case is related by Dr. Watts. Nor is Dr. Watts's case a singular one. M. Serres has described another much resembling it. A shoemaker, sixtyeight years old, of intemperate habits, after one of his debauches, exhibited a kind of drunkenness which surprised his friends. Instead of seeing objects turning around him, as a drunken person is apt to do, he thought he was himself turning, and soon began to revolve, and this lasted till he died; and when his head was examined, extensive mischief was found in one of the pedicles of his cerebellum. Again: M. Magendie noticed that when the upper part of the cerebrum is gently removed in birds and mammalia, they become blind; but no affection of the locomotive porvers is produced. No further result is occasioned by the removal of a portion of the gray matter of the corpora striata; but when the striated part is cut away, the animal immediately darts forward with rapidity, and continues to advance as if impelled by some irresistible force, until stopped by an obstacle; and, even then, it retains the attitude of one advancing. The experiment was tried, with the same results upon various species of animals-dogs, cats, hedgehogs, rabbits, Guinea-pigs, and squirrels. It seems that there are horses that can not back, although they make good progress enough in a straightforward direction. Now, Magendie says that he has opened the heads of such horses, and has always found in the lateral ventricles of their brains a collection of water, which must have compressed and even disorganized the corpora striata. It has been further ascertained, by the same experimenter, and by others, that certain injuries of the cerebellum cause animals to move backward contrarily to their will. If the tail of 
the animal so mutilated be pinched, he still persists in his retrograde course. Injuries of the medulla oblongata had the same effect. Pigeons, into which he forced a pin through that part, constantly receded for more than a month, and even flew backward. A section of the medulla oblongata, where it approaches the anterior pyramid, gives rise to a movement in a circle like that of a horse in a mill, the animal, in its walk or its flight, bearing round continually to the injured side. Surely we have, in these facts, supplied by experiments on living animals, and by observation of the phenomena of disease in the living human body, some of the materials for a more exact knowledge, both of the physiology and of the pathology of the nervous system, than we have reached. M. Magendie supposes that different portions of the encephalon are endowed with energies which tend to cause motion in various directions; that in the healthy state these balance each other, and that a preponderating impulse can be given to any one of these forces by the will; but that when the equilibrium is destroyed by disease, the will is not sufficient to counteract the tendencies which are then brought into play. Mr. Mayo offers a different explanation of the phenomena. He supposes that the injuries inflicted on the nervous matter produce a sensation analogous to vertigo, and that the animal conceives itself either to be hurried forward, and makes an exertion to repel imaginary force, or to be moving backward, or turning round in one direction, and endeavors to correct this by moving the corresponding muscles."

Treatment.-In stringhalt, it is nearly always safe for us to conclude that it must be treated on the same general principles which obtain in the management of other nervous disorders of a chronic character, viz .: in the use of tonics and anti-svasmodics. Take, for example:

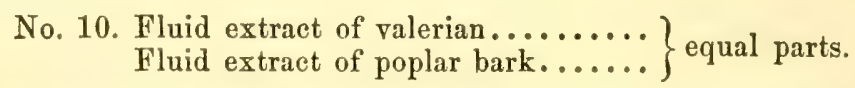

Dose, one ounce, morning and evening; to be placed on the tongue. The spine and affected limb or limbs should be rubbed every night, for a couple of weeks, with a portion of the following:

No. 11. Fluid extract of poppies.........6 oz. Proof spirit..................... 1 pint. Mix. 
Should the animal prove to be spavined, the following liniment is recommended:

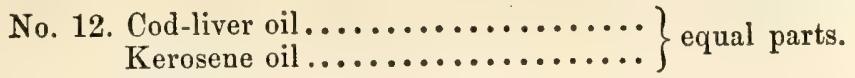

Mix.

A small quantity of this liniment should be rubbed on the seat of spavin, inside of the hock, once or twice daily, until counterirritation is accomplished, which shall be known in consequence of the hair falling off; then discontinue the liniment, and lubricate the affected parts with olive oil, and wait patiently, so as to see what Nature will do for the ease. "Patient waiters are no losers." The popular, or rather the ancient, method of treatment does more harm than good.

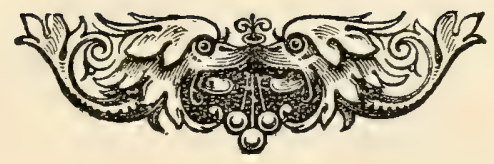




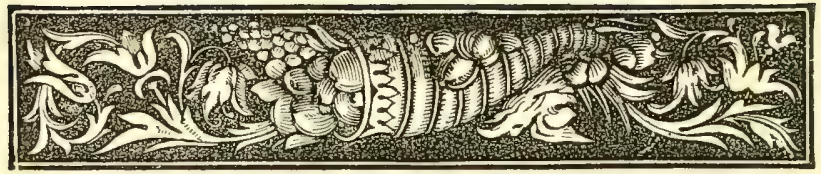

\section{SECTION IV.}

\section{DISEASES OF THE RESPIRATORY PASSAGES AND ORGA NS.}

Importance of Ventilating Stables-Brief Exposition and Description of the Function of the Lungs-Spasm of the Muscles of the Glottis and EpIglottis-Laryngitis, (Suppurative and Inflamatory)-Croup-Chronio Cough-Roaring-Polypus-Bronchocele-Influenza, or Epizootic Catarrh-Pneumonia, or Inflammation of the Lungs, including Typhotd Affections, Pleurisy, and Dropsy of the Chest.

\section{Importance of Ventilating Stables, in view of PREVENTING Disease OF THE Lungs.}

TT was the intention of the Creator that all animals, so long as 1 they were permitted to exercise their natural instincts, and thus comply with the requirements of physiology - the science of life-should enjoy health and long life. Hence a great amount of disease and death results from the evils of domestication.

One of the conditions which physiology imposes, in order that a horse shall enjoy health, is, that the atmosphere, at all times, and under all circumstances, shall be uncontaminated, so that the blood shall be decarbonized and purified of the defiling elements acquired in the course of circulation.

Let the reader understand that the lungs are something like a sponge, elastic, composed of a myriad of cells. In the former, however, these cells have a vast internal surface, communicating with each other up to their common origin, the bronchial tubes and windpipe. On their internal surface we find a delicate yet highly important membrane permeable to atmosphere. In extent, it is supposed to occupy a square surface equal to that of the external body. In contact with this membrane comes the atmosphere. If pure-zephyr-like-it fans into healthful blaze the flame of life, upheaving from the living Vesuvius arid lava, in 
the form of carbonic acid gas, almost as destructive to animality as that issuing from its great prototype proves to vegetation.

The stable atmosphere being pure, and the lungs in working order, the blood is well arterialized, capable of supplying the waste of the animal machine and renovating its tissues. On the other hand, should the atmosphere be impure, it fails to vitalize the blood. The latter is unfit for the purpose of nutrition, and may be considered a non-supporter of vitality. Hence the need of pure air, the breath of life.

But are horses always furnished with pure air? Let the owners of unventilated, crowded, filthy, down-cellar and low-roofed stables answer. Let those who have stables in the region of swamp, sewer, and stagnant pools of water answer. In such locations disease and death run riot, and the noble companion of man, instead of being within the ramparts of the science of life, is on the margin of death's domain. He may exist for several days without food and water, yet the consequent result is nothing when compared to that occasioned by breathing an atmosphere highly charged with emanations arising from his own body excrements and decomposing bedding.

A horse is said to consume in the lungs, in the course of twenfour hours, ninety-seven ounces of carbon, furnished by venous blood. In order to perform this feat, he requires 190 cubic feet of oxygen. Now, suppose there are ten horses occupying the stable. They require, in the same time, 1,900 cubic feet of oxygen, and consume 970 ounces of carbon. They are supposed, also, to give out from the lungs a volume of carbonic acid gas equal to that of the oxygen inspired; and supposing the atmosphere to be saturated with only five per centum of the former, it is a nonsupporter of life. Hence, a horse shut up in an unventilated stable must, sooner or later, become the subject of disease. The evil may be postponed, but the day of reckoning is sure and certain.

Diseases, such as horse-ail, influenza, catarrh, strangles, and glanders, often originate and prevail to an alarming extent in the unventilated stable and pest spot; while in other locations, favorable to the free and full play of vital operations, the favored ones seem to enjoy a remarkable immunity from the prevailing disease, or epizoötic.

Stablemen and husbandmen are often led to remark, that when 
they keep but few animals, disease and death, except in cases of accident or old age, are quite rare, but so soon as they crowded the same, sickness and death were the consequences. In view of supporting this theory, we may be permitted to remark that ship and jail fevers may be manufactured ad libitum, at any time when a large number of persons are congregated together in a given space, no provision having been made for the admission of pure air. The unfortunate prisoners in the Black Hole of Calcutta are an example, and the mortality occurring on board our emigrant ships furnishes another illustration.

A number of horses were once shipped from England to Spain, and on the passage, a violent gale arising, it became necessary to batten down the hatchway. The consequence was that most of them ultimately died of either glanders or farcy. We contend, therefore, that the active or morbid germ of disease enters the living citadel through the pulmonary tissue in an insidious manner, and, therefore, much oftener than the generality of men would be likely to realize. Therefore, it is a matter of vital importance that attention be paid to the ventilation of our stables. If proper sanitary regulations were established, and fully carried out in all our stables, glanders and other infectious diseases would be exceedingly rare. They are so among horses free from the control of man, whose stalls are broad as from ocean to ocean, their height ranging from earth to regions above, the space pervaded by a pure atmosphere concocted by the Great Chemist, pure as the pearly drops and refreshing as the morning zephyr. In such locations death has no terrors nor disease any victims.

\section{Brief Exposition of the Function of the Lungs.}

The principal function of the lungs is to arterialize or decarbonize the blood; that is, purify it. This arterialization of the blood, which goes the rounds of the circulation, is more essential to life than either food or water ; for men and animals can exist for several days, perhaps for two weeks, without food, yet the same can not live over a few seconds unless supplied with a sufficiency of atmospheric air. Hence, in a popular sense, pure air is the "breath of life."

The functional acts of respiration are necessarily divided into two parts; and in cattle the number of respirations are about 
twelve per minute, varying, however, according to the temperament of the animal and the condition he may be in at the time of making the observation; while in horses the respirations are more frequent, varying in health, and when at rest, from fourteen to trienty-five; yet, under excitement and disease, they sometimes number over one hundred.

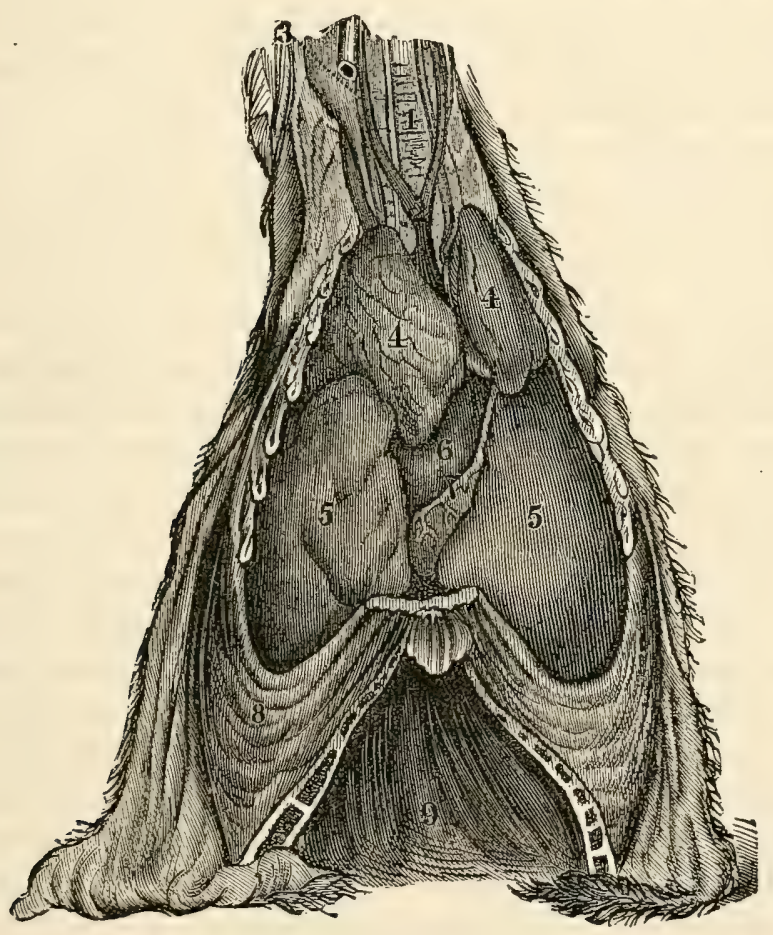

THE CONTENTS OF THE THORAX.

Explanation.-Fig. 1, Trachæ ; 2, Bifurcation of the carotid artery; 3, Internal carotid artery; 4 4, Anterior lobes of the lungs; 5 5, Posterior lobes of the lungs; 6 , The heart; 7, Coronary artery; 8, Cartilages of the false ribs; 9, The diaphragm.

When an animal is located in a pure atmosphere, and the lungs are in good working condition, all the impurities contained in venous blood are brought into the presence of oxygen through the lining membrane of the air-cells, and thus a change in the color and character of the blood is immediately effected. In the first place, the venous blood, as it appeared before having been submitted to the action of the atmosphere, was of a dark purple 
color. By union with the oxygen of the atmosphere, it has changed its color to one of scarlet. Next, the union of oxygen with the carbon of the blood liberates carbonic acid gas and vapor. The blood is now fit for circulation and for the renovation of the tissues. It appears, therefore, that as carbon exists in the venous blood, the lungs must be the pulmonary furnaces. The air-cells are flues or safety-valves, the membrane of the cells being permeable to oxygen, yet suffers not the blood to escape. Pulmonary combustion, therefore, bears some analogy to the combustion of carbon or charcoal in a stove; for, in that case, the oxygen causes the generation or evolution of carbonic acid gas.

\section{Brief Description of the Anatomy of the Lungs.}

The lungs, in common parlance, are known as the lights. They occupy the thoracic cavity, or chest. They are divided into right and left lobes, with a septum or partition between, which makes a double organ. This partition is a duplicature of the pleura, or membrane which completely lines the chest. When the lungs are healthy and properly inflated, they occupy the whole cavity of the chest; but when an action of expiration is effected, they are in a comparative state of collapse, occupying but a very small portion of the thoracic cavity. The lungs are composed of arteries, veins, absorbents, bronchial tubes, air-cells, and also what is known as their parenchyma, or substance. A healthy lung, when thrown into water, will float on the surface; while, on the other hand, a diseased lung, in a state of hepatization or condensation, sinks like a stone. In the fetal state, and when the lungs have never been inflated, they also sink when thrown into water.

\section{Spasm of the Muscles of the Glottis and Epiglottis.}

The aperture leading into the larynx and windpipe is termed the glottis. Spasm of the muscles of the glottis is one of the most terrible accidents that can possibly occur in either man or horse. I view it in the light of an accident, because it usually occurs without warning or any other premonitory symptoms, selecting its victims in the very prime of life, and carrying them off, usually, in the course of a very few seconds. The terms cramp and spasm mean the same thing. When a man, while bathing in the river 
or sea, is suddenly seized with cramp, it is nothing more nor less than spasm of the flexor muscles of his limbs. Sometimes, however, the extensor muscles are affected. In either case, unless assistance be at hand, the person is apt to find a watery grave. Cramp or spasm of the intestines is the same form of affection, only it is confined to the muscular fibers of the intestines; and whenever it occurs in the limbs or intestines, it is always accompanied by excruciating pain and torment.

Treatment.-As regards spasm of the muscles of the glottis, it is very apt to prove fatal, either in consequence of lack of knowledge of the proper mode of treatment, or in failing to apply the remedy which the urgency of the case demands. I allude to the operation of tracheotomy, which consists of making an incision into the windpipe and inserting a tube into the same. A tube may not always be at hand, but this must not deter us from operating; for, by some means or other, air must be admitted, even if it be necessary to dissect out a piece of the trachea, which I always do in the case of a horse, whether I have a tube by me or not. Very little pain attends the operation, and that only occurs when cutting through the skin; for the windpipe, being composed of cartilage, is comparatively insensible. It may be policy, when the subject is not in immediate peril of his life, to resort to some counterirritant and antispasmodic liniment (equal parts of spirits of camphor and tincture of lobelia); but when the danger is imminent, and the finger of Death is plainly on the patient, we only waste precious moments in the use of outward applications.

The following case, reported by J. B. Dosson, V. S., may possibly prove both interesting and instructive to some of our readers:

"At night a messenger came, saying the horse was very ill. Upon entering the stable, the animal presented the following symptoms: He was stretched out his full length in the stable, apparently in the agonies of suffocation; and such was the difficulty attending respiration that he positively screamed, in performing the act, so as to be heard at some considerable distance. I had not been with him many seconds, however, before he was slightly relieved, and in about ten minutes the spasm passed off, leaving him, with the exception, of course, of great exhaustion, otherwise as well as ever. Viewing the case as one of spasm of the muscles of the glottis, I applied stimulants, and ordered constant fomentations to the larynx, and left him with directions to be closely 
watched, in case the spasm might return. In the middle of the night I was again called, in haste; and this time the breathing seemed, if possible, worse than before, and it was evident that, if no relief were afforded, the animal must soon be suffocated. I accordingly, with some difficulty, owing to his struggles, performed tracheotomy. The relief was instantaneous, and he was soon on his legs, and anxious to feed. From this time the tracheotomy tube was kept in his throat for a week, when, considering that the glottis might have resumed its normal functions, I placed a cork in the orifice of the tube and sent him to work, as an experiment, ordering the cork to be removed if any symptom of suffocation should come on. We, however, found that the slightest effort at exertion brought on a fit of bad breathing, and it became evident that he would not work without the tube. The horse was accordingly sent to his regular work with the tube in his trachea, and, for three or four months, he did his accustomed labor with it in. At the end of that time, however, by an accident, the tube fell out, when he was some miles from home, and the horse coming home tolerably well without it, the horse-keeper neglected to inform me of it for a day or two, and when I saw my patient, the aperture in the muscles of the neck had closed. As the horse now seemed to work well without the tracheotomy tube, it was not reinserted, and from that time to this (more than two years) he had not another attack."

Should a case of this kind occur, and the consulted party have no tube at hand, he must pass a ligature through each side of the orifice, including a portion of skin and muscles. Each ligature is then to be passed over opposite sides of the neck, and tied at the upper part, just tight enough to keep the lips of the wound apart. In this way air is admitted into the windpipe, and thus the animal is out of danger.

\section{Laryngitis (Suppurative and Inflammatory).}

Laryngitis signifies inflammation of the lining membrane of the larynx. On applying the ear to the region of the throat, the locality of the affection is very evident, from the fact that no such embarrassed sound can be elicited in the lower part of the windpipe. Hence it must be inferred that the disease is located in the upper, or superior, passage of respiration. 


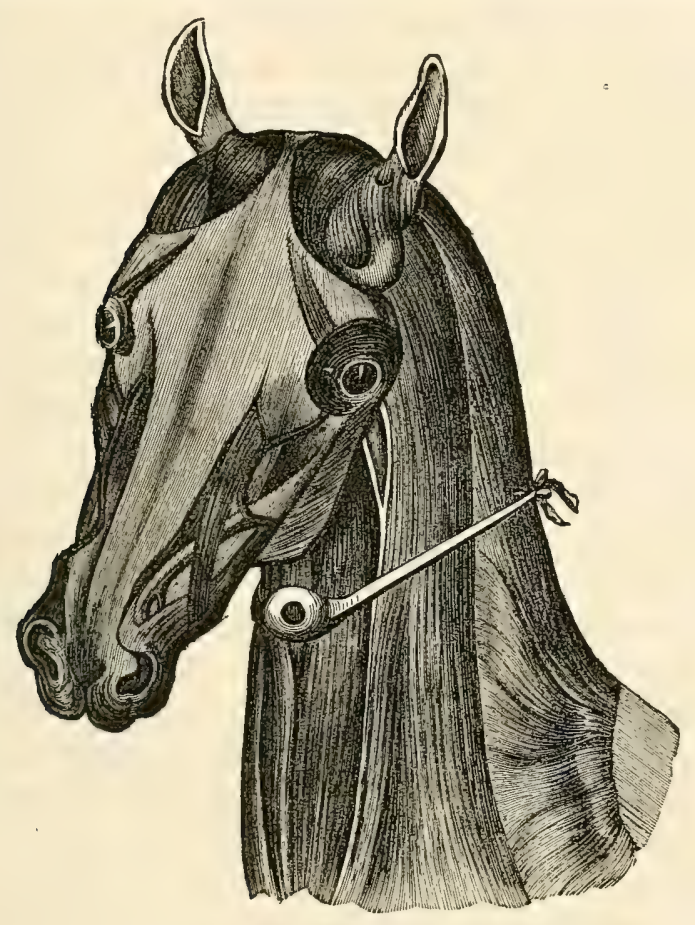

TRACHEOTOMT TUBE INBEETED IN THE WINDPIPE.

The inflammatory condition exists during a limited period, varying from one to five days. Then commences the suppurative stage, which is accompanied by a copious discharge, from both nostrils, of a secretion somewhat resembling pus, or matter.

Causes.-As regards the special cause of this affection very little is known. It may be supposed, however, to originate in a common cold, or catarrh; yet it is well known that horses are sometimes attacked with it that are not exposed to the ordinary influences which induce a catarrhal affection. Therefore we may infer that such disease will occasionally occur, either spontaneously or accidentally, as the case may be, in spite of our best efforts to prevent it. As the old saying is, "Nature is ever busy in maintaining the integrity of the vital forces of the animal economy. Any deviation from the prescribed laws which physiology imposes is a sure and certain cause of disease."

I now propose to introduce a case which, at the time of its occurrence, attracted much attention and curiosity, both on account 
of the great value of the animal, and the desperate state of the case at the time it fell into my hands. It will also serve to indorse the old aphorism that "where there is life there is hope."

Record of the Case.-The patient was a Patchen colt, aged four years, a very handsome and promising animal. He was purchased by Mr. McPhenson, of this city, for the sum of two thousand dollars, and, at the time of his sickness, was considered worth three thousand. But disease neither respects man nor horse. Each have, once in awhile, to suffer and groan, swallow drugs and get well, or die, as the case may be. And as regards the horse, the more costly shall he be, the more likely is he to get sick, from the fact that valuable horses are almost always overfed and petted, and receive too much care and attention. On arriving at the stable where the animal was located, I found him in a dangerous condition. He appeared to be gasping for breath. A loud stertorous noise, which could be heard at some distance, indicated the nature of the difficulty as depending on obstruction within the larynx. The pulse at the angle of the jaw was very indistinct. Both pupils of the eyes were dilated, or in a state of amaurosis. The extremities and external surface of the body were deathly cold. The tongue and visible mucous surfaces were livid, indicating speedy death. Once in awhile the animal would be seized with a convulsive or spasmodic fit of coughing, which, every time, seemed to threaten his life. In those fits of coughing he passed from the nostrils a sort of cheesy matter, which appeared to be mixed up with a diphtherial exudationyellow secretion and froth. Under the above circumstances, any attempt to administer medicine was deemed unsafe. I therefore decided to perform the operation known as tracheotomy, which was done, in the following manner: Having secured the services of a couple of assistants, the horse was led to a convenient spot in the center of the stable. An incision was then made through the skin, to expose the trachea, about midway between the jaws and breast-bone. The trachea was now punctured by means of a pointed scalpel, and a probe-pointed bistoury was used to dissect out a piece of the windpipe, corresponding to the size of the tracheotomy tube. This tube, after being inserted into the windpipe, was secured in place by means of elastic tape, which was passed around the neck and tied. On the introduction of the tube the alarming symptoms immediately subsided, and the act 
of breathing was performed through the tube. I then applied a counter-irritant to the throat, composed of cod-liver oil and spirits of camphor, equal parts; ordered a warm bran mash, into which was sprinkled one ounce of powdered chlorate of potass.

For several days a very copious discharge from both nostrils occurred; also from the orifice made in the windpipe; so that the tracheotomy tube had to be removed and cleansed several times, both during the day and night. Four days after the operation, at night, the tube accidentally slipped out of the windpipe, and the mishap was not discovered until morning, when I found that air from the lungs had escaped into the cellular tissue, and inflated it so that the soft parts in the region of the neck and head appeared much swollen. I gave the patient, twice daily, one ounce of hyposulphite of soda, and had the swollen parts well rubbed with liniment. On the fifth day I removed the tube from the windpipe, and allowed the animal to breathe through the artificial opening, he still being unable to respire through the nostrils. At the expiration of fifteen days from the commencement of the animal's sickness, the wound in the windpipe was closed, natural respiration having been restored. During the treatment of the patient, he was allowed, occasionally, flaxseed tea, with about one ounce of chlorate of potass per day. He had good attention, or, rather, good nursing, and finally was again restored to usefulness.

In the early stage of laryngitis, or, rather, the inflammatory stage, cold water bandages should be applied to the throat, and a few doses of fluid extract of gelseminum, at the rate of one fluid drachm per dose.

\section{Croup (Cynanche Trachealis).}

Croup (cynanche trachealis), or, as it is sometimes termed, tracheitis, manifests itself in the form of a violent and sudden disease affecting the mucous membrane of that portion of the air-passages which lies between the laryngeal cartilages and the primary passages of the trachea, or windpipe. CondIE contends that, in the majority of cases, the inflammation in croup commences in the mucous membrane of the larynx, and from thence extends into the trachea. The disease is, therefore, strictly speaking, a laryngeo-tracheitis. In many instances, however, we have reason to believe that the inflammation commences in the bronchi, 
and from thence extends to the trachea; but there are, we suspect, very few cases indeed, if any, in which the disease is confined to the trachea. In cases of croup that have terminated rapidly in death, the inflammation and pseudo-membranous exudation which form so striking a feature in this disease are found only in the larynx and upper portion of the trachea. When death occurs at a later period, the exudation is often present in the trachea alone, or in the trachea and bronchi. It is never found to exist in the latter tubes alone. It has been attempted to be shown, by JURIN and others, that in the ordinary form of croup the disease is, in its first stages at least, simply a tracheitis, and that in the more violent and rapid form (suffocating croup) the inflammation is confined to the larynx. Although this is not strictly true, as is proved by the result of numerous dissections, yet our observations have shown us that in cases marked by symptoms of great violence, which are sudden in their onset and rapid in their progress, the indications of inflammation are to a much greater extent, and the pseudo-membranous exudation more copious about the larynx, glottis, and upper portion of the trachea, than in cases in which the disease succeeds to bronchitis, runs a more protracted course, and is attended by symptoms of less violence.

The disease generally makes its appearance during the latter part of winter and in early spring, and most frequently occurs among horses just introduced to city life. The subjects such as the author has had oceasion to treat were young, most of them of the lymphatic temperament, having short, thick necks, which abounded in a profuse development of adipose and cellular tissues, which results in a rotund bodily conformation. This, perhaps, goes to show that some hereditary idiosyncrasy plays a part in the creation of the disease.

Causes.-The indirect causes of the disorder are embraced among the evils of domestication, which include errors in diet, impure air, and bad management. It usually dates its origin from what are generally considered as the causes of common cold. Several days previous to the attack, the animal appears " dumpish," drowsy, and inactive, having little desire for artificial food, and less for water. The eyes appear somewhat tumefied, and the respirations are laborious, having a peculiar, croupy sound, accompanied by a distressing cough. The respirations can be heard at a distance (as the disease progresses), and on making pressure in the region of 
the larynx, the animal evinces signs of uneasiness, suffocation, and pain. Soon the mucous, schneiderian, and conjunctivial membranes become reddened, the eyes protrude, the tongue tumefies, and external tumefaction in the region of the throat may be observed. Febrile symptoms are also present, manifested by coldness of the exterior and increased temperature of the interior parts. The nose appears dry, and the mouth is full of frothy saliva (sometimes lymphy mucus), and some difficulty is encountered in opening the latter.

History of the Disease.-The following case, from the author's note-book, will probably prove interesting to the reader: The patient, a roan mare, aged seven. Temperament, lymphatic. Just arrived, in company with several other horses, from Ohio. The property of Mr. Banford, Merrimac stable, Boston. Our patient had been "ailing" some three or four days before our services were secured, at which time she was laboring under "alarming symptoms" bordering on suffocation. The pulse was indistinct, small, thready, and difficult to number, yet probably exceeded sixty. The breathing was croupy, or stertorous, and could be heard at a distance of several yards. The nostrils were dilated, and from them issued a lymphy discharge, mixed with traces of blood. The submaxillary space was somewhat tumefied, hard, unyielding, and seemed to constrict the larynx and its associate muscles. The eyes were prominent, their membranes congested, and streaks of tears escaped over the inner canthus. The flanks were bedewed with a cold, clammy perspiration. The tips of the ears were quite cold, and the limbs the same. Auscultation, in the region of the larynx, revealed a very laborious and crepitating mucous rale. The trachea itself seemed to be free from obstruction. Thoracic auscultation elicited nothing tending to show that the lungs were involved.

The patient appeared to be in a deplorable, in fact dying, condition, making a sort of gasping effort to inflate the lungs. There was little time to spare; and being satisfied that the larynx was the seat of obstruction, we immediately cut down upon the trachea, about five inches below the thyroid cartilage, and amputated, from between two rings of the trachea, a piece, of an oval shape, corresponding in size to the caliber of Arnold's tracheotomy tube, which was the instrument used on this occasion. So soon as the opening into the trachea was effected, the patient experi- 
enced instantaneous relief from suffocation, and all danger from the same seemed to have immediately passed away. The tube was now introduced and secured around the neck in the usual manner, after which the patient received an aloetic enema, and had a strong counter-irritant applied to the submaxillary space and throat, after which the fauces were swabbed with a weak solution of alum, by means of sponge secured to a piece of whalebone. On examining the "swab," a sort of lymphy or albuminous concretion adhered to it; the mucous membrane of the larynx was either injected or œdematous. The pharynx was not involved, as the animal, shortly after the operation, drank two quarts of water, containing two drachms of nitrate potassa.

The tube remained within the trachea for a period of five days, during which time very little occurred worth recording, except that one night pneumatosis (distension of the cellular membrane with air) appeared, which yielded to a dose of hyposulphite of soda and an outward application of liquor ammonia acetatis; and, also, on the fifth day, a submaxillary tumor was punctured, which discharged freely. At the end of the above period, it was ascertained, by holding a lighted lamp to the nostrils, at the same time stopping up the orifice in the instrument, that the animal breathed through the usual channel. Then the instrument was removed, the parts cleansed, and secured together by suture. The wounds healed by the usual process, and, at the end of three weeks, the animal was disposed of, and went to work.

The operation of tracheotomy, formidable as it may appear to some, is unattended with danger; yet, like every other process of surgery or medicine, is only calculated to relieve certain states peculiarly adapted to the remedy. For example, the operation is admissible in cases of nasal obstruction, from the presence of nasal polypus or other causes; spasm of the larynx, threatening suffocation; suppurative laryngitis, when the animal is in great distress for "breath;" and in case of any foreign body oceupying the larynx, which precludes the possibility of continuing the process of respiration. On the other hand, the operation is worse than useless in eases of lung difficulty, bronchial obstruction, or when obstruction occurs within the trachea posterior to the usual point selected for the operation. 


\section{Cough in its Chronic and Acute Stage.}

According to veterinary jurisprudence, a cough, so long as it lasts, renders the animal unsound; hence it is very important that we should know something about its cause, nature, and treatment. Chronic cough is often the result of indiscretion in the treatment of influenza, distemper, and disease of the respiratory apparatus. It usually depends on a morbid and irritable condition of the membrane found on the interior of the respiratory passages. The cough is generally aggravated by over-exertion, especially when the roads are dusty. Food of an inferior quality, and that of a musty character, has the same effect.

It is generally supposed, by those persons who have not had the benefit of a medical education, that the presence of acute or chronic cough indicates diseased lungs, or disease in some parts of the organs of respiration. But this is not always the case; for, whenever the liver becomes diseased, the subject is very apt to be tormented with a harrassing cough, which lasts as long as that organ shall be the seat of disease. However, there are several symptoms to be observed in cases of functional or organic disease of the liver which are not present in lung disease; hence there is no difficulty in the way of making a correct diagnosis. And for the benefit of the non-professional, the author would inform them that, in all cases of liver disease, a marked yellow tinge will be observed on the visible surfaces within the mouth; the tongue slightly coated; the dung unusually dark colored, and voided in hardened lumps, and the urine also of a dark yellow color.

Treatment of Chronic Cough.-Give the patient daily one ounce of the fluid extract of Indian hemp (foreign), and offer him occasionally some flaxseed tea; or sprinkle in his food, every night, a handful of unground flaxseed. Should this fail to effect a cure, prepare the following:

No. 13. Fluid extract of bloodroot........4 $4 \mathrm{oz}$.

Fluid extract of pleurisy-root.......6 oz.

Common syrup................ $\frac{1}{2}$ pint

Mix.

Dose, two ounces per day.

The acute cough may be treated in the same manner as above, by substituting for the syrup half a pint of syrup of squills.

In the case of liver disease, accompanied by cough, give the 
animal one ounce of fluid extract of mandrake every six hours, until it operates on the bowels, or the membranes of the mouth lose their yellow tinge.

\section{ROARING.}

Roaring is usually the result of structural alterations within the larynx, or upper part of the windpipe bordering on the trachea. In mild cases of roaring, we usually find a thickened state of the membrane lining the upper portion of the respiratory passage; and when roaring is occasioned by thickening of this membrane, its degree depends on the ratio of decrease in the caliber of the tube breathed through. Roaring is a very aristocratic disease. Many of the very best and fastest horses in England were, and are now, notorious roarers. "Flying Childers," as fast a horse as ever wore horseshoes, was one of the worst roarers ever known. The story runs that when "Childers" was at full speed, his roaring resembled juvenile thunder! He could be heard when distant half a mile!

The worst form of this disease is whistling. This is the sharp shrill note not only occasioned by the thickening of the lining membrane of the primary passages of respiration, but by alterations in the form and structure of the larynx, the larynx being, in popular language, known as the "voice-box."

Roaring is more prevalent among stallions than mares and geldings, and the kind of horse most subject to it is the one having a thick, chunky neck, and having the angles of the jaws in very close proximity with the neck. Roaring scarcely, if ever, admits of a radical cure; and when of a hereditary or congenital origin, a cure is impossible. A roarer should never be incumbered with a check-rein; for it has the effect of causing undue pressure on the larynx, and thus augments the difficulty. Roaring: can, however, be relieved by an operation known as tracheotomy, which is performed at a point a few inches below the larynx.

At a meeting of the Imperial and Central Society of Veterinary Medicine, M. Leblanc read a communication on tracheotony which was performed on a carriage horse. The operation had been performed because the horse was a severe roarer; and he wore the tube eighteen years and a half, doing fast work all the time. The animal was destroyed at twenty-three years of age, the owner not desiring to make further use of him nor to sell 
him. Since the operation, Leblaxc had not observed any change in the horse, except a depression of the bones of the face. After death, the larynx was found very narrow, the mucous membrane and submucous cellular tissues were thickened, the epiglottis deformed, very obtuse, and everted at its free margin. The changes in the larynx were the original cause of roaring. The depression of the bones of the face was connected with constriction of the nasal chambers, and was evidently secondary to the change in the course of the air in the process of respiration. The parts of the trachea in contact with the tube had undergone a transformation into very hard tissue. It filled the trachea above the point where the tube had been introduced, and intermixed with this firm fibrous deposit was a cartilaginous and osseous tissue, which offered great resistance to the scalpel. Roaring, thick wind, whistling, etc., are often the sequel of strangles, influenza, laryngitis, and other affections of the respiratory passages, and hence have an accidental origin. In such cases, we may entertain a hope of doing some good by means of medicinal agents and counterirritation.

Treatment.-The medicines which have proved most successful in my practice are as follows:

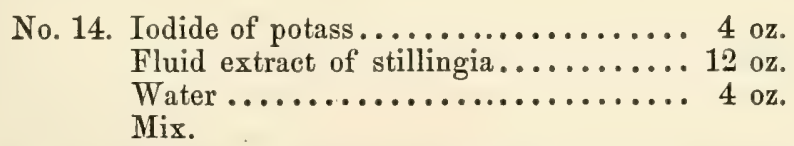

Give the patient two ounces daily, by means of a small vial; and rub the region of the throat every night with a portion of the following:

No. 15. Cod-liver oil

Spirits of camphor................

\section{Polypus within the Nostrils.}

Polypus is the name given to an excrescence, or tumor, which has its origin from the surface of the membrane lining the nasal cavities. It is designated as growing from a mucous membrane, having a narrow neck and body, resembling, in shape, a pear. The usual symptoms attending the presence of polypus in the nasal cavities are as follows: Difficulty of breathing, evidently occasioned by obstruction in the air-passages. 


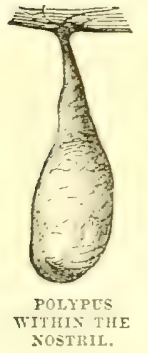

Percivalu informs us that the true polypus is attached to mucous membranes, and is usually found in the nasal cavities. He tells us that attending the difficulty of breathing is a mucous discharge from one or both nostrils, sometimes attended by a discharge of blood. Occasionally, however, pure blood runs continuously from the nose. Inspection in a full light discloses, higher or lower in the nostril, the rounded base of a polypus.

Treatment.-The services of a veterinary surgeon are, as a matter of course, here needed. The patient must be cast and the head fixed in a position so as to take advantage of the light. The operator then passes into the nostril and around the tumor an instrument called an ecraiseur, which will remove the tumor without loss of blood. If the instrument is not at hand, the surgeon will pass a ligature around the base of the tumor, and in the course of a couple of days it will be detached. PERcrVALL recommends that, in bringing down the tumor for operation, we must not use any great force. The pedicle being but a duplicature of the skin, and not a portion of the polypus itself, may be divided anywhere. In some cases, the polypus is so high up within the nostril that, in orter to get at its base, it becomes necessary to make an incision through the wall of the nostril.

Cinabert, in his "Veterinary Instructions," relates the following: "A horse in a cavalry regiment had been gradually losing flesh, and was quickly and painfully blown at every little exertion. Fetid matter began to run from his off nostril, and the gland correspondent enlarged. The horse was supposed by the sercent-furrier to be glandered, (there being no veterinary surgerms then in the French service,) and was treated accordingly. After at time, to the confusion and astonishment of the man, a fle hy subtance began to appear in the nostril, and which rapidly increated in size. At length a great mass protruded, and the farrier 'ut it otf'. To benefit followed; the nostril was still stopped, the breathing laborious, and the horse daily became thinner and wealier. After the lapse of a twelvemonth, the case attracted the attention of MI. Tears, the surgeon of the regiment. He cast the hores and slit up the nostril, when he not only found it completely filled with polypus, and the septum narium bulging into the other division of the carity, but, from long-continued inflam- 
mation and pressure, it adhered to the membrane of the nose in so many points, and so extensively, that it was impossible to get round it or move it. He contrived, at length, to pass a crucial bandage around it, and it was torn out by main force. Four considerable portions of the turbinated bones were brought away with it. The hemorrhage was excessive. He however filled the nostril completely with tow, and brought the divided edges of the false nostril together by sutures. In three days they were all torn out by the incessant attempts of the animal to get rid of the obstruction; but the horse eventually did well. The polypus weighed two pounds seven ounces."

GOHIER relates a case of a horse who had in his left nostril a polypus as large as a turkey's egg, of a grayish color and glossy surface, too high up to be reached with the finger, which prevented his breathing on that side, and gave rise to offensive effluvium, to enlargement of the lymphatic glands, but not to roaring. GoHIER slit up the nostril, and, with an iron rod with a notch upon its end, contrived to inclose its neck in the slip-knot of a ligature. In drawing this tight, however-which was, of necessity, done in an oblique direction - the pedicle was cut through. Little hemorrhage succeeded, although the tumor weighed twenty-four ounces. The slit nostril was sewn up, and. cold water injected into its eavity. A copious discharge from both nostrils followed, with swelling of the lymphatic glands. This was met by proper treatment, and in fifteen days the patient was sent out of the hospital.

\section{Bronchocele (COMmonly known as EnLARgement of The Thyroid GLands, or GoITrE).}

Bronchocele, or enlargement of the thyroid glands, is a disease which very frequently makes its appearance among certain breeds of horses and sheep, and is supposed to owe its origin to hereditary predispositions and influences. It is also very prevalent among members of the human family. It seems to acquire a home in certain localities in Derbyshire, England, where it is said to be a very common disorder; but its occurrence in other parts of that country is by no means frequent. Among the inhabitants of the Alps, and other mountainous countries bordering thereon, it is a disease very often met with.

The uncastrated animals seem to suffer, most. After they get 
to be about eight years of age, the tumors acquire such magnitude that they press on the vocal organs, so as to decrease the caliber of the larynx, and thus the animal becomes a "roarer." Judging from what we know of the disease in the human subject, the glands, while undergoing enlargement, do not occasion much pain. The danger arises from mechanical causes, and the death of the subject, if it occur, is due to asphyxia, or suffocation. Among horses there are very few fatal cases on record. The reverse is the case as regards sheep. When these glands are much enlarged, and the animal is near or past the adult age, it is very unsafe to attempt their removal by means of the knife; for at this stage they are highly vascular, and the arteries which run into them are much enlarged. The operation has been suc-

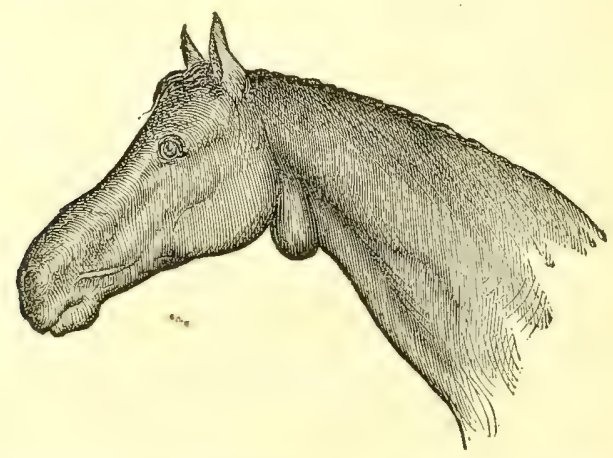

BHOWING THE TOMOR OF BEONOHOCELE IN THE REGION OF THE THROAT.

cessfully performed on lambs, but it must be done when they are quite young, and the artery must be secured before the gland is extirpated, or the animal will bleed to death in a few seconds. It is well known among the members of the profession that the disease is incurable; and the same remarks apply to all hereditary diseases, yet the growth of the glands may be retarded by means of local and constitutional treatment.

The thyroid glands are two ovoid bodies, varying in size from a filbert to an egg, located in the region of the thyroid cartilage (throat), one on each side of the trachea (windpipe). Their attachments are cellular. When cut into, they exhibit a porus texture, highly vascular, well supplied with blood-vessels. Very little is known of their physiology. They are called vascular 
glands without ducts or outlets, and thus they correspond with the spleen, thymus gland, and supra-renal capsules, all of which, when in active operation, are largely supplied with blood. As regards the function of each, they may be supposed to separate certain materials from the blood, and only differ from ordinary glands in not having a direct outlet. Consequently, not knowing the precise part which the thyroid glands play in the animal economy, it would be very unwise to remove them, when their enlargement, in some cases, amounts to little else than an "eyesore." Their enlargement is generally the consequence, and not the cause, of disease. Men, horses, dogs, and cattle of the scrofulous diathesis, are known to have what may be termed chronic enlargement of these glands, and this peculiarity would seem to indicate that the thyroid glands are somewhat associated with the lymphatic system. Then, again, we find these glands enlarged in cases of throat and lung difficulties-in catarrh, influenza, distemper, etc.-so that their extirpation would not remove the original difficulty. In such cases they decrease in size as soon as the original malady ceases. The enlargement is not then of a permanent character.

Treatment.-Should the enlargement appear to exist independent of febrile symptoms, twenty-five grains of the iodide of potassium may be given daily, in water (which the patient will not refuse to drink), and a small portion of the ointment of iodide of potassium may be rubbed on the enlargement, with decided advantage:

No. 16. Iodide of potassium.............. 1 part.

Lard....................... 8 parts.

Mix.

On the Action of Iodine.-Iodine and its compounds are the principal agents used by veterinary surgeons for the treatment of glandular affections, and they supersede, to a certain extent, the preparations of quicksilver, in the form of mercury, which are apt to be absorbed into the system, and thus produce mischief.

Morton, in his "Manual of Pharmacy," thus alludes to iodine: "The action of iodine and its compounds is markedly seen on glandular structure, and newly-formed and abnormal growths. For the latter they seem to manifest a decided preference, which renders them so valuable as therapeutic agents. Their influence is that of a stimulant to the absorbents; and by means of these 
vessels, when those agents have been long and injudiciously given, it is recorded that the mammæ of women and the testes of men have almost disappeared. A case illustrative of the effects of iodine on the glandular system was related by Mr. Wardle, who employs this agent largely, and with considerable success. He had been for some weeks exhibiting the iodide of potassium, and also applying it externally to a bull for an enlarged parotid gland. The reduction of the swelling having been accomplished, his attention was directed to the testicles of his patient, which had become so much diminished in size; and it was also found that the animal had no desire to copulate. Four months generous feed, however, effectually restored the parts to their pristine state.

In chronic enlargements of the submaxillary, parotid, mammary, and other glands; in tumors of long standing; for thickening of the integuments, and indurated swellings about the joints, in all our domestic animals, and for unhealthy ulcerated surfaces, the use of iodine and its compounds is indicated, combining both their internal and external employment. The latter should be accompanied with friction; and so soon as soreness is induced, the application of the compound must be suspended. A desquamation, or scaling off of the cuticle, or skin, usually follows this, and a reduction of the swelling will soon after be pereeptible. Unfortunately, it is too often the case that, if the expectations of the practitioner are not at once realized, the agent is discarded as useless. Now, the compounds of iodine rank among those substances whose operation is slow, but which, at the same time, constitute an important class, as their effects are permanent."

\section{Influenza, or Epizootic Catarrh.}

Influenza is a disease of a catarhal character, always accompanied by greht weakness or debility. It is apt to migrate from one tissue to another, and thus produce various complications. In stables that are badly ventilated, or over-crowded with horses, the malady is apt to make sad havoc, and assume a malignancy ending in typhoid or typhus fever.

In some cases the heart or lungs become congested, and thus their function is embarrassed. The blood accumulates carbon, the heart fails to propel, and the lungs to vitalize the blood. This state demands stimulants of a character that shail excite vital action, 
and tend to effect chemical changes in the blood. The most valuable agent, in this view, is carbonate of ammonia. In veterinary practice this agent is considered as one of the most valuable diffusible stimulants. It acts first on the nerves of the stomach, and through them excites the whole nervous system. It may be given in doses from one to three drachms, either in the form of bolus or drench. Its stimulating influence over the heart and nervous system may be increased by the addition of one or more drachms of good Jamaica ginger. This treatment may be continued until congestion subsides, which may be known by the fullness of pulse and heat of the external surface of the body, and by other symptoms which usually attend fever in its inflammatory stage.

The exciting or stimulating plan of treatment must never be entirely abandoned. To husband the powers of the system, and thus guard against subsequent prostration, must be our chief object. Although the case may require a sedative to-day, in the form of two or three drachms of fluid extract of gelseminum, yet, in the course of a very few hours, the prostration becomes so apparent that we are again compelled to exhibit life-sustaining agents. Excitants, or counter-excitants, are also, at times, needed externally. If the patient has a deep-seated cough, strong liniment, composed of oil of hartshorn (and sometimes oil of camphor), must be applied. Often I anoint the parts with a thick paste, composed of mustard and vinegar. The membranes of the fauces (throat) are excessively sore; then the same course has to be pursued. At the same time, a mucilaginous drink, composed of flaxseed sweetened with honey, must be allowed. The nervous system must also be aroused by the application of the above liniment to the spinal column. At another stage of the malady, diaphoresis (increased perspiration) must be excited by clothing the body with flannel, and drenching with a preparation known as solution of acetate of ammonia, to the amount of six or eight ounces per day, until the skin feels soft and warm. In case ædema (local dropsy) of the body or extremities sets in, the patient then requires diuretics. One or two ounces of sweet spirits of niter, or two ounces fluid extract of buchu, may be given in linseed tea, until the kidneys respond, and the secretion of urine becomes augmented. The bowels are sometimes inactive, but that inactivity may be corrected by adding to a bran-masb from four to six drachms of fluid extract of mandrake. Occasionally the patient becomes uneasy, paws with his fore-feet, and 
evinces signs of abdominal pain. In that event, he should have two or three drachms of fluid extract of Indian hemp. These latter symptoms indicate danger, showing that the bowels are congested, and the result may be mortification of the same.

The following case, occurring in the author's practice, goes to show that influenza may sometimes be followed by an acute disease of the brain, pleurisy, ete.: The patient was a bay gelding, aged about eight years. The groom informed me that the disease was ushered in by a shivering fit. Soon a discharge from both nostrils ensued. The throat became sore; cough and laborious respiration followed, and the animal became so weak that it was almost impossible to back him out of the stall. He ate but little, and in the act of drinking, the water returned through his nostrils. The treatment was commenced in the usual manner, by administering tonics and stimulants, which seemed to have a good effect on him. He also received enemas of soap-suds; yet, notwithstanding, symptoms of pleurisy supervened. He appeared to suffer much pain, and was very unwilling to have the region of the chest explored. The least pressure on the region of the intercostal spaces would cause him to grunt or groan with pain. The pulse was active and wiry, and the visible surfaces were much reddened. I administered a full dose of powdered niter, and twenty drops of aconite; and fearing effusion into the chest, I plastered the walls of the same with hot vinegar and mustard. The latter made the patient very uneasy, and he cut up all kinds of capers. In the course of a few hours I repeated the dose, and left the patient for the night. Next morning I again visited him, and was informed that he had made several attempts to get up in the manger. I noticed that his head was elevated, and the eyes fiery red. He acted wildly and showed decided symptoms of phrenzy. On attempting to administer a sedative, the animal went into convulsions, and I was compelled to make my escape from the stall as quick as possible. He soon got his fore-feet over the tops of an adjoining stall, five fect in height, remained there for a short time, when he scrambled over and fell like a log right into the next stall. I never expected to see him rise again; but after a few minutes, with some assistance, he got up. I gave him twenty drops of aconite. During this day the frantic animal went over a stall twice in succession, and fell very heavily each time on his back, yet did not appear to have suffered any injury. 
Most of the time he appeared frantic, yet unconscious; and just before going into a convulsive fit, he would stamp with his forefeet, continuously toss up his head, his neck being the seat of spasmodic twistings. Next day the symptoms had not altered much, and, during my examination, he pulled back, broke a portion of his manger to which he was tied, and fell head over heels on the floor. During the forenoon he became more calm, and lay on the floor, yet would occasionally jerk his head backward, and have slight convulsions. I continued the prostrating plan of treatment, only exhibiting smaller doses, and soon the patient began to improve. The membranes of the eyes, mouth, and nostrils assumed a more natural appearance; then the plan of treatment was altered, and the patient received tonics and stimulants, which soon resulted in convalescence.

Among all the cases that have come under my care and observation, I have neither found it advisable nor necessary to practice the antiphlogistic treatment, in so far as it relates to bleeding and purging; and I can not conceive a case, so depressing as influenza is known to be, that would require any such heroic treatment. The safety of our patient depends on the judicious application of remedies such as are here named, and, therefore, I would warn the farmer against the use of fleam and cathartic in the treatment of influenza. Yet, after all, the best treatment may fail in restoring a patient. We require aid in the form of pure air, suitable diet, and good nursing. Failing in the latter important adjuncts, our treatment avails but little.

Influenza is a disease accompanied by a discharge from the respiratory, conjunctival, and schneiderian membranes; and the organs of respiration themselves are more or less involved, as shown by cough and soreness of the throat, lassitude, fever, thirst, and loss of appetite. Now, if the term influenza means any thing, it signifies epidemic catarrh, and the eases alluded to were not of this character, but quite diverse, indicating a low congestive state. Still, the majority of eases that we have seen do appear to be nothing more nor less than influenza; but if the above diseases appear simultaneous with the latter, there is danger of persons making great mistakes in the treatment, for it is very apt to run into typhoid fever. 


\section{Typhus on TyphoId AfFections.}

The veterinary surgeons of England have hitherto maintained a remarkable silence on the subject of typhoid affections occurring among horses; and so late as the year 1850, Mr. Percivalu informs the world, through the pages of the "Veterinarian," that in London very little is known about such disease. In view, therefore, of lighting up the dark spots that exist in our department on this side of the water, we furnish a translation from the French, by Mr. Percivall. The article is a selection from a prize memoir written by a distinguished surgeon. It is preceded, as the reader will perceive, by a review from the pen of the translator, who says:

"In the 'Collection of Memoirs and Observations on (French) Military Veterinarian Hygiene and Medicine,' which we have so lately been engaged in examining, are contained two prize memoirs-one on Farcy, the other on Typhoid Affections in horses.* On the latter of these we would make a few remarks, if it were only for the reason of showing what is meant to be understood by such imposing titles. Typhus and typhoid are words but rarely heard in our own country in connection with veterinary, or at least with hippiatric, medicine. Our old writers on farriery described fevers in horses as very destructive in their character, requiring antiphlogistic treatment:

"Typhus Fever.-A disease touching which we (the author) are in possession of but few observations, and one that has been, and still is, in our opinion, mistaken for and confounded with either enteritis or gastro-enteritis-in cases, for example, in which its consequenees are of little importance-though, perhaps, with putrid fever, when, on the other hand, malignant and exhibiting extraordinary violence, its progress is rapid and its termination fatal. In its most benignant form, typhus fever, indeed, bears so great a resemblance to pure inflammation of the primary intestinal passages, that it is often difficult, very difficult, even to distinguish them. As for the cause which occasions it to be confounded with putrid fever, it is no matter of astonishment to us, since, in our opinion, one fever possesses, in many respects, so great an analogy

\footnotetext{
* The reader will fird an article on Typhus Fever, but in an enzoötic form, in "The Veterinarian," vol. xxii, p. 462.
} 
with the other; while typhus itself, through causes which remain latent up to the present hour, is, like the gastro-enteritis of 1825 , dangerous, fatal, and epizoötic. In general, typhus fever begins without any warning, though there are times in which its approach is marked by dullness and weakness, and a manifestation of bcing easily fatigued, sweating through little exertion, etc., with loss of appetite.

Of the first stage, the most remarkable symptoms are either a yellow redness of the pituitary membrane, or a dryness or a discharge of viscous matter, which adheres in thick incrustations around the alæ of the nostrils, with, occasionally, an appearance of drops of blood. The nostrils are more or less dilated, the respiration more or less frequent, according to the sharpness of the abdominal pains, or the existence of a lamentable complication of thoracic with abdominal disease. The ear applied to the windpipe or to the walls of the thorax, almost always detects a wellmarked mucous rale; also there is cough, with full, strong, and frequent pulse.

In the second stage, the mouth becomes dry, and sometimes displays aphthæ or cracks of some depth. The tongue is red about its point and along its sides, but its middle and base are of a deep yellow tint. 'The breath is tainted. When abdominal symptoms are present, which is not always the case, they are sufficiently well marked. The dung is either very hard, or it is, more commonly, liquid; sometimes it is bloody, and issues a fetid odor, the debility frequently being such at this period as to cause constant opening of the anus. The pituitary membrane is dry, and covered with bloody points. The conjunctival membrane is reddened and infiltrated, and, like the pituitary, presents petechial patches. The pulse has not its force and fullness, etc. Some veterinarians have remarked, at this period, upon the insides of the thighs those little vesicles to which the name of sudamina has been given. Urine scanty, possessing a remarkable fetor, and voided occasionally with great pain; great dejection, and occasional stupor; and, ordinarily, at this period it is that the ataxic or adynamic symptoms set in.

In the third stage, the parotids tumefy and ulcerate. Any setons or rowels that may have been introduced, or blisters, become so many causes of mortification of the parts in which they have been applied. And now the mouth becomes quite dried up; the tongue 
acquires a horny feel, and turns brown; the gums and teeth are covered with a dingy slime. Bleeding from the nose, which mostly appears during the inflammatory stage, now returns. The pulse becomes irregular and more feeble; œedema, sometimes considerable, of the extremities; great debility. The temperature of the body sinks. The neck, belly, flanks, axilla, and groins become covered with cold and clammy sweats; the tympanitis increases, the evacuations being bloody and fetid to a remarkable degree. The animal, continually in pain, exhibits symptoms of colic, often seeking to lie down; but if he does, he soon rises again. Respiration greatly accelerated; nostrils widely dilated; pulsations of the heart tumultuous. At length, beginning to stagger, he falls, either to die an easy death or to expire amid agonizing convulsions. When, however, recovery in place of death follows, such symptoms as we have last detailed do not occur, or with only modified force and character. When blood has been drawn for experiment, under such circumstances, at a time when adynamic and biliary symptoms prevailed, it has been found to contain but little hematosine, but, on the contrary, a large proportion of serum, of a light greenish hue. The disease may last from four, seven, or eight to thirty days. Relapse is extremely likely, even under every prospect of convalescence, unless great care be taken. The diagnosis at the commencement is difficult, even impossible, when the characteristic symptoms are but scantily and imperfectly developed.

The affections whose symptoms most resemble those of the disease before us are plegmasia of the intestinal tube, and particular of the brain. Should sudamina be detected, they are, as in human medicine, to be regarded as definitely characteristic of typhoid fever. Prognosis, for the most part, unfavorable; and the more so according as the nervous accompaniments are more numerous, more violent, and more early in showing themselves; the stupor great, the strength failing more, the pulse faltering, the diarrhea greater, more bloody, fetid, ete.

Our etiology is all at fault. In truth, we know not the determining cause of typhus. The post-mortem states are by no means agreed upon by those who have reported them. Most agree that the digestive canal exhibits the most constant and marked symptoms of disorder. The mouth presents marks of inflammation, and, on occasions, aphthæ or ulcerations, though commonly too superficial to destroy the living membrane. The pharynx sometimes 
the same as the mouth; at others, nothing. The stomach is frequently the seat of inflammation of a marked character. The intestines also reddened, to more or less extent. The cavity of the peritoneum, containing a citron-colored fluid, reddened, perhaps, the omentum being at times almost entirely destroyed. A liquid matter-a sort of bloody corruption of more or less consistence-is often found within the cavities of the small guts; and in those of the large, the cæcum and colon in particular, are sometimes to be remarked red and black spots, or ecchymoses, buds of smaller or larger size (glandes de Bruner) indistinctly planted over the mucous surface.

Malignant or Putrid Typhus-(Typhus Charbonneaux.)-These malignant and putrid affections, either constitutional, or, in some instances, but local in their origin, ever of a highly acute nature and of unequaled rapidity of progress, since not unfrequently they cause death in a few hours, are of more consequence for us to become acquainted with, as they are not only susceptible of being communicated from one animal to another, but even from animal to man, in whom, losing none of their malignity, they are likewise most commonly mortal. Frequently sporadie, sometimes epizoötic, but most commonly enzoötic, these diseases at times assume so redoubtable a form that nothing can arrest their progress. Invading farms or other extensive localities, they produce therein terrific ravages; for, being susceptible under various forms, almost equally fatal one with another, of prostrating a vast number of animals of the same or different species, these affections determine, under varying circumstances, losses which, on occasions, desolate and completely ruin the country where they have broken out.

Such are the two especial forms of typhus fever which have been observed among horse-kind-one of which would seem to occur at times in practice without being sufficiently heeded or recognized by veterinary surgeons in this country, while the other may be said to comprehend those malignant epidemics by which our cattle, if not our horses, have, of late years, been too fearfully visited." "

In the treatment of typhoid affections, we endeavor to give tone to the system by furnishing nutritious diet, tonic, and stimulating medicines. Ginger and golden seal, with a small portion of hyposulphite of soda, are the best remedies. 


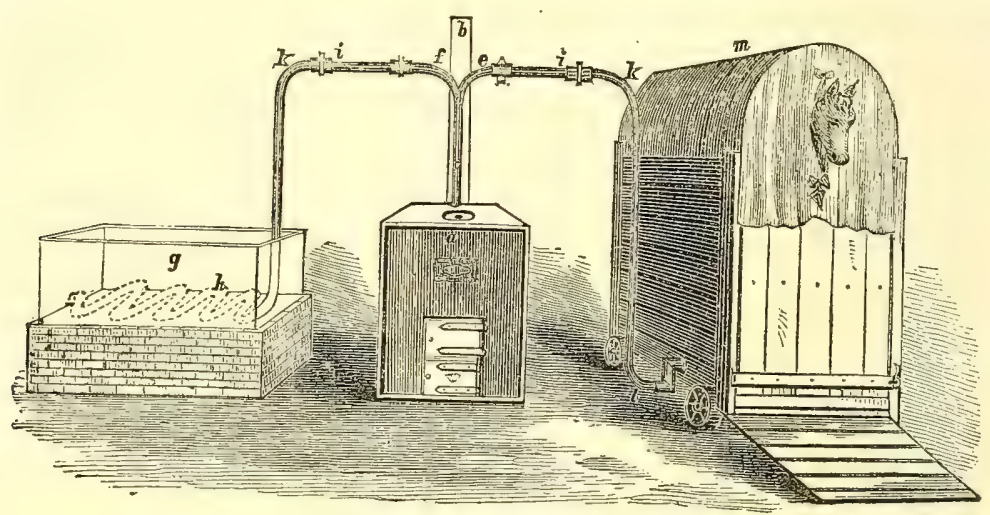

PLAN OF A VAPOR BATH USED IN THE TREATMENT OF LCNG AFFECTIONS.

Explanation, $-a$, represents a boiler originally erected for the purpose of supplying the infirmary with hot water; $c$, is the main pipe issuing from the top of the boiler receiving the steam, and conducting it, when not required for other purposes, into either a flue or the open air at $b ; e$ and $f$ are branch pipes from the main one (c), $f$ being that which conducts the steam into a worm $(h)$, winding through a condensing trough $(g)$; $e$, the branch pipe which conducts the steam (prevented by stop-cock from going in the other direction! into the bath, the place of admission ( $n$ ) being on one side, close to the floor, at a point intermediate between the horse's fore and hind feet while standing in the bath, with his head outside $m$ is the bath, being a horse-box, such as is used for embarking horses on board of ship. with the addition of a lining of flannel, a roofing of hoops and tilting, and curtains over the doors. front and back, to prevent the escape of steam. The box, being placed upon wheels, serves, besides being used as a bath, for the transport of sick or lame horses; and, having doors at both ends, and a moveable platform for the horse to walk in upon, is, in general, entered without any great deal of unwillingness.

\section{Pneumonia (Inflammation of the Lungs).}

Inflammation of the lungs, known to veterinarians as pneumonia, is not apt to be so prevalent among horned creatures as among horses, except, however milch cows, when they are located in filthy, unventilated milking establishments. In such places diseases of the lungs are often fearfully prevalent, raging as an epizoötic, as it did a few years ago in the swill-milk establishments of New York. It makes sad havoc when prevalent among a large herd that may be confined in a barn or stable of contracted proportions. All domestic animals require plenty of room, as well as light and pure air; for it is now pretty clearly demonstrated that the pleuro-pneumonia, which appeared in Massachusetts, in 1860, on the premises of Mr. Chenery, arose there spontaneously. His barn, or rather the basement, where the cattle lived, was a room fifty feet square and only eight feet in height. The walls on the north, and a portion of the 
east and west sides, were composed of brick, and the remainder of boards, with joints battened. On the south, east, and west sides were glass windows; on the north side a door opened into the vegetable cellar, and on the roof there was a sky-light. A stairway led to the hay-loft above, another to the manure cellar below, both closed by doors. The manure cellar was eight feet deep, and extended under the whole room. In the winter and spring of 1859 were confined forty head of cattle, arranged on three sides, with their heads toward the center; and within that center was another square, containing animals, so arranged that almost all were brought face to face. The manure cellar, about this time, contained from fifty to one hundred cords of manure, with from ten to twenty hogs in it. "This," says Mr. Chenery, "was my condition in the beginning of the year 1860. I had buried nearly half of my herd. I had experimentally acquired a knowledge of the fact that, in order to keep animals alive and in health, it was absolutely necessary that they should be supplied with pure air as well as with good food and pure water."

So far as the direct or indirect causes of pneumonia are concerned, we may safely infer that crowding and a bad system of ventilation includes them all. Yet, among horses, this disease often originates as the consequence of laborious work and feats of speed, which produces rapid and sometimes distressing respirations; but among cattle, whose powers of speed and endurance are not often put to the test, and whose natural respirations are slower, we infer that impure air, and perhaps exposure, too, are more operative than action or ill-usage.

The stimulating and morbid action of an impure atmosphere may produce a disease of this character by its irritating effects on the highly vascular membrane which lines the bronchi and aircells. But then we all know that impure air fails to decarbonize the blood; hence it is rendered unfit to enter the system. It has been noticed, however, by the drovers of the East, that when cattle have been driven a long distance without food, and in tempestuous weather, they are apt to become the subjects of diseased lungs. Fortunately for the poor brute, pneumonia is not so painful as bronchitis, pleurisy, or laryngitis ; and having passed through the acute stage, which is usually brief, it assumes a mild or subacute form, and ends in altered structure of the lungs, known as induration (hardening) or hepatization (liver-like), or it may end 
in pleurisy, and ultimately dropsy of the chest. I should judge that cattle, when the subjects of pneumonia, suffer less than horses, from the fact that the former will lie down during the progress of the disease, and the horse is scarcely, if ever, known to do so until shortly before he dies. In the horse, this malady occasionally takes a metastatic turn; that is, the disease is translated to the feet or brain, producing an affection of a very formidable and acute character. Among cattle, however, such translation is very rare.

Forms of Pneumonia.-Pneumonia is divided into several stages and forms, but as such refer more to its degree or intensity, I shall only inform the reader that pneumonia may exist either as a condition of congestion or of inflammation. Congestion signifies a distended or plethoric state of the blood-vessels of the substance of the lungs, and slow motion of the blood; and it sometimes sets in as suddenly as that form which is termed inflammatory; among cattle the former form is more prevalent. In the congestive stage the symptoms are those of embarrassment-the blood courses through its vessels sluggishly, and there is not that activity of the heart and lungs which is noticed in inflammatory pneumonia.

Symptoms of Inflammation of the Lungs.-There are many symptoms attending lung disease which are common to various other forms or affections. These must be inferred; for, by detailing them, I might only confound the non-medical reader, and render the diagnosis difficult, and, perhaps, impossible. The symptoms, in the early stages, are such as are usually observed at the commencement of febrile diseases, viz.: coldness of the extremities, and slight shivering fits; loss of appetite; labored respiration: pulse variable, and the mouth hot and clammy; the animal heaves at the flanks, is rather unwilling to move, and the fore-legs are widely separated, while the head is held in a drooping position. As the disease progresses, these symptoms vary more or less, and the appearance of the membranes of the mouth, nose, and eyes may vary from the color of bright scarlet to that of a leaden hue. In congestive pneumonia, the pulse is more voluminous, yet less active, than in health, or in acute disease. A cough, slight or active, as the case may be, is usually noticed; it is a sort of deepseated, half-suppressed one, and sometimes it is the first symptom which calls the owner's attention to the ailing animal.

Treatment.-It is important, at the commencement of the treat- 
ment, that the patient shall be placed in a comfortable shed or barn, where pure air abounds; for, under such circumstances, the coöperation of Nature in the cure of the malady is secured. Should the disease be of an acute character, it will be accompanied by quick or labored respiration, and a strong, wiry pulse. In that event, I should give two drachms of the tincture of gelseminum. The brisket and sides of the chest are to be rubbed occasionally with mustard and vinegar. This acts as a counterirritant, and diverts the blood from the lungs to the surface. It was customary, in former years, to bleed and purge almost all animals when attacked with acute pneumonia; but as the mortality was then very great, and less under a more rational system, we are led to believe that bad treatment was the cause of our want of success. Two or three doses of gelseminum, given at intervals of six or eight hours, will, together with the elapsed time, have a tendency to modify the affection. Then active medication is to be suspended, and we immediately give life-sustaining agents, which consist of pure air, water, proper food, tonics, and stimulants. The best tonies and stimulants that I know of are powdered golden seal and ginger, equal parts. Dose, half an ounce night and morning, as a drench; or two ounces, night and morning, of the fluid extract of resin weed may be substituted. The animal should have one ounce of powdered chlorate of potass every twelve hours, until it is evident that convalescence is approaching, or has actually set in, when it may be discontinued. The best way to administer the chlorate of potass, is to dissolve it in a few quarts of linseed tea, or an infusion of slippery elm, which should be kept in a bucket before the animal until he has partaken of the whole of it. The chlorate of potass is also indicated as a valuable agent in the treatment of all lung affections, including pleuro-pneumonia and pleurisy; its use is to be discontinued, however, when the urinary secretion becomes much augmented, or it may overwork the kidneys. Chlorate of potass acts as a sedative to the nervous system and to the circulation, and is a stimulant to the digestive organs and kidneys. For all cases of congestive pneumonia, or when the surface of the body is cold, as well as the limbs and ears, warm stimulants are indicated-ginger and golden seal; and, if possible, the body and limbs should be clothed, for the purpose of maintaining the natural heat of the skin, and of producing an equilibrium of the circulation; for when an 
equilibrium in the circulation of the blood is secured, then congestion ceases to exist.

I would caution my readers not to place too much reliance in art and medicine for the cure of disease. The physician does much good when he practices rationally-constitutes himself the servant of Nature, does her bidding, and does the system no harm in the use of poisons and meddlesome medication. In the treatment of many forms of curable disease it is often a question of time; they will "have their run." Hence, what we most need is patience, great faith in Nature, and a moderate degree of faith in drugs. The great trouble is, our instruction derived from textbooks and veterinary institutions of learning tends to develop heroic notions of medicine and its powers, and to depress those more potent remedies, the vitalizing and reanimating forces of Nature. A case of heroism of this kind, as an example, I must now relate, in order to illustrate my position:

Mr. S—, of Chicago, bought of Mr. P— a fine, promising young gelding, aged about eight years. The purchaser had used him during a period of five weeks with perfect satisfaction. Suddenly the newly-purchased animal became sick, and the services of a noted blacksmith were secured. The man of "tongs and hammer," realizing that the patient was the subject of the prevailing influenza, gave the patient "fits," in the form of the administration of a physic ball. He then abstracted two gallons of blood. The horse grew no better very fast. In consequence, he got more medicine. Purgation set in, which continued for twenty-four hours, when the horse died. The lives of many valuable animals are thus sacrificed. Finally, those men generally become the most distinguished who soonest emancipate themselves from the theory of the heroic schools of medicine.

The plan of treatment here recommended may appear to some of our readers as very simple. That is just what we claim for it. It is the simplicity of the practice which makes it really valuable, because such a system harmonizes with the law of Nature, and does not oppose her forces, which are always operating physiologically for the restoration of the sick and infirm; and it is our business, as rational physicians, to aid Nature in all possible ways. This is the kind of knowledge that we require-how to aid Nature, or give her the required assistance; and, in view of obtaining that information, we must read the great book of Nature, as its leaves 
are unfolded to us in the various departments of her great domain. We must altogether abandon a blind faith in doses of poisonous medicines and bleedings; otherwise, all our efforts are as but a withering simoom. For example, over seventy thousand head of cattle have lately perished of what has been called the "rinderpest," in and around the great city of London, which is claimed to be the model seat of veterinary learning for all British subjects. It is probable that the heroic practice there taught has something to do with this tremendous mortality; for we learn that a more simple plan of treatment has been inaugurated by another class of physicians, whose medicines are almost inert, and they have been more successful than the advocates of setons, blisters, sedatives, and narcotics, not forgetting their "sheet-anchor," calomel-as Napoleon observed, in derision, when on the island of St. Helena, "Bleeding and calomel for ever!" Simple, sanative medicines act potently, yet silently, after the fashion of Nature's workings; so that we should never attempt to force her to adopt our system of cure for that of her own.

\section{Pleurisy.}

Pleurisy signifies inflammation of the pleura. This membrane lines the internal surface of the chest, and furnishes a covering to the organs within the chest. It also serves to divide the chest into two cavities, which are known as the right and left. This membrane is similar in its texture and function to that which lines the cavity of the abdomen. Both are serous membranes, secreting, on their inner surface, a lubricating fluid, which guards against friction. This membrane is the seat of pleurisy, and pleurisy consists of inflammation of it.

Causes.-It may be presumed that the ordinary causes which produce affections of the lungs are operative in the production of this malady, viz. : exposure, by which the surface of the body becomes chilled; injuries in the thoracic region; over-work; an impure atmosphere; a plethoric state of the system; in short, any cause which is likely to produce an inflammatory affection in other parts of the body. I have known horses become the subjects of this malady that have been confined to the stable for weeks. Such cases are clearly traceable to plethora, want of exercise, and impure air. It is possible that some animals inherit 
a predisposition to pleurisy. It has been noticed that animals of the sanguine temperament are more prone to the malady than others of different temperaments. Hence, the only way to prevent pleurisy is to try and keep up a good standard of health in horses, by affording them a chance to have regular exercise, pure air in the stable, and a fair allowance of good wholesome food. Pleurisy in the horse is very apt to end in hydro-thorax, or dropsy of the chest; and the case that I am now about to offer will illustrate this, as well as the symptoms and treatment.

Case of Pleurisy and Dropsy of the Chest.-A few months ago I was requested to visit a gray gelding, the property of a Mr. Waldo. The animal had been out of health for a week or more, suffering from a slight attack of influenza, but was improving - so thought the person who was in medical attendance; but the owner thought otherwise, and decided to secure my services.

Symptoms. - On examination, the following symptoms were found present: Pulse, wiry; respirations, quick, laborious, and accompanied with a slight grunt, indicating pain within the chest. When pressure, ever so slight, was made on the sides of the chest, it elicited very decided symptoms of pain. The animal would occasionally turn its head toward the sides, as if to inform us of the seat of his sufferings. Dropsical swellings were observed under the chest and belly, and the hind legs were also dropsical, and the vascular surfaces of the mouth and nose were tinged slightly yellow; the feces were seanty, and the urine was high-colored; the appetite was much impaired, but the patient was very thirsty. On the strength of the condition of the animal, and the presentation of the above symptoms, and pereussion revealing water in the chest, I informed my employer that the case was one of pleurisy and dropsy of the chest. It is probable that water had been accumulating in the chest for several days, and that the pleura was involved at the commencement of the malady.

Treatment.-Dropsy of the chest is a very formidable disease to treat, and there are but few cases of cure on record; yet, I think if such cases came early into the hands of educated surgeons, and they use life-sustaining agents in view of husbanding the powers of the vital forces, more cures will hereafter be recorded. The treatment of this case was as follows: I rubbed the sides of the chest occasionally with a portion of camphor dissolved in olive oil, and gave, morning and evening, during a period of seventeen days, 
thirty grains of iodide of potass in two ounces of fluid extract of resin-weed root; an occasional dose of golden seal was given as a tonic. The iodide of potass acted on the system as a glandular stimulant, and thus increased the power of the absorbents and excrementitious vessels, to rid the chest of its fluid contents, while the resin-root acted as a diuretic, thereby carrying off some of the fluid by way of the kidneys. During the period of the sickness of this animal, the resonance of the chest gradually became clear, so that at the end of three weeks not more than a couple of pints of serum remained in the chest. The animal rapidly convalesced.

During the treatment of such a case as the above, I usually feed the animal liberally on oats and sweet hay; and, in order to obviate constipation, I order an occasional bran-mash. The animal, however, may not, at the commencement, have much relish for food, but after a few doses of the medicine here recommended have been given, the appetite will soon be restored. It will be noticed, during the progress of this malady, that the animal does not lie down, but stands with the fore-legs widely apart, although, at the very commencement of acute pleurisy, the subject will often get down, in view of mitigating the lancinating pain from which he suffers, in consequence of the distension of the vessels of the pleura. Pleurisy sometimes sets in as an accompaniment of influenza, which almost always ends in dropsy of the chest; and as a case of this kind is a good one to place on record, I here introduce one from my note-book:

Pleurisy ending in Dropsy and Death.-The horse, the subject of the above-named maladies, was the property of a milkman of Chicago. The animal had been previously treated for the prevailing influenza, accompanied by pleurisy, and had about recovered so as to take daily exercise; but finally the owner discovered that the horse was suddenly taken with a relapse. Feeling alarmed, he procured one pint of linseed oil, with which he drenched the animal. Still, the horse rapidly grew worse. At this period, my services were sought. On auscultating and percussing the chest, I found that the right cavity was occupied by a large quantity of water. Dropsical swellings were found in various parts of the body, viz.: the sheath, limbs, and region of the pectoral muscles. On applying the ear to the trachea, the respiration was of a mucous character, and tubular, showing that the lungs were much diseased. The examination revealed the fact that about one-half the left lung 
was involved in disease. The animal had no appetite, yet was thirsty; and when urged to move, would utter a grunt, indicative of pain. It was evident that the relapse was, or might be, considered a case of pleurisy, ending in effusion into the chest. The worst feature of the case was that the animal purged violently (liquid stools), having a very strong odor of linseed oil ; it covered a large space on the floor, and ran down his hind extremities. This seemed to me to render the case hopeless; for super-purgation of itself will often cause the death of an otherwise well animal. But this poor creature was laboring under a malady from which few ever recover. However, I thought that while "there is life there is hope," and I commenced the treatment by performing the operation of tapping for dropsy of the chest. I drew off three and a half gallons of fluid, of a light straw color, from the right eavity of the chest. On auscultating the chest, both sides appeared resonant, showing that the waters must have occupied both cavities; hence, the mediastinum (the membrane which divides the thorax into two equal eavities) must have been ruptured, thus permitting an influx and reflux of the fluid. Next, counterirritants were applied to both sides of the chest. The patient was properly clothed, tonics and astringents were given, and the ease was left in the care of an attendant for the night. Next day I found the patient was respiring very comfortably. The tapping of the chest seemed to have done much good, yet the purging still continued; so I ordered scalded milk and charcoal, and administered tonics and stimulants, as before. But it was very evident that the animal must die of super-purgation, and next morning I received word that death had taken place. It was unfortunate, under the circumstances, that the owner should have given linseed oil ; for it is a very powerful irritant and cathartic, and produces much irritation and inflammation on the surface of the interior of the stomach and intestines, as well as uncontrollable purgation. It is a remedy which is very quickly absorbed, and finds its way into the blood in a very few minutes, as the following case will show: I once gave a horse a dose of linseed oil for the purpose of experiment. (He happened to be in good health, or I should probably have lost him.) Ten minutes after administering the dose, I applied a linen handkerchief to the highly organized membrane lining the eyelid, and both the odor and color of the oil could easily be detected. On exposing the handkerchief 
to a moderate degree of heat, for the purpose of evaporation, a grease spot remained on the same; hence it is an agent that should never be used in pleurisy, nor in any disease occurring in the organs of respiration. Dropsical diseases, supervening on influenza, are never benefited by cathartics, for they always lead to debility and prostration. Influenza is a disease of very prostrating character; hence the proper plan of treatment is to sustain the vital power, by nourishing diet, tonics, and stimulants-ginger and golden seal. While commenting, however, on the linseed oil treatment, I do not wish to censure the owner of the animal; for I should be throwing stones into glass houses, having myself, in former years, used and recommended this kind of oil for various diseases; but, after many mishaps, I am satisfied that it is unsafe in the treatment of equine affections.

\section{Pericarditis (Inflammation of the Pericardium).}

The pericardium is a membranous sac, which surrounds and incloses the heart. It is composed of two layers, united by cellular tissue. The external layer is dense and fibrous, and is attached, by ligaments, to the sternum, or breast-bone and diaphragm, and above to the roots of the large blood-vessels. The internal layer exhibits internally a smooth surface, like the lining membrane of the cavity of the chest. From this surface is secreted a serous fluid, which lubricates the heart, and protects it from the friction which would otherwise occur. The pericardium is a wall of defense for the heart, and keeps it in its proper position.

Pericarditis signifies inflammation of the inner membrane of the pericardium. It is a disease which is almost unknown to the ordinary practitioner in this country, and entirely unknown to the husbandman; yet it is a disease that carries off a large number of horses and cattle, and often the parties concerned in the treatment and ownership of the animals do not even suspect the true nature of the malady. After the inflammatory action of the pericardium has reigned for several days, unsuspected and unchecked, an inordinate effusion from the serous lining takes place. We then have dropsy of the "heart-bag." Water accumulates around the heart, which interferes with its action so as to put a stop to circulation, and this results in the death of the animal. Pericarditis often accompanies, or is the sequel of, pleurisy and 
other diseases of the serous membranes; yet, fortunately for the patient as well as the practitioner, it does not require any special treatment other than that laid down for pleurisy and disease of other serous membranes.

Causes. - The causes of pericarditis are the same as those which are said to be operative in the production of pleurisy and disease in the serous membrane, viz.: sudden impressions of cold upon the external surface of the body; injuries; overtasking the powers of the animal by laborious and rapid work. Sometimes foreign bodies, such as pins and needles, have been found within the pericardium, which must have been taken into the stomach with the food, and have worked their way so as to penetrate the pericardium and heart. Such cases almost always prove fatal.

Symptoms.-The general symptoms of the disease are anxiety, shivering, coldness of the skin and mouth, loss of appetite; the jugular veins are congested, and they pulsate like an artery; there is a sort of tinkling or metallic sound, when the ear is applied to the chest and lower part of the neck. In the latter stages the respiratory murmur becomes very indistinct, and there is a sort of double expiration, and the ribs become contorted, the same as in dropsy of the chest.

Treatment.-The heat of the external surface of the body must be restored, and kept uniform by means of leggings and body clothing. Both sides of the chest are to be briskly rubbed, two or three times daily, with hot vinegar and strong mustard. One drachm of iodide of potass, dissolved in five ounces of warm water, should be given every four hours; but, should the case be seen, and the true nature of the malady discovered in its early stages, omit the iodide of potass, and substitute two fluid drachms of tincture of gelseminum. Two or three doses, at intervals of four hours, will suffice; then resort to the iodide of potass, or substitute for the same four drachms of chlorate of potass. So soon as the animal appears to improve, discontinue the above-named medicine, and give tonics. Four drachms of fluid extract of golden seal, twice daily, will suffice.

A fatal case of pure pericarditis is related by Mr. Woodener, V. S., in the London "Veterinarian." The autopsy revealed a healthy state of the lungs and pleura; yet the perieardium contained about three quarts of serum, highly colored. The walls of the pericardium were considerably thickened, and studded with 
merous bands of lymph, on the inner surface, of a bright yellow color. Several small patches of inflammation were likewise observed on the ventricles of the heart, near the valves of this organ. The heart itself was also larger than usual, and much softened.

\section{Heaves.}

A disease prevails among horses, more particularly in the Eastern States, known as heaves. The affected animal heaves at the flanks, or, rather, performs what is known as abdominal respiration, by bringing into play, at the moment of expiration, the abtominal muscles, for the purpose of aiding the lungs and diaphragm in the function of expiration and respiration. The disease somewhat resembles asthma, it being of a spasmodic character, and, after continuing for some time, will suddenly disappear. The disease may be defined as being great difficulty in breathing; continuous; aggravated, by dust on the road, musty hay, improper provender, impure air, and sudden atmospheric changes. On applying the ear in the region of bronchi, or to the upper sides of the chest, a wheezing sound of respiration is heard. These symptoms, together with the heaving motion at the flanks, constitutes heaves. When the disease is merely functional-that is to say, brought on by feeding musty hay and musty clover-a cure is affected by feeding prairie hay, which contain the well-known silphyum, "resin weed." The husbandmen who reside in the vicinity of where the resin weed grows are well acquainted with the properties of this plant, and they declare that it is a specific for the treatment of asthma or heaves. I have used the article in the form of fluid extract, prepared from the root, and I find it to be a very valuable remedy. The dose of the fluid extract is two ounces, morning and evening. There are some cases, however, which are incurable, owing to organic disease of the bronchi, or rupture of air-cells in the lungs. Such cases are known by the unnatural respiration when the horse is drawing a heavy load or traveling up hill. His breathing is then very distressing, and he not only brings into play the abdominal muscles, but also the muscles of the chest (intercostals). Such cases, although considered incurable, may be palliated by the daily use of fluid extract of resin weed. Provided prairie hay can not be obtained as food, the ordinary hay used should be sprinkled 
with salted water, in the proportion of a handful of salt to one bucket of water. Sprinkling the hay with weak lime-water has also a good effect. Sliced carrots, parsnips, or potatoes, fed occasionally, are also beneficial. But, of all our remedies, none equals a run at grass on prairie lands.

MLAN ARTERIES ON THE INSIDE OF ONE OF THE FORE-LEGS, FROM SHOULDER TO KNEE.

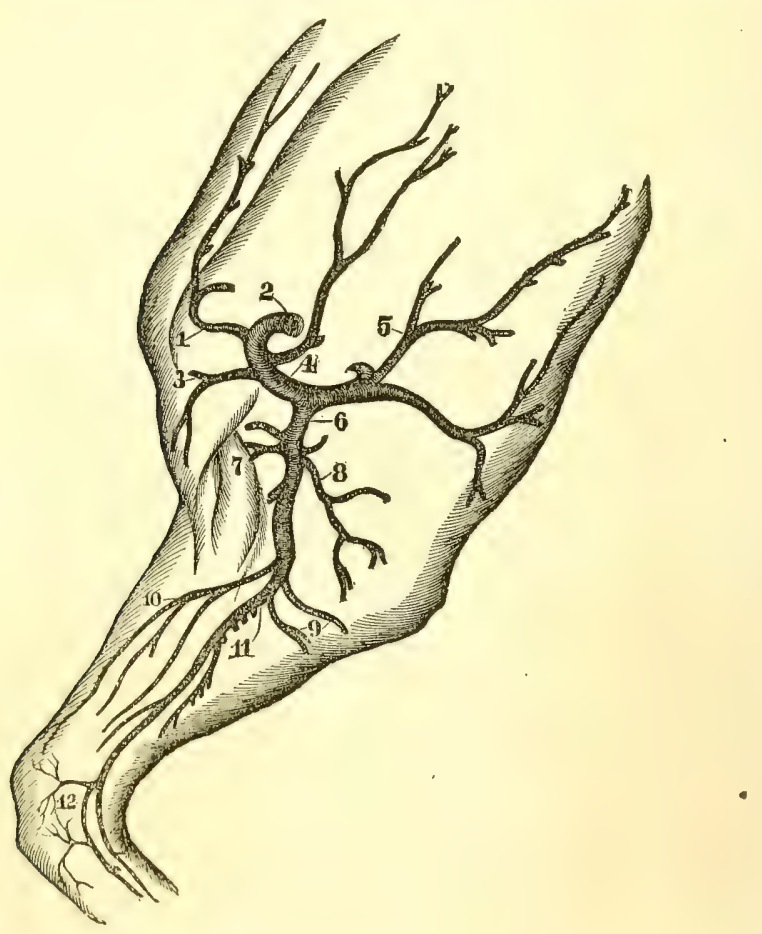

Explanation of Plate.

1. Humeral thoracic.

2. Axillary.

3. Internal thoracic.

4. Dorsal scapular.

5. External thoracic.

6. Humeral.
7. Brachial.

8. Branches of the triceps.

9. Ulnar branches.

10. Spiral branches.

11. Radial branches.

12. Metacarpal arteries, large and small. 


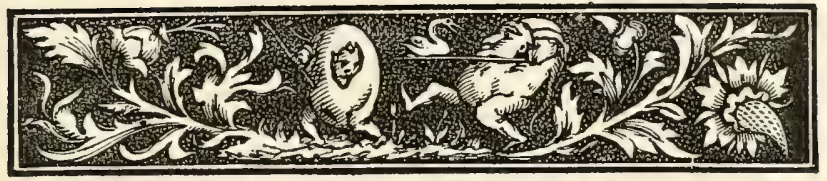

\title{
SECTION V.
}

\author{
DENTITION AND DIET.
}

The Horge's Age as shown by his Teeth-ON the Diet of Horses-The effects of variods kinds of Food-As regards the QUANTity of Food requiredAs regards changes in Diet-Conclusion.

The Horse's Age as shown by his Teeth.

WIGURE 1 is a representation of a foal's " mouth," as it ap1 pears at the age of twelve months; the temporary teeth are shown, and all, except the corner ones, are worn quite smooth. (See remarks on temporary teeth.) Figure 2, a two-years old; the two anterior, or front temporary teeth, are shed, and the permanent ones, with their deep pit in the center, and unworn edges,

Fig. 1.

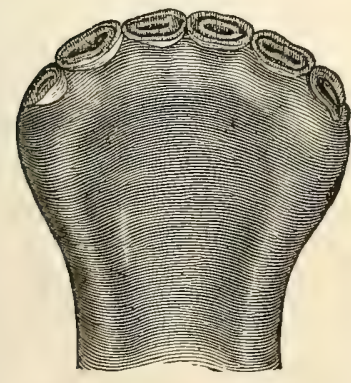

TEMPORARY TEETH OF THE COLT.
Fig. 2.

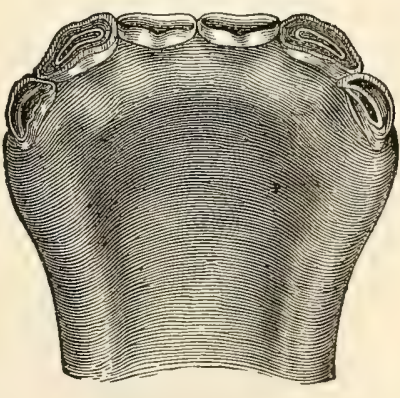

TWO YEARS OLD.

are approaching a level with the remaining temporary. Figure 3, a three-years old; the two permanent middle teeth are seen, with their deep pits, etc., and are nearly on a level with the two front permanent teeth, which are smoothed down. Figure 4, a fouryears old; the permanent corner teeth are now "cut," and the tushes have appeared, although the latter may not appear until 
five. Figure 5, a five-years old; the corner teeth are well up; the faces of the front and middle are worn more or less, and the tushes are more developed. Figure 6, a six-years old; the black marks have disappeared from the two front teeth, and a

Fig. 3.

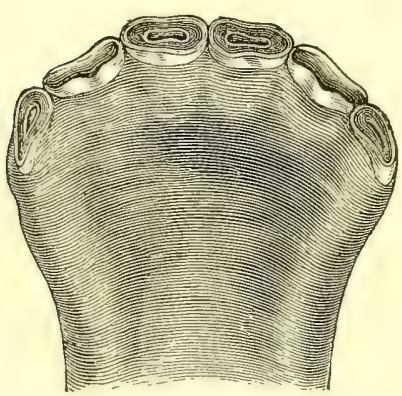

THREE YEARS OLD.
Fig. 4.

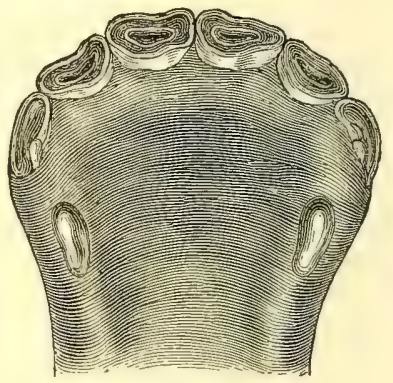

FOUR YEARS OLD,

brownish mark is perceivable. Figure 7, a seven-years old ; the black marks have now disappeared from the two middle teeth, and a brown spot is seen in the center. Figure 8, an eight-years old; the marks and cavities of the corner teeth are now effaced. Figure 9 is a horizontal view of "Black Hawk's" incisors, and, con-

Fig. 5.

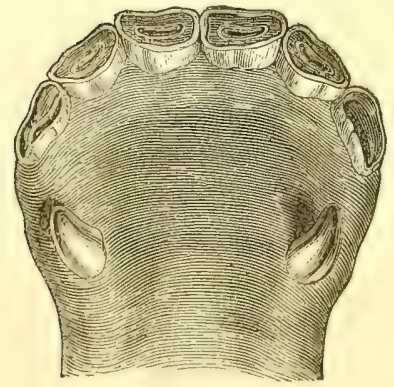

FIVE YEARS OLD.
Fig. 6.

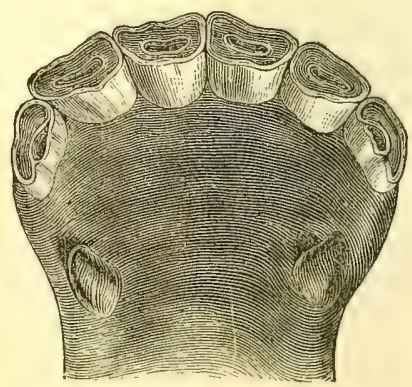

SIX YEARS OLD.

trasted with figure 10, (a young mouth,) shows the degree of inclination existing between the incisors of the aged and young animals.

The Colr's Mouth.-Remarks on the Temporary Teeth. (Incisors.) In the course of from ten to fifteen days after birth (varying 
occasionally), the front nippers in the upper and lower jaw make their appearance, and, during the period, which occurs between the third and fifth week, the middle incisors appear. Between the seventh and ninth month the lateral or corner teeth are cut. The

Fig. 7.

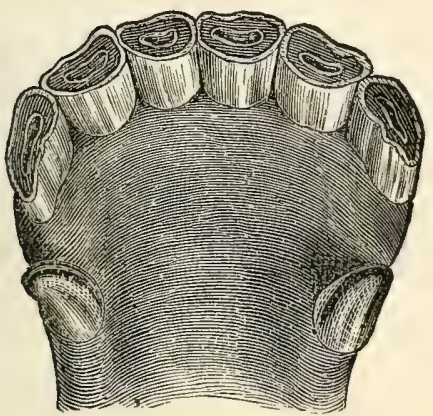

SEVEN YEARS OLD.
Fig. 8

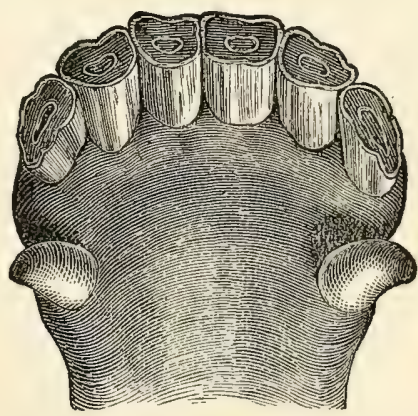

EIGUT YEARS OLD.

colt is now in possession of a full set of temporary incisors. These temporary, or "milk" teeth, differ from the permanent ones by being much smaller and (previous to shedding) whiter, having on their anterior or front surfaces grooves or furrows; also a welldefined body, neck, and slender fang. They also present oval surfaces anteriorily, or in front; and within the mouth they are concave. Their surfaces are generally uniform and smooth, and resemble the eye of an English horse-bean.

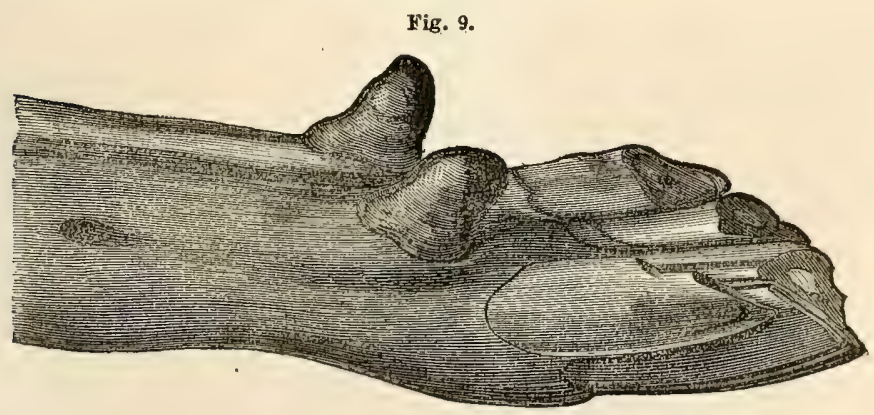

HOBIZONTAL VIEW OF OLD BLACK HAWB'S INCISORS AND TUEHES.

The Permanent Teeth.-Constitutional idiosyncrasies are often operative in hastening or retarding dentition, and the character of the food, whether it be natural or artificial; and the 
mode of obtaining the one or the other makes considerable difference in the wear and tear of the same, consequently we must bear in mind that the popular theory of "age" is sometimes more arbitrary than truthful, and allowances for the same must be made. At the age of $t w o$, or a few months over, the front permanent teeth ought to appear, and, consequently, at the age of three, varying a few months, the middle teeth are up. At four, varying as in the preceding cases, the corner teeth begin to show themselves, and are not up square with the others until the fifth year. The animal now emerges from colthood, and is known as a horse. As regards the female, during her minority she is termed "filly;" at five she enters the adult stage, and, consequently, is denomi-

Fig. 10.

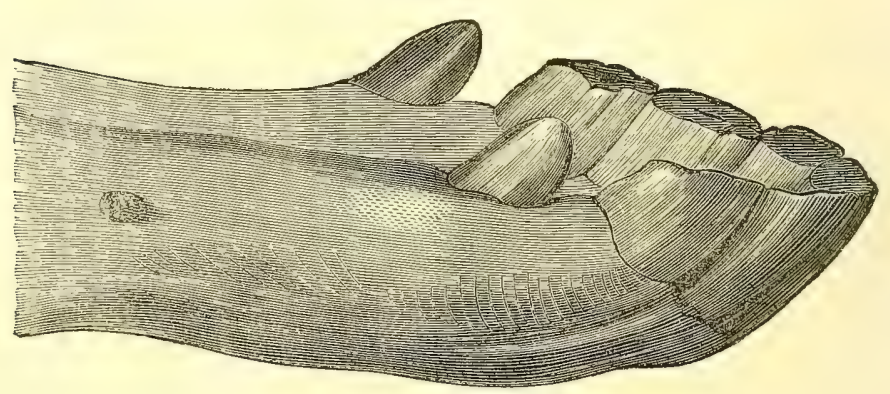

A YOUNG HORSE'S MOUTH.

nated a mare. Supposing the horse to have a full set of nippers, or incisors, at the age of five, the marks, or black incrustation, will have so worn away at six as to leave a faint brown mark. At seven, similar appearances have taken place in the two next, or middle, teeth. At eight the marks, or rather the cavities, of the two next, or corner teeth, are now about obliterated, and the face of the teeth are nearly level, and the central enamel is nearer the inward than the outward border. After the marks have all disappeared in the lower jaw, the exact age can not clearly be shown. At the age of nine the front teeth appear somewhat rounded; the middle and corner ones contract their oval faces, and the central enamel diminishes and approaches the inward border. At ten the middle incisors resemble those at nine, and the central enamel has approximated the inward border and is rounded. At eleven the middle teeth resemble those at ten, and 
the central enamel is almost worn off. At twelve the corner tecth take on the triangular form, and their central enamel is very contracted, and a yellow mark is left. At thirteen the sides of the front teeth thicken, and they begin to assume a triangular appearance, and the whole incisors of the lower jaw complete the triangular form at the age of seventeen. At eighteen the front teeth are somewhat flattened from side to side; the flattening goes on in the middle and corner teeth, and is completed at the age of twenty-one, giving them the appearance of a reversed oval. As regards the marks in the upper incisors, they disappear from the fiont teeth in the course of the ninth year, from the middle in the tenth, and from the corner or lateral ones in the eleventh year;

Fig. 11.

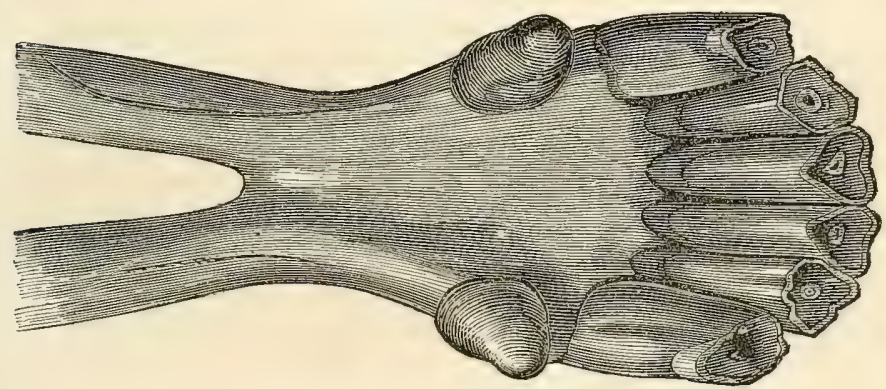

A PORTION OF THE LOWER JAW OF OLD BLACK HAWK, (Aged $t$ wenty-three years and eight months.)

and as the animal increases in years, the upper corner teeth become indented or notched. The inclination of the teeth enables us to form some idea of the horse's age. In a young horse, the teeth are upright (see fig. 10); after the age of eight they gradually become horizontal, (see "Black Hawk's" mouth,) and the upper teeth overlap the under ones, and thus wear off their outer edge.

The Tushes, or Canine Teeth.-Between the ages of four and six the tushes, which, in the male, are four in number, make their appearance. In shape they are conical, with a sharp point, and curved. On the inside there are two furrows, which commence at the base and meet at the apex, leaving a triangular eminence between them. The sharpness of the apex, the degree of curvature, and the distinctness of the furrows are good criterions of youth; for, as the animal advances in years, the tushes 
become blunt, less angular, and more rounded, and the furrows have disappeared. Supposing that the tushes are not completely evolved until the age of five; in the sixth year the apex of the cone is worn some; in the seventh the furrows grow shallow; in the eighth year they are obliterated, after which period the apex gradually wears away, and the body of the same becomes rounded and pointed, or blunt, according to circumstances. Aside from the teeth, an aged horse may be known by the deep pits above the orbital processes; the sunken eye ; by the prominence of the joints and loss of plumpness in the muscles; the lips are somewhat pendulous; the withers sharp; the back becomes arched; the teeth are lengthened, and become yellow.

Fig. 12 .

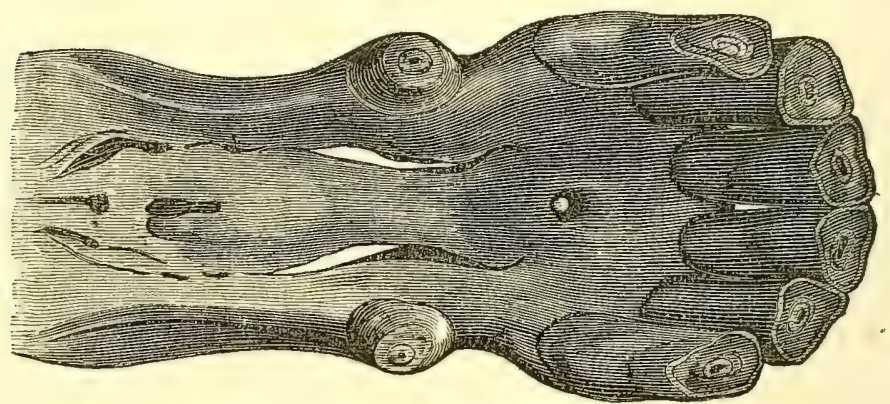

A PORTION OF THE UPPER JAW OF OLD BLACK HAWK,

(Aged twenty-three years and eight months.)

The Grinders, or Molars, afford but very little information as regards the precise age of a horse. As he advances in years, however, the outer edges become sharpened, so that it often becomes necessary to rasp them. As regards their development, it is understood that the foal is born with two upper and lower grinders in each jaw. At the end of a month, sometimes more, a third appears. At the completion of the first year, or thereabouts, a fourth grinder in each jaw appears. Thus the yearling has sixteen grinders. At the age of two, a fifth grinder appears, and at the age of three the sixth and last appear. It must be remembered that cribbers and voracious feeders are apt to deface their teeth, and thus some persons are apt to be deceived as regards the exact age; but a good judge, who takes into consideration not only the appearances of the surfaces, but also the marks, points, and the inclination of the teeth, will not be apt to make any grave mistake. 


\section{On the Diet of Horses-(Men often Dig their Graves} WITH THEIR TeEth, AND THE SAME IS TRUE AS REgardS Horses) - VARIETy oF Food NeCESSARY - EFfeCts oF CERTAIN KINDS OF FOOD-As REgardS THE REQUISITE QUANTITY, ETC.

It is admitted by medical men, and the experience of the reader may possibly have confirmed the fact, that a great proportion of the diseases occurring among live stock are produced, either directly or indirectly, by errors in diet; consequently the means of prevention should be known, and used accordingly.

No special form of diet can, for any great length of time, preserve the integrity of the animal system. In order to substantiate this assertion, we shall show the effect of simple elementary principles on the body of man, inferring, at the same time, that man's servant, the horse, is included. The reader has probably heard of the experiment made by Napoleon Bonaparte, which was that of trying to supply the nutritive wants of his system by living on a concentrated preparation of beef, in the form of jelly. He gave it a fair trial, yet came very near starving on the same; and he was led to remark that the stomach was a sort of scavenger, which required a given amount of rubbish to sort from, and select the necessary elements for renovating the tissues. $\mathrm{He}$ probably selected jelly because it is highly nutritious, containing more of the flesh-making principle than most articles of food. It was rich, not only in nitrogen, but also in oxygen, hydrogen, and carbon, four of the principal elements which compose the animal fabric.

The fact is, no single article of diet, let it be ever so rich in the elements of organized tissues, can long support life. A nation of men subsisting long on a simple form of diet, without variety, would soon become emaciated, and die of innutrition. Let the Grahamites abstain from milk, and live on bread and water, and they would soon exterminate themselves. Milk is a highly nutritious compound, and furnishes their systems with the equivalents that we obtain from beef and mutton. The inhabitants of the "Celestial Empire" (Chinese) are great consumers of rice. It is the principal article of diet among the poorer classes, many of whom are lank and lean, and would make very respectable 
walking lanterns, (if lighted candles were placed within their abdomens), only they happen, occasionally, to eateh a stray dog or pig, on which they make a savory meal, and thus furnish the material for the formation of muscle and fat.

Magendie has proved that even the canine race can not live more than forty days on any single article of diet, let it be ever so nutritious, for it is either followed by starvation or disease; hence the necessity for variety in food. In allusion to disease being produced by the long-continued use of a single article of diet, I would mention that the Scotch peasants are great consumers of oat-meal. This article is little inferior to wheat in the flesh-making principle, and we might naturally infer that an article of diet so valuable and palatable, when properly cooked, should tend to promote health. This, however, is not the case. Those who eat the most oat-meal are, according to medical testimony, the notorious subjects of intestinal concretions, and in the Edinburg Anatomical Museum is to be seen a vast and valuable collection of intestinal calculi, most of which eaused the deaths of confirmed oat-meal consumers.

Dr. Carpenter, an eminent physiologist, says that "no fact in dietetics is better established than that concerning the impossibility of long sustaining health and life on a single alimentary principle. Neither pure albumen, fibrine, gelatine, gum, sugar, starch, fat, nor oil, taken alone, can serve for the due nutrition of the body. This is partly due to their failing in supplying the waste of the tissues, and partly to the fact that single alimentary substances, long continued, excite such a feeling of disgust that the animals experimented on seem to prefer the endurance of starvation to the ingestion of the same."

The reader is probably aware that when a person has long been confined to any particular article of diet, a craving for something else is experienced, which very few persons can resist. This teaches us that, in order to preserve the health of live stock, we must vary the diet, and are not to be over-particular in selecting the most nutritious articles. But we want, as Napoleon says, a little rubbish-coarse rubbish. The internal surface of the stomach and bowels require to be irritated once in awhile, and this probably was the idea which Graham had when he first recommended coarse food. The stomach must be made to labor hard at times, or its function will deteriorate. Persons who complain 
of weak stomachs and dyspepsia are those who live on dainty viands, and seldom, if ever, distend that organ to a healthy capacity with coarse material. Consider, for a moment, the condition of Spanish and Italian peasants. They have not much of our national disease (dyspepsia) among them. The bread that they eat is made of coarse material; yet with that, and the addition of a little oil, wine, and a few vegetables, they can indure greater fatigue, and often carry a heavier burden than he who lives on more concentrated food. We may distend the horse's stomach with coarse food. and, perhaps, not impair its function so much as when overburdened with meal and concentrated food.

The stomach must be made to work for a living once in awhile. Hard work agrees with it, and coarse fodder stimulates and develops its latent powers, and augments the gastric secretion, which is the active solvent of the food. Labor operates on the stomach in the same manner and in the same ratio that it does on the brain or muscles-increases their capacity. Compare, for example, the brawny arm of the mechanic with that of the counting-house clerk, or the powerful muscles of the truck and farmhorse with those of the pet saddle-horse. This comes of work, hard work. See the king of birds, the eagle, towering above and beyond the sight and ken of man, darting, with almost lightning speed, from mountain to valley, buffeting the rude shocks of heaven's artillery. He acquires strength of muscle and wing by extraordinary exertion and desperate feats of flight. After the same fashion we develop the mental faculties, augment protracted mental labor, close thought, and study, light up the intellectual nature of man, and develop the latent powers of his brain; and the more his mind acquires, the greater and more varied are its powers.

It must be borne in mind, however, that the various functions of the body require periods of rest; for, should a horse be permitted to stand up to a full crib, and spend the greater part of the day and night in cramming his stomach, disease, sooner or later, must surely occur. The same is true as regards man. Let an individual gormandize through the day, and then indulge in a late supper, and continue the practice, he soon acquires a sympathetic headache, or the stomach grows refractory, and casts up the burden, for the simple reason that its function is overtaxed. It must have rest. The same rule applies to the muscular and men- 
tal natures. If, therefore, hard labor develops the function of either, rest, at suitable intervals, preserves their integrity, and, therefore, must not be disregarded.

In selecting food for working animals, we must remember that they require certain inorganic equivalents, which seem to be as necessary for the support of the system as nutriment. Salt, for example, is not nutritious; yet neither man nor brute can long exist without it. Common salt, chloride of sodium, is decomposed in the stomach, and is there found in the form of muriatic acid and soda. The former is supposed to aid digestion, and the latter eliminates bile. Neither is phosphorus (found in straw) nutritious, yet that article is absolutely necessary for the support of animal life. It is an element of both vegetable and animal organization. The former absorbs it from the soil, and, in turn, yield it to animals, by the process of digestion. Oats and beans are nitrogenous compounds, flesh-making equivalents, yet they furnish only one part in a thousand of the article we need-phosphorus; while cut straw, potatoes, and several other "inferior" vegetables, contain more than double the quantity of the same; so that a horse must eat such rubbish as straw, potatoes, carrots, beets, and "stubble," in order to supply the necessary material. Then consider that sulphur, iron, chlorine, lime, potassium, magnesium, and several other mineral substances, not in the least nutritious, are alike necessary for the support and integrity of the living organism, and, therefore, should be the elements of food. Some articles furnish the needful in abundance; in others there is a deficiency. This supplies another argument in favor of variations in diet.

A lecturer on physiology has remarked that "there exists a peculiar analogy between vegetable productions and living animals. Animal and vegetable fibrine-albumen of eggs and the gluten of wheat-contain about 15 per cent. of nitrogen, so that they are somewhat identical. If you take $100 \mathrm{lbs}$. of flour and wash it in water, frequently changing the same, you get $15 \mathrm{lbs}$. of gluten. This is the flesh-making principle, and represents 15 lbs. of the albumen of flesh. The gluten of flour, caseine of cheese and peas, albumen of eggs, and the flesh of an animal contain also a relative amount of carbon, hydrogen, and oxygen; so that the flesh of animals is already prepared for them in the vegetable world. The digestive organs of animals merely change the 
mechanical form and condition of the former ; their chemical composition remain about the same."

\section{THE EFFECTS OF VARTOUS KINDS OF Food.}

It is customary, in some stables, to feed horses, nearly all the year round, with what is known as "cut feed," which is composed of cut hay, meal, shorts, salt, and considerable water; the whole is mixed together, and server out, sometimes, without regard to quantity. This kind of food might, and sometimes does, agree with horses, but it is not right to feed them, year after year, on the same, for the reason just set forth. Another reason for objecting to this food is, that, in the stables alluded to, we hear of a great many cases of tympanitis and flatulent colic (diseases somewhat identical), arising, no doubt, from the presence of so large a quantity of water as some persons are in the habit of using. It saturates the food, and retards digestion. Not only this, but when dry food, highly charged with water, enters the stomach, the temperature of the latter causes the food to swellincrease in bulk-and distends that organ, and also favors fermentation instead of digestion; hence arises flatulency. We do not, however, mean to contend that such food is at all times the direct cause of indigestion, colic, etc., because many stablers are ready to testify that they have fed the same for many years without any apparent inconvenience to their horses; but we contend that it acts indirectly in the manner alluded to; and, although some horses may " get used to it," and others, having wonderful digestive organs, assimilate it, yet the day of reckoning may not be far off. We contend that water taken with food always retards digestion. The proper solvents of the food are the gastric fluids, and the horse has abundant facilities for supplying the requisite quantity. An ordinary horse is said to secrete, while feeding, fluid, of salivial and gastric characters, at the rate of one gallon per hour-enougl, we should judge, to saturate a common meal; therefore the water is not needed. We urge no objection against the more rational custom of merely sprinkling the food with salted water, in view of absorbing dust, which often abounds in inferior hay, but do seriously object to the practice of using a large quantity of culd water in the preparation of food for horses.

From experiments made by scientific men, it has been ascer- 
tained that parsnips, carrots, turnips, and cabbage, which contain from 80 to 90 per cent. of water, require over twice as much time to digest as when the food is free from water. Cabbage, for example, requires twenty hours, and broiled beef-steak only eight, to digest.

Turn a cow into a luxuriant pasture of grass or clover, and, after partaking of one or the other, she is liable to become "blown" or "hoven"-tympanitic; the abdomen becomes enormously distended with gas, (either carbonic acid gas, or sulphureted hydrogen,) and, unless the same be condensed or evacuated, rupture and death are sure to follow. This imperfect digestion and consequent generation of gas is due to the presence of vegetable fluids found in green fodder. Therefore, animals having weak digestive organs, predisposed to flatulency, should have the privilege of watering their own food with salivial fluid. The best diet for such an animal would be "dry feed," composed of ground oats, cracked corn, "fine feed," and a small quantity of sweet hay. On the other hand, a constipated state of the bowels always indicates coarse food; and in this view the English use chopped straw and coarse bran, with decided advantage. Animals should never be watered immediately before nor after meals, after the lapse of an hour from feeding time is the best.

\section{As regards the Quantity of Food Required.}

The adult horse does not require so much of the flesh-making principle as the young and growing animal, but he seems to require a greater variety. The adult merely requires enough to replace the waste-the wear and tear of his system. If he obtains more than this, the surplus is either excreted from the body, or else stored up within the same in the form of fat; and every body knows that a fat horse or fat man are not best adapted for a race nor hard labor, but of all others, (except those in a state of debility,) they are most subject to acute disease. With the young and growing animal the case is different. Here we require bone, muscle, and nerve. Oats, corn, and pollard furnish the same. The colt obtains from its mother's milk all the elements of its own organization in a concentrated form-all that seems necessary for developing bodily proportions and hereditary traits; therefore, when weaned, the colt must be furnished with the same equivalents in the form of fodder: ground oats, wheat bran, and meal furnish 
the same. It is the young and growing animal that requires our greatest attention. If our readers desire to raise colts that shall remunerate them for the trouble and expense incurred, they must feed the same, during their minority, with a liberal hand. Any neglect at this period can never be made up in after life. The subject will always remain lank and lean-living monuments of their master's folly or ignorance, as the case may be. In addition to the food required for the colt's growth, we must also furnish enough to supply the waste incurred by expenditure of muscular power. We all know that the young are very active and playful. Every muscular movement involves an expenditure of vital force, and thus exhausts the system; therefore, in view of developing their full proportions, and promoting the integrity of the living mechanism, they must have nutritious food, and plenty of it. They are not, however, to have a large quantity at a time, but little and often. Their stomach is small, not larger than that of a man's. Should it be overdistended with innutritious food, the organs of respiration and circulation become embarrassed, and the blood loaded with carbon. They require food often, because the digestive organs are very active, and soon dispose of an ordinary meal. Then comes the sensation of hunger, which every one knows is hard to bear.

The climate or temperature of the surrounding atmosphere has a wonderful effect on the animal machine. Let two horses be located in different stables, one of which shall be, like "Jack Straw's" house, neither wind-tight nor water-proof; the other built on the air-tight principle. The occupant of the former will require more food than he of the latter, because cold air has a depressing influence on the body, exhausting superficial heat. Our readers are probably aware that if a hot brick be placed in contact with a cold one, the caloric radiated from the first is absorbed by the latter, until a sort of equilibrium be established. The same is true as regards the body of a horse. He being in an atmosphere many degrees less than that of his own body, gives off the heat of the same to the surrounding medium. The heat thus given off has to be replaced by food, which is the combustible material. But it often happens that the digestive organs are deranged, incapable of assimilating a sufficiency of carbonaceous material to maintain even the normal temperature of the body. The consequences are, loss of flesh and health. On the other hand, a fat 
horse can endure the depressing influences of a cold atmosphere, because he has within the body a vast generator and non-conductor of heat in the form of adipose matter. It has been proved that the immediate cause of death in warm-blooded animals, when food has been withheld, was their inability to keep up that temperature necessary for the integrity of vital operations. The animal located in an air-tight stable is, probably, surrounded by an atmosphere almost equal in temperature to that of his own body, and, consequently, he does not require so much food as the former. Hence, the amount of food necessary in the one case might be too much for another, and, consequently, operate injuriously. A cold, bracing wind is said to "sharpen the appetite." The inhabitants of northern regions require more food than those of the southern. A horse, therefore, of weak muscular organization, the subject of debility, requires a comfortable stable, and food that will develop muscles. Now, it has been ascertained that horse-beans contain more of the nitro-albuminous principles than any other article of diet. English horses are very partial to the same, and in such the development of muscle is very remarkable; consequently, a fat horse requires less of the nitro-albuminous principle, and more of the aqueous, in the form of carrots, potatoes, and beets.

It must never be forgotten that some horses will keep in fair working order on a moderate supply of aliment. In such cases, we infer that the digestive organs are in a state of activity capable of extracting all the nutrient properties from the same. On the other hand, a horse performs less work than the former, consumes twice as much food, yet actually loses flesh. The digestive organs are then at fault. A horse, however, may lose flesh, become weak in the legs, etc., in consequence of the laborious nature of his employment. Therefore, any derangement occasioned in this way prevents the food from being converted into healthy chyme, chyle, or blood, and thus the necessary deposit of new matter is prevented.

Most voracious feeders are dyspeptic, and such are almost never free from intestinal worms. Their breath becomes feted; saliva, thick and tenacious; excrement, slimy; and should the subject be fed on oats, the same would be found, after traversing the alimentary canal, unmasticated. The principal remedy for indigestion is change in diet. Sometimes it may be proper to allow scalded oats, although cooking does not add to their nutritive quality, yet, more 
or less, completes the destruction of organization, and better prepares them for the action of weak solvents.

Changes in diet will sometimes work wonders in the restoration of a dyspeptic ; yet he will require, also, suitable medicinal agents, in view of augmenting the digestive function, for which purpose the following is recommended:

No. 17. Fluid extract of chamomile...........4 oz.

Fluid extract of ginger.............. $3 \mathrm{oz}$.

Powdered hyposulphite of soda......... 2 oz.

Water....................... 5 oz.

Mix.

Dose, a wine-glassful night and morning.

\section{Changes in Diet.}

Sudden changes in diet are not recommended. For example, should an animal have been previously fed on corn, meal, or oats, he should not be turned out to shift for himself, as the saying is, and depend entirely on grass for a living. Grass acts on horses unaccustomed to it as an aperient-scours them-which is a debilitating process. Grass may improve the health of a fat, lazy, or humory horse-reduce flesh and purify his blood; but the emaciated horse requires, in addition, a daily feed of oats or cracked corn, to make up for the deficiency of carbon in the former. For these, and other reasons that we might urge, the reader will perceive that changes in diet can only be made valuable in proportion to our knowledge of the wants of the animal economy.

A great proportion of our horses are too well fed, obtaining more food than they require. In this land of plenty, most of our valuable horses are overfed; and more especially does this happen among animals owned by wealthy and liberal individuals. The impression we wish to convey to the mind of the reader is, that the food of such is not propertioned to labor; in other words, there exists a disproportion between the amount of carbon taken, in the form of food, and the oxygen received, in the process of respiration. Now, to illustrate this, we will suppose that a man engaged in mercantile pursuits owns one or more horses. He has not the time nor inclination to give the one or the other the necessary amount of exercise. They stand up to a full crib, from day to day, enjoying, or, rather, gorging themselves with, a certain 
amount of fodder over and above what they actually require, and much more than they really need. The surplus is often stored up in the form of fat, and this induces acute diseases, and they die of too much food and care. It is very rare that we have occasion to recommend a man to feed his horse more liberally, but almost always the reverse. Starvation is said to be the cause for many equine diseases; but, so far as our experience goes, such cases are, in this country, very rare. We conceive the term starvation to be a libel upon civilization; and so unfrequent is its application among a nation of husbandmen, that it is omitted in our dictionaries. A man on a barren rock, or a horse in the deserts of Arabia, might probably starve; but the idea of the latter starving in the vicinity of a well-stocked barn or stable, within striking distance of a land of plenty, seems to us a very absurd conclusion. There are enough horses to be found dressed up in the garb of starvation, having tight skins, prominent ribs, and a cadaverous countenance, living, yet half dead. But they know nothing of the "famine in Egypt;" they get not only enough, but too much of the same kind. They probably require a change in diet, or else they are confirmed dyspeptics, laboring under a chronic form of indigestion; and if such should be the case, quantity is objectionable, and good quality more desirable. The stomach, however, is not at fault, its function being deranged. Or they probably get enough, and perhaps too much, for a weak stomach; hence loss of flesh, etc.

Some men are in the habit of bleeding their horses every spring. This is done in view of reducing fat and flesh, the subjects being humory (plethoric), their systems abounding in highly carbonized blood, which is proof positive that the same have been overfed. The racer, before he ean perform a feat of speed, must be prepared, as the saying is. This implies bleeding and physicking. Some works on farriery lay down regular rules for putting a horse in racing condition, and the remedies are fleam, physie, and bran, proof positive that such animals have had too bounteous a supply of nutriment.

Some horses-and the same is true of man-grow poor in consequence of having to carry abont a juvenile restaurant within their digestive organs. They probably become exhausted, or plethoric, as the case may be, in consequence of an overburdened stomach. A stomach overburdened reacts on the nervous system, deranges the physiological condition of the subject, and lays the foundation 
for hypertrophy (which is an abnormal increase of fat or tissue), or the opposite, which condition is known as atrophy - a wasting of the same. But most frequently an overburdened stomach induces diseases known as staggers, cerebral congestion, softening of brain, etc.

Many horse owners are continually devising means to excite the appetite of their horses, in order to get as much food as possible into their stomachs. Some men seem to think that an error in this direction can never occur; and should the animal refuse to consume the abundance thus placed before him, the liberal owner is apt to consider his pet sick, or think that he must have a poor appetite. Now, it were far better, for both man and horse if the former would only experiment in the opposite direction, and ascertain how small a quantity a horse may subsist on. A small quantity of good food, well digested, answers the purpose of nutrition much better than a large quantity, imperfectly so. It is very interesting to contemplate how efficient a little food proves for the promotion of health and longevity. For exåmple, a little barley and coarse fodder will suffice for the " courser of the desert." Shetland and Welsh ponies will live and grow fat on the mere vestiges of vegetation. The best cow in the world-the property of J. H. Kelly, of Cleveland, Ohio, weighing 1,350 pounds-is fed exclusively on hay and straw. Very poor fodder some may exclaim; yet in the course of ten months the same creature yielded 4,921 quarts of milk, and during a single month, after calving, she gave 620 quarts, and the least she gave during the winter months was 562 quarts. A pig will grow fat on small quantities of the right kind of food, and yet lose flesh when suffered to gorge himself with the same. Many swine, in the vicinity of coal mines, consume both coal and charcoal, and little else, yet they thrive well.

Evidences can be furnished going to show that both the superior and inferior orders of creation might subsist on much less food than they are in the habit of using, and without danger to themselves. Shipwrecked mariners have been known to exist several weeks without food; and there is a man now residing in Illinois, who, during a period of thirty-two days, never tasted food. The sleeping man of Rochester is another example; and a case is recorded in one of our medical journals showing that an individual once lived for several months on nothing but pure water. As 
regards horses, they form no exception to this peculiarity. We might introduce evidence, convincing and positive, of their ability to endure the privations of hunger, and, at the same time, show that they suffer but little from its effects. A single case will serve to illustrate this. We once treated a case of tetanus (lock-jaw). The subject never tasted food during a period of sixteen days; on the seventeenth the masseters relaxed, and the faculty of swallowing returned. At this period we might suppose him to be " hungry as a bear," yet, on offering him a few oats, he did not appear to be very ravenous, and partook of food subsequently offered him as if nothing had happened. These are extreme cases, yet they go to show that there is no cause for alarm because a horse happens to be "off his feed" once in awhile. Such condition may ultimately prove salutary, affording the stomach and its associates time to rest from their herculean labors.

The fact that most of our adult horses get more food than they need has been demonstrated by analysis of their excrement, which has been found to contain a large amount of nutritious material over and above what the animals actually need. We can develop the gormandizing powers of very many horses, by placing before them, from day to day, more than they require. Their appetites, like some of ours, are not proof against temptation; and the digestive organs may be trained to dispose of twice the quantity of food actually needed, and the habit, at first acquired, becomes permament, and the creature is known as a voracious feeder-a glutton. Hence, through indiseretions of this character; we can augment both function and capacity of stomach. We remember examining the stomach of a horse, the property of a baker, who was in the habit of feeding the former on brown bread. The animal died of chronic indigestion, and his stomach exceeded in capacity that of two ordinary horses. Great care, therefore, is requisite in regard to the proper feeding of horses; for, in their domesticated state, they have lost those natural instincts which serve to inform the untamed animal of the necessary amount of food which his system needs, and they are in the condition of a thoughtless child that will eat all day, and, on retiring to bed, will crave and cry for more.

Our readiers have probably heard of the gormandizing propensities of natives in the arctic regions. Some of them think nothing of bolting down twenty pounds of meat and oil per day, and 
making a good supper on tallow candles. A case is related, by Captain Cochraxe, of a Russian who ate, in the course of twentyfour hours, the hind-quarter of an ox, twenty pounds of fat, and drank a quantity of melted butter. He also states that he has seen three gluttons consume a deer at one meal. But we need not go beyond our own immediate vicinity to prove that the gormandizing powers of both men and horses are equally extraordinary. The corn-dealer's bill furnishes one illustration; and the lengthened meal which some of our young men indulge in, commencing in the morning and only ending at night, completes the evidence. Hence, with these facts before us, we may safely conclude that errors in diet are constantly occurring, and, consequently, a great many unnecessary diseases arise in consequence; therefore, we recommend our readers to make an experiment in the opposite direction, and ascertain how small a quantity of good food will answer the purpose of nutrition. Should the quantity be insufficient for the animal's wants, we shall soon be made aware of the fact by loss of flesh and other unmistakable signs. On the other hand, the error alluded to is not so easily corrected; for the animal may die, overburdened with fat, of an acute disease, before we can reduce his system.

\section{Conclusion.}

The reader will perceive that in the management and feeding of horses there opens a fine field of observation and improvement; yet, in order to apply that unlimited power which man seems to possess over his own organization and that of the inferior orders of creation, he must be conversant with animal physiology; for on this science alone do we base the problem of life.

Now, reader, after having presented this essay for your consideration, pray do not find fauit with the stable-keeper because your horse does not look fat and sleek. You had better trust to the discretion of the man who, having been long in the stable business, is perhaps better qualified than yourself to judge of the effects of food under the states of rest and exercise, and knows how to graduate the same accordingly. We frequently have occasion to notice that horses owned by stable-keepers are never so fat as the boarders-a very good proof that the latter get more than they require. Some men are in the habit of ordering a given quantity, say six or twelve quarts, of oats at a feed, whenever they put up, and the 
feeder gives it. At the same time he is well aware that the animal does not need it, yet he must obey orders; for should he suggest that the quantity be too great, his motrve may be questioned, and wrong inferences drawn. Depend upon it, therefore, that when the stabler, by a judicious system of feeding, prevents fat from ac- . cumulating on horses, he is conferring a favor on his patrons and benefiting the animal.

As regards the number of meals per day, our own observations satisfy us that working horses require three meals per day-a bountiful, yet very early breakfast; at noon, a light meal, composed of eut hay and oats; and at night the quantity may be increased equal to the morning meal. It is very poor policy to feed or water on the road when performing a short journey; yet, should a horse seem to stand in need of something, we should not object to a handful of oats and a few swallows of water occasionally.

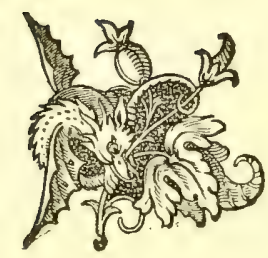




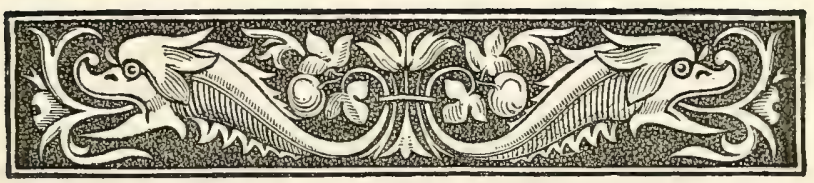

\title{
SECTION VI.
}

\author{
GLA N DERS AND FARCY.
}

Glanders-Gianders can be communicated to Man-Contagiousness of Glanders-Suspected Glanders-How came the Disease to be called Glanders? -Diagnostic Symptom of Glanders-Treatment of GlandersFarcy (Disease of the Absorbents).

\section{Glanders.}

O $\mathrm{NE}$ of the greatest evils which farmers and horse-dealers have been subjected to, during the prevalence of our late national troubles, was the sale of condemned army horses, many of them being afflicted, either insidionsly or actually, with that form of equine affection known as glanders; for it is a well-known fact that this terrible malady is, under certain circumstances or conditions of the animal economy, both infectious and contagious; hence the great evil.

For example, should a glandered horse be placed in a stable where the principles of ventilation and of the admission of light are entirely disregarded, the other inmates of the den, by continually breathing, over and over again, the contaminated atmosphere which must necessarily occupy the same, will (provided their systems are susceptible) take the disease by infection; that is to say, the miasmatic virus finds an introduction into the blood through the lungs and pulmonary circulation. On the other hand, glanders, under almost every circumstance, can be communicated by contagion or touch; that is, bringing the glandered nasal discharge in contact with an abraded surface, including cuts and scratches.

\section{Glanders can be communicated to Man.}

Within the last quarter of this century, two veterinary surgeons-one residing in Walworth, and the other in Wolverhamp- 
ton-are reported as having died from inoculation of glanders. This terrible disease is not often seen in Scotland, but very frequently in England, and still more so in Ireland. From the latter circumstance, the malady is often found to be imported about the west coast of Scotland. London has always been rather renowned for the prevalence of glanders among omnibus, cab, and other

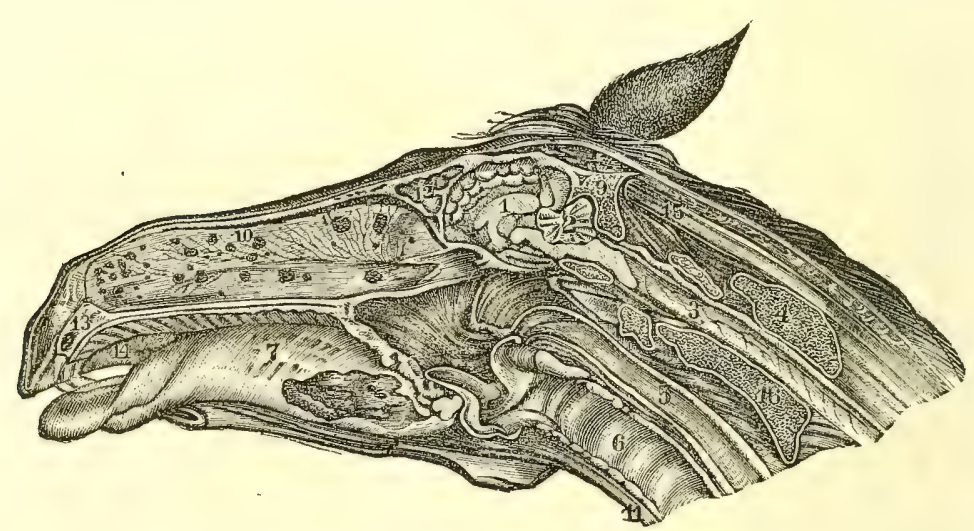

BECTION OF A HORSE'S IEAD SHOWING ULCERATION OF THE SCHNEIDERIAN MEMBRANE, WHICE CONSTITUTES THE DIAGNOSTIC SYMPTOM OF GLANDERS.

Explanatron.-On contrasting the above picture with the one on the opposite page, the reader will perceive, in the above, a number of dark spots on the lining membrane of the nose (schneiderian), showing the chancreous ulcerations which constitute the diagnostic symptoms of glanders.

1. The cerebrum, or anterior portion of the brain.

2. The cerebellum, or posterior portion of the brain.

3. The spinal cord.

4. Shows a section of the cervical vertebro, or bones of the neck.

5. The cosophagus.

6. The windpipe.

7. The tongue.

9. Section of the occipital bone.

10. The schneiderian membrane.

11. A muscle known as the sterno maxillarius. It is located beneath the neck, and is in. serted into the angle of the lower jaw and the front part of the breast bone. Its action is to aid in closing the mouth and drawing the head downward toward the breast; one muscle acting draws the head to one side.

12. 'The frontal sinuses.

13. Section of the anterior portion of the upper jaw.

14. The hard palate.

15. The strong ligament of the spine known as the ligamentum sufflavium.

16. Section of the inferior portion of the cervical vertebre

horses. A very strict supervision is maintained, and all glandered horses are destroyed when discovered; but nevertheless we can state, on good authority, that the omnibus horses of London have suffered very severely from this disease, and do so still. The par- 
tial measures adopted by companies are not sufficient to eradicate it, and the "glandered night train" is not altogether a thing of the past. The danger to human life is so great that we feel happy to seize any opportunity to urge the adoption of the most effectual measures for the suppression of any practice which tends to prolong the life of the glandered horse.

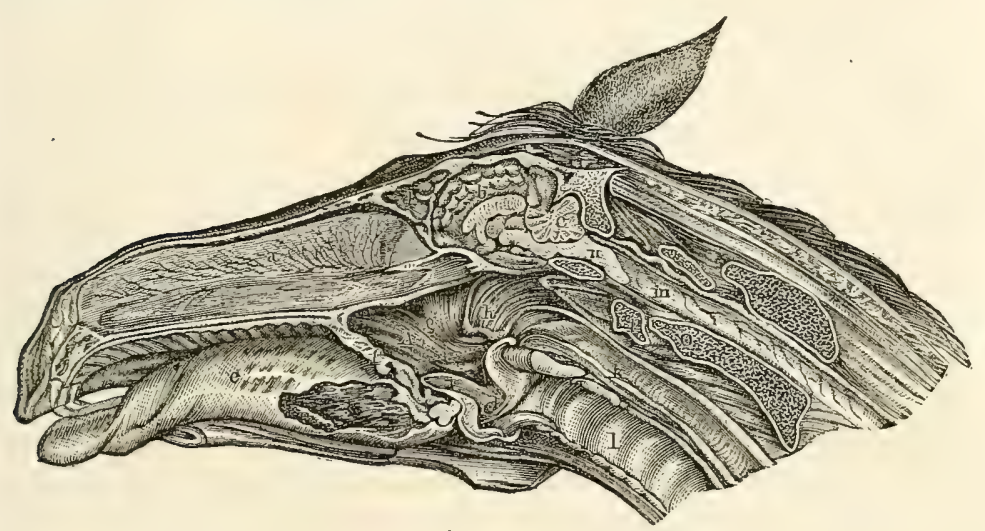

SECTION OF A HORSE'S HEAD IN A HEALTHY CONDITION-ITS ANATOMT.

Description.-a The lining membrane of the nose, known as the schneiderian membrane. On its surface are seen the vessels which furnish it with arterial blood.

$b$ The anterior portion of the brain, known as the cerebrum.

c The posterior portion of the brain, known as the cerebellum.

$d$ The frontal sinuses.

$e$ The tongue.

$f$ Showing the velum palate, or soft palate.

$g$ The back part of the mouth.

$h$ The pharynx, or top of the oesophagus.

$i$ The epiglottis, or cartilage at the root of the tongue.

$j$ The glottis, or opening intc the windpipe.

$k$ The œsophagus, or gullet.

$l$ 'The trachea, or windpipe.

$m$ The spinal cord.

$n$ Junction of the cerebellum with the spinal cord.

op Showing a section of the cervical vertebræ, or bones of the neck.

The dotted lines between $a$ and $g$, show the direction leading to the trachea and csophagus.

A fatal case of glander, reported in the Baltimore "Herald of Reform," is as follows: Mr. I. P. Burus, a grocer, died a horrible death in that city in consequence of poison communicated to his system from a horse afflicted with glanders. During the administration of medicine, Mr. Burus thrust into the animal's mouth his hand, a finger of which had been previously cut, and the flesh laid open. Through this wound the virus was absorbed, and mortification supervened. A surgeon was called upon to amputate 
the diseased member. Perceiving, however, that the poison had penetrated to every portion of the unfortunate man's system, he declined performing the operation, and stated that no earthly skill could save him. After lingering in great agony, death closed the scene.

Death of a Russian Lady from Glanders.-The awful death of Madame Palesikoff, one of the most charming among all that bevy of entertaining Russian ladies who sometimes gladden the winters of Paris, has created a terrible shock among the circles she so lately embellished by her presence. The unhappy lady left Paris but a short time ago on a summer tour to Germany. While stepping from the door of the opera-house in Berlin, to gain her carriage, she let fall one of her bracelets close to the pavement. Stooping to pick it up, she noticed, at the time, laughingly, that "one of the horses belonging to a carriage standing at hand, dropped his head so close to her face that he touched her, and left a moist kiss upon her cheek." In a few days the unfortunate lady was taken ill with that most horrible disease, glanders, and in a few days more, breathed her last, in spite of the attendance of the first physicians of Berlin, and every resource to be obtained by wealth, or by the ceaseless vigilance of friends.*

Still another case.-Sidney W. M., aged 23, a horse-slaughterer, residing at Plumistead, England, was admitted into Guy's Hospital, on March 13, 1861, under the care of Mr. Birkett. He had always enjoyed good health, but he lived freely. Six days previous to his admission he cut his right hand deeply over the dorsal aspect of the thumb. The wound bled freely, but he felt no inconvenience from it, of any consequence, until the 10 th. He then had pain in the part, extending upward to the axilla, and also a numbing pain in the calf of the right leg. His appetite was good, and he had so little constitutional disturbance that he went as usual to superintend the work which the aceident had prevented his carrying out. On the 11th the pain in the arm increased, and that in the leg, on the 12th, extended upward to the thigh. This had become considerably aggravated, and he then became generally indisposed, and was unable to stand. The following day he applied for admission. He stated that he was extremely ill, and that his limbs felt almost paralyzed. He was quite unable to

* Berlin Court Journal. 
stand. He had constant rigors, a burning skin, white tongue, and his pulse was 120. The wound in the hand was inflamed, and the calf of the right leg and the thigh were swollen, and evidently the seats of abscesses. These were freely opened, with relief. $\mathrm{He}$ was ordered effervescing medicine, and two pints of porter. The case was then regarded as one of pyæmia. On the 14th he was much relieved. He had slept tolerably. The abscesses.discharged freely, and he was able to take his food. On the 15 th he was seized with vomiting, bringing up a dark bilious fluid. His powers were less. Wine was given freely. On the 16 th the vomiting continued, and he complained of a pain in the left thigh. An abscess was discovered, on examination, which was freely opened. The man, at this time, was physically in a very low condition. He took brandy, eggs, and other liquid nourishment with avidity. His manner was peculiar, and somewhat excited. On the 17 th another abscess was opened in the left arm. On the 18th the left foot became the seat of an abscess, and on the 19th, for the first time, a pustular eruption was observed on the face and body, which suggested to $\mathbf{M r}$. Birkett the probability that some animal poison was the cause of all this mischief.

The eruption was peculiar, having no definite shape. It appeared to be more like small irregular blebs, containing pus, varying from the size of a pea to a sixpence. Some were round, others oval, and others of an irregular form. Toward evening, also, his breathing became much impaired, a bloody mucus obstructing the nostrils. Toward night he became delirious; his powers became less, the nasal discharge more profuse, and at 1.30 , on the 20 th, he died. The eruption, some hours before his death, in parts, had disappeared; but in others a fresh crop sprang up, particularly over the region of the sternum. These were, however, of the same character, but smaller. No post-mortem examination was made.*

\section{Contagiodsness of Glanders.}

Mr. Perctvalu submits the following deductions, regarding the contagiousness of glanders, as the result of facts gleaned from his own experience:

"1. That farcy and glanders, which constitute the same disease,

* Medical Times and Gazette. 
are propagated through the medium of stabling, and this we believe to be the more usual way in which the disease is communicated from horse to horse.

2. That infected stabling may harbor and retain the infection for months, or even years; and although, by thoroughly cleansing and making use of disinfecting means, the contagion might be destroyed, yet it would not be wise to occupy such stables immediately after such supposed or alleged disinfection.

3. That the virus, or poison of glanders, may lie for months, in a state of incubation, in the horse's constitution before the disease breaks out. Of this we have had the most positive evidence.

4. That when a stable of horses becomes contaminated, the disease often makes fearful ravages among them before it quits; and it is only after a period of several months exemption from all disease of the kind that a clean bill of health can be rendered."

From the preceding evidence, it is probable that the reader will entertain but little doubt of the contagious character of the malady; yet it is very important that every one, either directly or indirectly interested in horses, or having any regard for the welfare of mankind, should be familiar with all that is important and useful as regards the cause and nature of the awful malady now under consideration. It is often mistaken for other diseases that (in so far as contagion is concerned) are perfectly harmless; yet many valuable human lives have paid the forfeit, and many priceless animals have been sacrificed on an altar of ignorance which the light of science has but recently illuminated. Hence, correct information is what the people require, in consequence of the emergency of the peril; and this is what the author aims at in offering this article for the consideration of the reader.

Many hundreds of times, during the professional career of the author, have horses been brought to him for treatment, declared by their owners to be the subjects of glanders, simply because they had enlarged glands under the jaw, and a nasal discharge; and by the successful treatment of the same, he has got the credit of curing glanders, a feat which he never pretends to have accomplished. Mr. GaMgeE has very lucidly illustrated this part of the argument, as regards the mistakes made in diagnosing glanders. He states that glanders may be suspected, instead of being a reality; hence, many supposed cures are on record. The following are his remarks, under the caption of "suspected glanders": 
"Glanders is, fortunately, a rare disease in this country (Scotland), thanks to the pole-ax. Englishmen have long since advocated and practiced the shooting of suspected animals, rather than trusting the lives of men and horses to the chances of escape, wherever cases of chronic nasal discharge are met with. The command officer and veterinarian of a British cavalry regiment would consider it a great disgrace if such a disease acquired any firm hold in their stables ; and in spite of occasional introductions of the disease when a number of remounts may be purchased, the unrelenting order to kill rather than attempt to cure, saves the public purse and the reputation of those responsible for the health and condition of our troop horses. I am as great an advocate for the slaughter of glandered horses as I am for the slaughter of cattle affected with rinderpest. Glanders is more incurable than the cattle plague, as not even ten per cent. recover, but its communication is less certain and swift. It never could and never did destroy its tens of thousands over a country in the short space of time in which the steppe murrain spreads over the land, but it is, nevertheless, wise and proper to stamp it out. We have not indulged, as do our neighbors the French-who manage this matter, at all events, worse than we-in wild theories as to the transmissibility of acute and not of chronic glanders. We admit it to be always contagious and always deadly, and prevent it killing by shooting its victims.

We must not, however, forget that there are hundreds-nay, thousands-of cases of chronic nasal discharge which admit of some diagnosis on the part of skilled veterinarians, and which are erroneously set down as cases of glanders. Many of these cases are condemned because they baffle the attempts to restore them for a great length of time; and, unfortunately, in this country many forms of nasal disease have been rarely cured, simply because their nature has not been understood, and bold surgical operations have been dreaded. I could relate the histories of many cases which have yielded to radical measures after several veterinarians had pronounced the animals incurably glandered-more to get rid of them, perhaps, than from a conviction that they were suffering from the disease. I have seen as many as half a dozen animals, in a stable containing a score of horses, given up by practitioners because they had chronic discharge and swollen glands; and in ten days or a fortnight all the animals 
have recovered under the influence of nasal injections, and the internal administration of tonics.

It is impossible to enter into details as to the diagnosis of a great variety of cures. I may mention, however, that the curable discharges set out as incurable forms of glanders may be grouped under six heads.

1st. Fetid discharges from the nostril, owing to a carious tooth and caries of the upper jaw. The fetor is characteristic.

$2 d$. Intermittent discharges from an abscess in one of the turbinated bones within the nasal chamber. The swelling of the nasal bones and flow of pus when the head is jerked upward are diagnostic.

3d. Continuous or intermittent discharge from one or both nostrils, from accumulations of pus in the frontal and superior maxillary sinuses, indicated by the shape of the forehead and absence of resonance on percussion.

4th. Irregular discharge from the guttural pouches, brought on by exereise, or seen when an animal is made to eat hay, oats, a turnip, or carrots off the ground. As the head is depressed and jerked, a somewhat fetid and often abundant purulent matter flows freely.

5th. Discharges kept up by foreign objects in the posterior nares, which are continuous and fetid.

6th. Chronic regular nasal discharge-pure ozena-dependent on constitutional causes and defective management of acute catarrh.

All the foregoing varieties include the innumerable cases of suspected glanders for which animals are wrongfully destroyed. It is not every practitioner who can or would venture to open the guttural pouches, or practice dissection on the bones of the face to such an extent as we find requisite in overcoming deformities and curing long-standing diseases. The longer these cases are treated by any but the right plan, the greater the difficulties encountered when surgical operations are determined upon; and for this reason even those who would not dare to cut should always strive correctly to diagnose the form of disease presenting itself. It is not necessary to refer at greater length to the method of dealing with the very various forms of disease above indicated." 


\section{How came the disease to be Called Gianders.}

PERcrvalu is our authority for the following explanation: "The derivation of our word glanders is traceable through the French language, from which we appear to have borrowed it, to the Latin roots glandula and glans, the latter signifying any fruit kernel, such as a chestnut or acorn; the former, its diminutive, any small fruit kernel; and both afterward used in medicine to denote the glands of the body, many of which-such as were then so called-are small and comparable, both in shape and size, to acorns or other kernels. Celsus applies the term glandula to a swelling in the neck, supposed to be glandular; and Vegetius uses the same to denote swollen glands 'between the cheek-bones and lower jaws:' from his saying, however, that the glandules are 'especially troublesome to foales,' it would appear the disease he meant to describe was not glanders, but strangles. The French veterinarians, following the ancient phraseology, called a horse exhibiting any submaxillary tumor or enlargement, glande; not with any special reference to glanders, but simply because his glands or ' kernels,' as our farriers denominate them, had become enlarged; hence, with the French, a horse was said to be glande de gourme, as well as glande de morve and glande de farcin. It seems to have been our English writers on farriery who have restricted the application of the term to the foul and malignant disease now known under that appellation. Before then, glanders appears to have had no other meaning save that the horse had tumefied glands, or that, in the farrier's phrase, 'his kernels had come down.' The French call the disease la morve. A horse, however, in the estimation of Lafosse, is not to be regarded as having la morve proprement dite, unless he be glande, or have tumefaction of his glands."

Diagnostic Symptoms of Glanders.-Glanders consists in a discharge, from one or both nostrils, of matter which, by transfer or inoculation, will produce the same disease in another animal (of the equine or human species), and which discharge is, sooner or later, accompanied by vascular injection and chancrous ulceration of the schneiderian membrane of the nostrils, and tumefiction of the submaxillary lymphatic glands, and by farcy; so that a horse can not be considered as the subject of glanders until these symptoms are made manifest. 
On consulting “ Hippopathology," I find a paragraph, credited to a French surgeon, who very accurately describes the symptoms of glanders. It reads as follows: "The signs by which the disease may be known are, when a horse, already too old to be troubled with strangles, without a cough, voids matter by the nose, and has a kernel sticking to the bone; and, besides, in glanders the matter usually flows from one nostril, whereas, in a cold, it runs always out of both. Some cast the matter that is voided by the nostrils into water, and, if it swim on the top, they conclude the horse to be free of this distemper; but if it sink to the bottom, it is a sign of glanders, the principal use of this experiment being to distinguish the pus. But you must not depend on the certainty of this sign; for if the matter stick to the nostrils, like glue, it is a bad sign, and you may conclude the disease to be the glanders, though the matter do swim on the top. When either the breath or matter that comes out of the nostrils stinks, the disease is almost always incurable. I have seen horses troubled with this distemper without kernels, or, if there were any, they were small and moveable; and the only sign by which we could discover it to be glanders, was the glueyness of the matter discharged from the nasal outlet."

Treatment.-The author knows of no remedy for the cure of glanders. He considers it an incurable disease. In fact, most of our educated veterinarians contend that the disease, like pulmonary consumption, is incurable.

MAYHEw, one of the most intelligent veterinary teachers of the present period, informs us that "no medicine can restore the parts which disease has disorganized. There is no cure for glanders, which is essentially an ulcerative disorder." And this opinion is indorsed by others of equal eminence in the profession, who were employed lately, by the members of an agricultural society in England, to ascertain if there was any specific for the disease known as glanders, and the verdict was that no specific could be found. So soon as glanders is discovered in the horse, he should, by all means, be destroyed, and buried deep in the earth.

\section{Farcy (Disease of the Absorbents).}

This disease is usually met with among horses of the scrofulous diathesis, which diathesis is known by a proneness to diseases of 
the skin, and does not readily yield to medical treatment. Such horses are noticed as belonging to the nervous temperament, having thin, spare muscles, wiry neck and limbs, very quick and active in their movements, and having a very narrow chest. Yet it should be remembered that the scrofulous diathesis may exist in any horse, without regard to his conformation or temperament, provided he inherit, from either sire or dam, the requisite predisposition.

The disease sometimes steals on in a slow and insidious manner, so that it is not known to exist until, all at once, the animal becomes lame, and, in the course of a few hours, his legs swell; large inflammatory farey-buds appear just as suddenly; a stinking discharge takes place from the nostrils, and, unless the animal be destroyed, he soon dies. This form of farcy may, very properly, be denominated malignant farcy. When the disease manifests itself after this fashion, it is dangerous for a person to handle the subject. He should be destroyed and buried.

A case of this kind occurred very recently in a large stable, the facts of which are as follows: The author was requested to visit a bay gelding, of the nervous temperament, supposed to be the subject of a catarrhal affection. He lid not show any very marked symptoms of ill-health, except a slight discharge from both nostrils. He had performed labor up to within a few hours of the time of my visit, and had partaken of his usual amount of food. This was toward evening. I directed the foreman to rub the throat with stimulating liniment. Two ounces of fluid extract of resin weed were given him; his legs being cold, they were bandaged, and a warm bran-mash was placed before him. Next day he was a pitiable sight to behold. His limbs and other parts of the body had become dropsical; his face and eyelids had become so tumefied that the eyelids were almost totally closed, and scalding tears ran profusely down his cheeks. In various parts of the body the lymphatics were tumefied, presenting unmistakable farcybuds. A stinking discharge ran from his nostrils, and he was so lame that it was almost impossible to get him out of the stable. The case being diagnosed as farcy, the animal was shot.

Mr. Percivall alludes to the lameness and rapid tumefaction in the following language: "I have known horses so lame from farcy before the disease had, in any local or characteristic form, declared itself, that shoes have been removed and feet 
searched, etc., to discover the seat and cause of lameness, no suspicion having existed at the time that farcy was present in the animal's system. It may so happen, however, that some of the preliminary symptoms are observed or observable; that, on the contrary, farcy at once develops itself in an attack on some locality-most probably one hind limb. Indeed, so sudden, sharp, and severe are attacks of farey, in some instances, that, in the course of one night, the horse's limb will be swollen to a frightful size, so as to incapacitate him almost from turning in his stall and walking out of the stable. Ordinarily, the development of farcy plainly accounts for the halting or lameness, yet the lameness may appear without any ostensible cause."

Syimptoms. - The ordinary symptoms of farcy are, slight impairment of the general health; feverish symptoms; a snall, quick pulse; swelling or tumefaction of one of the hind legs, generally the left, with much lameness; tumefaction of the lymphatics, on various parts of the body, and in the groin. On the inside of the thighs, along the course of the femoral vein, corded, nodulous swellings will be found. Sometimes one or both hind limbs will swell below the hock, and an abscess may form in the vicinity of the fetlock, and isolated blotches will break out, suppurate, and terminate in farcy ulcer. No swelling of a hind limb, or any other part, constitutes a case of farcy apart from the unequivocal signs of lymphatic disease. There must be present corded, nodulated swellings, buds in some form or other, together with actual or appronching tumefaction of the lymphatic glands, or the case is not farcy. The disease evidently affects the absorbents. When it commences in those which are deep-seated, it usually ends in glanders. Glanders and farcy are considered the same disease; yet, unlike glanders, many cases of cure of mild farey are on record, but the author knows of none. A disease known as lymphatitis is often mistaken for farcy, and, as the former is curable, it is often recorded that the latter affection has been successfully treated.

Trectment.-I do not know that it is good policy for me to recommend any form of treatment for the disease known as true farcy. It is against the laws of this country, and the interests of society for any one to keep or treat a case of declared farey; therefore, the advice offered in regard to the treatment of glanders applies also to farcy. To show the reader that the author is not 
singular in his opinion as regards the treatment of this disease, a quotation from MAYHEw is here introduced:

"Farcy is, by the generality of practitioners, regarded as a more tractable disease than glanders. Certainly the course of the disorder is arrested much easier; but, to cure the malady, there is a constitution to renovate and a virus to destroy. Is it in the porrer of medicine to restore the health and strength, which have been underfed, sapped by a foul atmosphere, and exhausted by overwork? Tonics may prop up or stimulate for a time; but the drunkard and the opium-eater, among human beings, can inform us that the potency of the best selected and the choicest drugs, most judiciously prescribed, and carefully prepared, is very limited. Sulphate of copper, iron, oak bark, cayenne pepper, and cantharides, probably, are the chief medicines the practitioner will give. With such the horse may be patched up; he may even return to work. But at what a risk! He carries about the seeds of a disorder contagious to the human species, and in man even more terrible than the quadruped. Is it lawful, is it right, to try to save an avaricious master the chance of a few shillings, and incur the risk of poisoning an innocent person? The author thinks not. Therefore he will give no directions how to arrest the progress of farcy. The horse once contaminated is, indeed, very rarely or never cured. The animal, after the veterinary surgeon has shaken hands with the proprietor and departed, too often bears about an enlarged limb, which impedes his utility, and, at any period, may break forth again with more than the virulence of the original affection."

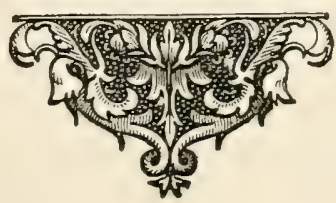




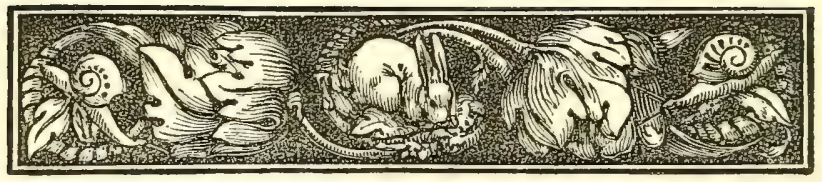

\title{
SECTION VII.
}

\author{
DISEASES OF THE DIGESTIVE ORGANS.
}

Lampas-Spontaneots Salivation-Bots-Inflammation of Stomach-Rup. TURE of Stomach-Gorged, or over-distended Stomach-Meteorization -Fuatulent Colic-Spasmodic Colic-Inflammation of the PeritonumAscites-Inflamation of tine Intestines-Strangulation of IntestinesWounds penetrating the Abdominal Cavitx-Splenic Apoplexy-Functional Disease of the Liver-Pancreas; its Fonction-Parasites which AFFECT THE INTESTINES.

\section{LAMPAS.}

$\mathbf{L}$ AMPAS is the name given to a slight tumefaction of the soft palate of the horse's mouth. It usually occurs during the period of dentition, at which time the mouth is hot and tender. At the time when the tushes are about making their appearance, the membrane over the crown of the tooth becomes tense, so as to cause the root of the tooth to press on the tental nerve. This causes much pain. In such cases we make a slight incision over the region of the tooth, which immediately relieves the animal. But most men overlook this difficulty, and, because the palate happens to be tumified, it is at once attacked with the barbarous firingiron. This treatment was condemned by Percivald, who, in writing of it, says:

"Are lampas disease? The complaints, frequent and grievous, which are daily reaching our ears, are enough to persuade us that they are disease. Every groom that has an unthriving horse, or one that does not feed, is sure to search for lampas; and, should he find any, the cause of loss of appetite, etc., is detected, and the remedy obvious-burning them out. Many a poor wight of a horse, even when suffering from a constitutional disease, has been subjected to this torturing operation, with a view of demonstrating the sagacity of the groom, and thereby has got added to his other 
ailments a foul, sloughy, carious sore upon the roof of his mouth. This may be said to be the fruits of the removal of lampas.

Supposing that the existence of lampas is owing to the teeth, surely the teeth should be removed, and not the bars of the mouth. In cutting or burning away the lampas, we are mistaking the effect for the cause. If it be contended that lampas do not owe their production to the irritation of teething, then I should like to be informed what does give rise to them; and, let what will give rise to them, I do not imagine there is any veterinarian hardy enough

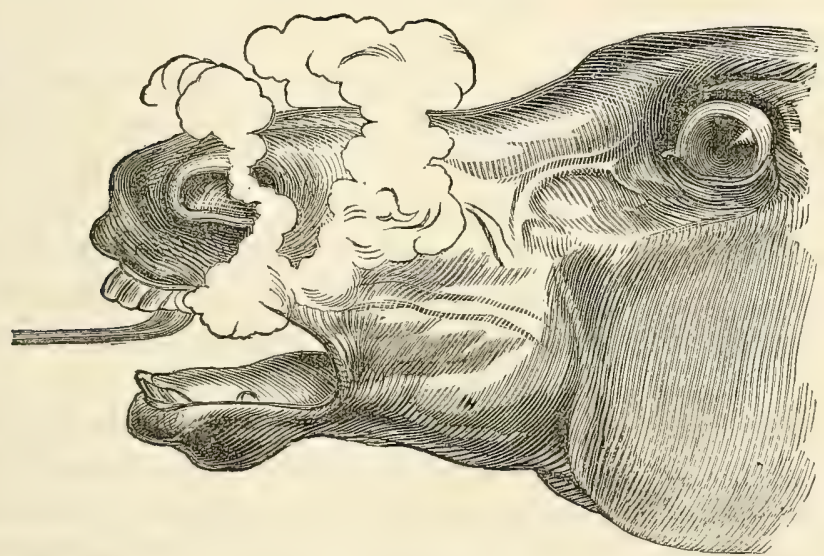

BURNING A HORSE'S MOUTH FOR LAMPAS.

(A cruel and unnecessary operation.)

to contend that the cause resides in the palate, and becomes removed by the hot iron. Those who are entering private practice, and find themselves compelled, at all times, to belie their consciences by the performance of unnecessary operations to please their employers, may be told that burning out lampas is, after all, preferable to lancing or cutting the bars; for, unless the palative artery is wounded, very little blood is obtained by stabbing the mouth; and the wounding of this vessel, which will certainly take place, should the punctures be made along the sides of the palate, or extend forward beyond the fourth bar from the front teeth, is not always a very safe proceeding."

It is an unfortunate occurrence that men can be found who will perform barbarous and unnecessary operations just to please their employers. If men were true to themselves and their profession, neither threats nor promises of reward would tempt them to prac- 
tice cruelty on animals. It is a fact that some men are often apparently compelled to perform unnecessary operations even when the inward monitor admonishes them that they are about to outrage the feelings of a poor dumb brute. Yet, for the honor of our profession, let it be recorded that there are but few, if any, of our educated surgeons that can be induced to perpetrate this outrage. If medical men will only take the pains to explain this matter, and prove to the people that the enormity here complained of is both absurd and barbarous, then the evil will soon cease; but so long as horse-owners believe lampas to be a disease, and men can be found ready to "burn them out," just so long will the evil exist. The more intelligent portion of husbandmen are now beginning to lend their influence to the work of rebuking ignorance, and of reforming the barbarous customs of bygone days, and, erelong, it will be a difficult job to convince them that their horses have lampas, and that the firing-iron is the remedy indicated. Surely, if the distinguished Percivalu calls lampas a "supposed malady," and sets his face against the means used for its removal, the wise men of the fleam should indorse his sentiments.

Treatment.- Should the colt's mouth, while teething, be hot and tender, it should be sponged often with a portion of equal parts of vinegar and water; and should any of the teeth appear imprisoned by the membrane of the mouth, a slight incision over the point of the tooth will give immediate relief.

\section{Spontaneods Salivation-" Slatering."}

Persons who own and handle horses must have noticed, occasionally, a foaming at the mouth, and sometimes a constant and profuse discharge of saliva. This, when occurring without any assignable cause, is termed spontaneous salivation. The disease is usually occasioned by some irritation in the immediate vicinity of the salivary glands, or from perverted function in the glands themselves. The preparations of antimony and calomel, when given to horses for any great length of time, in the form of condition powders, are very apt to produce salivation. Musty clover, and hay that is intermixed with lobelia, will also produce it; so, also, will sharp, projecting teeth; and diseased teeth have the same effect. In such cases, the cause being discovered, the removal of the same is the first step toward the cure. Then drench the animal, twice daily, with the following: 
No. 18. Powdered chlorate of potass........ 1 oz. Water...................... $\frac{1}{2}$ pint.

Should the animal show signs of debility or weakness, give one ounce of fluid extract of golden seal every morning, before feeding. Should the mouth appear to be sore, from the constant flow of saliva over its surfaces, or from any other cause, then the parts should be sponged two or three times daily with a portion of equal parts of powdered borax and honey, or with an infusion of bayberry bark.

When spontaneous salivation occurs in an aged horse, it denotes debility, and thus points out the propriety of sustaining the patient's strength by administering tonics and diffusible stimulants. The following is the best remedy for this state of the system:

No. 19. Fluid extract of chamomile........ $1 \mathrm{oz}$.

Fluid extract of ginger........... $\frac{1}{2} \mathrm{oz}$.

Give as a drench, night and morning. The patient should have a fair allowance of good nutritious diet.

Secretion of Saliva in Horses.-From experiments lately made, it appears that a healthy horse secretes from the paroted glands, which are situated beneath the ears at the posterior angles of the lower jaw, during active mastication, about one gallon of saliva per hour, and other glands in the vicinity of the throat and mouth secrete, in the same amount of time, three gallons, making in all four gallons per hour, which is mixed with the food during mastication. The following experiment was lately made on an aged horse, 15 hands 3 inches in height. The tubes leading from the paroted glands were divided on either side, and so fixed as to throw their contents into vessels held for that purpose; the animal was then fed on oats, which he masticated during a period of thirty minutes, at the end of which time half a gallon of saliva was caught. This calculation, however, will not hold good in all cases; for ravenous feeders, who do not thoroughly masticate their food, fail to secrete the necessary amount of saliva; hence the food is not properly masticated nor insalivated, and can not, therefore, undergo proper digestion; and when food is not thoroughly digested, it imparts but little of its nutriment to the body. Slow feeders, therefore, are more likely to accumulate flesh than the voracious ones. 


\section{Bots (Stomachic and Hemorrhoidal).}

Some persons contend that bots are always injurious. The author dissents from this dictum. It is possible that, as in the case of intestinal worms, which are now recognized as the scavengers of Nature, that the bots are Nature's hirelings, created and commissioned to do her bidding, to maintain the integrity of her physiological laws. The parent of the bot, as Bracy Clark remarks, "selects her subjects," or, in other words, pounces on those who are not in rapport with Nature, and hence have no business to enjoy good health, nor even to live.

This was the case when the people of the great city of London were afflicted with the terrible plague, which ran riot and carried off about one-fourth of the inhabitants. The sanitary emissary of Nature, whose shield was emblazoned with the motto, "Thus far shalt thou go and no further," pounced upon selected subjects, the intemperate, licentious, and the gluttonous, and those who had violated Nature's laws by wallowing in filth in down-cellar locations, where the breath of life-pure air-scarcely ever entered. These were the selected sufferers. The same is true as regards the mortality attending the yellow fever, which made such sad havoc in the city of New Orleans some years ago. The medical authorities contend that the disease carried off over one thousand of the inhabitants, without touching a single sober or temperate person; hence the plague, the yellow fever, and the cholera may be said to be the forces which Nature employs to maintain the integrity of her laws. Intestinal worms, found in the intestinal tube of the emaciated and the glutton, are said to be Nature's scavengers, and the same perhaps is true of bots. They may be the agents of $\mathrm{Na}$ ture, employed to keep tha balance of power within her own hand, for the purpose, sometimes, of preventing a too rapid multiplication of the species; at others, to avenge her for crimes committed against the laws of physiology.

Let us, for example, inquire into the history and habits of some of the inferior orders of creation, and we may be led to infer that the presence of bots in the stomach of a horse is no deviation from the general rule which seems to pervade all creation. Our tenure of life depends on the use which we make of it, and the same is true as regards the horse.

In the study of physiology, we discover that animals and insects 
require the operation of certain forces, in order that their peculiar vital properties shall be manifested. They all require food, water, and oxygen-food for the development of organized tissues, water to maintain an equilibrium between the solids and fluids, and oxygen for promoting various changes, uniting some particles for the benefit of the whole fabric, and disengaging others destined for excretion. These have to be obtained under various circumstances. The number of the different species of reptiles known to naturalists is about 1,300 , and there are at least 160,000 species of insects. Among this vast assemblage of animate forms, a great proportion obtain food, water, and oxygen in a situation and at a temperature which is most congenial to each species, each one of which, as species, exhibit great varieties in physical organization and habits; and hence the necessity for that diversity in geographical distribution which seems to surprise some men. Each species of animal and insect carry about with them, in their own organization, the fertile embryonic habitation for successive parasitic development, and all are, to a certain extent, dependent on each other for both food and life. It has been truly said that there " is life within life." Begin with the body of man, for example, and we shall find that it is infested with thirty-nine distinct species of entozoa. These are not confined to a single location, as the bots to the digestive cavity of the horse; but some are to be seen in the eye, bronchial glands, kidneys, liver, gall, bladder, intestines, muscles, and even in the blood. There are several other species of entophyta, to the number of ten, inhabitants of the skin and mucous surfaces. So that the master can boast of a larger number of living parasites within and about his body than we have yet been able to find in his servant, the horse. And if the former can carry about in the living citadel such a myriad of living, active creatures, without inconvenience, and he being the weaker party, why should not the horse, who is stronger, be able to furnish nutriment for some half dozen or more bots that are occasionally found in his stomach, and to perform his 2.40 gait without inconvenience? Some of the inferior orders of creation are the receptacle of a still greater amount of parasites. The grasshopper, for example, is infested with a species of gordius, a sort of hair-worm, which some persons have erroneously supposed to be a transformed horse-hair. Several of these coil themselves into the digestive cavity of the former, at times penetrating the 
abdomen, thorax, and cranium. Their bulk and weight often exceeds that of an ordinary grasshopper ; still you see and hear them, skipping, jumping, and chirruping, notwithstanding this immense parasitic mass, just as freely as those not infested.

Then consider the condition of the hog. We frequently find, in his liver, a vesicle filled with fluid, apparently possessing no further organization. But examine it carefully, and we shall find within its enveloping tunic others, the rudiments of successive cells, in various stages of growth. These are all young hydatids, which contain still others, which increase in size until the parent sac is so distended that it finally bursts, and thus liberates a multitude of parasites, which, in their turn, undergo the same evolution, becoming each a parent hydatid, producing a subsequent generation, which diffuse themselves over the whole body of the pig; and hence arises that peculiar feature in pork known as measles.

Examine the same animal after he is slaughtered, and you may possibly find in the intestines a large number of the ascaris lumbricoides (the common worm of the human intestines); and they are so prolific that naturalists have calculated sixty-four millions of ova within the body of a single female, which are, at the proper season, deposited within the intestinal tube of the pig, who, notwithstanding, grows fat, and furnishes a savory meal for the lovers of pork. Sheep, also, are infested with a species of entozoa termed the "fluke," besides other different species; and it is only when the latter become very numerous that they can be considered injurious.

We might go on to show that every living being is more or less infested with parasites, and that parasites are, in their turn, the local habitation for other parasites. The very atmosphere we breathe, and which is the purificator of the vital current (the blood), teems with an innumerable host of living organized creatures. The water which serves to quench the thirst, that plays so important a part in our economy, and in the arts and sciences, whether it be the ocean, lake, river, pond, or gully, all contains crowds of parasites, or animalcula, at times, so numerous that several hundred thousand have, by means of a magnifying lens, been discovered in a single drop of this fluid. Yet such is good and pleasant to the taste; and the water is not injured thereby; neither is it, in turn, injurious to man. Dr. LEIDY states that he 
has, at various times, purposely swallowed large draughts of water containing myriads of animalculæ, without ever perceiving any effect; and he combats the notion that diseases are produced or propagated by these parasites taken into the body. The most curious feature in the history of parasites is their extraordinary powers of multiplication, which is doubted by some; but it is well known to others that some species are capable of producing a hundred repetitions of themselves, and the process can be repeated ten times in a season. The common white ant is capable of depositing eggs at the rate of 80,000 per day, for several successive weeks, and the common flesh-maggot can be generated by the million in the course of a few hours; and as regards growth and development, the common flesh-fly and the eaterpillar increase in weight 200 times in the course of twenty-four hours.

But the bot is a creature that does not multiply nor increase in bulk at this rapid rate. He may be said to be a "slow coach," and when once located in the stomach of a horse, he generally makes it his abode for a season, at least. $\mathrm{He}$ is a sort of aristocratic entozoa. He lives in the upper region, the stomach. $\mathrm{He}$ seldom condescends to mix with the lower orders that infest the alimentary regions. The little creature seems to exercise considerable tact in selecting his location. Although he has but a squatter's title to it, yet it is the best and safest in the whole diggings. $\mathrm{He}$ is in the upper part of the stomach, where the fluids (poisons or medicines) with which you are about to coax or drive him off, are inoperative, (for they merely give him a sort of shower-bath,) and pass immediately through the stomach into the intestines, where all the fluid a horse drinks is generally found; therefore they can not act on the bot. Then, again, he is located on the cuticular coat of the stomach, a membrane as insensible as the horse's hoof, and, therefore, not liable to become diseased, nor to be acted on by either medicine or bot nostrums. You may kill the horse by the same, but the bot, being within his own castle, can refuse whatever you offer him.

We can not make medicine act on the external surface of the bot, for it does not absorb fluid; it is impervious. These creatures have been put into muriatic acid, and kept there for a time, without being injured. You may put them into new rum, and keep them for weeks, and, on taking them out and exposing them to the sun's rays, they will manifest vitality. 
We often hear wonderful stories related of bots burrowing through the coats of the stomach. This, we think, rarely takes place while the horse is alive. That cavity is the home of the bot, its natural habitation; for we know of no other. Here it generally remains until it is capable of exercising an independent existence. In this situation the little creature is too comfortably located to burrow through the stomach into a cavity where it might perish for want of food. If the time has arrived for it to vacate its stronghold, instinet teaches it the most safe and expeditious route, which is through the alimentary canal. Turn a horse out to grass in the spring, or give him some green fodder in the stable, and the bots will soon leave him, if they are matured; otherwise they must remain until that period arrives, unless Nature has some work for them to perform. We shall not contend that bots are never found in the abdominal cavity, for some persons have testified to the fact; but, during a practice of several years, and having opportunities of making many post-mortem examinations, we have not yet been able to observe the phenomena, except in cases of ruptured stomach. Still, a few solitary cases are on record, and hence it remains for us to explain how they got there.

We all know that the moment a horse dies his whole body is subject to the common law of decomposition; but the central organs, where the greatest activity prevailed during life, are generally the first to succumb. Our business is with the stomach, the great chemical laboratory, the center of sympathies-an organ that is very seldom permitted to rest, consequently an active one. Its powerful solvents, during life, were busy in transforming hay and grain into chyme, chyle, and blood; but now that death has the victory, the gastric fluid ants on the coats of the stomach, and thus its decomposition is effected; so that what was previously good food for bots is now their bane, and, unless they escape, their death is sure and certain.

The peristaltic motion of the intestines, which favored the exit of the bots through that channel, has ceased; they are too weil acquainted with its intricate labyrinthian outlet (their usual route) to even attempt its passage. No! the same energies of one Eternal Mind, "pervading and instructing all that live," suggests the only means of escape from threatening dangers. The stomach being partly decomposed, offers but little opposition to their en- 
croachments. They burst their prison-house, and hence are found in the abdominal cavity; and when there, they may be said to have jumped from the "frying-pan into the fire!" Open a horse immediately after death, and, provided his stomach be in a healthy condition, we shall find that the bots have not penetrated beyond the cuticular coat of it; but if he shall not be examined until some hours have elapsed, the bots may be found to have passed through the walls of the decomposed stomach and its peritoneal tunic.

We can imagine, also, that a large number of bots might congregate at a given point in the stomach of a horse, and, aided by disease, occasion a loss of continuity in the fibers of that organ; then, on the slightest distension by wind, its walls might be ruptured and its function paralyzed, and thus the bot be involuntarily driven from its home, to seek shelter and food in another location.

We contend that the stomach of a horse is the natural habitation of the bot during its minority, and, at the proper season, the digestive canal is the usual channel for its introduction into the external world; and if these parasites are ever found in any other situation within a horse's body, they are there by the force of circumstances, owing to disease or rupture of the stomach, or from some morbid condition in the gastric fluids, which arouses a set of involuntary actions in response to a stimulus; because, during the whole period of their minority, that is, the larveal state, (a term which, in the language of entomology, applies to the bot from the time of emerging from the egg, or nit, up to that period when it vacates the horse and assumes the form of a gad-fly), they are in the same condition as a new-born babe or an idiot-the one imbibing its mother's milk, and the other performing unnatural antics, both appearing to lack that train of mental operations which implies knowledge, motive, or the consequences resulting from such actions. We very much doubt if the bot can, at any time, by voluntary act, vacate the body of the horse. Reasoning from analogy, we are led to the conclusion that the result is accomplished through their instinctive properties, which are common to many insects and parasites-a perfect adaptation of means to an end-by which they perform a certain set of operations without choice, purpose, or intention of their own, yet, in many cases, producing results which man, through the aid of his superior intellect, has not been able to surpass. 
Persons who have paid any attention to the study of physiology, are aware that these instinctive or involuntary movements, performed without consciousness, are the birthright of a vast number of the inferior orders of creation; therefore, it is not likely that the bot would, even if he possessed the power, voluntarily vacate a location favorable to its growth and development.

Veterinary surgeons have long since discarded the absurd notion that bots are the cause of any pain or suffering to horses. In fact, some of the most distinguished of them assert that these little creatures, with their rough exterior, are rather beneficial than otherwise, and that, by friction and irritation, they arouse the sluggishness of the stomach, and thus promote digestion. Persons unacquainted with these facts are, therefore, apt to attribute effects, during life, to causes which happen after death, and, consequently, the poor horse has to be dosed with all sorts of nostrums.

So popular has been the belief that bots are injurious to horses, and, therefore, must be expelled at all hazards, that almost all the old works on farriery contained some favorite recipe for their expulsion. Popular opinion, too, has been so much in favor of the theory, that Mr. Percivalu thought it his duty, as a public teacher, to make use of the following language:

"You may boldly assert that bots are in nowise injurious. Still, you can not persuade the world so, and, therefore, you must be prepared to meet the complaints of those unbelievers, who will, now and then, declare that their horses have bots, which must be got rid of. But I know of no medicine that has the power of destroying; and even if we possessed such, I am not sure that we could, even when dead, detach them from the cuticular coat of the stomach, to which they are attached by small horns."

In allusion to the parasites which infest the human body, WATsoN remarks:

"It is a curious fact that numerous parasites do crawl over the surface of our bodies, burrow beneath our skin, nestle in our entrails, and riot and propagate their kind in every corner of our frame, producing ofttimes such molestation and disturbance as to require the interference of medicine. Nearly a score of animals that have their dwelling-place in the interior of the human body have been already discovered and deseribed, and scarcely a tissue or an organ but is occasionally profaned by their inroads. Each, also, has its special or its favorite domicile. One species chooses 
the heart for its place of abode; another inhabits the arteries; a third, the kidney. Myriads of minute worms lie coiled up in the voluntary muscles or in the areolar tissue that connects the flesh fibers. The guinea-worm and chigoe bore through the skin, and reside in the subajacent reticular membrane. Hydatids infest various parts of the body, but especially the liver and brain. A little fluke, in general appearance much like a minature flounder, lives, steeped in gall, in the biliary vessels. If you squeeze from the skin of your nose, what is vulgarly called a maggot (the contents of one of the hair-pellicles), it is ten to one that you find in that small sebaceous cylinder several animalcules, extremely minute, yet exhibiting, under the microscope, a curious and complicated structure. Even the eye has its living inmates; but it is in the alimentary tube that we are most infested with these vermin."

It is evident, from competent testimony, that these, as well as other kinds of parasites, are always more or less injurious; hence the same may be true as regards the bot in a horse's stomach. The best authority we have for the origin and history of the bot is BRACY ClaRK, V. S., a selection from whose works is here introduced:

"The Estrus Equi, or the Stomach Bot.-When the female has been impregnated, and the eggs sufficiently matured, she seeks among the horses a subject for her purpose; and approaching him on the wing, she carries her body nearly upright in the air, and her tail, which is elevated or lengthened for the purpose, curved inward and upward. In this way she approaches the part where she designs to deposit the egg, and, suspending herself for a few seconds before it, suddenly darts upon it, and leaves the egg adhering to the hair. She hardly appears to settle, but merely touches the hair, with the egg held out on the projected point of the abdomen. The egg is made to adhere by means of a glutinous liquor secreted with it. She then leaves the horse at a small distance, and prepares a second egg, and, poising herself before the part, deposits it in the same way. The liquor dries, and the egg becomes firmly glued to the hair. This is repeated by these flies till four or five hundred eggs are sometimes placed on one horse.

The skin of the horse is usually thrown into a tremulous motion on the touch of this insect, which merely arises from the very great irritability of the skin and cutaneous muscles at this season of the year, occasioned by the heat and continual teasing of the flies, till, 
at length, these muscles appear to act involuntarily on the slightest touch of any body whatever.
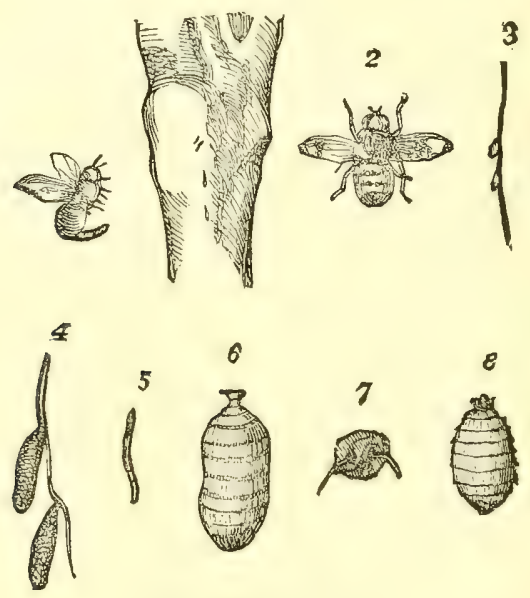

THE ESTRUS EQUT.

1. The female fly, about to deposit an egg.

2. The male fly.

3. The egg; its natural size.

4. The egg magnified.
5. The newly-hatched bot.

6. The bot full-grown.

7. The head of a bot magnified.

8. The chrysalis.

The inside of the knee is the part on which these flies are most fond of depositing their eggs, and next to this on the side and back part of the shoulder, and less frequently on the extreme ends of the hairs of the mane. But it is a fact worthy of attention that the fly does not place them promiseuously about the body, but constantly on those parts which are most liable to be licked with the tongue, and the ova, therefore, are always scrupulously placed within its reach.

The eggs thus deposited I at first supposed were loosened from the hairs by the moisture of the tongue, aided by its roughness, and were conveyed to the stomach, where they were hatched; but on more minute search, I do not find this to be the case, or, at least, only by accident; for when they have remained on the hairs four or five days, they become ripe, after which time the slightest application of warmth and moisture is sufficient to bring forth, in an instant, the latent larvæ. At this time, if the tongue of the horse touches the egg, its operculium is thrown open, and a small active worm is produced, which readily adheres to the moist surface of the tongue, and is thence conveyed with the food to the stom- 
ach. At the first hatching, as we observe, it is a small, active worm, long in proportion to its thickness; but as its growth advances, it becomes proportionably thicker and broader, and beset with bristles.

Bots are very frequent in horses that have been at grass, and are, in general, found adhering to the white insensible tissue or coat of the stomach. They usually hang in dense clusters to the white cuticular lining of the stomach, and maintain their hold by means of two dark brown hooks, between which a longitudinal slit is seen, which is the mouth of the larvæ. When removed from the stomach by the fingers, by a sudden jerk, so as not to injure them, they will, if fresh and healthy, attach themselves to any loose membrane, and even to the skin of the hand. For this purpose they sheath or draw back the hooks almost entirely within the skin, until the two points come close to each other. They then present them to the membrane, and, keeping them parallel till it is pierced through, they expand them in a lateral direction, and afterward, by bringing the points downward toward themselves, they include a sufficient piece of the membrane, to remain firmly fixed for any length of time, as if at anchor. These bots pass the autumn, winter, and spring months in the stomach, and arrive, about the commencement or middle of the summer, at their full growth, requiring a year to fully complete their structure.

The Estrus Hemorrhoidalis, or Fundament Bot.-The parts chosen by this insect for this purpose is the lips of the horse, which is very distreissing to the animal, from the excessive titillation it occasions; for he immediately rubs his mouth against the ground, his fore-legs, or sometimes against a tree, with great emotion, till the animal, at length, finding this mode of defense insufficient, quits the spot enraged, and endeavors to avoid it by galloping away to a distant part of the field; and if the fly still continues to follow and tease him, his last resource is in the water, where the ostrus never is observed to pursue him. These flies appear sometimes to hide themselves in the grass, and, as the horse stoops to graze, they dart on the mouth or lips, and are always observed to poise themselves, during a few seconds, in the air, while the egg is preparing on the extended point of the abdomen. When several of these flies are confined in a close place, they have a particularly strong, musty smell; and I have observed both sheep and horses, when teased by them, to look into the grass 
and smell it very anxiously; and if they, by these means, discover the fly, they immediately turn aside and hasten to a distant part of the field. I once saw, in a meadow or field, upon the cliffs of Margate, a fly of this sort teasing a horse that was confined to a small space by a spike stuck in the ground, to which a cord was tied. He could not get away from its attack, and became quite furious, for in kicking at the fly with his fore-foot, which he did vehemently, he often struck the bone of the lower jaw, creating excessive pain ; for in that direction, while grazing, the fly comes to the beard of the lower lip.
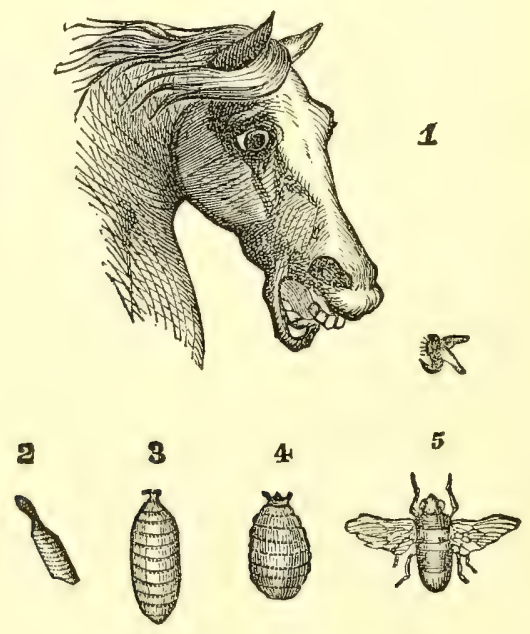

THE GSTRUS IEMORRHOIDALIS.

1. The femalo about to deposit an egg.

2. The egg magnified.

5. The wale fly.

3. The bot.

4. The chrysalis.

The eggs of this species are difficult to be seen upon the horse's skin or beard, owing to the agitation of the beast, and from the color of the egg being dark, like that of the skin of the horse. The animal has been generally too impatient, while undergoing this operation, to let me examine them very well. I ascertained, however, its form by pressing one of these eggs from the abdomen.

The larvæ, or grubs, of this species inhabit the stomach as the former, generally adhering to the white lining, and are disposed promiscuously in dense clusters, after the same manner. They may, however, be distinguished from them by being, in general, smaller and longer in proportion to their bulk. 
The larva of this species may be obtained from almost any horse that has been much the preceding year at grass, and exposed to these flies, and will be found, during the summer months, sticking more or less within the verge or opening of the anus, adhering to its soft lining, and producing considerable irritation and uneasiness. Indeed, I once well remember being on a tour of pleasure in the Isle of Wight, and experiencing much annoyance from these larvæ. The little horse I had hired for the journey became so lazy and unwilling to go on, and moved so awkwardly, that I could not keep pace with my company, and I was at a loss how to proceed; but, on casually taking up the tail, I discovered three or four of these insects hanging to the rectum, and their removal instantly proved a cure."

It is very likely that the fundamental or hemorrhoidal bot does produce some irritation within the rectum, but not more so than the ascaris, or the filaria, which inhabit the intestines, including the rectum, of both man and horse. Taking a rational and scientific view of this subject, the author is inclined to believe that all parasites infesting the bodies (or living citadels) of the superior orders of creation are only, in their essence, so many modes of death, more or less sudden or gradual, as the case may be, or as the exigencies of Nature require. In some cases it is supposed to be a beneficent provision that some horses, as well as men, should have a gradual exit out of life, when, by virtue of their constitution, they inherited no long lease of it. The parasites here alluded to, and others not mentioned, may be classed as so many diseases, which never prove mortal unless the individual's lease of life has expired, and then, as before remarked, he has no tenure on longevity. In taking this view of the subject, the reader will, of course, infer that all extraneous or accidental causes of death are excluded.

Disease, which probably includes the presence of all parasites, in the bodies of men and animals, may be considered as the trials of pilgrimage life, and, therefore, may be classed as the checkmates to Nature. In order to play the game of life so as not to get checkmated, we must understand the law of rational physiology, and use our intelligence accordingly, to preserve health. In the ordinary acceptation of the term disease, it does not mean much of any thing; for it does not express the manner in which the animal is affected. It simply means that he is sick, from 
some cause or other. A man or animal may be diseased without our expression of the manner in which either are affected, just as a flower may be said to be colored without designating its particular hue. In each case we receive definite, although negative, ideas-in the one, that the creature is not in health; in the other, that the flower is not colorless. And so the same reasoning may apply when bots become the subject of our consideration. In a given case, they may prove injurious (according to our ideas), and in another they may remain, for a certain length of time, perfectly harmless; so that the animal infected or infested may or may not suffer any infliction, but trot on through the journey of life just as long as horse-life is worth wearing.

As regards the several modes of death to horse flesh, in which bots are said to play an active part, they may thus be stated: Bots sometimes congregate, in large numbers, at the superior or inferior inlet or outlet of the stomach, and thus produce mechanical obstruction to the passage of both semi-fluids and solids; and the result is, equine death. 'The author once held an autopsy, which revealed the presence of a cluster of bots, numbering about one hundred, located in the cardiac region of the oesophagus. They completely obstructed the passage of food into the stomach. The horse died suddenly, as if suffocated, from distension of the œesophagus and pressure on the trachea.

The following case, furnished for the "Veterinarian" by Surgeon MATHER, illustrates another mode of death from the presence of bots :

"The inclosed tongue and larynx I took from a foal this evening, which I shall feel obliged to you for examining, as at the base of the tongue you will find about a score of what appear to me to be perfeet specimens of a small bot. I have only made a partial examination, as I was anxious to send the specimen off to you before it became decomposed, as the weather is very hot. The history of the case is as follows: Two days ago I was requested to go to Lord Glasgow's breeding establishment, to see a foal that was unwell. I inquired of the stud-groom how long the animal had been ill, and he informed me that three days ago it appeared to have a difficulty in swallowing, and that he noticed some saliva issuing from its mouth; and that, thinking it had a sore throat, he had rubbed some embrocation upon the parts. On examining the animal, I found it in a very debilitated state. Pulse, very 
weak, and numbering 80 ; ears and legs, extremely cold, associated with shivering fits of the entire body; mouth, filled with partly masticated hay and grass; tongue, furred. The animal had seemingly a desire to eat, but was unable to open its mouth more than about an inch. It was likewise unable to swallow any thing but liquids, and these only in very small quantities. In spite of all I could do, the power of swallowing got worse, and this morning it died, very much to their disappointment, as it was a very highbred animal.

Could this foal have taken in the ova of the gad-fly, and some of them become hatched, and, instead of passing into the stomach, have become adherent to the base of the tongue, and there set up such an amount of irritation as to cause the parts to swell so as to prevent the animal from swallowing?

Observations on the. Case.-We are indebted to Assistant Professor Varnell for the following remarks upon this singular case:

'The morbid specimen forwarded by Mr. G. Mather, is of very rare occurrence. We have never met with such an one before, nor are we aware that a similar instance has ever been recorded. The parts came to hand in a good state of preservation, although the temperature at the time was very high, so that we were enabled to form a pretty correct opinion as to the nature of the changes that had taken place during the life of the animal. The dorsum of the tongue, its root, the whole of the surface of the fauces, the velum palati, and the margin of the glottal opening, as well as the lining membrane of the larynx and pharynx, were very much inflamed, and, in places, considerably thickened. These parts were likewise covered with a deposit seemingly consisting of a mixture of mucus, lymph, and pus.

The most remarkable feature, however, of the case was in the cause that had given rise to the inflammatory state of the throat; namely, the existence of a number of small bots-the oestrus hemorrhoidalis (the 'red-tailed horse bot' of Bracy Clark)—which were found to be firmly adhering, by their tentacula, to the mucous membrane. Besides the number which were still in situ, many others had apparently been attached to the parts, but which had, most likely, been lost in the handling they had undergone.

By a little reflection, we may probably form a correct opinion as to how these parasites became located in this unusual situation. We imagine that the foal, by licking, gathered the ova, in a state 
of perfection, from the hair of its dam, on which they had been deposited, rather than from its own coat. The perfected ova, being thus taken into the mouth, became first entangled in its mucus, and finally lodged in those shallow recesses at the root of the tongue, where they became hatched. Some of these creatures measured as much as three-eighths of an inch in length, and rather less than one in diameter. They were firmly fixed, by their little hooklets, apparently to the inner surface of the mucous follicles. Their free extremities were of a deep red color, and their bodies of a yellowish brown."'

Treatment.-The only remedy for ridding the system of bots is a run at grass, in the month of May.

\section{Gastritis, or Inflammation of the Stomach.}

Acute inflammation of the stomach seldom, if ever, occurs among horses as a spontaneous affection. It is usually occasioned by irritating medicines or poisons, or food of an inferior quality. When an irritating poison has been received into the stomach, and excites inflammation there, or when acute inflammation arises from any cause, the symptoms which mark that inflammation are pain, distress, and restlessness ; a loathing of food ; for, if any thing be given by the mouth, it creates increased pain. The animal breaks out in a cold sweat, lies down and quickly rises again, becomes early and greatly prostrated in strength, and has a pulse usually quick and much oppressed. Sometimes purging sets in; at others, the bowels are constipated. The disease is very apt to run into gastro-enteritis-inflammation of the stomach and bowels.

Treatment.-The general treatment consists in the administration of slippery-elm mucilage, and half-ounce doses of chlorate of potass, two or three times per day. Should gas accumulate in the stomach or bowels, give one ounce of hyposulphite of soda, dissolved in a pint of warm water. The ordinary drink should consist of hay tea, to which a small quantity of molasses may be added.

\section{Rupture of the Stonach.}

Rupture of the stomach is always a fatal malady, over which neither art nor nature has any control; yet, the accident being one of frequent occurrence, it becomes necessary for us to know some- 
thing regarding its cause and symptoms, which are all detailed in the following case, from the author's note-book:

Death of a Horse from Ruptured Stomach.-The subject, a black gelding, nine years old, had been driven a distance of twelve miles into the city; on arriving at the "City Hotel stable," he first manifested signs of uneasiness, by pawing with his feet and turning his head toward the belly. He finally got down, rolled, tumbled about, and passed considerable flatus, etc. His owner, supposing him to be the subject of colic, administered a dose of " nire-water," in the form of gin and cayenne pepper, followed by three ounces of spirits of niter. He urinated freely, passed considerable flatus, yet grew no better very fast. Four hours from the commencement of the attack our attention was called to him. The case then appeared hopeless, consequently we declined prescribing. The following were the symptoms: Pulse, small and thready; in number, fifty-six; respirations, sixty-laborious, emitting, occasionally, a sort of grunt, indicative of pressure, from a distended stomach, on the diaphragm and lungs. The eyes were amaurotic (pupils dilated and loss of vision); sclerotica (white of the eye) slightly injected; conjunctival membrane, within the eyelids, surcharged with blood. 'The buccal membrane, within the mouth, intensely inflamed, induced, probably, by the fire-water drench. The tongue was of a leaden hue, and the nasal membranes were somewhat reddened. The bady, or external surface, was the seat of profuse perspiration, which ran down the limbs like rain. They, as well as other parts of the body, remained comfortably warm until within a few minutes of death. There appeared to be considerable distension in the region of the stomach, and less at the flanks, from which circumstance we concluded that the patient was laboring under tympanitis (windy distension) of the stomach. At the moment of death, the reverse was the case, which circumstance seemed to confirm this opinion; for then, the stomach being ruptured, the gas would diffuse itself in the abdominal cavity and increase in bulk.

Aspect and motions of the body.-The head was kept within striking distance of the ground; the tail was elevated, and kept in a quivering condition (involuntary). The fore extremities were advanced, one leg frequently crossing the other; the hinder parts maintained a crouching attitude, and the penis hung pendulous. When not in this position, the patient would revolve in a circle; 
then lie down flat on his side, turn his head toward the ribs, and, after a short time, raise the forward parts, and sit on his haunches like a dog. At last he fell over, gave two or three convulsive struggles, and died, just thirty-five minutes from the time we first saw him.

Next morning, in company with Dr. Wood, we proceeded to Ward's establishment, but came very near being too late; for the metamorphosis of the horse into grease, food for swine, etc., had commenced. We however had an opportunity to make a casual examination of some of the organs, and found a rupture of the stomach of some six or eight inches. The contents, some of which were found in the abdominal cavity, were very imperfectly (crudely) masticated. It may be proper, therefore, to name the disease indigestion; its consequences, tympanitis of the stomach ; the cause of death, rupture of the stomach. The loss of vision referred to is one of the symptoms which generally attends acute indigestion.

A person, who was present during the last moments of the animal's life, asked if we were not going to give or administer something. Our reply was, that we never tortured dying horses by forcing drugs down their throats. One of our duties, as a physician, was to know when to withhold medicine-when to do nothing; and another equally important one was to be able to discriminate between, a hopeless and curable case. The one in question was beyond the aid of science, and, therefore, in view of "clearing our skirts" of being the cause of death by administering the last dose (a charge that is often ungenerously laid at the door of medical men), we preferred to watch the dying animal, and give directions calculated to lessen the pains of death.

Unfortunately for us and our patients, many employers form an estimate of medical qualification in proportion to the amount of medicine administered. They have an idea that the more medicine we give, the greater are our efforts to control the disease, and the more willing are they to pay for the same, when, in ninetynine cases out of a hundred, the very reverse is the case; for many diseases to which horses and cattle are subject have a definite type and limited duration, and would terminate favorably with less medicine; provided, however, the patient have the advantages of pure air, suitable diet, and proper management. Many medical men are compelled to swerve from the line of their own convictions merely to satisfy their employers. This should not be. 
If a man employs a physician, (quacks are not here considered,) he should have unbounded confidence in the ability of the former to treat the case. If any thing occur to lessen that confidence, let the attendant be discharged immediately. The employer has no right to dictate how or in what way his horse shall be treated when he abandons him to scientific men. The science of medicine is never a "God-send." No man is thus favored. It is the result of diligent application and study, in and out of the dissecting-room, and the legitimate student of medicine is a person entitled to the full confidence of reasonable men for its legitimate practice. Therefore, we contend that the qualifications of a veterinary surgeon should never be estimated in ratio to the quantity of drugs administered, nor the length of his newly-invented syringe, nor by the number of instruments contained in his pocket-case; but by his works let him be judged.

There is one practice, prevalent among some horsemen, which we very much deplore; that is, the drenching of sick animals with gin, pepper, and molasses. This is a universal stable panacea in certain quarters, and is often the cause of much pain and annoyance to the animal, and more frequently of an aggravated form of disease. Gin, or the fire-water sold under that name, is not always to be procured pure. A great deal of it appears to us to be composed of spirits of turpentine, new rum, and spirits of juniper, which, of course, in certain cases, excites inflammatory action. We have seen a horse's mouth in a most shocking condition-the interior surfaces almost completely denuded-after a drench of the same; consequently, in cases of inflammation of the stomach or bowels, (which the stable oracles are apt to mistake for colic, or "bots," as they term it,) the remedy (poison) is certainly objectionable, because it not only operates unfavorably on the horse by aggravating the disease, and sometimes changing a simple into a grave one, but much valuable time is lost, in not sending for a competent person to treat the case. Besides, if a medical man be called on after the animal has been improperly treated, he then has two things to contend against: a medicinal disease and the original one; and the former is sometimes more difficult to control than the latter; and this is the reason why some medical men, ourselves included, refuse to take charge of what we term a second-hand case.

The above case illustrates our argument. The remedies were 
not adapted to the emergency. Even the simple article molasses was oljectionable, because it is an acid. The case required alkalies, in the form of ammonia or chloride of lime. Gin was objectionable, beeause it is an excitant of the brain and nervous system, when, instead of the brain, the stomach ought to have been stimulated. Niter, which was given, is an agent that increases urinary discharges only, and, therefore, was not needed. The trouble was in the stomach-to arouse its function and condense the gas which distended the same. Alkalies and tonies should have been administered, followed by such other treatment as the nature of the case demanded. We look upon this gin and molasses treatment as decidedly unprofitable to owners of live stock. It savors too much of our grandmothers' castor-oil practice, which often proves more heroie than salutary, does more harm than good, and, at the present day, is decidedly unpopular.

Our advice to horse-owners, therefore, is, if you have a sick animal, and can not comprehend the nature of the disease, never attempt to prescribe for him, but call in the aid of a professional man. We give this advice under the conviction that domestic animals are subject to many of the diseases that afflict our race; that the treatment should be somewhat analagous, and the tact and skill not inferior, but superior, to that of the human practitioner.

\section{Gorged or Over-distended Stomach.}

Gorger stomach is usually the result of overfeeding, by which means the stomach becomes overtaxed in its function, or overburdened in its capacity; so that, in the first place, digestion is partially or totally suspended; and, secondly, the stomach, being distended beyond its physiological capacity, can not perform the mechanical action, viz.: contraction and expansion, so necessary for the physiological process of digestion. The act of digestion is consummated through the aid of Nature's chemistry, by means of the salivial fluids, bile and pancreatic juice. These are the solvents of all the digestible food. This chemical action receives much aid in the mechanical action of the muscular fibers of the stomach, which, by alternate contractions and expansions, kcep not only the stomach in motion, but also its contents, and thus the food is digested. As regards overfeeding, however, it may be proper to remark, lest the groom or feeder take offense, that an animal may 
be regularly fed from meal to meal (not carelessly overfed), yet the stomach, being disordered, does not digest the usual meal. A portion of it is "left over," as the saying is, and thus gradually accumulates in that organ, until over-distension, with its consequences, commands our attention. The reader must also bear in mind that horses sometimes free themselves from the halter at night, or when the groom is absent, and voluntarily gorge themselves; therefore the person who has charge of and feeds the subject is not always at fault. When engorgement of the stomach occurs, and is associated with flatulency, the sufferings are doubly severe, because then the intestines are also the seat of distension, and the pain from that alone is sometimes terrific.

The following case, which lately occurred in the author's practice, fully.illustrates the condition just alluded to. The subject was a bay gelding, aged about seven years, the owner of which informed me that the animal had, in the early part of the morning, broken loose from his stall, and immediately commenced a desperate attack on a bag of oats, which happened to be within reach. The horse was known as a ravenous feeder, and the probability is that he did not spare the oats, but took a pretty large dose, so as to over-distend his stomach. The services of the animal being required, a few hours afterward he was hitched up. He soon, however, showed symptoms of distress, attempted to lie down, and finally got into a profuse perspiration. Shortly after this, my attention was called to him. I found the patient in great distresshis head pendulous; breathing, much embarrassed; pulse, very rapid; body, bedewed with a chilly perspiration; legs and ears, cold; continual eructations of gas; at the same time efforts to vomit, which brought away nothing more than salival secretion, which, however, was quite profuse. The patient rolled and tumbled about considerably, and would occasionally stand up for a few seconds at a time.

Treatment.-It appeared that the indications, in view of relief, were to arouse the action of the stomach, and, at the same time, to arrest fermentation. I was under the impression that the contents of the gorged stomach could only be got rid of in the ordinary way, viz.: by digestion. I am aware that it is customary to give drastic cathartics, in view of getting rid of the contents of the stomach; yet I would not advise the reader to do this, for the horse may die ere the medicine operates. The following drench was administered: 
No. 20. Fluid extract of ginger............ $1 \mathrm{oz}$.

Fluid extract of golden seal.......2 oz.

Hyposulphite of soda..........2 drachms.

Water................... 4 oz.

After the exhibition of the above, I threw into the rectum a couple of quarts of soap-suds, to which was added a handful of salt. In the course of about an hour the animal appeared to be somewhat relieved, and passed a large quantity of oats, whole. I repeated the dose; also the enema. The patient very soon afterward passed a large quantity of excrement, mixed with oats, and so rapidly improved that I left him, and did not see him until the next morning, when he appeared to have entirely recovered. With the exception of a bran-mash or two, the above comprises the whole of the treatment.

It was lucky for the horse as well as the parties concerned that the animal did not get corn instead of oats; for the same quantity of corn would have surely caused death, from the fact that when this article of fodder is submitted, within the stomach, to the action of heat and moisture, it increases in bulk in a ratio of about five to one, and the usual result is rupture of the stomach. Still, should any of our readers be called upon to treat a case of the latter kind, there is no other plan of treatment with which $I$ am acquainted that will be likely to succeed in saving the animal than the one here indicated.

Another case.-The patient, a gray gelding, aged about eight years, the property of Messrs. Wright \& Bros., of Chicago, had performed an ordinary day's work without showing any symptoms of ill-health. About five o'clock in the evening he was unharnessed and put up for the night. In a few minutes it was noticed that he began to bloat, and that the abdomen increased in size very rapiclly. A messenger was immediately dispatched for me. On arriving at the stable, I found the animal in a very dangerous condition. The abdomen was enormonsly distended, and an eructation of gas from the stomach, by the mouth, was continually occurring. The respirations were laborious and accelerated, and very much quickened; pulse, very indistinct; extremities and surface of the body, quite chilly; rectum, protruding; and the animal was very uneasy, and appeared to suffer much pain. Occasionally he would get down and make desperate efforts to roll on his back, yet he did not succeed, for he was round as a barrel; and when he could 
scarcely stand alone, in fact, required the services of three men to steady him, he could walk, and seemed most at ease when kept in motion. I commenced immediately to inject warm soap-suds into the rectum; but so soon as the fluid entered the same it was immediately ejected, there being no room for it within, in consequence of the distension of the walls of the intestines, which pressed on the rectum. I next attempted to administer a colic drench, composed of ginger, golden seal, and hyposulphite of soda, but did not succeed in getting much of it down; for as soon as the fluid entered the rsophagus, it was met by a volume of eructating gas, which brought the fluid back by way of the mouth and nostrils. I learned, however, that, previous to my arrival, Mr. Smith, the foreman, had succeeded in drenching the patient with a full dose of the colic mixture. This probably had the effect of limiting the generation of gas; otherwise the animal might have died ere my arrival. The case now appeared almost hopeless; so I procured a trocar and canula, and plunged them through the flank into the large intestines. (See puncture of the intestines). On withdrawing the cutting instrument, a steady volume of gas issued through the canula for a period of ten minutes. The relief was immediate. I next gave a dose of colic drench, and left the animal in charge of the owner for the night. Early next morning I visited the animal, and found that he was all right. He was fed lightly for a few days, and, without any other medical treatment, was again put to work.

The dangers to be apprehended in cases of this character are either rupture of the intestines or diaphragm. In either case, death is sure and certain. Sometimes, however, neither of these ruptures occur; then the distended intestines exert such pressure on the diaphragm and organs of respiration that the animal dies of suffocation and loss of pulse. When, therefore, it becomes impossible for the patient to swallow medicine, in a case of this character, recourse must be had to the trocar and canula, in view of liberating the imprisoned gas. The lack of a little knowledge on this subject has been the cause of the loss of very many valuable horses. 


\section{Meteorization or Sudden Distension of the INTESTINES.}

Meteorization is the name given to a disease known as bloat or hoven in cattle. When it appears in a horse, it receives the above name, or else is termed acute tympanitis. This form of windy or tympanitic distension is always sudden in its attack; animals apparently in the very best of health all at once show symptoms of enormous distension of the abdomen, and, in the course of a few moments, their lives may be said to be in peril. The distension of the stomach and intestines is due to the presence of an immense volume of gas-probably carbureted hydrogen-which augments either by spontaneous gencration, or in consequence of the food running into fermentation instead of being digested.

Cause.-As regards the cause of this malady, we may, with safety, assert that it is the result of a temporary derangement of either the digestive function or the organs of digestion; and such condition is inaugurated by overtaxing that function, or those organs which carry it on, either by feeding indigestible food, or in feeding with too liberal a hand. I have noticed that many animals have periodical attacks of this malady. Such are said to be voracious feeders. Their abdomen is unusually large, and out of proportion to other parts of the body; hence, in so far as their conformation is concerned, they may be said to be predisposed; yet the disease sometimes occurs accidentally, and without the intervention of any hereditary tendency.

Physiologists contend that very many diseases which afflict horses are due to hereditary tendencies. For example, diarrhea and colic are, to a certain extent, hereditary, inasmuch as they are very prone to attack animals of particular form and constitution, as those with narrow loins, large sides, and of what is generally termed a "washy" appearance. If such animals be overworked, especially soon after being fed, if their food be suddenly changed, or if they be allowed an unusual quantity of water, they are almost certain to be attacked by purging or tympanitis. The tendency of these diseases appears, in such cases, to depend on a want of adjustment among the different organs of the body - a want of balance among the functions of digestion, circulation, and respiration.

The following case will give the reader some idea of the symptoms and treatment of this malady: 
History of the Case.-The horse had performed his usual daily labor, without any fault-finding on the part of his driver, when, all at once, he commenced to bloat, appeared uneasy, and, by his actions, demonstrated very conclusively that he needed some assistance. On examination, I found that the abdomen was distended to its utmost eapacity. The walls of the abdomen were rigid as a plank, and the respirations, in consequence of the great pressure on the diaphragm and lungs, were very laborious and much accelerated, amounting to about one hundred per minute. The pulse was thready and indistinct; the tongue, livid; eyes, glassy and protruding; surface of the body, bedewed with a cold, clammy perspiration; paralysis of the optic nerve had set in, and the animal was "blind as a bat;" in fact, he was dead to all intents and purposes. This was not the worst feature of the case. He was continually vomiting from both nostrils, and, as the story goes, "a horse that vomits surely dies." Occasionally the animal would fall on the ground with violence, and then assume a variety of positions, in view of finding some relief, but, alas! to no purpose. His agony was intense, and there seemed to be no relief for him, unless by a surgical operation. Medicine was out of the question; the animal had lost the power of swallowing, and an attempt to administer a drench would have choked him to death; so I selected the most salient or tympanitic spot on the left flank, and then sent a trocar and canula through the walls of the abdomen into the large intestine. So soon as I had withdrawn the trocar, the gas escaped very rapidly through the tube, making a noise like a steam-whistle. The animal obtained almost immediate relief, and the bad symptoms rapidly subsided, so that I was enabled to give a colic drench. This operated to prevent fermentation, and gave tone to the digestive organs. Two subsequent drenches, of four ounces each, were given, and at the end of twenty-four hours the animal was taken home. The slight wound made through the walls of the abdomen into the intestine healed readily, so that I had no trouble with that; and, to conclude, I would inform the reader that puncture of the intestine is the only rational plan of treatment in a case of this character.

Colic drench is made up of the following ingredients :

No. 21. Fluid extract of ginger............. 2 oz.

Fluid extract of golden seal............ 2 oz.

Hyposulphite of soda.............. 1 oz. 


\section{TYMPANITIS.}

Case in Illustration.-At midnight, June 16, 1865, I was called upon to visit a horse afflicted with this disease. On arriving at the stable, I found the animal on the floor, apparently in great agony. The abdomen was distended with gas to an immense capacity; the pulse was feeble; respirations accelerated, and very laborious; body was bedewed with a cold, clammy perspiration; tips of the ears, cold; extremities in a similar condition. Eructations of gas from the stomach, by the way of the mouth, were constantly occurring, indicating that the stomach, as well as the intestines, were occupied with gas. The animal had bruised himself very badly in struggling, and extensive abrasion of the skin had taken place in various parts of the body. I was informed that the horse had just returned from a very long journey, and had not tasted food for sixteen hours. On arriving at the stable, where I found him, a bountiful supply was placed before him. In his weak and exhausted condition, this was about the worst that could have been done; for the stomach and digestive organs, sharing, either by direct sympathy or otherwise, with other parts of the body, were not in a fit state, until a period of rest had occurred, to digest even a small quantity of food. The cravings of hunger, or a morbid appetite, induced the animal to devour most, if not all, of what was placed before him. The consequence was, the food, instead of undergoing digestion, ran into fermentation, and generated gas known as sulphureted hydrogen.

Treatment.-The animal was urged to rise. I then gave him a colic drench, composed of two ounces of fluid extract of golden seal, and one ounce of hyposulphite of soda. The surface of the body was then rubbed with wisps of straw, which produced some reaction, so that the surface of the patient became warmer. Occasionally the animal was led about for a short distance, and then was led back again to the stall, where he would get down, and roll and tumble about, as if in great agony. I administered enemas of soap-suds and salt, but did not succeed in bringing away either feces or gas, and all the gas which escaped from the alimentary cavity passed by the mouth. Two hours after the administration of the first dose, finding that the animal was still unrelieved, I repeated the dose of colic drench, and threw soap-suds into the rectum. Soon after some feces were voided, and with them quan- 
tities of gas. A slight improvement was soon perceptible, yet it appeared that the fermentation was still going on, as the abdomen did not decrease in volume; so I gave two ounces of hyposulphite of soda, in view of arresting the generation of gas. This had the desired effect; for, as the gas was liberated, the abdomen decreased in size. It was not, however, until a lapse of ten hours from the attack that the patient obtained complete relief from his sufferings. This case only differs from meteorization in the suddenness of the attack of the former.

Persons who attempt to treat cases of this character must exercise due patience. The animal must suffer a certain length of time, notwithstanding the best-selected remedies are exhibited. In other words, the reader is advised not to be in too much of a hurry in getting the animal on its legs again. Take good care of him ; give him plenty of bedding to rest or roll on; in short, nurse him as if he were a child, and, above all, do not give him too much medicine.

\section{Flatulent Colic.}

This is a disease of very frequent occurrence among horses. It is known by a distension of the intestines and abdomen, with flatus or gas. In the early stage, no perceptible abdominal distension occurs, yet it very soon manifests itself. One way of satisfying ourselves of the presence of flatus is, to apply the ear to the abdominal region, within which a sort of active rumbling is heard, often accompanied by a tinkling or metallic sound. But while making our examination, we may, perhaps, perceive that the horse passes flatus by the anus, or eructates it from the stomach by the mouth. In either case, our doubts, if we have any, are set at rest. This feature of flatulency, accompanied by others which will be alluded to, complete the chain of evidence.

In some cases the gas accumulates in the large intestines until the abdomen resembles, in size, that of an ox when "hoven." It is very distressing to witness the sufferings of an animal in this condition; and when the intestines are so enormously distended as to threaten rupture, the only chance of relief is to send a trocar and canula through the walls of the abdomen, into some portion of the distended intestine, and thus liberate the gas.

Symptoms. - It may be well to bear in mind that flatulent colic 
is always sudden in its attack, and some horses are liable to it under every variety of circumstance-in the stable, on the road, or at grass. At the commencement of the attack the animal becomes uneasy from pain, and commences to paw with his fore-feet. $\mathrm{He}$ soon gets down, and, if space be sufficient, he commences to roll from side to side, often remaining for a few moments on his back, in which position he seems to obtain temporary relief. Sometimes, as quick as thought, he is on his legs again, gives the body a shake, and then anxiously regards his flanks, by turning his head toward one side or the other, as much as to say, "Here is the seat of my trouble." Soon he is down again on the floor, rolling and tumbling about. Now and then the animal remains quiet for a time, in a sort of crouching attitude, the limbs being gathered beneath the body, until the distension is so great, or the pains so severe, that he must shift his position, when again we find him rolling, or standing with his hind extremities stretched backward, and the fore ones advanced, thus representing the attitude of a horse in the act of urinating. Supposing, at this period, that there be no flatulency present, yet the respirations are hurried, the pulse wiry, the exes glassy, and the patient excessively nervous and uncontrollable, the case is then of a spasmodic character (see Spasmodic Colic); but should the animal pass flatus, or the abdomen increase in volume, the case is unmistakable-it is flatulent colic.

Causes.-I shall now offer some brief remarks on the cause of colic. If we could only stretch the imagination, so as to take for granted all the popular causes assigned for the production of colic, then their name would be legion. Some writers inform us that a drink of cold water, when the animal is heated, is the most potent cause. Then we must infer that the horses ridden by the Russian Cossaes, in a country where cold water is abundant, should be the subjects of colic; but the very reverse is the case. The disease is almost unknown among them.

Previous to the introduction of Cochituate water into the city of Boston, colic occurring among horses was partially attributed to the cold well-water then used; but now they all drink the former, yet colic is just as prevalent, and, indeed, more so, than at the time referred to.

There are no people that pay more attention to the watering of horses than the English grooms, and it is customary, when a horse is taken from his home to a distant race-course, to remain away 
only a few days, to have sufficient water transported with him to last during his stay, such a dread have English grooms of "strange water," as they term it. Now, it is a notorious fact that English horses are more subject to bowel affections than those of this country. From this circumstance, I infer that bowel affections are hereditary, or, in other words, a predisposition to such is transmitted from parents to offspring.

I can well remember the time when physicians would not allow a fever patient to taste water; but now they act more in accordance with reason, and allow the sick just what they crave for. It is not good policy, however, to allow a horse, when performing a journey, a bountiful supply of water, because active exercise somewhat suspends the digestive function, and, therefore, the water may remain in a portion of the large intestines without undergoing the usual transformation. It then occupies space, and, being weighty, may, in the rapid movements of the animal, operate unfarorably in various ways, more particularly on the gut itself, it being pendulous from the spine.

A horse is often brought into the stable in a state of profuse perspiration, and, of course, is somewhat exhausted. Now, it would be decidedly wrong to either feed or water him until he is rubbed dry, and has rested awhile; for, at such times food is just as likely, and, I think, more so than water, to operate unfavorably on an exhausted animal. Many horses, however, even when in this exhausted state, will fill their stomachs with food and water, and yet enjoy immunity from colic. Therefore, should colic occur in a horse after he has had a hard drive, he having partaken of a draught of water, it is rather difficult to decide which of the two, if either, was the cause of the malady. One fact is certain, and that is, the stomach is deranged, and, therefore, fermentation precedes digestion; hence the gas.

Notwithstanding our best efforts to prevent it, colic will occasionally occur. Green grass, clover, carrots, and turnips are saic to occasion it. Then, again, it appears in stables, where nothing but corn, oats, and hay are used. One horse is attacked immediately after a draught of cold water; another has the chill taken off his, yet he is often found in the same predicament. Warm water is the most insipid and nauseating drink that you can offer a horse; and many would prefer to continue thirsty for some time ere they would imbibe it. 
It is well known to plysiologists, and I presume the reader must have observed, that both men and animals inherit peculiar idiosyncrasies. Each are predisposed, either through parental defect, temperament, or conformation, to certain forms of disease. This peculiarity, or predisposition, is said to lurk in breed, and those conversant with the horse's structure and temperament can readily determine whether he be predisposed to certain forms of disease or not. For example, a horse predisposed to flatulent colic is often observed to have a capacious belly, voracious appetite, and does not properly masticate his food; and he is not over-particular as to the kind of diet, for we often find him devouring, with apparent relish, the filthy straw that has served as bedding. Often he proves to be a "crib-biter." (Cribbing is a defect, either inherited or acquired.) Ordinarily the salivial fluid is augmented, yet it does not look healthy; it is more like soap-suds, and of a dirty color. The tongue is also foul, and the breath somewhat fetid. One or more of these peculiarities generally predominate in colicky horses. I contend, therefore, that some horses are predisposed to colic; and this explains the reason why the ordinary exciting causes, such as cold water, exposure, fatigue, irritating food and medicine, are operative on the system of one horse and inoperative on that of another. Colic is the heritage of some of the best horses in the world.

Now, should the question be asked, What are the causes of colic? I answer, It is occasioned by predisposing, direct and indirect causes, operating conjointly or not. In all cases of colic, the function of the stomach is either disturbed or partly suspended.

Treatment.-The principal objects in the cure of colic are, to restore the tone of the stomach, by means of stimulants and tonics, and to arrest fermentation and absorb the gases of the alimentary canal. I shall not undertake to say that the remedies here alluded to are the very best; but, as I have met with remarkable success in their use, I can safely recommend them. I have an impression that when a horse is the subject of colic, he requires to be treated just as any intelligent physician would treat one of us. There is no necessity to convert his stomach into a "slop-shop." The patient should be treated in a rational manner, by the same means and with the same skill as if one of our race were concerned.

I do not like to sce an animal trotted up one street and down another, followed by a biped, whip in hand, and a crowd of idlers, 
when he is the subject of excruciating abdominal pains, and the sweat is pouring off him like rain, from sheer agony. The custom is decidedly wrong. Our reasoning faculties confirm this opinion; and what reason teaches, man should endeavor to put in practice. No practitioner of human medicine dare advise such a course as compelling a man to hop off a bed of sickness, and start on a fast trot up and down stairs; for, should he so advise, he would very soon entitle himself to a ticket of leave. Should, however, the attack be slight, a little walking exercise does no harm; and if the patient appears disposed to roll, it were better to let him do so-on the ground, rather than in a narrow stall.

My usual directions are: Give the patient a wide stall and plenty of bedding. Let him lie down, rise, and tumble about just as much as he pleases, only watch and see that no accident happens to the animal. The colic drench used by the author, during the past ten years, is composed of the following:

No. 22. Fluid extract of Jamaica ginger....... 2 oz.

Fluid extract of golden seal......... 1 oz.

Powdered hyposulphite of soda....... 1 oz.

Water...................... 4 oz.

Dissolve the hyposulphite of soda in the water, then add the other ingredients to it. The dose may be repeated, if necessary.

A good wisp of straw, vigorously applied to the belly and flanks, and also to the limbs, may do some good, because we thus preserve the equilibrium of the circulation. Enemas of soap-suds should be administered often, and, provided the case be a curable one, the horse will soon recover.

\section{Spasmodic Colic.}

The usual causes of spasmodic colic are, mental emotions, external chilliness by exposure, cold water drank hastily, drastic cathartics, poisons, etc. Spasmodic colic is usually confined to the small intestines, and they become cramped and contorted. In this condition the patient is much tortured. The pain occasionally remits, and the patient will appear easy, but only for a short time. In some cases the patient will stamp and paw almost continually, and strike his belly. He throws himself violently on the floor, and occasionally rolls on his back. 'The abdomen is not distended, as in the case of flatulent colic, but is rather tucked up, and the 
abdominal walls are rigidly contracted. In the commencement of the malady, the pulse is usually.hard and wiry, but varies as the disease progresses; and the same is true of the respirations, viz.: they are accelerated and laborious. The patient occasionally suffers much pain in consequence of retention of urine and overdistension of the bladder, which is the result of sympathetic spasm in the region of the neck of the bladder. Such are the notable symptoms of spasmodic colic.

Treatment.-The disease being of a spasmodic character, requires the exhibition of medicines of an antispasmodic character, such as assafetida, lobelia, sulphuric ether, and copious enemas of warm water, or an infusion of lobelia. The latter is the most valuable antispasmodic now in use. Should the ease, however, be a desperate one, I advise the reader to follow the treatment indicated in the following ease, taken from my note-book:

On making an examination of the animal, the following symp- toms were observed: Pulse, very strong and wiry, averaging twenty beats above the natural standard; membranes of the mouth, nose, and eyes very vascular, or highly reddened; the surface of the bodly was bedewed, in patches, with perspiration; there appeared to be much rigidity of the muscles, especially in the abdominal region. All at once the animal would throw himself violently on the floor, and throw his limbs about in the most wild and reckless manner. He would assume all sorts of positions, yet seemed to obtain most ease when flat on his back; still, if closely approached or touched, he would kick and strike with fury, as if he were intent on mischief. In short, the horse cut up such antics that it was almost impossible to approach him; yet I finally succeeded in delivering an antispasmodic drench while the animal lay on his back, in which position I also gave an enema, composed of warm water and lobelia.

The animal kept growing more restive and uncontrollable, till, at last, it became very evident that convulsions had set in. The breathing had become fearfully laborious and rapid; his nostrils were dilated to their utmost capacity; the sight had become so affected, through temporary paralysis of the optic nerve, that temporary blindness had set in.

There seemed to be little hope for the animal, and, fearing that he might either kill himself by violence, or injure those in attendance, I concluded to chloroform him, and thus put a stop to his 
dangerous performances. He had had the best of us long enough, and now it was for me to show what science had in store for such otherwise unmanageable cases. I procured a mixture, composed of four ounces of chloroform and the same quantity of sulphuric ether; next, a sponge was tied to a broom-handle, and covered with a towel. Thus we were enabled to chloroform him at a safe distance.

The patient did not seem to relish this mode of practice. $\mathrm{He}$ fought for some time, tried to strike and kick me, but, being temporarily blind, I had the advantage of him. It was soon evident that the chloroform was beginning to do its work. The patient gradually settled himself on the floor, and was soon completely etherized. As it is dangerous to keep a horse under the full effects of chloroform any great length of time, I now removed the sponge, and only applied it occasionally, slightly saturated, so as to insure a sort of incomplete state of insensibility. It was an encouraging sight to behold the once powerful and furious animal now lying free from pain, and deprived of the power of injuring himself or those in attendance; and it is also gratifying to know that science ministers to the wants and necessities of the inferior as well as the superior orders of creation.

At the expiration of an hour, during which time the animal was more or less under the anæsthetic agent, he was allowed to rise. He gave himself a few shakes, and seemed very much relieved and more tranquil. I now gave a drench of spirits of niter and infusion of lobelia; administered, also, a lobelia enema, and left the patient to the care of his attendants. A few hours afterward I again visited the patient, and learned that he had a slight spasm occasionally, for which I prescribed two drachms of powdered assafetida. I then introduced the male catheter into the bladder, drew off a small quantity of urine, and this completed the whole of the treatment. The patient recovered.

Another case of Spasmodic Colic, treated by Inhalation.-The subject was a large and powerful stallion, aged about eight years, the property of a gentleman residing in Chicago. The horse was attacked in the street with symptoms of acute abdominal pain, and, in consequence, was hurried home to the stable. The moment he arrived there, he threw himself down and commenced rolling, and grew excessively uneasy and irritable. In the course of an hour our attention was called to him. He then exhibited 
symptoms of acute abdominal pain. The eyes appeared wild, and their membranes congested ; the nostrils were dilated; pulse, about sixty ; extremities cold; body, bedewed with perspiration ; no tympanitis, yet the animal anxiously regarded his flanks. He would paw with his fore-feet, and strike forward in a most reckless manner, and fall down, as if in convulsions; then he would roll on his back, curve his head to one side, and, as quick as thought, jump up again.

He was now so spiteful and unmanageable that it was impossible to administer either drench or ball. We tried every means to get something down, but all to no purpose; he was determined to make us keep beyond the reach of his fore-feet and mouth. It now became evident to us that the patient must be mastered, and, in this view, not knowing at the time that the agent we were about to use would act beneficially on the disease, we procured three ounces of chloroform, and the same quantity of sulphuric ether. An old sheet and a sponge were used as an inhaler. Watching an opportunity, when the horse was down, we got two men to secure him there by the neck. We then saturated the sponge with the mixture, and applied the same to the nostrils, enveloping them with the folded sheet, leaving an orifice for the admission of air. The patient struggled violently at first, but soon became quieter, and, in the course of two minutes, trembled, breathed deep and loud. The pupils then became dilated, the breathing stertorous, and the animal was fully etherized. Having him completely under control, we were not disposed to let him up in a hurry. The sponge, however, was removed, and so soon as he showed signs of partial consciousness, it was again replenished with ether, and applied.

Supposing that the case might be running on to intussusception, or entanglement of the bowels, we thought there could be no harm in keeping the animal under the influence of an agent that seemed to act so well; consequently he was kept under its influence for fifty-five minutes. His head was then brought toward the door, and a few drops of cold water were sprinkled on him. Soon he raised his head, looked bewildered, yet showed no symptoms of pain nor uneasiness. Shortly afterward he got on his legs, but had to be supported by several men, who soon rubbed him dry. He was then drenched with laudanum, five drachms; sweet spirits of niter, one ounce. He was led to a stall, entirely free from pain, 
partook of some gruel, and rested well during the night. Next morning he got five drachms of aloes and one of ginger, in solution; the bowels responded to the same, and four days after he was at work. We would not, however, have the reader suppose that the same agent shall suit all cases of this character; for another similar feat might prove a failure, and a third equally unmanageable. A case might yield to the ordinary sedatives, however, provided the patient could be induced to swallow them.

\section{Inflammation of the Peritoneum (Peritonitis).}

The peritoneum is a serous membrane, which not only gives a covering to the intestines, but also a lining to the abdominal cavity. Our pathologists teach that the peritoneum, like other serous membranes, is very ready to take on inflammatory action upon the operation of certain exciting causes. WATson informs us that "acute inflammation, beginning in one spot, is almost sure to transfer itself to any other spot that happens to be in contact with the first, and is very apt to extend itself rapidly to the whole membrane. The disease tends to the effusion of serum, and of coagulable lymph. It is of the adhesive kind, and its effects are those of distending the peritoneal cavity with fluid, or of gluing its opposite surfaces together, so as to obliterate that cavity, or of forming partial attachments. In all these respects the analogy between inflammation of the peritoneum and inflammation of the serous membranes of the chest and the covering of the heart is perfect."

Peritonitis is generally a manageable disease when treated according to the principles of rational medicine; but when treated according to the dictum of BLAIN and others, who have never done any original thinking, in so far as veterinary science is concerned, it is decidedly the reverse. For example, here is a passage from BLAIN's works :

"The treatment of peritonitis is much the same as for enteritis. The only difference is, that the animal will lose more blood before he displays any symptoms of uneasiness. We may also be more bold with purgatives. The three ounces of solution of aloes may be increased to five, the pint of oil enlarged to a pint and a half; and if this produces no signs of its having acted on the bowels, it may even be repeated when another three hours and a half have elapsed. All the other measures are alike, in both cases." 
Those who follow such advice as this would be very apt to lose their patients, either through the direct influence of meddlesome medication, or by converting peritonitis into dropsy of the abdomen. But let us see what Watson says on the subject of the treatment of peritonitis :

"Most writers whose worth I am acquainted with recommend purgatives as highly serviceable in peritonitis. I do not think the good which they are calculated to do, as antiphlogistic remedies, can at all be put in competition with the harm that I am persuaded they may produce, by increasing the peristaltic action of the intestines, and so causing additional friction and tension of the inflamed membrane. I believe that in all cases of well-marked and pure peritonitis, when the inflammation is limited to the serous membrane, it is far better and safer to restrain than to solicit the internal movements of the alimentary tube."

In human practice, physicians, instead of giving purgatives, resort to the use of narcotics, in view of tranquilizing the system, and for the purpose of mitigating the intense pain which exists during the acute attack.

Symptoms.-The most marked symptoms of peritonitis are as follows: In the early stage, the animal paws slightly, but not with that sort of viciousness which is characteristic of colic or strangulation of the intestines. Soon, he shows febrile symptoms; the pulse runs up to seventy or more, and a shivering fit sets in. When pressure is made on the abdomen, the animal evinces signs of pain. He will sometimes get down and lie on his back, but he soon finds out that the best way of lessening his pains and aches is to keep as quiet as possible. His tongue is usually coated, and the bowels are inactive.

Treatment.-Give the patient mucilaginous drinks, as in the case of enteritis, and drench him occasionally with an infusion of poppyheads, in pint doses. Empty the rectum by means of soap-suds enemas, and then throw in a quart of infusion of hops. Cloths, wet with warm water, should be applied to the abdomen, and the surface of the body should be kept warm by means of blankets. This kind of treatment, with good nursing, will probably restore the animal to health. Should symptoms of dropsy set in, give two ounces of fluid extract of buchu, daily. 


\section{Ascites, or Abdominal Dropsy.}

Ascites signifies a collection of serous fluid within the abdominal cavity. The subject of this disease is noticed as having a symmetrical and uniform enlargement of the abdomen; and, if the patient be a mare, some persons are likely to infer that she is pregnant, but examination, by pressure, will suffice to determine the conditions; for, if pressure be made with the fingers, in the abdominal region, a waving or fluctuating motion is discovered, similar to that which follows the forcible displacement of liquid. This fluctuation is the discriminating symptom between ascites and pregnancy, for in the case of pregnancy no such fluctuation can be elicited.

Ascites is usually the result of some chronic or acute disease of the peritoneum (lining membrane of the walls of the abdomen), or, rather, a loss of equilibrium between the local functions of secretion and absorption. This disease, like many others, may be rapid or insidious. If rapid or acute, it is apt to prove fatal in the course of a few days; if insidious or chronic, the animal may survive for a long time, as the following case will show:

Case of Dropsy of the Abdomen, ending in Rupture of the Stomach.-The subject of the above-named affections was a mare, owned by the North Chicago Railroad Company. She had been in their employ during a period of three years, and had always performed the work required of her without any manifestation of disease; in fact, had gone her usual trips up to within a few hours of her death. She had just partaken of a hearty breakfast, when, all at once, she began to falter, and the surface of the body was soon bedewed with a profuse perspiration; she trembled, the head became pendulous, and the case assumed an alarming aspect.

This happened at about six o'clock in the morning. At eight; my attention was called to her, when the following symptoms were observed. Pulse, small and indistinct; respirations, humid, and rather laborious; abdomen, quite tense from distension ; percussion over the region of the abdomen yielded a dull sound, yet fluctuating, showing that the abdominal cavity was occupied by some kind of fluid. From the peculiar conformation of the abdomen, I at first suspected that the animal was pregnant, which proved not to be the case.

On percussing over the region of the stomach, a tympanitic 
sound was elicited, showing that the stomach contained a large quantity of gas, and was over-distended. Her extremities were cold, and other symptoms were present which indicate death. I suspected that the food was running into fermentation, and, in view of arresting it, I gave one ounce of the hyposulphite of soda, and the same quantity of fluid extract of ginger and golden seal. The body was bathed with new rum, and she was rubbed vigorously, by three men, for about half an hour, from which treatment she seemed to rally some. Several injections of salt and soap-suds were thrown into the rectum. They brought away a slimy substance, but very little feces. Finally nothing came away but that which was injected, and from this I was led to infer that, either from pressure or stricture, the small intestines were closed.

I now determined to send a trocar into the abdomen, for the purpose of discovering what it contained, and, accordingly, selected a point in the lower middle of the same. In withdrawing the cutting instrument, a fluid commenced to run, which, in color and consistence, resembled urine. For an hour and a quarter this fluid continued to run through the tube, and the amount obtained must have been over three bucketsful. I was in hopes that the withdrawal of this large amount of fluid might remove the pressure on the intestinal tube, and thus the gas, which was pent up in the stomach, might find its way to the anal outlet. Not so, however; for, notwithstanding all our efforts and attention, the mare died, at noon, with rupture of the stomach.

The autopsy revealed a rent, or rupture, in the stomach, of about eight inches in length, through which a portion of the food and fluids of the stomach had escaped into the abdominal cavity. The small intestines were the seat of stricture in several places; their external surface, or, rather, peritoneal covering, was studded with albuminous and fibrous deposits, resulting from abdominal dropsy ; otherwise, the contents of the chest and abdomen presented the appearance of perfect health. I should judge that the water had been accumulating in the abdominal cavity for a long time. The foreman at the stable observed that the animal always appeared very portly, so much so that the abdomen was square with the hips, which had led some persons to suppose that her hips were "knocked down."

Most authors contend that when a horse is the subject of rupture of the stomach or intestines, he squats on the haunches, like a dog; 
but this animal did not exhibit any such symptoms, but stood up until within a few minutes of death.

Treatment.-A case of this kind requires the exhibition of tonics and diuretics, and the following is the best remedy which can be given :

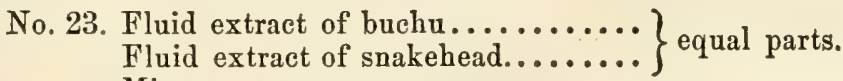
Mix.

Dose, two ounces per day.

\section{Inflammation of the Intestines (Enteritis).}

This disease is located on the inner or mucous coat of the intestines, sometimes, however, involving the muscular portion of the same. The causes are various: improper management, bad food, worms, irritating medicines, musty hay or clover, chilling the body by cold water, exposure to rain, etc.

Symptoms. - The disease often commences with a shivering fit, to which succeeds heat of the skin, restlessness, loss of appetite; the mouth hot and dry; the inner membrane of the eyelids and nostrils are redder than usual. As the disease progresses, the pain increases, and the animal will lie down. There is no intermission of pain; it is persistent. The breathing is accelerated, and pressure in the abdominal region elicits symptoms of pain. The bowels are usually constipated, and if any feces are voided, they consist of small, hardened balls.

An examination after death reveals a gangrenous state of the small and sometimes the large intestines. Often extravasated blood is found within the intestinal tube. So soon as the pain ceases, and the animal appears no better, we infer that gangrene has set in ; cold sweats set in, and the animal shortly dies.

Treatment.-The treatment of enteritis does not, and should not, differ from that of any other acute affection. Mucilaginous drinks, chlorate of potass, hyposulphite of soda, in doses as recommended for inflammation of the stomach. Occasional enemas of soap-suds are to be thrown into the rectum, so long as the feces remain hard, or are voided with difficulty. The objectionable method of backraking - so highly recommended by some authors-should never be resorted to; it is a disgusting affair, and actually unnecessary; for, let the rectum be ever so impacted, it can be evacuated by 


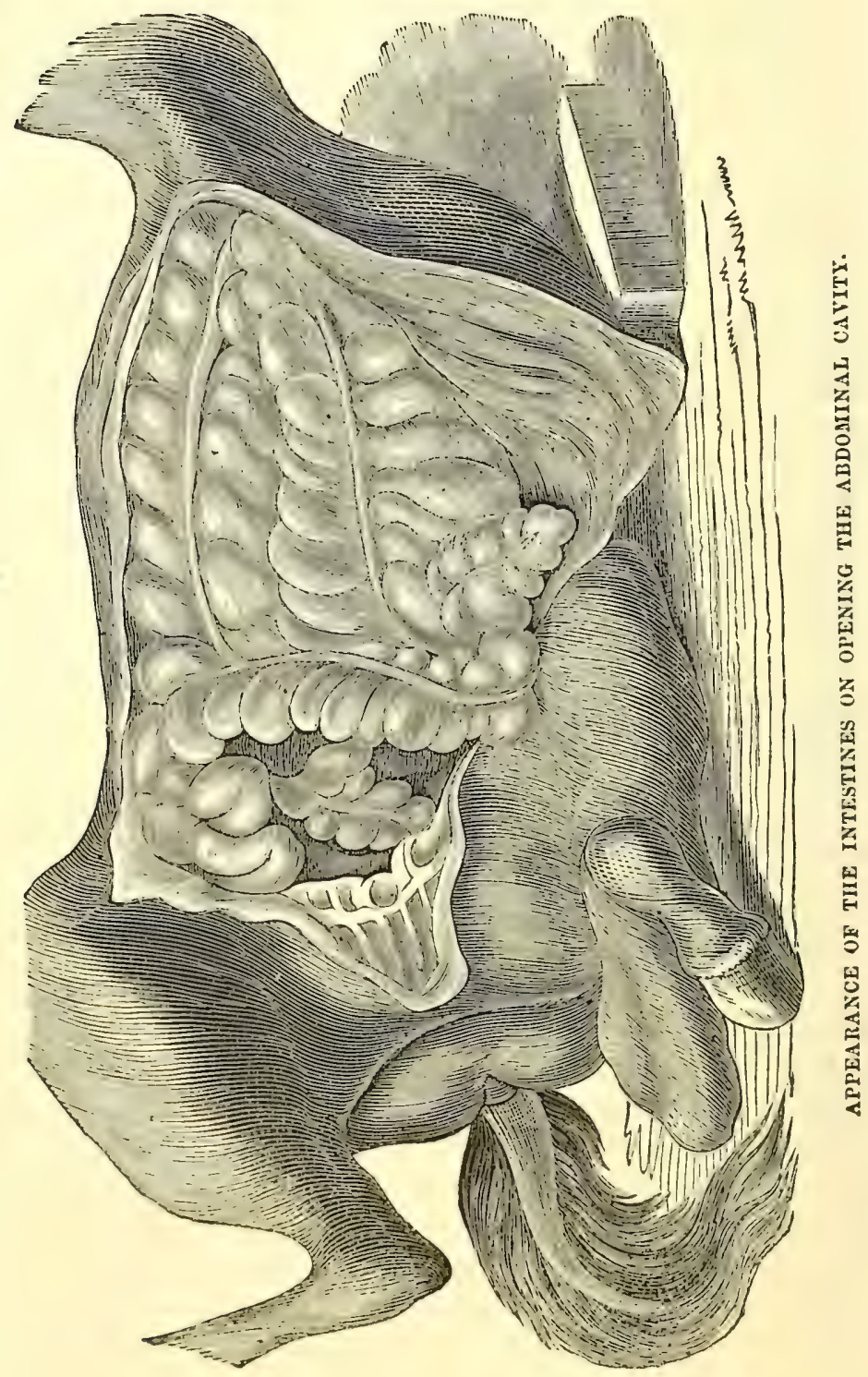


enemeta, composed of soap-suds, to which add a little ginger or lobelia. If the weather is not too cold, cloths wet with warm water may be applied to the abdomen, over which throw a blanket, so as to prevent evaporation and chill. The outrageous treatment recommended by some authors is a disgrace to the profession. They recommend bleeding until the horse faints or falls; then to seorch the sides with ammonia and cantharides; and, lastly, to empoison what little blood he may have left in his system, so that death may occur secundum artem.

Should the subject of inflammation of the intestines suffer much pain, a drench of half a pint or more of infusion of hops, or poppies, may be given occasionally; or one ounce of the fluid extract of one of these agents may be substituted, to be given two or three times during twenty-four hours.

The treatment of inflammation of the intestines, as well as inflammation of any other part of the body, consists in using those means and agents which ward off or remove any agent which, in its effects, does, or tends to, deprive any of the organs or tissues of the living body of their vital action. The remedial means used to accomplish this object must be such, and such only, as sustain the weakened or increase the reduced vital action in any of the organs or tissues. This is the doctrine which the author attempts to teach in this work. And is the doctrine not plain? Could any thing accord more directly with reason, or commend itself more clearly to the common sense and unbiased judgment of intelligent people? If the vital action of any of the organs or tissues become weakened or enfeebled, should they not be strengthened? Can this be done by any other agent and means than those which increase and strengthen this very vital action? If this action, in any of the organs or tissues of the animal body, becomes reduced or impaired, must it not be increased by agents and means which coöperate with the vital power? Then, how can agents and means which always impair vital action (poisons) cure disease?

Different remedial means have different effects upon the organs and tissues of the system; but all poisons and agencies which impair the integrity of the organism of a well animal must have a disastrous effect on one that is sick and suffering. Unhealthy and poisonous agents impair and reduce the vital action of certain organs and tissues according to the nature and tendency to affect various parts of the living structures. 
Now, if it can be made to appear that disease consists, in its nature and effects, in increased vital action or increased health of any portion of the body diseased, then will it be proved that poisons are, and of necessity must be, medicines or the agents to cure disease. But what are we taught by our brethren of the heroic school upon this point? "In spontaneous disease," that is, disease which occurs without any apparent cause, Husren contends, "we know of no method that will entirely quiet or remove the inflammatory action or disposition. All we can do, probably, is to lessen every thing which has a tendency to keep it up. Inflammation is either an increase of life or an increase of a disposition to use it."

Again: "One of the means of curing this inflammation," says the same author, "is by producing weakness. The means of producing weakness of an absolute character are, bleeding, purging, and the exhibition of poisonous agents. The inconvenience, however, arising from this practice is, that the sound and healthy parts must suffer nearly in the same proportion with the diseased; for, by bringing the inflamed parts on a par with health, the sound parts must be brought much lower, so as to be too low. The first method, namely, bleeding, will have the greatest, the most permanent, the most lasting effect; because, if it have any effect at all, the diseased action can not be soon renewed; the second, purging and poisoning, will act as auxiliary, so that these processes go hand in hand; but neither these nor sickness can possibly lessen the original inflammatory disposition. Lessening the power of action belonging to the inflammatory disposition can only lessen or protect its effects, which, however, may be of service, as less mischief will be done, and this will often give the inflamed disposition time to wear itself out. But this practice must not be carried too far. It must be followed with great judgment. Nothing debilitates so much as purging, if carried beyond a certain point. Even one purging may kill when the constitution is much reduced. The internal medicines generally ordered for the cure of inflammations are such as have a similar effect to that of bleeding; namely, lowering the constitution or the action of the parts. As inflammation has too much action, which action gives the idea of strength, such applications as weaken are recommended."

Here, then, are the reasons for the use of poisons to cure dis- 
eases, as believed and taught by our brethren of the heroic school. Such agents and means we do not want, for the plain reason that disease can be cured quicker and better without than with them. Our system of practice, and that recommended in this work, tends to sustain the vital powers during the progress of disease; and this is accomplished by resorting to sanative medication. This sanative system of medication must, eventually, commend itself to the candid and to the unprejudiced reasoner and scientific inquirer after truth.

\section{Strangulation of the Intestines.}

When an animal becomes the subject of strangulation of the intestines, it is very difficult, at first, to diagnose the case, for the symptoms very much resemble those of spasmodic colic; yet as the treatment of the two diseases does not differ much, the mistake in itself is of little consequence.

Symptoms.-The difficulty is, at first, accompanied by uneasiness. The animal moves about in his stall, and commences stamping with one of the fore-feet. Frequently he gets down and rolls on his back, in which position he will remain sometimes for several minutes. So soon as he rises, he commences to paw and stamp with a vengeance. As the disease advances, he totters, and sometimes drops on the floor. The pulse is usually irregular, the mouth cold, and visible surfaces pallid; the tongue appears of a bluish east; and the body is generally bedewed with sweat. Finally, the animal makes great efforts to evacuate the rectum, and in so doing strains violently, so as to cause blood to escape from the rectum; and sometimes the rectum itself protrudes. Should the symptoms not improve, the animal soon dies of strangulation and mortification of the intestines.

Treatment.-This must be of an anti-spasmodic character. An ounce or two of fluid extract of lobelia should be given, every two hours, in a pint of warm water; the body should be enveloped in bandages, wet with warm water, and enemas of assafetida or lobelia should be thrown into the rectum; but if the rectum protrude, enemas are inadmissible. On some occasions of the kind, I etherize the patient, with marked relief. 


\section{Wounds penetrating the Abdominal Cavity.}

The treatment of a penetrating wound into the abdomen will depend somewhat upon the nature of the same. Should the intestines protrude through the wound and exhibit a puncture, put on the twitch and Rarey-strap. Then return the intestines into the abdominal eavity, and proceed to suture or sew up the intestinal rent, by means of a small needle, armed with sewing silk-first, by means of sutures through the abdominal muscles, one end of the suture being left long enough to hang out of the wound; second, close the skin (the sutures should be distant from each other about one inch); next place a cold water pad on the parts, and encircle the body in the injured region with a cotton-roller, over which place a sureingle, and securely fasten it. In the event of the intestines being swollen and distended, it may be necessary to cast the patient; then roll him on his back, and thus the protrusion may be returned; but should they still prove too large for the orifice, it must be dilated by means of a probe-pointed knife.

\section{Splenic Apoplexy.}

Mr. Gamgee has made some translations from foreign authors on the subject of Splenic Apoplexy, and he tells us that "this is the disease described as carbuncular fever by Renault and Rcynal. It is most common in hot and tempestuous seasons. In its carefully-observed symptoms, M. Anginiard is induced to regard the disease as dependent on miasmatic exhalation. Until recently, the author had studied the malady only in sheep, but has frequently observed it, of late, in the horse. It is not so suddenly fatal in the latter as in the former. The prostration and stupor are very striking. The horse can barely stand, and is very listless; the muscles of the limbs tremble, and partial sweats bedew the body. In some parts the skin is dry, and the coat staring; the breathing is slow and irregular; the conjunctiva, or lining of the eyelids, is white, and indicative of an anemic or bloodless state; the pulse is feeble, wiry, and accelerated, offering a remarkable contrast to the tumultuous action of the heart; the loins are very sensitive; and there are often indications of pain on pressure on the abdominal walls. Colicky symptoms are frequently noticed. At first, all appetite is lost, but, as the disease advances, 
the animal eats and drinks freely. In attempting to bleed an animal, great difficulty is experienced in filling the vein; and when the blood flows, the animal instantly manifests great weakness. The blood is black, and does not coagulate as in health. The symptoms rapidly grow worse, the breathing labored, the eyes sunken, the prostration extreme, and progression impossible, until the animal reels over and dies. The disease runs a rapid course, and, on an average, death occurs within fifteen or eighteen hours. There is an absence of the external swellings characteristic of ordinary carbuncular fever; and the internal distensions of organs, particularly of the spleen, so ably described by Renault and Reynal, are sufficient, according to M. Anginiard, to distinguish the two diseases. In the treatment of this disease, Anginiard trusts mainly to quinine. He administers a drachm and a half as soon as possible, either in an infusion of coffee or other aromatic mixture. If the symptoms do not subside two hours afterward, two drachms are given. Generally this is sufficient. If not, a third and fourth dose have to be exhibited. Bleeding is to be condemned. The body of the animal may be rubbed with a wisp, or a turpentine liniment may be employed as a derivative. Free ventilation, and leaving the animal uncovered, to permit free exhalation, are essential points in the management of these animals. M. Dumesnie has tried this treatment, and found it most successful. Anginiard believes in small doses, and not often enough repeated."

Apoplexy is a disease which, if not discovered early, is very apt to prove fatal. Whether discovered or not, in its early stage it generally baffles the skill of those who, in this country, have attempted to cure it. The incurability of such malady may, under the circumstances, be accounted for on the belief that it often rages as an enzoötic, affecting animals in isolated spots, without regard to locality, and attacking only those susceptible to its influence. What the conditions are which favor its propagation I am unable to determine, for I have known it to affect equally those which appeared healthy as well as others unhealthy in appearance; hence it is very difficult for any one to point out the direct causes of the affection. I have not seen many cases of either enlargement of the spleen, or apoplexy of the same, occurring in cattle, from the fact that my practice has been mostly confined to horses in cities, where cattle were not very numerous; but a friend 
of mine, located in a cattle-raising district in the State of Maine, informed me that this disease, accompanied by enlargement, often prevails there enzoötically, and that most of the animals thus affected die. I have had considerable experience in the treatment of such affections in horses, but, as I have just observed, the result is very unsatisfactory. I believe I have but one case of cure on record, and the medicines used on that occasion were stimulants and tonics, with forty grains of iodide of potass per day. The spine was also rubbed, night and morning, with equal parts of cod-liver oil and spirits of camphor.

Symptoms.-The symptoms of enlargement of the spleen in horses (they do not differ much in cattle) are as follows: Feeble pulse; respiration not much disturbed; the tongue is usually coated; mouth, hot, and the breath has a feted odor; the membranes lining the mouth and eyeballs have a slight yellow tinge; the head droops, and the tips of the ears and lower parts of the limbs are chilly; the patient is rather unwilling to move, and, when urged to do so, exhibits a staggering gait, and sometimes falls never to rise; the urine is scanty, and, in the last stages, blood oozes from the anus and nostrils; colicky pains attend the disease.

In a case that terminated fatally, I made the following record of the post-mortem examination: On opening the abdominal cavity, the spleen was found to occupy a very large space. It was of immense proportions, and weighed nearly twelve pounds. The ordinary weight is three pounds. It presented the appearance of a spleen in the chronic stage of disease, being altered in structure, of a dark, pitchy color, and surcharged with dark, venous blood. Before death, I percussed the left side, in the locality of the spleen, and the sound elicited was of a solid character, which indicated enlargement of the same.

The early symptoms of splenic apoplexy do not differ much from the above, only they are of a more acute character. In the latter stages, the malady is complicated with a painful affection of the bowels. The diagnostic symptom of splenic apoplexy, with enlargement of the same, is a notable enlargement on the left side of the abdomen, well up toward the ribs. When standing behind an affected animal, and casting one's eyes carefully along the sides of the abdominal walls, a perceptible eminence will be seen, which can not be mistaken. The region of the spleen is between the stomach and the false ribs on the left side. 
In view of rendering this subject more valuable and interesting, I now introduce the following, which was written for the "Edinburgh Review :"

"Splenic Apoplexy.-This disease broke out in the year 1858, on a farm characterized by its extreme richness of soil, in the north of Northumberland. In 1859 the malady reappeared, and did not cease until twenty-three head of cattle had been affected. Splenic apoplexy is a malady that has not hitherto been observed in the north of Britain, and its occurrence recently is evidently to be attributed to the special method of farming and feeding stock where it has, for the last two years, proved so destructive.

The farm is a peculiarly dry one. About three hundred head of cattle are annually fattened on it. One lot was made ready by Christmas, and sold as fat beef in Neweastle; a second was kept back until January, when they were allowed an abundance of turnips, meal, and the best oat straw. The cattle affected were three years old; and it would appear that those fed on Swedish turnips, especially from a certain field, suffered most; but some fed on yellow or white turnips have been affected, and we should regard the meal, and perhaps the oat straw, as more likely to produce this disorder. According to Delafoud's researehes, leguminosæ, or forage very rich in nutritive principles and deficient in water, from artificial pastures, are frequent causes of splenic apoplexy. The farmer in Northumberland suspected that the artificial manures had produced the trouble, and the following season he dressed his land with home manure; but the properties of the last crop have proved as deleterious as those of the preceding one. The fact is, the crop had nothing to do with the development of the disease.

The malady stopped suddenly, about the middle of February, and this was probably due to the cattle suffering only when the system was taxed by change from rather moderate to very high feeding. It is an interesting fact that in cows the disorder only affects those which are approaching the period when the secretion of milk is stopped, and when there is a tendency to lay on flesh, they then being liable to plethora. Cows fed on food capable of producing splenic apoplexy die of it.

Change of diet proved of no avail at the farm referred to (showing very conclusively that the food was not the special cause). Common salt was recommended as a preventive; but Delafoud 
has shown that salt is not a reliable remedy in such cases. The malady stopped spontaneously, and the point now to settle is the prevention of disease during the future seasons.

For the benefit of our readers who may not know the symptoms of splenic apoplexy, we may mention that often there are premonitory signs - the animals apparently enjoying the best of health early in the morning, may be dead some time before noon. Sometimes there are symptoms of excitement; the eyes are prominent, and the visible mucous membranes are injected or reddened. Symptoms of uneasiness suddenly manifest themselves, and colicky pains indicate abdominal disorder. The urine voided is highcolored and red, and there may also be blood in the feces. The back becomes arched, and the animal fixes himself, hanging on to any thing by which he is tied, or he will press his haunches on some resting-place in the corner of the stall. After this the animal soon drops, and is seized with convulsive pains and twitchings. In addition to the discharge of feces and urine, tinged with blood, there is a red, frothy liquid, which escapes from the nostrils; the animal bellows, moans, and soon dies."

Treatment.-Tonics, alteratives, and glandular stimulants are indicated. Take of

No. 24. Fluid extract of blood-root.........4 oz.

Fluid extract of golden sea.l......6 oz.

Chlorate of potass............. 4 oz.

Dissolve the potass in one pint of boiling water ; then add the extracts. Give the patient a wine-glassful every six hours, until amendment is perceptible, when the dose may be decreased to two ounces every twelve hours. Rub the body with a portion of the following:

No. 25. Proof spirits................ 1 pint.

Tincture of eapsicum........... 4 oz.

Two or three applications, in the course of twenty-four hours, will suffice. Should the animal suffer much from colicky pains, dissolve one ounce of hyposulphite of sorla in four ounces of hot water, to which add half a table-spoonful of powdered ginger, and drench with the same. Pure air and a light diet will aid in restoring the animal, provided the case is curable. 


\section{Functional Disease of the Liver.}

The liver, like other parts of the digestive apparatus, is liable to become deranged, in either function or structure, by errors in diet, which is the most potent cause of both functional and organic disease of the same. In almost all cases of liver disease, we observe a yellow tinge of the visible surfaces-membranes of the eye and mouth. In order to account for the yellow tinge, we must bear in mind that bile is formed in the blood; that the office of the liver is to absorb or withdraw it from the circulation constantly, as fast as it is formed. Disease of the liver, simulating jaundice, manifests itself whenever the due separation of the bile from the blood is not accomplished; hence the accumulated bile seeks other outlets, namely, the kidneys and intestines, and excretory gland of the skin.

When animals become jaundiced suddenly, we may infer that the difficulty, whatever it may be, in the liver, is of an acute character; that is to say, it is recent in origin and active in form. Jaundice is a phenomenon of several diseases peculiar to horses. During the progress of the epizoötic known as influenza, a jaundiced state of the visible surfaces is always present, which disappears when the patient convalesces, showing that the liver is only functionally deranged. When jaundice accompanies indigestion, and disappears under the action of alteratives or changes in diet, we may also infer that, if not absolutely functional, it is, at least, not purely inflammatory.

It is plain, therefore, that jaundice is of but little consequence in prognosis, since the symptoms with which it is often associated declare, much more positively than itself, the gravity of the affection in which it occurs as a symptom. It sometimes happens, however, that the function of the liver becomes suddenly interrupted, causing pain, after the fashion of what accompanies the bilious colic of man.

The following case will probably give the reader some idea of the nature of acute functional disorder of the liver. The patient, a gray gelding, aged nine years, was suddenly attacked with symptoms of colicky pains, for the relief of which the owner had given a colic drench, which seemed to afford temporary ease; but soon the symptoms reappeared, the animal got down, and scemed to be the subject of acute pain. Under these circumstances, the owner sent for me. 
Symptoms.-On arriving at the stable, I found the patient standing, his head somewhat pendulous, appearing dull and sleepy. The membranes within the eyelids and mouth were of a bright yellow tinge; pulse, rather feeble; bowels, constipated ; in short, the usual appearances attending functional derangement of the liver were present; yet the "key-note," or diagnostic symptom, was the bright yellow tinge of the visible surfaces.

Treatment.-The following drench was given :

No. 26. Fluid extract of mandrake.......... 3 oz.

Fluid extract of golden seal ........ $1 \mathrm{oz}$.

Carbonate of soda............... $2 \mathrm{dr}$.

Water...................... $\frac{1}{2}$ pint.

An injection of soap-suds was thrown into the rectum, which brought away a small quantity of hardened, dark-colored excrement. Next day I found the patient in about the same condition, and repeated the dose of medicine and injection. In the course of a few hours the bowels responded, evacuating a large quantity of semifluid, dark-colored feces, mixed with a substance resembling shreds of albumen. Next day the animal appeared much better. I then gave two ounces of fluid extract of golden seal, and one drachm of hyposulphite of soda, and thus ended the treatment.

This case is an illustration of a simple mode of treatment far superior to the old-fashioned system, which contemplates strong doses of aloes and calomel, agents which usually intensify the morbid phenomena, often producing a medicinal affection, which requires much time for the animal to recover from. It is the author's opinion that a vast number of very fine animals in these United States die from the heroic system of practice, which overlooks the best means of aid presented by the resources of mild and sanative medication.

We have long been an advocate of the physiological method of treating disease, and we contend that is more philosophical, safe, and the surest means of restoring an equilibrium of vital action in the animal economy, in all forms of morbid conditions; and we earnestly entreat our readers to exercise great faith in the powers of Nature to aid and assist in the cure of all maladies, and place less reliance in the power of art.

In view of strengthening the faith of young converts to sanative medication, we quote the language of Sir JoHn Forbes, a man of undoubted authority. 
"Such has ever been the want of trust in Nature, and the overtrust in art, prevalent among the members of the medical profession, that the field of natural observation has been, to a great extent, hidden from them-hididen either actually from their eyes or virtually from their apprehension. The constant interference of art, in the form of medical treatment, with the normal processes of disease, has not only had the frequent effect of disturbing them in reality, but, when it failed to do so, has created the belief that it did so, leading, in either case, to an inference equally wrong-the false picture, in the one instance, being supposed to be true; the true picture, in the other, being supposed to be false."

In all cases of liver disease, it is good policy to change the diet, and offer the animal green vegetables-sliced carrots, and grass if it can be had. In all cases of chronic disease of the liver, a run at grass is the most potent remedy for the cure of the same.

It was formerly supposed that the function of the liver was merely to eliminate bile, but modern physiologists have discovered that the blood itself is changed while circulating through that gland, which elaborates fibrine from albumen, and fat from sugar. The liver stores up fatty matter, so that, should the food be defieient in fat, the liver yields up what it contains. It is very curious to notice the peculiarity in the circulation of the liver. It is supplied, like other organs, with arterial blood for its own support; and it also receives an immense quantity of venous blood through veins commencing on the gastro-intestinal cavity, which terminate in a vessel named vena porta. This vessel, on entering the liver, ramifies in various directions, like an artery, and ultimately terminates in the veins peculiar to the liver; so that the blood, instead of flowing directly from the stomach and intestines, is made to circulate through the liver, and thus the various transformations are effected. The liver must, therefore, perform the double function of secretion and excretion. It secretes bile, and excretes carbon and hydrogen from the system. Should an animal labor under diseased lungs, he may, under proper medical treatment, recover, provided the liver be healthy. This organ can, for a time, elininate carbon and hydrogen; but, instead of passing off as they do from the lungs, in the form of carbonic acid, they accumulate in the liver, in the form of fat, or else the liver increases to an extraordinary size. In Strasburg they prepare a favorite dish from the livers of geese, artificially enlarged by the cruel process of depriving them of 
light and liberty, confining them in a warm room, and stuffing them with food. We find that, under such barbarous management, there is a disproportion between the oxygen respired and the carbon taken in the form of food. The respiration which active exercise increases and rest diminishes are, now that the animal is confined, slow and uniform. The lungs fail in eliminating carbonic acid, and hence carbon and hydrogen are driven back to the liver by means of venous circulation. An excess of carbonaceous material in the liver is liable to result in jaundice, which is a simple functional derangement of that organ. It occurs generally in the warm months, and is usually sudden in its attack.

Among the most prominent symptoms are high-colored urine, yellow tinge of the visible surfaces, languid pulse, and slow action. If the liver be the seat of inflammatory action, the pulse will be quick and bounding, respiration hurried, the patient feverish, and pressure over the region of the liver elicits symptoms of pain. If simple functional derangement cxists unchecked for any length of time, it leads to organic lesions and structural disease. When the bile accumulates, it is very apt to thicken and produce gallstones or calculi ; if these accumulate in the gall ducts, the subject, unless relieved, soon dies.

Diseases of the liver have hitherto been considered the bane of tropical climates, but they are equally prevalent in cold and moist regions. Horses and men are as frequently attacked with it in northern as in southern latitudes. The celebrated sheep-breeder, Bakewell, knew that early disturbance of the liver led to the accumulation of fat, and, in order to derange the liver, he was in the habit of folding his sheep in wet pastures. Now, the English agriculturists are well acquainted with the fact that water meadows have a tendency to produce that almost incurable disease termed rot. Rot originates from a diseased liver, and, in the early stages of it, the animal accumulates fat very fast; so that by rotting sheep he was able to bring fat ones early to market, and thus steal a mareh on his more conscientious neighbors. This state of the liver termed rot is associated with the existence of parasites termed distoma hepaticum, commonly denominated flukes, and these parasites are considered the cause of rot, when, in fact, they are the results of deranged functions of the liver. The rot, therefore, is not local. It can be produced in any country by exposing animals to the debilitating effects of moisture and 
neglect. It is not only prevalent in England, but also in Asia, Africa, and America. In Egypt, on the receding of the Nile, it spreads to a fearful extent. In warm climates the livers of men and animals become diseased, not so much from local causes as from stimulating drinks and diet. If a man attempts to consume the same amount of food in warm that he has been accustomed to in cold climates, he is apt to become bilious, and this is a premonitory indication of perverted function of the liver. A very important fact has lately been discovered in reference to the function of the liver. A French chemist has ascertained that one function of the liver was to produce sugar out of the animal organism. This is the secret of the formation of the fat. Chemically speaking, sugar and fat are nearly allied, the chief difference being that one contains a small portion of oxygen.

Many valuable animals are lost from organic disease of the liver, the origin of which may, in many cases that have come to our knowledge, be traced to simple functional derangement, which has existed for some time. Its symptoms are either unobserved or, if perceived, disregarded. Some of the subjects that have been put under our care for the treatment of diseased liver had a strong predisposition for the malady, their color being black and temperament bilious. Such horses are the ones that require special attention whenever they appear to be "ailing." It is remarkable to observe, however, that many horses of a temperament diverse from the bilious are often attacked with organic and functional derangement of the liver, and this peculiarity ean only be accounted for on the ground that the horse has no gall-bladderno receptacle, such as is found in cattle, for the accumulation of bile; consequently, the horse's liver must be an active organ, especially when the animal is permitted to make but one meal per day, and that meal occupies a period from sunrise to sunset, and daily and weekly continues to occupy no less time. The consequence is, the organ is overworked, and is the seat of local exhaustion. The liver requires regular periods of rest to recover from fatigue or functional duty, yet how is it possible to secure the same when a horse is permitted to make a perpetual hay-rack and corn-bin of his stomach? The herculean feat of converting a hay-stack and corn granary into bone, muscle, and nerve at the rate of 2.40 is beyond the physiological capacity of any vital organ; therefore, in the language of the sailor, the vessel sinks 
from local inundation-the liver caves in from overwork, too much food, and too little discretion. A hard pull on an omnibus, or a similar feat in a marketer's wagon, would be the very best medicine in the world for a plethoric horse, or one predisposed to hepatic derangement.

\section{The Pancreas and its Function.}

The pancreas is a glandular body lying across the spine, in the epigastric region, underneath the diaphragm, near the small curvature of the stomach. The body of this gland is pierced by the vena porta (known as the gate rein); has one attachment to the spleen, and another to the left kidney. It is divided into head, body, and tail. Structure, similar to salivary glands. Color, pale red, speckled. It is composed of many lobules. Every lobe has a distinct set of nerves, arteries, veins, and ducts. Every lobe is, therefore, considered as a distinct gland, and the same is true of the salivary glands. The duct pierces the duodenum (known as the second stomach), alongside of the hepatic duct.

The pancreatic arteries are derived mostly from the hepatic. Several, however, come from the splenie, in its course to the left side of the abdomen, and one or two from the gastric. The veins are tributary to the vena porta. The nerves come from the ceeliac plexus. The fluid secreted by the pancreas is a colorless, limpid fluir. It forms an emulsion of fat; hence it dissolves the fatty matters of the food. It is an active agent in the preparation of chyle. That the pancreatic juice possesses the property of emulsifying fat is proved from the fact that when the pancreas is destroyed, and the animal fed on food containing fatty matter, the latter passes with the feces, just like fat in an unchanged state.

Mr. GAugee says: "The pancreatic juice has another important use which remains to be spoken of, and that is a peculiar action on nitrogenized substances. If raw meat be placed in some of the juice, the meat speedily softens and putrefies. The same occurs with albumen or caseine in the raw state; but if the azotized principles are previously boiled, or acted upon by the gastric juice, the pancreatie therefore serves many purposes, and acts on every kind of aliment.

Circumstances affecting the digestive function in general have a special influence on the pancreas, and modify its secretion. Such 
is not the case with the salivary glands and their products. The pancreatic juice may, like every other secretion, be found to contain adventitious substances that have accidentally entered the blood. Thus, iodide of potassium may be eliminated from the system by the pancreas. It is not so with every salt, the prussiate of potash, for example, never being seen in the pancreatic juice.

The pancreatic secretion is formed during infra-uterine life, but we are at a loss to account for its uses there. It is difficult to determine what nervous influenees affects its production. A dose of ether excites it, and pressure on the abdominal viscera likewise tends to its increase ; the efforts of vomiting stop it."

\section{On Parasimes which infest the Intestinal Canal.}

There are various forms of parasites which infest the alimentary canal of horses and cattle which, no doubt, are the cause of some annoyance to the infested; but really they are not, at all times, so injurious as some writers make them out to be. They are rarely if ever found in the intestines of healthy animals, and their presence is generally due to a deranged condition of the digestive organs. They very frequently originate spontaneously.

"Certain independent organisms, both vegetable and animal, are found in the body. The vegetable growths are all microscopic, and belong to the lowest order of plants, the algre and fungi. They are never met with except upon cutaneous or mucous surfaces, nor while these surfaces remain healthy, usually. A secretion of fibrine or mucus, undergoing decomposition, forms the soil in which they grow. In some cases, they are believed to be the media of contagion.

Animal parasites are very numerous. Many of them are infusorial. Many belong to the class of insects and mites, as fleas, lice, bugs, and the acari, of which the most important one is the itch-mite. A class of higher consequence comprises several sorts of worms. Those which infest the intestinal canal are extremely common, and are the oxguris vermicularis, or thread-worm, which inhabits the rectum; the trichocephalus dispar, or long threadworm, which is found in the large intestine, and especially in the cæcum; the ascares lumbricoedes, or round worm, whose ordinary residence is the small intestine; the tape-worm, or tænia, which also affects the same part. The kidney is occasionally the seat of 
a round worm, called ths strongylus gigas, measuring from five inches to three feet in length, and from two to six lines in thickness.

The diseases with which even large intestinal worms are connected appear to be sometimes the cause and sometimes the effect of the presence of these parasites. Very often they exist in considerable numbers without producing the least disturbance of the economy, but in other cases they are unquestionably the cause of much suffering and ill-health. How far they are themselves the result of a morbid state of the organs in which they appear is still an undecided question.

The origin of parasites is extremely obscure, and has long been a mooted point among naturalists. It may not be inappropriate to present a summary of the opinions which are entertained respecting a subject of so much interest, but, in doing so, we shall confine our remarks to the parasitic animals which inhabit the interior of the body, or entozoa.

It is evident that these animals must originate in one of two ways; that they must be derived directly or indirectly from without, or be created out of materials existing within, and furnished by, the body. No other supposition is possible. If an entozoön is in any manner derived from without, it must be admitted that this takes place either through the reception of the animal itself or of its ova. If either opinion be assumed, it follows that the parent animal must exist somewhere external to the body. But the parasites in question have never, in any case whatever, been detected except within the organism. If it is objected that many of these animals are so minute that they might easily elude discovery in the elements around us, the argument fails when applied to the giant strongylus, the stout lumbricoid worm, and the tænia, measuring many yards in length. Besides, even admitting for a moment the possibility of the parasites which inhabit the intestine, and other mucous cavities, having once existed externally, the insuperable difficulty still remains of explaining the entrance of entozoa into shut cavities and parenchymatous structures-into the eye, or the muscles, for example, and their presence in the unborn child, and even in the bodies of larger entozoa of a different species. On the other hand, if it is maintained that the ova are alone received, it must still be shown that the ova exist external to the body, which has never been done. Nor would the 
admission of this explanation be sufficient; for many of the entozoa are not propagated by eggs, but belong to the viviparous class, so that in regard to them the difficulty remains undiminished. But granting the existence of ova without, and their reception into the body, it is still impossible to explain the development from. them of the animals found in the parenchyma, in the embryo, etc., without, at the same time, admitting that the ova are not only carried to these localities through the blood-vessels, but actually pass through the walls of the capillaries. Such an admission would be a physiological absurdity; for the extreme vessels will allow of the passage of a single blood-globule at a time, and no more, and will not permit any denser fluid than the plasma of the blood to permeate their walls. How, then, could they afford a passage in any manner to ova, the least of which is ten times as large as a blood globule?

If the hypothesis now presented is untenable, it only remains to adopt the alternative one, to-wit: that entozoa are generated or created anew out of the materials or the products of the living organism. It may be urged affirmatively, in support of this doctrine, that each organ possesses its own entozoa-the kidney, a species different from those of the intestine, which are, again, unline the parasites of the liver. Even more: the several parts of the same organ generate dissimilar animals. The small intestine produces the round and the tape-worms; the large intestine, the two species of thread-worms. These facts seem to show that some extremely local concurrence of circumstances is essential to the production of the several entozoa. It may also be argued, and we think the argument unanswerable, that if spermatic animalcules, which exist in the testicle, are there spontaneously generated, no violence is done to probability in supposing parasitic animals to be produced in the same manner. It will hardly be denied that spermatozoa are literally evolved from the constituents of the semen ; but it is objected to the doctrine of spontaneous generation that it is against analogy, which every-where supports the famous dogma, omne vivum ex ovo. This objection is a mere begging of the question. The decision of the case in hand involves the truth of the theory just quoted, and, as we believe, must be allowed to show that this theory is not absolutely universal in its application. Other facts, also, among which are the following, tend to invalidate it. Nothing can be more certain than that all organized 
beings were, at some time or other, created. Geology proves that successive genera and species have been thus created, at long intervals apart; and the history of disease renders it probable that one affection, at least (syphilis), which is only propagated by direct descent, $e x$ ovo, as it were, is really of comparatively recent origin.

In conclusion, after the review of the preceding outline of an argument upon the generation of parasitic entozoa, we feel obliged to admit that the weight of facts and probabilities is wholly on the side of the doctrine of spontaneous generation. At the same time, we can not but look with interest to the results of future observations in this field, nor altogether suppress the hope that the simple law of Tature, omne vivum ex oro, may even yet be found to embrace the classes which now appear to form so striking an exception to its provisions."

Symptoms.-The usual symptoms indicating the presence of worms in the intestinal canal are, sometimes, a scurfy, yellow mucous accumulation around the margin of the anus; the appetite is not uniform; the bowels are irregular, sometimes loose, and sometimes constipated; and the feces are often shrouded with shreds of mucus, of a yellow color. When ascarides are present (located in the rectum), the horse is much inclined to rub his tail against any post or fence he can get at, and he employs himself at such feats against the sides of the stall, in view, probably, of allaying the intolerable itching which usually attends the presence of these parasites.

The lumbrici, or round worms, sometimes exist in the intestines in great numbers. They are then apt to be hurtful. In such cases, the coat looks unthrifty, and the hair has lost its natural glossiness, and appears rough to the sense of touch. It has been noticed, also, that when horses are afflicted with intestinal parasites, their breath gives out a fetid odor, and they have a sort of dry, hacking cough. It is well known, however, that some horses, enjoying, apparently, the very best kind of health, are often the subjects of worms.

Trectment.-Nost of the remedies recommended in the ancient works on farriery are just about as likely to kill the horse as the parasites, and consequently are, to say the least, unsafe. The true theory is this: worms are the result of indigestion; hence our 
object, by way of prevention, is to give tone to the digestive organs; and soon after the worms have congregated in the intestinal canal, bitter tonics and alteratives are obnoxious to them. They then die, are digested, and pass from the anus as fecal matter.

Suppose we give the infested horse powerful purges, tartar emetic, calomel, turpentine, etc., as the books recommend. We may succeed in their expulsion, but that does not mend the matter; for the agents just named are all more or less prostrating, and create a worse state of digestive derangement than that which previously existed, and soon, by virtue of spontaneous generation, a new crop of parasites make their appearance.

When a horse shows any of the symptoms here alluded to, indicating indigestion, or the actual presence of worms, I recommend that an occasional dose of the following be given:

No. 27. Powdered poplar bark (populus tremuloides). )

Powdered sulphur.....................

Powdered salt .....................

Mix.

Dose, one table-spoonful, to be mixed with bran or oats.

EXAMPLES OF VERMIFUGES.

No. 1.-Fluid extract of wormwood, four drachms; to be given in the morning, before feeding time, for several successive days.

No. 2.-Give one ounce of fluid extract of pink-root, every morning, before feeding, for a week.

A GOOD REMEDY FOR THE TAPE-TORM.

Give four drachms of the solid extract of male fern (aspidium felix mas) every other morning, during a period of about ten days. This is said to be a specific for tape-worm.

Finally, as regards the treatment of worms, Nature has endowed these parasites with such tenacity of life, that no matters known to us will effect their destruction, though a few may answer the purpose of their expulsion. Bots are so hardy as apparently to survive immersion in oil, in alcohol, spirits of turpentine, and even porverful solutions of mineral acids. The continued use of salt, mixed with the food, appears, however, obnoxious to them; for sometimes, under its use, their hold gives way, and they are ejected. Bitters, purgatives, and the mechanical irritation of 
pointed bodies, as pewter, tin, etc., have no effect whatever upon bots; but with regard to the other parasites, rather more success may be expected from medical aid, in the form of vermifuges. It has been attempted to effect the removal of worms mechanically, by dissolving the mucus they are supposed to be imbedded in, for which purpose lime-water, oil, solutions of aloes, etc., have been injected by clyster up the rectum, and which practice is most to be depended on for the ejection of ascarides when in the rectum. This practice of washing away the mucus of the intestine, and thus to deprive the intestine of the secretion given for its protection, is not to be recommended, though oil, for this end, would be harmless. Strong purges are given with the same intent, which may remove them also from the whole alimentary track. Remedies have likewise been exhibited to destroy them within the body, by the mechanical irritation of their spiculi, under which view tin, brass, iron, and pewter are thought remedial. The Indian caustic barley and Indian pink are reputed vermifuges against the teres and ascaris. The oil of turpentine has also been strongly recommended as an excellent general vermifuge; but, except for the destruction of the tænia, or tape-worm, it certainly does not appear to deserve that character."'*

*Baine's Outlines."

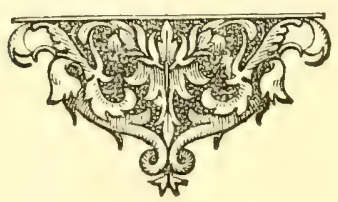




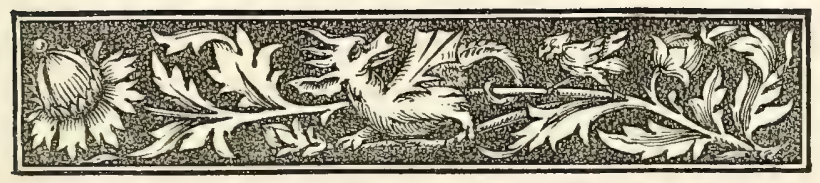

\section{SECTION VIII.}

DISEASES OF THE URINARY ORGANS.

Inflamiation of the Kidneys-Inflammation of the Bladder-Stone in the Bladder-Supprfssion of the Urine-Retention of the Urine-Diabetes, or Profuse Stalling-Rupture of the Bladder-Albuminous UrineHematuria, or Bloody URine.

\section{Inflamation of the Kidneys (Nephritis).}

A NEPHRITIC affection is often mistaken for what some A persons term "sprain across the kidneys" (lumbar sprain). It is a mistake of some importance, from the fact that when sprain or strain is suspected, people are apt to resort to the use of irritating embrocations or liniments, which may do much harm, as their action is to augment heat and pain, which, in case of nephritis, is to be avoided.

Symptoms.-Hard and accelerated pulse; quickened respiration, indicative of pain; back, arched; legs, straddling; the head is often turned toward the loins, or region of pain; the animal is unwilling to describe a circle with its body, and, while the acute stage lasts, scarcely if ever gets down on the floor; the urine is reddened and scanty; finally, the animal crouches when pressure is made over the region of the loins, and, as is the case in all acute affections, thirst and loss of appetite are observable.

Treatment.-The treatment of nephritis, in the acute or inflammatory stage, is just such as would be proper supposing the case to be one of enteritis, or peritonitis. Twenty or forty drops of fluid extract of gelseminum may be placed on the tongue two or three times, at intervals of four hours; fomentations of hops or poppy-heads (warm) should be applied to the loins, and occasional enemas of warm water may be thrown into the rectum. The drink should consist of what is known as flaxseed or slippery- 
elm tea. Soon the inflammatory symptoms will subside, the patient will manifest some relief from pain, and the color of the urine will change, become lighter and thicker in consistence. We then discontinue the above treatment, and administer one ounce of fluid extract of buchu, morning and evening. This treatment, aided by rest and good nursing, usually completes the cure.

Should it be suspected that the animal has a fit of pain, caused by the "gravel," or passage or presence of urinary calculi, then two drachms of muriatic acid should be mixed in the ordinary drink, every time the animal is watered. He should also have a small quantity of powdered slippery-elm or flaxseed mixed with the food. Horses the subjects of urinary calculi pass urine which, on being caught in an earthen vessel, deposits phosphates and other earthy matter. When this occurs, and the animal has a fit of pain or gravel, we may infer, in the absence of more positive proof, that urinary calculi are present in some portion of the urinary apparatus.

\section{Inflammation of the Bladder (Cystitis).}

The principal symptom of inflammation of the bladder is frequent urination, accoinpanied by straining and pain. Sometimes the urine dribbles away, involuntarily or not, as the case may be. It appears that the least distension of the bladder causes pain; hence the effort to keep it empty. The urine is usually highcolored, or, rather, of a dull red color. The animal stands with his hind limbs widely separated. The treatment is precisely the same as that just recommended for inflammation of the kidneys.

\section{Stone iN the Bladder.}

One very remarkable symptom attending the presence of calculus, or stone in the bladder, is, that after the urine is voided there comes a painful sensation, which causes the horse to groan. This is caused by the walls of the bladder coming forcibly in contact with the calculus, which is now a foreign body. Occasionally the horse is urinating a full stream, when, all at once, the stream is suddenly arrested, the animal still straining until urination again commences. This is a pretty sure sign of stone in the bladder. Stones that have been taken from the bladder, after death, present a highly-polished surface; hence they do not produce that amount 
of irritation which proceeds from intestinal calculi, the surfaces of which are always rough.

Mr. YouatT says that "the symptoms of stone in the bladder much resemble those of spasmodic colic, except that, on careful inquiry, it will be found that there has been much irregularity in the discharge of urine, and occasional suppression of it. When fits of apparent colic frequently return, and are accompanied by any peculiarity in the appearance or the discharge of the urine, the horse should be carefully examined. For this purpose, he must be thrown. If there is stone in the bladder, it will, while the horse lies on his back, press on the rectum. Several cases have lately occurred of successful extraction of the calculus, but to effect this it is always necessary to have recourse to the aid of a veterinary practitioner."

It is not necessary to cast the horse in view of ascertaining the presence of calculus. The hand can be introduced into the rectum while the horse is standing. The rectum should, however, first be evacuated by enemas of warm water. But we have a better plan than this. A sound or jointed catheter has been invented, which, when passed into the bladder, determines, by percussion, the presence of calculi. The operation of lithotomy can only be performed by one skilled in the science; yet the reader may desire to know something about the mode of performing it; therefore I introduce a case of the kind, which occurred in the practice of Mr. FIELD. The following is the case:

"The animal was cast, in the usual manner, and both hind legs were drawn to the shoulders, as if for castration. Read's new flexible catheter being passed into the bladder, a quantity of warm water was injected sufficient to distend that organ and the urethra moderately. The catheter being withdrawn, and holding the penis with the left hand, a slightly-curved grooved staff, two feet long, was introduced, so as for the curved part to come into the subanal portion of the urethra, above the posterior edge of the ischium, extending toward the sphincter ani. An assistant, kneeling on the left side of the horse, drew the penis forward with his left hand, and gently pushed the staff backward with the right, at the same time keeping the groove exactly beneath the raphe. This elevated the portion of the urethra to be incised. I then made an incision, a line from and on the right side of the raphe, through the skin and fascia, extending the length of from three to four 
inches; and, pushing the penis a little on one side, I gradually divided the muscular and spongy portion, and exposed the mucous membrane of the urethra, when the finger readily detected the groove of the staff, into which a small incision was made sufficient to admit the bistoire cachee, following which with the index finger of the left hand, the membrane was divided to the rectum. Very little blood flowed, and the water of the urethra gushed out. The staff being removed, I easily introduced the small forceps through the urethra into the bladder, and grasped the stone, a portion of which flaked off. The large forceps were then employed, and, my brother holding the handles, I directed the blades upon the stone, my left hand being in the rectum. Having placed the stone in a proper position, I grasped it with the forceps, and, with both hands, gave it a half-turn, so as to place its widest axis between the pubis and rectum; and thus, with a moderate force, I gradually and evenly drew it out, the neck of the bladder readily dilating. Two stitches were inserted in that part of the incision nearest the anus, the lower part being left to itself."

\section{SUPPRESSION OF URINE.}

Suppression of urine signifies that condition in which no urine is either secreted or voided. The affection is due to either functional or organic disease of the kidneys, or it is an accompaniment of various forms of disease, and, in such cases, is termed functional. For example, I lately treated a horse the subject of jaundice; he did not pass a drop of urine for two days. I inferred that the kidneys were inactive, and did not secrete urine, therefore he had none to pass; but after this period, a slight improvement in the disease (jaundice) having taken place, he urinated, yet at first only in small quantities.

When the affection comes on periodically, it indicates organic disease of the kidneys, and, finally, is apt to prove fatal. WАTsoN says "that if no urine be separated from the blood, coma soon supervenes, and death. It is believed that these consequences result from the detention of urea in the system. Urea is a mere excrement, which, in health, is removed from the blood by the kidneys as fast as it enters that fluid. When it is not so carried off, it accumulates in the blood, circulates with it to every part of the body, and acts as a poison, especially upon the brain. 
To render it thus poisonous, however, its decomposition in the blood appears to be requisite.

This is one of the numerous instances showing that the carrying fluid of the body may become the vehicle of disease and death, if it be not duly purged of deleterious matters which pertain to the unceasing processes of organic life. If carbonic acid be not extricated by the lungs, the animal functions are as certainly and almost as speedily extinguished by that gas as the flame of a taper might be, and we know that when the outlet of the liver is shut up, when the blood is not pumped from the excrementitious bile, the powers of animal life are weakened and sometimes utterly and rapidly destroyed."

Frequent complaints are made to me, by horsemen, that their horses do not urinate sufficiently, and I am requested to furnish diuretics. I usually advise the former not to feel alarmed, for Nature regulates these matters, and, in time, all will be well; provided, however, good food is furnished, and proper care is taken of the animal. The subject being one of importance, both as regards man and horse, I here introduce the following article from WATSON's "Lectures:"

"Suppression of urine, for a considerable time, is not, however, necessarily and universally fatal. Patients laboring under the epidemic cholera would secrete not a drop of water for some days, and yet recover. It was remarkable how entirely free such patients were from any approach toward coma. Was the urea here drained off from the blood in the enormous and unnatural flux from the stomach and bowels? I think it probably was; but chemical search has not detected that substance in the fluids so effused. Schmidt thinks, indeed, that it would be found but for its rapid decomposition into carbonate of ammonia. There are, however, some very singular instances on record of persons who have passed days and even weeks without secreting urine, and without showing any other indication of impaired health. What degree of credit such narratives deserve I do not know; but assuming that there was neither fraud nor mistake, it may be suspected that either the natural secretion was compensated by some vicarious or supplemental discharge, or that a small quantity of urine was actually separated by the kidneys. 'If any water, however small the quantity,' remarks Sir Henry Halford, 'had been made in these cases, I should have thought it possible that the patient 
might have recovered; for it has often surprised me to observe how small has been the measure of that excrementitious fluid which the frame has sometimes thrown off, and yet preserved itsclf harmless. But the cessation of the excretion altogether is universally a fatal symptom in my experience, being followed by oppression on the brain.' The same eminent physician states that in three of his five cases there was observed a remarkably strong urinous smell in the perspiration for twenty-four hours before death. This I believe is of common occurrence in such cases. Other patients have vomited, or passed by the bowels, watery matters possessing some of the sensible qualities of urine; and a urinous fluid is said to have been found in the ventricles of the brain in some of the fatal cases.

I have spoken of suppression of urine as a malady, though it probably is never any thing more than a symptom; yet it is one of those symptoms which, from our uncertainty respecting their origin and determining cause, we are obliged to treat and to study as if they were substantive diseases. In the only well-marked instance that I have seen of suppression of urine coming on in an apparently healthy person, some blood had appeared in the urine for a day or two before the secretion was totally suspended, and the kidneys were found gorged with blood. Extreme congestion or inflammation of the substance of the gland is probably at the bottom of many of these cases. The same train of symptoms supervenes not unfrequently upon organic renal disease. They happen, too, sometimes, when the ureters become impervious from disease, or from impacted gravel. In this condition urine continues to be secreted, for a time at least, and distends the ureter behind the seat of the obstruction. The apoplectic state which ensues may arise from a reabsorption of the secreted fluid; or, in consequence of the obstacle, the secretion itself, after going to a certain point, may stop, and then the case becomes a case of suppression."

Treatment.-Persons desirous of administering medicine for the treatment of this affection, are advised to give half an ounce of powdered chlorate of potass, dissolved in the drink, every night, and half an ounce of fluid extract of buchu every morning. 


\section{Retentron of Urine.}

Retention and suppression of urine are terms very often confounded with each other. We have just shown what is meant by suppression, and now it remains for us to understand the true nature of retention. Retention signifies an undue delay of natural discharges. The secretion of urine may be active as ever, yet the animal has not the power to void it. The bladder soon becomes distended beyond its physiological capacity. Its muscular fibers are overstretched, and thus have not the power to contract again until the fluid is evacuated by means of the catheter; hence the treatment of such a case as this pertains more to the art of surgery than that of medicine.

Treatment.-In most of these cases we find the neck of the bladder spasmodically contracted. The spasm may be either primary or it may accompany spasmodic colic, which is often the case. In fact some animals, when suffering from retention of urine, act just as if they had colic; hence it is, in such cases, highly necessary that the bladder be examined by introducing a hand into the rectum. By this means, should the bladder be distended, it can easily be discovered. The catheter must then be introduced, or the animal will die from rupture of the bladder. Provided no catheter should be at hand, I should throw into the rectum copious enemas of warm water, and administer one or two ounces of tincture of assafetida as an antispasmodic, which may possibly have the desired effect.

\section{Diabetes, or Profuse Urination.}

Many veterinary writers contend that diabetes is caused by the administration of diuretics, or else in consequence of improper food. In some instances this may be true. Errors of this kind may produce an excessive flow of urine, but this does not constitute diabetes.

Symptoms. - The characteristic symptom of diabetes is a most remarkable change in the quality of the urine. It becomes loaded with sugar. It has been found that in true diabetes the urine is never without sugar. The most ready test for sugar in urine is as follows: Invert a test-tube, filled with urine, to which a small quantity of yeast has been added, into a saucer, also containing 
urine; set the mixture in a warm place, and, if sugar be present, fermentation will soon commence, and carbonic acid rising in the tube will depress the upper surface of the urine.

Very few experiments have ever been made on the diabetic urine of horses; but large quantities of saccharine matters have been obtained from the diabetic urine of man, by a process of evaporation. WATsoN tells us, in his "Lectures," that he has seen large flat cakes of beautifully crystallized diabetic sugar. "It differs somewhat from common sugar, the produce of the sugar-cane, and approaches more nearly to the sugar of grapes. This kind of sugar, which may also be produced artificially from starch, chemists have named glucose. By rapid evaporation of the water, a thick syrup is produced, resembling treacle; but Dr. Macintyre, who has presented to our hospital museum some very fine specimens of this sugar, prepared by Dr. Blandford, informs me that to get it well crystallized, the evaporation in a steambath should be stopped while the urine is of thin consistence. It may be quickly reduced to one-half, perhaps, of its original quantity; then it should be set aside, in shallow plates, and in the course of ten days or a fortnight the sugar will be deposited."

An animal the subject of diabetes is usually very thirsty. The urine is light-colored, almost transparent. It has not the ordinary odor of common urine, but something like musty hay.

Treatment.-The indications in the treatment of this affection are, to give tone to the system and sustain the general health. A drachm or two of the sulphate of iron may be occasionally mixed with the oats (the latter must be of the best quality), and one ounce of the fluid extract of buchu may be given every night.* Good wholesome food and an occasional drink of slippery-elm tea are also indicated. Should the disease not yield to such treatment as this, the case may be considered incurable.

Cause.-In regard to the cause of diabetes, very little is known; but, to set the matter right in the minds of some who believe that nothing but diuretics and inferior provender excite it, I offer the following quotation from the pen of the author just named. I think, however, that bad food is most likely to produce diabetes.

* The buchu is not a direct diuretic, like resin or niter; therefore it may be given with safety. It acts as a tonic and sudorific, and operates physiologically on the kidneys. 
"It is probable enough that the exciting cause of diabetes may sometimes lie in the digestive organs, as Mr. McGregor supposed. The results of his experiments do not conflict with M. Bernard's. Mr. McGregor, you may remember, found sugar in the partlydigested food brought up from the stomach of a diabetic patient. He detected it also in the saliva; and in the feces, which, when allowed to dry spontaneously, became covered, after the lapse of some time, with distinct crystals of sugar. And yeast having been administered to two diabetic patients, in ounce doses, after each meal, had soon to be discontinued, because the patients, to use their own expressions, felt as if they' 'were on the eve of being blown up.' There being sugar in the blood, we need not be surprised that he met with it in the gastric and intestinal secretions from the blood. Dr. Harley has observed that by injecting irritating matters into the portal vein, (ammonia, ether, chloroform, alcohol,) a saccharine condition of the urine may be artificially produced. It is conjectured that these substances act upon fibers of the pneumogastric nerve, whence an impression is transmitted to the nervous centers, and thence is again reflected upon the liver through the splanchnic nerves. It is not difficult to imagine that irritating substances may find their way into the portal blood through a faulty digestion, or through the use of certain kinds of food or of medicine. Again : since contrived irritation of the brain at the origin of the pneumogastric nerves will make the urine saccharine, the cause of diabetes in the human subject may reasonably be placed, in some instances, within the skull; and we may understand how injuries or diseases of the brain, or even mental disquiet and dejection, operating through the brain, may produce it. Some striking cases have been published by Dr. Goolden, in which head symptoms were accompanied by saccharine urine, and in which the diabetic symptoms were checked or removed by remedies addressed to the head affection-by blisters especially, and by purgatives. Nay, we may ask whether there may not, in fact, be two varieties of diabetes mellitus, in one of which the animal and in the other the regetable form of sugar may be present in the urine, and whether the one of these varieties may not be more hopeful of cure or recovery than the other. Bearing in mind the name and the distribution of the pneumogastric nerve, may we not indulge the conjecture that disease or injury of the brain near the origin of that nerve may directly affect the functions of the stom- 
ach, and thus prevent its digestive power, or the functions of the lungs, and thus interfere with the chemical destruction of sugar in those organs? This last notion might seem to receive support from the frequent association of pulmonary disease or disorder with saccharine urine."

\section{Rupture OF THE BLADDER.}

It is well known that rupture of the bladder may arise from over-distension with urine; yet it may oceur from other causes, as the following article, contributed by Dr. C. M. Woon, will show :

"September 1.-My subject was a bay horse, seven years old, in high condition; that is, fat. On inquiry, I was informed, by the man who had the care of him, that when he entered the stable, at four o'clock, A. M., he found the horse rolling and tumbling, and in great pain. I asked, What has the horse been doing? and was answered that, having fallen into other hands, he had done little more than merely exercise for the past four months. On exanination, the following symptoms were observed: The animal was standing, with the near fore extremity raised and extended, and in the act of pawing, but he did not disturb his bedding. He would suddenly place one foot down and raise the opposite one, when he would continue to paw, in like manner, for two or three minutes. He would then cease pawing, and extend himself in his stall, with the head elevated, and a fixed stare, as if in the act of urinating. He would then perhaps lie down at full length in his stall, or throw his head back upon his side, and remain in this position a few minutes; would again rise and commence pawing, as before. When standing, his hind extremities were wide apart. His pulse was forty-two; mouth, hot, but moist; the visible mucous surfaces slightly injected; his respiration little disturbed; in fact, his symptoms were not violent. I gave an antispasmodic drench, and left him. At eight, A. M., again visited my patient. Found him standing, apparently relieved, yet he oceasionally manifested slight symptoms of a kind of dull abdominal pain. His bowels being costive, I gave him one ounce of cathartic mass, one drachm of ealomel, and half a drachm of pulverized opium, in a ball. At noon he was still the same. Ordered him a bran-mash, which he did not eat; nor did he drink. Gave an enema, stim- 
ulated the abdomen, and left him. Six, P. M.-He has laid down quietly for two hours; stands easy, but neither eats nor drinks. Nine, P. M.-Still the same; lies down occasionally. When standing, paws considerably. Has had no evacuation of the bowels or of the urinary passages since the man discovered that the animal was sick. Examined the rectum, but found no feces therein. Gave half a drachm each of Venice turpentine and copaiba, in an infusion of linseed; gave also an enema, and left him for the night, leaving a pail of water in the manger before him.

September 2, six o'clock, A. M.-On entering the stable, my patient appeared much the same as when $\mathrm{I}$ left him the previous evening. I inquired how he had been during the night. The groom informed me that 'he had remained easy until about one o'clock, when he commenced pawing, as before, but without touching his bedding; that he would lie down for a few minutes quite easy, when he would throw himself at full length in his stall, and remain in that position a short time; then rise up and extend himself, as if to relieve the urinary passages, but only a few drops dribbled away, as usual.' As usual? said I. Have you never observed any difficulty in his making water before? He answered, 'I have taken care of this horse for over two years, and have never seen him make more than a wine-glassful of water at any one time. He passed a little every ten or fifteen minutes. His stall was constantly wet, but I have never seen any trouble on account of it.' This statement was fully corroborated by several other persons. I now examined my patient per rectum, and found the bladder empty. Upon introducing the catheter not a drop of urine was obtained, nor even could the odor of urine be detected on the catheter. I now concluded that he had a ruptured bladder, and gave up all hopes of his recovery. However, from the pain at intervals, and apparent relief of the animal occasionally, I directed my treatment to the kidneys and bladder, by giving oily laxatives, emollient enemas, and counter-irritation, in the hope, not of cure, but of palliation, and each, in turn, gave relief for a short time. Nine, P. M.-Have seen my patient several times during the day. His symptoms increasing in frequency and violence. Pulse, sixty-six; respiration, hurried; body and extremities, still warm. He paws, rolls, and tumbles violently. Gave an enema, which had the effect to remove the contents of the rectum, consisting of four or five small pellets of feces, covered with 
mucus. He now, for the first time, drank six or seven swallows of water, but had no desire for food. I ordered a pail of water to be placed before him, and that a man should watch him during the night.

September 3, six, A. M.-Has drank the water. Still paws, lies down, suddenly rises, paws again, and thus continues. The body is still warm; he is not at all violent, his pain being, as it were, dull; the abdomen rapidly increasing in size. Nine, P. M.Have seen my patient several times since morning. See no possible chance for recovery. His respiration is quick and laborious; pulse, seventy-four; visible mucous surfaces highly injected; head and neck bedewed with a cold, clammy perspiration; tremor of the fore extremities; still paws, but does not lie down. He puts his nose in the bucket, plays with the water in it, but does not drink. Abdomen appears to increase in size. Notwithstanding every means was used for his relief, the symptoms continued to increase in severity, and he died at one o'clock, A. M., on the fourth.

Made an examination thirty-six hours after death, the knacker neglecting to remove the horse before. On opening the abdomen the following appearances were visible: A slight blush of inflammation pervaded the whole of the intestinal canal. Extreme distention of the cæcum and colon, but their contents were pultacious. The bladder quite empty, and contracted at its cervix into firm rugœ; in substance its walls thickened to half an inch; its apex bore marks of ulceration, with a rupture of one and a half inches through its parieties. Had it not been ruptured, I think the bladder could not have been made to contain more than a quart; and we think this condition of the organ is sufficient to account for the constant dribbling spoken of by the groom. There was an accumulation of several gallons of fluid in the abdominal carity, and the peritonem bore evident marks of inflammation, which, doubtless, had been rendered less solvent by the medicines administered. The lining membrane of the bladder was clothed with a kind of mucus, thick, muddy, and of a dark color. The kidneys were almost destitute of the investing membrane. What remained rubbed off with the slightest touch. In attempting to remove them from their connections, they were torn with very little force. They were of a brick red color, extremely soft, so that they could be squeezed between the fingers like a paste; or, 
when laid upon a board, they flattened like soft dough. The liver was softened, or, as it is generally called, rotten. It appeared pale, was of a clay color externally, and could be broken down with the slightest pressure. The stomach was healthy, and contained about four quarts of fluid. The thoracic viscera healthy; heart, large and firm ; lungs, sound, but discolored, doubtless from lying so long after death.

An idea suggests itself in regard to the case now under consideration: that the liver did not properly prepare the blood for the secretion of the urine; hence the morbid condition of the kidneys and bladder. But, although these organs are shown to have been exercising a diseased secretory action, yet doubtless the primary seat of the affection was in the digestive apparatus, the stomach, and more particularly the liver. This question is not only a very important one in a pathological point of view, but it is also one of peculiar interest to the practitioner in his treatment of disease. It directs him to the proper remedies to employ, and the action of these remedies on the organs affected. In the treatment of all diseases, it is not only necessary to understand the cause, but to know the organ or viscus affected."

\section{Albuminous Urine.}

This is rather a rare disease among horses; yet, as it does occasionally occur, it may be proper to take some notice of it. Those cases which have come under the author's notice have occurred in animals of the scrofulous diathesis, most of which animals had a scurfy skin, and were otherwise unthrifty, just the subjects for disease of the kidneys; for it is well known that when the excrementitious function of the skin is interrupted, the kidneys have to perform double labor. Their function is then overtaxed; hence, disease.

Albuminous urine is often associated with various forms of disease, or, rather, is the result of disease, or may follow the use of improper medicines or bad food. Such are said to be the cause of this malady (functional) in the human; and, reasoning from analogy, we infer that the same causes operate on the horse, for all the functions of his body are carried on after the same general plan that obtains in the body of man.

WATSON teaches us " that some articles of food, and some medi- 
cines, have the effect, in some cases, of rendering the urine for a time albuminous. Perhaps it would be more correct to say that certain forms of indigestion may cause this change. Albumen has, also, been detected after a blister upon the skin, or under that general state of irritation of the skin called eczema rubrum, which is produced by the use of mercury. In the crisis of some febrile disorders, in some cases of pregnancy, of heart disease, and in epidemic cholera, the same phenomena has been observed. Whenever blood, proceeding from the long track of mucous membrane which lines the urinary organs, mingles with the urine, that fluid, of necessity, contains albumen, and coagulates, if tested by heat or by nitric acid." There is no albumen in healthy urine; neither can we recognize its presence by mere inspection. Horses often pass urine of a thick and ropy character, but that does not prove that it is albuminous. It may be loaded with morbid or excrementitious matter, yet contain not a particle of albumen. Healthy urine, when recently discharged, possesses the ordinary temperature of the body, is transparent, of a straw color, and exhales a peculiar ammoniacal odor, which it loses in cooling. About ninety-three parts in one hundred of healthy urine is water; the remainder are made up chiefly of urea, saline and organic matters. Albumen, being similar to the white of eggs, passes from the fluid to the solid state by boiling; therefore, in order to detect albumen in urine, it is only necessary to heat the suspected urine to the boiling point, when the albuminous opacity becomes visible.

A horse the subject of albuminous urine usually has a straddling gait; will stretch backward his hind legs; is stiff, and makes short turns with difficulty. He is usually thirsty and feverish, has a quick pulse, and the feuces are hard and dark-colored. When the disease is of long standing, and the animal loses flesh, and the mucous membranes of the mouth appear pale, the probability is that the disease is organic, and the case may be considered incurable. In such cases, the morbid appearances after death denote degeneration, or structural change in the secreting surface of the kidneys, and in the glands also.

Treatment.-The best remedy for the treatment of this affection is fluid extract of buchu, two ounces per day. Green vegetables and carrots are also indicated. 


\section{Hematuria (Bloody Urine).}

Hæmaturia consists of extravasation of blood from the urinary organs, which is mixed with the urine, giving to the latter a bloodred tinge. Small quantities of blood is very often excreted from the kidneys, in consequence of congestion of the renal blood-vessels. The affection often follows injury or sprain in the lumbar region, and may occur in consequence of active disease, scattered in either the kidneys or the bladder. Strong diuretics, such as turpentine, juniper berries, etc., are operative in producing hæmaturia. But many cases are very obscure. Blood is sometimes voided with the urine without any assignable cause. I have known mares, when menstruating, to pass considerable blood with the urine, which ceased on the termination of menstruation.

Treatment.-The proper method of treating this affection is to apply warm-water dressings to the loins, and drench the patient with one ounce of fluid extract of matico, night and morning. Mucilaginous drinks are also needed, which may be composed of, slippery-elm, flaxseed, or gum arabic.

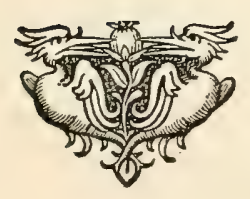




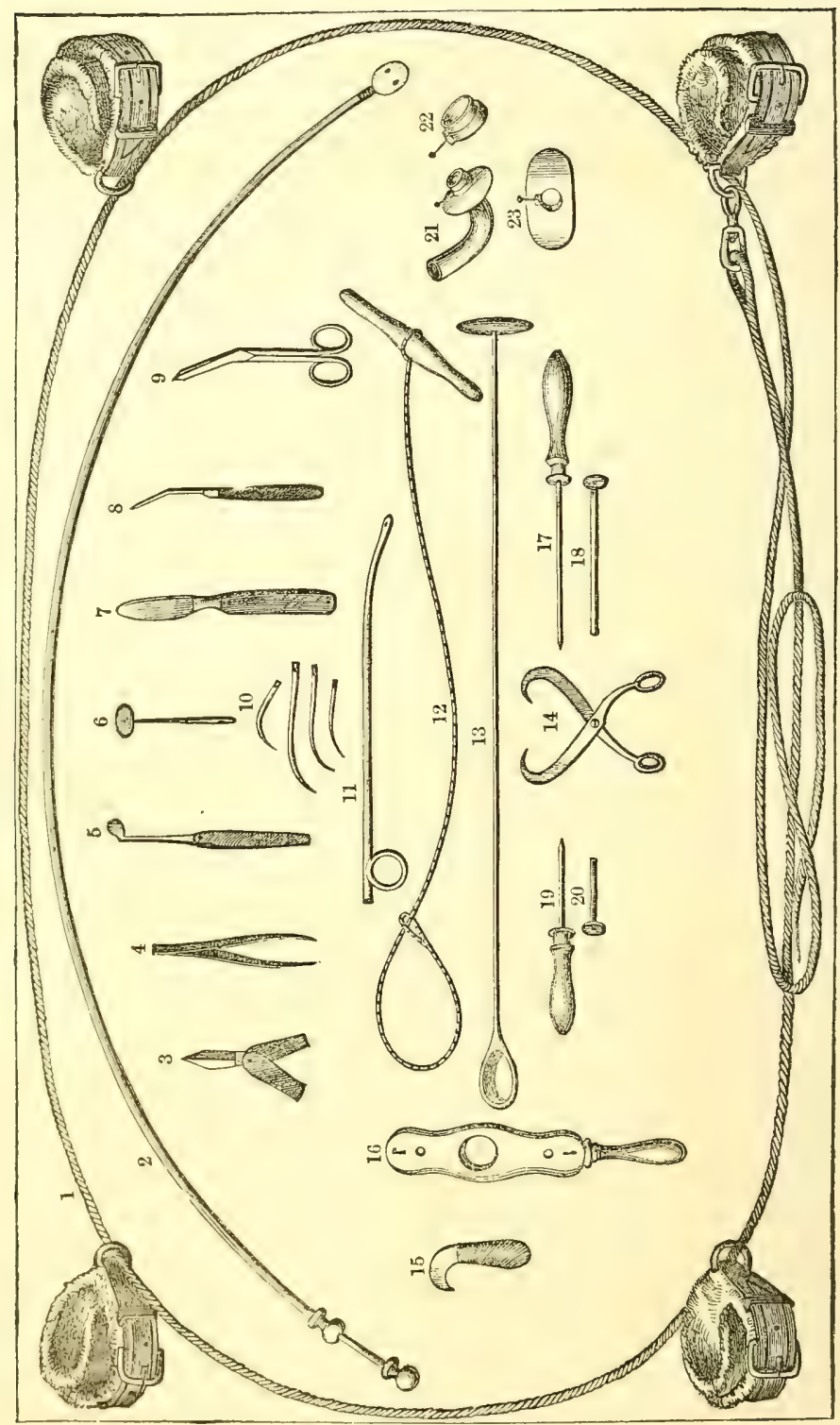

\section{SURGIOAL INSTRUMENTS USED IN VETERINARY PRACTIOE.}

Explanation.-Fig. 1, Hobbles for casting; 2, Probang, for the removal of olstruction within the osophrgus; 3, Thumb lancet; 4, Artery forceps; 5, Gum scarificator; 6, Tube used for obstruction in the teats; 7, Castrating knife; 8, Beak-pointed scalpel; 9, Curved scissors 10, Suture-needles; 11, Female catheter; 12, Parturient slip-noose; 13, Parturient laver ; J4, Embryotomy forceps, or clasp-hooks; 15, Embryotomy knife; 16, Mrouth-gag; 17, Trocar; 18, Canula or cover of the trocnr; 19, 20, Trocar and canula for puncturing the chest; 21, 22, 23, Views of the several parts of the tracheotomy tube. 


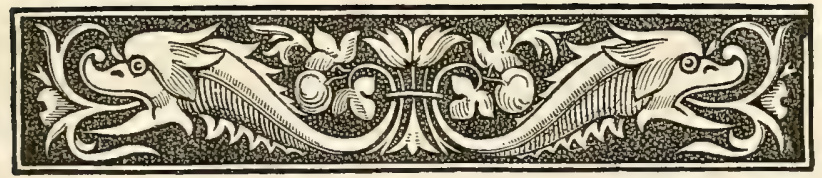

\title{
SECTION IX.
}

\author{
SURGICAL OPERATIONS.
}

Of Surgical Operations and the various Restraints it is sometimes NeCessary to Place the Horse under for their PERForMance-CastingSutnging-Castration-French Method of Castration-Castration by

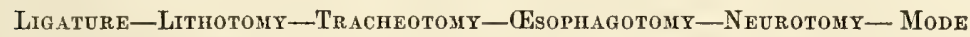
of Performing Neurotomy-Periosteotomit-Division of the Flexor Tendons-Amputations-Amputation of the Penis-Amputation of the.TailNicking-Firing-Blistering-Ammoniacal Blister-Roweling-SetonsAbstraction of Blood, or Bleeding-Division of the Temporal arteryBleeding by the Palate-Bleeding by the Toe-Phlebotomy-Bleeding by the Jugular Vein.

Of Surgical Operations and the Various Restraints it IS SOMETIMES NECESSARY TO PLACE THE HORSE UNDER FOR THEIR PERFORMANCE.

" HEN it is necessary to perform any painful operation on so powerful an animal as the horse, it is of consequence to subject him to a restraint equal to the occasion. Horses are very dissimilar in their tempers, and bear pain very differently; but it is always prudent to prepare for the worst, and few important operations should be attempted without casting. Humanity should be the fundamental principle of every proceeding, and we ought always to subject this noble animal to pain with reluctance; but when circumstances absolutely call for it, we should joyfully close our hearts to all necessary suffering. The resistance of the horse is terrible, and it is but common prudence to guard against the effects of it. The lesser restraints are various. Among them may be first noticed the тwIтсH. The twitch is a very necessary instrument in a stable, though, when frequently and officiously used, it may have the ill-effect of rendering some horses violent to resist its application. In many instances blindfolding will do 
more than the twitch; and some horses may be quieted, when the pain is not excessive, by holding the ear in one hand, and rubbing the point of the nose with the other. A soothing manner will often engage the attention and prevent violence; but it is seldom that either threats or punishment render an unruly horse more calm. Inexperienced persons guard themselves only against the hind legs; but they should be aware that some horses strike terribly with their fore-feet. It is prudent, therefore, in all operations, to blindfold the animal, as, by this, he becomes particularly intimidated, and if he strikes he can not aim. When one of the fore extremities requires a very minute examination, it is prudent to have the opposite leg held up (it may, in some cases, be tied); and when one of the hinder feet is the object of attention, the fore one of the same side should be held up, as, by this means, the animal is commonly prevented from striking. If this precaution be not taken, still observe to keep one hand on the hock, while the other is employed in what is necessary, by which means, if the foot become elevated to kick, sufficient warning is given, and the very action of the horse throws the operator away from the stroke. Without the use of these arts, the practitioner will expose himself to much risk. The TREVIS is the very utmost limit of restraint, and is seldom used save by smiths, to shoe very violent and powerful horses. Whenever recourse is had to it, the greatest caution is necessary to bed and bolster all the parts that are likely to come in contact with the body. On the Continent we have seen horses shod in this machine, and apparently put into it from no necessity greater than to prevent the clothes of the smith from being dirtied. Horses have been destroyed by the trevis, as well as by casting; or their aversion to the restraint has been such, they have died from the consequences of their own resistance. The SIDE-LINE is now very generally used, not only in minor operations, but also in those more important. Many veterinarians do not use any other restraint than this, in which they consider there is safety both to the horse and to the operator. It is applicable to such horses as are disposed to strike behind, and consists in placing a hobble-strap around the pastern of one hind leg, and then carrying from a web collar, passed over the head, the end of a rope through the D of the hobble, and back again under the webbing round the neck. A man is then set to pull at the free end of the rope, by which the hinder leg is drawn forward without elevating it from the ground. 
By this displacement of one leg the horse is effectually secured from kicking with either. Occasionally it is thus applied: hobbles are put on both hind legs, and the rope is passed through each of the rings. According to this last method, the horse is actually cast, as he must fall when the ropes are pulled. Take a long rope, and tie a loop in the middle, which is to be of such a size as it may serve for a collar; pass the loop over the head, letting the knot rest upon the withers; then take the free ends, pass them through the hobbles, and bring it under the loop. Let two men pull at the ropes, and the hind legs will be drawn forward.

\section{Casting.}

The objections to this practice arise from the dangers incurred by forcing the horse to the ground. Mr. Bracy Clark simplified casting by inventing some patent hobbles, having a running chain instead of rope, and which, by a shifting $\mathrm{D}$, made the loosening of all the hobbles, for the purpose of getting at a particular leg, unnecessary. These were still further improved by Mr. Budd, so as to render a release from all the hobbles at once practicable. Hobble leathers and ropes should be kept supple and pliant with oil, and ought to be always examined previous to using; nor should the $\mathrm{D}$. or ring of the strap be of any other metal than iron. Brass, however thick, is brittle, and not to be depended on. To the D ring, or ring of one pastern hobble, a chain of about four feet long is attached; to this a strong rope is well fastened, and, according to the way the horse is to be thrown, this hobble is to be fixed on the fore-foot of the contrary side. The rope is then passed from the hobble on the fore-foot to the $\mathrm{D}$ of the hind foot of that side, then to the other hind foot, and, lastly, through the D of the other forefoot. After this, much of the ease and safety of the throw depends on bringing the legs as near together as possible. This should be done by gradually moving them nearer to each other, without alarming the horse, which will very much facilitate the business, and is really of more moment than is generally imagined. A space sufficiently large should be chosen for the purpose of casting, as some horses struggle much, and throw themselves with great violence a considerable way to one side or the other; and they are able to do this if the feet have not been brought near together previous to attempting the cast. The place should be also very well 
littered down. The legs having been brought together, the assistants must act in concert. One, particularly, should be at the head, which must be carefully held throughout by means of a strong snafile-bridle; another should be at the hind part, to direct the fall, and to force the body of the horse to the side which is requisite. Pursuing these instructions, the animal may be at once rather let down than thrown, by a dexterous and quick drawing of the rope, the whole assistants acting in concert. The moment the horse is down, the person at the head must throw himself upon that member, and keep it secure; for all the efforts of the animal to disengage himself are begun by elevating the head and fore-parts. The rope is tightened. The chain is fixed by inserting a hook through one of the links, of sufficient size not to pass the hobbles. When the operation is over, the screw which fastens the chain to the hobble, first put upon one fore-leg, is withdrawn. The chain then flies through the D's of the other hobbles, and all the legs are free, save the fore-leg first alluded to; the strap of this has to be afterward unbuckled. There are also other apparatus used in casting, as a strong leathern case to pass over the head, serving as a blind when the animal is being thrown, and as a protection against his rubbing the skin off his eyes when down. Then a surcingle is also used. This is fastened round the horse's body, and from the back hangs a broad strap and a rope. The strap is fistened to the fore-leg of that side which it is desired should be uppermost. The line is given to a man who stands on the opposite side to the generality of the pullers. On the signal being given, the men having hold of the hobble-rope pull the legs one way, while he who has hold of the rope attached to the surcingle pulls the back in a contrary direction, and the horse is immediately cast.

\section{SLINGING.}

This is a restraint which horses submit to with great impatience, and not without much inconvenience, from the violent excoriations occasioned by the friction and pressure of the bandaging around his body. Graver evils are also brought about by the abdominal pressure. Some horses stale and dung with diffieulty when suspended, and inflammation of the bowels has not unfrequently come on during slinging. The slings are, however, forced on us in some eases, as in fractured bones, the treatment of open joints, 
and some other wounds where motion would be most unfavorable to the curative treatment. Suspension may be partial or complete. Suspension of any kind will require the application of pulleys and ropes affixed to the beams, that the whole body of the horse may be supported. A sling may be formed of a piece of strong sacking, which is to pass under the belly, the two ends being fustened firmly to pieces of wood, each of about three feet long, and which are to reach a little higher than the horse's back. To the pieces of wood cords and pulleys are to be firmly attached, by which means the sacking can be lowered or raised at pleasure. To the sacking, also, are to be sewn strong straps, both before and behind, to prevent the horse sliding in either direction, without carrying the sacking with him. Upon this so-formed cradle he is to recline. If horses when they are fresh should be placed in this machine, most of them would either injure themselves or break through all restraint. However, by tying up their heads for three or four nights their spirit is destroyed. The slings may then be applied without the fear of resistance. It is the best method not to pull the canvas firm up, but to leave about an inch between the horse's belly and the cloth, so that the animal may stand free, or throw his weight into the slings when he pleases. In this fashion a horse may remain for months in the slings, and at the end of the time display none of the wear and tear so feelingly described by old authors.

\section{Castration.}

This practice is of very ancient origin, and is as extensive as ancient. It is founded on the superior placidity of temper it gives. The castrated horse no longer evinces the superioritics of his masculine character, but approaches the softer form and nflder character of the mare. Losing his ungovernable desires, he submits to discipline and confinement without resistance; and, if he be less worthy of the painter's delineation and the poet's song, he is raluable to his possessor in a tenfold degree. In England, where length in the arms and of the wide-spread angles of the limbs is absolutely necessary in the horse to accomplish the rapid traveling so much in rogue among us, the exchange of the lofty carriage and high action of the stallion is absolutely necessary; and when we have added the lessened tendency of the gelding to some diseases, as hernia, founder, cutaneous affections, etc., we may be 
content to leave the sexual type with the racer for his breed; also with the dray-horse for his weight, and the fancy of his owner.

Supposing it, therefore, eligible to castrate our horses, what is the proper age for the operation? What are the relative advantages and disadvantages of the different methods of performing it? The proper age to castrate the young horse must depend on circumstances, as on his present appearance, his growth, and the future purposes we intend him for, observing, generally, that the more early it is done, the safer is the operation; for, until these organs begin to secrete, they are purely structural parts, and, as such, are not so intimately connected with the sympathies of the constitution. Some breeders of horses castrate at twelve months; others object to this period, because they think the animal has not sufficiently recovered the check experienced from weaning before this new shock to the system occurs. In the more common sort of horses, used for agricultural purposes, it is probably indifferent at what time the operation is performed, this consideration being kept in view : that the earlier it is done, the lighter will the horse be in his forehand; and the longer it is protracted, the heavier will be his crest, and the greater his weight before, which, in heavy draught-work, is desirable. For carriage-horses it would be less so, and the period of two years is not a bad one for their castration. The better sort of saddle-horses should be well examined every three or four months, particularly at the ages of twelve, eighteen, and twenty-four months, at either of which times, according to circumstances or to fancy, provided the forehand be sufficiently developed, it may be proceeded with. Waiting longer may make the horse heavy; but, if his neck appear too long and thin, and his shoulders spare, he will assuredly be improved by being allowed to remain entire for six or eight months later. Many of the Yorkshire breeders never cut till two years, and think their horses stronger and handsomer for it. Some wait even longer; but the fear in this ease is, that the stallion form will be too predominant, and a heavy erest and weighty forehand be the consequence. Perhaps, also, the temper may suffer. Young colts require little preparation, provided they are healthy and not too full from high living. If so, they must be kept somewhat short for a few days; and, in all, the choice of a mild season and moderate temperature is proper. 
When a full-grown horse is operated on, some further preparation is necessary. He should not be in a state of debility, and, certainly, not in one of plethora. In the latter case, lower his diet; and it would be prudent to give him a purgative. It is also advisable that it be done when no influenza or strangles rage, as we have found the effects of castration render a horse very obnoxious to any prevalent disease. The advanced spring season-previous, however, to the flies becoming troublesome-is the proper time for the performance of the operation upon all valuable horses; and be careful that it be not done until after the winter coat has been shed, which will have a favorable effect on the future coating of the horse, independent of the circumstance that at a period of change the constitution is not favorable to unusual excitement.

Castration is performed in various ways, but in all it expresses the removal of the testicles. There are methods of rendering the animal impotent without the actual destruction of these organs; for if, by any other method, the secretion of the spermatic glands is prevented, our end is answered.

Castration by cauterization is the method which has been principally practiced among us; but this by no means proves it the best. On the contrary, many of our most expert veterinarians do not castrate by this method. Mr. Goodwin, and many other practitioners of eminence, never castrate by cautery.

A preliminary observation should be made previously to casting, to see that the horse is not suffering from a rupture. Such cases have happened; and as in our method we open a direct communication with the abdomen, when the horse rises it is not improbable that his bowels protrude until they trail on the ground. Hernia, as a consequence of castration, may easily occur by the uncovered operation; for, as already observed, it makes the scrotal sac and abdominal cavity one continuous opening. It is not to be wondered at, therefore, if the violent struggles of the animal should force a quantity of intestine through the rings into the scrotal bag. Should we be called on to operate on a horse which already had hernia, it is evident we ought not to proceed with it unless the owner be apprised of the risk, and willing to abide by it. In such cases, we would recommend that the method of Girurd be practiced; that is, to inclose the tunica vaginalis within the clams, (sufficiently tight to retain them, but not to produce death in the part,) pushed high up against the abdominal ring, and then 
to remove the testicle, being very careful to avoid injuring any portion of intestine in the operation. When a discovery is made of the existence of hernia after an opening has been already made for the common purpose of castration, should the operator continue his process, and castrate? We should say, By no means; but, on the contrary, we would greatly prefer the method recommended by Mr. Percivall: firmly to unite the lips of the external wound by suture, allowing the testicle itself to assist in blocking up the passage, with a hope, also, that the inflammation caused by the incision might altogether stop up the scrotal communication with the abdomen. But, in the appalling ease of immense protrusion of intestine, what is to be done? Mr. Coleman, in such a case, proposes to make an opening near the umbilicus large enough to introduce the hand, and thus draw in the bowels. Mr. Percivall would prefer dilating the cxternal ring. But the testicle must be very firmly retained, and even permanently fixed against the dilated ring, or the bowels would again descend. The intestines probably would become inflated in any such erse.

As unbroken young horses are the most usual subjects of this operition, and as such often have not yet been bridled, if a colt can not be enticed with oats, etc., he must be driven into a corner, between two steady horses, where, if a halter can not be put on, at least a running hempen noose can be got round his neck; but whichever is used, it should be flat, or the struggles, which are often long and violent, may bruise the neck, and produce abscess or injury. When his exertions have tired him, he may then be led to the operating spot. Here his attention should be engaged while the hobbles are put on, if possible; if not, a long and strong cart-rope, liaving its middle portion formed into a noose sufficiently large to take in the head and neck, is to be slipped on, with the knotted part applied to the counter or breast. The long pendent ends are passed backward between the fore-legs; then carried round the hind fetlocks, brought forward again on the outside, run under the collar-rope; a second time carried backward on the outer side of all, and extended to the full length in a direct line bohind the animal. Thus fettered, Mr. Pereivall says his hind feet may be drawn under him toward the elbows. It has been, however, often found that, at the moment the rope touches the legs, the colt either kicks and displaces the rope, or altogether displaces himself; but his attention can generally be engaged by one fore-leg being held 
up, or by having his ear or muzzle rubbed, or even by the twitch; if not, the rope may be carried actually round each fetlock, which then acts like a hobble, and this rope may be gradually tightened. This last, however, is a very questionable method, and the others, therefore, ought to be long tried before it is resorted to. In this way people have succeeded with very refiactory colts; but it requires very able assistants, and, if possible, the man who has been used to the individual colt should be present. In either way, as soon as the rope is fixed, with a man at each end of it, behind the colt, let themy by a sudden and forcible effort in concert, approximate his hind legs to his fore, and thus throw him. Before the colt is cast, however, it should be endeavored to ascertain that he is free from strangles and hernia.

Being satisfied that no hernia exists on either side, proceed to cast the colt, turning him, not directly on the left side, but principally inclining that way; and, if possible, let the croup be very slightly elevated. It is usual to place him directly flat on the left side, but the above is more convenient. Next, secure the near hind leg with a piece of hempen tackle, having a running noose; or, in default of this not being at hand, make use of the flat part of a hempen halter, which should, for safety, be put on before the hobble of that leg is removed-as may be readily done, if the hobbles having shifting or screw D's (as described in casting) are made use of. Every requisite being at hand, the operator, having his scalpel ready, should place himself behind the horse, as the most convenient way to perform his manipulations; and, firmly grasping the left testicle with his left hand, and drawing it out so as to render the scrotum tense, he should make an incision lengthways, from the anterior to the posterior part of the bag. The resistance of the cremaster muscle has to be overcome before the testicle can be forced to the bottom of the scrotum, and this is the more readily accomplished if the animal's attention be engaged. The incision may be carried at once through the integuments, the thin dartos expansion, and the vaginal coat of the testicles with a sweep of the scalpel; but with one less dextrous at the operation, it will be more prudent to make the first incision through the scrotum and dartos only, to the required extent, and then to do the same by the vaginal coat, thus avoiding to wound the testicle, which would produce violent resistance, and give unnecessary pain. 
We, however, take this opportunity of noting that cases have occurred when the tunica vaginalis was divided no testicle followed, firm adhesions between this tunic and the tunica albuginea having retained it fast. In such cases the scalpel must be employed to free the testicle, by dissecting it away from the vaginal sac. When no such obstruction occurs, the testicle, if the opening be sufficiently large, will slip out; but the operator must be prepared, at the moment of so doing, to expect some violent struggles, more particularly if he attempt to restrain the contractions of the cremaster, and, by main force, to draw out the testicle. Preparatory to this, therefore, the twitch should be tightened; the attendants, especially the man at the head, must be on the alert; and the testicle itself, at the time of this violent retraction of the cremaster, should be merely held, but not dragged in opposition to the contraction. If the clams have been put on over the whole, according to Mr. Percivall's method, they will assist in retaining the retracting parts; but they must not be used with too much pressure. The resistance having subsided, the clams must now be removed; or, if they have not been previously in use, they must now be taken in hand, and, having been prepared by some tow being wound around them, should be placed easily on the cord, while time is found to free from the grip of the pincers the vas deferens, or spermatic tube, which is seen continued from the epididymis. The Russians, Mr. Goodwin informs us, cut it through when they operate. Humanity is much concerned in its removal from pressure, because of the excess of pain felt when it is included.

It is necessary, before the final fixing of the clams, to determine on the part where the division of the cord is to take place. To use Mr. Percivall's words, 'if it be left too long, it is apt to hang out of the wound afterward, and retard the process of union.' On the other hand, if it be cut very short, and the arteries happen to bleed afresh after it has been released from the clams, the operator will find it no easy task to recover it. The natural length of the cord, which will mainly depend on the degree of the descent of the gland, will be our best guide in this particular. The place of section determined on and marked, close the clams sufficiently tight to retain firm hold of the cord, and to effectually stop the circulation within it. There are now two modes of making the division : the one is to sever it with a sealpel, and then to sufficiently sear the end of it as to prevent a flow of blood; the 
other, and in some respects the preferable method, is to employ a blunt-edged iron, which is to divide by little crucial sawings, so that, when the cord is separated, it shall not present a uniform surface, but ragged edges, which will perfectly close the mouths of the vessels. This done, loosen the clams sufficiently to observe whether there be any flow of blood. Gently wipe the end of the cord, also, with the finger, as sometimes an accidental small plug gets within the vessel; this had better be removed at the time. Retain a hold on the clams a few minutes longer; and, while loosening them gradually, observe to have an iron in readiness again to touch the end of the cord, if any blood makes its appearance. Satisfied on this point, sponge the parts with cold water. No sort of external application is necessary, still less any resin seared on the end of the cord, which can only irritate, and will never adhere.

On the after-treatment much difference of opinion has existed, and even yet exists. The powerful evidence of accumulated facts has now convinced us of the necessity and propriety of some motion for the newly castrated horse, as a preventive of local congestion. Such practice is common in most countries, and seems salutary in all. Hurtrel d'Arboval, thus impressed, recommends the horse, immediately after the operation, to be led out to walk for an hour; and it is a general plan in France to walk such horses in hand an hour night and morning. Mr. Goodwin, in proof of its not being hurtful, informs us that whole studs of horses, brought to St. Petersburg to be operated on, are immediately traveled back a certain portion of the distance, night and morning, until they arrive at home. We have, therefore, no hesitation in recommending a moderate degree of motion in preference to absolute rest.

The French method of castration is advocated by Mr. Goodwin, and it is sufficient that it receives his recommendation to entitle it to attention. It is rendered the more so, as he observes, on the method in general use among us, "that the operation performed by the actual cautery always induces, more or less, symptoms that often become alarming, and that it can not be performed on the adult without incurring more swelling and severer consequences than attend other methods of operation. If I ever use the actual cautery, it is for the sake of expedition, and then only on a yearling or a two-year old; but I am resolved never to em- 
ploy it again on an adult.' These observations, as emanating from such a source, must be deemed important.

Mr. Goodwin then offers the description of the French method of operating, from Hurtrel d'Arboval: 'Castration by means of the clams is the method in general use, if not the only one now employed. It is the most ancient, since it was recommended by Hieroclius among the Greeks. It is performed in two ways, the testicle being covered or uncovered. In the former, the exterior of the scrotum, formed by the skin and dartos muscle, is eut through, and the testicle is brought out by dissecting away the laminated tissue, the gland being covered by the tunica vaginalis. The clam is then placed above the epididymis, outside the external peritoneal covering of the cord. In the uncovered operatirn, the incision is made through the servus capsule of the testicle. The tunica vaginalis being divicicd, the testicle presents itself, and the clam is placed well above the epididymis, on the cord. The operation, performed in either way, requires us to provide ourselves with a scalpel, a pair of clams, a pair of long pincers, made purposely to bring the ends of the clams together, and some waxed string. The clams may be formed of different kinds of wood, but the alder is considered the best, and generally made use of. To make a clam, we procure a branch of old and dry alder, whose diameter should be about an inch, and whose length should be from five to six inches; of course, the dimensions must, at all times, be proportioned to the size of the cord we have to operate on. At the distance of half an inch from each end, a small nick, sufficiently deep to hold the string, must be made, and then the wood should be sawed through the middle lengthways. Each divided surface should be planed, so as to facilitate the opening of the clams, either when about to place them on or take them oft. The pith of the wood is then to be taken out, and the hollow slionld be filled with corrosive sublimate and flour, mixed with sufficient water to form it into a paste. Some persons are not in the habit of using any caustic whatever; then, of course, scooping out of the inside of the clam is not necessary. Notwithstanding, the caustic, inasmuch as it produces a speedier dissolution of the parts, must be useful, and onght not to be neglected.' The addition of the caustic, however, Mr. Goodwin oljects to, with great reason, remarking that, unless it be a very strong one, and therefore dangerous to employ, it can not be of any use to parts com- 
pressed and deprived of circulation and life. He further informs us that he has operated in six cases in succession with the same effect, without any escharotic matter whatever. An experimental case of Mr. Percivall's terminated fatally. By the use of caustic the cord was greatly inflamed, as high as the ring, and which, unquestionably, produced the unfortunate result.

'The covered operation,' continues Mr. Goodwin, 'is the one that $I$ am about to advocate, and which differs only insomuch that the scrotum and dartos muscle must be cantiously cut through, without dividing the tunica vaginalis. It was Monsieur Berger who was accidentally at my house when I was about to eastrate a horse, and who, on my saying that I should probably do it with the cautery, expressed his surprise that I should perform the operation in any other way than on the plan generally approved of in France. Being a stranger to it, he kindly consented to preside at the operation, and, after seeing him perform on the near testicle, I did the same on the right, but, of course, not with the same facility. After opening the scrotum, and dissecting through the dartos, which is very readily done by passing the knife lightly over its fibers, the testicle and its covering, the tunica vaginalis, must be taken in the right hand, while the left should be employed in pushing back the scrotum from its attachments; and, having your assistant ready, as before, with the clam, it must be placed well above the epididymis, and greater pressure is, of course, necessary, as the vaginal covering is included in the clam.'

Mr. Goodwin further observes that in Russia he has seen hundreds of horses operated on, even after the human fashion, with safety; and, he remarks, it certainly produces less pain, the animal loses less flesh and condition, and is sooner recovered than when operated on by the actual cautery.

Castration by ligature is a painful, barbarous, and very dangerous practice, and consists in inclosing the testicles and scrotum within ligatures, until mortification occurs, and they drop off. It is practiced by some breeders on their young colts, but it is always hazardous and disgracefully cruel. The substance of the testicle, in some countries, is also broken down, either by rubbing or otherwise by pressure between two hard bodies. This is practiced in Algiers, instead of excision, and tetanus is a frequent consequence of it. In Portugal they twist round the testicle, and thus stop the circulation of the gland. Division of the vas deferens has been 
performed, it is said, with success, on many animals, and is proposed as a safe and less painful process than the emasculation of the horse. It consists in a longitudinal section through the scrotum, dartos, and vaginal sheath, so as to expose the cord, from which the vas deferens is to be separated and severed from the artery and vein. There is a certain consent of parts, by which the sympathy of an organ remains after its functional offices are apparently destroyed. There can be little doubt but the nervous excitcment would continue, the vein and artery remaining entire. There are certain nice conditions of the organ necessary for propagation. Thus, the horse who retains his testicles within his abdomen, possesses all the roguish qualities of him with one perfectly evolved; he is lustful, and can cover, but is seldom fruitful.

Of the morbid consequences of castration we have little to say. By early evacuations, green food, a loose box, a cool air, moderate clothing, but, particularly, by walking exercise, swellings of the parts may be prevented; if not, bleed and foment. Should suppuration follow, and sinuses form, treat as directed under those heads; and if tetrnic symptoms start up, refer to that article. There has been lately practiced in India a novel mode of castration, which is said to be the invention of a Boer, settled at the Cape of Good Hope. The cord is exposed in the usual manner. From the cord the artery is singled out. This vessel is scraped through with a coarse-edged, blunt knife, when the other constitutents of the cord are cut away, and the operation is finished. This method is much praised by those who have adopted it, and is said to be always attended with success.

\section{Liтнотому.}

Hurtrel d'Arboval's account of the progress of lithotomy in veterinary practice commenees in 1774 ; the second case was successfully operated on in 1794; and at later periods other veterinary surgcons have also performed it. In monodactyles there are two methods of operating for the stone-one through the rectum, the other through the bladder. The first, which consists in laying open the bladder hy a longitudinal incision made through the parietes of the part of the rectum adherent to it, by means of a straight bistoury, is easily practiced, but in its consequences is dangerous in the extreme; in fact, it is an operation never to be 
adopted but in a case where the magnitude of the stone precludes its extraction through the neck of the bladder. In all other cases, lithotomy by the urethra is to be pursued. For its performance are required a straight probe-pointed bistoury, a whalebone fluted staff, and a pair of forceps, eurved at the extremities. The animal should, if practicable, be maintained in the erect posture. The tail plaited and carried round on the right quarter, the operator feels for the end of the staff introduced up the urethra, and makes an incision directly upon it, from above downward, an inch and a half or two inches in length. Next, he introduces the sound, and passes it on ward into the bladder. Now, placing the back of the bistoury within the groove of the sound, by gliding the knife forward, the pelvic portion of the urethra, and also the neck of the bladder, becomes slit open-the latter in two places, in consequence of a second cut being made in withdrawing the bistoury. The opening made being considered of sufficient dimensions, the operator introduces the forceps into the bladder, and seizes the calculus, one hand being up the rectum, to aid him in so doing. The forceps, clasping the stone, are now to be withdrawn, but with gentleness, and with a vacillating sort of movement of the hand from side to side, in order more easily to surmount any difficulties in the passage, and the more effectually to avoid confusion or laceration. M. Girard tells us 'that the cut through the pelvic portion of the urethra ought always to be made obliquely to one side. The operator should hold his bistoury in such a direction that its cutting edge be turned toward the angle of the thigh. By this procedure we shall gain easier access to the bladder, and not only avoid wounding the rectum, but also the artery of the bulb, as well as the bulb itself, and suspensory ligaments of the penis.

The parts eut through in the operation are, 1st, the fine thin skin of the perineum, smooth externally, and marked with a raphe; densely cellular internally; $2 d$, adhering to the tissue, the fascial covering, derived from the fascia superficialis abdominis, which has here become fibrous, it forms the common envelope to the parts underneath, and is closely connected with the corpus musculosum urethræ; $3 \mathrm{~d}$, the corpus musculosum urethræ, that penniform band of fleshy fibers which springs by two branches from the ischiatic tuberosities embracing the sphincter ani, and concealing the arteries of the bulb, whence they unite, and proceed to envelop the urethra; 4 th, the corpus spongiosum urethræ, 
the part immediately covered by the muscular envelope, and which here is bulbous (it is more particularly worthy our remark, from two arteries penetrating the bulb, which come from without the pelvis, ascending obliquely outward to reach the part); 5th, the suspensory ligaments of the penis, pursuing the course of, and adhering to, the tendinous union of the erectores. An attention to the relative position of these parts will demonstrate the advantages of the lateral oblique incision over one made directly along the raphe. By pursuing the latter, we necessarily cut through the suspensory ligaments and into the bulb, wounding thereby the arteries; whereas, by the former, all this danger is avoided, besides that it renders the operation more simple and facile.

\section{TracheOTOMY.}

Cases occur when this operation is required, as in strangles, when the tumors threaten sufiocation, or when any substance has remained unswallowed in the osophagus, the pressure of which obstructs respiration. In a distressing case of gunpowder bursting immediately under a horse's nose, the effects of which tumefied his mouth and nostrils, so as to prevent free inspiration, the animal owed his life entirely to our exeising a portion from the tracheal rings, about ten inches below the angle of the throat. The operation is a very simple one, and may consist either in a longitudinal section made through two or three of the rings, or a portion, occupying about an inch round, may be excised from the anterior cartilaginous substance. The proper mode, when it can be done, however, is to make a circular opening with a very narrow knife, removing a portion of two cartilages, or taking a semicircular piece from each; and this last, although it is seldom performed, is by fur the best method. The integuments should be first divided in the exact center of the neck, three or four inches below the obstruction; then the skin and tissues should be sufficiently separated to allow a tube adapted to the size of the trachea to be introduced, the tube having an acute turn and a rim, which must be furnished with holes for the adaptation of tapes, to secure it around the neck. There are several instruments of this sort in use, of which that adopted by the French, or the one invented by Mr. Gowing, of Camden Town, is to be preferred. The operation has been also performed in cases of roaring, under an idea 
of dividing the stricture which impeded respiration; but, unless the exact situation of this were discovered, it would be but an experimental attempt.

\section{CEsophagotomy.}

It was long thought that a wound in the oesophagus must be necessarily fatal, but we have now sufficient proofs to the contrary on record, so that we are not deterred from cutting into the œsophageal tube when it is necessary; but it is an operation requiring skill and anatomical knowledge, and its future results are sometimes very serious. The cases that call for cesophagotomy are the lodgment of accidental substances within the tube. An apple once so lodged was removed by incision by a veterinary surgeon at Windsor. Carrots, parsnips, beets, ete., are liable to produce such obstruction when not sliced. Too large a medicinal mass, also, has lodged there; and a voracious eater has, by attempting to sivallow too large a quantity of not salivated bran or chaff, produced an obstruction, which pressed on the trachea and threatened suffocation. In all cases of obstruction of this kind, we will suppose that a probang, well oiled, has been previously attempted to be passed, and has completely failed. The probang for the horse, however, differs materially from that used for the cow. It is formed after the fashion of the one adopted by the human practitioner, consisting of a pliable piece of whalebone, having a sponge tied to one end. The operation being determined on, may be practiced standing. If the swelling be large, no fear need be entertained about cutting important organs, as the enlargement will push them on one side. Cut down, therefore, directly upon the center of the impacted substance. If the horse be cast, which is quite unnecessary, have him, of course, thrown with his left side uppermost. It will also be necessary to command a good light. The part of the neck chosen for the opening must, of course, be governed by the obstructing mass. A section should be made through the integuments and cellular tissue beneath them, right into the œsophagus, if possible, with one cut, and into the center of the pipe. If this be not done at once, and it requires some dexterity so as to effect it, mind to make all future incisions in a line. with the first opening, as it is important that the cellular tissue should be little interfered with. The œsophagus, fairly cut into the impactment, should jump forth. Should it not do so, do 
not manipulate, or attempt to force it out, but enlarge the opening, and the substance will come through when that is long enongh; but no fingering could compel its exit while the opening is too small. The end gained for which the incision was made in the csophagus, the wound may be then closed by the interrupted sutures, each holding a small piece of tow above the orifice, and having their ends hanging out of the external opening, which should also be brought together by sutures. The after-treatment should be to interdict all dry food; the animal ought to subsist on very thick gruel for three, four, or five days. If the condition appears to suffer much, allow malt mashes, and when so doing watch the wound; and if the matters taken in are seen to ooze out, wash them away frequently with warm water, to prevent lodgment, which might encourage sinuses to form; and after each washing, syringe with some very mild stimulant, as a very weak solution of sulphate of zinc (white vitriol), etc.

\section{Neurotomy (Division of the Sentient Nerves of THE FОот).}

Neurotomy has now stood the test of very extensive application. Our writers offer innumerable proofs of its restoring almost useless animals to a state of much utility; and if there are chances that it may oceasion such injury as to hasten the end of some horses, it is usually in such as the disease would have done the same for at no distant period. Having stated thus much in its favor, it must not be supposed that we recommend it as an unqualified benefit, even where it succeeds best. No neurotomized horse ever after goes with the same freedom, nor with equal safety, as he did before the operation was performed. Indifference to the nature of the ground gone over is said to have fractured legs; it is quite common to batter the feet to pieces; and, although horses have hunted afterward, and hackneys have carried their riders long distances, yet it is more calculated to prove beneficial to carriage than to saddle-horses. This we believe to be a just statement of its merits; but there are benefits which it offers to the animal of a more extensive and constitutional kind. Those gained by the bodily system generally have been, in some cases, very marked. Thus, an aged and crippled stallion, from the irritation constantly kept up, became so emaciated as to be unable to fecundate; but, 
being relieved from a constant state of suffering by neurotomy, improved in health and condition, and was again used to cover. It happened, also, that a mare, similarly circumstanced, ceased to feel œstrum; but after neurotomy it again returned, and she resumed her character of a brood-mare. It appears to act with most certainty when a portion of the irritated nerve is excised. One case has actually occurred where the tetanus, occasioned by a wound in the foot, was arrested and removed by neurotomy. It also promises much in the painful state of some cankers, where the irritation has rendered the application of dressings almost impossible. Here, by depriving the foot of sensibility, we deprive the horse of that which is injurious to him. The sore itself is often amended by it; but in every instance the dressings can be effectively applied, and the healthy processes can not be at all suspended.

With respect to whether the lower or upper incision ought to have the preference, the decision should be guided by the circumstances, as regards the intensity and the seat of the disease. The operation commonly leaves, for a considerable time, some enlargement around the spot, the effects of the adhesive matter interposed between the severed portions of the nerve, and which can be remedied by no application of bandages. This bulging remains so long as life continues; and, however cunningly the incision be concealed, this can be felt with ease, and tells the truth ever after the operation has been performed. Such a circumstance has, however, led some practitioners, when it has been wished to make the upper section, and yet to avoid the chance of detection, to operate on the metacarpal nerve on the outside, and on the pastern or plantar nerve on the inside.

Mode of performing the operation.-The situation of the section through the skin being determined on, a guide to which may be gained from the perforatus tendon, and having firmly secured the leg to be first operated on, cut the hair from the part. This being done, and the exact course of the artery being ascertained by its pulsation, make a section close to the edge of the flexor tendon. Let the cut be near, but rather behind, the artery, if below the fetlock joint. The cellular substance being cleared away will bring the vessels into view, and the nerve will be readily distinguished from them by its whiteness. Elevating it from the ressels and its membranous attachments, by means of a crooked needle, armed 
with thread, pass a bistoury under it, as near to the upper angle of the section as possible. The violent spasm the division of the nerve produces may be somewhat lessened by pressing the nerve between the finger and the thumb, when an opportunity may be taken, either with the scalpel or scissors, of dividing it; then, taking hold of the lowermost portion between a pair of forceps, excise about three-fourths of an inch of its trunk. Having finished, if both feet are affected, proceed to operate on the contrary side of the other leg; after which turn the horse, and repeat the operations on the like parts of each leg as they come in succession. The integuments may be now drawn neatly together, and secured by a twisted suture, the whole being properly covered by a light compress. Tie up the head for a day or two, after which put on a cradle. Keep the horse very quiet and low; give mashes, to. open the bowels; but we should avoid physicking, from the fear that griping might occur, which would make him restless, or probably require exercise.

\section{Perrosteotomy.}

This operation consists in having the horse thrown upon his side, and the leg to be operated upon released from the hobble, and extended upon a sack, filled with refuse hay or straw. This is done by means of a piece of webbing passed round the hoof, and the end given to a man to hold, who pulls rather violently at the member. The operator then kneels down and feels for the exostosis he intends to perform periosteotomy upon. This may be a splint or a node, and commonly exists upon the metacarpal portion of the fore-limb. The operator having found the excrescence, snips just below it with a pair of rowelling scissors. He then takes a blunt seton-needle and drives it through the cellular tissue, and immediately over the enlargement. Next, another slit in the skin, above the exostosis, is made with the rowelling scissors, and through this last opening the point of the seton-needle is forced and then withdrawn. Into the free space thus made a curved knife is introduced. The point of this knife is blunt, and the blade curves upward, the cutting part being below. Some persons use a very diminutive blade, but the editor prefers a rather large instrument, as being more under the command of the hand. Having introduced this knife, he turus the cutting edge downward, 
and with it incises the enlargement, sending the blade right through the periosteum, and also through the substance of the exostosis, if it be not too solid for the knife to penetrate. This latter fact is only to be ascertained by actual experience, and no opinion formed after an external examination can be of any value, such being much more the guess of a pretender than the judgment of a surgeon. The age of the animal may be some guide, but even this it is better not to depend upon too entirely. It is true that young horses freely east forth exostoses, which aged animals mostly absorb; but this rule, though very general, hos exceptions, and by no means is to be absolutely depended upon.

The enlargement being cut through, next take a seton-needle, armed with a tape, and draw it through the channel already made. Tie a knot at either end of the tape, large enough to prevent its being pulled through the opening at either end, and the business is over. The affair is very simple, and the horse may be at once let up. It is, however, in some cases, and only in some, of so much benefit that the horse, being thrown 'dead lame,' gets up and trots off quite sound. However, ere you adopt the operation, apprise the owner of the risk incurred, and that it is by no means a certain cure. Leave the choice with him, but be sure and tell him the opening made for the entrance and exit of the seton commonly leave a blemish behind them; and where the seton traveled, often there remains a thickening, which it may require months to obliterate.

The after part of the treatment consists in merely having the seton daily moved to and fro, though some persons apply an active blister all over the parts immediately in the neighborhood of the seton, under the idea that the vesicatory renders the operation of greater effieacy, which, however, is very questionable. When periosteotomy acts at all, it mostly does so at once; and when its benefits are not immediate, it is better to withdraw the seton, to prevent after-blemish, rather than hazard further and useless treatment by the application of a stimulant to skin already deprived of any connection with the deeper-seated structures.

\section{Division of the Flexor Tendon.}

There are so many accidents and diseases that may produce contraction of the flexor tendons, that we only wonder we do not 
more often meet with them, which we should certainly do, but that the attendants, despairing of being able to afford relief, advise their destruction. The operation consists in making a longitudinal incision, of about three inches in length, along the inner lateral edge of the tendon, dissecting each portion from its cellular attachments, so as to expose the nerve, artery, and tendons. This opening will allow the perforans to be freed from the perforatus, when a division should be made by a scalpel applied to its surface. It is evident that this should take place below any thickening, or adhesions, which may have permanently connected the tendon with the neighboring parts. Any lesser attachments will be broken through, by forcing back the foot to the just position. By Mr. Dick this was done 'by placing his knee against the front or projecting part of the pastern, at the same time laying hold of the foot with one hand and the upper part of the leg with the other, and using considerable force; and this appears to be necessary, in order to break any adhesions that may have formed.' The limb should now be placed in a poultice; and if any fear of future contraction should arise during the cure, lengthen the toe of the shoe proper to the foot operated on. Some slow exercise, after the first week, may be allowed, but previously to that the horse should be confined to a stall, during which the bowels must be kept open with mashes.

\section{Amputations.}

These have been hitherto confined principally to the tail, the ears, and other parts of minor importance in the animal frame; but veterinary surgery now takes a wider field, and the extremities are amputated with a certainty of making horned cattle still serviceable for the purpose of yielding milk; and, without doubt, the same might be done with the brood-mare or stallion, particularly in fractures of the fore extremities.

Professor Dick, of the Edinburgh Veterinary College, furnishes a case, sent to him by one of his pupils, to the following effect: 'I performed amputation upon a cow on the 7 th of July. $\Lambda \mathrm{fter}$ having properly secured the animal, and applied a torniquet above the carpus, I made a circular incision through the integuments round the leg, a little below the carpus; and, having separated the skin so as to allow of its being pushed up a little, I cut through the sinews, and lastly sawed off the stump. The parts are now 
completely whole, although she has been going at grass all the time, and, now that she has got the advantage of a cork stump, makes a wonderful shift for herself, and yields a good supply of milk to her owner.'

Mr. Dick also notices another case of amputation of the fore-leg of a two-year old heifer; and of a third, where the hind leg was removed above the tarsus. Such operations have occasionally occurred from time immemorial, with a few enterprising charactcrs. We have heard of them, but they were mostly regarded as mere matters of curiosity or wonder, and, therefore, were not imitated. We shall, however, probably, erelong have them more common, in cows, at least; for, occurring below the carpus and tarsus, they are as easily performed as nicking or docking; and there is no doubt but, were a hollow padded stump applied, such low operations might be prudent in many cases. Fractures, with great comminution of bone, considerable ravages of disease within the foot, or extensive gangrene, are the cases which might call for amputation.

Of the method of amputation little need be added to the above. The principal practical points are, the fixing of a torniquet of sufficient force, which should be padded to make its principal pressure on the leading arterial trunks, while its general circumference will act on the smaller vessels. A ring should be cut lightly below the intended place of operation, only through the integuments, which, when separated from their cellular adhesions for about six inches, should be turned back; and a circular incision may then be made through the muscles, etc., taking up by ligature such vessels, both venous and arterial, as display a disposition for much hemorrhage. The section thus made, free the bone from the soft parts by the scalpel, where the adherences are very intimate, and, having, by means of a crucial bandage, retracted the soft parts altogether above the bone, saw it through. Finally, effectually secure the principal vessels, when, bringing the soft parts and skin over the bone, retain them there by proper bandaging, which suffer to remain without disturbance until the third or fourth day.

Amputation of the Penis.-Amputation of the penis is not unknown among us. It has been performed several times, and it is found that no canula is necessary to keep open the urethra, the force of the urinary flow breaking down any incipient cicatrization of its 
orifice. The sheath is first forced back, and the penis brought forward to its greatest possible extent. Whatever portion it is intended to remove is now cut through, by means of an amputating knife, when the remainder is retracted within the sheath, and little hemorrhage has afterward occurred, except at the time of passing the urine; but there appears to have been no alarming quantity of blood lost.

Amputation of the Tail, or Docking.-We are most happy to state this filthy and unnecessary operation is now discarded. It never consisted of more than the cutting off a portion of the stump with brute force, and the cruel application afterward of a hot iron to the small artery of the tail.

\section{NICKING.}

We should be grateful that this barbarous and dangerous process is no longer numbered among the necessary operations. It is so beset with accidents, which no skill or prudence can prevent, that no one who has a free will ought to mutilate a horse by nicking.

\section{Firing.}

The practice of firing was not always confined to quadrupeds. On the contrary, it probably was first used on man; and to this day, in many countries, it is a very popular remedy among human surgeons. In India it is applied over the abdomen for the cure of scirrhosity of the liver.

Firing, in veterinary practice, has, by Mr. Coleman's pupils, been justified as only men will justify a favorite operation, the virtues of which have been impressed upon their minds by an eloquent teacher. When Coleman was the chief of the veterinary profession, firing, under his rule, was used for any and every occasion. It was ridiculously supposed to act as a permanent bandage, as if a few strokes with a heated iron could destroy the elastic property inherent in the skin. It was the favorite styptic of these practitioners, and was applied to arteries (as of the tail) as though it possessed within itself some medicinal virtue. It was used to promote absorption, as in callus; and was likewise resorted to for checking absorption, as in ulceration. It was called into action to promote granulation, in broken knees; and was also a 
favorite agent to check granulations, when they were too luxuriant. In short, there was no folly which a hot iron did not cover. It has now, happily, fallen into disuse. Most modern practitioners will now confess that their chief reason for exercising the iron is to satisfy the proprietor, not to benefit the animal. After such an acknowledgment, who would submit to have his patient servant's skin scored and burnt with red-hot metal?

The mode of cauterization differs according to circumstances. As a general rule, it ought, of course, to be applied in the direction of the hair, by which the blemish is lessened; but this rule can not be arbitrarily followed, although it ought to do away with all the false pride of displaying the taste in the figures scored upon a prostrate beast. The Veterinary College recommends that the limbs be always fired in perpendicular lines; others advocate all manner of fanciful marks. Some cast the horse; many surgeons perform standing. The irons used are of various shapes and dimensions. Some recommend the firing, of all things, to be very light; others persist there is no virtue in hot iron unless it burns very deep. The operation consists in having irons of some substance made red-hot, and then drawing them mechanically along, or twisting them about upon the skin. The figures are various; so is the depth of the incision. Both must be decided by the taste, judgment, or heartlessness of the operator.

\section{Blistering.}

This is an operation of very great utility, and is, perhaps, compared with its benefits and importance, the safest that is performed. When a vesicatory becomes absorbed through the pores of the skin, it inflames the sensible cutis underneath, the consequence of which is, an infusion of serum through the part, which, in the human subject, elevates the cuticle into a bladder equal to the surface inflamed, but in the horse, from the greater tenacity of the cuticular connections, it becomes separated in the form of small distinct vesicles only. "If the irritating cause be quickly removed, the serum may be reabsorbed, and the surface restored by a slight effort of adhesive inflammation. If the irritant act in a still minor degree, it simply irritates the vessels of the cutis to an infiltration of fluid through the sensible pores, but produces no desquamation of cuticle. Such has been called a sweating blister. 
But when, by continued irritation, the cutis is exposed, suppuration succeeds, and the part is fully blistered.

The salutary action of blisters is exerted in several ways-in promoting absorption, in combating deep-seated inflammations, and in aiding others. As a stimulus to the absorbents, they act beneficially in the removal of injurious deposits, as the coagula remaining after inflammatory lesions; but it is to be remarked that when any existing deposit is of long continuance, or is osseous, it requires that the action of the vesicatory be kept up. Mercurial blisters have been thought to have a superior infuence in accelerating absorption. Mercurials, rubbed in some weeks or days previously to blistering, are certainly great assistants, and should always be employed in the treatment of obstinate osseous or ligamentary enlargements. Blisters are very important aids in inflammatory affections, as counter-irritants, derived from a law in the animal economy, that two inflammations seldom exist in the vicinity of each other; therefore, when such an affection has taken place in any part, and we wish to remove it, we attempt to raise an artificial inflammation in the neighborhood by means of blisters, which, if persevered in, destroy, or at least lessen, the original one. Occasionally, also, we blister the immediate inflamed part, with an intention to hasten the suppurative process by increasing the activity of the vessels, as in deep-seated abscesses, and also in those which attack glandular parts. We therefore employ blisters to hasten the maturation of the tumors in strangles. When the flagging powers vascillate between resolution and suppuration, as they often do in the phlegmonous inflammations of glandular or of deep-seated parts, blisters may either hasten the resolution, or they may add their influence to the attempted suppuration, and thus bring it to maturity. But we carefully avoid, in other cases, applying a vesicant to a part immediately in a state of active inflammation. Particularly we should avoid what is too often done, that of blistering over the tendons, ligaments, and articulatory surfaces of a tumid limb, laboring under a congested state of the parts from excess of vascular action. Here we should do great injury were we to blister, by causing a greater deposit of lymph, and by hastening its organization into an injurious bond of union between the inflamed parts.

The vesicatory, or blister, for general use in veterinary medicine, as a simple stimulant, should be principally composed of 
Spanish flies. Cheaper substitutes are used, but they irritate violently. In extensive inflammatory affections, they are, on this account, perfectly inadmissible; and wherever a case requires any thing more, it will be noted. The mode of blistering with the Spanish fly is sufficiently known. The hair should be cut or shorn as close as possible from around the part; the blistering matter should then be well rubbed in for ten or fifteen minutes. If the pasterns and fetlocks are the parts to be blistered, previous to rubbing in the ointment, smear some lard, tallow, or melted suet over the heels, and within the hollow at the back of the small pastern. This will often prevent some troublesome sores forming, from the blistering ointment falling on these sensitive parts. While a blister is acting, the litter should be removed from under the feet, or it will tickle the legs and irritate; but, above all, the head ought to be most carefully secured, for two days and nights, to oppose lying down, more especially to prevent the horse biting the blistered part. On the third evening he may be permitted to repose; but a prevention should even then be continued, by means of what is called a cradle. This apparatus may be bought at every turning shop, or may be made of eight or ten pieces of round wood, an inch and a half in diameter, and two feet long. These are strung at each end on a rope, and fastened around the neck. When it is intended to blister repeatedly, the effects of the first should have subsided before the second is applied, the seurf and scabs also be cleared away, and the part well washed with soap and water. In all cases, the third or fourth day after the application the part should be thoroughly painted over, by means of a long-haired brush (such as are in use with pastry-cooks to glaze their crusts), with lead liniment, which should be repeated every day. And when it is proposed to turn a horse out, it should never be done until the whole blistered surface be quite healed; otherwise dirt, flies, etc., may prove hurtful.

It remains to observe that, instead of repeated active blistering, it is, in some cases, preferable to keep up a continual slight irritation on the original blister by means of stimulants, as iodine ointment, mild blistering applications, etc. Caution is, however, necessary, to avoid forming an eschar, and thereby a permanent blemish; but when a blemish is not of consequence, this plan will be found often more efficacious than firing, as in splints, spavins, etc. Some practitioners blister mildly one day, and on the next 
wash off the blistering matter, thereby saving loss of hair; but there is more of apparent than real good in this plan. If a blister be necessary, it requires all its activity.

Ammoniacal blister.-Spanish flies are only efficacious when the animal can afford to wait their action, which is rather slow. In most of the acute diseases, the horse would perish before the blister began to rise, wherefore resort has been had to boiling water and red-hot iron. The action of these last coarse and brutal measures was alone controlled by the violence of the internal inflammation, and, if the practitioner was mistaken in his estimate of the immediate danger, extensive and lasting blemish was the consequence. We have in the liquor ammonia an agent quite as formidable as boiling water or heated iron, but it is rather longer in displaying its force; wherefore, it allows time for watching its action, and of ehecking it the instant it has sufficiently blistered the skin. It is true the liquor ammonia upon the skin can not be removed, neither need it be counteracted. Ammonia is like steam, only powerful when confined. The ordinary soap liniment, if covered over, would, because of the ammonia it contains, produce a lasting blemish; but every veterinary surgeon knows how very harmless a preparation that is when simply rubbed upon the surface. So, when we desire the active effects of liquor ammonia, we double a blanket or rug four or five times and hold it over the liquid. It takes from ten to twenty minutes to raise a blister, and it consequently can, from time to time, be observed; and when its action has reached the wished-for point, all we have to do, effectually to stop it, is to take away the rug or blanket. That removed, the free surface and the heat of the body occasions the ammoniacal vapor to be dispersed, and the animal is safe.

\section{Rowelting.}

Rowels acts as foreign substances within the body. They cause irritation and suppuration, whereby more deep-seated inflammations are supposed to be removed. They are, however, often very convenient, because they stand as sign-boards to show the proprietor that something has been done. The common mode of making a rowel is after the following manner: A slit is first made by means of the rowel scissors, on any part of the integuments, held between the finger and thumb. With the handle of the scissors 
separate from its cellular connections a circle of two or three inches in diameter, into which introduce something to prevent the reunion of the skin. A piece of circular leather, tolerably stiff, with a central hole, is a very common substance used, but is objected to by some on account of the difficulty of changing it without injuring the skin. Tow, as more pliant, is frequently introduced into this cavity. If the rowel runs freely, it should be dressed every day, by changing the plug, if of tow, and by cleansing it, if of leather. No rowel should go undressed beyond the second day, for the comfort of the horse. They are very favorite applications with farriers, and frequently abused by being employed indiscriminately, but are falling into disuse, setons having almost superseded them.

Setons, in their action, resemble a very mild form of rowel, but are more convenient in their application. There is hardly a part of the body where a seton may not be conveniently placed. They have been put around the eye; they have also been entered at the withers, and brought out between the humerus and the sternum, so extensive or so diminutive can they be made. In sinuous ulcers of the withers and of the neck they may be placed; through the heels, in foot diseases, they have been inserted. In cavernous sores, they are entered at the sutperior part, and are brought out at an inferior, so as to form a depending orifice. The formation of a seton is very simple. A skein of thread, or a piece of tape, of a convenient size, may be used. At the one end place a large knot; arm the eye of a corresponding-sized seton-needle with the free end of the tape; introduce this into any proposed part, and, bringing it out at some other, either make a second knot, or tie the two ends of the tape together; which last method of fastening is, however, often objected to, from the danger of its catching in something and being torn out, to the disfigurement of the horse. When a seton is placed in a sinuous track, for the purpose of inflaming, it is moved twice a day, frequently, and moistened each time with some stimulant, as oil of turpentine, tincture of aloes and of benjamin. All setons require daily cleaning and moving. When they are required to act more quickly, the tape is infused in terebinthinate of cantharides, or small pieces of black hellebore are servn within it. An old material, composed of woollen, flax, or cotton and hair, is also used instead of tape. Setons, however, are of small service in acute cases. They are chiefly in use for disturbances of a chronic description. 


\section{Abstraction of Blood, or Bleeding (Arteriotomy.)}

Blood is abstracted by opening the conducting vessels, arterial and venous. When taken from arteries, the process is called arteriotomy; when by the latter, phlebotomy. Some bleedings include both these operations, as general searifications of the soft parts, bleeding at the toe point, divisions of the vessels of the cornea, etc. Blood-letting is called local when it is practiced on or very near the affected part; and it is supposed to act more immediately than general bleeding because it produces more effect with the loss of less blood. Local bleeding is, therefore, usually practiced on the minor branches of the arteries and veins, as on the temporal artery, the plate vein, the vena saphena, etc. Leeches are a means of local bleeding not often used by us in veterinary practice; but there is no reason whatever why they should not be enployed. When applied to the eye, and occasionally to other parts, also, they adhere readily, abstracting blood rapidly, and, therefore, might be valuable aids in violent local inflammation. Cupping is also practiced, in France and other parts of the Continent, with very large glasses, and it is there supposed to act remedially in many local inflammations. By general bleeding we understand the depletion of the system at large, and this we practice in extensive inflammations.

Division of the temporal artery.-The proper spot for either its puncture or division is directly where the vessel leaves the parotid gland, to curve upward and forward around the jaw, a little below its condyle. When it is punctured, it usually affords much blood; and in such case, enough having been obtained, divide the trunk, when, the receding portions becoming pressed by the integuments, and lessening by their own eontractility, the hemorrhage is stopped. It should be punctured by a lancet; a fleam may fix itself in the bone. Its division can be readily made, also, either by a lancet or scalpel.

Blecting by the pulate is also a species of arterio-phlebotomy, and is a very favorite spot for abstracting blood with most ignorant persons, who vehemently recommend it in spasmodic colic or gripes, and in megrims. In such cases, however, a want of knowledge of the anatomy of the parts has occasioned a serious hemorrhage to oceur; it may prove a fatal one if the artery proper to the part be divided incompletely. The palatine artery and nerve run 
near each other, on each side of the roof of the mouth, so as to divide the inner surface of the hard palate into three nearly equal portions. No other than a direct division of the vein should ever be made; therefore, when bleeding is determined on at this place, do it by plunging a lancet or even a penknife in a direct line across the rugæ, one inch within the mouth, exactly between the middle and second nippers. There these vessels form a curve, which curve will be divided, and will then yield three or four pints of blood. If the instrument enter too much on one side, as about the middle of the second nipper, then a partial and longitudinal division of the artery may be made, and an alarming hemorrhage may follow. In this case, the section must be enlarged and deepened inwardly-that is, away from the teeth-which completely severs the vessel, and its retraction will stop the hemorrhage. A moderate or slight flow of blood from the palate may be obtained by light scarifications of the rugæ. But all bleedings here, except under circumstances of the most urgent necessity, had better be avoided.

Bleeding by the toe is also arterio-phlebotomy. By no means cut out a portion of the sole at the point of the frog, which frequently occasions abscess; but, with a very fine drawing-knife, cut down exactly in the line of union between the crust and the sole; then, by puncturing the part with a lancet, a vast flow of blood may be obtained, the benefits of which, in some cases, are very marked, particularly in acute founder. If the blood should not flow with sufficient freedom, place the foot in warm water. The bleeding finished, cover the puncture with some tow and a little tar, and lightly tack on the shoe. There are, however, other methods of bleeding from the toe. Mr. Maver uses a drawingknife with a long curve, so that one sweep of the blade may cut a piece out of the foot. This appears to us bad practice, as it leaves Nature a space to fill up, instead of a simple incised wound to heal. Others take away none of the horn, but merely make a slit through the outer covering on to the vascular portion of the foot. The flap of horn they hold up, so long as they desire blood, by the insertion of a piece of wood; and when they have abtained blood enough, they take out the wood, so as to let the horny flap down. This last method, of all others, appears to us the easiest and the best.

Sometimes the plantar vein is opened as a substitute. Scarifi- 
cations are, also, occasionally practiced, which, of course, divide both venous and arterial branches. In France extensive scarifications used to be made into indurations before the suppurative process had commenced, which, in some cases, prevented that from going on, and the remedial wounds made were healed by adhesive inflammation, or by healthy granulation. The same method has also been occasionally practiced here, but it is not now often attempted.

Phlebotomy, or the puncture of a venous branch, is the most usual mode of drawing blood in veterinary practice, and may be employed on any point of the body; but some vessels are much more frequently opened than others, and most of all the jugular vein.

Bleeding by the thigh vein.-The saphena is a prominent vein, continued from the inner part of the hock, and may be opened by the fleam, but with much greater safety and propriety by a lancet. The opposite leg being held up, the operator, placing himself in front of the thigh, and steadying himself and the horse by placing one hand on the hock, may fix the vein with the little finger of the other, while the lancet, held between the thumb and fore-finger, punctures it. This vein should never be opened save upon absolute necessity, as it is often troublesome to pin up. A horse has been cast for the trivial matter of stopping the hemorrhage.

Bleeding from the plate vein.-This vein is frequently opened to abstract blood after injuries of the fore extremities.

The superficial brachial vein is a continuation of the superficial division of the metacarpal veins, and in the passage upward receives more than one branch. Its principal trunk ascends along the inner side of the radius. It may also be well to remark that, when taking blood from the superficial veins of the arm or fore-arm, if any difficulty is experienced in obtaining a sufficient flow, the lifting up of the other leg, by throwing the muscles of the punctured one into action, will force the blood from the inner to the outer set, and an increased quantity may be obtained. The plate vein, or external thoracic, is often opened, as it emerges from behind the arm, and is pinned up without any difficulty.

Blceding by the jugular vein.-The situation of this important vessel is well known, but its internal connections are not so familiar, though such knowledge is essential to the uniform safety of the operation. The horse has only external jugular veins, a right 
and a left one. As each emerges from the chest, it is found deepseated, and approaching the trachea. It then passes forward, in company with the external carotid artery. Toward the middle of the neck it becomes more superficial, and is now distinctly seen progressing rather above and without the carotid artery and trachea, or windpipe. The carotid, therefore, in the future course of the jugular, is situated a little below and more deep-seated than the vein. The jugular is also separated from the carotid by a slight muscular band, derived from the levator humeri. Its further track is marked in the hollow formed by the inferior edge of the levator humeri, where it is covered by the panniculus carnosus and integuments only, when, having nearly reached the jaw, it makes its well-known division into two portions. Bleeding by the jugular is usually practiced with a lancet or with a fleam. The proper spot for the puncture may be found anywhere between two inches and six from the division of the vein. This latitude is here mentioned, because it is prudent to avoid puncturing directly over a former bleeding-place, known by the scar and enlargement. It should also be avoided where a little knot in the course of the vein will sometimes denote the existence of one of the venous valves. But in all ordinary cases, where these hindrances do not appear, operate at two or three inches from the division of the vein, which will be sufficiently evident when it is pressed on below the place punctured. Avoid operating low down in the neck, as there the vessel is deeper-seated, and near to important parts.

First moisten the hair and smooth it down; then, steadying and enlarging the vessel with one hand, with the other plunge the point of the lancet into the integuments, so as just to puncture them and the vein; then, by a slight turn of the wrist, carry the instrument obliquely forward to finish the cut. For opening the smaller veins, the lancet should always be used. In all but the practiced hand the fleam is the safest for bleeding from the jugular. It is always prudent to have the eye of the horse covered. Unless the eye be covered, the horse will be likely to flinch at the moment of the stroke, and the puncture may be made in any place but where we wish. The hair being first wetted and smoothed, and the fleam being retained in the left hand, the unemployed fingers pressing on the vein so as to fix and swell the ressel, let the point rest exactly in the middle of the swelling. Strike the fleam sufficiently hard to penetrate the skin and vein. A blood- 
stick is preferable for the purpose of striking the fleam. There is a vibration between two hard bodies when they meet, which, in this instance, is favorable to a quick and moderate puncture of the vein. After the vein has been opened, moderate pressure with the edge of the can which catches the blood is sufficient to keep up the flow. It may also be encouraged by putting a finger within the horse's mouth. The requisite quantity of blood being drawn, remove the can. The remaining process of securing the vessel is of equal importance. The sides of the orifice are first to be brought in opposition, without pinching them, and without drawing them from the vein. The same cautions should also be observed when the pin is introduced. Let it be small, with an irregular point, and, when inserted, wrap around it a few hairs or a little tow.

Common, however, as this operation is, and qualified as every one thinks himself to perform it, yet there are very serious accidents which do arise occasionally: It has occurred that the carotid artery has become penetrated. When the puncture has been made through the vein, the accident is known immediately by the forcible and pulsatory gush of florid arterial and dark venous blood together. In one instance of this kind, which occurred to a French practitioner, he immediately thrust his finger into the opening through the vein, and thus plugged up the artery, intending to wait for assistance. In this state he remained, we believe, an hour or more, when, removing his finger, to his surprise, he found the hemorrhage had ceased, and did not again return. In another case, where an English practitioner accidentally opened the carotid, he placed a compress on the orifice, and had relays of men to hold it there for forty-eight hours, when it was found the bleeding had stopped.

The admission of air is also another serious accident that now and then attends bleeding. It sometimes happens from the sudden removal of the fingers or blood-can, or whatever was used to distend the vessel by obstructing the return of the blood. This, being suddenly taken away, allows the escape of the blood toward the heart, and occasions a momentary vacuum, the air being heard to rush with a gurgling noise into the vein through the orifice; it then mixes with the blood, and oceasions, in some instances, almost immediate death. The animal begins to tremble; he next staggers, and finally falls in a state of convulsion. If the quantity of air 
taken in has been considerable, death ensues. The remedy must, therefore, be instantaneous, and consists in again opening the orifice, or making a new one, to gain an immediate renewed flow of blood, which will, in most cases, renovate the horse, who has been found afterward to be tormented with an intolerable itching." *

* Blaine's "Outlines."

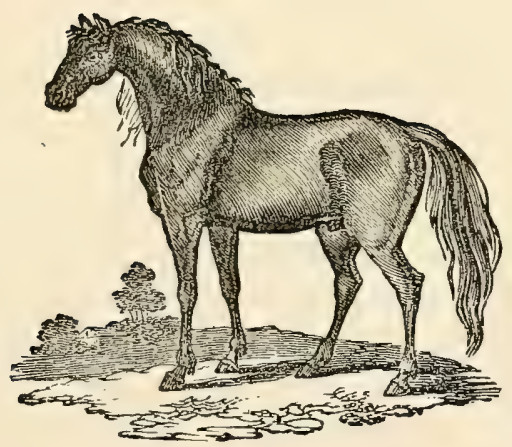




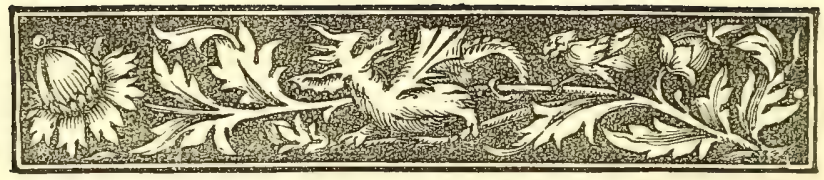

\section{SECTION $\mathbf{X}$.}

DISEASES OF THE SKIN AND SUB-TISSUES.

Itch and Mange-Lice on Animals-Grease and Scratches-Surfeit-Dropsical Limis - Scarletina - Ringworm - Prurigo - Pole-evil - Fistulous Withers-Warts on the Skin-PuRpura Hemorrhagica-OUt of CondTION-HIDE-BOUND-HERPES.

\section{ITCH AND MANGE.}

TTCH, mange, and scabies are essentially local affections of the 1 skin, and are occasioned by the presence of parasites known as "sarcoptes-equi." The eruption ensuing on the skin of a horse, when subject to this affection, is very

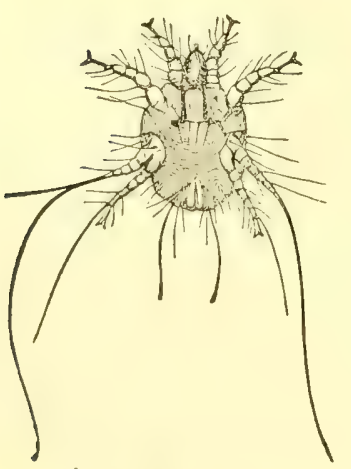

ACARES, OR RARCOPTRS-RQTI, TIIE ITCH PAKASITE (MALAIFIED). similar to the appearance of itch in man, and probably is just as tormenting to the animal as in the case of his master. Numerous cases are recorded of transmission of itch from horse to man, and, when so acquired, it is impossible to distinguish it from the human itch. Some persons suffer severely when attending mangy horses, but a clean person or clean horse are not apt to take the disease. It is well known that a healthy and clean horse may stand for wceks near a mangy one without taking the disease, showing, very conclusively, that the best preventive measures are those which promote health and cleanliness. As the English groom s.rys, "plenty of elbow-grease, by means of brush and curry-comb, on the skin of the horse; regular daily exereise, pure air in the stable, and a sufficiency of good digestible food are sovereign remedies against itch and mange." This, we presume, is all the 
reader wants to know with reference to the cause of itch and mange.

Symptoms. - When a horse is affected with this malady, in either of the forms here named, the owner's attention will be aroused by the animal rubbing himself, whenever he can get a fair ehance to do so; and, should the limbs or heels be affected, he will rub the-fore-legs one against the other, and, at night, will constantly strike the floor with his hind extremities. The hair looks unthrifty, has lost its gloss, and does not lay smooth on the body. It falls off in patches, and an eruption is observed similar to the appearance of itch on man, yet not so distinctly recognized. A few pimples, scabs, and a slight reddening of the parts is all that may, perhaps, be seen. The skin of some horses, however, is more vascular and more delicately organized than others. In the latter case the disease is more marked, creates greater irritation, and the nervous system suffers more from sympathetic irritability than in the case of an animal having a coarse and comparatively insensible "hide." In addition to these symptoms, the affected animal will be observed to be more or less fractious and unmanageable.

Treatment.-The disease being of a local character, requires topical (local) remedies, and the best medicine is that which most quickly destroys the life of the itch parasite. It has been found that preparations of creosote kill the parasite in a few seconds, and GERLACH recommends the following preparation:

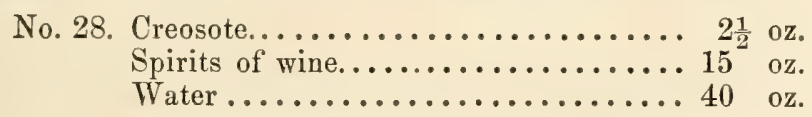

Previous to the application of this, the same authority advises that the horse be smeared all over the body with soft soap, washing it off some time afterward with warm water, and having the animal well brushed; or a wash may be afterward used, consisting of one part of caustic potass to fifty parts of water; or, lastly, greasing the animal with linseed oil, train oil, ete., and, one or two days afterward, washing with soap and water or potash ley; then apply the preparation of creosote.

The cheapest, and probably the safest, plan of treatment, and that which has proved most successful in the practice of the author, is as follows: 
No. 29. Unslaked lime................ $1 \mathrm{lb}$. Flour of sulphur............... 2 lbs. Water....................... 12 pts.

Put these ingredients in a stone jar, set it on the stove, or in a regular water-bath, until it boils. During the interval, the mixture must be constantly stirred, both in view of insuring a complete mixture, and of keeping the lime and sulphur from-depositing in the bottom of the jar; for, should this occur, the jar will crack. The mixture must be stirred with a wooden spatula or glass rod. Keep the mixture boiling for about ten or fifteen minutes; then set it aside for twelve hours, at the end of which time pour off the clear liquor, bottle tightly, and cork the bottles. These should be put away in a dark closet, as the mixture soon decomposes when exposed to the sun's rays. This remedy will cure the itch on horses and cattle. It is, also, a sure cure when used on man. It is not adapted to the cure of the malady in sheep, as the sulphur is supposed to be injurious to wool. The only objection to its use is, that it has a very unpleasant odorsmells like sulphureted hydrogen. Before applying this mixture, the animal should be thoroughly washed with warm water and common brown soap; then wipe the animal dry, and apply the sulphur mixture, by means of a sponge. One application, if properly applied, will usually suffice to kill the parasites. Should it fail in the first instance, a reapplication will do no harm, as the preparation is perfectly inocuous.

Scabies is to be treated in the same manner; in fact, all diseases of the skin, supposed to be dependent on the presence of parasites, are to be treated after this fashion. GERLaCH, of the Royal Veterinary School of Berlin, has made a series of experiments with various remedies for the destruction of the itch parasite, and the following table shows the result of his labors: 


\begin{tabular}{|c|c|c|c|}
\hline AGENT. & $\begin{array}{r}\text { Dea } \\
\text { Hours. }\end{array}$ & Minutes. & OBservations. \\
\hline 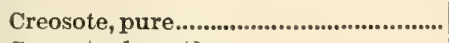 & ............... & $1 / 4-1 / 2$ & \multirow{38}{*}{$\begin{array}{l}\left\{\begin{array}{l}\text { Mathieu. } \\
\text { White arsenic, } 1 \text { part. } \\
\text { Sulphate of iron, } 10 \text { parts. } \\
\text { Water, } 100 \text { parts. }\end{array}\right. \\
\left\{\begin{array}{l}\text { Mathieu. } \\
\text { White arsenic, } 1 \text { part. } \\
\text { Alum, 10 parts. } \\
\text { Water, 100 parts. }\end{array}\right.\end{array}$} \\
\hline $\left.\begin{array}{l}\text { Creosote, } 1 \text { part } \\
\text { Spirit, } 10 \text { parts }\end{array}\right\}$..................................... & & & \\
\hline Water, 30 parts $\}$ & & $1 / 2-11 / 2$ & \\
\hline 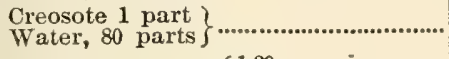 & & $2-21 / 2$ & \\
\hline 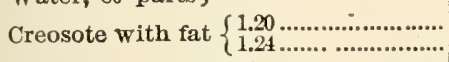 & ........... & $\frac{1 / r^{-1} 1 / 2}{31 / 2-5}$ & \\
\hline 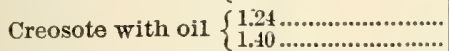 & ............... & $31 / 2-7$ & \\
\hline 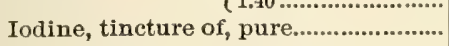 & (n............ & $\begin{array}{l}51 / 2-9 \\
1-2\end{array}$ & \\
\hline Iodine, tincture of, with water, $1.4 \ldots$ & ................. & $4-6$ & \\
\hline Iodide of potassium with water, $1.2 \ldots$ & ................. & 9 & \\
\hline Iodide of potassium with water, $1.4 \ldots$. & .............. & $20-26$ & \\
\hline Caustic potash, 1 part; 24 do. & ............... & $2-2 \frac{1}{4}$ & \\
\hline $\left.\begin{array}{l}\text { Caustic potash, } 10 \text { part } \\
\text { Water, } \\
\text { Tar, } \\
2\end{array}\right\}$ & & $11 / 4$ & \\
\hline Tar, & & & \\
\hline $\begin{array}{l}\text { Caustic potash, } 1 \text { part } \\
\text { Water, } \\
\text { Tar, }\end{array}$ & & $7-12$ & \\
\hline $\begin{array}{l}\text { Hartshorn oil, } 2 \text { " } \\
\text { Sulphuret of } 5 \text { with water, } 1.10 . . . . . . . . . . .\end{array}$ & & $15-3$ & \\
\hline 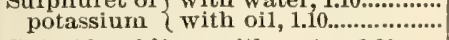 & $10-20$ & & \\
\hline Chloride of lime with water, $1.30 \ldots . . .$. & .............. & $15-30$ & \\
\hline Hartshorn oil, pure................................... & ............. & $3-4$ & \\
\hline Hartshorn oil, with water, $1.10 . . . . . . . . . . .$. & .............. & 30 & \\
\hline 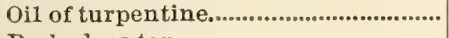 & (............... & $5-9$ & \\
\hline Barbadoes tar................................ & (................ & $\begin{array}{c}5-9 \\
8-13\end{array}$ & \\
\hline Tar (Pix liquida) & 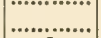 & 7 & \\
\hline 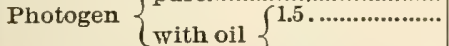 & 1 & & \\
\hline Concentrated vinegar, pure............................ & $\cdots \cdots \cdots$ & $1-1^{3 / 3}$ & \\
\hline Concentrated vinegar, with water 1.1 & ................ & $2-31 / 2$ & \\
\hline Acetic acid with water, $1.1 \ldots \ldots \ldots \ldots \ldots \ldots . . . . . . . .$. & .................. & 2 & \\
\hline $\begin{array}{c}\text { Concentrated sulphuric acid }\left\{\begin{array}{l}1.24 \ldots \ldots . \\
\text { with water }\end{array}\right. \\
1.48 \ldots \ldots . .\end{array}$ & .............. & $\begin{array}{c}7-8 \\
32-35\end{array}$ & \\
\hline Decoction of tobacco $\left\{\begin{array}{l}1.5 \ldots \ldots \ldots \ldots \ldots \ldots \\
1.10 \ldots \ldots \ldots \ldots \ldots \ldots \\
1.50 \ldots \ldots \ldots \ldots \ldots \ldots\end{array}\right.$ & $\begin{array}{c}2-5 \\
4-10\end{array}$ & $10-20$ & \\
\hline Tessier's arsenical bath............................ & ................ & $7-25$ & \\
\hline Mathieu's arsenical bath....................... & ................ & $15-16$ & \\
\hline $\begin{array}{l}\text { Over-saturated solution of arsenic in } \\
\text { water } 1.6 \ldots\end{array}$ & $2-3$ & . & \\
\hline 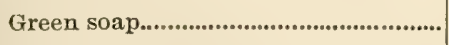 & $1 / 2-1$ & ............. & \\
\hline 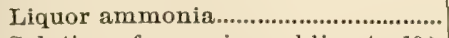 & $1 / 4-1$ & ............. & \\
\hline $\begin{array}{l}\text { Solution of corrosive sublimate, } 10 \\
\text { grains, one ounce }\end{array}$ & ............. & $15-45$ & \\
\hline $\begin{array}{l}\text { Infusions of henbane, belladonna, } \\
\text { and Persian insect powder, } 1.16\end{array}$ & $12-16$ & & \\
\hline $\begin{array}{l}\text { Decoction of black and white helle- } \\
\text { bore, } 1.16\end{array}$ & 6-36 & & \\
\hline Infusion of digatalis, $1.16 \ldots \ldots . . . . . . . . . . . . . .$. & $24-36$ & & \\
\hline $\begin{array}{l}\text { Walz's ley, containing caustic pot- } \\
\text { ash, hartshorn oil, and tar }\end{array}$ & $6-48$ & .......... & \\
\hline
\end{tabular}




\section{The Skin Diseases of Domestic Antmals.}

A celebrated writer on the diseases of the skin of domesticated animals, thus discourses on the subject:

"Few topics have engaged the attention of the successful breeder of our improved domesticated animals more than skin diseases produced by insects, in consequence of the manner in which both the breeding and feeding qualities of stock are affected by them. This arises from the little progress scientific inquiry has experimentally made in the physiology of these tiny parasites by means of the microscope, and the consequent paucity of reliable scientific works on the subject. Hitherto half the conclusions of our veterinary surgeons relative to their natural history have been drawn from the traditionary philosophy of our forefathers, and not from actual examination, either with the eye of the modern physiologist or morbid anatomist; while our entomologists have had too much to do to overtake the branches of zoölogical science, to fill their cabinets with specimens of the class in question-a class far more varied than the kingdom itself, every different race of animals being not only infested with a different brood of insects (mites), but almost every organic substance, animal and vegetable.

A very superficial acquaintance with these facts must convince the reader of the importance of the lecture on this subject, delivered by Professor Simonds, before the council of the Royal Agricultural Society of England, accompanied with drawings, exhibited on the wall, and specimens under his microscope. The former, drawn to a greatly magnified scale, illustrated, in a very conspicuous manner, the different stages of vitality, from the ovum to the insect, in all the vigor of matured life. But to us, and several other members who examined them, the latter appeared the most interesting, as nothing can exceed the fidelity with which the mieroseope exemplifies Nature, though all but invisible to the naked eye, confirming, in the most satisfactory manner, the soundness of the conclusions at which the Professor arrived, differing, as they did, in many respects, from those hitherto drawn, both by veterinary surgeons and entomologists.

Referring to the report of Mr. Simonds' first lecture, the task which devolves upon us is to apply it to the daily practice of the farmer, so as to profit by its deductions. These were principally confined, it will be seen, to scabies and ætrus in sheep and other 
animals, with a few observations on the dog-flea (pulex canis). Scabies is a pestiferous disease, whether it affects the horse, the ox, the sheep, swine, or poultry, inflicting a loss not easily estimated; hence the maxim of every intelligent farmer is, to avoid. With him "prevention is better than cure," and, therefore, his grand desideratum is to guard against contagion. Sheep are, perhaps, more subject to it than any of the other animals, arising as much from the nature of their skins and coats as from the fecundity of the acarus ovis, and the greater vicissitudes of the weather to which they (the sheep) are exposed. "Certain parts of the body are more liable to be affected than others; and so is an unhealthy skin than a healthy one. Indeed, it has been said that an unliealthy skin will itself produce scabies (?), but this conclusion does not appear to be well founded; for a disease dependent upon the presence of living parasites can never arise spontaneously, but must be effected by contagion, either by means of their eggs, or the insect in some other stage of its existence.

Now, from what has just been said, it will appear obvious that cleanliness, a healthy skin and state of the body, and a separation from foul animals and ground, are the means necessary to avoid contagion. The truth of this will, perhaps, be better understood if we first review the important distinctions which Mr. Simonds makes between the habits of the acarus scabiei of the human body and the acari of our domestic animals, the former burrowing in the skin, but the latter living on its surface, clinging to the skin, hair, or wool with their trumpet-shaped, vesicular-cushioned feet, to prevent their being thrown off by the animal when shaking or nibbling itself. Hitherto distinctions of this kind have been overlooked, writers generally concluding that the acari of quadrupeds burrowed in the skin like those of man, thus proving the little use which had been made of the microscope in examining the former, as it shows them to be incapable of living in the skin, from the configuration of their bodies. Indeed, to have made similar acari for naked skins as for those covered with hair, wool, or feathers would have been an oversight on the part of Nature; while the fact that the acarus scabiei will not live on the horse, nor acarus equi on man, or acarus ovis on the ox, or acarus bovis on the sheep, and so on, proves that greater differences than the mere configuration of the animal structure exist, all pointing to the above means as necessary, in every case, to avoid so great a 
pest-one which appears to form an integral part of that curse inflicted on the whole creation by the fall of man.

The importance of cleanliness, and its concomitant health, to prevent contagion, may be further illustrated. We have just seen above that an acarus, although a loathsome pest, is yet very nice in its taste, and particular about a nidus in which to deposit and hatch its eggs. It enjoys the highest degree of prosperity on the unhealthy skin, multiplying there fastest; so that if it creeps from it to the opposite-the sleek, healthy one of the horse or ox, or dry wool of the sheep-it feels itself from home, and, before it even reaches the skin, may be bruised or shaken off. If, however, it creeps upon the unhealthy animal with its staring coat, it soon reaches the skin, and commences its direful work, every thing there being congenial to his happiness; hence the incredible speed at which it propagates its species, until it either consumes its victim alive, or is arrested at its fatal work by the timely unguent of the veterinary surgeon.

Again: when a dirty animal shakes itself, as it invariably does after rubbing itself against any thing, less or more scurf, dandruff, and dust is thrown into the atmosphere, and carried to a distance by high wind. Now, under such circumstances, when affected with scabies, it is manifest that the smallest of these puny insects, as well as their eggs, will be blown from one pasture to another; that the latter will lodge in the dirty staring coat of the unhealthy animal, when they will be blown off that of the clean sleek one, or be brushed off before they reach the skin, or any nidus capable of hatching them. In this manner we can trace contagion from one animal to another, and thus account, in harmony with entomological science, for what has hitherto been termed spontaneous eases of scabies in some of our domesticated animals, while others have escaped the disease, though all herding together in one field. We can also account for the fact why the disease is more liable to break out among sheep than horses and cattle, without coming in contact with strange flocks, because the coats of the latter are more likely to be impregnated with eggs than those of the former, while they afford a better nidus for hatching them.

With regard to health, it has even been said that the blood of scabbed animals is diseased; nay, that the blood of all animals is loaded, more or less, with the eggs of acari, and that they are 
hatched under certain cutaneous affections, thus accounting for spontaneous cases differently from the atmospheric distribution of eggs. Hence the reason why sulphur and mercury are taken, internally as well as externally, to get rid of the disease. But this sanguiferous doctrine of distribution is more difficult to reconcile with entomological science than the atmospheric one.

Contagion by direct contact of clean animals with diseased, or where the former enter foul ground, will be difficult to avoid so long as the commerce of live farm stock is conducted as at present; for diseased animals will be sent to market, although contrary to the spirit of the law, thus not only communicating the disease to all they come in contact with, but also infecting the market-place. According to the experiments made by Mr. Simonds, acari left upon a post, hurdle, or hedge by a scabbed beast will survive for fourteen days, and at the end of that time lay hold of any animal coming in contact with it, and thus communicate the disease; consequently our weekly and fortnightly markets are little better, during the warm months of summer, than nests for propagating malignant parasites. It would be well, then, if very stringent statutes were enacted, confiscating all diseased animals offered for sale, and that microscopical examinations were enforced in all suspicious cases, so as to guarantee its healthy operation. It will thus be seen that the means for preventing contagion may be summed up in two words-improved management.

At a very early period in our history, acarus scabiei was much more common among our forefathers than among their posterity of the present day. The change which has taken place is obviously attributable to progress in dietetics, clothing, and medicine; and among our domesticated animals it is no more than reasonable to conclude that similar causes will produce similar effects. In point of fact, improved management has already greatly reduced the prevalence of scabies among both our herds and flocks, thus holding out every encouragement to persevere in obtaining further progress. There is, perhaps, no branch of husbandry where greater advances can be made than in cattle-cookery, household accommodation, including grooming, etc., and medicine ; and when we contemplate how much cutaneous diseases are dependent on management in each of these three respects, it were difficult to estimate what influence further improvements in them may have on the disease in question." 


\section{Lige oN Horses.}

Lice, in a majority of cases, may be attributed to want of cleanliness; for they are rarely if ever seen on the body of an animal with clean skin. Brush and curry-comb, and clean stables, are, therefore, the preventives.

A short time ago I had occasion to visit a stable, located in Waukegan, Ill. There I saw a horse almost perfectly hairless, except the mane and tail. I asked the owner what occasioned the depilation of the hair. He informed me that the horse's body was completely infested with lice; that he had tried all sorts of remedies without success, and, at last, thought he would try kerosene oil, and, accordingly, rubbed the body all over with it, using about two quarts. He said "it lilled all the lice, but came very near killing the horse. It made him perfectly crazy ; his limbs became swollen to the proportions of a young elephant, and the hair all fell off."

The remedy I have found most efficacious is composed of

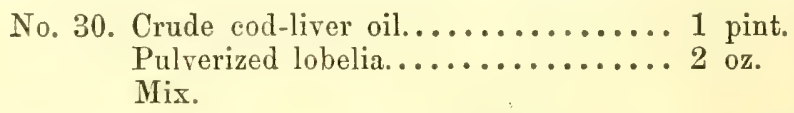

This should be thoroughly rubbed all over the body by means of a stiff brush; at the expiration of four hours, carefully wash the horse all over with soft soap and warm water. It may be necessary to make a second and even a third application, on successive days, ere the parasites are all killed. The parasites can be seen with the naked eye; therefore the owner of the horse must be the judge as regards the number of applications needed.

At a meeting of a Farmer's Club, "Lice" formed the subject for di-cussion. Dr. J. R. Surth explained that lice, as well as all sinilar insects, breathe through holes in the body. These holes are mimute spirules, constantly kept open by an elastic ring, and surrounded by a fringe of extremely delicate hair, which prevents the intrusion of any solid particles. To kill the insect it is only necessary to close these breathing-holes, and this is done by smearing them with any kind of grease or oil. You may catch a caterpillar and examine him with a magnifying glass, and you will find these spirules arranged in two rows, one on each side; then, if you take a moth or butterfly, you will find the 
breathing-holes in the body corresponding with those in the body of the caterpillar from which it was produced-the same body, in fact, remaining after the wings are developed. If you dip a feather in oil, and smear the two spirules nearest the tail, the lower portion of the body will be paralyzed so far as these holes. Proceeding upward, you may paralyze the whole body until you come to the last two, which are situated just below the jaws. So long as these remain open, the insect will continue to breathe, but if these are now closed, he dies immediately. To exterminate lice upon any animal, it is only necessary to cover the animal completely with grease or oil. The simplest and cheapest oil is best-lard, fish oil, or any other that is at hand. When the lice have not become so numerous as to produce the disease known as pityriasis, or mange, this application, undoubtedly, is the best that can be used. In addition to being effective, it is perfectly harmless. Stock-breeders should always recollect that prevention shows wisdom, and saves annoyance and expense; and, by neatness, cleanliness and comfort, the health of the animal will be insured, and the animals will not become infested with the troublesome parasites.

Poultry lice affecting horses.-Horses, when located near a henroost, are apt to be infested with poultry lice. The remedy is the same as in the case of the common louse.

\section{Diseases of the Heels (Grease, or Scratches).}

In olden times grease, or scratches, and cracked heels, were not only very prevalent but very formidable affections. Before veterinary surgeons were employed in the British Army, many thousands of valuable horses vere condemned as iseless for active service in consequence of the prevalence of that loathsome affection known as inveterate grease; but now, in consequence of the presence of veterinary surgeons, and the consequent improved system of management in camp and stable, grease is almost unknown. Most veterinary writers contend that grease almost always arises from improper management of the horse, yet I have seen it occur in horses that were well cared for and properly treated. The truth is, some horses are predisposed to affections of the skin of the heels and the sebaceous glands of the same; and, although cleanliness and good management may stave off an attack for a long while, yet when the system abounds in morbific 
matter, the same is very apt to gravitate toward the heels, (they being most remote from the center of circulation,) inducing inflammation of the skin of the heels, distension of the sebaceous glands, and a stinking and unhealthy deposit on the surface, and a purulent discharge through ulcerated cracks. This constitutes grease.

Cause.-It is contended by Mr. BuAin and others that grease has local weakness for a cause. As fluids press, not in proportion to their diameter, but to the height of their column, the venous blood must find some difficulty to its ascent. Debility is, therefore, more felt in the distended vessels remote from the influence of the heart, under which circumstance the effects productive of grease necessarily ensue. Some horses are constantly the subjects of swelled legs, and, if the heels happen to be accidentally abraded, the latter often operates as the exciting eause of a very formidable affection of the heels. Associated with swelled legs is a scurfiness of the skin beneath the fetlock. These conditions, together with the fact that the animal is of the lymphatic temperament (gray color), are sure indications that predisposition to maladies of the above character are present. Having such horses under our care, the chief object should be to ward off an attack, by dietetic and hygienic measures. Too much washing of the heels, without drying them by rubbing, is just about as bad as if the filth was allowed to remain. The practice chills the part by a process of slow evaporation, and the result is local congestion, etc.

I now propose to illustrate, for the benefit of the reader, the treatment of grease or scratches. I was requested to see a gray gelding, the property of the Transfer Company of St. Louis. On arrival, I examined the animal, and found him to be a large, flabbily-organized creature, having a large amount of loose tissue under the skin. I found that there was a bad odor arising from some ulcerations and exudations about the heels and sides of the same. Intermediate of the uleers were dry, horny scabs; the hair about the parts pointed straight out; the heel was excessively tender, quite vascular, and blood escaped from its vessels. The moment a hand was placed upon the locality, the animal would catch up the limb and appear to suffer much pain.

Treatment.-In the first place, I had the parts well cleansed with soap and water; then, after wiping them dry, they were wetted, three times daily, with a portion of the following solution: 
No. 31. Nitrate of potass (saltpeter)........ 2 oz. Water........................ 1 pt. Glycerine................. 4 oz.

Every time the solution was applied, the parts were dusted with pulverized charcoal. This charcoal, completely covering the abraded parts, and being a good antiseptic, had an excellent effect in excluding atmospheric air, and in correcting feted odor. I administered, as an alterative, to correct the morbid habit,

No. 32. Iodide of potass.............. 20 gr.

Sulphur..................... $4 \mathrm{dr}$.

Powdered sassafras bark............ $2 \mathrm{dr}$.

Gentian...................... $1 \mathrm{dr}$.

These were mixed in food, and the same quantity was given during a period of four days, and the dressings were also continued. On the fifth day the animal had very much improved, stood fair on the foot, and seemed to suffer but very little pain. The limb was somewhat swollen, partly from want of use, and otherwise from slight effusion into the cellular tissue. I discontinued the medicine, and ordered the following mixture, to be applied twice daily:

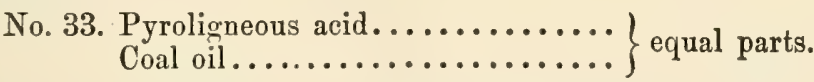

After each application the charcoal was reapplied.

The following case serves to illustrate that the virus of grease can be transmitted from horse to man:

Transmission of the Virus of Grease from the Horse to Man.Drs. Madnoury and Piciot have published an interesting series of experiments, tending to prove the identity of grease and cowpox. This doctrine, which was always maintained by JENnER, has received confirmation from the observations of Loy, Godine, and others. The following is a summary of the facts, related by Drs. Maunoury and Рichot:

"Francis Barthelemy B—, aged twenty-eight, of lymphatic temperament, a farrier, presented himself to Dr. Pichot on the 5th of March, 1856. He had not been vaccinated. On the backs of his hands, which were red and swollen, were several confluent opaline pustules, depressed in their center, and having all the appearance of vaccine pustules of the eight or ninth day. The inflammation with which the pustules were surrounded had ap- 
peared on the second; the pustules themselves preceded the inflammation some days. This man had not been in contact with any cow, but on the 11th of February he had shod a horse suffering from grease. There existed, at the time, numerous cracks about the hands. The disease from which the horse was suffering was certified by a qualified veterinarian. Various inoculations were practiced with the liquid taken from the pustules presented by $\mathrm{B}$ - , with the effect of reproducing the same disease. The most perfect set of experiments were made by M. Maunoury, who transmitted the virus through four sets of cases. The following are the results of his observations:

1. That virus obtained from the hands of the farrier $\mathrm{B}-$, and inoculated on the arm of an infant, produced a full pustule, having all the character of a vaccine pustule-form, evolution, termination.

2. That lymph taken from this pustule, and inoculated on the arm of three persons, had produced identical pustules, which are truly vaccine.

3. That the transmission of the virus by successive generations has not diminished the intensity of the force of the poison. One of the set of cases presented large pustules, depressed in the center, and filled with matter; each pustule served for several inoculations and the charging of several sets of glasses.

4. That from these facts it is evident that the virus taken from the pustules of the farrier was identical with the vaccine."*

\section{SURFEIT.}

The term surfeit is used to designate an eruptive affection of the skin, which usually makes its appearance very suddenly. There is nothing dangerous about it, and it sometimes disappears, in the course of a few hours, without any medical treatment. . It usually appears in warm weather, and especially among horses that are in a state of plethora, from the use of a large quantity of meal, or what is known as eut feed.

Symptoms.-A horse may be taken out of the stable apparently in perfect health, except that he is fat, and, after being driven a

*Generales de Mèdècine, April, 1857, pp. 365, 398, from the British and Foreign Medical Chiurgical Review. 
short distance, he is brought back with his neck and sides covered with blotches or elevations. On pressing them with the fingers they crepitate, showing that they are gaseous elevations or distensions, originating in the cellular tissue beneath the skin, from the spontaneous generation of gas. The gas probably arises from fermentation of the food, and instead of accumulating in the intestines, finds its way to the surface of the body.

Treatment.-Dissolve two ounces of hyposulphite of soda in one pint of warm water, and drench the animal with the same. Then sponge the body with a portion of the following surfeit lotion:

No. 34. Aqưa ammonia................ 2 oz.

Animal glycerine.............. 1 oz.

Water..................... 1 quart.

If taken in time, the affection will disappear in a few hours. The patient should be kept on bran-mashes, well salted, for a ferv days ; or a couple of weeks' run at grass, if the season permits, will be of great service.

\section{Dropsical Lmibs (Swelled Legs).}

It is a very serious annoyance to the owner of a valuable horse to find that, after the animal has been standing in the stable for only a period of twelve hours, his hind limbs have wonderfully increased in size; and equally mortifying is it to see some animals with their limbs continually tumefied, and without any other apparent sign of disease.

Dropsy of the limbs generally proceeds from congestion. The serum, or more fluid part of the blood, transudes through the walls of the blood-vessels, and accumulates in the cellular tissue, under the skin. But dropsy takes place not only in consequence of venous congestion, but accompanies very many forms of disease, such as scarletina, influenza, farcy; and the cure of such diseases also accomplishes the cure of dropsy.

Causes.-The cause of that form of dropsical limbs which seems to occur without any other visible disease is now the subject for consideration. When a horse's limbs swell persistently, after a few hours' rest, and disappears under exercise, we may infer that there exists a dropsical diathesis in his system; that, by virtue of his constitution, he is predisposed; and such cases are designated as chronic local dropsy. 
Treatment.-The remedies are hand-rubbing, exercise, and stimulating liniment. The best liniment for local dropsy of this character is as follows:

No. 35. Fluid extract of wormwood......... $4 \mathrm{oz}$.

Fluid extract of ginger........... $3 \mathrm{oz}$.

Spirits of camphor.............. 1 pint.

Rub the region of tumefaction with a portion of this liniment every night.

When swelling of the limbs do not assume a periodical character, and suspicion of predisposition can not be entertained, then, in addition to the application of the liniment, give the animal, morning and evening, one ounce of the fluid extract of buchu.

\section{Scarlatina.}

Scarlet fever, or scarletina, as it occurs among horses in the United States, is a febrile disease of a very prostrating character; yet it is not considered by the author a contagious malady. It has only lately, however, been recognized in this country as a distinct equine disease, and, being of rather rare occurrence, we know but little about it. The cases that have come under the author's treatment were unattended by ulceration of the throat, and this may account for the non-contagion; for, in the human subject, when ulceration of the throat takes place, the case becomes malignant.

WATSON says: "The malignant sore throat may be caught from a patient who has mild scarlet fever; and mild scarlet fever may, in like manner, be contracted from one who is suffering under the malignant sore throat. The two forms graduate insensibly, in different cases, toward each other; and it would be impossible, even if it were desirable, to draw any strict line of separation between them. Many would say, and probably with truth, that the difference was this: in the one form, the poison of the disorder is seeking its vent, principally, by the throat; in the other, by the skin." It appears, therefore, that in the human subject the disease is capable of being communicated at any stage.

The cases that have come under the author's notice since he first recognized the malady have all been of a mild form; that is to say, non-malignant. The limbs, sheath, and pectoral regions were excessively dropsical ; the skin was the seat of rash or minute ele- 
vations, and scarlet spots or patches were always present on the visible surfaces of the mouth and nostrils; yet the patients always drank and ate mashes freely, which they could not have done so readily had their throats been sore and ulcerated.

The author would intimate, however, that, should the disease ever become malignant in the horse, it may also become, as in the human subject, contagious and infectious. Perhaps the reason why it has not attained a malignant type in this country is, because it is of recent origin; whereas, among the members of the human family, scarlatina has been known to exist for several hundred years, and may have acquired intensity and malignancy with age. In anticipation of such an event, the author recommends that scarlet-fever patients be separated from other inmates of the stable.

The following case is from the author's note-book, and may serve to instruct the reader on the theory and practice of the malady: A short time ago, a bay gelding, aged about nine years, was admitted into the Chicago Infirmary. On examination, the following symptoms were observed:

Symptoms. - The hair about the neck and fore and hind limbs was elevated in blotches, with corresponding elevations on the skin beneath; the membranes within the cavities of the nostrils had scarlet spots, variable in size; all the limbs were more or less swollen; the breath was hot and fetid; the animal was unsteady in his gait, apparently very weak; had great thirst, yet little appetite. The elevations on the surface of the body, and the scarlet spots on the nasal membrane, constituted the diagnostic symptoms of scarlet fever.

Treatment.-During the first three days, I drenched the animal with three ounces of liquor acetate of ammonia, in an equal quantity of cold water. The elevations on the surface of the body were sponged once a day with a lotion composed of two ounces of aqua ammonia and one quart of rain-water. A sloppy branmash was occasionally furnished, of which the patient partook freely. This tended to keep the bowels loose, and moderate the febrile symptoms. On the fourth day all the limbs became very much swollen, from dropsical effusions, which feature of the case is very apt to present itself. I now changed the treatmentomitted the lotion bathing, and gave, night and morning, twoounce doses of compound fluid extract of buchu and juniper ber- 
ries. A slight discharge of glairy mucus now cornmenced running from both nostrils; the scarlet spots had become more diffused, and of a brighter tint; the appetite had returned, and a few oats and a smill quantity of hay were oceasionally allowed, which were eaten. The limbs were hand-rubbed often, and the body was clothed with warm blankets. This treatment was followed up for a period of five days, at the end of which time he had improved very much, yet the limbs were considerably swollen.

On the tenth day I commenced to give four ounces of fluid extract of resin-weed per day, with an occasional dose of fluid extract of golden seal, under which treatment he convalesced very rapidly, so that on the sixteenth day all medicine was discontinued. I now turned the animal into a small inclosure, where he seemed to take pleasure in rolling and exercising himself, which had the effect of reducing the dropsical swelling of the limbs, and on the fourth of December the patient was sent home-not exactly to go to work, but to receive care and attention until he had recovered from the effects of the disease, and had gained his ordinary strength.

It is very difficult to say to what extent scarlet fever among horses prevails in the United States; for very little, if any thing, has ever appeared in print of American origin, except that which has appeared from the pen of the author of this work. On the other side of the water, however, the disease is of frequent occurrence, and is well understood. The first account of it published in England was from the pen of Mr. Percivalu, in the year 1843, who says that "the existence of scarlet fever has received such confirmation from other quarters as to leave no doubt in my mind that, rare as the malady acknowledgedly is, and hitherto undescribed as it has remained, it will one day find a place in our established veterinary nosology."

Since the above date, Surgeon HAYcock and several other writers have noticed the disease, treated it, and minutely described its symptoms. They all agree that when the disease does make its apperance, it is usually the sequel of epidemic eatarrh, or influcnza; and this was probably the case regarding the patient the sulyeet of this paper, for the owner informed me that the animal had previonsly shown symptoms of distemper. 


\section{RINGTORII.}

This disease usually makes its appearance on the shoulders and sides of the horse, in the form of circular patches, attended by scurfiness and loss of hair. Small vesicular eruptions can also be detected, by means of a magnifying glass. It is usually spontaneous in its origin, but probably is generated by filth.

Treatment.-In view of treating the disease successfully, the body should be well cleansed with soap and water, and afterward rubbed dry; then apply daily, by means of a sponge, a portion of the following:

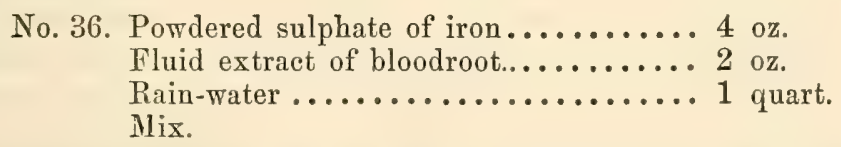

Give, as an alterative, half an ounce of the fluid extract of mandrake, in half a pint of water, night and morning.

When the disease has been neglected, the parts are apt to ulcerate; in that event, the following preparation is recommended:

No. 37. Pyroligneous acid.............. 6 oz.

Linseed oil.................. 5 oz.

Spirits of camphor............... 2 oz.

Mix.

Dress the ulcerations twice daily.

A liberal supply of cut grass, sliced potatoes, or carrots should be allowed, if they can be obtained. Particular attention should be paid to the matter of keeping the skin clean. The treatment must be continued until all vestiges of the disease have disappeared, or it may break out again.

\section{Prurigo.}

This is a disease of the skin, accompanied by a terribly annoying itching sensation. The torment experienced by animals suffering under this form of malady is scarcely describable. They often rub and abrade the skin until blood appears on the surface, and they are rendered perfectly miserable. There are said to be various forms of prurigo, but they do not differ in kind, only in degree. Heat aggravates the malady; therefore horses located in a warm and unventilated stable are apt to suffer most. And the 
same remarks apply to many other forms of disease, which shows how important it is that stables should be properly ventilated.

Symptoms. - The skin in those parts where the affection is located shows on its surface small elevations, known as papulæ; but they are difficult to discover in some horses, as they are of the same tint as the skin. After awhile the rubbing tears away the summits of the papulæ, and a secretion exudes which soon forms very minute scabs.

During the past four years the disease has prevailed very extensively among army horses, and it usually proved very obstinate, from the fact that very few of the army farriers understood its true nature; neither were they acquainted with the moctus operandi of treatment. The authorities contend that prurigo is not a contagious affection, like itch and mange, and, therefore, it may be classed as a local affection, brought on by inattention to cleanliness. In order to prevent it, the author advises a free use of the brush and curry-comb.

Treatment.-The best mode of treatment is as follows. Sponge the affected parts thoroughly with the following lotion:

No. 38. Powdered borax.............. 4 oz.

Rain-water ................... 2 quarts.

After using the above quantity, at one or two dressings, then rub the affected regions dry, and lubricate the same with a portion of the following:

No. 39. Kerosene oil................. 4 oz.

Pyroligneous acid............. 12 oz.

Mix.

It is usually good policy to put the patient under a course of alterative treatment; therefore the author recommends that he have, daily, thirty grains of iodide of potass, dissolved in four ounces of water, to be given in the form of drench, every day, until the disease has disappeared.

\section{PoLL-EVIL}

The name of this disease originated in England, in consequence of it being more prevalent in that country than in some others; hence, was a great evil, and occurring about the nape of the neck or region of the poll, it was called "poll-evil." Much of the poll- 
evil occurring in former years was unnecessary, and occurred in consequence of neglect, injuries, and abuse. The disease first makes its appearance in the form of a local tumor, of an inflammatory character. Soon it runs into the suppurative stage; pus or matter is secreted, and we then have a common abscess, which may result in fistula of the poll.

Causes-The disease may arise from blows and bruises. Horses when located in low-roofed stables are apt to strike the poll against the ceiling, or a beam, or the upper part of the doorway, and frequent repetition of the act either ends in induration (hardening) or poll-abscess. Some grooms are in the habit of occasionally jerking the animal's head by means of the halter, and some horses are very restless in the stall, and are often seen to jerk their heads upward, forgetting that they have any halter around their necks, until they feel the pain which they themselves inflicted. The injury, slight as it may be, often remains undiscovered or unattended to until considerable tumefaction or even suppuration has set in. The case may then become protracted, and exhaust the patience of all concerned. Excessive friction on the nape of the neck from bridle or halter, or pressure from either one of these causes, produces poll-evil; and the halter, by being fastened too tightly, is a fertile cause in producing this affection. Dirt and filth are operative, also, in producing this malady. It is well known that the poll is a part which very seldom makes the acquaintance of the brush or curry-comb, yet it is the locality of considerable dust and scurf; and sometimes, owing to accumulations of the same, a cutaneous eruption arises, and the itching sensation consequent causes the horse to fret and rub whenever he can get a chance. The evil goes on, until what was at first superficial now becomes deepseated. The bungling and cruel manner of sometimes forcing a small collar on a large horse often does mischief, and lays the foundation for poll-evil. Some persons are in the habit of bracing the horse's head downward with the martingale, so as to bring the lips and chest in close proximity, yet forget that the strain comes on the poll and bruises the soft parts.

Treatment.- Should the tumor be discovered in its early stage, before matter has formed, the parts should be bathed often with a portion of vinegar and water, equal parts; or else a cold-water dressing or pad may be applied, which should be reapplied as fast as it becomes dry. Half an ounce of powdered chlorate of potass 
should be given night and morning. This may be dissolved in half a bucket of water, which the horse will drink. In the course of a couple of days, the practitioner will be able to determine whether or not the tumor is likely to suppurate; if so, it will have increased in size, and have a soft fluctuating feel.

Just as soon as matter can be detected, the abscess must be punctured at its base, by means of a thumb lancet. It will not do to allow the matter to accumulate; for, if so, it will burrow and spread in various directions, making a very extensive and formidable abscess. It would be necessary to make a free opening into the abscess large enough to admit a man's finger, and if, in the course of a day or two, the opening should partly close or contract, it must be dilated with a knife. So soon as the abscess is laid open, all the matter must be squeezed out, which process must be accomplished by the use of sponge and hot water. We then, in view of removing every particle of matter, carefully inject the cavity with a quantity of pyroligneous acid and water, equal parts. A glass syringe is best, as the acid has a bad effect on a metal one.

It will be necessary to sponge the cavity once daily for several days, or until matter ceases to form. In the mean time the animal must be put under treatment, for it is very rare that this disease can be cured by local treatment alone. My usual custom is to give the patient thirty grains of the iodide of potass, twice daily, in half a bucket of water. The animal will not refuse to drink it. Should he be weak and emaciated, tonics and stimulants are indicated. Half an ounce of powdered golden seal and the same quantity of ginger, given with a small quantity of water, as a drench, daily, will answer the purpose. Sometimes it is advisable to put a pad of cotton eloth, or a large wad of oakum on the poll, in view of keeping the skin and sub-tissues in contact, by which means they more readily unite. The pad may be secured to the part by passing a cotton roller, five inches wide and three yards in length, around the neck. At the poll the bandage is to be further secured, by tying a lock of the hair of the forehead with a lock of the hair of the mane. This precaution prevents the bandage from slipping backward. The bandage should be removed and readjusted every day, and the parts are to be cleansed and syringed, in the manner just alluded to, as long as necessary. After the lapse of a few days, should the secretion of matter decrease, then the chances are in favor of a cure. 
Cases, however, will sometimes occur which set at defiance all our skill. In such we find that either the ligamentary, tendinous, or fleshy parts, or perhaps the bones, are involved, and it often happens that fistulas or pipes run in various directions. Such cases require the services of a surgeon, who will dissect out the fistulous pipes, and remove all the diseased portions of the flesh and bone. The after-treatment is then the same as we have just indicated.

\section{Fistulous Withers.}

This disease resembles poll-evil, and, in a majority of cases, owes its origin to the same exciting causes; namely, external bruises, or injury inflicted by a bad-fitting collar or saddle. The treatment is the same as that recommended for the cure of pollevil.

\section{WARTS ON THE Skin.}

Warts are of two kinds. One of these makes its appearance upon, and consists in part of an increased development of, the outer scurf or scarfskin; the other kind is contained under, and, while it elevates, does not grow from the scarfskin. As it frequently becomes desirable to remove these growths, and to counteract that condition of skin on which their reproduction depends, we shall briefly describe them. The most common kind of wart, and that called the true angleberry, is the first of the two to which we have alluded. It has always a hard, horny sort of investment externally. This is formed of scales of scarfskin matted together in great quantities, and prolonged from the true skin, to which they are attached. As this outer covering increases in growth, it dries and splits up into shreds or fibers, which generally become more or less pointed at their free ends. Many of these shreds may grow from one common stock or pedicle, called the "neck." In other cases there is no well-defined neck, and a large surface will be covered with a coarse, rough, and horny crop of warts, all, as it were, united where they originated from the skin.

Cause.-Warts are always produced from what mar be considered as greatly-developed knots, or papillæ, within the true skin. Blood, in greater quantities than natural, is sent to these, and the papillæ continuing to grow, forms perceptible tumors, which are somewhat sensitive, painful if exposed to friction, and form or 
throw off the scaly covering which eventually forms the main part of warts. We see many instances of warts on the human hands and other parts of the body, and always find that the dry, horny part grows from the skin somewhat thickened and increased in vascularity. So great, indeed, is the quantity of blood which some warts receive, that they bleed much more profusely than other portions of skin when wounded.

The other kind of wart to which we alluded does not cause any external roughness or scaly appearance. It is formed within the outer skin, and commonly appears as a circumscribed round tumor. On being examined by the hand, it is felt to be contained in a sack or pouch, within which it is easily moveable. It is almost insensible, and consists of a quantity of white fibrous and sometimes grisly substance, which does not possess blood-vessels in its interior. We often meet with this kind of encysted wart near the outer organs of generation in the horse or mare. The horny wart which we first noticed is common in horses, cattle, and dogs. The encysted wart is comparatively rare in cattle; it is more common in dogs, but is most frequently found in horses.

Treatment.-In treating warts with a view to remove them, and prevent their reproduction, it becomes necessary to destroy, in a measure, the surface from which they sprout. When an angleberry has.a "neck," this should be cut off flat and close with the skin, and the raw surface thus made should be touched occasionally and freely with caustic or a heated iron. If the neck be so large as to endanger much bleeding if divided at once, a ligature of thin, strong string may be tied round it very tightly, in order to cut off its supply of blood. The ligature should be applied frequently-daily, if convenient. Where a large surface is covered by warts which do not possess any definite necks, their horny parts should be cropped or eut closely down to the true skin, with scissors or other convenient instrument, and the surface thus exposed should then be touched with a hot iron, or rubbed with caustic potass. Such application is to be continued till the horn ceases to grow. Encysted warts are removed by cutting the skin of the sack and squeezing them out. The exposed cavity should be dressed daily with digestive ointment. 


\section{Purpura Hemorrhagica.}

A disease not unfrequently makes its appearance among horses termed purpura hemorrhagica, known in human practice as the "purples." It consists of congestion (extravasation) blood of and effusion of serum (water) into the cellular tissue. The disease probably owes its origin to a depraved condition of the blood.

Symptoms.-On making an examination of the affected animal, we find that the cellular tissue, in various parts of the body, is distended with serum and blood. Local swelling will appear in various parts of the body, more particularly about the face, lips, and limbs. The disease also affects internal parts. Blood is sometimes passed with the urine and feces; respiration is embarrassed; the heart palpitates, and abnormal cerebral symptoms set in. In the human subject the disease is considered strictly as a hemorrhage. Small round spots appear on various parts of the body and legs, of a dull crimson or purple color. Pressure upon them does not efface the color, nor render it fainter, as it does that of common inflammatory spots of the skin. There is scarcely any prominence of the purple stigmata ; but they are sometimes intermixed with livid blotches, with appearances exactly resembling bruises, and they undergo, before they disappear, the same changes in color which attends the disappearance of a bruise. In fact, the anatomical condition of a bruise is exactly the same, with the diffused condition as in purpura. In each case the color is the result of echymosis (effusion of blood beneath the skin).

In the human subject, also, the disease is not confined to the skin. WA'TSON informs us that " the spots are not confined to the skin, nor to the subcutaneous tissues, but are found, occasionally, upon all the internal surfaces also, and within the substance of the several viscera (internal organs of the body). I have seen these purple spots in the mucous surface of the mouth, throat, stomach, and intestines; in the pleura and pericardium; in the chest; in the peritoneal investment of the abdominal organs; in the substance of the muscles, and even upon the membranes of the brain and in the sheaths of the large nerves, and I have known them to be accompanied with large extravasations of blood in most of the vital organs of the body."

The same appearances have been observed in equine autopsies, which accounts for the immobility and deranged condition of all 
the functions. Sometimes the throat is so badly affected that tracheotomy has to be resorted to. Now and then the tongue becomes livid and swollen, so that the animal can not partake of food. In such cases the tongue may be freely swabbed with warm vinegar, until its volume is reduced.

Treatment.-So soon as the disease is discovered, the animal should be put under the following treatment:

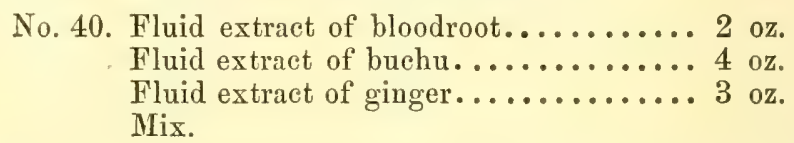

Give one ounce of this mixture night and morning, in about a gill of water. Should there appear to be any trouble with the throat, lubricate it, twice daily, with a portion of the following:

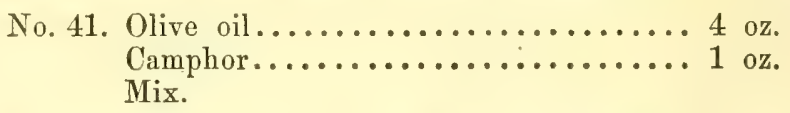

In preparing the above, it is best to pulverize the camphor, when it will dissolve more readily in the oil. A lotion must now be prepared, with which the whole surface of the body should be sponged daily.

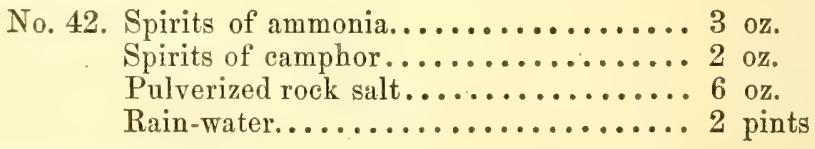

Dissolve the salt in the water, allowing the impurities to settle; then add the clear liquor to the camphor and ammonia, previously mixed.

It occasionally happens that the skin, in various parts of the body, cracks, and portions slough off. Should there be any appearance of the kind, discontinue the above lotion and substitute the following:

No. 43. Pyroligneous acid.........................................

Every time the patient is watered, (say about three times per day,) half an ounce of hyposulphite of soda should be dissolved in the pail. Vegetables, also, are indicated, if they are to be had. I should give tomatoes, sliced earrots, potatoes, and green grass. Bleeding, purging, and all kinds of poisonous and sedative medicines are inadmissible. 


\section{Out of Condition (Hide-Bound).}

Either from derangement of the digestive function, or in consequence of the animal being fed on poor provender or worked beyond his strength, debility and emaciation ensue. A horse out of condition usually loses flesh; the hair loses its glossy appearance; the skin becomes unhealthy and scurfy, and he can not perform his ordinary labor without apparent fatigue.

Treatment.-The best remedy, if the season permits, is a run at grass, taking care to give a good feed of oats every night; otherwise the grass will not improve his condition. In the stable the treatment is as follows: Give the animal one ounce of the fluid extract of camomile flowers every morning, before feeding, and at night mix one ounce of powdered poplar bark with the oats. This, together with good food and rest, will complete the cure.

\section{Herpes.}

Herpes is a disease of the skin, manifesting itself in the form of vesicles, which contain a small quantity of aqueous fluid effused beneath the true skin. Sometimes they are thinly scattered over the surface, and sometimes collected into clusters. The vesicles appear in irregular succession. They terminate, also, in various ways : by the reabsorption of the fluid, and, in bad cases, falling off of the hair, and peeling of the skin, in some places. In the human subject the malady is considered transient and non-contagious, consisting of red patches, of irregular form and variable size, upon each of which stands a crop of vesicles.

Treatment.-I have been very successful in the treatment of this malady by using the following lotion :

No. 44. Glycerine.................... 2 oz.

Tannin..................... $2 \mathrm{dr}$.

Rain-water................. 4 oz.

Apply once or twice daily, by means of a soft sponge.

In the horse the affection sometimes assumes an epizoötic form. In that event it can be communicated to man, as the following cases will show:

"At the commencement of 1849, Count Faverges invited Professor Papa to the valley of Borne, in Savoy, to see a disease affecting animals, and which even spread to men. Every facility 
was offered by Government to Professor Papa, and many persons having affected animals were requested to permit their inspection, and, indeed, threatened with a fine if they did not. Papa saw about three hundred horses and mules affected. The disease appeared in circular patches of furfuraceous scales, with grayishwhite scabs. These patches had usually well-defined margins, about the size of a dollar or five-shilling piece. Usually they were isolated, but at other times they were confluent, or running together in groups. The head, neck, withers, shoulders, and loins were the parts chiefly affected. More rarely the upper portion of the extremities, and never on the lower part of the limbs, chest, or belly. The malady commences with a violent itching, and an eruption in small circumscribed points, about the size of a lentil, is witnessed. The scabs form, with the exudation drying and entangling cuticle and hairs. In the vicinity of the first, other eruptive spots appeared, which, widening, became confluent and run into one another, especially where the skin is folded and animals have a chance of rubbing themselves. A scab forms on the sore surface, and the surface beneath it is red and tumefied, but in a little time desquamation occurs. A very careful microscopical examination failed to indicate the existence of any acari.

The disease is contagious, and Papa says all those who come more or less in contact with herpetic horses or mules, and especially the conductors of the same, were covered on the arms, legs, chest, and face with pruriginous eruptions, limited and circumscribed, sometimes isolated, occasionally confluent, in the form of red patches covered with papulæ and vesicles, which become incrusted with brownish-yellow scabs, beneath which purulent deposits formed. In consequence of the violent pruritis'attending this disease, it was believed to be scabies or itch by the people, and, though in many houses individuals were affected, they were ashamed to confess it, and it was with great difficulty that Papa collected information on the subject; but, having gained confidence on the latter, the people more freely related their cases to him. The first to be affected were those intrusted with dressing the diseased animals. The parts first attacked were the articular regions, about the forearm, arm, face, and rarely the lower limbs.

Papa describes one of many cases of direct contagion. It occurred in a lad of sixteen, who had jumped on the bare back of an affeeted horse, to take it to a watering-place. Two days afterward, 
on the inside of the thighs and legs, from the pubis to the calf of the leg, there was extreme pruritis. The skin became of reddishbrown color, and vesicles, full of yellowish lymph, formed, which gave way to vesicular patches or scabs, rough to the touch, first isolated, and afterward confluent.

The transmission occurred from horse to horse, horse to man, and from man to man; in fact, persons who never touched an affected horse were infected from individuals they came in contact with. A soldier, having arrived in perfect health from his regiment, slept with his brother, who suffered from the disease, and became affected. The wives of carters not only took the disease, but communicated it to their sucking infants.

Papa visited the district at a season when the fetid hellebore was growing abundantly. He caused some to be gathered, and obtained decoctions of the roots. With this he mixed equal parts of water, and distributed the lotion in different parts, to be applied to men and animals that were affected. Without any internal treatment, this application had the best effect. He tried tobacco decoction, but not with a similar amount of success. Papa regards the disease as the herpes tonsurans, which has been spoken of by many veterinarians as common in the live animals."*

* Edinburg Veterinary Review.

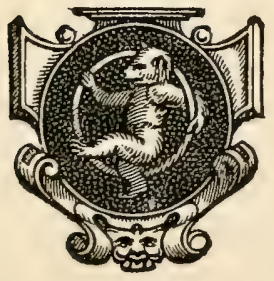




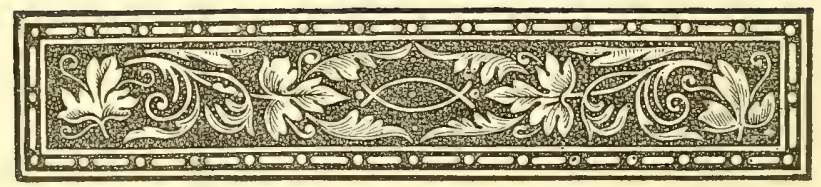

\section{SECTION XI.}

ON BREEDING, AND THE PRINCIPLES OF THE, SAME

Breeding-The Principles of Breeding-In-and-In Breeding-Permanent Varieties-Animals become Parents too Early-Objections to In-andin Breeding Answered-The Advantage of In-And-in Breeding-Thg Importance of Studying Anatomy and Physiology.

\section{BREEDING.}

TVHE choice of a sire and dam is a point of the utmost conse1 quence in breeding horses (or indeed any other animal), as the offspring will be found, in almost every instance, to inherit the qualities of its parents. Peculiarity of form and constitution is inherent, and descends from generation to generation; hence the necessary attention to those niceties which breeders are often apt to forget. Nor is it sufficient that one of the parents be good and the other indifferent; for the perfection of the sire may be lost through the deficiencies of the mare, and vice versâ.

In the selection of a stallion many things should be observed. There should be general uniformity and compactness in every part. The height should depend on the occupation the foal is destined to fill. The legs should be particularly examined, and disease should pervade no part of the system. Fat, heavy horses, with thick legs, and coarse, unseemly heads, should always be avoided. Horses should be free from specks on the eye-partial or total blindness. Temper is an essential point, for vice is sometimes hereditary. Stallions that cover too many mares in a season, in the latter period produce weak offspring.

As regards the mare, it is well known that the dam contributes more to the integrity of the offspring than the sire. It is essential that she be in full possession of her natural strength and powers. The vigor of the constitution determines much in favor of the 
foal. It is a great error to suppose that a mare that has once been good, and capable of great exertion, should, when old, and no longer fitted for work, produce offspring equally efficient as when in her prime. The foal will certainly inherit some of the weakness of the present nature and broken-down constitution. Mares should never be put to the stallion untii they have arrived at maturity, which takes place about the fifth year. Mares are bred from earlier, but it is a bad practice, for strength and beauty are absent; and thus not only is the dam rendered inefficient sooner than in one that is allowed to come to maturity, but the foal can never be expected to be either healthy or strong in constitution. The period of going with foal is eleven months. After the sixth month, great care and gentleness should be exercised toward them. Moderate exercise is essential. Hard work in harness, over bad roads, is likely to produce abortion, and mares that have once aborted are very liable (if the same causes are in operation) to a recurrence of the same.

The proper time for copulation is when there is a discharge of a whitish fluid from the vagina. She neighs frequently, and exhibits great desire for the horse. When she has conceived she shows no further desire, and the discharge from the vagina stops. Some writers recommend that the mare be put to the stallion early after foaling. This is bad policy, for the simple reason that the dam, if she become impregnated, has to nourish both the foal and the embryo. This is excessively weakening, and tells on either dam, fretus, or foal.

Disproportionate copulations are also objectionable, as, for example, putting a large horse to a small mare. When improvement in size is the object, it should be attempted by regular gradations. This is the best method of securing size and beauty, as well as strength. The best mode of preserving the breed and making improvements is, to make selections of the best on both sides. Much judgment and circumspection is necessary, at all times, in crossing the breed, and many errors arise in consequence of a want of knowledge on this subject.

When the period of foaling draws nigh, the mare should be separated from her companions. Having foaled, she should be turned into a pasture, if possible; otherwise, into a loose box inclosure, or empty barn. The foal may be weaned at the age of six months, more or less, as circumstances seem to require. A 
weak foal should run with its mother for a longer period than one that shows signs of vigorous health. Should the foal die at the period of parturition, humanity would seem to suggest that the mare be excused from duty for a week or two, by which time she will have recovered from the effects of parturition.

\section{The Principles of Breeding.}

It is a law of Nature that peculiarities of form, size, color, etc., shall be transmitted by parents to offspring, ("like begets like,") although, under certain circumstances, a modification of this law is to be expected. If, for example, we liberate an animal from domestication and its influences, which are known to operate very markedly on animal organizations and habits, the creature thus liberated loses its acquirements, and, in successive generations, gradually returns to the original type. This is a modification of the above law, and, supposing our pecuniary interests are the object of the experiment, it will be an improvement in the wrong direction.

On the other hand, take a wild animal; bring him under the influences of domestication, and he gradually loses all his distinctive characteristies of size, form, and instinct, and, in popular language, becomes a new creature, improved or not, as the case may be, under the direction of his lord and master; so that the inferior orders of creation are really the creatures of circumstances. These changes are the result of man's experience or non-experience. These are general propositions which "precede beauty and symmetry."

Beauty and Symmetry.-If we examine into the methods pursued by some of the most successful raisers of live stock, we shall see that they paid particular attention to the selection of wellformed, beautiful animals. 'They very naturally supposed that external conformation was transmissible; that if they happened to obtain a good calf or foal from inferior, diseased, or malformed parents, it was purely accidental, and out of the ordinary course of Nature. In selecting beautiful animals, they naturally excluded those of narrow chest, which peculiarity is indicative of predisposition to pulmonary affections and founder (the latter term signifying a worthless or ruined condition, which, in the eye of the law, renders them actually unsound), because they have that about them which may impair their future usefulness. Hence, for more 
reasons than one, such are unfit for breeding purposes, unless, however, the morbid phenomena be neutralized by blood, in one of the parents, of a superior order, free from taint, as it appears in the other.

In my adventures in search of the beautiful, I should pass by all animals having any peculiarity of conformation, general or local, which experience teaches me are sure to reappear, either directly or indirectly, in future progeny. This appears to be the proper course to pursue; yet many, otherwise intelligent men will invest their money in the purchase of defective animals, merely because they can be bought cheap, when every body knows, or ought to know, that cheap animals, like cheap watches, cost more, in the end, for repairs, etc., than a commodity of more real value. The same rule applies to horses. Some men, if they happen to be in possession of a broken-down mare, unable, from faults, defects, old age, and disease, to perform ordinary horse duties, commence a speculation in a different enterprise. The reproductive organs must be exercised. Just at this time some cent-and-dollar calculating jockey advertises and exhibits a well-fed, prancing steed, bearing a popular name; but his whole carcass is redolent of faults and defects, which the owner is not expected to know any thing about, and if he does, it is not for his interest to point them out. The price of copulation is dirt cheap, and the foolish speculator expects to obtain a smart colt, that will eventually command a high price. But, alas for such milk-and-water calculations! the expected specimen of equine animality inherits and exhibits its parental infirmities and deformities, and ultimately becomes a living monument of the folly of its owner.

There is no beauty in the faults and defects which must necessarily occur through the sexual congress of faulty and defective animals; neither can there be grace in the motions of an overfed or ill-conditioned animal. Beauty, symmetry, grace, and soundness are defunct in the ill-conditioned creature, or otherwise defective one-a mere apology for the handiwork of Nature. The muscular system of a horse or cow may, in some regions of the body, be well-developed; but should their limbs be unnaturally curved, or any part of their system be out of proportion to the other parts of the body, then beauty has never seated itself on their thrones. Yet we can improve the appearance of such animals by artificial means and costly adornments. This artificial 
kind of beauty is what most men seek after, and, at a high price, to their sorrow, obtain it; whereas the real beauty of form and endowments, which practical husbandmen really require, must originate unartificially in the living citadel-must be the work of Nature and not of art.

It is a fact, well known to many men, that animals of faultless form, having symmetrical proportions, are exempt from many diseases which are the heritage of faulty and inferior animals, and insurance companies take advantage of this knowledge, and insure the former at less rates than the latter. As an illustration of the above, I may be permitted to remark that the "Arabian Courser," English "blood horse," American "Black Hawk," and pure "Devon" cow-all remarkable for good points, beauty, and endurance-are exempt from many of the common and unnecessary diseases and abortions. Beauty and symmetrical proportions contribute to health, because there is a certain degree of relationship between external and internal organs and functions. For example, a fine exterior, good depth of chest, well-proportioned muscles, and graceful limbs are generally coëxistent with good "wind and bottom," and the subjects, of course, possess active respiratory organs, a finely-balanced circulation, and 2. digestive apparatus that ean digest every article in the shape of fodder. In selecting the beautiful, we therefore obtain material for perpetuating health, long life, and valuable offspring.

One of BLAKEw ELL's great secrets was, "Breed from the beautiful." I allude to him because he was the most successful "breeder" that England ever boasted of. At the onset he paid great attention to "beauty of form." Having developed a fine exterior, he engrafted on it the useful. He was well aware that beauty and utility were not always combined, but, being in possession of the former, he could produce the latter to "order ;" and he accomplished his object in the following manner. He required cows that would yield a large quantity of milk; consequently he selected those whose dams had long been celebrated for their excellent milking qualities, and from among such he chose the very best female of the family, and united her with a beautiful male. Having, in the production of offspring through the above union, accomplished his object, he then paid less attention to beauty, and more to the milking qualities, so as to render the latter permanent in the breed. Still, in all his experiments he rejected uncouth, 
unthrifty, and defective animals. I am aware, however, that the evils of domestication are operative, to a certain extent, on all animals. Still, I contend that the "well-bred" animal can resist the insidious encroachments of disease, and survive longer under its depressing influence than "ill-favored" creatures. Illustrations are not wanting to prove the correctness of BLAKEWELL's theory; therefore, I advise the reader to follow the example of one who has been styled the "Napoleon of breeders."

\section{IN-AND-IN BREEDING.}

We now inquire, What is breeding in-and-in? I answer, It implies consanguinity-breeding from animals of the same blood, or propagating in a close degree of relationship. Some persons have an idea that this system is pernicious, and leads to degeneracy and premature decay; but that is a matter of argument, and, as I shall attempt to prove, depends on the skill of the "breeder," and his ability to make wise selections. The human race-the sons and daughters of Adam and Eve-afford a stupendous illustration of the practicability of in-and-in breeding. The millions terrestrial all originated (so the Good Book informs us) from our common parents, and, consequently, we are all "blood" relations; and this fact goes to show that in-and-in breeding is in accordance with the laws of physiology, and does not conflict with the intentions of "Him who doeth all things well." It is a part of the great scheme of creation, a physiological law, the problem of life, to solve which God has endowed us with reason, "God-like reason," the exercise of which puts us in possession of the fruit of the "tree of knowledge." The non-exercise of the same furnishes us with forbidden fruit.

Ill-assorted marriages-consumption mating with consumption, and scrofula with scrofula-seeks at the altar an introduction to an early grave. The same is true of animals. Bad selections thwart the intentions of the Creator, mar his handiwork, and if a friendly epizoötic sweeps them from the face of the earth, it is more of a blessing than a curse. From the great human family I select a branch to illustrate this principle, and I choose to refer to the Jews. So long as they are so in faith and practice-marrying their own kindred-they are a living illustration of the above principle. Have they degenerated? No. The distinctive 
characteristics of the race are just as remarkable as they ever were. The vicious and defective find an early grave, but the "race" remains pure.

Now, as regards horses and cows, the same is true; they all had a common origin. The different varieties that now exist are the work of time, circumstances, and the art of man. The famous horses mentioned by Mahomet, in the "Koran," resulted from in-and-in breeding. They have existed and multiplied for three thousand years without the slightest intermixture of foreign blood, and, from the time of Solomon up to the present day, their pedigree has been watched and chronicled with great care, so that no doubt exists as regards their consanguinity. Their fine form, splendid action, endurance, spirit, speed, and docility can only be retained by preserving the race pure, and this is an argument in favor of in-and-in breeding.

No breed can be preserved pure unless the in-and-in system be pursued. Take the Suffolk pig, for example. So long as we put Suffolk to Suffolk we get " pure" Suffolk, and, if proper selections have been made, good Suffolks are the result; but deviate from direct lineage, and the breed degenerates, for better or worse, as the case may be, and they lose their permanency of type, and cease to become pure bloods.

'From the brave descend the brave.

The Suffolks in this country are notorious for a cutaneous disease simulating scrofula, and many suppose that this arises in consequence of the in-and-in system of breeding; but this I think is an error. It results from the evils of domestication, and our want of knowledge in making proper selections. There often is one or more animals in a litter incapacitated, by fault, defect, or debility, to perpetuate the stamina and remarkable points of the breed; these are to be rejected. If we fail to do so, the next generation, or the next to that, furnish more convincing proof of error, which I contend exists in making "bad" selections, and not in the above system.

Turn for a moment to the history of the French Merino, and we shall find that Victor GrlberT-a name familiar to America's most successful sheep raisers-practiced no other than the inand-in system of breeding. A lot of Merinos were sent, in 1786, by the Queen of Spain, to the King of France. The latter, in 
order to benefit the agricultural community, sent half of them to Rambouillet, and the other half to Croissy. The climate happened to suit them, and they were considered superior to the existing breeds in France. In the year 1800, VICTOR GILBERT bought at Croissy a four year old ram and eight ewes. He bred from those animals during a period of ten years, occasionally, however, "renewing the stock," as he termed it, by purchasing annually from two to four sheep. In 1821 he purchased some of the Rambouillet stock, and asserts that, "from a union of the same, he obtained great advantages." Now, the reader will perceive that there was an alliance of blood. The two flocks were of the same lineal descent, belonged to the same "family," and of course the system of breeding was on the in-and-in principle.

Now, as regards French Merinos obtained from the above source, and imported into this country, we have only to refer the reader to Tatntor, of Hartford; DeForrest, of New York; SAnford, of Vermont; Parker and Howard, of Ohio, and many others, who are ready and willing, we presume, to testify as regards the prosperity of the above breed in this country, the result of in-and-in breeding. S. W. JEWETT, of Vermont, has purchased, up to the present time, over three hundred bucks and ewes, the offspring of the Croissy and Rambouillet breed, and this is proof positive of the correctness of our theory.

Now, as regards the advantages of in-and-in breeding, what are they? When Victor Gilbert purchased the first ram, he carried but 9 pounds of wool; he finally realized 24 pounds from the rams, and from the ewes 18 pounds, a tangible illustration of the advantages. A very fine French Merino, originating from the above source, was very recently exhibited at a sheep-shearing in Wisconsin; the animal weighed 325 pounds, and carried 40 pounds of wool. Now, the originals, as I have already shown, carried but 9 pounds; so that the standard, as regards a particular quality, has been raised, and that is what we understand by "improvement."

Mr. V. BARFord, of England, has demonstrated the propriety of in-and-in breeding. He is a man, however, of superior judgment, and he culls and casts out from his flocks and herds all animals showing the least predisposition to any defect. A writer in the "Mark Lane Express" considers Mr. VAlentine Barford " one of the most successful in-and-in breeders of sheep in the king- 
dom; for he has bred from the pure Blakewell blood for sixtyfive years, without the aid of any other sort or kind, and bred from his own flock for upward of fifty years, maintaining size, weight, and constitution. I have known Mr. Barford's flock for upward of half a century, and they appear as strong in their stamina as when I first saw them. Mr. Barford does not use a ram unless he has a wide loin, a large breast and collar, and very wide between the forelegs, all of which points denote a hale constitution. Although in-and-in breeding has beat thousands to a stand, Mr. Barford still shines in it."

Therefore I contend that great advantages are derived from preserving the breed pure and in making proper selections. I have been very much pleased in perusing some remarks made by SANFORD How ARD, and published in the "New England Farmer;" and, as they happen to have a direct bearing on our subject, I here give them:

"What is breeding in-and-in? While some apply the term only to animals distantly related, he conceived the only true idea of the matter to be that it applied to creatures of the same blood. The consequences of breeding in-and-in, he believed, might be either good or bad, and depended wholly on the skill of the breeder. As proof that breeding in-and-in was not contrary to Nature, he referred to birds, the buffalo, ete., in a wild state. It is known that they breed in-and-in constantly, and yet no deterioration takes place. He had known geese to be propagated in this way for forty years, and not the slightest depreciation in size, quality, or feather was visible in them. Still cases could be cited where breeding in-and-in had produced bad results, yet these might be attributed to imperfections in the parent stock. It is only necessary to select perfect specimens."

Having now attempted to show that in-and-in breeding is in accordance with Nature, and that the birth of inferior and defective animals, under this rule, resulted from the breeder's ignorance or neglect in making proper selections, I shall next introduce some remarks of a general character.

Permanent Varieties.-In-and-in breeding should be practiced in view of some specific object; namely, perpetuating species capable of constantly and permanently transmitting characteristic peculiarities, such as may be observed in any particular permanent variety. As an illustration of the same, we offer the French 
Merino sheep, Black Hawk horse, and Devon bull. These are permanent varieties, the type of each having been established by a long course of in-and-in breeding. We are told, however, that many of the imported French Merinos (so called) have produced offspring scarcely worth the trouble of raising-far inferior to our common native stock. The principal reason is, many sub-races of Merinos, existing in France and elsewhere, are the result of cross-breeding. They are nothing less than mongrels, destitute of that permanency of type indicative of pure blood. But there are other causes for the deterioration complained of, which will be noticed hereafter.

By preserving the race pure through a certain period, we thus obtain the requisite permanency of type, which can then be transmitted indefinitely. Let us see, now, if we understand what is meant by a permanent variety, and inquire if such do really transmit their permanent peculiarities. A permanent variety (Black Hawk breed, or Devon cow, for example), are animals whose peculiarities were not coeval with the tribe, but have arisen, or been engrafted on the same, subsequent to the advent of their existence; and, therefore, what some may term distinct species are, in reality, nothing more than permanent varieties. The Shetland pony, therefore, is a permanent variety. Circumstances have made him just what he is. He, being an inhabitant of a stormy, tempestu- ous region, comparatively destitute of shelter and artificial food, is left, in many instances, like the cattle of Norway, (whose diminutive size is notorious,) to seek such food as the county affords ; and, owing to their being secluded from other horses, frequent intercourse among themselves have rendered their small and peculiar forms permanent in the race ; and, with unerring precision, "like begets like."

The permanent varieties that now exist among the cattle of the British Isles is due to local circumstances and the art of man. Most of the fine breeds now owned by English husbandmen are named after the location in which they are said to originate. These are permanent varieties, yet the most of them (in fact all, except importations) are descendants of the "Wild or White Forest breed." Hence the Highland breed, dispersed over the mountains of North Britain; the Alderney, of the Norman Isles; and the Devon, from the Bristol Channel, etc., are all permanent varieties, originally of the White Forest breed. Yorkshire is 
celebrated for the production of a permanent variety of horses known as "Clevelands." They are an ancient and unmixed race. Their bodies have peculiar markings, and if a foal should be born without them, the owner would give orders for its immediate destruction. I presume it is now understood what is meant by permanent variety.

\section{Animals become Parents too early.}

VICTOR GiLbert never allowed ewes to have lambs until they had passed their third year, and the bucks were never used until they had arrived at full maturity. He, as well as many other sagacious stock-raisers that I might name, are probably conversant with the fact that, during the period of growth and development up to maturity, the reproductive organs are dormant, while, at the same time, the nutritive function was wholly engaged in elaborating chyle and blood for the development of bone, muscle, and nerve; and that by calling into requisition the reproductive or generative organs before the animal had attained full growth, must necessarily divert the elements of matter intended for nutrition from their legitimate channel, and direct them to the reproductive organs. This is precisely what takes place. A too early use of the purely animal functions induces weakness and stunted growth.

The author remembers that, in his school-boy days, it was eustomary, so soon as a boy had accumulated a few pence, to invest the same in a rabbit (a favorite animal at that period); consequently we had a community of juvenile rabbit-raisers; and from the results of past experience, as observed by the older boys, a rule was adopted among us that the doe should not be put to the buck until she had attained full maturity. The reason assigned for this rule was, "Early breeding prevented the doe from growing." The facts were evident; still we knew not the why nor wherefore, but acted on the spur of experience. Physiologists have assigned the above as the true cause, and have, in their writings, pointed out the woeful results which often follow a too early use of the purely animal functions and organs. I seriously urge farmers to give this subject that attention which it evidently demands. By so doing they will prevent many of the unnecessary abortions which are almust constantly occurring. Among the higher orders of 
creation the same law holds good. In fact, there can be no deviation from it without incurring the hazard of paying the penalty.

Heifers should never be put to the bull until they have attained the age of three or four years. At this period they are in their prime. If they happen to have acquired too much fat, their daily allowance must be reduced.

Human growth, according to the best authority, ceases between the ages of twenty and twenty-five. In very warm regions, however, where development and decay are universally allowed to be more rapid, the inhabitants come to maturity much earlier. A superior class of human beings can only be produced by selections and exclusions similar to those so successfully employed in rearing the inferior orders. We may rejoice in a Fulton, Franklin, or Webster occasionally, (by mere accident,) the parents of such being absolutely ignorant of the first principles of physiology; but, in the breeder's language, such are in possession of the " prerequisites." In the first place, they had not entered the marriage relationship prior to the age of reason. The parents, if we mistake not, were full-grown men and women, not boys nor girls. They possibly possessed a sound mind and healthy constitution, free from hereditary defect of mind and body, which stunted growth, aided by artificial modes of life, are almost sure to entail. Until within a few years, a wise and salutary law was in operation in the British Isles which interdicted marriage until the candidates had arrived at the age of twenty-one. That law has been set aside, and, consequently, the mass of the population of the present day will not bear comparison with that of the past.

One safeguard, therefore, against stunted growth and ill-health is to avoid a too early use of the reproductive function. And herein we are not safe unless proper selections have been made, and faulty animals or persons rejected. I am persuaded that if husbandmen of these United States could all view this important matter in its right light, a very decided improvement would soon be the result. The subject will, however, eventually command the attention of all.

Many persons urge that the offspring of blood relatives are weak, nervous, deficient in intellect, complete idiots, etc., and that further intercourse in the same direction only makes bad worse. These are facts which can not be controverted, yet the weakness, nervousness, etc., does not occur from the relationship 
which exists between the parties, but is the result of their defective constitutions. Many of the cousin-marriages are entered into without the least regard to the rational theory of selection and rejection. The question is one of interest, not of utility. These blood connections are consummated merely to retain property in families, and to satisfy the caprices of doting parents, superannuated uncles, and disappointed maiden aunts; and thus many an idiotic or emaciated sprig of aristocracy unites his destinies with a fashionable cousin the very counterpart of himself, both inheriting family defects of precisely similar character. Should they be blessed (cursed, rather) with offspring, may we not expect a miniature of the originals? "Without due attention to avoiding disease, it will increase in the progeny."

Among the human species, therefore, where the objects are of such immense importance to present and future generations, the principles of selection and rejection are entirely overlooked. We pay more attention to the principles of breeding in cattle than we do to those applicable also to their lord and master. Hence the faults and defects alluded to are not the result of close affinities, but result from our indifference or ignorance of the laws of life.

\section{Objections to In-And-In Breeding Anstwered.}

The two preceding paragraphs are brief answers to the objections against the sexual congress of blood-relations; yet, as other evidence than my own, and of a more general character, may be demanded by the reader, I have thought it best to introduce other testimony. The following quotations are from the pen of P.S. Humbrickson, of Ohio, published as a "Prize Essay :"

"Many object to what has been termed in-and-in breeding. Indeed, with most of those who do so, their objections assume the form of a very strong prejudice. This is attributable to a certain confusion in their minds, by which reasons, wherein there is no proper connection, are made, with the aid of imagination, to assume a form of mutual dependence and coherence in support of their theory. In the human family, marriages within the Levitical degree, or, as they are also called, incestuous marriages, are forbidden. But the prohibition rests exclusively upon moral, and not at all upon natural or physical reasons; yet the direct physical calamities are imagined to pursue the infringement of that law. 
Now, it would seem that where the moral reasons in favor of an enactment are sufficient not only to justify but to demand it, no more should be required. And this is in accordance with the soundest rules of philosophizing. Moreover, if no such moral reason existed, the authority of the Lawgiver, in the case of the divine law, is a sufficient foundation and warrant for it ; and the human law is bound by and but follows the divine. Shall we, then, take the step beyond, and apply to the inferior animal the rule that was intended for rational, accountable man? We know that, if left to his own observation and experience, and being himself the judge, the objector must avouch the fact that, in the state of Nature, there is no restraint, and that such connections are and must be continually taking place.

\section{The Advantages of In-and-In Breeding.}

"And if there are advantages arising from the having placed in the line of the direct ancestry, near and remote, of our flocks, a great number of approved individuals, both male and female, as has been seen, it follows that there must be far greater advantages arising from the having the same one individual (if he be of marked superiority) placed in that line the greatest possible number of times. This is done by in-and-in breeding, and is the object of it. Now, it is easier to find this one unsurpassed individual than to find many; for in the many there will, most certainly, be one to be preferred to all the rest. Then, under the operation or the principle of atavism, the chances that the resemblance of such unequaled ancestor will be obtained must be in the ratio of the number of times that he occurs in the ascending lines; hence greater uniformity and greater excellence in all the progeny. An apt illustration of this is found in the frequent occurrence of the Godolphin Arabian, in the pedigrees of all our best blooded horses, carried back to him as their founder."

In conclusion, the author offers the following, in view not only of benefiting the horse, but his owner also.

The Importance of Studying Anatomy and Physiology.

There is an aphorism which is said to have emanated from a Divine source, and it appeals to the intelligence of this enlightened 
and progressive generation with peculiar force. It is, "Know thyself." To understand our moral, intellectual, and physical natures and tendencies should be the business of every one. The cultivation of, and proper direction given to, the former, bring us within the halo of health, purity, and peace. A knowledge of the physical or physiological laws of life, and in practicing fidelity to what they teach, places us on the high road to health and long life. Anatomical and physiological acquirements are needed by every one, in order that we may know ourselves, and thus be able to preserve our wondrous mechanism, "the harp of a thousand strings," in its normal condition; for without health we can not enjoy life, nor answer the purposes for which we were created.

We have the testimony of learned men, and our own reflective minds confirm the facts, that an alarming number of premature deaths, and an untold amount of physical infirmities, are the result of either our ignorance of, or indifference to, the uncompromising laws of Nature. The rational being, free from hereditary taint, of mental or physical deformities, comes into the world with all that is essential to his future life and happiness. He has within his organization a radiant volume of intellectuality, organized, compiled, and bound by the Divine hand, the first glimmer of which reveals something adapted to present and future wants and necessities. The intellectual spark, once ignited, is capable of an endless increase. We can add ray to ray, power on power, until the Grod-like man acquires the mental greatness of a Webster, or the mechanical skill and distinction of a Fulton. We do not expect that all can become Websters and Fultons, because we do not all practice that invariable perseverance and stern energy characteristic of giant intellect and mechanical skill. We are not all willing to toil, mentally or physically, with that perseverance and industry so necessary to success; and if we were willing, after having attained maturity or manhood, our minds and bodies being trained and molded for station and eircumstances, are then not so well adapted, as in youth, for increase and power. Still, at this period of life, we are not destitute of the means of self-culture.

We are living illustrations of progressive mental capacities which often transpire in men past the meridian of life, who, before that period, never considered themselves favored in this line. How unfortunate it is, therefore, that the generality of mankind should be so indifferent about the science of life, and be 
so slow in making it the basis of rational action in warding off disease, and in promoting the integrity of their existence! The reason is, our education is incomplete. We are all that Nature intended in elementary ability, and only deficient in its use. The means are anatomical and physiological studies; the time is in youth, when the mind is pliant, capable of receiving permanent impressions. The place for the engrafting of the same is in our common schools and at the fireside, the mother and the teacher wielding a common scepter of instruction.

Some people do not realize that our organizations are the result of the most positive laws of Nature, and that our ailments are the result of our own ignorance or folly. The mass of mankind are not aware that our physical systems are capable of improvement analagous to the mental. No; they generally think and act on the false and ruinous proposition that our diseases, aches, and pains are so woven into the filamentary mechanism of the living citadel as to be beyond the power of either art or science to eradicate-a proposition that should never, for a moment, be entertained; for, if we live right up to the laws of life, we are then within the impregnable ramparts of physiology, where our acquired and fashionable maladies can not obtain.

Within the bulwarks of physiology certain conditions are imposed upon us, and we must observe them. For example, we require a pure atmosphere, at all times, to vitalize the blood, and thus deprive it of those defiling elements acquired by venous blood, and which would otherwise operate, as they often do, in our crowded assemblies and unventilated sleeping-rooms, as the germs to excite unnecessary disease. Next, the body should be kept erect, so that the muscles and organs may acquire and maintain natural action. We require vigorous daily exercise of all the muscles of voluntary motion; freedom from all compression by dress; apparel that shall afford needed protection; a quantity of food and drink, at intervals, that shall furnish materials for the wants of our systems, and that control of feeling which enlightened reason and virtue demand. Our time should be distributed into daily periods of labor, rest, and amusement; and, above all, we must bear in mind that infirmity wedded to infirmity is a sin against our nature-a wanton violation of the law of Nature and of our existence, to which a fearful penalty is appended, even unto the "third and fourth generations." 
The faithful observance of the physiological laws of Nature result in health and strength, and promote longevity. Such is the fruit which the tree of knowledge beareth. The non-observance of these laws of life places us on a par with the poor idiot, when sporting with the implements of self-destruction, and furnishes to us the forbidden fruit. Health depends entirely on the use which we make of the various organs and functions of the body. Health never comes to us or our live stock by virtue of good luck. None are healthy by special Providence or Divine favor. Neither can health be found in the popular nostrums of the day on the proceeds of which quacks grow fat and wealthy, and patients often become poverty-stricken, and finally sink into the arms of Death, in blissful ignorance of what brought them there. Much of what is here written is equally true regarding horses and cattle. Many of their diseases spring from the same causes which are operative on the human subject; hence, knowledge of the above description is what all interested should seek after.

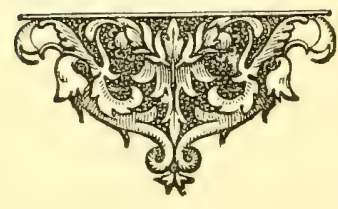




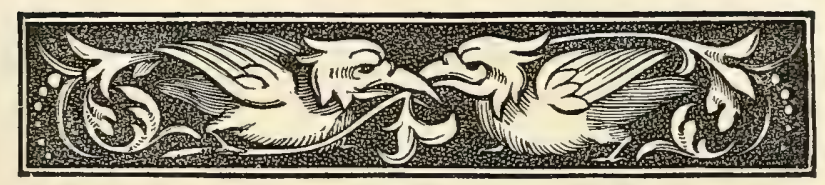

\title{
SECTION XII.
}

\author{
IAMENESS FROM VARIOUS CAUSES.
}

\begin{abstract}
Corbs-Elbow Tumor-Capped Hock-Varix, or Bog Spatin-Atrophy, or Wasting of Muscles-Thorough-pin-Navicular Disease, or LamenessPleuro-dynia - Acute Rheumatism - Chronic Rheumatism-Ring-BoneSplint-Spavin-Osteo Porosis-Curvature of the Spine-Hoof-BoundLaminitis-Sandcrack-Quarter Crack-Navicular Thritis-Stifle OUT -Contraction of the Hoof-Bowed Legs-Corns-Lameness.
\end{abstract}

\section{Curbs.}

CURB consists of sprain of the posterior ligament of the hock, known as the calcaneo-cuboid ligament. It is thus named in consequence of being inserted into the oscalcis and cuboid bones. The tumefaction is usually confined to the skin and subcellular tissue. At first the part is hot and painful, and the animal is lame-does not flex the hock in a natural manner; yet, when standing still, the horse keeps the lower part of the limb flexed, so as to relieve the overstrained tendon. The liability or predisposition to the disease lurks in breed. Such horses are observed to have ill-shaped hocks. Such have been denominated "cowhocks." The parts are not symmetrical. There is an obvious disproportion between the width of the parts above and below the hock, which gives it an angular appearance; and the more angularity there is, the more liability is there to sprain, because there is too much tension on the calcaneo-cuboid ligament.

Causes.-The exciting causes of curb are sprain, occurring in the act of backing a heavy load, or in leaping; in short the performance of any feat that violently calls into play the flexor muscles and tendons of the hind extremities; hence stallions with faulty hocks, when used for procreation, are very apt to become the subject of curb. 


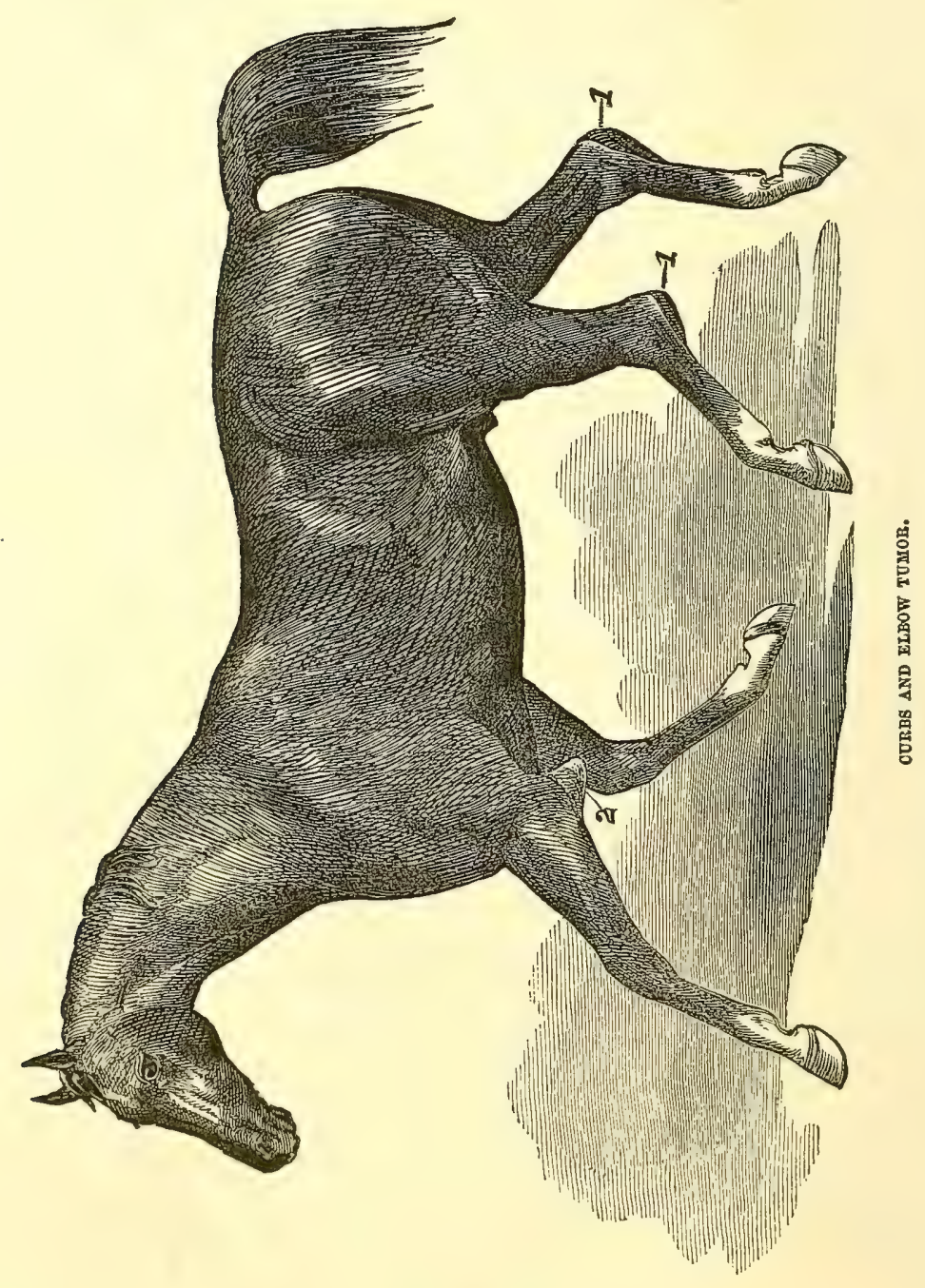

ExplaAation.

11. Curbs, or enlargement in the region of the back or posterior part of the hock, an affection known to veterinarians as sprain of the calcaneo-cuboid ligament.

2. Elbow tumor, or enlargement at the point of the elbow. The point of the elbow corresponds to the olecranon of man. 
Treatment.-So soon as the injury is discovered, the part should be fomented often with either an infusion of hops or poppyheads. It is best to apply the infusion cold, and by means of a sponge, the animal being kept at rest during the acute stage. Sometimes, in view of relieving the tension of the sprained ligament, it is customary to tack on a high-heeled shoe. This may or may not be necessary, and depends on the severity of the case. After having modified the inflammatory action, the high-heeled shoe may be dispensed with. Then, in order to get rid of the chronic enlargement, the part must be daily anointed, for a short time, with a portion of the following:

No. 45. Powdered iodine................ $1 \mathrm{dr}$.

Simple ointment................ $7 \mathrm{dr}$.

Mix.

After a short time, the animal may be put to light work, still making an occasional application of the iodine ointment.

Should the parts about the hock degenerate into a hard callous, friction with cod-liver oil may be employed. Should this fail to have the desired effect, then make a few applications of the acetate of cantharides, prepared as follows:

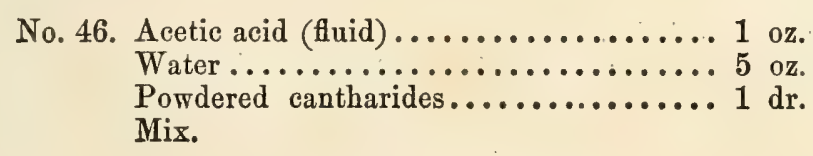

\section{Eubow Tumor.}

Elbow tumor (see cut on preceding page, fig. 2,) is usually recognized as "capped elbow." It sometimes grows to an enormous size, and not only becomes unsightly, but seriously interferes with the action of the elbow and its articulation. At first, the part is hot, inflamed, and tender. Soon effusion takes place; then the part is soft and fluctuating; next, albumen is deposited, and this, finally, is metamorphosed into fibrine, at which stage the tumor is large and dense; the walls of the skin are also very much thickened ; in fact, when dissecting out the tumor, it appears that the skin is in a state of hypertrophy, or morbid growth. However, there is usually a central orifice in the tumor which seems to indicate that the first trouble originated in a bursal sac, from external injury, either by the calking of the shoe or the toe of the 
hind foot; or it may arise from a bruise inflicted in the act of lying down on a hard floor.

Treatment.-In the early stage, and when there is nothing but fluid in the sac, an opening should be made into the lower part of it, by means of a thumb lancet. After the fluid has been evacuated, by pressure, inject into it an astringent, composed of pyroligneous acid and water, equal parts; and rub the exterior of the tumor, twice daily, with a small quantity of cod-liver oil. The cod-liver-oil will act as a glandular stimulant, and thus aid in the absorption of the tumor. A seton, inserted through the tumor, sometimes has a very good effect; but if the tumor be large and unsightly, the services of a surgeon will be required for its removal, by means of the scalpel.

\section{CAPped Hock.}

Capped hock consists of tumefaction, or enlargement at the point of the hock. Occurring, as it does, at a very prominent and exposed part of the limb, it is almost sure to attract notice; hence is not only an eye-sore, but a serious defect, and, if accompanied by lameness, the animal may be pronounced unsound, during the period of lameness. The tumor is the result of enlargement of a synovial sac which lies between the bone and the tendon gastrocnemii. This sac contains synovia, and it is furnished for the purpose of lubricating the parts for the prevention of friction. The skin, also, becomes thickened, or, rather, is in a state of hypertrophy (morbid growth), which add largely to the size of the tumor.

Causes.-Capped hock is generally produced by a blow or bruise. It is almost always occasioned by the whiffle-tree coming in contact with the parts. The animal may, however, injure the parts in the act of kicking. The parts may also be bruised in the act of lying down or rising in the stall. Therefore, not knowing any other cause for capped hock, we may safely infer that it is the result of external injury.

Treatment.-In the early or inflammatory stage, refrigerating lotions are indicated. Take of

No. 47. Sal ammonia.................. 1 oz.

Nitrate of potass................ 1 oz.

Water...................... 1 pint. 
Sponge the part quite often, for a day or two, until the heat and attendant pain subsides; after which the affection will run on to the chronic stage, and perhaps acquire magnitude; then the treatment must be altered. The object now is to excite absorption. In that view, I recommend the following:

No. 48. Powdered iodide of potass......... $2 \mathrm{dr}$.

Yellow basilicon............... 1 $1 \frac{1}{2}$ oz.

Mix.

Apply a portion of this ointment to the enlargement, morning and evening.

\section{Varix (known as Bog Spavin).}

Varix, or "bog spavin," consists of an enlargement on the inside of the hock, corresponding to a disease of the same character in the human subject known as varicose veins. It very rarely produces lameness, and most writers contend that it is nothing more than an eye-sore. Be this as it may, it is a defect, and, in the eye of the law, constitutes unsoundness, simply from the fact that soundness consists in perfection of function and structure, and, also, because sound horses do not have defects of this character. The enlargement does not generally impair the usefulness of the animal for ordinary work ; yet sometimes, in consequence of overwork or sprain, the tumor acquires magnitude.

It is always dangerous to attempt to remove varix, or to puncture it; and the only proper method of treatment, in view of limiting its growth, for it can not be cured, is to bathe it often with the following astringent lotion :

No. 49. Fluid extract of witeh-hazel....... 2 oz.

Fluid extract of bayberry bark...... $4 \mathrm{oz}$.

Proof spirit.................... 1 pint.

Mix.

Bog spavin is an indication of weak and faulty hocks, unless it can be shown that the animal has been made to labor too early in life, or has performed excessive work beyond his capacity. Then the affection can be accounted for, and may not owe its origin to either fault or defect. 


\section{Atrophy, or Wasting of Muscles in the Region of the Shoulder-blade, (known as Sweeny.)}

Atrophy signifies wasting of muscles, in which diseased parts become notably smaller than those of the natural, without other alteration in texture. The author has had several opportunities of examining horses that have died in consequence of disease or old age, who, during life, were the subjects of what is known as sweeny, and found that the muscles of the shoulder had quite a pale appearance, somewhat resembling those of a calf which had been bled to death. A condensation or wasting of muscular fiber was very apparent; the cellular membrane under the skin had almost disappeared, merely by condensation and contraction of the skin over the region of the parts, which contraction occurs in consequence of shrinkage of the muscles beneath the skin.

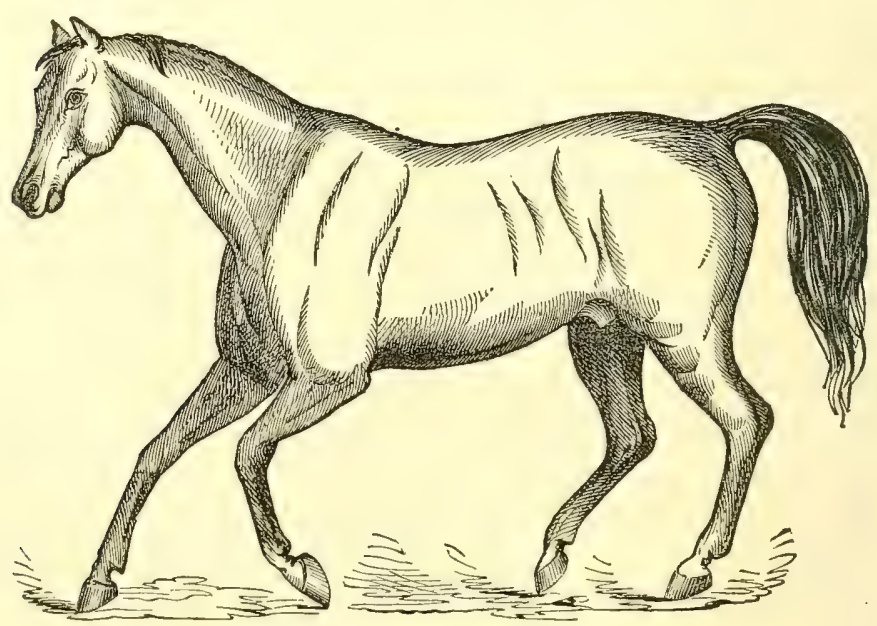

A FOUNDERED OR DEAD LAME HORSE, (See p. 336.)

Causes.-As regards the cause of atrophy, it is a well-known fact that inaction of a muscle produces a notable decrease in size. The muscles of laboring men decrease in size whenever, from choice or necessity, the individual leads a sedentary life. A limb kept at rest for any length of time undergoes more or less atrophy (wasting). In some cases, the foot diseases, so common, are of that character as to cause the subjects much pain in progression; 
and, in order to relieve the feet, the horse spares the muscles, which, for want of free and full action, leads to diminished nutrition and wasting (atrophy). Some of the readers of this work must have noticed the fact of the shrinking of the muscles of the haunch in case of disease or severe lameness of one of the hind feet. This shrinkage often occurs, as in the case of the shoulder, from want of natural action and full play of the muscles concerned. We have ample proof that wasting is the result of want of action, in the fact that when a horse becomes the subject of a painful disease in the hind foot, he neither advances nor rotates the limb any more than he can possibly help, but holds the foot up, sometimes points it. On examination, the atrophy, or wasting, is confined to the muscles which perform these two actions; and in the case of pain or lameness in one of the fore-feet, the same faulty action is observable, and the same class of muscles (whose action is almost identical with that of the hind parts) are the ones affected.

The author deems it of great importance that husbandmen should fully understand this subject; for it will enable them to perceive that, while inflating horses' shoulders with a quill, or practicing any other absurd treatment in the vicinity of the wasted or sweenied shoulder, they are overlooking the real malady (in the foot), and at best are only treating symptoms. On careful examination, however, should it appear that the patient is free from any disorder of the foot or feet, and, on the other hand, it is clearly evident that myositis or myalgia (muscular pain) exists, then the treatment must be directed to the affected parts. The facts in either case can only be determined by a competent practitioner.

The author has often noticed that when horses have been overchriven or overworked, they become stiff and lame in those parts of the body most susceptible to the ordinary influences. For example, one horse may perform a long journey, with a weighty man on his back, and the next day show symptoms of lameness in the lumbar region. His back is arched; the limbs are brought under the center of the body, and every movement indicates that the animal suffers tormenting pains, which are located in the muscles of the back. This is myositis, or inflammation of the muscular fiber. Sometimes, however, the malady constitutes myalgia (muscular pain and spasm), unaccompanied by inflammatory action. Another horse may be put to a similar kind of work, and, in a 
few hours afterward, be found stiff and lame. But the symptoms are not the same as those just recorded. The pathology is probably identical ; it is the same disease, only it has a different locality. It is myositis of the pectoral muscles, involving, also, the muscles of the shoulder-blades. And the intelligent owner of the afflicted animal will notice that the fore extremities are unnaturally advanced; the foreparts of the body are unnaturally hot and tender ; the fore-feet are feverish, and the hoofs are hotter still. The advanced position of the fore extremities and shoulders gives a very marked hollowness to the forepart of the chest, and many men, on seeing such a case, would declare that the animal was chestfoundered, this being the name which is sometimes given to such a condition; but it is really owing to myalgia (muscular pain and stiffness).

It is well-known that all museles are subject to inflammatory action and muscular pain from work disproportionate to their strength. For example, let any man undertake to saw wood, practice with heavy dumb-bells, or ride on a horse-feats that he has never been accustomed to-and, whether the labor be excessive or not, the individual will shortly complain of more or less muscular pain; and if he be a weak man, the more excessive will be that pain. Now, the muscles of the horse, being just as susceptible to pain as those of man, are just as easily operated on through the well-known exciting causes.

Symptoms.-In the case of a horse, when the work has been excessive, and of a character to bring the muscles of the shoulders, their tendons and coverings, into a state of over-exertion, it will often be found that some of the muscular fibers in the region of the shoulder are fractured, small blood-vessels are ruptured, and other pathological changes take place. This is followed by inflammation (myositis). Suppose, therefore, a horse shows symptoms of myositis after excessive work, and, in the course of a few days, it is noticed that the muscles of his shoulders are wasting away, and continue to waste, so that all persons who examine the animal pronounce him sweenied, we may then infer that the wasting (sweeny) is not symptomatic, but is the result of myositis.

Treatment.-The principal treatment is rest; afterward diminish the work, and, by proper diet and tonics, increase the power to do it. The muscular parts affected should be bathed, morning and evening, with a portion of the following: 
$\left.\begin{array}{l}\text { No. 50. Fluid extract of wormwood...... } \\ \text { Fluid extract of poppies....... }\end{array}\right\}$ each $2 \mathrm{oz}$. Proof spirits.................... 1 pint. Mix.

Should the feet prove to be very hot and feverish, frequent bathing with cold water, or a cold infusion of hops, will have a very good effect. In the mean time give the animal two drachms of fluid extract of gelseminum, once or twice per day, until the feverish symptoms subside. Then, when the case passes into the chronic stage (sweeny), the shoulders and wasted parts are to be well rubbed, often, with a portion of the following:

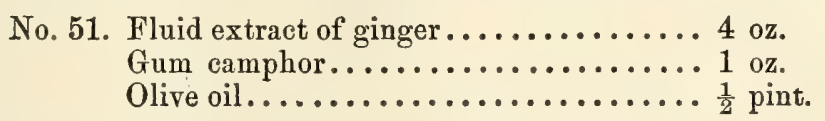

Pulverize the gum ; add it to the oil, in which it will soon dissolve; then mix with it the ginger, and the preparation is fit for use.

Should the muscles of the shoulder waste, in consequence of chronic disease or altered structure about the hoof or within it, such as ring-bone, ossification of the lateral cartilages, etc., then a strong counter-irritant must be applied, for a week or so, around the coronet. At the same time we should treat the shoulder as above. A good counter-irritant for the coronet may be thus prepared:

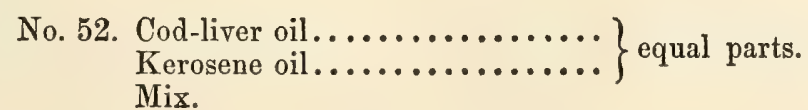

The great trouble in chronic cases of this character is, that it usually takes a long time to free the animal from lameness, and to restore the muscles of the shoulder to their original size; and, after all we can do, it may be necessary to let the animal have a run at grass. It will be advisable, in all chronic cases, unless the horse be very lame, to insure regular exercise, which will tend to develop the shrunken muscles.

The best way to prevent sweeny is to keep the horse in good condition by a rational use of his muscular powers, being careful not to overtax them, and by giving proper attention to his feet, both in the stable and at the blacksmith's shop. 


\section{THOROUGH-PIN.}

The seat of thorough-pin is between the popliteous (fig. 2) and the point of the hock, near where the tendon is severed, as shown in the accompanying engraving. The disease is called thoroughpin, simply because the fluid contained in the bursal sac can be squeezed from one side to the other.

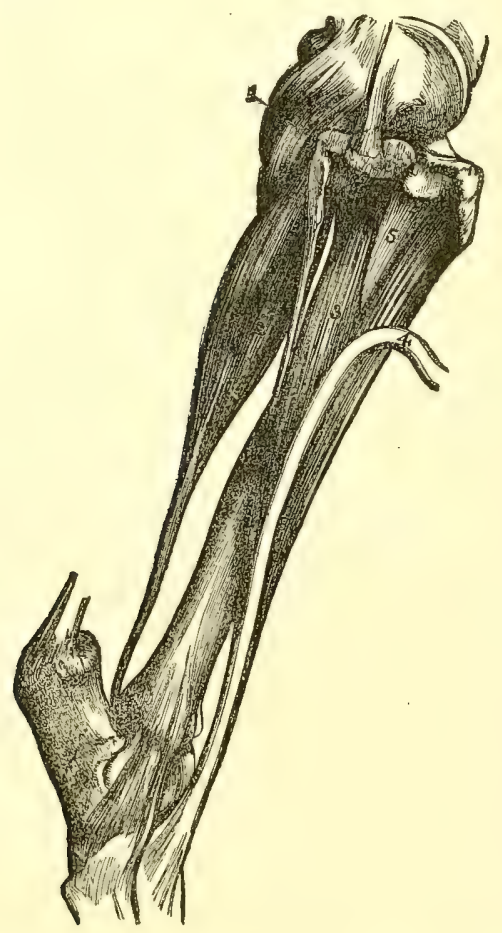

VIEW OF SOME OF THE DEEP-SEATED MUSCLES IN THE REGION OF THE HOCK AND STIFLE.

Explanation.-1, Popliteous; 2, Flexor pedis accessorius; 3, Flexor metatarsi magnus;

4, The tendon, common both to the flexor metatarsi and extensor pedis; 5, The groove in which the extensor plays.

Symptoms.-The disease is similar to bog spavin and wind-galls; namely, enlargement of a synovial sac. It seldom if ever lames a horse, yet, when large, is apt to interfere with the free action of the joint.

In former years it was customary to open thorough-pins by means of a lancet, in view of evacuating the fluid; but many fatal 
cases having occurred, the practice is now discontinued. Among the many fatal cases recorded I select the following, which has been recorded in the "Veterinarian," by W. A. WHEATLEY, V.S. :

"The subject was a five-year old cart-horse, the property of the South-eastern Railway Company, admitted on the 1st of September, 1855, with an immense thorough-pin on the off hock. The animal being then in a very high condition, a support shoe was placed on the foot of the diseased limb, and the frequent application of cold water to the diseased parts resorted to, the diet being restricted to mashes.

October 6.-The horse was considered in a fit state for the operation. Accordingly, I had the animal secured by a side line, and first punctured the enlargement with the exploring-needle, in four different places; but, as the imprisoned fluid did not escape so readily as I expected, I determined upon opening the part with the lancet, which being done, the fluid escaped to the amount of a small tea-cupful. It was of a thin consistence, and of a pale straw color. I then applied bandages dipped in cold water, and kept the hock constantly wet.

October 7.-The swelling had very slightly returned. I gave some aperient medicine, and continued the bandages with cold water, and applied a suitable truss to the hock.

I need not give a report of the case from day to day. Suffice it to say that the cold water and the truss were continued in use, and the animal never manifested the least inconvenience from the operation.

November 21.-He was considered cured, not the least appearance of the enlargement being left.

November 29.-On this day the horse was attacked with abdominal obstruction, and, despite all our remedial measures, consisting of active purgatives, as solution of aloes, calomel, oleum lini et crotoni, with the frequent use of medicated enemata, counterirritants, etc., he continued to grow worse till the 1st of December, when death took place. This was the fourth attack of this kind that the animal had experienced while under treatment for the diseased hock."

The danger to be apprehended in cases where an opening is made by the lancet arises from the liability to synovitis, (inflammation of the synovial membrane,) which reacts sympathetically on the system, producing derangement of some of the organs or func- 
tions of the body. Therefore I consider it injudicious to lay open a synovial capsule in any part of the body.

Treatment.-The safest method of treatment is to puncture the sac, by means of small needles. After having evacuated the fluid through the sieve-like needle-holes, the part is to be smeared with ointment of iodine-one drachm of iodine to seven drachms of simple ointment. Then apply a spring truss, so constructed as to press on both sides of the hock. By this means the internal parts of the sac are kept in contact, and they finally unite. Thus the enlargement is obliterated. If, at any time during the treatment, the parts become hot or tender, discontinue the ointment, and substitute a pad on each extremity of the truss, which are to be kept constantly wet with cold water until the inflammatory action disappears.

\section{Navicular Lameness}

Navicular lameness is of very frequent occurrence among horses. It usually depends upon strain or injury of the tendon, known as perforans, just where it passes over the navicular bone. Sometimes the navicular bone becomes diseased, ulcerates, and finally becomes fractured; at other times the bone becomes the seat of incrustations, or roughing, and thus irritates the flexor tendon. Such affections always cause pain and lameness. Horses with narrow heels, or those laboring under contraction at the quarters and heels, suffer much pain in the posterior or navicular region, in consequence of hoof pressure on the sensitive parts of the foot.

A disease known as navicular thritis (inflammation of the parts in the navicular region) often prevails. This is an inflammatory affection, very painful, and much resembles laminitis) fever in the foot).

Symptoms.-Pointing of the affected foot; the animal, while standing, throws the affected foot forward, by which means the weight of the forepart of the body is transferred to the other limb; the animal has a short, tripping gait, and steps cautiously; the muscles of the shoulder waste; the spine of the shoulder-blade becomes quite prominent; and this condition of the muscles is often called sweeny. We usually find, also, that the heels are pinched in, and the walls are thin and weak. The liability to navicular lameness is very great in horses with narrow chests, upright pasterns, and outturned toes. Other defects in the loins exist. Hence 
it is supposed that a predisposition to this affection may lurk in the breed; in fact, it is known that a tendency to it exists in the progeny of certain horses. It is related that the progeny of "Young Musician," a thorough-bred horse, all showed a tendency to navicular disease.

Treatment.-We first ascertain whether the difficulty is of an acute or chronic character. Should it have made its appearance very suddenly, and the part feels hot and painful, then we class it as the acute stage. Should the trouble, however, be of long standing, it will be in the chronic stage. In the acute stage, cold water bandages must be applied to the region of the fetlock, which should be kept wet with water, to which may be added a little vinegar, merely to acidulate the former; the patient should be kept at rest, and fed on a light diet. In the chronic stage, counter-irritants are indicated, yet organic disease may be the result; in that event, the horse has always a sort of groggy walk, and is never sound.

Neurotomy has been practiced in view of treating this affection, but I can not perceive how such an operation can benefit a horse having an organic difficulty. Neurotomy merely destroys, for a short time, the sensibility of certain parts of the foot; but so soon as the divided nerve reunites, sensibility is again restored. There may, however, occur some form of lameness which might be benefited by neurotomy, but I think the same thing might be achieved by counter-irritation, and without the use of the knife. The counter-irritant I have found most useful is as follows:

No. 53. Cod-liver oil ................... 2 oz.

Kerosene ..................... 3 oz.

Cantharides ................... $1 \mathrm{dr}$.

Mix.

Apply a portion of the above to the navicular region, between the heels and fetlock, on the back part, once or twice daily, until the part is well blistered, which will take place at the end of a week. The more thorough the blistering, the more chance is there of doing good. No after-dressing need be applied, unless the parts become very sore; in that event, I should lubricate with olive oil.

It will be necessary, in chronic cases, to secure the services of a good blacksmith, so that the animal may have a shoe affixed properly adapted to the necessities of the case. If any signs of 
contraction exist, the heels are to be opened, and the walls at the quarters must be rasped thin, so as to remove the lateral pressure on the sensitive parts.

It may be necessary, in the event of the patient not being benefited by the above treatment, to reapply the counter-irritant; this matter is left discretionary with those who take the case in hand. In the treatment of such cases, the owner of the afflicted animal must be prepared to exercise due patience, for the day of recovery may be far distant; often it is a mere question of time, and not one of skill.

\section{Pleurodynia (Painful affection of the Pleura and Muscles of the Chest).}

Pleurodynia (in human practice) signifies pain in the side, from a rheumatic affection of the pleura. In horses the malady not only affects the pleura, but also the intercostal muscles. It is a very painful malady, and some persons are in the habit of calling it "founder." Pleurodynia, however, differs in its origin from founder, inasmuch as the latter may arise from various causes, whereas pleurodynia is almost always the result of an excessive use of the muscular powers, or when the animal has been subjected to long-continued and very severe exertion.

Symptoms. - It will be noticed that the animal moves in a very stiff manner; steps short and quite slow-he appears about used up, as the saying is; the back is arched, and the skin is over the service of the body, and is not only hot but very tender; on making pressure over the regions of the sides of the chest, symptoms of pain will be elicited, showing conclusively that the intercostal muscles between the ribs and pleura, or lining membrane of the cavity of the chest, are involved; the respirations are shor't and jerking, and the number of pulsations range from forty to sixty per minute. Both fore-feet are usually advanced, and, on applying a hand to them, it will be found that they are unusually hot, and that they are inflamed; the appetite is not good; the urine is high-colored, and the bowels constipated. Such are the most observable and common symptoms of this painful malady. It is something like rheumatism in its pathology, and often migrates to the brain, or its investing membranes. When this happens there remains but little hope for the patient. 
Treatment.-In view of illustrating the treatment, I introduce the following case: The animal had been hired of Mr. P- of Chicago, by a person who appeared to have but very little respect or compassion for horse-flesh; for on the return of the animal to the stable, he showed unmistakable evidences of shameful abuse. He was completely jaded, and could scarcely stand on his feet. He received proper care and attention during the night, and next morning I visited him. On making a careful examination, I found most of the above symptoms present. It seemed, however, that his feet were more affected than other parts of the body, and it was impossible to get him to move. I had his feet bathed constantly with cold water, and his joints, back, and sides were rubbed with a liniment composed of equal parts of cod-liver oil and spirits of camphor. The medicine administered was one ounce of powdered niter, night and morning, for three days. The pulse had decreased to thirty-six per minute, and the respirations were more tranquil; so I discontinued the niter, and commenced an alterative plan of treatment, by giving one ounce of fluid extract of stillingia, night and morning, at the same time continuing the local applications until they were no longer needed. One ounce of fluid extract of buchu was occasionally given, in view of exciting the kidneys to action, and this is all the medicine the patient received until convalescence was established, when he was placed under tonic treatment. The tonic used was one ounce and a half of fluid extract of golden seal, daily. The treatment occupied a period of two weeks, during which time the animal stood but little on his limbs, seldom rising except to partake of food, which consisted of bran and water, a few oats, and small quantities of hay. So soon as the patient had regained some strength, he was led to pasture, where he soon got well. It appears that in the treatment of a disease of this character, light diet, a few simple medicines, and good nursing are all that are indicated.

\section{Acute Rheumatism.}

The faithful servant of man, the horse, does not enjoy immunity from this distressing disease. The same causes which operate on the system of man to develop a disease of this character are, with unerring certainty, operative on the inferior creature. Rheumatism is mostly confined to the fibrous tex- 
tures, or faschia of muscles, and the inter-articular cartilages of the joints, and, finally, the articulating surfaces of the bones become involved. The disease is always characterized by great

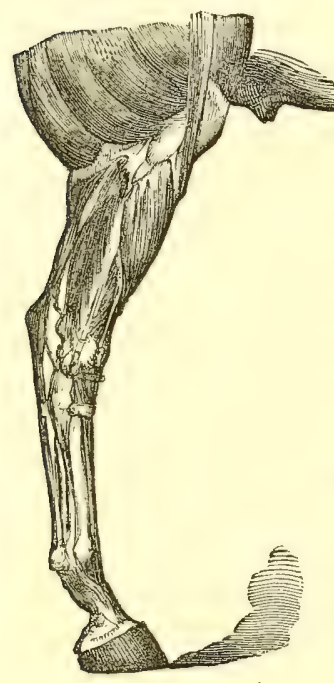

EXTERNAL MUSCLES AND TENDONS OF TIIE OFF-IIIND LEG. pain, and sometimes swelling in the region of joint. It is accompanied by a quick and wiry pulse, and by other symptoms, denominated febrile. It occurs among men and horses at all seasons of the year, yet at the period of sudden transitions from heat to cold it is most prevalent. Animals when heated by exercise, and then suffered to "cool off," without ordinary care, are very apt to become the subjects of this malady, so that prevention, to a certain extent, is within our power, and, in the exercise of preventive measures, we may, in common parlance, "stave off," for a time, this disease, although it may be latent in the system as an hereditary affection. Mr. F. Dun, who is good authority on the subject, says:

"Rheumatism is neither so common, nor are its symptoms so well marked, in horses as in cattle. When, however, it does occur in the horse, it manifests the same well-known appearances which characterize it in all animals. It affects the fibrous tissues of joints, the coverings of muscles, tendons, valves about the heart, and larger vessels, and manifests a peculiar tendency to shift from one part of the body to another, often affecting, in succession, all the larger joints-at one time chiefly in the neck, at another, in the back and loins, while, in many of its more acute attacks, it appears to involve almost every portion of fibrous and fibro-serous tissues throughout the body. In all its varied types it exhibits a full, strong, hard, and unyielding pulse, caused by the inflammation involving the serous and fibro-serous tissues of the heart and circulating vessels. During its existence, various excrementitious matters accumulate in the blood, and the fibrinous constituents of the same exceed their normal proportions, as indicated by the production of the buffy coat on the blood. In severe or badlytreated cases, the inflammation is very apt to be transformed from 
the joints and muscles to the heart, and its investing membranes, and it is the danger of this change in the seat of the disease that renders rheumatism so formidable, and often so fatal. It always leaves the parts affected so altered as to be extremely predisposed to subsequent attacks, and it is more than probable that this altered condition is reproduced in the progenies of rheumatic subjects, and constitutes in them the inherent tendency to the disease.

Horses sometimes suffer from rheumatic inflammation in the fibrous sheathing envelopes of the muscles of the neck, constituting what is popularly known as the chords. When thus affected, the animal is very stiff, remains as much as possible in one position, and is unwilling to bend his neck either one way or the other, or to elevate or depress his head. There is always more or less fever, with a strong, full pulse. Sometimes, as in lumbago, in the human subject, it affects the muscles of the back and loins, causing stiffness, tenderness, and pain, which are especially evinced on moving or turning the animal. These rheumatic affections are very readily produced in predisposed subjects by exposure to rain and cold, especially when accompanied by overheating or exhaustion.

Rheumatism sometimes occurs in horses as a prominent symptom of that epizoötic affection which usually receives the muchabused title of influenza. In such cases the rheumatism is of a somewhat more subacute or chronic character than common, and is accompanied by that low, debilitating fever so often the concomitant of epizoötic maladies. It usually affects all parts of the body susceptible of the rheumatic inflammation, is attended particularly by those symptoms which indicate disease of the heart and pericardium, as an intermittent pulse, etc., and often terminates fatally by effusions into the pleura or pericardium, thus causing death by arresting the motions of the heart."

Treatment.-In the treatment of rheumatism simulating an inflammatory type, our first object is to produce a sedative effect on the heart and its vessels of circulation. With this object in view, we administer one or two drachms of fluid extract of gelseminum every four hours, until the pulse becomes softer. In the mean time a few doses of nitrate of potass * should be given in the water

* Nitrate of Potassa.-In a case of synovial rheumatism this remedy was given by a Boston physician, in a single dose of one ounce, dissolved in a pint 
which the animal drinks, at the rate of one ounce per day, divided into doses in proportion to the number of times the patient is watered. So soon as the animal evinces signs of improvement, the above treatment is to be discontinued; then give six drachms per day of fluid extract of prince's pine. The joints which seem to be affected are to be bathed occasionally with camphorated vinegar, in the proportion of one ounce of camphor to eight ounces (fluid) of vinegar

\section{Chronic Rheumatism}

Chronic rheumatism is usually the sequel of the acute kind. It is generally obstinate and lingering, and prone to recur. Unlike the acute kind, pain is alleviated by counter-irritation and exercise; and when the patient gets warmed up, as the saying is, he either forgets his pains or becomes relieved. Bathing with warm vinegar has a good effect in the treatment of this disease, but I have found the following liniment useful in almost all the cases that have come under my care:

$$
\begin{aligned}
& \text { No. 54. Oil of cedar................... } 2 \text { oz. } \\
& \text { Sulphuric ether................. } 1 \text { oz. } \\
& \text { Proof spirit................... } 1 \text { pint. }
\end{aligned}
$$

First, mix together the oil of cedar and sulphuric ether; then add the proof spirit, and, after shaking it awhile, it is fit for use. Give the animal one fluid drachm of the fluid extract of colchicum-root, night and morning, for three days; after which give, daily, twenty-five grains of the iodide of potass, dissolved in half a gill of water. Should the animal show any signs of debility or loss of flesh, tonics, stimulants, and nutritious diet are indicated.

and a half of barley-water. This was followed by one grain of opium. In fifteen hours the pulse was found reduced, and the pain absolutely gone; and in a few days the tongue was clean, and the swelling entirely abated. The remedy caused neither emesis nor catharsis, but passed off by the kidneys. In another case of acute synovial attack, following chronic rheumatism, the same dose was prescribed without any good effect, causing active catharsis. Again, half an ounce of nitrate of potassa, largely diluted, was given every two to four hours, until the patient took three ounces in eighteen hours, with two doses of opium of one grain each, with entire relief to the pain and fever. When the potash was reduced to drachm doses, nausea and vomiting followed, which were relieved by resication with aqua ammonia, the blister being sprinkled with half a grain of morphine. The patient recovered. Another physician had used the remedy to the extent of half an ounce in twenty-four hours, for three successive days. 
The authorities teach us, and my own experience has confirmed the fact, that iodide of potass is a valuable agent in the treatment of chronic rheumatism. It is most sure to act beneficially when the periosteum (fibrous covering of the bones) is affected. There is no doubt that chronic rheumatism often cripples the joints by producing disease of the bones in the region of their articulations. This makes a poor horse dead lame, and renders him almost useless. When the bones become ulcerated or carious there remains but little hope of cure.

\section{RING-BONE.}

Ring-bone is a form of disease the pathology of which is similar to that of spavin and splent. It is located just above the coronet or crown of the hoof. The enlargement is called exostosis (a morbid enlargement of bone), and when the large pastern becomes united to the smaller one, the case is called anchylosis (permanent rigidity of the joint).

The term ring-bone is far behind the times as regards our present knowledge of nosology (the doctrine of the names of diseases); yet, to the unprofessional, it is suggestive, and, therefore, we are compelled to retain a name which all can interpret. It signifies a complete or partial eminence around pastern bones. The ring is formed by incrustations, or osseous deposits in the pastern, or involving the joint, as the case may be; yet, in order to make out a pure case of ring-bone, that comes strictly within the meaning of the term as horsemen interpret it, there must exist a complete ridge of bone around the anterior part, extending from side to side. A pure case of ring-bone generally originates on the surface of the bone, on or beneath the periosteum. Commencing at the lower margin of pastern and upper part of the coronet bone, the disease spreads, involving both capsular ligament and joint. The remote cause of ring-bone lies in hereditary idiosyncrasies, transmitted through the sexual congress. The disease itself is sometimes directly transmitted. I once bought an unweaned colt, and brought it up by hand. A short time after purchase, I observed enlargements on the hind pasterns. He ultimately turned out to be a ring-boned animal, and had stiff joints. I found out afterward that the mother had large ring-bones on both hind pasterns. The disease was, therefore, congenital.

Mr. Percivald, whose opinion on such matters is unquestion- 
able, says that his attention to the hereditary origin of ring-bone was first aroused from a remark made by an extensive dealer in horses, in reply to a question put to him, How it happened that but few ringbones were met with, compared to the number that attracted notice in times past? The reply was, "Because no breeder of horses nowadays will send a mare to a horse having ring-bone." A very good example for American horsemen to follow, for the disease is very prevalent in some parts of this country. A vast number of our best as well as inferior horses are the subjects of this infirmity. The disease lurks in breed, after the fashion of scrofula and consumption in the human subject. When both parents are affected, the disease in the offspring is doubly severe.

The author just quoted remarks that " a coarse or half-breed, fleshy or bony-legged horse, with short and upright pasterns, is the ordinary subject of this disease; and there exists satisfactory reasons why we should expect him to be so. The pastern and coffin bones constitute the nethermost of the column of bones composing the limbs, and being so, they receive the entire weight and force transmitted from above. The pastern, being long and oblique in position, receives the superincumbent weight on such an indirect line that, bending toward the ground with the fetlock, nothing like jar nor concussion follows. The very reverse of this, however, happens every time the foot of a limb, having a short, upright pastern, comes to the ground. In such, instead of the weight descending obliquely upon the sessamoids, and the fetlock bending therewith, it descends directly, or nearly so, upon the pasterns, making this bone entirely dependent on the bone beneath it for counteracting concussion; and should any thing occur to diminish this, or to throw more weight on the bones beneath than they can counteract, jar of the whole apparatus ensues; and an effort of Nature to strengthen the parts, by investing them with callous and ossification, is likely to be the ultimate result; for we would view ring-bone, disease though it must assuredly be called, a recourse of Nature to strengthen weak parts, the bones being unequal to the exertions or efforts required of them."

Another quotation may possibly interest the reader:

"Ring-bone is an exostosis (a growth of bone from bone) situated around the coronet, mostly near the pastern joint, at other times just above it; and not unfrequently the joint becomes anchylosed, 
owing to the spread of ossification; that is, the coffin bone and pastern bone become united together by bony growth. Ringbones are the result of hereditary predisposition, structural organization, and accident. As to hereditary predisposition, there is no doubt but that some sires, the subjects of spavin, ring-bone, and other forms of exostosis, father foals which often, sooner or later, themselves become victims of the same disease. The same law applies to dams. Breeders of horses are fully aware of this fact, and are, therefore, more careful than formerly in the selection of parents for the purposes of breeding from. As to form, many living cases which now exist prove the truth of the statement that low-bred, coarse-limbed horses, with short, upright pasterns, are frequently subjects of ring-bone. Why such should be the case with animals with upright pasterns is easily explained, as follows: The pastern bones in a well-formed limb ought to form an oblique angle with the cannon-bone (metacarpus) placed above them; whereas, if the pastern is upright, the column of bones from the elbow-joint to the foot is, necessarily, placed in one and the same straight line, causing, at every step made by the animal during progression, a severe concussive shock to be produced on the whole column of bones, but more especially on the pastern bones, owing to the superincumbent weight of the bones above them. Thus one or more of the pastern-bones become injured and inflamed, and, finally, as a consequence, exostosis ensues, the coronet becomes involved, and the disease denominated ring-bone is set up."

Mr. Pencrvall quotes from Gibson: "When a well-formed, high-bred horse happens to have a ring-bone, we may conclude it to proceed from some accident rather than from any natural fault. The disease may also be produced by a blow to the part, and from the efforts made to increase speed, when concussion causes injury, inflammation, and consequent exostosis to the bones of the pastern. As to treatment, we should recommend the proprietors of young horses, subject to ring-bones, to place their horses in a state of nature, by removing the shoes from their feet, and by turning out to grass. If this, after a fair trial, fail, then the treatment for spavin and other exostosis must be resorted to, such as blistering the affected part and keeping the animal on a low diet. No disease is more troublesome to treat, or more demands the strict attention of a skillful veterinarian."

Causes.-The exciting causes of ring-bone are ligamentary 
sprains, brought about by overwork, extraordinary feats of strength in drawing heavy loads, etc. In short, either sprain, injury, blow, or bruise may act as the pathological spark to bring about the discase.

Regarding the treatment of ring-bone, it would be interesting, and perhaps amusing, to call atterition to some of the heroic methods of combating this malady; but the animal has nerves of sensibility, and is as keenly alive to pain as one of us; therefore it is not really pleasant to dwell on the atrocities which are not unfrequently practiced on this noble animal. A very common error exists in the minds of some people that ring-bone is fed by a bladder at the posterior part of the pastern, which has about as much to do in the production of the disease as the author of this work. This error would not amount to much, however, only it has led to the infliction of a cruel operation, without the least advantage; namely, the cutting out of the bladder. Now, this socalled bladder is, in reality, a bursal sac, the use of which is to secrete synovia, to be used for the purpose of guarding against friction; therefore, the extraction of it must be disadvantageous to the parts.

It seems that ring-bone is not enough torment for the poor brute to suffer, but he must, in addition, submit to a species of cruelty unheard of in the annals of human medicine. Such treatinent is wrong, and every man who loves a horse should set his face against it, and use every effort for the purpose of putting a str $p$ to the practice of all barbarity in the treatment of domestic animals.

Treatment.-The question to decide, when we undertake to treat a case of this character is, whether the disease is in t'ie acute or chronic stage. Experience teaches us that nearly all $r$ iseases may be divided into two classes: First, the acute, or thosf which come on suddenly, attended by pain and fever, or, rather, ebrile symptoms; for there are no primary fevers in the horse. Equine fevers are always called into existence, or accompany a pa'hological condition which frequently existed, and the fever is $\mathbf{i} 1$ exact ratio to the intensity of the disease. Cure the disease and the fever ceases. Acute diseases also tend to termination within a short perrod of time, or else run into the chronic stage. Seco:Illy, the chronic stage. Chronic diseases are those of slow progress, and exceed in duration the ordinary existence of the acute class. They may arise in consequence of acute disorders, or be duveloped originally, 
in the form which they long afterward preserve. For the most part, chronic disease and lameness are not marked by any violent symptoms, nor are they accompanied with much pain, if the horse be kept at rest.

Suppose the disease to have first made its appearance, the parts will be hot and painful; but the idea of curing ring-bone-that is, restoring the parts to their normal condition-is really absurd; for, when once the bones are anchylosed, the joint never can perform its natural function again. The new growths (exostosis), and the changes of structure that occur within and about the region of disease, are a part of Nature's own handiwork, in view of strengthening a weak bone or joint. Our object in the treatment, therefore, is merely to aid Nature. Excuse the animal from work, and apply sedative lotions (cold-water dressings) so long as the acute stage lasts; then, when the case passes into the chronic stage, apply counter-irritants for a week or two, and, finally, turn the patient out to grass. The following will be found an effective sedative lotion:

No. 55. Spirits of camphor............. $2 \mathrm{oz}$.

Fluid extract of wormwood........ $1 \mathrm{oz}$.

Vinegar.................... 5 oz.

To be applied twice daily.

The following is an excellent counter-irritant for ring-bone in its chronic stage :

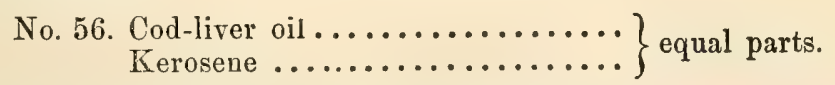

Apply once or twice daily, until a crop of pustules appear ; then omit the application, and reapply it at the expiration of two or three intermitting periods.

The remedies used by veterinary surgeons generally, are oil of cantharides, acetate of cantharides, blistering ointment, ointment of biniodide of mercury, and, lastly, the actual cautery, or heated iron. I have tried all except the latter, which savors too much of barbarity, and I never found any thing to equal the preparation here recommended-namely, cod-liver oil and kerosene.

\section{Navicularthritis (Navicular Disease).}

Navicularthritis is an acute disease, supposed to be confined to the navicular region, or joint, which is in the posterior part of the 
foot. The navicular bone (sometimes called the shuttle-bone) is so placed as to strengthen the union between the lower pastern and the coffin bone, and to enable the flexor tendon, which passes over it, in order to be inserted into the bottom of the coffin bone, to act with more advantage. There is a good deal of weight thrown on the navicular bone, and from the navicular bone to the tendon, and there is a great deal of motion or play between them in the bending and extension of the pasterns. It is very easy, therefore, to conceive that from sudden concussion, or from rapid and unrestrained motion, the delicate membrane which covers the bone, or the cartilage of the bone, may become diseased. The bone sometimes becomes ulcerated and fractured, so that it renders the horse almost perpetually lame.

Symptoms. - The principal symptom of this affection is pointing of the foot. If the horse be watched, when standing still in the stable or on the street, it will be noticed that the affected limb is advanced or pointed in such a manner as to relieve the navicular bone of any pressure from the flexor tendon, and this occurs in the chronic stage as well as in the acute. It will be observed, also, that the animal, when traveling, takes short steps, goes on the toe, and tries to favor the heel, or posterior parts, as much as possible. The favoring of the heel tends to destroy the function of expansibility of the hoof, and contraction of the same is the result.

Treatment.- Should the disease be discovered early, that is, in the acute stage, the foot should be placed in a boot lined with a sponge. The latter is to be kept constantly wet with a portion of vinegar and water, equal parts. This may be continued for a perior of twenty-four hours, at the end of which time bathe the coronet and heels with tincture of arnica. Light diet and perfect quiet, as in every other variety of inflammatory disease, are always necessary.

In old chronic cases we resort to counter-irritation, through repeated applications of the acetate of cantharides. Should there be any appearance of contraction, the heels of the hoof must be well opened, and the walls of the hoof, near the heels, must be rasped thin, so as not only to favor the expansibility of the foot, but also with a view to relieve the navicular region of any undue pressure. 


\section{Stifle Out (Dislocation or Luxation of the Stifle BONE).}

The patella of the horse, or stifle bone, as it is familiarly known, corresponds to the knee-pan of man, and answers the same purpose; that is, to facilitate motion, and protect the two extremities of the bones which compose the stifle-joint. It is retained in situ at the anterior part of the inferior extremity of the thigh bone and superior extremity of the tibia.

An accident of this character is generally accompanied with considerable pain and uneasiness; but the most notable symptom is, the bone is thrown off its pulley-like articulatory surface, and thus the recti and vasti muscles (the latter being direct extensors of the leg or thigh, and the action of the former being similar) are, for the time being, paralyzed; that is to say, their action is suspended. If they act at all, it is under great disadvantage, and at the expense of their muscular integrity. The liability to luxation of the stifle bone (patella) is not so great as some persons would have us believe. The bone is secured in its position by a number of strong ligaments-has a complete articulatory joint of its own, whose capsular ligaments have strong attachments to surround them. The muscles, tensor vaginæ, rectus, vasti faschia, panniculus carnosus, subcellular substance and integument, all operate favorably against the chances of luxation. The accident, however, does sometimes occur-not, perhaps, from any disarrangement of the mechanical order of the parts by violence, such as might be operative in dislocation of the thigh or arm bones, but often from spasm, cramp, etc., of certain muscles in the immediate vicinity of the parts.

"I think his stifle is out," is a very common complaint made by horse-owners when a horse becomes lame, and the "neighbors" can not discover the exact location of the same. They hastily conclude, if the lameness is in the hind extremity, that the "stifle is out" (luxated). Here is a case in point. I received a message some time ago, to visit a horse said to have " his stifle out." On making examination of the foot on the lame side, I found it much hotter than its fellow on the opposite side. The owner, at the moment, remarked that there was " no trouble in the foot." $\mathrm{He}$, however, was mistaken; for I found that a nail had entered the posterior part of the cleft of the frog, which I extracted, and this was the sole cause of lameness. I might relate a number of mis- 
takes of this kind, all going to show how very apt people are to be deceived regarding the seat of lameness in horses. In short, judging from my own experience, and the testimony offered by other professional men, it may be confidently asserted that luxation of the patella is quite a rare accident. These remarks are intended for the instruction of the non-professional reader. Persons conversant with the anatomy of the horse are not expected to make such a mistake as the one just alluded to; hence they do not stand in need of the author's advice.

Method of reducing dislocation of the patella.-The means of reduction are very simple. The patella will be found on the lower and outer condyle of the thigh bone. With one hand let the operator grasp the leg, just below the hock, and raise it from the ground in an upward and forward direction; the other hand embraces the stifle bone, and by a simultaneous action of pressing the latter inward, and the bones that compose the joint backward, the reduction is soon effected. The bone, in returning to its natural situation, emits a sound such as usually follows that of a dislocated bone just at the moment when slipping into the socket. A recent dislocation, when reduced, needs no after-treatment, except the occasional application of an astringent and a couple of days' rest; but, should the bone have remained unreduced for many hours, we may infer that the muscles and ligaments have been stretched or distended too long in one direction, and that, on reduction, the fibers of the muscles concerned may not immediately contract; hence the bone is apt to slip off its articulatory surface again. To guard against this mishap, a man must be stationed beside the horse. He must keep his hand on the bone, and by pressure, when the horse moves, retain it in its proper place. At the same time, the part is to be bathed with some astringent. An infusion of white-oak bark will answer; or, failing to obtain this article, apply alum-water, in the proportion of two ounces of alum to one quart of water.

\section{Contraction of the Hoof.}

Contraction of the hoof is so easily recognized by persons accustomed to handle horses, that very little needs to be written on that subject. A contracted foot "speaks for itself," as the saying is. The heels are approximated too closely, and this mars the beauty 
and form of the foot. Contraction is almost always, in its first stage, accompanied by lameness. Faulty shoeing is said to have considerable to do in the production of contracted feet; yet there are plenty of horses, having strong, thick, walled hoofs, very wide at the heels, often shod in the worst possible manner, and their heels never contract. It is certain, therefore, that a predisposition to contraction lurks in some breeds of horses, which is shown by their having weak quarters and a thin, shelly hoof. A contracted hoof, which is very thin at the heels, may not occasion lameness, because it is more elastic than the thick, walled hoof, and gives to the lateral pressure of the foot when it comes to the ground.

Percivall contends that "shoeing must be regarded as the main cause, indirect though it be, of contraction ; and to the modification or correction of it must we look for the prophylactic. Shoeing, as it respects horses, has been said to be 'a necessary evil.' Without shoes, upon our artificial roads, we can not make use of horses; and no shoes have been found to answer save such as are hard and inflexible or metallic, and as are fixed to the hoofs with nails. Here, therefore, we find ourselves in an awkward dilemma. We can not do without horseshoes; and from the moment we nail them to the hoofs, the feet begin to undergo more or less alteration in form, and, in too many instances, to experience harm from their application. The art of shoeing has given rise to a wonderful deal of difference of opinion and controversy, some thinking one shape of shoe answered best, some another; while some prefer one mode of nailing it upon the hoof, others a different one. As far as my own experience has served as a guide to me through this labyrinth of opinion, I have ever found that method of shoeing the preferable one which approached the nearest to Nature, or, in other words, which interfered the least with the economy of the foot. If we could do without them, horseshoes would, undoubtedly, be best abolished altogether; but, since this is impracticable, let us adopt such shoes and modes of attaching them to the hoofs as are found to work the least mischief to the feet. On this principle it is that a half shoe is to be preferred to a whole shoe, and for the same reason it is that tips, of all the horseshoes that were ever invented, are the best; that is, the least objectionable. If those in the profession would come forward and inform us of their experience (if they have had any) of tip-shoeing, I believe it would uniformly be found that, whatever objection might be urged against 
the use of tips, no one would deny their tendency the least to interfere with the operations of the foot. If there be any horseshoe calculated to prevent contraction, and navicularthritis as well, I feel no hesitation myself in pronouncing that horseshoe to be the tip. In saying so much, I am fully aware that tip-shoeing can not be introduced into general practice for reason of the roads horses have to travel and work upon, and of the numbers of horses having hoofs of too weak and brittle a fiber to stand work without chipping and breaking and wearing too rapidly away. On horses, however, whose hoofs are strong and hard enough, and whose work is light enough to admit of their wearing tips for any length of time, or in situations where the roads or parts of the country they have to do their work upon enable them to wear tips constantly, no wholly-shod horses' feet will ever bear a comparison with theirs.

Pressure to the frog.-Coleman's favorite prophylactic against contraction (considering shoeing to be an indispensable evil) must certainly be regarded as next in importance, as a preventive, to getting quit of the shoe itself, or of part of it. The frog being a body which in action operates in the expansion of the hoof, the removal of it, or even the impairment of it, must, necessarily, give facility to contraction. It therefore behooves us, in ordinary shoeing, to look well to the preservation of the integrity of this important part of the foot.

The cutting away of the bars in shoeing, through robbing the hoof of a couple of stays operating against the closure of its heels, conduces to its contraction. Nature gave the bars as a sort of buttress against either heel of the hoof, to oppose its drawing inward, while the frog, placed between the heels, is operating in forcing them asunder; consequently, if the bars be removed, the expansive or counteractive powers of the hoof lose an agent they can, in many cases, ill afford to be deprived of.

The contracting effects of heat and drought on the hoof may be guarded against by keeping the horse's stall free from fermentable litter, while the atmosphere of the stable is maintained cool and unpolluted. The practice, also, of stopping horses' feet (or, what I believe to be better, of wearing swabs in the stable) will likewise tend to guard against the contracting effects of these agents. We now come to the

Treatment of contracted feet. - The first thing to determine, wnen- 
ever a case of contraction is submitted to us for treatment, will be whether it be one of the pure or mixed description. If the former, the horse not therefore lame, and his feet be submitted to our inspection simply from the apprehension of his becoming lame, and the contraction be on this account desired to be removed, the simple and best means of doing so will be to substitute tips for the horse's ordinary shoes, and to order that he stand with his contracted feet in cold water (or, what is better, in a bed of clay) for a couple of hours, once or twice a day, he being allowed to lie down, as usual, at night. By such a simple plan of treatment as this will his hoofs, giving sufficiency of time for Nature to carry out her operations, become restored to their pristine condition."

The author's method of treating contraction is, to soften the hoof by soaking it often in warm water; then open the heels by means of the drawing-knife; rasp the quarters of the hoof; then pare the foot, but do not touch the bars nor frog; then put on a light shoe, and use the animal, daily, at light work. It will be proper, also, to anoint the hoof, frog, and sole occasionally with a portion of fish oil and spirits of turpentine, equal parts. This will soon soften the hoof, and render it elastic.

\section{HOOF-BOUND.}

This term is usually applied to hoofs that appear to be dry, brittle, unyielding, and having the appearance of more or less contraction at the heels. The hoof, under such circumstances, "pinches the foot," as the saying is, or, rather, embraces the soft tissues too closely. The consequence is, pain, fever, lameness, and perhaps, finally, organic disease of the foot. The condition of the foot termed hoof-bound usually occurs in horses laboring under some predisposition to the same; that is to say, they have faulty feet, which fault may be either functional or structural, and thus the foot may be affected accordingly whenever the ordinary causes of disease are in operation.

Causes. - The ordinary or exciting causes of diseases of the feet are said to be faulty shoeing; allowing horses to stand on plank floors, or in any situation which is likely to pervert the function of the foot; sometimes the feet are very much neglected, and scarcely, if ever, make the acquaintance of sponge and water, and it is very certain that uncleanliness has a bad effect upon the feet, 
as it is known to have on other parts of the body. "No prince ever died of the plague," shows that the ablutions daily practiced by persons of refinement, occupying an exalted position in society, operate as a preventive against even a mortal malady. Yet, notwithstanding all this, it is proper for us to remark that some horses are occasionally the subjects of foot maladies, notwithstanding the very best system of shoeing is practiced on them; and others, that never stood on a plank floor or inclined plane, are in the same predicament; so, also, are some that have their feet washed often. This goes to show that if animals under the very best care and management get hoof-bound, or contracted feet, it is due to a predisposition which they have either inherited or acquired, and, as the old saying is, they would not enjoy bodily health unless they were hoof-bound.

It is very evident that many diseases of the feet are traceable to bad shoeing. Some persons seem to suppose that the foot of a horse is as insensible as stone; and, when we see the blacksmith wrenching off shoes by main force, often fracturing the hoof or breaking off a portion of the bars, frog, and sole, with as little care as a man would rough-trim a log, and afterward applying a red-hot shoe to the thinned sole, we might be led to infer that the foot is really insensible. The Hon. Marshalr P. Wilder thus denounces such bad practice:

"I know of no greater evidence of inhumanity to that noble animal the horse, than the almost universal custom of paring down the foot and burning on the shoe. It has ever, to my mind, been among the barbarisms of the age, which I trust will be no longer countenanced by civilized society; and I sympathize with the efforts of every one engaged in correcting such dangerous practices."

The practice, also, of using a pair of pinchers on the feet, in view of discovering some real or supposed lameness, is equally barbarous, and often results in bruises of the sole, and should, therefore, be discountenanced.

Treatment.--The blacksmith should so prepare the foot and fit the shoe that the frog may be brought in contact with the ground. over which the horse travels. This not only prevents undue concussion, which would otherwise occur, but tends, also, to expand the heels, and preserve the natural tread and function of the foot. Having prepared the foot in a proper manner, we then, if the sea- 
son of the year permits, apply cold water to the hoofs, often and freely. At night the feet are to be wiped perfectly dry, and, by means of a sponge, a portion of the following mixture must be rubbed on: Kerosene oil and honey; equal parts ; or neats-foot oil and turpentine, equal parts. Notwithstanding the very best treatment, the restoration of the foot to comparative or natural soundness will be a work of time, and sometimes, in bad cases, or one of long standing, an impossibility; yet the above treatment is the best we are acquainted with. Some persons prefer to use moistened clay, cow-dung, etc., but they are of no value, only in consequence of the moisture they contain, and clean water is far preferable.

The Mechanism of the Hoof.-A brief exposition of the mechanism of the hoof may possibly interest the reader. The external covering of the foot may be divided into four parts ; namely, the wall, bars, sole, and frog. The external portion, or wall, serves to defend the sensitive tissues within. The wall, or hoof, is composed of small filaments, or hollow tubes, consolidated in such a manner as to preserve their canals distinct. These canals constitute the excrementitious outlets of the hoof, from which morbid materials find an outlet, and in these canals are found the vessels by which horn is secreted or produced. The small vessels arising from the vascular and nervous tissues of the foot proper enter, also, into these canals. The bars are a continuation of the external portion just described. They form an angle at the heels, which terminates toward the toe. They thus serve to give strength and durability to the hoof, and also to prevent contraction at the heels. When these bars are cut away or demolished by means of the butteris, then the foot often goes to "rack and ruin." The sole is much more elastic than the crust, and is a medium of the sensitive faculty, through which, together with its powers of electricity, the percussion of the foot against the ground is regulated. The frog is much more elastic than either of the parts just described. Any unnecessary paring of the same is a monstrous evil.

On the internal portion of the above-named parts we find a beautiful set of leaves (laminæ), resembling those found in the under surface of a mushroom. Their number is said to be five hundred. These articulate with a like number given off from the sensitive tissnes of the foot proper, each laminæ having two sides and an edge, from a series of articulations, numbering three thon- 
sand. The whole surface of these articulations measures four square feet; hence, the horse having four feet, his body rests on sixteen square feet of surface, all contained within his hoofs.

The reader will probably realize that a knowledge of the anatomy and physiology of the foot is absolutely necessary, in order to practice, with any degree of success, the art of shoeing. How is this knowledge to be obtained? Can it be furnished at the forge, in the blacksmith shop? Can such a complicated piece of mechanism be thoroughly understood by the craftsmen of the forge, who have never made the subject one of scientific inquiry?

Let it not be understood that I am in a fault-finding mood. The fact is, the husbandmen of this country have failed to put their shoulders to the wheel for the purpose of establishing and endowing veterinary schools in this country, and if blacksmiths and "vets" do not understand the anatomy of horses feet, the former, who have vast interests at stake, must come in for a share of the blame. On the other hand, we are disposed to be charitable toward blacksmiths; and, taking into consideration the unyielding nature of our paved streets, and the rough ground over which some poor animals are forced to travel, the wonder is, that their feet are not oftener diseased.

\section{Laminitis, or Acute Disease of the Laminæe of THE FOOT.}

Laminitis, or fever in the feet, is an acute affection, confined to the sensitive tissues within the hoof. It is one of the most painful maladies to which the horse is subject.

Symptoms.-It manifests itself, very frequently, after a long drive or race. The horse may be put into the stable at night all right. In the morning he is observed to be all in a heap, as the saying is-his flesh quivering, pulse very active, respirations quick and jerking; the flanks are tucked up, the back is roached, and the fore-feet are sent forward, so that the animal treads on his heels; and, when compelled to move, he endeavors to throw the weight of his body on the hind legs. He is thirsty and feverish, but has no appetite. All appearances about him indicate that he suffers great pain. When a hand is placed upon the fore-feet, they feel unnaturally hot. Such are the most prominent symptoms of laminitis, or fever in the feet. 
Treatment.-The principal objects to be accomplished are, to keep the feet cool by frequent sponging with cold water; next, the bowels must be kept loose my means of bran-mashes, or, if necessary, a dose of Glauber salts-dose, twelve ounces, dissolved in a pint of warm water, to which add half a gill of syrup-and an occasional enema of soap-suds, and also a few doses of fluid extract of gelseminum, say two drachms night and morning. This is the kind of treatment that the author has found most successful during a long period of practice.

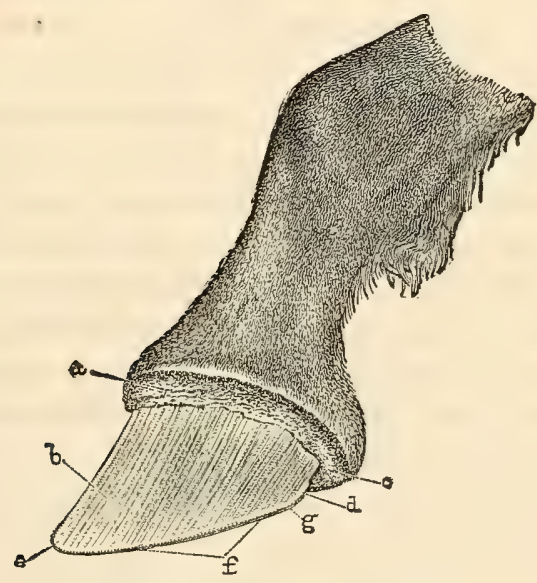

EXTREMITY OF ONF OF THE FORE LIMBS.

Explanation. $-a$, The region of the coronet; $b$, The sensitive laminæ; $c$, The point of the toe; $d$, The quarters; $e$, The heel; $f$, The sole; $g$, The solar border.

Many who treat such diseases are apt to do too much. YouATT, and several other writers, recommend repeated bleedings, blisters, and purges, and even tell us to bleed in the chronic stage. This is all wrong; and such outrageous treatment is almost sure to end in suppuration, founder, or ruin. (See article on Inflammation.) The patient must be kept at rest, and, if he should lie down, must not be disturbed. I never remove the shoes, because the patient is in so much pain that he can not stand on the frog or sole, and the shoes are a protection to the frog. 


\section{SAND-CRACK.}

"The name of sand-crack seems of questionable application. It is, evidently, a compound of the word sand and crack, as though it denoted a crack with sand in it, or a crack occurring in a sandy country, or in a dry, sandy season, which several derivations have been ascribed to the term. May not the word sand admit of resolution into its primitive signification, and mean in this, as in other instances, a sundered crack? A sand-crack may be defined to be a longitudinal division in the fibers of the wall of the hoof, amounting to a flaw simply, or else to a cleft or fissure through the substance of the horn.

The direction of the crack is slanting, from above downward, and from behind forward, following the course of the fibers of the hoof. A sand-crack in the side of the wall slants more than one in front, owing to the greater obliquity of the course of the horny fibers, as we proceed from the toe to the heel of the foot.

There are two kinds of sand-crack, quarter sand-crack and toe sand-crack, the former occurring in the fore, the latter in the hind foot. At least this is generally the case. It is rare to find the reverse, though there are occasions on which we meet with sandcrack in the toe of the fore-foot and the quarter of the hind foot. It is possible for cracks to occur in other parts of the hoof; but in these two situations it is that veritable sand-crack occurs, and there are here, as we shall find hereafter, special causes for their production. Let us first consider

\section{Quarter Crack.}

The situation of this crack is the slanting line of the wall of the hoof, directly opposed to the extremity of the wing of the coffin bone; and it is oftener found in the inner than in the outer quarter, added to which the hoof in which sand-crack occurs is always a contracted one, quarter sand-crack, no more than toe sand-crack, never happening in a hoof disposed to obliquity and flatness. The same description of foot which is predisposed to contraction is, for the same reasons, predisposed to sand-crack. There is an obvious connection between contraction and quarter sand-crack. The light, near-the-ground stepping horse, with strong, narrow, upright hoofs. will be equally likely, under cer- 
tain conditions, to have the heels of his fore hoofs becoming contracted, and exhibiting quarter sand-cracks. Hot stables conduce to this, but, more still, hot climates. Hurtrel d'Arboval informs us that at the time the French army was in Egypt, their horses were continually having sand-cracks; and he adds that long voyages on board of ship are, on some occasions, attended with like results.

The proximate cause of quarter sand-crack is, then, contraction. The horn, from dryness, or other cause, rendered cracky and fragile, breaks at the quarter of the wall, from being at that part bent across the edge of the ala of the coffin bone, during the curving in of the heel, the result of contraction; and this oftenest happens to the inner quarter, from its being the thinner and weaker one, as well as from being the one which is the first and most disposed to contract-not that sand-crack is the necessary consequence of contraction, but that contraction becomes a necessary precursor to sand-crack. If this were not the explanation of the case, sand-crack would be as likely to occur in any part of the wall as at the quarter, and on the outer as often as on the inner side of the wall. This likewise accounts for the comparative unfrequency of quarter sand-crack at the present day among our nag and cavalry horses, since that which has tended to diminish the frequency of contraction has had the same effect in regard to the occurrence of sand-crack. Greater attention to shoeing, and increased care about the condition of the hoof itself, has, no doubt, had a very beneficial effect in the prevention both of contraction and sand-crack.

The origin of sand-crack is usually sudden, both in the fore and hind feet, though in the former case, from its situation in the inner quarter, a part not exposed to transient view, it is possible for a crack to exist for some time, unless lameness happens to arise from it without being discovered. The crack first takes place through the superior or coronary border of the hoof, that being composed of newly-formed horn, and, consequently, thin and fragile. Quickly it extends downward, through the thickest part of the wall, stopping, as it does in some instances, at least for a time, half way down, and afterward reaching nearly or quite to the bottom.

Sand-crack is either penetrant or non-penetrant. It is usually penetrant, by which we mean the crack extends completely through 
the thickness of the hoof, and produces lesion of the sensitive parts underneath the horn, giving rise to some little hemorrhage in the first instance, and subsequently to more or less inflammationoccasionally, even to suppuration, depending, of course, on the extent and nature and duration of the lesion, as well as on any treatment, or aggravation from non-treatment, it may have received since being occasioned. When the crack does not extend through the substance of the wall-which it sometimes does not at first, though it may do so afterward-it may be said to be nonpenetrant; and this, when it happens, seems to be referable to a sort of natural fusion there certainly exists between the solid horn, as it descends from its secreting gland, (the coronet,) and the horny laminæ (new matter), which become glued to it in its course downward. It is in this uniting horny medium that seedy toe commences and progresses.

Lameness is the usual accompaniment of penetrant sand-crack, but not the invariable one. When penetrant it is said to arise from the sensitive laminæ getting pinched between the sides of the crack. However this may be, inflammation following the lesion has certainly its share in causing pain during action. In general, the lameness does not amount to much, nor is it of long duration, provided the sand-crack receive proper and timely attention; but if the crack is not heeded, action of the foot will much aggravate the malady, and end in lameness increased so much as to forbid further use being made of the animal.

\section{ToE SAND-CRACK.}

Occurring in the hind foot, as this crack almost invariably does, occupying a different situation in the hoof, and arising altogether from a different cause, toe sand-crack may be regarded as almost a distinct affection.

The subject of it is not the light horse, but the heavy one-the cart and wagon-horse, the dray-horse, the latter especially; and in particular, I believe, in paved cities, they are the sufferers from this disease. I say 'sufferers,' because it is only those veterinary surgeons whose practice lies among horses of this description that have any conception of the amount of pain and irritation with which sand-crack-simple as it may seem to those who are in the habit of meeting quarter crack only-is found to be accompanied. 
The cause of toe sand-crack is violence. Shoeing, also, may have something to do in its production. The horses who are the subjects of it are those employed in laborious and straining draft. The toe of the hind foot is the grand fulcrum through which the hind limbs, the propellers of the body, exert their power; and it is in some violent and forcible effort that the hind hoof, strained as it is to its uttermost, and in particular at the toe, splits, commonly first at the coronet, the same as in the fore-foot, where the horn, but newly-formed, is then unresisting, the crack subsequently extending gradually down the wall, even as far as the point of the toe. Digging the tip of the toe into the ground, or stamping it hard down upon the pavement, and especially when this stress upon the forepart of the wall is at all times promoted by high eaulkings to the shoe, must certainly, one would think, be the main producer of toe sand-crack-an opinion still further favored by the observation which has been made of shaft-horses in drays being more subject to the accident than trace-horses. Still, however, for all this, it behooves me to say that, with the best judges of such matters, the point is one not yet set free from doubt and difference of thinking. Short and upright pasterns, with clubby prominent hoofs, indicate a predisposition to toe sandcrack, the disease being in no instances seen in flat, shelvy, oblique hoofs. It is said sand-crack may originate in tread. Undoubtedly any lesion of the coronary body, sufficient to injure or destroy its secretory apparatus, may occasion imperfect or morbid formation of horn, or loss of horn altogether; but I do not believe this to be a very common cause of sand-crack.

The consequences of sand-crack in the hind hoof are, as I have before hinted, apt to be of a much more serious nature than any usually arising from a quarter sand-crack. Whether the crack extend to the bottom of the wall or not, being uniformly of the penetrant description, lameness, to greater or less degree, is the invariable result. And when the fissure does reach down to the toe, the wall opens and exposes the laminæ, probably the whole way from the coronet downward, the consequence of which is inflammation and suppuration of those parts, and sometimes even mortification and sloughing of them; and not of them alone, but of the bone to which they are attached as well, which not unfrequently runs into a state of caries, ending in defalcation of sub'stance, to be filled up by the effusion of callus, which usually 
terminates in exostosis, coated with some tissue very imperfectly representing the original laminated structure.

Mr. Braby, the intelligent veterinary surgeon to Messrs. Barclay and Perkins' establishment, to whom I am indebted for much of the information I possess on this part of my subject, has had many cases of this description, one of which, of extraordinary character, I shall relate here. One of his dray horses had suffered long and severely from toe sand-crack in one hind foot, but, at length, had recovered, and returned to work. Some time afterward, however, during the season of influenza, he was attacked with a violent laryngitis, which increased to a degree to call for the operation of tracheotomy, to save him from suffocation. Notwithstanding this temporary salvation, however, the patient, in the end, succumbed to the disease. His post-mortem examination became doubly attractive, owing to the circumstance of the long-standing. and obstinate sand-crack he had suffered from heretofore, and the result in this latter respect proved extremely interesting. The coffin bone, along its front, occupying the line of the surface be-tween the coronal process and the toe, exhibited a channel or loss of substance half an inch in breadth, and fully the same in depth, thereby robbing it of a quarter of an inch of its solid diameter. This, of course, left the bone considerably weakened, the result of which subsequently was, transverse fracture in two places, the fractures commencing upon the articulatory surface, whence they extended directly crosswise through the middle of its body, so as to become apparent upon its concave surface underneath. In addition to this, growing from the laminated interior of the wall of the hoof, opposite to the middle or deepest part of the channel in the coffin bone, was a projection of hard, horny, callous substance, having a covering of imperfectly-formed horny laminæ. At the time this horse was suffering in the greatest degree from this extraordinary product of sand-crack, constitutional irritation ran so high as even to create alarm for the animal's life. The treatment of sand-crack, whether it be in the quarter or in the toe, will have to be conducted upon principles applicable to both forms of the disease, though one must be regarded as of much more consequence than the other. The treatment of quarter sand-crack, generally speaking, is but comparatively a simple affair ; indeed, so lightly is it looked upon by horsemen in general, that we should run some risk of their displeasure, and our own reputation as 
well, were we to talk about laying a horse up for so trifling an accident. Nay, some horses, with non-penetrant sand-cracks, or with sand-cracks that have been penetrant, but have become horned over, showing little or no lameness, continue to work on without evincing any pain or inconvenience from them. Whether a horse be lame or not, however, should he have a sand-crack, and we be consulted about it, it becomes our duty to arrest the extension of the crack so long as it be but partial; and, besides that, to take measures for the cure or permanent removal of the crack. The owner of the horse should be given to understand that no flaw or crack in the hoof can, by possibility, unite the same as a wound in a vital part does, but must, as the saying is, grow down; that is, must be replaced by new horn, and be itself, by degrees, renewed, as it continues to come under the operation of the drawingknife every time the horse is fresh shod; so that, in fact, the cure, or obliteration of the crack, is necessarily a work of some months, though the lameness may be cured in as many hours or days.

Paring out the crack, the shoe being taken off the foot, is the first thing to be done. The cutting cautiously away of its rugged edges, and the scooping out, with a light hand, (probably with the back of the drawing-knife, of its cavity, will enable us to examine into the condition of it. Should there be no lesion or exposure of the laminæ detectable, nothing further will be needed from the drawing-knife than the cleaning out of the crack.

Firing the crack is the next operation. Cross-firing will be advisable below or above, or in both situations, according to circumstances. So long as the crack has not reached to the bottom of the wall, it will be requisite, with an ordinary firing-iron, at a red heat, to burn a deep but short fissure, or 'mark,' across its lower extremity; and whether a similar operation be required across the superior termination of the crack, must depend upon its extension or not through the horn at the coronet. If there be any interval of sound horn between the hair and the crack, of sufficient breadth and substance to bear firing, a very slight burn may do good. In all cases it is the practice to finish the firing with running the sharp edge of the iron down the crack; and this certainly proves beneficial in destroying any tendency there may be (supposing the laminæ to have become denuded) to abnormal action, as well to stimulate any vascular parts exposed to issue horny matter to cover in the bottom of the crack. 
Binding up the crack is a good practice after firing. With a wax-end of sufficient length (such as shoemakers use) bind round the wall of the hoof, so that any tar or pitch-plaster it may be deemed advisable to place in or upon the crack may be maintained there; at the same time the hoof itself is, by the tight binding, restricted in any tendency it may have to expand, and thereby open wider the crack.

A bar-shoe is the preferable one for a sand-cracked foot. By it, the bearing being taken off that part of the wall which is opposite to the crack, the pressure and jar, so continually splitting afresh the new-formed horn over the crack at the coronet, is put a stop to, the formation of an undivided coronary horny band being the commencement of the radical cure of the sand-crack. As I said before, horn being an inorganic substance, no union whatever can take place in the crack itself. Permanent cure can be effected only through obliteration by the growing out or down of the crack. This, I repeat, is the reason why a sand-crack occupies so long a time in its removal; though, by way of compensation, a horse is not kept out of work while cure is being effected; for, after the crack has been bound up, and the hoof shod with a barshoe, it is quite surprising to find how soundly and firmly the animal sometimes steps upon the foot of which he had but now been so lame.

The treatment adopted by the late Mr. Read, V. S., of Crediton, carries the same object into execution, through a different method of procedure. This, as detailed in the volume of the 'Veterinarian' for 1848, consists in simply isolating the fissure within the segment of a circle, by means of an ordinary firingiron. The best plan is to operate with the heel of the iron, beginning at the coronet with either extremity of the segment, and bringing the iron to a finish at the center. The iron should be at a strong red heat, and be carried through the horny crust until it touches lightly the sensible laminæ, and so throughout the entire semicircle. As you recede from the coronet, so, in proportion, you will require to deepen the fissure in the crust. The iron ought to be applied every week or ten days. The first effect desirable to be produced is a bulging of the crust around the coronet within the segment, and when once this is fairly established, the cure may be said to be effected, it being seldom necessary to apply the cautery afterward. The old method of making a line with 
the iron across the fissure can not prevent the opening and closure of the fissure during the action of the foot; whereas, isolating the fissure (or part of it) within the segment of a circle completely effects the object. No tar-cord or strapping, or alteration of the shoe, is required to limit the motion of the crust, all motion being suspended within the segment, and especially after bulging has commenced.

The objects of treatment, after what has been stated, will clearly appear to be, first, to place the hoof in such a condition as shall not render it liable to crack again; second, to remove that state of it which, in the first instance, disposed it to crack, if it were not of itself the immediate cause of cracking. The way in which the first object is effected has been already shown, and when this has become accomplished, past all risk of return, we may set about to bring into effect the second. A bar-shoe, from its taking the bearing off the quarters and placing it upon the frog, will, in a measure, give facility to what we now are desirous of preventing; namely, the expansion of the heels of the foot; but a tip (providing it can be worn, which it frequently may, with great advantage, after a bar-shoe) will bring about greater reform stillwill, in fact, by persistence in its use, bring about, in time, that improved form of hoof" which will be no more liable to quarter sand-crack.

The treatment of toe sand-crack is, in some respects, a different affair from that of quarter sand-crack. This disease is not only different in its relative situation as regards other parts of the foot, but it arises, as we have seen, from a totally different causation. When once it has occurred, it becomes, compared with the other, a serious affair. The horse is lame, too lame to continue his work, probably, and we have a penetrant crack to deal with, extending all, or nearly all, the way from the coronet to the toe, discharging blood, or, perhaps, matter of some ichorous, offensive description, plainly calling upon us for, not binding up, etc., as in the former case, but for opening, cleansing, and dressing the same. The shoe being taken off, let the crack be pared out, and freed from all horny rags and asperities, and laid completely open to view, so that the bottom can be inspected and dressed with whatever may be deemed requisite. In fact, when once the fissure is dilated into a clean and open channel by the drawing-knife, warm baths or poultices, or dressings of any kind, as may become necessary, are 
now applicable, the case being in this stage no more than one of dilated sinus in the foot, similar to what might, in another situation, be called quittor. As with the quarter sand-crack, the cure will, of course, be tedious in its duration, since we know that all complete repair can only come from the coronet. 'The sensitive laminæ having the power of secreting horny laminæ, may, as in the quarter crack, issue a sort of horny covering-in of the bottom of the fissure; but fissure or cleft will ever remain so long as an integral formation of horn does not grow down from the source of secretion.

Whenever the horse is in a condition for work, a bar-shoe, so made that at the toe the sides of the shoe remain unjoined together, an interval being left of sufficient width to receive the crack in front of the hoof, is, perhaps, the best. Binding the hoof up with circles of wax-end, as in the case of quarter sand-crack, with some plaster or dressing underneath it, will also now become advisable. It will restrict the spreading inclination of the hoof, as well as keep dirt and wet out of the crack. Repetition of this, and the continuance of the bar-shoe, will be required so long as there appears any risk of an extension or renewal of the crack." "*

The diseases of the hoof here alluded to are very apt to occur in horses whose walls of the hoof are thin and brittle. They are, to a certain extent, non-elastic, and, therefore, do not ward off the necessary amount of concussion when the animal is traveling over hard roads. At the same time there is always a liability to incipient and permanent contraction in such hoof. More especially is this the case when the foot is mutilated by paring or cutting away the bars (the main stages or supports which prevent the heels approximating). These are the kind of feet on which the butteris-knife and rasp should be used with great caution ; for, being naturally weak, and perhaps congenitally defective in the function of secreting horn, and the necessary amount of moisture to keep the part pliant, any unnecessary tinkering with such tools is inadmissible. Such hoofs lack moisture; therefore they should be well soaked, night and morning, in warm water; afterward wiped dry, and then smeared with a portion of the following:

No. 57. Neats-foot oil............. 8 oz.

Kerosene................ 4 oz.

Lamp-black............... 1 táble-spoonful. 
Mix well, and apply twice daily, by means of a common varnishbrush.

Very many diseases of the feet, which are of daily occurrence, might be prevented by proper attention to the feet. "No foot, no horse ;" hence the feet need to be kept as free from filth as other parts of the body. I think that a great majority of English grooms pay as much, if not more, attention to horses feet than to other parts of the body, and thus many forms of foot lameness are avoided.

\section{Curvature and Disease of the Spine.}

The annexed engraving, represents a case of curvature of the spine in a downward direction, known in stable language as hollow back. It usually, when it makes its appearance, occurs in aged horses, and probably arises from putting too much weight on the animal's back.

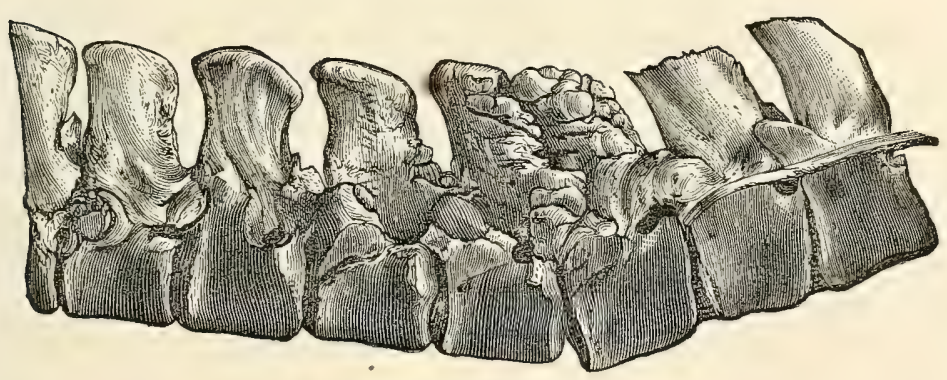

SECTION OF THE SPINE.

It will be observed that two of the superior spinous processes of the vertebræ are united (anchylosed), and ossific deposits are thrown out on the surfaces of the spine. On the lower part, beneath the diseased spine, a cleft is seen, showing that there is an incomplete dislocation, and the capsular ligament was probably dislocated or torn.

It may be interesting to the reader to know, if he does not already, that the bones, like other parts of the body, are composed of arteries, veins, absorbent vessels, nerves, and a cellular texture. They are endowed with vitality, are nourished, grow, waste, and are repaired, and undergo various mutations, according to the age of the animal; and they are subject to disease analagous to the 
soft parts. To the phosphate of lime, which is, more or less, distributed in their texture, they owe their solidity; and perhaps it is to the same earthy substance that the difference in their vital properties, and in their diseases from those of the rest of the body, is to be referred. In fact, this particular organization and inferior vitality of the bones are generally supposed to account for the small number, peculiar character, and general slow progress of their diseases.

Treatment.-So soon as a horse shows any symptom of undue curvature of the spine, he must not be used under the saddle; but, provided he can work, he may be used lightly in a buggy. If unfit for work, the owner knows what is best to do with him. I should give an animal afflicted with this disease a wine-glassful of cod-liver oil, two or three times per week, and one table-spoonful of flour of bone, every night, in the food. If this article can not be procured, substitute for it phosphate of lime.

\section{Osteo Porosis (known as Big Head and Big Jaw).}

The terms big head and big jaw are used by husbandmen and others in Ohio, and, indeed, throughout the Great West and South, to designate a disease of the bony structure of the horse-a disease about which very little of a reliable character has ever been said or written; yet the subject is one of great importance to the people of this country, for the simple reason that the evil is one of alarming proportions; and should the disease multiply in a ratio equal to that of the past few years, it will be a terrible blow to the laudable and hitherto profitable enterprise of raising colts in sections where this disease prevails.

We do not wish to create any unnecessary alarm, but urge the husbandmen of the South and South-west to give this subject their earnest consideration ; for the disease probably has an hereditary origin-is "inbred"--and that, as the saying is, "constitutes the root of the evil." The disease having been pronounced incurable, our only hopes of success in putting a stop to its propagation and development is, to adopt preventive measures, by rejecting, as breeders, all animals (sire and dam) that show the least predisposition to or for this affection.

Let the reader understand that the disease itself is incurable in so far as the enlargement, dilatation, and softening of the jaw- 
bones are concerned; yet, like spavin, ring-bone, and various other diseases, it is accompanied, in certain stages, by pain and lameness, and our services as physicians are only secured in view of removing this pain and lameness, so that the animal may be enabled to perform the ordinary equine duties. This is what some persons please to term a cure, whereas it almost always happens that some alteration in the structure of the parts remains, which actually renders the horse unsound, because he has that about him which may, from overwork or other exciting causes, impair his usefulness.

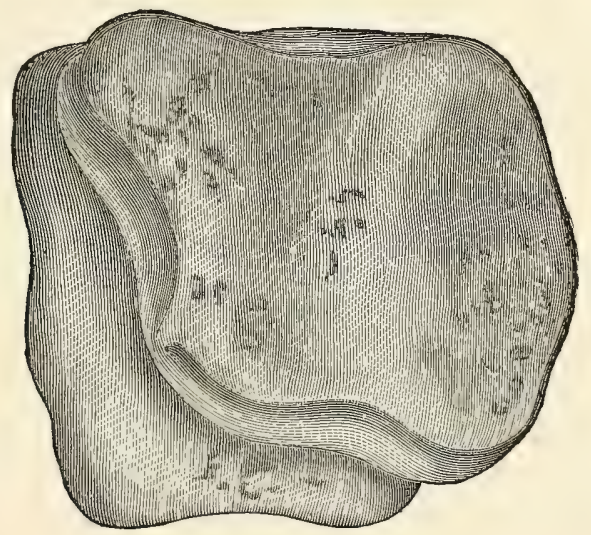

THE PATELLA, OR STIFLE BONE.

This cut shows the appearance of disease on articulatory surfaces when the animal is the subject of Osteo Porosis, or Big Head.

As regards the "alarming" features of big head or big jaw, we would inform the reader that almost every lame horse examined by us, during a period of six months, in Ohio, had either one or the other jaw enlarged. In some cases one angle of the lower jaw was the seat of thickening and enlargement, but among the majority both angles were affected. These remarks do not apply to other obvious or accidental lamenesses which are constantly occurring.

A short time ago we visited, in company with a practicing veterinarian whose attention had never been called to this affection, three horses, the subjects of lameness, which was said to be occasioned by eating Hungarian grass. To the astonishment of the parties concerned, we demonstrated that they were all the subjects of enlargements of the lower jaws. From the history of the cases, 
and the character of the lameness, it was very evident that it was of an inter-articular character, and this is a peculiar feature of this affection. Then, again, the reader must bear in mind that the jaws of animals do not dilate nor enlarge suddenly, nor make their appearance all at once, like a meteor in the regions of space. The affection is insidious, progresses in an almost imperceptible manner, and usually escapes the notice of those who daily have charge of and handle the animal, as the following case, which is selected from among others, will serve to illustrate:

A few years ago a gentleman purchased what then appeared as a fine family horse-a sorrel gelding, aged five years. About the beginning of March, one year after the purchase, in attempting to walk the animal from one stable to another, but a short distance off, he became suddenly lame in one of the hind limbs, low down, toward the foot, and it was found almost impossible to urge him forward. Finally, assistance was procured, and the animal had, literally, to be carried into a stable. Our services having been secured, we visited the sufferer, and found him standing on three legs, the near hind one being placed resting on the toe. The parts around the coronet were hot, and the patient was very unwilling to have the parts handled. He seemed to suffer much pain, was breathing hurriedly, and the pulsations were quick and wiry. The near approach of any person seemed to terrify the animal, and he actually trembled, from fear and pain, when urged to move. On making an examination of the jaws, we found that both angles of the lower jaw were enlarged to about two inches in thickness. This explained the nature of the sudden and otherwise mysterious lameness. The articulating surfaces of various bones, and the bones themselves, had become diseased, as is often the case in big jaw. The owner assured us that the horse had never before been lame, and he was very much surprised to find the lower jaw enlarged.

Here the reader will perceive that the animal had been in the owner's possession one year; had performed ordinary duty, yet was the subject of a gradual enlargement of the jaw and a constitutional disease, which probably existed, yet in a slight degree, at the time of purchase. Hence we contend that the disease, in its early stages, is of a hidden or insidious character, and requires some tact and knowledge of the subject in order to detect it.

Nature of the disease.-Big head and big jaw are but one form 
of disease, only located in different parts. When the upper jaw becomes the seat of affection, an enlargement of the facial bones will be observed, and the examiner's attention will be attracted by the unnatural appearance of the face. As the disease progresses,

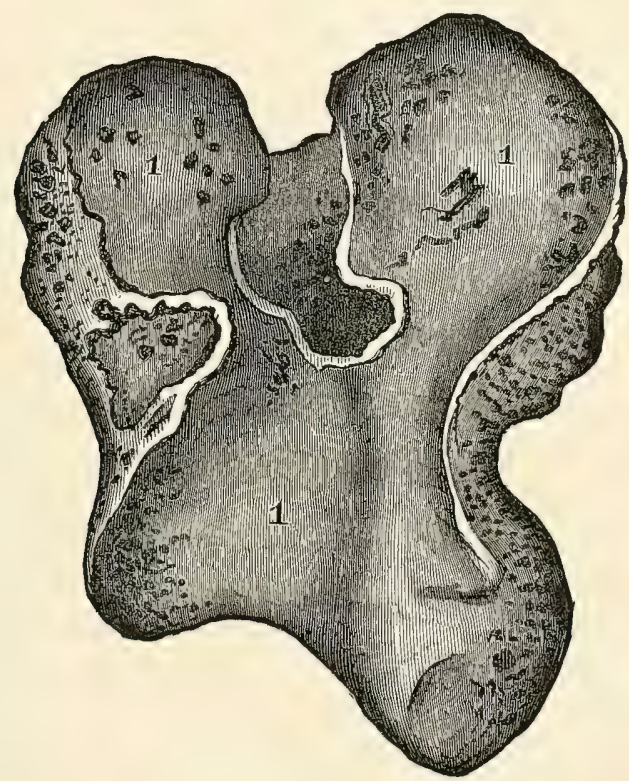

ARTICULATING SURFACE OF THE THIGH BONE.

Explanation-111, Articulating surface of the lower or inferior part of the thigh bone occurring in the disease known as Osteo Porosis, or Big Head.

the face looks more like that of an elephant than a horse; and now, the disease being incurable, the subject should mercifully be put out of his misery. The enlargement of the lower jaw is discovered by manipulation. On removing the skin from the sides of the face of such a subject, and dissecting the muscles and periosteum (covering of bone), the bones appear to have undergone a softening process, and particles can be separated by means of the finger-nails. At the same time, if a transverse section of any of the shaft-bones be made, they will be found to have undergone structural changes. For example, they will not only appear softened, but honey-combed, and many of the laminæ and cartilaginous braces will have been decomposed or dissolved, so that the bone looks like fibrous net-work. In most cases, the vacuities 
and canals are filled with material resembling soft cheese, which is probably fatty matter. This occasionally degenerates into pus.

Dr. Gordon forwarded me, some time ago, the head of a horse who had long been the subject of this malady. The transverse diameter of the bones of both upper and lower jaw was very much enlarged; the periosteum was very highly organized with bloodvessels; the bones were softened and elastic, and could be easily cut with a knife. On submitting them to the acid test, earthy matter was found in excess, and, by burning them, it was found that they contained very little animal matter; hence we were led to infer that the disease was one of mal-nutrition. The articular surfaces of various bones, and sometimes all the bones of the body, are often ulcerated and studded with incrustations. This explains the why and wherefore of the sudden and tormenting pains which animals suffer, and goes to show how little of benefit can be expected from the ordinary treatment, which is principally directed to the enlarged jaws.

On removing these diseased cartilages from the articulating surfaces of the bones, the latter are found to be affected-in fact, ulcerated. In some cases the ligaments and tendons are separated by decomposition, or necrosis of bone, and the animal "breaks down," as the saying is.

The subjects of this disease sometimes show lameness in the vertebral region, and in that of the head of the thigh bone. In such cases, we may safely infer that the lameness is of an articular character, and the interticular cartilages, as well as the surfaces of the bones, are incrustated and ulcerated. In this condition, the animal is liable to become useless, or "break down" at any moment. The following case will illustrate what is meant by breaking down:

We were requested, a short time ago, by Prof. Muzzy, to examine an aged gray gelding, the subject of lameness in the hind feet. Examination revealed considerable heat, tenderness, and tumefaction around both the hind coronets, and both angles of the lower jaw were much enlarged in their transverse diameters. We advised the owner to let the animal have a run at grass. Accordingly he was sent into the country about thirty miles, which was too long a journey for him. A few days afterward, Prof. Muzzy received a letter that the horse's strings (tendons) became loose, and he broke down and traveled on his fetlocks, and was then 
dead. We infer that necrosis took place, and the extensor tendons lost their attachment, which accounted for his breaking down.

Prof. VArnell lately consulted Dr. Harlex, of London, in reference to this formidable malady, which is said to havé been almost unknown in England until November, 1859, when several cases occurred among some horses the property of Mr. Champion Calcot, near Reading. Three of this gentleman's animals had died, a fourth was not expected to live many days, and two others were laboring under the same disease, in a less acute form. The most singular feature of the disease, as it appeared on the premises of Mr. Calcot, was, that it did not appear to owe its origin to any perceivable cause, and it was not known to be prevalent in any other part of England. Still, our opinion is that it does exist in that country, only, not being suspected, is not sought for. It prevails very extensively among horses brought to Ohio, and hundreds of horses have been purchased for the American Government having more or less enlargement of the lower jaw, yet we do not believe that either seller or purchaser suspected the same. This arises from a lack of knowledge on the subject, and the same remarks, as far as our ignorance of the subject is concerned, may apply to England.

In answer to questions put to Mr. Calcot on the subject of management, etc., he contends that the affected animals had plenty of exercise. There were no chemical factories or works in the vicinity. One horse affected was purchased at a distance; the rest had been bred on his farm. The sires and dams all appeared free from the disease, and they were not all got by the same sire. The food consisted of good grass, hay, pollard, oatmeal, and roots. Other horses were on the farm, fed in the same manner as the affected ones, yet they had no symptoms of the disease. (Perhaps they will show symptoms when the jaws shall be examined. They may have the disease, yet not be lame nor apparently ailing.) Mr. Calcot never saw a case of the kind before.

From the above testimony, we may infer that the causes of this affection are not yet discovered in England; but our impression is that overfeeding has a good deal to do in the production of the malady. It appears to me that this disease, as I have already stated, is one of malnutrition, or defective nourishment; hence may be considered as a scorbutic affection, like that affecting the human subject, which is known to be the result of faulty nutri- 
tion, and which often results in division of bony parts which were once immovable (the epiphysis of the pelvis, for example); also in the separation of cartilages from the ribs, and the shaft bones softened and ruined by caries (ulceration or death of bones). Big head and big jaw are terms sufficiently explicit in ordinary conversation; but, in view of scientific inquiry, it is necessary to employ terms indicative of the pathology of the disease. We can not expect, however, to select any one name that shall apply to all the pathological conditions during the rise, progress, and termination of this peculiar malady. The condition of the bones is a state of enlargement, (porousness and softening;) hence the name osteo porosis.

Causes.-We have already informed the reader that the disease may have an hereditary origin ; otherwise, we can not account for its universal prevalence. In certain localities, under the ordinary modes of feeding and general management, we grant that at first the disease might have had an accidental or spontaneous origin, and finally become permanent and transmissible. For example, glanders and farcy afford illustrations of a spontaneous disease becoming contagious and transmissible. There must have been a time when neither of the two latter diseases existed; hence, when the first subject became glandered, he could not have taken it by infection or by contagion, but it must have had a spontaneous origin, and, finally, became permanent. We do not pretend to urge that the active disease itself is transmissible; yet, in certain cases, a predisposition is transferred to the progeny. This may be called the predisposing cause; the ordinary exciting causes are those which disturb and derange the digestive function. The digestive or nutritive function is deranged, both by excessive and defective functional labors, or by the animal existing on food that does not contain the necessary amount of nitrogenous or musclemaking matter. This is the case when Indian corn is used as food for a great length of time. It is hard to digest, is deficient in nitrogen, and almost always over-distends the stomach; for, when submitted to the action of heat and the gastric fluids, it increases in bulk to about five or six times its original capacity. I have noticed that where much whole corn is fed, as in Ohio and Indiana, the disease is most prevalent. Associated with the predisposing causes are others-for example, hard usage, sore abuse, and bad stable management. 
This disease is rarely ever heard of in England, probably from the fact that the food of horses is rich in phosphates and nitrogen, while corn contains more of starchy matter, which, instead of furnishing material for the preparation of muscular or animal matter, merely furnishes that which is consumed in process of respiration.

Treatment.-The ordinary treatment, as practiced by some persons, is to bore into the jaw-bone, and inject the same with some corrosive poison. Others expose the jaw-bone, and saw out a section of the same. Some persons blister, or apply preparations of iodine. Such treatment, I think, only tends to create unnecessary irritation and pain, and can not possibly be of any benefit; for I contend that the disease is not local, but constitutional, and the reader will probably, after perusing this dissertation, come to the same conclusion. The disease has extensive ramifications in various parts of the bony fabric, and, therefore, the local treatment must fail in curing the malady. Cases may arise which require surgical operations, and if so, I have no objections to offer. I have often been told that horses, after having all sorts of barbarities practiced on them, have recovered. This, so far as the treatment is concerned, is in accordance with the spirit of the old error, "He got well after taking my medicine; therefore, in consequence of taking it." This is assuming a falsehood for a fact, and then giving fanciful reasons for it.

In view both of prevention and cure of this organic disease of the bones and their articulations, more is to be accomplished by regimen than by medicine. The animal should always be provided with wholesome diet, and whenever green vegetables can be obtained, they should be fed liberally, or, what is much better, provided the season permits, allow the animal to roam in a, pasture. Whenever I have a case of this kind under treatment, I furnish apples, beets, carrots, cabbage, or any other kind of vegetable that I can procure. I find that such articles are usually devoured with good relish. The object in feeding green food is to combat the scorbutic diathesis which usually exists. The patient should also be allowed from five to seven quarts of oats per day.

When a horse with an enlargement of the lower or upper jaw is suddenly attacked with acute lameness, he should be placed in a wide stall, and the parts where the lameness appears to be located, as well as the jaws, should be diligently rubbed, twice daily, with a portion of the following: 
No. 58. Spirits of eamphor.............6 oz.

Cod-liver oil................... 4 oz.

Oil of cedar.................. 2 oz

Diluted acetic acid............... 1 pint.

Mix.

Then procure the following:

No. 59. Chlorate of potass............. 2 oz.

Powdered ginger.............. 4 oz.

Gentian..................... 3 oz.

Podophyllum................. 2 oz.

Poplar bark.................6 6 oz.

Mix.

Dose, one ounce night and morning, to be incorporated in the food.

This treatment usually palliates the lameness. Should it not do so, the owner must be patient, and give Nature time to restore the animal to comparative usefulness. Flour of bone or phosphate of lime may be given, at the rate of a couple of ounces per day. Such agents are always indicated in the treatment of the above malady.

\section{Spavin.}

Spavin consists of an exostosis of the hock, commonly locater on the inner side, whereby bones before moveable become cemented and fixed together, and which is often productive of ulceration of the articular surfaces of one or more of the joints of the hock. Sometimes, however, spavin makes its appearance on the anterior or front part of the hock, and sometimes there is no enlargement to be discovered. Such cases, unaccompanied by enlargement, are called inter-articular spavin.

Symptoms. - "The symptoms of spavin," says Mr. Percivali, "are, in general, plain, simple, and unequivocal. The horse manifests lameness in one of his hind limbs, and, on examination, a circumscribed spheroid tumor, of the magnitude of half a walnut or more, is evident enough both to the sight and feel of the man practiced in such matters. Lameness, however, the effect of spavin, may be present without any detectable tumor.

My own observation has led me to note two kinds of lameness in spavin-one, the effect either of the ordinary subacute or chronic inflammation, or of partial anchylosis of the parts, is but comparatively slight, and consists, for the most part, in stiffness on motion, 
or in defective flexible power of the hock-joint; the other, the effect of acute inflammation of the spavined parts, or else of ulceration of the joints, consists in a sort of spasmodic catching up of the spavined limb the moment the heel of the foot comes down upon the ground, something after the manner of string-halt. Stiffness may not unfrequently be observed even in the horse's side move-

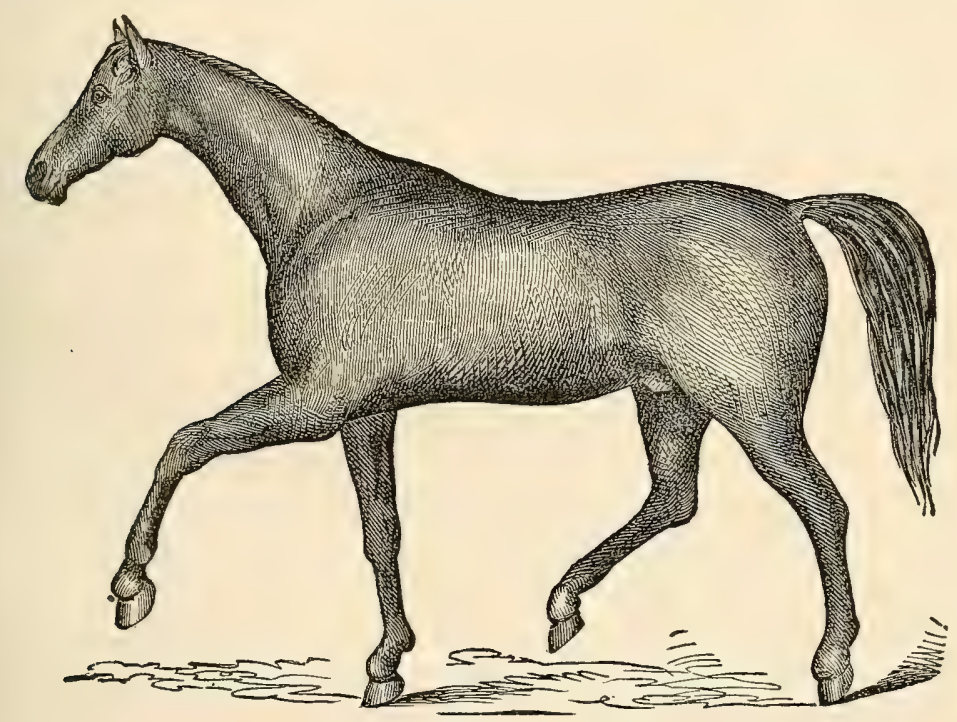

SPATINED IN OFF HIND LEG-RINGBONE IN ALL THE PASTERNS.

ment in his stall. With such characteristic lameness as this, and with spavin present as well, evidently hot and tender to pressure, there can exist no doubt about the case. Where, however, the lameness is but slight, although a spavin is present, yet, from the absence of any heat or tenderness in the swelling, as well as from its duration, many doubts arise as to the cause of the lameness. In such a case as this, we should take advantage of the wellknown fact of the fluctuating or evanescent character of spavin lameness, to which end it is advisable to have the horse under examination hard ridden or driven, or otherwise exercised, until he be in a profuse sweat, and afterward kept standing tied up in a stall, until he be cold and stiff in his joints, and then trotted out again. A knowing vender of a spavined horse would take care to 'warm' him by a good ride or drive before he took him to show to the presumed purchaser; and then, while under ex- 
amination, by dint of whip and spur, and management in the bridle-hand, he might pass his merchandise off to an unwary buver as sound. Indeed, so much is sweating work, or exercise approaching thereto, apt to prove a foil to showing lameness, that one is almost inclined to say no horse ought to be examined under such circumstances; certainly no horse suspected of spavin. The time, of all others, that a spavined horse will be apt to manifest his lameness will be the day following after a hard day's work; and when he makes his first egress from the stable in the morning is the critical period for examination. Horses that go limpingly lame from spavin, lame at all times, and lamer still when they work, often experience pain in the seat of disease to a degree which, in the language of Solleysell, causes them 'to pine away, especially about the flanks.' They have probably been blistered and fired, perhaps setoned; have had their hocks frightfully scarred, and yet are lame to that degree that they are unable to do more than gingerly put the toe of the foot of the spavined limb to the ground, and so painfully hobble along; and, although they may still maintain their appetite, yet they are low in condition, tucked up in their flanks-evidently, in short, ' pining away.'

Such pitiable subjects, it is true, may be kept at work. The little, however, they can do, when put to any thing requiring strength of action or pull, together with the wretched condition they are generally in, is a fact so well known to coach and omnibus proprietors, and horse-keepers in general, that at the horse auctions such animals fetch little or nothing. Even for agricultural work such laborers as these prove of but little worth. Now and then, however, it happens that the spavined horse, although treatment has failed to render him sound, continues, in respect to his disease, in that state in which he appears to suffer no local pain at all while at rest, and but little while at work, and so is able to do a considerable amount of some kinds of labor, lasting in it perhaps for years. Still, such a horse is more likely than another to receive injuries, to experience aggravation or relapse of disease in his already diseased hock; and, under such return or augmentation of ailment, unless great care be taken, and frequently with all the care we can take, may and will fail altogether.

Spavins exist which occasion no lameness. How this comes to pass will appear when the time arrives to consider the reasons why spavins in general cause lameness, and, on occasions, very great 
pain as well, which can not be done before we come to treat of the pathology of spavin. It is sufficient for our purpose here that we note and establish the fact that lameness is not a necessary consequence of spavin. Nothing is more common than to meet with horses, colts even, who have what the dealers call 'knots' in their spavin places; and the time was when such 'knots' (which have always been regarded as spavins) were certificated as constituting unsoundness.

Lameness arising from spavin is sometimes present without the outward appearance of spavin. This is a form of disease better known to veterinary surgeons in general, I believe, under the denomination of occult hock lameness. My own attention to the subject was first drawn so long ago as in the year 1815, though then I was quite in the dark as to the nature of the case. On my return from Belgium, after the battle of Waterloo, I had in my possession a bay blood mare, who was lame in one of her hind legs (I forget which), but whose lameness was of that nature that no external sign whatever was apparent to account for it. The limb had been searched over and over again, by myself and some other veterinary surgeons, and the mare had been trotted and walked, circled and paced, and put to all other known trials and tests, without the examinations ending in any thing like concurrent opinions respecting either the seat or the nature of her lameness. The mare returned home, marching with the troops, led by a man on horseback-for, notwithstanding her lameness, she walked very well-and, as soon as she arrived at head-quarters (Woolwich), I showed her to my father, at the time senior veterinary surgeon of the Ordnance Department. He examined her, and without hesitation pronounced her 'lame in the hock,' and she was treated accordingly; and the result was, at no great distance of time, her complete restoration to soundness.

It is true, so far as the case above related goes, that the only proof that the mare's lameness was in the hock, was her restoration to soundness after the application of remedies to that joint. There is, however, tr be said, in addition, to induce us to believe that it was so, that, of all the joints of the hind limb, no one is so frequently or so likely to be deranged as the hock; and, consequently, from this fact alone, is a prima facie case made out. Moreover, we have, to assist us in our diagnosis, the stiff or imperfect flexion of the hock-joint in action, and the wearing away 
of the toe of the shoe, showing that the heel is rarely or but very gingerly put to the ground; also the circumstance, often observable by the groom, of the animal resting the lame (hind) limb in the stable; on occasions, perhaps, knackling over upon it, and so bearing the weight upon the toe alone. And it has happened before now that, while doubt was impending as to the locality and nature of the lameness, a spavin has made its appearance, and dissipated all further conjecture; and with this development of the spavin, the lameness, so far from being augmented, is not unlikely to become better. This is an observation made so long ago as the time of Solleysell. This admirable observer, in one part of his chapter on spavins, says: 'At their first piercing, they, generally speaking, make a horse halt, and afterward the swelling growing bigger, the horse halteth no more with it." "

I have noticed that spavined horses are usually the subjects of ill-shaped hocks; hence this peculiarity of conformation would seem to indicate that such animals have a certain amount of predisposition lurking in their systems. The ordinary exciting causes are sprain or strain, and overwork.

Now, what will cure spavin? This question was propounded to the author, some time ago, and the following answer was elicited. The answer is as good to-day as when first written. "What will cure spavin?" I answer: Time, rest, and the application of remedies adapted to the various stages of the disease. The usual remedies, in the early stage, are rest, frequent use of the shower-bath, and refrigerating lotions. In the chronic stage, and when the disease has commenced on the articulating surfaces of the hock-joint, or an ossific bony enlargement has made its appearance, then we resort to counter-irritation, by using some one of the preparations of cantharides or iodine. "What will cure spavin?" is a tough question for a medical man to solve; for, if he has been properly educated, he knows well enough that bone spavin, like hip-joint disease in the human subject, is, to all intents and purposes, incurable-I mean in so far as the physiology and pathology of the parts are concerned. Soft tissues, once having been changed into bone, never can be remetamorphosed into the original condition so as to possess their inherent qualities of elasticity (contraction and expansion). At the same time we are all horsemen enough to understand what the term "cure" means when applied to the disease known as spavin. It signifies 
freedom from sensibility and lameness, and a partial disappearance of the enlargement, or "eye-sore." As the reader may like to refer to the opinion of some well-known author, as well as what I here advance, I select a quotation on the treatment of bone spavin from the pen of Mr. Blaine:

"This disease does not differ from splint, except that it is much oftener a cause of serious lameness. Occurring, however, as it commonly does, in older horses, it also proves more obstinate, and the treatment required, therefore, should be more active. Among the old farriers, who, like some of the moderns, thought nothing too strong for a horse, violent mechanical operations were resorted to, as the mallet and chisel to chip it off, boring the exostosis with a gimlet, punching it with a hot iron, or applying caustics. The first removing it mechanically, and the three latter methods destroying its vitality, promoted its exfoliation. As might be expected, for one case which succeeded (and in some it certainly did succeed) in many it increased the lameness, or ended in anchylosis and sometimes death. It is not improbable that instruments may yet be devised which will operate on these bony enlargements without risk, though the chances are fewer in the hock, from its connection with capsular and bursal ligaments, than in any other parts.

Treatment.-The treatment pursued by veterinarians of the present day varies somewhat. Those bordering on the old school still rub with some violence, and then stimulate them with oil origanum, oil terebinth, etc. Those of a later day blister and fire. At the Veterinary College setons are used, by ripping up the integument and pushing a needle around, with tape through it, so that the seton within the skin exactly opposes itself to the spavin. If the skin is tender or tumefied, it is more proper to make an opening above and below the exostosis, and to push a blunt setonneedle or eyed probe from one opening to the other, armed with a tape smeared with mild blistering ointment, or common turpentine. In this way, report says, the College practice has proved very successful. Blistering we have, however, found, when repeated over and over, commonly prove equal to all the benefit these obstinate cases can receive; for, when the bone deposit is fully formed, it is in vain to expect its entire absorption. Even its partial absorption is frustrated. The removal of acute lameness is generally the only benefit which can be anticipated. Our 
principal hope lies in preventing the increase of the bony deposit, and likewise in removing that inflammation which is the existing cause of much of the pain, stiffness, and tumefaction in the surrounding ligaments. Our hopes of success must also greatly depend on the time the evil has existed. When it is early attended to-that is, before the bony deposit has gained its full soliditystimulants act more favorably, and lessen it more materially. It remains to add that, when repeated blistering fails, the firing-iron generally concludes the business, but commonly answers no further purpose than being a publication of the animal's infirmity, and a warning to practitioners as regards the previous treatment."

The remedies and means used by the author of this work for the treatment of spavin are the same as recommended for ringbone. (See article on Ring-bone.)

\section{- Fracture of the Pelvis.}

My attention having lately been called to two cases of fractured pelvis, I am led to make the following remarks for the benefit of those who feel an interest in matters pertaining to veterinary science. The accident of fracture of the pelvis is of very common occurrence, and I think that many such cases might be prevented by the exercise of a little judgment on the part of horse-owners. For example, the great majority of cases with which I am acquainted have occurred in the stable, after the animals have been put up for the night "all well," as the owners have informed me, and to them the matter seemed very mysterious and unaccountable. But I think that the mystery can easily be explained.

If a horse is put up for the night all well and free from lameness, and in the morning is lame, and it is observed that the hip is knuckled down, as the saying is, (fracture of the pelvis,) or, in other words, he is "hip-shot," the evidence then seems conclusive that the accident occurred within the stable. Then the question arises, How dicl it occur? We all ought to know that the stalls in our city stables are too narrow; many of them do not exceed four feet in breadth. Now, let a large horse throw himself violently down, as, they often do when tired or in pain, and he is very apt to strike the point of the hip against one side of the stable. The point of the hip being but imperfectly protected from external or lateral injury, except by the common integument, the 
jar or concussion thus received often occasions fracture. I know that this is the case; for, on making examination of some of these fractures, I found the skin over the region of the point of the hip bruised and abraded. Then, again, horses frequently get cast at night, and are the subjects of colic, or other aches or pains. They struggle violently, get imprisoned, as it were, in their narrow apartments, and, when released by assistance, fracture of the pelvis is often discovered.

This is not all. Sometimes sufficient bedding is not furnished to protect the animal from the effects of hurriedly reclining, or slipping down, on a hard plank floor, and the result is often the same as that just alluded to. Then, in view of prevention, these facts suggest the propriety of having wider stalls, and a liberal supply of shavings or sawdust for bedding; that is, when straw can not be obtained; yet I think that when sawdust can be obtained, it is the most wholesome and economical bedding for horses. Taking a pathological view of the matter, I have to inform the reader that a great proportion of these fractures occur among horses well advanced in years; and it may be that their bones, in some cases, are brittle and very easily fractured, as is the case with some aged members of the human family, whose thigh bones have been easily fractured by a slight fall, or jumping out of bed in a hurry, many such cases being on record.

The horse is occasionally subject to a disease of the bones known as fatty degeneration, which is said to be partly occasioned by an exclusive Indian corn diet. In such cases the vacuities in some of the bones are filled with oily mätter, are light, and easily fractured. He is also subject to a disease known as rickets, which may depend either on hereditary predisposition or imperfect nutrition. The bones are defective in early constituents, and, consequently, give way under the weight which they ought otherwise to sustain. The remedy is, a nutritive diet, partly composed of oats, and occasional doses of phosphate of lime.

Horses very frequently fracture the pelvis by falling in harness, but for this I know of no remedy except careful driving; yet sometimes, in spite of due care, they must fall when traveling, on slippery pavements, and when improperly shod.

Treatment.-As regards the treatment of fracture of the pelvis I have but little to offer. It is all contained in the words rest and counter-irritation (seton or blister), yet requires time for 
Nature to unite the bones after her well-known fashion. If the horse is a valuable one, and the fracture slight, he should be placed in slings. The hair may then be shaved off the region of fracture to the extent of one foot in diameter; then smear on, while warm, a strengthening plaster, composed of pitch, resin, and beeswax. But should the fracture be of a very grave character-the bones broken in several places, the animal down and in great agony - then the sooner he is put out of his misery the better.

\section{Splent, or Splint.}

Splent, or splint, makes its appearance on the inner and lower part of the knee-joint. It consists of a bony tumor (exostosis), and, finally, the small bone known as the metacarpus purvus becomes united to the cannon or shank bone. This constitutes anchylosis, so that the disease is of the same nature as ring-bone. Splent sometimes appears on the outside of the leg; but the disease is oftener found on the inside, from the fact that it is nearer the center of the weight of the body than the outer, and receives the greatest amount of concussion. The inner bone is said to receive nearly the whole weight transmitted to the small bone of the knee. A splent on the inside is often the result of a blow inflicted with the opposite foot, which bruises the soft parts and periosteum, and, finally, the bones become affected. Faulty shoeing has also a direct agency in the production of splent. For example: if one caulk of the shoe be higher than the other, or should the foot be pared more on one side than the other, the effect will be to throw the limb into a false position, which may result in ligamentary lameness and splent. Splent, however, appears sometimes without any assignable cause. On dissecting the limbs of aged horses, we frequently find the splent bones firmly united to the shank. The following is the opinion of Prof. Coleman in regard to splent:

" ' A splent once is a splent always.' The two bones being once united by ossific matter, this connection, whatever we may do or apply, will never be altered. It used to be the custom to remove the splent with a hammer and chisel, but the production of a large blemish, from the destruction of skin, was the consequence of this barbarous method. Another practice, as ancient and barbarous as the former, was the application of corrosive sublimate, 
A PERFECT FORE-IJEG.

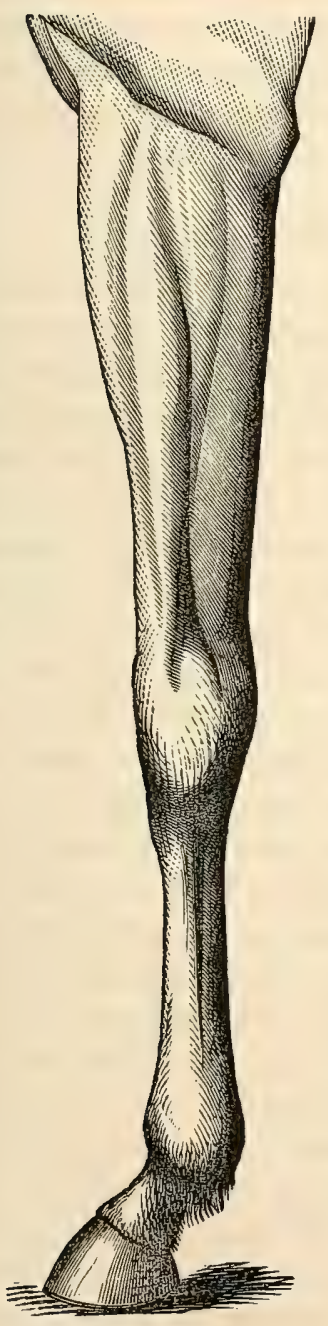

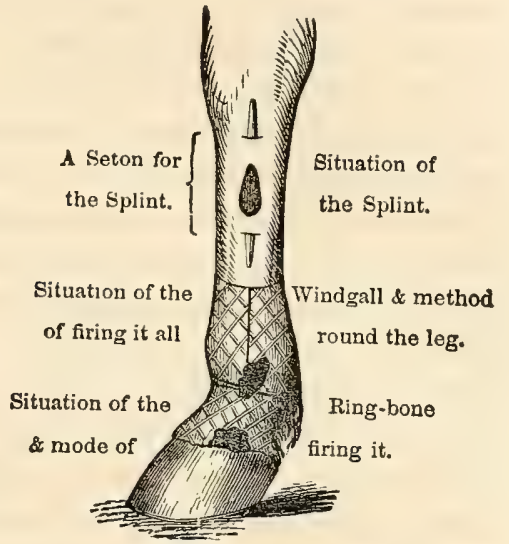

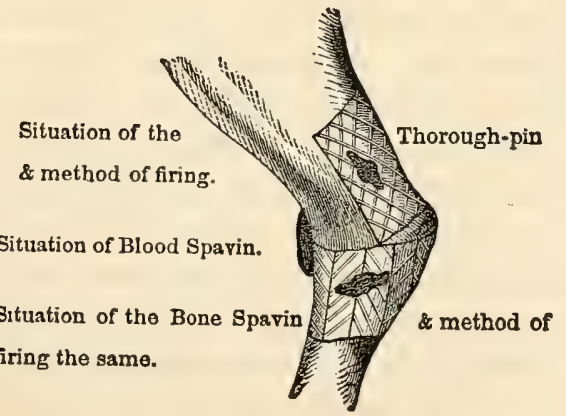

BOTTOS OF THE HOOF.

$a$ a the crust. $b b$ the sole.

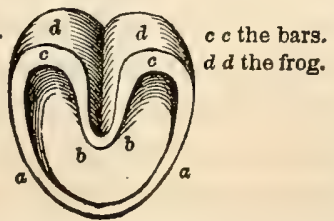


or other strong caustic, so as to occasion a slough. In this case, too, the remedy was as bad as the disease; the horse was disfigured for life. Better and more recent treatment than this was either firing or blistering. The objection to the former, however, was the marks it produced; and to the latter, in common with the former, that though the lameness was removed, it very frequently returned with the resumption of labor.

The more modern, successful, and scientific treatment of splents consists in the operation of periosteotomy, or division of the periosteum, which covers the bone.

Professor Sewell has introduced, within the few past years, an excellent mode of performing the operation. The horse is cast, the leg properly straightened and secured, and then a small opening is made just below the splent sufficient to introduce a long, narrow, convex, probe-pointed knife, the edge of which is on the convex side. The knife is then passed up under the skin, and by drawing it backward and forward on the splent, pressing firmly at the same time, the periosteum is completely divided. A small opening is then made through the skin above the splent, and a narrow seton passed from one orifice to the other, after which a bandage is placed on the leg, and the horse released. The seton should be moved and dressed daily with digestive ointment, and at the expiration of a week removed, and the wound permitted to heal. I have found the operation succeed whenever I have adopted it. In the very numerous cases that occur of splents being unattended with lameness, it is 'better to let well alone;' but when the lameness is slight, and the horse can not be spared from work more than a few days, it is well to apply a mild blistering application, such as the tincture of cantharides, about a tea-spoonful of which will be sufficient for one application, which may be repeated according to the action it produces and the benefit it oceasions."

Treatment.-Our practice in this country is to treat splent on - the same general principles that obtain in spavin and ring-bonenamely, in the acute stage, when the accident of striking has happened, we apply sedatives and refrigerents, and in the chronic stage, counter-irritants and absorbents. For the treatment of the acute stage, a selection from the following articles may be made: Arnica, infusion of hops or poppies, cold water, or equal parts of vinegar and water. In the chronic stage, and in view of lessening the tumefaction, I recommend the following: 
No. 60. Powdered iodide of potass.......... $2 \mathrm{dr}$.

Simple Cerate................. 1 oz.

Cod-liver oil................. $\frac{1}{2}$ oz.

Rub these ingredients together in a mortar, and when thoroughly mixed it is fit for use. A daily application of this ointment for a short time will suffice. There are many cases of splent that do not occasion the least lameness - therefore they had better be let alone.

\section{Bowed Legs or Sprung Knees.}

The following case will illustrate the method of cure for sprung knees. The subject of this operation had met with an accident, which resulted in "bowed limb," and had so progressed within the period of a few months as to render the limb the most deformed one I ever saw ; for when the toe touched the ground, the heel was elevated about four inches. At the same time, the inferior extremity of the cannon overlapped the large pastern something like three-quarters of an inch. Such a deviation from the perpendicular line would, as a matter of course, bring a tremendous strain on the extensor tendons in front and the suspensory ligament, and this appeared to be the case; for the tendons and ligament were rigid as a bowstring.

The horse was cast on the off side, and, after securing the limbs, the near fore one was released from the hobble and held by assistants. A puncture in the outer lateral region of the flexor tendons was made through the integument. A convex, probe-pointed bistoury was then introduced, and, by a gentle sawing movement, the flexors perforatus and perforans were divided. On straightening the limb, which was done without the least difficulty, the edges of the tendons had separated almost two inches. These would, of course, after awhile, become reunited by granulation and interstitial deposits. Thus tendons would, finally, gain in length something less than two inches, which would render the leg almost straight again. The puncture through the skin being small, a single suture sufficed to close the orifice. This being accomplished, a cold-water bandage was neatly applied, and then the patient was permitted to rise. He walked to the stable tolerably well, yet still had a slight "knuckling over" at the fetlock. This was probably owing to the distended or lax state of the capsular ligament and extensor tendon. A shoe was now applied to the 
foot, having a straight piece of iron, eight inches in length, welded on to the ground surface of the toe of the shoe. The object in applying this contrivance was to prevent flexion, to keep the divided ends of the tendon apart, and to guard against direct union of the same. The operation finally proved successful.

The following case occurred in the practice of Professor BRACHET, as given in the "Veterinary Record:"

"This most extraordinary case was observed in September, 1856, three months after the inundation of the Rhone, on a farm near this river. The subject of the disease was a filly, three months old. She was born perfectly straight on her legs, but about fifteen days after birth the fore-limbs became bent, and the deformity increased so rapidly that when Brachet saw the animal it moved on its knees. It was with great difficulty that, by efforts to extend the limb, the acute angle formed at the knee could at all be widened, and such an experiment gave great pain to the animal. The dam being taken out of the stable, Brachet was astonished to see the filly walking after her on her knees, almost as fast as if progressing naturally. On the front of the knee the skin was hard and callous, the tendons behind it were rigid and immovable, and Brachet decided on performing tenotomy. The animal was cast and the near fore-leg operated upon. An incision was made, about an inch in length, at the back of the arm, in a line with the upper part of the carpus; the tendon of the flexor metacarpi externus was first cut, then one of the medius, and, lastly, that of the internal flexor of the metacarpus, when the limb straightened. A splint was placed in front of the leg. Eight days afterward the off limb was operated upon; fifteen days later the splints were removed, and, in the course of a month, the filly stood well, but in moving carried her fore-legs forward in a piece. Flexion at the knee could not be effected, but the young animal improved rapidly; the limbs became strong, and though slightly bowed, their action was perfect. Brachet believes the deformity arose from rheumatism, induced by the damp on the occasion of the overflowing of the river near the farm."

The following is Mr. Pencrvalc's method of performing tenotomy for sprung knees:

"The operation of tenotomy, though a formidable one for the patient, is not a difficult one for the operator. The object is section of the flexor tendons, the effect of which, as we have seen, is to 
let down the heel of the foot, not the fetlock, to the ground. The flexor tendons support the pastern and foot-joints principally, the fetlock-joint having the additional strong support of the suspensory ligament, which it still retains after the tendons have been cut through. This accounts for the heel of the foot, without the fetlock, being let down by the operation of tenotomy.

Having cast the horse, and so secured the limb to be operated on that there is not much chance of any interruption being oceasioned through its motion, I recommend that a longitudinal incision, three or four inches in length, be made through the skin, along the back of the leg, down upon the middle portion of the flexor perforatus tendon. This incision freely and boldly made at once, the operator will be able to stretch the mouth of the wound he has made round to the inner side of the leg, in which stretched position the skin is to be held by an assistant, while the operator introduces the fore-finger of his left hand, to push back the bloodvessels and nerve (which run along the inner borders of the tendons) against the suspensory ligament, so that they be safe out of the way, while with the right hand he insinuates his bistoury between them and the flexor tendons. Opposing now the cutting edge of the bistoury to the tendons themselves, he commences incising them by a steady but firm and strong sawing movement, until both be completely divided. I say he is to hold the bistoury (which ought to be a stout one) firmly, and to use it with some force, since such is the dense and tough texture of these tendons that they are not cut completely through without some determination. Complete division being made of them, the heel of the foot may not (in all probability will not) come down without some extension of the leg; and this must be made, not by such violence as Mr. Goodenough found himself compelled to use, still with such force as will, if possible, make the limb straight, by stretching or even tearing through adhesions of moderate standing, so far as this can be effected without the risk of rupturing ligament or fracturing bone. The giving way of adhesions, in such cases, is frequently attended with a sort of snap or jerk, denotive of their being overcome, and by this the end is known to be answered, such adhesions and impediments to extension being commonly situate about or in the vicinity of the fetlock-joint. The usual and most effective mode of accomplishing the extension is, to place the knee against the front of the fetlock, and, grasping 
the back of the foot with one hand, and the upper end of the leg with the other, to use such steady and moderate force as will accomplish the object without doing harm thereby. This done, the diviced ends of the tendons recede from each other, leaving a gap between them of one or two inches, or even more, dependent upon circumstances."

\section{ConNs.}

What is known as corns, occurring in horses, is very different from that affection in the human subject, which bears the same name. In the horse the affection is caused by bruise of the sole, and consists of extravasation of blood. On making an examination in the angle between the bars and quarters, the horn of the sole will appear red. This may not be seen, however, until the blacksmith has pared away a flake or two of the sole.

Treatment.-Neglected corns are very apt to end in suppuration (formation of matter within the hoof). This matter sometimes burrows in an upward direction, and breaks out at the top of the hoof. It then forms what is known as a quittor, the remedy for which is to make a small opening into the sole, and thus allow the matter to escape in a downward direction. After making the orifice in the sole, it should be dressed with a paste composed of equal parts of sugar and brown soap. As a dressing for common corn, without suppuration, I recommend equal parts of tar and olive oil. This I conceive to be one of the best dressings ever used for corns.

Many corns are produced by suffering the shoe to remain on too long. It thus becomes imbedded in the heel of the foot; the external crust then grows down on the outside of the shoe, and the bearing is thrown in the angular portion of the sole. In unusually troublesome cases of corns, recourse should be had to the bar-shoe.

YouatT says: "Mr. Spooner, of Southampton, very properly states that the corns occasionally fester, and the purulent matter which is secreted, having no dependent orifice, ascends, torturing the animal to a dreadful extent, and breaks out at the coronet. These cases are very troublesome. Sinuses are formed, and the evil may end in quittor. A large and free dependent orifice must then be made, and a poultice applied, to which should succeed a solution of sulphate of zinc, with the application of the compound tar ointment. 
The cause of corn is a most important subject of inquiry, and which a careful examination of the foot and the shoe will easily discover. The cause being ascertained, the effect may, to a great extent, be afterward removed. Turning out to grass, after the horn is a little grown, first with the bar-shoe and afterward with the shoe fettered on one side, or with tips, will often be serviceable. A horse that has once had corns to any considerable extent should, at every shoeing, have the seat of corn well pared out, and the butter of antimony applied. The seated shoe should be used, with a web sufficiently thick to cover the place of corn, and extending as far back as it can be made to do without injury to the frog.

Low, weak heels should be rarely touched with the knife, or any thing more be done to them than lightly to rasp them, in order to give them a level surface. The inner heel should be particularly spared. Corns are seldom found in the hind feet, because the heels are stronger, and the feet are not exposed to so much concussion; and when they are found there, they are rarely or never productive of lameness. There is nothing perhaps in which the improvement in the veterinary art has relieved the horse from so much suffering as shoeing. Where corns now exist of any consequence, they are a disgrace to the smith, the groom, and even to the owner."

\section{LAMENESS.}

We have already considered the nature and treatment of various forms of lameness, occurring in consequence of strain or sprain of different parts of the body; also that attending rheumatism and diseases of the feet. It only remains to offer a few remarks on some special forms of lameness. Lameness occurs in one of two forms. We either find it in the acute stage, when, from injury or other causes, it comes on suddenly; or else in the chronic stage, that form whieh has existed for some time. Therefore, there being only two forms of lameness, there are only two indications to fulfill, namely: in the acute stage, we endeavor to lessen the activity in the circulation, heat, and pain of the parts by rest and cold-water bathing; and when pain exists, we mitigate it by bathing the affected parts with cold infusion of hops or poppy-heads. When the affection assumes a chronic type, we apply stimulants and counter-irritants. The following is the best remedy in use: 
No. 61. Aqua ammonia.............. $3 \frac{1}{2}$ oz. Spirits of camphor........... $3 . .$. Salt (bay or rock salt).........8 oz. Water.................. 2 pints.

Dissolve the salt in the water, with a few drops of aqua ammonia; allow the impurities to settle, and add the clear liquor to the camphor and ammonia, the latter having been previously mixed. A portion of this should be rubbed on the region of lameness morning and evening. Sometimes the actual seat of lameness may be somewhat obseure, yet we can generally find out which limb it is in ; then, to make sure that we shall hit the mark, the whole limb may be treated. Should counter-irritation be necessary, strong liniments and blisters, such as are in general use, are recommended.

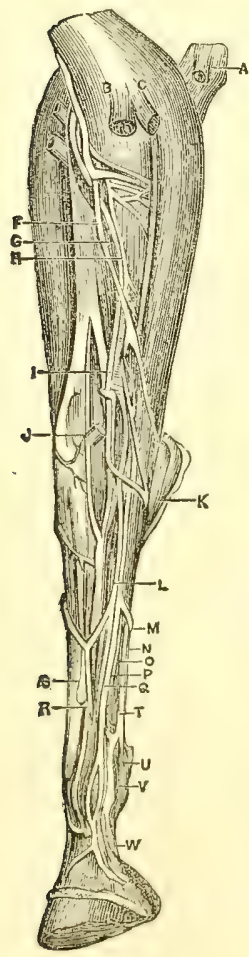

This plate represents the fore extremity, cut off a little above the condyles of the humerus, with the flexor metacarpi internus, medius and the ulnaris accessorius muscles removed.

A, The origin of the ulnaris accessorius muscle.

$B$, The flexor metacarpi internus.

C, The flexor metacarpi medius.

$\mathrm{E}$, The radial nerve.

$G$, The radial artery.

H, The radial rein.

I, The superior suspensory ligament, cr band.

$J$, The tendon of the flexor metacarpi internus muscle.

$\mathrm{K}$, The cut edge of the posterior annular ligament turned back.

$L$, The inferior suspensory band.

M, Oblique branch of nerve crossing the perforatus tendon.

$\mathrm{N}$, The perforatus tendon.

$\mathrm{O}$, The perforans tendon.

$P$, The inner metacarpal nerve.

Q, Metacarpal artery.

$\mathbf{R}$, Suspensory ligament.

$\mathrm{S}$, The metacarpal vein.

$\mathrm{T}$, One of the lumbrici muscles.

$U$, Cut edge of the thin, dense membrane whtch invests the perforatus from a little above the front of the fetlock downward.

$\mathrm{V}$, The perforatus tendon.

W, A portion of the tendon lined internally with synovial membrane, removed, to expose the perforans as it passes through the bifurcation of the perforatus. 


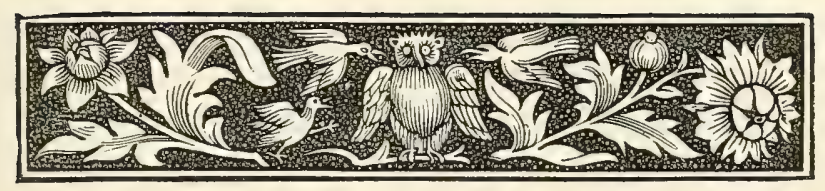

\section{SECTION XIII.}

HORSE MANAGEMENT, ETC.

Prolapsus Ani-Hemorrhords, or Piles-Pricking and Nicking Horses Tails -Docking Horses-Anemia-The Use and Abuse of the Curry-combHorses and Cattle shodld have a supply of Pure Water-Galled Back -The Heart-The quantity of Blood contained in a Horse's BodyRapidity of the Circulation-The transfusion of Blood-Management of the Horses pUt in the Stable-Strangles.

\section{Prolapsus Ani (Falling of the Fundament or Rectum).}

$\mathrm{P}$ ROLAPSUS ANI, or protrusion of the rectum, is a calamity much to be dreaded. Fortunately it very rarely occurs, yet when it does, a valuable horse is almost sure to be the subject; hence it is a matter of importance that persons who own valuable horses should know something about the nature and treatment of this mishap. The best authority on this subject is Mr. Percivall, from whose works the following is quoted:

"It consists in inversion and prolapse of the mucous coat of the rectum, either confined to the mucous membrane itself, or otherwise involving more or less of the entire substance of the intestine along with it. The aspect of the protrusion will mainly denend on the nature and volume of the parts ejected, and upon the time such evolution has been in existence. When recent, so readily does tumefaction (swelling) of the parts follow their protrusion, that this speedily opposes all attempts at return, should the animal make any, though, in truth, his efforts in general have the effect of straining more gut out, and through his straining, in some cases, tearing the membrane, and thus augmenting instead of diminishing the evolution as well. In this manner arises a large (sometimes enormous) rotund, red, wrinkled redness of skin, consisting of cylinders of mucous membrane, having an aperture 
closed through constriction in its centre, though, when open, issuing a mucous and sanious matter, especially at such time as the animal is straining afresh. The circumflex action of the sphincter ani around the neck of the swelling, together with its distension with gas, and the action of the air upon its surface, all add to its increase of volume and change of color; while the straining, at the same time, increases the difficulty of any effort the practitioner may make toward effecting a return of the protruded gut. With its augmentation of volume comes a deepening redness, turning, through the constriction of the sphincter, to a darker, and even purple hue, the membrane becoming, at the same time, inflated and swollen, and gradually changing its color from red to yellow and to brown, though still having a humid shining aspect, now becoming glairy from albuminous secretion, which is not only mucous, but at times purulent.

Causes.-These may be summed up in irritation, either direct or sympathetic, of the mucous membrane lining the anus and rectum. It is possible that prolonged constipation, from giving rise to violent straining efforts at expulsion of dry and hardened feces, might bring it on, or even the strains of parturition. The action produced by excessive purgation may cause it, but more especially irritation of a mechanical kind, arising from direct injury to the membrane or rectum during the operation of raking or manual exploration of the gut, or of clystering. It may prove an accompaniment of an enteritic, colicky, or diarrheal condition of bowel. It is possible it may ensue on nicking, though I never knew it to follow that operation. Violent struggles of any kind, as in such a case as is mentioned in the 'Veterinarian,' by Mr. J. Brown, V.S., London, wherein the horse 'had forced out nearly a foot of the rectum in struggling violently to release himself,' while being cast.

Treatment.-The formidable, and indeed awful, aspect of this disease is apt to operate in the mind of the owner of the animal favorably for the veterinary surgeon, inasmuch as it gives rise to his being called to the case sooner than he otherwise would have been. Should the summons be an early one, at the time that the protrusion is recent, and its volume nothing so very alarming, judicions exercise of the taxis, if employed at the moment, may succed in the return of the gut. Distributing the fingers of both hands over the wrinkled and swollen body of the protrusion, 
steady, firm, and forcible pressure ought to be maintained against it for such length of time as appears to afford any chance of success, augmenting the force used whenever there be any remission of the straining. Should the first efforts of this description fail, the protruded mass may have its bulk lessened and constricted as much as possible by local application of some sort, than which none offers a better chance of succeeding than such as follows from sudden and intense cold, though to a part so sensitive and vascular the practice is not devoid of danger. Ice may be powdered and inclosed in a linen bag, so as to form a sort of bolster, that may be held or braced with firmness upon the tumefaction. The contrary of such treatment as this, however, though the object be the same, is generally preferred-namely, fomentation, medicated or not, with scarification of the exposed membranethough in other cases astringent applications are employed, with a view of causing contraction and diminution of the mass, such as lime-water, decoction of oak bark, solution of alum, etc.; and while such proceedings are going on, it is advisable to have the hind parts raised. Supposing we succeed in accomplishing the reduction, the chances are in favor of the relapse of the prolapsus, and these chances seem great in proportion to the facility with which the return of the inverted gut has been effected. Indeed, so constant and troublesome does the return in some of these cases become, that it is necessary to contrive some sort of truss to oppose the descent, the same as is done for rupture in man. $\mathrm{Mr}$. Dycer's truss consisted of ' a new wet chamois leather, a breastplate, and a hip (human) truss.' Should constipation be thought to operate against reduction, in addition to clysters, we may exhibit cathartics and aloes in solution, and should there be plethora and great irritability in the membrane of the rectum and anus, a blood-letting will be advisable, while an opiate or belladonna clyster will be advantageously administered, in order to allay all local irritation as much as possible.

As a dernier and effective mode of procedure in prolapsus ani, when the case proves either irreducible, or, after return, continually protrudes on any slight effort, such as coughing afresh, we have recourse, with safety and certainty, to an operation consisting in excision of more or less of the inverted membrane enveloping the protruding portion of the gut. This is an operation of ancient date, though to Dupuytren is ascribed the credit of 
introducing it into human practice, and to the French veterinarians that of transplanting the same into veterinary practice. Formerly the actual cautery was the instrument employed for the removal of the parts obstructing reduction; but in our own day this has been thrown aside for the scalpel, an instrument quite as effective, while the simple act of cutting with a knife gives so much less pain than cutting or dividing with the actual cautery. In general, it is considered necessary to cast the horse for the operation; but Mr. Gregory, V. S., did not cast his patient, but merely put on a side-line, and had one leg held up and the tail kept on one side; and, most assuredly, the standing position of the patient is one which, in such an operation, offers no mean advantages to the operator, provided he can avail himself of it without any personal danger. A great preservation against refractoriness in an operation of the kind, at the moment when any pain is felt, is a twitch, well put on, and well and timely turned. The horse secured, with his tail turned out of the way, an incision, forming a circle, is to be carried around the protruding mass, at sufficient distance from the constricted part of the neck of the protrusion to leave behind it such parts as are distended from tumefaction or infiltration, whose removal will render the retraction of the gut an easy and natural effort. In making incision, it may be advisable to take up and tie with a silk ligature any vessel met with of size enough to issue a current of blood, though sometimes no such precaution is required. In the case mentioned by Mr. Gregory, not more than a quart of blood was lost. The incision ought to be made of sufficient depth to penetrate completely through the substance of the mucous membrane, however morbidly thickened that may be, without, however, running a risk of wounding the muscular coat beneath it, the object being to dissect the former away and strip it off the latter, so as to lessen the bulk of the mass to that degree that return becomes a voluntary and facile action of the animal himself. So soon, however, as this denudation has been carried near to the inverted anus, care must be taken not to dissect or otherwise injure the sphineter of that part, lest we leave the horse with an imperfection in closing an outlet of so important a function. A soft or mash diet should, for a few weeks, succeed the operation, with abstinence from hay, which, from its fibrous, prickly nature, must be particularly offensive to the denuded gut. Occasional emollient clysters are also 
recommendable, especially when there appears any pain or difficulty in giving exit to the feces."

\section{Hemorrhoids, or Piles in the Horse.}

The following case of piles, occurring in the practice of Mr. P. WALS, V. S., is here introduced from the "Veternarian:"

"On the evening of March 13, 1851, I was summoned hastily to attend a brown carriage-mare, belonging to an employer about a mile distant from Norwich, which was reported to have something 'bloody' hanging out of her rectum. The coachman who came said it had made its appearance two or three times during the day after dunging, but had always returned again, until just before he left home, when a portion of it was left out, and the mare became very uneasy. On my arrival, I found, as reported, the mare in pain, indicated by a peculiar switching or lashing of the tail, and stamping of the hind feet; but she had not lain down, although she made several attempts to do so. It first struck me as a case of prolapsus ani, but, on examination, it proved to be a true case of piles. The tumor protruding was about the size of a duck's egg, presenting precisely the appearance of a pilous grape in the human subject, only, of course, much larger. I had some difficulty in returning it, in consequence of the powerful action of the sphincter ani, which, no doubt, accounted for the pain; but, having accomplished it, the mare became easy, and continued so for some little time. I thought it right, however, to have her removed to my infirmary, as I anticipated a return of the tumor when she again dunged, in which I was not deceived, since, on her arrival at my stable, I found it out again. On making a more minute examination, I discovered smaller tumors round and about the larger one, the latter, however, being the only one that protruded. The mare being naturally of an irritable temperament, and consequently dunging more frequently than one less so would have done, induced me to have a careful assistant remain up with her during the night, for the purpose of returning the tumor whenever it made its reappearance, which it invariably was said to do after each evacuation, pain being constantly present at such time.

Reflecting on the case next morning, and observing considerable mischief was being done by the necessary manipulation, I 
foresaw I should have a very troublesome case, unless I could hit upon some plan of dilating the sphincter so as to allow of a return of the tumor without so much handling of it. This I accomplished in the following manner: I had the mare placed in a narrow stall, the back part of which was well bedded with litter, so that, in standing, her hind quarters were elevated six or eight inches higher than the fore ones. I then procured a pair of glovesticks, such as glovers use for opening the fingers of gloves, and, having dipped their ends in oil, gently introduced them about an inch or an inch and a balf into the rectum, underneath the tumor, letting it, in fact, rest upon them. In this way, by pressing the handles, dilatation took place, and the tumor receded with little or no difficulty. This treatment I persevered in for four days, in conjunction with a constant application of cold water to the parts, at the end of which time such great improvement had taken place that the use of the sticks became no longer necessary. In four days more the parts appeared to have become quite reinstated, which, of course, rendered any further treatment unnecessary. I gave no medicine, except a very mild aperient in the first instance, but kept the bowels lax with bran, linseed, carrots, etc. The mare being sold shortly afterward, I lost sight of her, and am unable to say, in consequence, whether any return of the difficulty has taken place, but I should think such an occurrence not improbable."

\section{Pricking or Nicking Horses' Tails.}

The English method of nicking horses is, instead of inserting a knife and making a subcutancous section of the muscles of the tail, known as the depressors, to make three straight incisions at equal distances right across the tail. By this method the muscles of the tail are effectnally or completely divided. The first incision is usually made about two inches from the root of the tail, and the other two at equal distances from the first, in a direction toward the end of the tail. The tail is then kept perpendicular, in the usual manner, by pulleys. It is said that by this method a more graceful curvature of the tail is secured, which I believe is a fact, yet the operation is any thing but pleasant to the horse. It is one of the fashionable barbarisms of civilization.

The English method of nicking, although the most unsightly and barbarous, is still the safest, as there is less liability to lock- 
jaw. It is well-known that lock-jaw more frequently ensues from a punctured wound than a clean cut or incision; yet when nicking or pricking, after the American fashion, (if care be taken to make the orifice through the skin sufficiently large, so that pus, afterward secreted, may escape,) I think there is no danger. The danger arises from the imprisonment of pus by closure of the orifice in the skin. In healthy subjects the orifice made through the skin will often unite in the course of a few hours; then, should morbid matter accumulate within the substance of the tail, it burrows, forms an abscess, or else becomes absorbed, producing lockjaw. Therefore, I would advise persons having pricked or nicked horses under their care, to pay strict attention to such, and see that the orifices are kept open, which may easily be done by occasionally inserting the point of a penknife. The skin should be the last to heal. The healing process must be perfected in the interior first, and, lastly, the skin. Should fungus or "proud flesh" appear, apply powdered bloodroot, burnt alum, or red precipitate. When an abscess forms at the base of the tail after pricking, apply a poultice of flaxseed; and when the tail is much inflamed and swollen, it should be bathed frequently with the following:

No, 62. Acetic acid.................. 1 part.

Water...................... 7 parts.

Mix.

When horses are nicked in warm weather, the tail should be moistened with tincture of aloes; this will keep the flies off.

Persons who are not experts in the art of pricking are apt to wound, and sometimes completely sever the coccygeal arteries, thereby causing an unnecessary and dangerous hemorrhage. In such cases, I should advise the operator to crowd into the orifice a small piece of dry sponge. This will arrest the hemorrhage, and, at the same time, keep the divided ends of the muscles apart, so that they can not reunite; hence it is not necessary to put the tail into pulleys for some hours, or until bleeding has ceased. When this is the case, the sponge may be removed by means of a small pair of forceps.

The success in securing what is denominated a fashionable tail depends altogether on the skill of the operator, who must make a complete division of the muscles beneath the tail only, as their action is to compress and depress that useful appendage; for, 
should the incision be made too high on the side of the tail, the curvators may be partly or wholly severed. Then the tail will ultimately diverge laterally, or, in stable language, it will be said that "the horse does not carry his tail straight." To remedy this, the curvator on the opposite side must be divided; then the tail has to be forcibly drawn and kept in an opposite direction from the curved side, so as to prevent the muscle from reuniting. In the course of a short time granulations are thrown out from the surface of the divided ends of the muscle. It then acquires length, and thus the tail becomes straight.

\section{ANATOMY OF THE TAIL.}

For the benefit of those who wish to practice the art of " nicking or pricking" understandingly, I now propose to give a brief exposition of the anatomy of the tail.

Bones of the Tail.-The bones of the tail, numbering fifteen, sometimes sixteen, are situated at the back of the rump-bone, or sacrum. They are not a continuation of the vertebral bones, but are an appendage to the haunch bone. The bones entire are termed coccyx (from the Greek, which signifies cuckoo, the bill of which bird the appendage to the sacrum, or haunch-bone of man, is said to represent); and, in order to prevent confusion in anatomical terms, veterinary surgeons have applied the same name to the tail bones of the horse. Hence, when the terms coccygis or coccygeal are used, they have some relation to the tail. The bones are roughened on their surfaces, and have depressions and eminences for the insertion of ligaments, tendons, and muscles.

Muscles of the Tail. - These muscles are divisible into four pairs. They are most distinctly seen at the root of the tail, but, in their course toward the end of the same, they become blended one with another.

The first pair of coccygeal muscles are situated on the upper part of the tail. They are attached to the sacrum and to the bones of the tail. Their action is to raise or erect the tail.

The second pair are termed depressors coceyx, and are situated at the under part of the tail. They are attached to the underneath part of the sacrum, and to the same locality on the bones of the tail. They are antagonistic to the first pair, and their direct action is to depress the tail. These are the muscles which should be divided in the process of pricking or nicking. 
The third pair are termed curvators coccyx. These muscles are situated on the sides of the tail, between the first and second pairs. They are attached to the fourth and fifth lumbar vertebra, and to the sides or transverse processes of all the bones of the tail. Their action is to curve or flex the tail toward the quarter and sides, and the division of one of these muscles causes permanent flexure of the tail in an opposite direction. Considering the tail as a means of defense in switching off flies, etc., these muscles are the principal ones called into action.

The fourth pair are termed compressors coccyx. They are located at the sides and root of the tail, and are inserted into the back part of the hip bones' (ischium), and into the five first bones of the tail. The action of these muscles is to maintain the tail forcibly against the anus. In the mare, these muscles protect her from ravishment.

The arteries of the Tail are termed coccygeal arteries. Those located at the sides are called lateral coccygeal, and the one found in the lower or inferior region is called inferior coccygeal.

Nerves of the Tail.-As with the muscles, so with the nerves. There are four pairs of coccygeal nerves. They are not a continuation of the true spinal cord, but are given off from it. They are distributed to the muscular filaments, to the skin, and at the extremity of the tail.

\section{Docking Horses.}

All persons familiar with the old tactics of "jockeyism" and the tricks of horse trade, will understand what is meant by the above caption. It is a most cruel and barbarous outrage on one of Nature's menials, and I feel inclined to indorse the sentiments of the profound poet Tupper, when he says, "In that great day, when crime is sentenced, cruel man shall not go uncondemned because no complaining brute stands there accusing, for our $\mathrm{Fa}$ ther careth when a sparrow dies."

Horses should never be deprived of any part of that valuable weapon of defense known as the tail. It is a part and parcel of the organization of the horse, and to deprive him of what Nature gave him, after the fashion of "doing all things well," is an outrage which should be considered beneath the dignity of a gentleman to perpetrate. Besides the cruelty which is acknowledged 
to be identified with the operation, there are other reasons, on the grounds of physiology, why the tail should be left entire, especially in the case of a mare; but, as the reader is probably conversant with the facts in the case, I shall not enter into a discussion of the same, but prefer to introduce the following paragraph, written by a person who aims at reform:

"I am glad that the abominable practice of docking and nicking horses is going out of fashion. It prevails in no country in the world besides England and the United States. We got it from the mother country, and the sooner we leave it off the better. It is wonderful how any body but an ignorant, narrowminded blockhead of a jockey should ever have thought of it, being as offensive to good taste as it is a violation of every humane feeling. Has Nature done her work in such a bungling manner in forming that paragon of animals, the horse, that a large piece of bone should be chopped off with an ax to reduce him to symmetry, or that beauty and grace can be obtained only by cutting a pair of his large muscles? 'The docking and nicking of horses,' says an intelligent writer on farriery, 'is a shockingly cruel practice, and ought to be abandoned by the whole race of mankind. Every human being, possessed of feeling and a magnanimous mind, must confess that it is cruel and entirely uncalled for ; but that creature called man attempts thus to mend the works of his almighty, wise Creator, in doing which he often spoils and disfigures them. What is more beautiful than a fine horse, with an elegant, long tail and a flowing mane waving in the sports of the wind, and exhibiting itself in a perfect state of nature? Besides, our Creator has given them for defense as well as beauty.' The same author relates an instance of a fine hunting horse, owned by an Englishman, which would carry its rider over the highest fivebarred gate with ease; but he thought that the horse did not earry as good a tail as he wished; he therefore had him nicked, and when the horse got well, he could scarcely carry over two bars. 'Thus,' said he, 'I have spoiled a fine horse; and no wonder, for it weakened his loins.' Any man of common sense would give ten per cent. more for a fine horse whose tail had never been mutilated, than for one which had been under the hands of a jockey." 


\section{Anæmia.}

This signifies an impoverished state of the blood, usually occasioned by want of nutritious food and exposure to inclement weather. I was called, a short time ago, to see a yearling, said to be ailing and unthrifty, and had been so for some time.

Symptoms.-The principal symptoms were as follows: The head, face, and eyes were in a dropsical condition and much swollen; underneath the heart, and along the lower part of the abdomen, dropsical swellings were also observed; the membranes of the mouth and nose appeared pale, almost bloodless; the pulse was quite slow, and the action of the heart feeble; the respirations were somewhat hurried, yet the lungs were not expanded to their full eapacity. I was informed that the urine was thick, and of a cream-like color, and the feces hard and scanty. The muscular system was spare and wiry; the ribs could be easily counted, and the animal was unsteady on his legs. From such symptoms as these the reader will perceive that the case was one of debility, and from the fact that the animal had been confined to an innutritious diet, or, rather, like a hen, had to scratch for a living. It will also be inferred that the debility was the result of partial starvation.

Treatment.-I gave the animal one drachm of sulphate of iron, and three ounces of the fluid extract of resin weed per day, during a period of ten days. At the same time, he was liberally fed on oats and good timothy hay, under which treatment he rapidly gained in health and strength, and soon recovered.

\section{The Use and Abuse of the CURry-comb.}

I do not advocate that the curry-comb shall be entirely dispensed with ; for, in the first place, it is needed for the purpose of removing scurf and dirt which has been removed from the external surface of the body by means of the brush, and, probably, this was the intention of the man who first invented a currycomb; and, in the next place, the curry-comb may be needed to scratch off or remove mud that may have become dry and stiff on the limbs of horses ; and for these reasons, if for no others, I suppose we can not dispense with the curry-comb. There are some parts where the curry-comb should not be used-for example, on 
the inside of the thighs, flanks, chest, and face, where the skin is not so thick, and more sensitive than some other regions of the body. Some horses, especially those of a strongly-marked, nervous temperament, have very sensitive skins, and can not even bear a fly to light on their bodies without." evincing signs of pain and irritation. On such the curry-comb should be used gently and in a spirit of mercy. There are other horses, however, especially those of the lymphatic temperament, and whose skins are less sensitive, who can bear the action of the curry-comb without much flinching, and it is the duty of a merciful man to consider this matter when cleaning a horse. Thoroughbred horses, which include a vast number of the sanguine temperament, have a very active circulation over the whole surface of the body, and this vast circulation, through the superficial vessels, argues a delicate sense of touch. To them the curry-comb, unless used very gently, is severe torture.

\section{Horses and Cattle should have a Supply of Pure Water.}

Although few persons think it worth while to pay much attention to this department of husbandry, yet a little reflection will convince any reasonable person of the value and importance of furnishing animals with a constant supply of pure water. Pure, cool water is said to be a God-send to a thirsty throat; and as animals are apt to have thirsty throats, they should be permitted to enjoy a luxury which costs but a trifle, and operates very favorably in promoting their health. All classes of domestic animals have as great an aversion to impure, filthy' water as ourselves, and the former will often turn away with disgust from the filthy stuff called water which is often found in water-troughs on the roadside, and within the precincts of the barn and in some pastures. The common, stagnated pond-water, which many poor creatures are compelled to imbibe, is often the exciting cause of disease, especially in the Western States, where decayed vegetable matter abounds.

Pure water will never injure an animal. I do not believe the stories which are told about horses becoming foundered in consequence of drinking pure water. In a majority of cases, Mr. Fastman is blameable. He has probably either overdriven or over- 
worked the creature, or else has suffered it, when heated, to cool off without the necessary care and attention which should always be observed when animals are fatigued or perspiring freely. Hard usage, willful neglect, and wanton cruelty are more likely to produce disease than the universal beverage, so acceptable to the palate of a weary or thirsty horse. How often do we see a "let" horse come into the stable all exhausted and used up, scarcely able to advance one limb before another! Examine into the facts, and we shall find that the powers of the subject have perhaps been overtaxed. He has been driven too far, or at too rapid a rate, for the present state of his constitution to endure, and perhaps he has not had sufficient nourishment to repair the waste incidental to the living mechanism under the states of rapid and protracted labor. Is not this enough to account for the used-up condition? Is it not more rational to suppose that abuse of the respiratory organs and those of locomotion operates far more unfavorably on the horse than water? It is. But Mr. Fastman must, if there be any blame rightly belonging to him, try to shift the same from his shoulders, and, therefore, he avails himself of a popular error"He drank too much water ;" yet the individual had no means of ascertaining the precise quantity needed.

We might say, as regards some horses, whose labors are very fatiguing, that they come from their work, and, as soon as unharnessed, go to the trough, and imbibe from one to three buckets without any bad effect. Some animals need more water than others. The kind of work, the temperature of the atmosphere, and the nature of the food, whether it be wet or dry, all tend to diversify an animal's wants. The domesticated horse requires a bountiful supply of good water. His body is composed of seventyfive per cent. of the same, and he can no more exist without it than he can without food. A cow or ox is probably the best judge as regards its own wants as to the quantity of water needed. It is not the quantity which a rational animal imbibes which does harm, but it is the quality that demands our attention.

Thirsty people drink all the cold water they need. Then why deprive a cow or horse of what they actually require? Consider the condition of the inhabitants of populous cities during the summer season. Thirst amounts almost to a disease, and, in view of quenching it, the thirsty are continually imbibing water, rendered cold, hot, sour, sweet, or alkaline, just as fancy dictates or as fashion 
prevails. Cold icings and other fixings are called into requisition to smother the fire of thirst which rages within. Every body partakes freely - the young and the aged, the exhausted and vigorous, the laborer wearied by a hard day's work, and the rich man of no work-each and all are doing their best to see the bottom of the pitcher, and to pitch their bodies into the watery element. Yet, after all, how few persons complain of any bad effect from it.

Inquire into the history of some of the acute maladies that are supposed to arise from water-drinking, and it will be found that many of the sufferers have a peculiarity of constitution which renders them amenable to the laws of primogenital disease, which, although latent under ordinary circumstances, can, by disturbing the life forces, through neglect, cruelty, and overwork, be developed at almost any time of life.

Animals should never be allowed to drink pond-water. They should either have access to a running stream or a clean watertrough.

\section{Galled Back.}

Galled back, or sore back, is usually occasioned by bad-fitting saddle or harness. If that be the case, the shape of the same must be altered so as to correspond to the shape of that portion of the back which comes in contact with it. This can be done by padding the concavities with lamb's wool, and in chambering the convexities so that there shall be no unequal pressure nor causes for irritation. Yet, after all, it may be more economical to procure a new article and a better fit; and this may also be the most humane policy.

Treatment.-So soon as an abrasion is discovered on the back, the animal should be excused from duty for a few days. The abraded part should be lubricated two or three times daily with common tar and olive oil, equal parts, and this will soon restore the parts to soundness. Should there be no abrasion, yet tumefaction, heat, and tenderness, a cold-water pledget, renewed as occasion seems to require, will improve the condition of the parts, when recourse must be had to the tar and oil lotion. Sometimes, in consequence of neglect, the integument becomes callous or indurated. This condition was termed by ancient farriers "sit-fast." The treatment for this consists in smearing the callous with the following : 
No. 63. Iodide of potass............... $1 \mathrm{dr}$.

Simple ointment............... $6 \mathrm{dr}$.

Glycerine................... $2 \mathrm{dr}$.

Mix these ingredients well together, and they are then fit for use. A few applications of this ointment will have the effect of removing the callous, when a healthy surface will appear.

Some animals, owing to a peculiarity of constitution or of form, will chafe in those parts which come in contact with the collar and saddle, and no human foresight nor mechanical contrivance can always succeed in preventing the same. For example, some horses are humory, as the saying is-perhaps have a taint of scrofula about them-and if they perform a hard day's labor in the warm season of the year, notwithstanding they be harnessed in the best possible manner, they will come home at night with either galled shoulders or sore back. For such cases as these the harness-maker, with all his skill and ingenuity, has no other remedy than that of a palliative character.

\section{The Heart.}

The current of blood is said to be put in motion by a muscular organ known as the heart, which is located within the thorax, or chest. The blood is distributed to all the various ramifications of the body by means of vessels called arteries, and it is returned to the heart by the veins. The red color of the blood is due to the presence of red corpuscles, which are always to be found in the blood of the vertebrata. In some of the invertebrata the blood is white, or resembles the color of chyle.

Mode of Circulation.-The blood, having been propelled from the left or posterior ventricle of the heart to all the various ramifications of the body, passes through the extreme vessels (capillary) into the veins. These veins terminate in a vessel knorvn as vena cava, anterior and posterior, both of which sections terminate in the right or anterior auricle. This auricle contracts, and the venous blood thus finds its way into the right ventricle, where the pulmonary arteries have their origin. By aid of the muscular power of this ventricle, the blood is sent through the pulmonary arteries to the lungs (aeration and oxygenation.) The color of this blood is dark, almost black. It is highly carbonized, abounds in morbific matter and the worn-out materials of the body, and in this state is a non-supporter of vitality. In this condition, 
then, venous blood enters the pulmonary tissues. The arterialization of the blood which follows is a process more essential to vital integrity than the assimilation of food; for animals can live a long time without food, but they can only exist a short time unless the blood be oxygenized.

The pulmonary arteries branch off into minute ramifications on the surface of the air-cells of the lungs, and where these minute divisions terminate others commence, termed venous radicles, which ultimately become large veins. By the pulmonary veins the oxygenized blood is returned to the left auricle and ventricle of the heart. This route of the blood from heart to lungs, and back again from lungs to heart, is termed the lesser circulation. The left ventricle delivers the blood into the great aorta, and thence to every part of the body. This is called the greater circulation.

Difference between Veins and Arteries.-The veins differ from arteries in being less dense, having no muscular nor ligamentary tunics like the arteries, and, therefore, offer but little resistance to tight collars and tight-fitting harness and circingles, which are apt to interfere with the return of blood to the heart. The incapacity of veins returning blood to the heart in the same ratio in which it is carried by the arteries, is proved from the fact that the former are twice as voluminous as the latter. The veins also differ in their internal arrangement from the arteries, the former being furnished, at proper distances, with valves, which guard against a retrograde venous action.

\section{The Quantity of Blood Contained in the Body of A Horse.}

Mr. Percivalu, who is our chief authority on this subject, contends that, for many reasons, the quantity of blood contained in an animal body may be made matter of speculation, but can not, for many reasons, be ascertained with any degree of precision; for, says he, "if we attempt to draw all the blood out of the body, the animal sinks and dies long before its vessels are evacuated; and as we possess no means of measuring what remains behind, any calculation we may make from the quantity that has flowed must necessarily turn out vague, if not altogether incorrect." $\mathrm{He}$ contends, however, that the following experiment offers a datum, or guide: 
"The weight of an animal being ascertained to be seventynine pounds, a puncture was made with a lancet into the jugular, from which the blood flowed in a very free stream, and was collected. The vein having ceased to bleed, the caroted artery of the same side was divided, but no blood came from it. In a few seconds afterward the animal was dead. The weight of the carcass was found to be seventy-three and one half pounds; consequently, the animal had sustained a loss of 5.12 pounds, precisely the measure of the blood drawn.

It appears, therefore, from this experiment, that an animal will lose one-fifteenth of its weight of blood ere it dies, although a less quantity may so far debilitate the vital powers as to be, though less suddenly, equally fatal. In the human subject, the quantity of blood has been computed at about one-eighth part of the weight of the body; and as such an opinion has been broached from the results of experiments on quadrupeds, we may fairly take that to be about the proportion of it in the horse; so that if we estimate the weight of a common-sized horse at about 12 cwt., the whole quantity of blood will amount to 84 quarts, or 168 pounds, of which about 45 quarts, or 90 pounds, will flow from the jugular vein prior to death, though the loss of a much smaller quantity will sometimes deprive the animal of life."

\section{The Rapidity of the Circulation.}

In 1827, Hering,* a German physiologist, performed the experiment of injecting into the jugular vein of a dog a harmless substance, which could be easily recognized by its chemical reactions, and noted the time which elapsed before it could be detected in the vein of the opposite side. This gave the first correct idea of the rapidity of the circulation ; for though the older physiologists had studied the subject, their estimates were founded on calculations which had no accurate basis, and gave very different results. The experiment of HerING is often roughly performed as a physiological demonstration, and we have thus had frequent occasion, in a general way, to verify its accuracy. If, for example, we expose both jugulars of a dog, inject into one a solution of ferro-cyanide of potassium in water, and draw a specimen of 
blood from the other, with as little loss of time as possible, it will be found that, in twenty or thirty seconds after the injection, the salt has had time to pass from the jugular to the right side of the heart, thence to the lungs and left side of the heart, and from this, through the eapillaries of the head and face, back to the jugular on the opposite side. Its presence can be determined by the distinct blue color produce $i$ on the addition of the perchloride of iron to the serum, if the specimen be allowed to stand, or a clear extract of the blood be made by boiling with a little sulphate of soda and filtering, treating the colorless liquid thus obtained with the salt of iron. The experiments of Hersag were evidently conducted with great care and accuracy. He drew blood at intervals of five seconds after the commencement of the injection, and thus, by repeated observations, ascertained pretty nearly the rapidity of the circuit of blood in the animals on which he experimented. Others have taken up these investigations, and introduced some modifications in the manipulations. VIERORDT collected the blood as it flowed, in little vessels fixed on a disk revolving at a known rate, which gave a little more exactness to the observations; but the method is essentially the same as that employed by Hering, and the results obtained by these two observers nearly correspond.

Herrag made observations on horses by increasing the frequency of the pulse, on the one hand, physiologically, by exercise, and, on the other hand, pathologically, by inducing inflammation. He found, in the first instance, that in a horse, with the heart beating at the rate of thirty-six per minute, with eight respiratory acts, ferro-cyanide of potassium injected into the jugular appeared on the vessels on the opposite side, after an interval of from twenty to twenty-five seconds. By exercise, the number of pulsations was raised to one hundred per minute, and the rapidity of the circulation was from fifteen to twenty seconds. The observations were made with an interval of twenty-four hours. The same results were obtained in other experiments.

\section{The Transfusion of Blood.}

J. FArrell, V. S., has lately been experimenting in an interesting department of veterinary science; namely, the transfusion of equine blood in diseases attended with low, vital action. Trans- 
fusion signifies the transfer of blood from one living animal to another, by means of a hollow tube inserted into the jugular veins of each horse. His method was as follows:

"I commenced a series of experiments, so as to discover the simplest, safest, and most effectual method of conveying blood from one animal to another. I first tried the transfusion syringe, which has been used by medical practitioners for this purpose; but, whether from want of skill in its use, or from some defect in the instrument, (which had been recommended to me as one of the best, ) or from some other cause, I can not tell, but certain it is I was in no case so successful with it as I was with a more simple apparatus. I fancied that the blood lost much of its vitality by being exposed to atmospheric action, and also by its being forced and compressed within the cylinder of the instrument. These impressions as to the cause of failure induced me to undertake several experiments, with a view to the construction of an instrument which would fulfill the requisite indications; namely, to allow the blood to pass freely from the vein of the healthy into that of the diseased subject, without coming in contact with the atmosphere, and without alteration of its temperature. I at length adopted an exceedingly simple apparatus, which may be described in a few words. It consists of an India-rubber tube, some two and a half feet long, and three-eighths of an inch in diameter; that is, about the caliber of the vein in the adult horse. To either end of this is fitted a silver tube, curved somewhat like a syphon, so that the end, which is slightly rounded at the point, might be easily passed into the vein, both tubes being exactly alike. A narrow zine or tin trough is required, to contain hot water, in which two-thirds of the tube should be immersed during the time that the blood is flowing. This completes the apparatus; and, being prepared, and the horses ready, held by assistants, the jugular vein of the healthy horse from which the supply of is to be taken may be opened, and into it one of the silver tubes carefully passed, point upward, so as to receive the current of blood as it flows back from the head-the operator holding the other end, and, having previously opened the corresponding vein in the patient, he should wait till the current is passing freely down the tube from the healthy horse, and then bring it in contact with that which is now flowing slowly from the patient. He should pass the end of the tube carefully into the vein, point downward, by which means the 
possibility of any air getting into the tube is avoided. The quantity to be transfused is readily ascertained by watching the expression of the eyes and noting the pulse carefully. So long as there is no dilatation of the pupils, and so long as the heart's action is not very much affected, the blood may be allowed to flow on uninterruptedly; but as soon as the pupils become dilated, it is necessary to lessen the supply gradually, by compressing the tube with the finger and thumb. If the dilatation disappear after a minute or two, the blood may be again allowed to flow; but if it increases, further transfusion must be stopped, or, otherwise, injurious consequences will result.

In the cases of two animals which I purchased for the purpose of experiment, I purposely allowed the blood to flow after dilatation of the pupil had manifested itself, in order that I might see the result, and I found it to increase; and in one of the eases, after winking both eyes three or four times, in rapid succession, the horse reared up and fell back. In the other case, I forced the blood in from an India-rubber enema bottle. The pupils first became still more dilated; the breathing very quick and difficult; the eyes assumed a wild, agonized look, and the animal, with a sudden bound, fell forward dead. I had my finger on the artery during the entire time, and observed a great unsteadiness and fluttering of the pulse, which increased in frequency until the instant before he fell. In the first of these cases, I did not force the blood into the vein, but allowed it to flow until I perceived the injurious effects upon the horse. He tottered and fell, but in a short time recovered, and was walked back to his stable. He died in the course of the night, and, on examining his head the next day, I found considerable congestion of the brain. The tube used for the experiments transferred about three quarts of blood in eight minutes; at least it might be so inferred, from the fact that when used as a syphon, it passed three and a half quarts of water (and a little less of oil) from one vessel to another in that time.

I think, however, that I am correct in saying that this disease is by no means so likely to occur in horses as in human beings, and is certainly not so formidable; but, nevertheless, I am quite sure that it may happen as a consequence of the operation, if the instrument be not kept scrupulously clean, and also if great care be not taken in its introduction in the vein. Having, from these and other experiments, not necessary to detail, determined on the mode 
of operation most likely to be successful, I shall now proceed to state the result of four eases in which I have operated, and which, I think, I may look upon as having been followed with entire success. In three of the cases the patients had been bled a short time previous to my having seen them, and were so much weakened that they could scarcely walk. In the fourth case, a drastic purgative had been administered, causing superpurgation and great prostration of strength. In each of the four cases, the condition of the patient was so similar that the description I have already given may answer for all. Having selected a healthy young horse from which to obtain the blood to be transferred, I opened the jugular vein in the patient and in the healthy subject; and having inserted the tube, as before described, into the vein of the healthy horse, I placed the India-rubber tube in the tin trough containing hot water, to maintain the right temperature, and the other curved tube into the descending portion of the vein in the patient. As soon as the current from the healthy horse had completely expelled all atmospheric air, the instrument being thus arranged, the blood flowed freely from the vein of one horse into that of the other in an unbroken current. The average quantity of blood transferred in each of these cases was about three quarts. I observed no particular symptoms to follow from the transfusion, until two quarts or more had passed from the healthy to the diseased subject; but as soon as about that quantity had flowed, there appeared to be produced an amount of stimulation, indicated by an increased action of the heart; at the same time the pupils began to dilate, and the countenance evinced an anxious expression. $\mathrm{My}$ former experiments led me to watch with great care the progressive dilatation of the pupil, and I deemed it expedient, in each case, when this symptom was well developed, to compress the tube so as to diminish the current, and allow the transfusion to proceed more gradually and slowly. Occasionally I almost completely interrupted the current until the subsidence of this symptom, and I found that when about three quarts had been transfused, any additional quantity was followed by unpleasant symptoms, which indicated the necessity of stopping the operation. On removing the tube and elosing the vein, all symptoms of irritation gradually subsided, and the pulse, from being rapid and irritable, became slower, stronger, and fuller, gradually approaching the healthy standard. 
In each of these four cases the reaction was steady and progressive. The natural warmth of the extremities was gradually restored, and, in the course of ten or twelve hours, the patient presented other equally unmistakable symptoms of amendment, such as returning appetite, more quiet and steady respiration, cheerfulness of countenance, and a willingness to move about. From this point there was a gradual improvement, and, in a short time, they were pronounced cured.

I have been induced to submit these few remarks, much less with a view to record any little snceess I may have had in performing the operation myself, than with a hope that others, far more capable, will take up the matter, and test it thoroughly; for, whatever obstacles and objections there may be to its performance in the human subject, there are none to prevent its becoming a most valuable agent in veterinary science."

Soon after the discovery of the circulation of the blood by HARVEY, transfusion was attempted on animals, and afterward on man. In some cases the experiment was successful, while others failed to afford relief; in fact, a majority of the cases terminated unfarorably; and of late years transfusion has been abandoned, and regarded as "useless and even dangerous." We can conceive, however, of cases which might occur-when, for example, a horse has lost a large quantity of blood accidentally-to warrant us in resorting to transfusion; and if any of our veterinary friends are disposed to make experiments of this kind, we urge them to do so, notwithstanding the opinion of the Parliament of France against it, and that of some scientific men who have pronounced it "useless and dangerous."

One among the many reasons which may be urged against transfusion is the fact that venous blood is a non-supporter of vitality. It is saturated with the excrementitious material absorbed from the intestinal canal, fibrous membranes, and cellular tissues, and a vast amount of morbific matter is mixed with the venous blood in the course of its circulation, so that it can not be of any benefit to the system of a sick or dying horse until it shall become oxygenized or vitalized, or changed from venous to arterial blood, which change can not possibly take place until this fluid has reached the air-cells of the lungs. It would appear, therefore, that the transfusion of vitalized arterial blood, if the process can be conducted with the relative amount of safety at- 
tending that of the transfer of venous blood, would be a procedure more consistent with the known laws of physiology thin is the case when venous blood is transfused. It is possible that some animals might be benefited by the transfusion of venous blood, provided the heart and lungs of the patient are in a healthy state, so as to insure sufficient oxygenation; otherwise, the rapid consumption of the little oxygen absorbed would be likely to end in asphyxia. If the function of the lungs be impaired by disease, then the process of aeration of the blood is checked, and the blood can not circulate, notwithstanding the healthy state of the heart. In verification of this fact, as regards the very important function of respiration and aeration, we may mention that Dr. AUstin FLint, JUn., has lately demonstrated that the condition of oxygenation of the blood is necessary to the performance of the vital functions, and that a want of capillary power (occasioned by imperfect oxygenation) throws all onus on the heart, and that the heart is insufficient for the labor. In one of his experiments, after capillary circulation had entirely ceased, the chest was opened and the heart found beating regularly.

\section{Management of the Horse's Foot in the Stable.}

It is my firm belief, based on a knowledge of the physiology of the foot, that soft bedding, containing the usual amount of filth, is, in many cases, the exciting cause, not only of tenderness and contraction, but also of many muscular affections. I rejoice to find horse-owners exercising their reason in this department of stable management, because it is one step in the right direction, and will put a stop to those glaring absurdities which are constantly practiced just because they have the sanction of antiquity, or because Neighbor So-and-so recommends them. Reason teaches that the health of the whole animal fabric is best promoted by cleanliness and ventilation (by which a pure atmosphere is insured), and by food suitable in quantity and quality to the special wants of the animal. Hence, what reason teaches, man should practice.

It is unreasonable, therefore, to suppose that a horse can be benefited by standing on a soft bed, composed in part of the defiling excrements of his own body, which are constantly decomposing and forming gaseous vapors unfit for respiration. If dirt 
and filth are innoxious, in what does the value of our sanitary regulations consist? Why do our city authorities spend so much money to purify the pestiferous cesspool and sewer, and to rid our streets of accumulating rubbish and filth? Let human beings wallow knee-deep in muck, and revel shoulder-deep in an atmosphere saturated with ammoniacal and carbonic acid gases (as some horses are compelled to), and death would run riot-our cities would be converted into immense charnel-houses, fit receptacles for a race of beings that would not adopt the means which reason and experience suggest for averting the calamity.

Horses that have no better care than that alluded to, are in close proximity with disease. That they are often found dead in their stalls from the effects of carbonized blood I can testify, and many more would die, only they are permitted to take a little of the breath of life during the day, which, to some extent, dilutes the poisonous gases with which their system has been saturated during the night, and thus their life, which, under the best circumstances, is a weary toil, is prolonged.

Without attempting to prove the general effects of impure air and filth on the system of a horse located in a stall from one to two and a half feet deep of soft bedding, let us consider, in a brief manner, the loeal phenomena. Our readers are all aware that the combined action of heat and moisture tends to relax-enervates the tissues of the body, and, if carried beyond a certain point, ends in decomposition. Take, for example, a common poultice, apply it to a horse's foot, and renew it as soon as it becomes dry. In the course of two or three days the hoof will separate from its matrix, the frog and heels soften, the tissues be in a state of relaxation, and, if the poultice is continued, the hoof will separate from the sensible parts; if the foot is already diseased, the separation is accelerated. Warm water has the same effect. Applied externally for any length of time, it relaxes and prostrates; applied internally, it relaxes and vomits. Hence the soft, (which implies moist,) hot bedding, tends to create morbid action in the feet, and whatever disease the horse may be predisposed to in those parts will generally manifest itself. Some animals, however, escape the evils alluded to, owing to their insusceptibility; for disease of the foot can not occur without a susceptibility to it and the application of a cause. Soft bedding, cow dung, and other unmentionable filth, are often resorted to as remedies for con- 
traction, but they are classed among the irrational barbarities of the past, with which they ought to have sunk into oblivion. Veterinary science has discovered that if such articles possess any virtue, it is owing to the moisture they impart to the foot. Therefore, on the score of decency, and in view of relaxing the contracted foot according to the principles of reason and modern science, we should avoid every kind of filth, and resorts to pure water or wholesome poultice; or take off the shoes, wash the feet daily, and let the horse run to grass, so that he may bring the sole and frog in contact with the ground, and thus promote expansion of the heels.

As regards the manufacture of tender feet, in both the young and aged, it makes but little difference whether they have the range of a filthy barn-yard or are confined in a bedded stall; the effect is tenderness of foot, and subsequent lameness; and the same, or a disposition to it, may be transmitted through the sexual congress to future offspring. In view, therefore, of preventing diseased feet, strict attention must be paid to cleanliness. The stall floor should be composed of brick or plank, having just sufficient declivity to conduct fluids into a gutter, running along the entrance to the stall, which should terminate exterior to the stable, so that the ammonia, in which the urine is abundant, is carried beyond the stable atmosphere. The bedding, which, according to long custom, is stowed under the crib, there acting as a sort of noxious smelling-bottle to the horse's nostrils, should be spread out in the open air, sorted, the refuse and excrement removed to a dung-heap, located as far from the stable as possible; for the common manure receptacle, under the stable floor, is one of the worst features of stable economy. The stable floor should be washed clean as often as circumstances permit.

\section{Strangles.}

This disease is most prevalent in young horses. After the age of eight the disease is more rare. I have, however, seen some well-marked cases occurring in horses whose ages varied from five to eight years. Strangles is, without doubt, one of the evils of domestication, and often results from errors in diet and management, which, if we make some exceptions, will generally bear improvement. 
Stmagles is supposed to be a disease to which all horses are subjer once in their lives, yet Mr. Percivalu contends that many horses escape the disease. Hence, if many escape, it is very natural for those who know the value of pure air, natural food, and exercise to conclude that the colt, while enjoying these great luxuries in the open air, by the side of its mother, guided by her superior instinct, is not liable to be attacked with a disease which, as already stated, we believe to result from depriving animals of those blessings which Nature has in store for them in their unrestrained state. But it often happens that young colts, after running a season with their mother, partaking of the invigorating country air, grow up to be strong and robust, and then the period arrives for weaning them. How changed the scene! Instead of being permitted to gambol in their native element, they are confined to a small space, not large enough to swing a cat round, and perhaps as dark as the grave; and the animal, after fretting for a season, and making unsuccessful efforts to escape from its prisonhouse, tamely submits to the discipline, not, however, until he has cut and bruised and otherwise injured himself. I was called, a short time ago, to visit a young colt that had lacerated his head, breast, and forc-legs in a most shocking manner, in making an attempt to escape through a window from the horrors of confinement. His companions were about a dozen cows, more calculated to alarm and render his position a perilous one than otherwise; and the impure atmosphere, rendered so by the emanations from the excrements and from the lungs of his companions, was a source of great mischief. Then, who can blame such an one for attempting to escape and regain liberty? If strangles should appear in such a subject, it would not be surprising.

Then, again, take a colt from its mother, whose milk contains all the elements for sustaining life and developing the organization of the young subject, and place it upon a diet of hay or like innutritious trash, a whole truss of which would not afford one-half the quantity of nutriment contained in a quart of its mother's milk. However profitable and well-adapted hay may be for stock of mature growth and powerful digestive organs, it is a sad mistake to suppose that it will do for the young. A case of this kind "came under my observation last year. The subject, aged two and a half years, died in a state of marasmus (a gradual wasting of the system without any apparent disease). A post mortem examina- 
tion failed to detect any disease other than general emaciation. On making inquiries concerning the food, the owner replied, "The colt has been fed on good sweet hay and corn-stalks ever since it was weaned." This was capital food for the poor thing, provided, however, its stomach had a grist-mill within it; otherwise, it was hard fare, and must derange the digestive function, and, ere the colt has attained maturity, dyspepsia, in either a mild or aggravated form, has secured a victim.

Mr. Percivall has defined strangles to be "a diffusible swelling under the jaw. The tumor consists in a circumscribed inflammation, having all the characteristies of simple phlegmon, attacking the subcutaneous cellular substance included between the branches of the jaw, which, in consequence, become gradually filled and distended with effusions of lymphy and serous matters, acquires a firm and solid feel, tenderness on pressure, and a sense of unnatural heat. This commonly proceeds to suppuration, ending, to all appearances, in a common submaxillary abscess. Now, this, and this alone, constitutes strangles. There are in the books a variety of other symptoms described, but they are all concomitant or accidental, none but these being, properly speaking, essential to its existence. The usual concomitants are, membranous inflammation, giving rise to soreness about the throat; reddening and discharge at the nose, and perhaps cough; tumefaction of the salivary glands, producing pain and difficulty of deglutition; and, lastly, some slight febrile commotion of the system."

Contagiousness of Strangles.-We learn from the "Veterinarian" that M. REynat, clinical professor at the Alfort School, submits a number of observations corroborative of the contagious character of strangles. He states that "young horses having strangles, and put into stables with horses of adult age, doing their duty, have communicated the disease to those of the latter, who have stood in adjoining stalls, though some few have only exhibited the disease in a catarrhal form. Even the foal has been known to suck the disease from its dam. Moreover, experiment has been had recourse to to inoculate for strangles. M. Damalix smeared with a sponge, impregnated with matter taken from the abscess of strangles, twice daily, both sides of the pituitary membrane and the internal surfaces of the linings of the eyelids, in a sound horse, about to be cast for spavin. This was continued for seven days. On the eighth, he remarked that the horse had lost 
his appetite, had commenced running from both nostrils, coughed softly and loosely, and had swelling under the jaw, which ended in resolution, all the symptoms terminating eight days from their commencement.

It has been remarked that strangles is more surely communicated at an early than a late stage, and in a certain form more readily than in others. Strangles will assume the herpetic character, simulate farcy and glanders, settle in the mesenteric glands, or may follow castration. In regard to contagion, may be mentioned, as most readily communicable, that form of strangles which assumes the character of eruptions on the lips, nose, and pituitary membrane.".

The Tumor of Strangles is not always confined to the submaxillary space, as will be observed from the following case, which occurred in the author's practice, a short time ago, in a gray mare, and which speedily ran to a fatal termination. The subject had previously suffered from some internal disorder, and, on recovery from which, did not appear to regain its usual health, but remained in an unthrifty condition. A few days before our attention was called to the case, a tumor made its appearance on the near hip, which suppurated and discharged. On the morning of our visit, the animal had been attacked with abdominal pain, for which remedies had been prescribed. They not appearing to afford relief, the owner called in the aid of some person, who inserted a knife into the mouth, and wounded the palatine artery, from which the blood had flowed for several hours, but was now arrested. We found the extremities icy cold, and the pulse very feeble. The eye appeared glassy, mucous surfaces pale, and the lips hanging pendulous. There was a painful tumor on the near hind leg, close to the stifle, and another on the flank of the same side. The animal was exceedingly stiff and lame in the hind extremities, and appeared to be failing very fast. Considering the case a hopeless one, we merely recommended some restorative cordial and a few quarts of gruel. The animal died in the course of a few hours, and its death, no doubt, was hastened by the loss of blood; for, as the owner expressed himself, "the horse had some life in him before being bled," thereby intimating that the abstraction of blood deprived him of that life.

Treatment.-So soon as matter can be detected in the tumor or abscess beneath the jaw it should be laid open. This will liber- 
ate a quantity of pus. The orifice thus made must not be allowed to close, but must be kept open for a few days, or until matter ceases to run. During this period, a liniment, composed of codliver oil, half a pint, and powdered camphor, two ounces, must occasionally be rubbed about the region of the jaws. Half an ounce of powdered chlorate of potass should be mixed in the drink, or with some flaxseed tea, if the patient will drink it. A couple of drachms of fluid extract of pleurisy-root may be given, night and morning, for a short time. Active medicine is not indicated; good nursing, aided by pure air and voluntary exereise in an inclosure, will soon restore the patient to health.

We close our labors in the Equine Department by entering our solemn protest against the unwarrantable use of the lancet or knife in Veterinary Practice. It is a disgrace to humanity, an outrage on the rights and privileges of that noble animal, the horse, over whom God has given us dominion and power-not to abuse and render their life a weary toil, and extort front them all the labor we can, but to protect them, to administer to their wants and necessities, and render their condition as happy as possible. A moral responsibility rests on every man to see that the claims which these poor creatures have upon our race are respected; that their rights are not violated; that, when sick and in a dying condition, they shall be treated with mercy, and be protected from their tormentors, the unmerciful phlebotomists. Any misguided man who can draw from the veins of an emaciated or dying horse the little blood they contain should be sent to the insane asylum, and never again be permitted to disgrace the noble nature of man. We can respect the man who conscientiously practices blood-letting; but where is the medical man who can conscientiously bleed a dying brute with a view of saving life? 



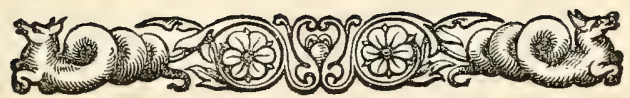

\section{BOVINE DEPARTMENT.}

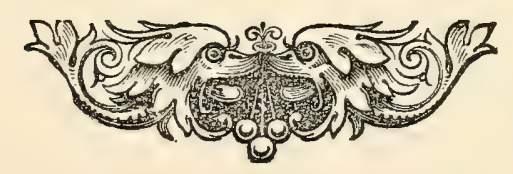




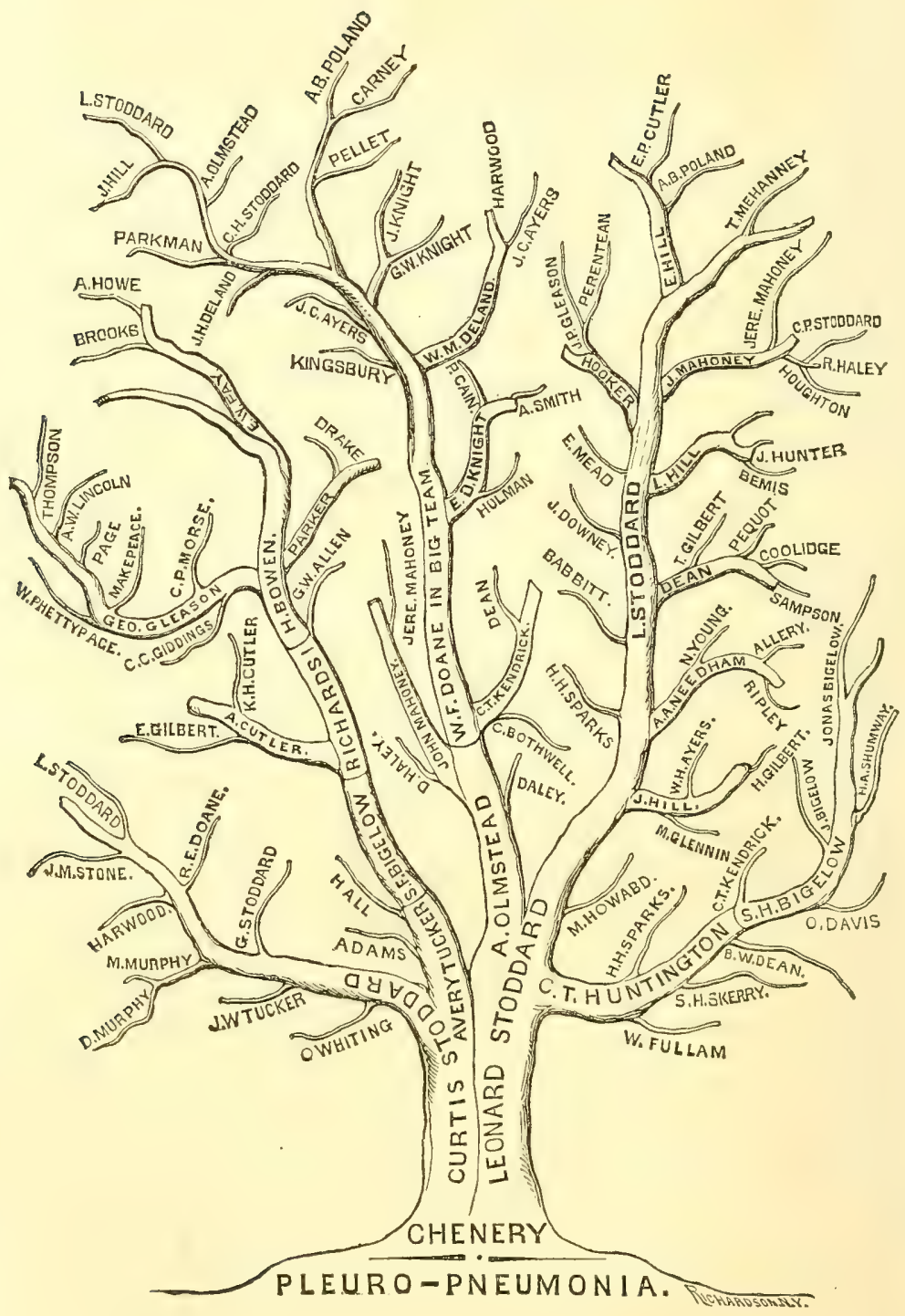

\section{EXPLANATION.}

The above cut represents the geneology of pleuro-pneumonia, as it occurred in Massachasetts, a few years ago. It commenced with Mr. Chenery's herd, and spread in the above order. The names are those of the parties whose herds took the disease by infection or contagion. (See article Pleuro-pneumonia.)

(418) 


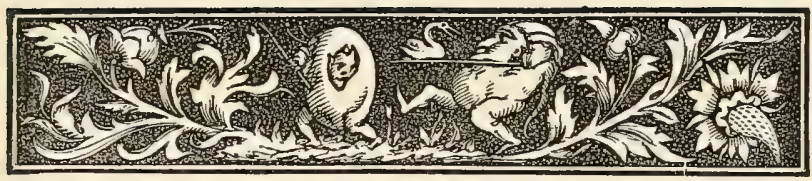

PART SECOND.

\section{DISEASES OF CATLE.}

\section{SECTION XIV.}

THE RINDERPEST, OR CONTAGIOUS PLEURO-

PNE U MONIA.

Origin of the Disease in Massachusetts-Spread of the Disease-The Cattle Plague in Kentucky.

Origin of the Disease in Massachusetts.

TN the early part of the spring of $1859, \mathrm{Mr}$. W. W. Chenery 1 sent to Holland for three cows and one heifer. They arrived in Boston on the 23d of May. Two of the cows were found to be in very bad condition. The first died at the end of a week, and was buried; the second one died two days afterward, and was also buried. About the 26th of June the third cow was found to be sick; she died the 29th day of June. The next cow found to be diseased was taken sick in August. This cow was imported from Holland in 1852. She died in about a fortnight from the time she was first taken. The disease then spread among the rest of the herd owned by Mr. Chenery, of which he lost a large number.

I examined the two cows that were landed sick. They did not appear to manifest any symptoms of pleuro-pneumonia, but seemed to have suffered terribly from the effects of the voyage. They had got down during the latter part of the voyage, and the external surface of the body was so bruised and abraded that some of the bones were visible. But perhaps Mr. Chenery's own history of 
the origin of the disease in Massachusetts may be the best that can be given, which I here present to the reader:

Boston, Mass., March 7, 1860.

DEAR SIR: In compliance with your request, I take pleasure in furnishing you a succinct account of my last importation of cattle from Holland, and also of the disease that subsequently prevailed in my herd at the Highland Farm, in Belmont, as is now generally supposed, in consequence of that importation.

It may be remarked that I had previously made two importations from Holland, and the extraordinary superiority of the animals received from that source, in comparison either with our native or any of the foreign breeds heretofore imported, induced me to increase my stock of Dutch cattle by further importations, rather than await the natural increase from those already imported. Accordingly, in December, 1858, I transmitted an order to my correspondents in Schiedam, Holland, instructing them to employ a competent person expressly to proceed to the north of Holland and make selections of the best animals that could be obtained, without regard to the expense. Following my instructions, four cows were procured from Purmerend and the Beemster, and were duly shipped at Rotterdam, on board the barque " J. C. Humphreys," which vessel sailed from that port early in April, and arrived here on the $23 \mathrm{~d}$ of May, 1859.

Upon examination, the cows were found to be in an extremely bad coudition-very much bruised and emaciated-one of them, as the mate of the barque stated, not having been on her feet during the trenty days preceding her arrival, while another one was totally unable to walk, and these two animals were accordingly carried to the farm in wagons. The remaining two were driven out. Deeming it impossible for the first-mentioned cow to recover, she was, on the 31st of May, slaughtered, and on the $2 \mathrm{~d}$ of June following the second cow died. At that time, in common with every one knowing the facts in the case, I was so fully persuaded that the bad condition of these animals was caused by neglect on the voyage, that I libeled the vessel, and went through a course of one hundred and fifty dollars' worth of law in my endeavors to obtain indemnity for the loss; but the law is uncertain, and the barque went on her way. My mind, however, remains unchanged with regard to the treatment the animals received on the voyage. The third cow of this importation seemed to be doing well until about the 20th of June, when she became sick, and died in ten days after. The fourth cow, "Lady Beemster," has, down to this time, shown no indications of sickness, but is, on the contrary, in a thriving condition. 
Early in the month of August following, symptoms of disease were observable in the Dutch cow, "Lady Louise" (imported in 1852). She died on the 20th of the same month. About this time several other animals were taken sick, in rapid succession, and then it was that the idea was first advanced that the disease was identical with that known in Europe as "epizoötic pleuro-pneumonia." From that date every possible precaution was taken to prevent the spread of the supposed distemper, strict orders having been given that no animals should be allowed to leave the farm, nor any strange cattle to come upon the premises. A temporary building was erected, in a pasture at considerable distance from the farm barn, to which the animals not infected were removed, and disinfecting agents were used about the premises, etc.

The following extract from my letter-book, in answer to an application for stock during the prevalence of the disease, will serve to show my views with regard to it at that time: "I am at present obliged to decline any applications for stock, owing to an epidemic disease in my herd. The disease is that known as 'pleuro-pneumonia,' and I have already lost seventeen head, and have ten more very sick. I am using every precaution to prevent the disease from spreading, and you will, of course, see the propriety of my refusing to allow any animal to leave the farm until the infection has ceased."

It is manifest that the means used to prevent the spread of the distemper have so far proved eminently successful, inasmuch as, notwithstanding I have lost some thirty animals (nearly half of my whole herd), there has not been a single case of the disease elsewhere in the neighborhood of my farm.

As there seems to be an impression abroad that the disease in my case was confined exclusively to the Dutch stock, I would remark that such is not the fact; that, on the contrary, they have, comparatively, been more exempt from it than any other breed. It is not, however, confined to any particular breed, or to any age or sex, as the full-grown $o x$, the mature cow, and the young calf have been alike prostrated by it; and of the animals lost, five were thorough-bred Dutch, five Durham, one Ayrshire, one Guernsey, two Jerseys, five Devons, two natives (all the natives on the farm), and the remainder were grade Dutch.

There has been no new case of sickness in my herd since the death of the mammoth cow "Lady Washington," which occurred on the Sth of January, and I am, therefore, induced to believe that, so far as my animals are concerned, the disease has run its course.

From the foregoing remarks, it will be perceived that some mouths ago, when the disease was raging, I believed it to be infectious or contagious; but I confess that more recently the conviction has forced itself upon me that it was of an entirely local character; that the 
lungs of the cattle had become diseased by breathing carbonic acid gas, generated in the cow-house in consequence of insufficient ventilation. The recent developments with regard to the spread of the disease in North Brookfield and vicinity - in tracing its origin to the calves that went from my farm in June last-together with the information I have just received in a letter from Holland upon the subject, will, I fear, force me back again to the belief that it is the veritable European scourge.

With regard to the treatment of the disease, I can only say that several of our most eminent veterinary surgeons have availed themselves of the opportunity to study the disease, by post mortem examinations and otherwise, and are, doubtless, competent to give valuable information upon the subject. My own private opinion, based upon costly experience, is, that whenever an animal becomes really sick with this distemper, the best teatment is to slaughter and bury forthwith.

In concluding this long letter, I beg to say that, if it shall prove that through my efforts to improve the breeds of neat cattle, I have been the means of introducing this disease into the country, I am sure no one can possibly regret it more sincerely than myself. At the same time, I feel some consolation in believing that, provided the disease cau be restricted to any thing like its present limits, the benefits conferred by the introduction of the Dutch cattle here will more than outweigh the losses incurred by the introduction of the disease.

Respectfully,

Winthrop W. Chenery.

\section{Spread of the Disease.}

The disease was introduced into North Brookfield from Belmont. Mr. C. STODdard, a young man of North Brookfield, purchased of Mr. Chenery three calves. After arriving (by rail) at North Brookfield, they were driven toward the place of destination (about five miles). On the way, one of the calves was observed to falter, and at the end of the journey seemed to be quite sick. In two or three days, the father of the young man took the calf away to his own barn for treatment. In this barn were about forty head of cattle. The calf growing no better, the son took it back again to his own residence. In about ten days it died. Soon the senior Stoddard observed that one of his oxen was sick; it soon died. Two weeks after, a second was taken sick and died. Finally, in the course of a short time he had lost eight oxen and cows. Young Stoddard lost no animal by the infection 
at that time. He sold, however. eleven heifers, and retained nine of the most valuable himself. These nine were four oxen and five young cattle. The four he took to his father, three of the others to his uncle, and the remaining two he left with his fatherin-law. It was finally discovered that wherever these animals went they scattered the infection, without a single failure, showing that the disease was of a contagious character. It turned out, finally, that the herds of both father and son were badly diseased. Out of the nine formerly belonging to the son, seven were the subjects of pleuro-pneumonia. The elder Stoddard lost fourteen of his animals before the commissioners for the extirpation of the pest were appointed. The elder Stoddard kept about eight oxen, which he employed in teaming. He was drawing some lumber, and stopped over night at a neighbor's named Needham. The disease was communicated, and Mr. Needham finally lost his whole herd. Many died, while others were destroyed by order of the commissioners. Finally, Mr. Stoddard sold an animal to Mr. Woodis, of New Braintree, who had twenty-three fine cows. Seven or eight died before the commissioners got there, and the whole herd had to be condemned. Next, Mr. Stoddard sold a yoke of cattle to Mr. Olmstead. At the end of five days they had infected the whole herd. Finally, these cattle came into the hands of Mr. Doane. They were put in with twenty-two yoke of cattle, and employed a day and a half in moving a building from Oakham to North Brookfield. It was afterward proved that the whole of these cattle took the infection. Belonging, as they did, to eleven different herds, they carried the infection to eleven different farms, and thus, by contagion or infection, the cattle for miles around became the subjects of pleuro-pneumonia. The disease assumed such proportions that the commissioners had not funds sufficient (ten thousand dollars) to perform the operations required by law-namely, extirpation. And here it may be proper to inform the reader that the commissioners had no power to order an attempt at medicinal treatment. They were ordered to extirpate the malady - to kill and bury all infected herds; and this has, finally, proved to be, both in this and other countries, the cheapest way of getting rid of contagious pleuro-pneumonia.

The following is a brief record of the autopsies made by veterinary surgeons employed by the State of Massachusetts :

Autopsy 1.-This cow appeared badly. She coughed, but, it 
was said, ate well. The posterior lobe of the lung adhered to the diaphragm, was indurated, and contained a cyst, in which was a very small quantity of pus and a mass of detached lung of the size of a quart measure. This was connected with the main portion of the lung by bronchi, some of which had been cut off in such a manner as to admit air into the cyst. The mass was offensive, friable, and, on incision, did not present the bright, mottled, organized appearance of similar masses lying in air-tight cysts. There was no serum in the chest.-Dr. Martin, April 12, 1860.

Autopsy 2.-An ox, 7 years old. The lung was extensively and firmly adherent in various parts, and almost "as hard as a stone." On incision, there was found a large cyst, containing pus and lymph.-Drs. DADD and ThAYER, April 19, 1860.

Autopsy 3.-A steer, 3 years old. The owner said that this animal was but slightly, if at all sick. The left lung was adherent to the pleura costalis and diaphragm. On incision, there was found, in the middle, a large cyst, containing pulmonary tissue. The right lung was adherent, but its substance healthy.-Drs. DAdD and Thayer, April 19, 1860.

Autopsy 4.-A steer, 2 years old. Marked adhesion of the posterior superior portion of the lung to the diaphragm. Some consolidation of the lung. The right lung contained a cyst, in which was a mass not entirely separated.-Drs. DADD and Thayer, April 19, 1860.

Autopsy 5.-A cow, 8 years old. The owner said that this animal was one of the most healthy in his herd. At the apex of the left lung was a very large slough. There was also red hepatization.-Drs. Dadd and Thayer, April 19, 1860.

Autopsy 6.-An ox, 7 years old, owned by Curtis Stoddard, and exposed to the Chenery calf during the illness of the latter. The superior anterior portion of the right lung was much enlarged, and contained a large cyst, in which was a quantity of pus and a mass of detached pulmonary tissue. Left lung adherent to the diaphragm.-Drs. DADD and Tyler, April 20, 1860.

Autopsy 7.-The anterior lobe of the right lung adhered to the sternum. A portion of the pulmonary tissue was separated and contained in a cyst.-Drs. Bates, DAdD, and Thayer, April 21,1860 .

Autopsy 8.-A yearling heifer. Much serum was found in the chest, and the pleural surfaces were extensively adherent. The 
substance of the right lung was completely* marbled, some parts having suppurated, while others were only indurated.-Dr. TrLER, April 27, 1860.

Autopsy 9.-Killed a cow which had been sick nineteen days. She was feeble; had but little appetite; diarrhea, cough, and shortness of breath; hair rough, etc. Percussion dull all over the left side of the chest, with absence of normal respiration. The left pleural cavity contained several gallons of serum. Over the costal pleura was a firm layer of lymph, resembling the velvety parts of tripe. The whole lung was indurated, especially at its base, and brittle, like liver. No pus. Right side and lung healthy.-Dr. Martin, April 11, 1860.

Autopsy 10.-Killed a cow, 10 years old. Sick since January. Dullness over the left side. On opening the chest, there flowed from it a stream of fetid matter as thick as tar. Strong adhesions to the ribs. In the lung was a large tumor, enveloped in a cyst, or sac. Right lung indurated.-Drs. DADD, Thayer, and TYLER, April 19, 1860.

Autopsy 11.-Killed a calf, 3 weeks old. In the right pleural cavity was a quart of serum. Lymph, easily separated, was spread over the surface of the lung. The left lung adhered to the ribs, sternum, and pericardium.-Drs. DAdD, Thayer, and Tyler, April 19, 1860.

Autopsy 12.-Killed a cow, 10 years old, which had been sick three or four months. The posterior superior portions of the right lung adhered so strongly to the ribs and diaphragm, that the knife was necessary for their separation. On opening the posterior part of the lung, a detached mass was found, very soft, surrounded by pus, and contained in a sac. Left lung healthy.-Drs. Bates, Dadd, and Thayer, April 21, 1860.

Autopsy 13.-Left lung healthy, but slight adhesions to diaphragm. Right lung firmly adherent to the diaphragm. In it was found a mass of consolidated lung, not entirely separated.Drs. Bates, DAdD, and Thayer, April 21, 1860.

Autopsy 14.-Killed a calf, 4 weeks old. Right lung inflamed. Left chest filled with serum. Lung covered with lymph.-Drs. Bates, Dadd, and Thayer, April 21, 1860.

Autopsy 15.-Killed a cow, 9 years old, and mother of the calf. In the right lung was a cyst of the size of a pullet's egg, filled with pus. The left lung contained a small cyst, filled with the 
same. Heart flabby.-Drs. Bates, Dadd, and Thayer, April 21,1860 .

Autopsy 16.-Killed a stag that was in the "big team." Three months before, he had been sick for four weeks, but had recovered, and had been working very hard. Serum in the right pleural cavity. Lung indurated, and adherent throughout so strongly to the ribs and diaphragm, that it was necessary to cut or scrape it off. In this lung was a small cyst.-Examined May 9, 1860.

Autopsy 17.-Killed the other stag. Left lung useless. Serum in the chest. Right lung in the acute inflammatory stage.

These stags were taken December 3, 1860, in exchange for a yoke of oxen sold at the same time to William F. Doane, and kept with Olmstead's diseased herd. They were in the "big team" December 19.-Examined May 9, 1860.

Many other autopsies were made by members of our sister profession, human medicine, and the disease was always found to be seated within the chest. It was not of so formidable or malignant a character as it has proved to be in some parts of Europe, and probably this is owing to the fact, if fact it be, that in the transplantation of the disease from Holland to this country it has undergone some modification.

From my own experience, I am led to believe that the Holland cattle inherit in their systems an idiosyncrasy or predisposition to pleuro-pneumonia, and that when the circumstances are favorable for its development-namely, impure air and bad managementthe latent disease to which, by virtue of their constitution they are liable, is thus fanned into a flame. Being in its character contagious, it then spreads, according to the laws, or after the fashion which govern or obtain, in other epizoötic contagious maladies. This must have been the case as regards Mr. ChenERY's herd. Before his Dutch stock left Holland, they were carefully examined by competent surgeons, well acquainted with the national discase pleuro-pneumonia, and a clean bill of health was rendered. It was also ascertained that the malady was not then prevailing in the localities where the animals were purchased; hence it manifested itself in the manner here deseribed. The same is true as regards its introduction into the Cape of Good Hope. The animal was in perfect health on leaving Holland, but on arriving at the place of destination manifested the disease, and communicated it to others. 
The history of the disease in South Africa is quite interesting; hence I introduce the following testimony, as given before a committee of the Legislature of Massachusetts, by Rev. D. LINDLEY, lately a missionary in South Africa. He said:

"The disease, whatever be its name, and it has different names, was introduced into South Africa a few years ago. It was introduced from Holland, imported in the body of a bull. A gentleman in Cape Town, wishing to improve his stock, made that importation, and with it that disease which has been to South Africa the severest scourge that has ever fallen on its property interest. It was about six weeks after the animal landed (he having been on board the vessel on the passage about two months) before any sign of sickness appeared in him. At the time, it was not suspected that the disease was the lung contagion, so long known in Holland. However, he died. He communicated that disease to a considerable number of cattle, and before the people became aware of the evil that threatened them, it had scattered about them very extensively.

"The question may arise in the mind of the committee, Why was it not at once exterminated there, as you propose to have it here? The answer to this question will be found in this statement that I must make, in order that you may understand the circumstances of that country. You will imagine New England, and a great part of the United States, divested of its woods, its forests, leaving, here and there, thickets and jungles, and a grass country that is without fences or any inclosures, and all this country spread over with cattle by the thousand (for the property of the inhabitants of the country consists in cattle and in sheep). I have seen 1,600 in one herd, but generally the herds are from one hundred to five hundred. In those parts of the country where the lions and tigers have been exterminated, these cattle are allowed to roam, night and day, where they please, and they wander considerable distances, sometimes miles around. In addition to that, all the produce of the country that is brought to market, whether to supply the city of Cape Town or Port Elizabeth, or other towns lying along the coast, is brought down from the interior in large wagons, drawn by oxen. All the goods imported into the country and taken inland are conveyed on these wagons, drawn by oxen; and to each wagon the custom of the country gives six pairs of oxen. 
The country is large, it being from Cape Town to the extremity of any civilization in the interior, twelve hundred miles, and across the plains to where I live, twelve hundred miles more. Well, this country is passed through, up and down, crosswise, and backward and forward, by hundreds of wagons and thousands of cattle every day. They have no railroads, no rivers-no other way of transporting goods from one point to another but this ox-wagon. Well, they are great sheep-raisers in this country, having five to ten thousand sheep in a flock, and I have seen as many as fourteen thousand in one flock. Their elips of wool are all sent down in these wagons to the coast.

"In a country of this kind, where there are so many cattle, and where every thing is done by means of cattle, and they are traveling night and day, there is no possibility of killing out this disease by extirpation. The seed had been so widely disseminated before the people knew what the matter was, that such a system was looked upon as hopeless, and the Government adopted no measures to stay it, and every man was left to look out for his own interests. I will say that, after it had got fairly spread abroad to a considerable extent, the inhabitants very generally resorted to inoculation; and I will say, in passing, that we are indebted to that for about all the cattle we have left. We should have been flat on the ground, and no man could have got to the coast with his products or returned with his merchandise. Inoculation has saved us what we have, after six years. The disease was still at work when I came away, but it was more under subjection. It has killed hundreds and thousands of cattle, and I can assure you, gentlemen, that where it has come into a flock it has not left more than five out of a hundred. I was happily surprised when I heard Dr. Loring state that in the past year, in this State, not more than twenty per cent. had died.

"With us, when an animal is known as having the disease, we look upon it as already dead; and I can affirm, without hesitation, that where it has got into a herd of cattle, not more than five out of a hundred have been spared. Occasionally one has passed through, and has not had the disease at all; and a few, on the other hand-two or three in a hundred-have recovered, and no more. I know of one man who had five hundred head of cattle. The disease got in among them, and, finally, he had but five left. If I speak with emphasis, it is because I have had sad 
experience; and I have been afraid that the good citizens of Massachusetts might not be aware of the evil that I do most firmly believe threatens their property interest more than any thing that ever threatened it yet.

"The disease has spread in every direction from Holland, and by contagion. I will give you facts on this matter of contagion. Well-meaning men-men of science, and who hold high and influential stations-said it was not contagious; that it was impossible for a lung disease to be contagious, and, through their influence, some herds suffered that might, to my certain knowledge, have been saved. One of the commissioners appointed was a man of some science, and he said, 'Poh! poh! it can not be contageous,' and the cattle were left, and the consequence is, it has spread over all the country around them.

"I will tell you how the disease came to my particular neighborhood. A native went out as a peddler, over the Cathumba Mountains, into the interior, nearly three hundred miles. There he took cattle in payment for goods. He brought down a herd of oxen to the eastern coast. While on the way down, some of his oxen became sick, and he quietly put them out of the way; for he could travel one or two days, perhaps, and not see a single person, and the dead cattle were not likely to attract attention. He had that failing which we can pardon in others, as we see it in ourselves, that he cared a little more for himself than he did for his neighbors. He put the sick oxen out of the way, and brought down the rest and sold them. They were bought by a gentleman who had about one hundred and twenty oxen. The peddler's cattle, looking apparently well, were put into that herd. Presently the disease broke out. It was in that instance that this doctor had the influence to prevent the slaughter of that herd, because he said the affection was not contagious. These cattle were running about in the neighborhood-out on the plain twenty miles square, without fence and without tree, save here and there a bush-where were grazing thousands of cattle, and they ran just where they pleased. From this flock the contagion was communicated to all the cattle in the region. Oxen were traveling through the country every day (at least a hundred passing in a day), and in that way it was carried widely through the country. Until it was brought from a contaminated region in the interior by these oxen, the disease had never been within three hundred 
miles of us. I might give a thousand facts just equal to this, but I am mentioning what occurred in my neighborhood.

"The disease had not crossed to the northward, to the Ungani River, until this happened: A man wished to convey a boat from Port Natal to a place about sixty miles to the northward. He put the boat on a wagon, and took his six yoke of oxen to draw it. He traveled one day, and camped just outside of a village through which he had passed. In the morning he found one of his oxen sick. He had camped on a piece of ground where cattle grazed every day, and in a place where the people had thought themselves safe. Finding his ox sick, he quietly took him and his mate out of the wagon, and, leaving them there, started on. These oxen remained through the day, and mixed with the many cattle owned in that village. The second day after they had been there, it was discovered that there was a sick ox in the field. The inhabitants were all out at once. They killed the ox, and, from the description, they saw that he had the disease they had dreaded. They immediately inoculated their cattle, and saved a goodly number of them. Now, in regard to that, I wish to make this statement. I made a statement which was honestly reported, I suppose, but mistakenly as a statement, that they had saved ninety per cent.; in some instances not more than thirty per cent. Between this and ninety is probably the average percentage saved. In this case, I mentioned that there was a clear, distinct instance where the sickness had been brought from the interior three hundred miles, and in the last case it was earried twenty miles. In another instance, two natives were trading, and brought the disease from the country where they went, two hundred miles, and set it down in a perfectly healthy region, in a herd of about eighty cattle, and there it spread, and they were every one carried off.

"Another fact, and one with which I had to do myself. A native, a stupid heathen, was working for an Englishman in an infected region. He took his pay in cattle-two calves, I think, a year or a year and a half old. He carried them into a healthy district, where the disease had been kept out, and within twenty miles of which it was not known. Presently these calves fell sick and died, and the eattle with which they were placed began to be sick. I had in my service a young man belonging to that village, which was twelve miles from where I lived. A messenger came to this young man to say, 'Your cattle are sick.' When I 
heard that, I began to inquire if any cattle had been brought from within the infected region to his kräal. They said such an one (meaning the native before mentioned) had been working with a man, and taken two head of cattle for his pay. He came back, a little more than two months ago, with these cattle, and they took sick and died, and now our other cattle are sick. I saw at once what the matter was; for I knew that the region where these two cattle were taken from was wholly contaminated. I said, 'Your cattle will all die; you ought to tell your neighbors to keep their cattle away from you.' I asked him, further, if his cattle had mixed with other cattle, and he said, 'There are three kräals that have mixed with ours; so it was too late, and the result was, they all died. I told the young man whom I sent to go and warn the neighbors. He did so, and they took their cattle in the opposite direction to grass, and for two years before I came away not a single herd of the cattle around them had taken the disease. Just those that were exposed to the contagion, and no others, died. The neighbors' cattle continued in a state of perfect health for two years after those four herds (one hundred or one hundred and thirty head) had died, right out there in the heart of a healthy region, a region as large as a county.

"I can not doubt that the disease was communicated by contagion, and that if the animals can be cut off, the discase will be kept off. It was kept off in the region in which I lived in this way. The chief with whom I have lived occupies a considerable extent of territory, and he is fortunately fortified on one side by a range of mountains, and on the other by a precipice some hundred feet in height. He had assembled his tribe for another purpose, and, wanting my advice in reference to some political difficulties, he sent a messenger to tell me of his trouble. I went to him, and, after that matter was settled, I took occasion to tell him that the sickness was within some forty miles of us. I told him what the disease had done and would do, and I said to him, 'There is just one thing to do, and that is, to keep your cattle where they are, and not allow any to go out or come in.' The people there love their cattle, as they say, better than they love their lives. They took the alarm, and every effort that was made, on the part of any one, to bring cattle into the country was immediately and stoutly resisted. The intruder was met with spear and shield, and threatened with death and destruction to himself 
and his cattle if he came a step further, and so was made to go back. Only half a mile off, within sight of these cattle, dead animals were lying unburied that had been exposed to this contagion. The disease was brought there by the oxen of an individual who had been into the interior, and when he came home his oxen died. They communicated the disease to all the cattle in that neighborhood, and I never saw more complete destruction. There was not a single head left in all those kräals. Those cattle came up to within half a mile of our boundary, and you could look down and see herds of them lying dead. That was three years ago, and yet, when I came away, the disease had not got one inch over that line.

"These are facts that I have seen and know; and in that country, if you should ask us, 'Is the disease communicated by contagion?' we would say 'Yes,' and we should just as soon doubt that the sun made daylight. There are thousands upon thousands of facts to prove it. We have no more questions to ask on that subject. You will see how widely the disease might spread in a country like that, where cattle are so abundant, where the travel is continued day and night, and where thousands of oxen are on the road every twenty-four hours. It has been to that country a great scourge. Thousands and hundreds of thousands of cattle have died, and many of the people have been made poor by the ravages of the disease, and the only hope they have of securing a comfortable subsistence, and recovering a comfortable position in respect to property, is through sheep. They have given up all idea of grazing cattle, and are now turning their attention to sheep; for the disease is so widely spread, that they have no hope that it will ever be exterminated.

"I do not know that I have any thing further to state. I might repeat hundreds and hundreds of facts of precisely this character. If I have appeared earnest in my statements-somewhat as if I was making a speech, which is, perhaps, my profession-I hope you will not attribute it to any other motive than a wish to make you fear as I think you ought to fear. Massachusetts has enacted some glorious history, whereof you have famous monuments, and I hope that pluck will not be wanting now."

From such evidence as the foregoing, there can not be any doubt regarding the contagious element of the disease. 


\section{The Cattle Plague in Kentucky.}

The Cattle Plague of Kentucky is known in Texas as "Texas Fever." There are, at the present time, about one hundred thousand head of cattle pastured along the eastern and north-eastern limits of the State of Louisiana, destined for the markets of the Mississippi. They are detained where they are by the statutes of neighboring States, which forbid their being driven through them before the 10th of November, the reason being a disease called the "Texas," or cattle fever, which prevails in the summer months, and is contagious.

Some of these Texas cattle were brought into the State of Kentucky early in August, 1866, and shortly after the disease made its appearance, the details of which were communicated to the "Lexington Observer and Reporter" in the following letter:

\section{Scotт Countr, Kr., September 1, 1866.}

\section{Cot. L. J. Bradford, President of Kentucky. State Agricultural Society:}

DEAR SIR-This section of the country has been much disturbed, in the last few weeks, owing to a frightful disease which has made its appearance among the cattle, killing many, making some blind, and otherwise affecting a large number. There was no disease among the cattle of these interior counties prior to the introduction of a large lot from Texas by General R. Gano. It has been asserted, and believed, for a long time, that the Texas cattle would propagate a peculiar and destructive disease. Missouri, in consequence, established a cattle quarantine, and, I believe, passed a prohibitory law; hence, for some time, that State has not been visited with this fatal malady. Kentucky has it now, and there can be no doubt, in the minds of those who have been informed of the facts, that it was introduced by the Texas cattle.

Reason.-My mother-in-law, Mrs. James K. Duke, who lives nine miles from Lexington and four from Georgetown, in this county, on one of the best and most beautiful farms in the State, on which no disease of any kind had ever occurred, took upon pasture, the 28th of June last, over three hundred of these Texas cattle. None of her own ran with them. They remained until the $3 d$ of August, looked healthy, and grazed well. Within five or six days after they had been taken away, a lot of Kentucky cattle (a great many raised on the farm) were turned on the pasture which had been occupied by the Texas cattle. No danger was apprehended, but, within a week or ten 
days, it was discovered that the disease had broken out among them. Attention was immediately given. All were removed from the pasture, and the sick separated from the well. Two or three died almost immediately.

External Symptoms.-Dull, stupid, stiff; separation from the herd; drooping of the head; disinclination to eat; trembling, cramping, staggering, falling, and, in many cases ( $\mathrm{I}$ do not know that there is an exception), compression of the jaws. Some are made perfectly blind-none on this farm, but on an adjoining one, that of Mr. Charles Herndon, whose milch cows, calves, and a few steers were affected first in the eyes (some in one, some in both); and it was a week or ten days after the disease manifested itself in this way before these or others began to seriously sicken and die.

Internal Symptoms, as far as Observed.-Blood, very black and thick; heart, only slightly affected; lungs, perfectly sound and healthy; the manifold, or second stomach, very much contracted, with the fecal matter, hard, dry, and compact; the paunch, or first stomach, almost empty, and with no apparent change; bowels, contracted, nearly empty, and extremely costive; bladder, generally containing only a small quantity of thick, high-colored urine. In a few cases they discharge a considerable quantity of bloody urine.

Up to the present time Mr. Herndon has lost eight or ten out of a lot of twenty-five or thirty; Mrs. Duke, with a herd of seventy-five, has lost twenty. Another neighbor, Mr. James Kenney, where only Texas cattle remained, lost two. A gentleman near Oxford, Scott County, lost twelve; another near Midway, Woodward County, lost sixteen, from turning on a pasture where the Texas cattle had been but one night and part of a day. Many others have died in the wake of these Texas cattle, yet it is a remarkable fact that the disease has broken out nowhere in a malignant form save where these imported cattle have been. On the farm of Mrs. Duke, her milch cows and calves, which were kept near the house, and in the central part of the place; and not allowed to mix with these foreign cattle, or run on the pasture where they had been, are and have been free from, the disease, and milk and butter used all the time.

What is the Disease? - I think it is nothing less than the cattle plague, of which we have heard so much of late as raging with such terrible fatality in Europe. In England it is called the rinderpest; in Russia, eattle plague; in Mexico and Texas, the Spanish fever. The symptoms are very similar. In almost every case thus far it has proved fatal. The rinderpest could not be worse.

How is it Communicated?-Dr. J. Burden Sanderson, of England, discovered that the blood of the animal affected with eattle plague 
contained the poison of the malady, so that serum obtained from it would give the disease by inoculation. Now, how did these Texas cattle communicate this disease, when they were apparently healthy? Spanish fever is an endemic disease of Texas, and cattle have been for years dying with it, sometimes, in certain localities, prevailing as an epidemic. No disease, however, even in the form of epidemic, is always fatal; but I am sure, from the natural course of things, the discovery of Sanderson, and from facts herein given, that some of those Texas cattle had this disease in their systems, and brought it to Kentucky, so that the poison has inoculated our cattle and produced the disease from which they are now suffering.

Our grasses seem to have had the effect to eliminate the poison through their kidneys, bowels, and, perhaps, through the lungs, which, being deposited on the pasture, was left ready for the first that followed after them. Our Kentucky cattle, of course, not being acclimated, are readily susceptible to the infection of the poison, and have suffered accordingly. The disease is entirely distinct from any we have ever had in the State before-attacks suddenly, affects singularly, and kills in almost every case. One who has ever seen a case can point out the cattle affected with it as soon as they begin to sicken.

Thus far, Mrs. Duke has lost twenty out of twenty-six cases. On the 25th of August she commenced feeding green corn. Since that date only one has died-five getting well, and no others sickening. Every case, with the exception of blindness at Mr. Herndon's, one case of purging at Mr. Kenney's, and two of bloody urine, were affected precisely alike; so much so, that the description of one would answer for all, the symptoms being more uniform than in any epidemic I have ever seen or known. The fact that this disease has been brought here by Texas cattle I regard as perfectly certain, no such disease having ever appeared here prior to their coming into the State, and none occurring, so far as ean be ascertained, save where they have been.

It is well known that they introduced the same disease into Missouri for several consecutive years, and the State was only relieved of the pestilence by the Legislature passing prohibitory laws, making it a heary penalty to bring a single one into the State. It is true they come here seemingly healthy, but may they not, as I suggested before, bring it in their systems, and, through the effect of grasses upon their bowels and kidneys, deposit the poison upon our pastures by their urine and dung? The theory that the disease is communicated through the feet, or by the ticks, I think fallacious, and will not discuss it. Their feet are healthy, smooth, and sound, and the ticks on them can be found almost anywhere in Kentucky. I have seen them often. 
Prior to using the green corn, I am informed, by Dr. A. B. Duke, that salt and ashes, soda, sulphur, copperas, bleeding in the mouth, neck, ears, and tail were resorted to without any good effect; but it is not conclusive that green corn will be of any material service. It was only an experiment, did good in a few cases on Mrs. Duke's farm, but has not yet checked the cases on Mr. Herndon's. It is impossible to give a remedy so soon. It requires much experience and experiment, and it would be well for farmers in Kentucky to look into the matter and prepare for it.

An Agricultural College has been established near Lexington. Would it not be well to have Veterinary Surgery studied-in fact, have a special chair for that profession? Many young men will study it, and it is as important as any other. The diseases of cattle, horses, hogs, and sheep are as little known and understood in Kentucky as though stock never did suffer from disease. Kentucky has long been known and noted as a cattle or stock-breeding, raising, and feeding State. If she would maintain prominence, and protect her greatest interest, she must establish quarantines and pass laws shutting out this terrible pestilence from her borders, and prepare for the cure and relief of her fine stock.

Most respectfully your obedient servant,

G. Clay Sirith.

The following is my reply to the above:

G. Clay Suith, EsQ.:

Chicago, Ilu., September 13, 1866.

Dear SrR-Through the kindness of R. W. Carroll \& Co., of Cincinnati, I am in possession of an article, over your signature, having reference to the "Cattle Plague in Kentucky." I have carefully perused the article and compared the symptoms, as described, and find that the disease bears no analogy to the rinderpest in Europe, nor the pleuro-pneumonia of Holland, which was imported into Massachusetts about six years ago, where I had ample opportunities of studying it, having been appointed by Governor Banks as surgeon to the commission appointed to extirpate the pest.

The Texas bovine malady, just introduced in Kentucky, is peculiar to the breed of Texas. In that country the disease has acquired a home in the constitutions of animals bred and reared there. The disease lurks in the breed in a latent form, and, when circumstances are favorable for its development, it manifests itself, and becomes both contagious and infectious; and thus it spreads after the fashion of the rinderpest and other bovine epizoöties. 
Your suggestion in regard to establishing a special chair for Veterinary Science in the Agricultural College of Kentucky is a move in the right direction. The husbandmen of Kentucky have great interests at stake in the ownership of a vast number of some of the finest stock in the world. They are subject to most of the diseases that afflict the superior orders of creation, and the theory and practice of veterinary medicine and surgery rests upon the same intelligent basis as that which obtains in human medicine. It makes no difference whether we prescribe for a man or an animal, the laws of the animal economy are the same; each one is susceptible, and can be benefited by the same plan of treatment. Hence the need of reterinary schools of learning in the United States for the instruction of those who shall engage in the practice of our art. The science has been sadly neglected in this country, but a period has arrived when we must have educated men to prescribe for our flocks and herds. Humanity demands it-our own interests reiterate the demand. Thousands upon thousands of valuable animals die annually, prematurely and unnecessarily, in consequence of a want of knowledge of the nature of the disease and the modus operandi of medicine.

I know of no better locality in the United States than Kentucky for the establishment of a rational system of teaching veterinary science at an Agricultural College; and permit me, dear sir, to urge you to use your efforts for the above purpose. Such an undertaking, if successful (and I have no doubt of it), will reflect great credit on you, and finally crown your State with the laurel of veterinary fame.

Yours respectfully,

$$
\text { G. H. DADD, V. S. }
$$

Causes.-There is a great deal of doubt in regard to the manner in which infectious and contagious principles invade the economy, yet I think we shall not miss the mark in contending that it is chiefly through the medium of the lungs that they are introduced into the system; and, finally, the pernicious substance is absorbed into the blood, which it, more or less, quickly vitiates, and thus occasions the subsequent symptoms. All contagious diseases differ from one another. Every one possesses its own peculiar powers, by which, when they have come into bodies favoring their action, through the proper channel of communication, they every one piocreate their own distinct form of disease by a constant and uniform law; hence the contagion of rinderpest produces its specific effect on the system, spending its force principally on the respiratory organs. The virus of glanders will not produce any other dis- 
ease than glanders. The same remarks apply to rabies, scarlet fever, small pox, ete. ; so that the virus of Texas fever produces that disease and no other. The agents through which the virus is most readily concocted are animal emanations, or secretions, particularly the effluvia arising from the excretions of infected animals, and that arising from the carcasses of those that have died of the complaint. Hence, all that succumb to the same. should be buried or burned.

When a large number of animals are crowded together, and the disease makes its appearance, the location may be designated as the center of infection. In that event they need more space, and must have it, or the disease will run riot among them; yet the cordon sanataire must be drawn around them, for, if fresh cattle come within breathing distance, the chances are that the malady will spread.

Treatment. - In regard to the treatment of this malady I have but little to offer. Medicine has but little control over diseases of this character, yet I should recommend isolation, pure air, and a free use of chlorate of potass, hyposulphite of soda, sulphate of iron, and ginger. Take of

No. 64. Powdered chlorate of potass.......... 3 oz Powdered hyposulphite of soda...... $4 \mathrm{oz}$. Powdered sulphate of iron.......... $2 \mathrm{oz}$. Powdered ginger............... 7 oz. Mix.

Dose, one ounce, morning and evening; to be mixed each time in half a pint of flaxseed tea.

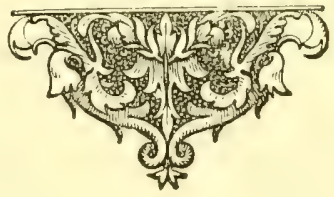




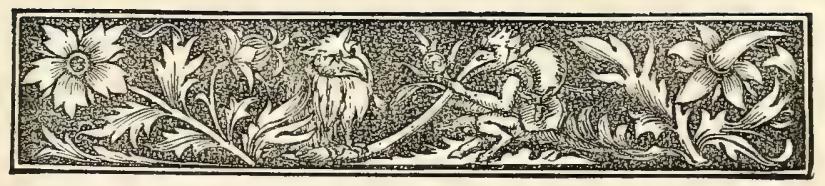

\section{SECTION XV.}

DISEASES OF THE ORGANS OF RESPIRATION.

Crodp-Laryngitis-Bronchitis-Inflammation of the Lungs-Hoose or Common Catarrh-Epizootic Catarrh-Consumption-Pharyngitis (Sore Throat)-Pulmonary Apoplexy-Pledrist, and Description of the Pleura.

\section{Crour.}

THIS disease is generally supposed to occur among young ani1 mals, but ClaUde relates a case of false membranes in a nine-year old ox. Young calves are frequently attacked with "laryngitis," and very many die from obstruction in the airpassages. These, after death, are found to be occupied by semiorganized lymph. Such cases are known to veterinary surgeons as membraneous croup, and, in the latter stages of the disease, the false membranes are found to occupy the intestinal canal, as well as the air passages.

Symptoms.-The animal is observed to be in imminent danger of suffocation; there is some discharge of morbid matter from the nostrils, yet it is evident that the respiratory passages are fast filling up with the morbid secretion. The only chance of saving the animal is to perform the operation of tracheotomy; but this operation, in order to be successful, must be performed in the early stage of the disease, or when false membranes are confined above the point selected for the operation; for when the obstruction exists at or about the lower end of the trachea, in the vicinity of the bronchial tubes, the case is hopeless. The only medicine of any value, in the early stage of this affection is

No. 65. Glycerine.................... 2 oz.

Tincture of lobelia................

Water........................ 2 oz.

A second dose may be given at an interval of two hours. If 
the patient does not improve, and the danger appears imminent, tracheotomy must be performed.

\section{LARYNGITIS.}

This disease often commences as an ordinary cold; but soon the throat becomes husky, followed by prolonged sonorous respiration. The respiration soon becomes quickened, and, finally, the patient may die of suffocation. Laryngitis consists of an inflammatory affection of the submucons cellular membrane of the larynx, often ending in cedematous laryngitis. This is one of the most dangerous of all diseases, for, after œdema has once set in, the animal is liable to die of asphyxia (loss of pulse) at any moment.

Symptoms.-Commencing, as it often does, as an ordinary cold or sore throat, it has some symptoms in common with the latter. The animal protrudes the nose, so that the head, instead of being pendulous, is thrust forward, and he very rarely turns his head sideways. The region of the throat is usually tumefied and tender, and pressure on the larynx occasions great distress. These symptoms, associated with the alarming character of the breathing and livid appearance of the visible surfaces, will enable any one to determine the true nature of the disease.

Treatment. - The first object is to endeavor to prevent effusion. In this view, I recommend that the patient be carefully drenched with the following:

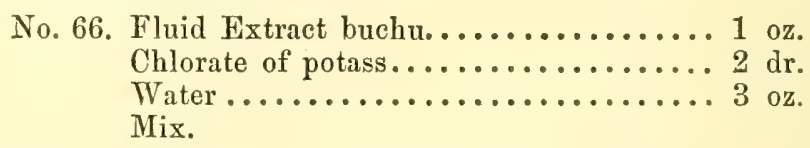

At the end of three hours, repeat the dose. In the mean time bathe the jaws and throat with a strong infusion of lobelia; then apply a cold-water bandage around the jaws and throat. The body and limbs should be well rubbed with a wisp of straw, so as to keep up an active circulation on the surface of the body. An enema, composed of soap and water, may be thrown into the rectum. Should the symptoms become more alarming, the services of a veterinary surgeon will be required, to perform the operation of tracheotomy, which consists in taking a circular piece of cartilage out of the trachea, and inserting therein a common tracheotomy tube. If the disease be confined to the parts above 
the seat selected as the place of operation, the animal may yet be saved. When the disease passes into the chronic stage, we have a purulent discharge from the nostrils. This discharge may be modified by giving a few doses of the following:

No. 67. Tincture of matico.............. 2 oz. Syrup of garlic................ 8 oz. Sweet spirits of niter............. 2 oz. Mix.

Dose, two ounces, morning and evening. The throat should be rubbed occasionally with a small quantity of tincture of bloodroot or hot vinegar. It often happens that chronic laryngitis ends in thickening, or altered structure, of parts within and around the larynx. Should this be the case, the patient should be put on a course of iodine. Twenty grains per day of iodide of potassium may be given in a small quantity of water; and the region of the throat should be anointed every night with the following ointment:

No. 68. Powdered iodide of potassium....... $2 \mathrm{dr}$.

Simple ointment................ 2 oz.

Mix.

Description of the Larynx.-The larynx is seated at the upper part of the windpipe, to which it is joined. It occupies that part known as the throat, between the broadest part of the angles of the jaw. It is composed of several cartilages, which are under the control of the laryngial muscles. These cartilages are so contrived as to be movable on each other, in various directions.

The first cartilage is named thyroid, or shield-like. It forms the most extensive part of the larynx, and protects the other parts from external injury.

The second cartilage is named cricoid, or ring-like cartilage. It overlaps the first ring of the trachea, or windpipe, in the form of a helmet.

Thirdly, there are two ewer-shaped cartilages, termed arytenoid. They are found on the upper and back part of the trachea. They form a canal which leads to the glottis.

The fourth cartilage is named epiglottis, in consequence of being situated upon and over the glottis. It is the door-keeper of the larynx, and every particle of food or drop of water which the animal swallows must pass over it. If it fail to perform its function 
for a single moment, when the animal is either drinking or eating, death is sure to follow. But the ox is rather more favored in the mechanism of the epiglottis than the horse. The epiglottis of the horse is just adapted to the caliber of the glottis, he being a nonruminant; but in the ease of the ox, the rough, unmasticated particles of food have to be returned to the mouth for a second mastication. This would peril the life of the animal if he had no other protection than that found in the larynx of horses. The fact is, the epiglottis of cattle, instead of being confined to the caliber of the rim of the glottis, overlaps it, and this securely protects the parts from the accidents which may occur in the process of remastication.

The larynx is lined by a membrane very susceptible to irritation. It is abundantly supplied with excretory glands and orifices, for the emission of a secretion peculiar to itself.

\section{Bronchitis. -}

Bronchitis is a disease of the bronchial mucous membrane. In its early stage, the term acute has been applied to it; this having subsided, it assumes a chronic type. It is very rare that this is a primary affection, for it is generally preceded by cough or catarrh, or else is an accompaniment of an abnormal condition of contiguous tissues. Its existence may be demonstrated very readily by applying the ear to the trachea, in the region of the point of the breast-bone, the peculiar sound differing from that in any other part of the trachea, having what is termed a sibilant, or whistling sound.

Treatment.-The treatment will be about the same as that recommended for common catarrh, with the addition of a counterirritant to the sides of the chest. A little mustard and vinegar will answer the purpose. A bronchial difficulty, of a very alarming character, sometimes prevails as an epizoötic, and this must be treated the same as epizoötic catarrh.

Description of the Bronchial Tubes.-The bronchial tubes are a continuation of the trachea. It having entered the thorax, becomes forked or bifurcated. They are constituted of several pieces, making up so many segments of the circle, overlapping each other so as to admit of extension and contraction in the respiratory acts. These are connected together and invested by an elastic cellular 
substance, which imparts to them both strength and elasticity. A further subdivision of the bronchial tubes takes place as they penetrate the substance of the lungs, so that they become very numerous. As they proceed onward their caliber continually grows less, until they end in the extreme ramifications known as air-cells. The bronchial tubes are lined by a membrane common to the trachea.

\section{INFLAMMATION OF THE LUNGS.}

Inflammation of the lungs, known, also, as pneumonia, is not usually so prevalent among the bovine as it proves to be in the equine species, excepting, however, milch cows, located in unventilated milking establishments. In such locations diseases of the lungs are often fearfully prevalent, raging, at times, as an enzoötic affection, which generally proves fatal when a large number of animals are confined in a small space.

Among horses this disease is often occasioned by laborious work and feats of speed, which produce rapid and sometimes distressing respiration; but among cattle, whose powers of speed and endurance are not often put to the test, we may reasonably infer that the exciting causes vary in their general character.

The stimulating and morbid action of an impure atmosphere may produce this disease by first creating irritation on the lining membrane of the respiratory passages. It has also been noticed that this disease frequently appears among cattle that have been driven a long distance, and have also been compelled to go hungry and thirsty for many hours. Fortunately for the poor brute, this disease is not so painful as bronchitis, pleurisy, and laryngitis; and, after having passed through the acute stage, it assumes a sort of mild, subacute, or chronic type, which, apparently, appears less dangerous than the acute kind; yet, after all, is more so, as it is apt to terminate in altered structure, hepatization, induration, and tubercles.

Pneumonia, now and then, terminates by metastasis ; that is, by translation of the formidable lung difficulty to one equally formidable, which locates in the feet, known to veterinarians as laminitis (fever in the feet). Among cattle, however, this termination is rather rare, yet very frequent among horses. When the disease does not take this course it often ends in "resolution," which signifies a return to health, without leaving any perceivable evidence 
of altered structure; so that, after awhile, the animal may become sound as ever.

Pneumonia is divided into several forms or stages, but, as they all have reference to its degree or intensity, it seems unnecessary to refer to them. It may, however, be proper to inform the reader that pneumonia may exist either as a state of congestion or of inflammation. Congestion signifies a distended or plethoric state of the blood-vessels of the parenchyma of the lungs, and slow motion of blood. Congestive pneumonia sometimes sets in as suddenly as that which is termed " inflammatory," and among cattle the former is most prevalent. In the congestive stage the symptoms are those of embarrassment, the blood courses through its channels sluggishly, and there is not the activity of heart and lungs which is perceivable in pneumonia.

Symptoms. - The symptoms, in the early stage, are such as are generally observed at the commencement of any inflammatory affection; namely, coldness of extremities and shivering fits; loss of appetite; labored respiration, quick pulse, slight cough ; mouth hot and clammy. The animal will not lie down, and refuses to move; the head is extended, perhaps drooping, and the fore-legs stand wide apart. As the disease progresses these symptoms vary, and the appearance of the membrane of the mouth, nose, and eyes vary also, from the color of bright scarlet to that of a leaden hue. In the congestive stage, the pulse is more voluminous, yet less active, and the visible surfaces are highly congested. A cough, slight or active, as the case may be, is usually noticed; it is a sort of deep-seated, half-suppressed cough, and sometimes is the first symptom which attracts the owner's attention to the ailing animal. - Treatment.-I have little faith in the heroic remedies so highly recommended by YouATT and others, and even by myself only a few years ago. I now have more faith in Nature and in regimenal means, and find that more cases are cured in this way than by the old method. It is very important, at the commencement of the treatment, that the patient shall be placed in a clean, comfortable location, where pure air abounds; for, under such circumstances, the condition favorable to the operation of Nature in the cure of the malady are secured. Should the animal labor under accelerated respiration and full, strong pulse, I should administer one ounce of powdered niter in a quart of cold water; after which, four ounces of the liquor acetate of ammonia may be given, every 
four hours. This agent, also, should have a quart of cold water added to it at every dose. The brisket and sides may be rubbed with a portion of the following: Powdered mustard and strong vinEydr, enough of each to form a thin paste. When mixed, a small quantity of oil of cedar may be added. This application should be repeated two or three times in the course of twenty-four hours. Nauseants are next administered, in view of relaxing capillary and muscular constrictions, and this is desirable, as such conditions tend to equalize the circulation of the blood, and prevent an undue quantity accumulating in the pulmonary organs.

In cattle practice, having had a case of this character under treatment, I prefer to administer the nauseating remedies by the anus; hence, a couple of quarts of infusion of lobelia may occasionally be thrown into the rectum. Considerable of the active principle of lobelia will be absorbed within this gut, and, under the circumstances, it is much better to introduce the medicine into the system in this way than by the stomach. The proportions of the lobelia to the water are-

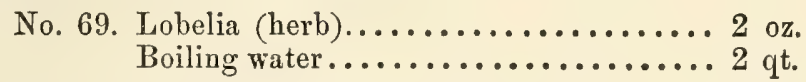

When cool, strain, and it is fit for use.

It may be necessary to give a dose of medicine; if so, I should use the following:

No. 70. Glauber salts................ 12 oz. Ginger...................... $1 \mathrm{dr}$. Warm water................... 1 qt.

It should be known to all husbandmen that a disease of this character, located in such important organs as those of respiration, is very prostrating, and operates very unfavorably on the inherent vitality of parts; hence, so soon as the activity of the morbid phenomena is somewhat subdued, all active medication should cease.

The affection is likely to continue for some days, and all we have to do is to try to keep the patient alive while the disease is running its course. Careful nursing, pure air, and light diet are the remedies. After the first twenty-four hours, I pay little attention to the pulse, but more to the patient, for I can conceive of but two conditions in this disease. One I call acute, the other chronic. The very moment the acute condition subsides, it merges 
into the chronic, and requires life-sustaining agents. After the first twenty-four hours, I generally resort to the following medicine:

No. 71. Glycerine................... 8 oz.

Powdered bloodroot............... $4 \mathrm{dr}$.

Powdered golden seal............. 1 oz.

Water.................... 4 oz.

Mix.

Dose, one table-spoonful, to be smeared on the tongue night and morning.

Management of the Patient white under Treatment.-As I have already intimated, a full supply of pure air must be insured; for a practitioner would be more likely to save an animal in the open air (provided the weather was not too cold or tempestuous) than in the unventilated cow-house. Should the limbs at any time be cold, they are to be hand-rubbed and bandaged; the body being in the same condition, must also be clothed. I should also give the chilled patient some warm ginger-tea, or any other non-alcoholic stimulant or carminative, in view of arousing the action of the heart and capillaries, by which means the red arterial and life-sustaining blood would be forced to the external surface and extremities, imparting to them a genial warmth, and thus insuring an equilibrium of the circulating fluid. The patient should be furnished constantly with a bucket of pure, cold water. When morbid thirst prevails, the water must be acidulated with either lemon-juice, cream of tartar, or acetic acid. Any symptoms of debility or lassitude are to be opposed by a few doses of some vegetable tonic. Tincture of golden seal, or tincture of matico, in ounce doses, every twelve hours, are the best remedies that I am acquainted with.

My experience in the treatment of this formidable disease is, that in ninety-nine cases out of one hundred, the patient dies of a meddlesome medicinal disease; in fact, he dies secundem artem. In view of furnishing a logical argument to support this theory, I refer the reader to Youatr, Pencrvall, and others of the orthodox stamp, (very learned men,) who are apt to place too much confidence in art, to the exclusion of Nature.

It is my opinion, after many years study and practice, that diseases are not cured by art; but art may so modify the diseased condition that the recuperative powers of the system can thereby induce salutary changes, without which they can not so readily be 
effected. Thus art, when understandingly applied, may be said to aid Nature in the cure of disease.

\section{Hoose, or Common Catarrh.}

Cattle, as well as horses, are subject to a catarrhal affection, known among English farmers as "hoose." This consists of a defluxion from the nasal cavity, accompanied with cough, loss of appetite, and loss of flesh. In popular language, it is nothing more than a common cold, induced by the ordinary causes, such as exposure, errors in diet, and management. It generally appears during spring, or toward the latter part of autumn, when the temperature of the atmosphere undergoes the most sudden changes; and it generally selects its subjects; for many animals, subjected to the ordinary causes of cold, enjoy immunity from the same; hence, I infer that a predisposition to this affection is hereditary, and manifests itself at periodical intervals, without the intervention of the common exciting causes, although they may prove operative in developing a latent disease.

I conceive that it sometimes has an hereditary origin, from the fact that some breeds are more subject to it than others. In some cases, however, this hereditariness exists only so far as the animal is of a peculiar temperament, so that, when removed from a warm to a colder region, it is apt to contract catarrh. This is the case with many of the Alderney breed of cows imported into the northern region of the United States. Ere they have been here long, they have an attack of catarrh, which often runs into the chronic stage, and ends in consumption.

Symptoms.-The first symptom which the farrier will observe, is loss of appetite. Succeeding this are febrile symptoms, such as quick pulse and respiration, heaving at the flanks, dry muzzle, glairy discharge from the nostrils, reddening of the visible surfaces. The limbs are generally colder than usual, and the hair loses its glossiness and appears roughened. The animal will occasionally snort and discharge more or less of glairy mucus, and some soreness of throat may be observed. Such are the early and most noticeable symptoms of this disease; and this is the most proper period for the animal to receive attention, in order to prevent the malady running into the chronic form; for, should it do so, ten chances to one if the case is not called "horn-ail," and 
thus the poor animal has to submit to a routine of barbarisms, such as boring horns, letting daylight, pepper, and turpentine into the frontal sinuses, much to the annoyance of a sick brute, and very significant of the ignorance of the itinerant cattle-slayer.

Treatment.-The treatment depends somewhat on the condition of the patient, as regards the preponderance of fever or debility. A high-fed animal, abounding in morbific material, and necessarily of a febrile diathesis, must have aperients. Eight ounces of Glauber salts, dissolved in warm water, and sweetened with molasses, may, without the least danger, be administered, followed by a liberal supply of warm, sloppy, bran-mashes; and, should the pulse be voluminous and excessively active, thirty grains of powdered niter may be added to the above, which, in all probability, will reduce the action of the heart. In the mean time, we keep the patient quiet. Withhold all fat and muscle-making food, and allow the patient to breathe a pure and cool atmosphere; for a cool atmosphere is, perhaps, a better sedative than niter, and certainly more requisite; and, after the medicine shall have had time to traverse a portion of the intestinal surface, say a lapse of five or six hours, an enema of Glauber salts may be given, in the proportion of half a pound to half a gallon of water.

In the early stage, and having a plethoric subject under treatment, it may, by some persons, be considered necessary to resort to the fleam; and some highly-educated physicians decide this to be the best course. There may be cases, occurring in pampered and stable-fed animals, which demand a prompt use of the above instrument; but the author has never seen a case of catarrh which, in his judgment, demanded the abstraction of blood. Bleed by the bowels, if there be need of depletion. This is my doctrine. Catarrh, whether it be simple or epidemic, in one feature resembles influenza occurring among horses. It is a prostrating disease, inducing debility. However, I have no desire to force my opinions on any man. Try sanitive medicines; if they have not the desired effect, the judicious practitioner has no other remedy.

We have now only to keep the patient alive while the disease is running its course, and this is accomplished by means of "good nursing." There are a great many remedies that might be recommended, in view of hastening convalescence, but "good nursing" supplants the whole. A sore throat may accompany the malady, and, if so, I recommend the following: 
No. 72. Olive oil................... 6 oz.

Oil of cedar.................... 1 oz.

Spirit of ammonia................

Tincture of capsicum.............. 1 oz.

Mix.

Apply a portion to the throat twice daily.

In order to promote a discharge from the nasal outlets, we resort to vapor, which may be generated by dropping water or vinegar on a hot brick; and to insure the full effect of the same, I envelop the head with a cloth or blanket, so as to direct the current of vapor through the nasal passages. In view of promoting a nasal discharge, a small quantity of bayberry bark (pulverized) may occasionally be blown up the nostrils, from a quill or a hollow tube of paper. The after-treatment will depend upon the observable symptoms. While a febrile diathesis continues, we depend on sedatives and aperients. In the chronic stage, tonics and alteratives are indicated, and must be resorted to, in view of warding off a chronic cough and its consequences.

\section{Epizootic Catarkh.}

Epizoötic catarrh is infectious, yet animals having once had an attack of the same may enjoy immunity from it thereafter. It usually appears and spreads over various parts of the country when great variations in the weather are noticed. It generally appears in the spring, and disappears when the weather becomes warmer and more uniform.

Symptoms. - The symptoms of epizoötic catarrh, in the early stage, do not differ materially from those alluded to in the preceding article (common catarrh). In a very short time, however, the animal begins to grow very weak, and becomes debilitated. Tumors form in various parts of the body, emitting, when pressed, a crackling sound; the glands in the region of the throat are enlarged; the neck, stiff; the odor from the breath and feces is very offensive; the animal loses flesh very fast, and, unless relieved, will surely die.

Causes.-The direct causes of this, like that of any other epizoötic and endemic affection, are involved in obscurity. Speculation is rife as regards the causes of cholera and the potato rot, which probably have analogous origins, but it is very difficult, if 
not impossible, at the present time, to define the precise character of the morbid germ which, "like a little leaven," leavens the whole body.

Treatment.-The principal objects in the treatment of this malady are to sustain the vital powers, and thus guard against the subsequent prostration and decomposition which, under the orthodox treatment, is sure to occur. The proper mode of treatment is to drench the animal with the following:

No. 73. Tincture of matico.............. 1 oz. Hyposulphite of soda.............6 $6 \mathrm{dr}$.

Powdered golden seal............. 2 dr. Warm water.................. 1 pint.

Having administered the above medicine, anoint the throat and all tumefied parts with a portion of the following counter-irritant:

No. 74. Oil of cedar................. 1 oz.

Oil of sassafras................

Cod-liver oil.................6 oz.

Mix.

Apply by means of a small piece of sponge. Should the breath become fetid, and the odor from the evacuations almost intolerable, as is often the case, very powerful antiseptics will be needed, to arrest the morbid fermentation. The most efficient and valuable article for this purpose is pyroligneous acid. A couple of ounces of the same may be given in a quart of oatmeal gruel, every four hours, until the odor is exterminated. The diet should consist of well-salted, scalded shorts, sliced carrots, and parsnips. In the absence of rumination, give a drachm of powdered golden seal and half a drachm of carbonate of soda, twice in twentyfour hours.

\section{Consumption.}

This disease, as it appears among cattle, is supposed to be the sequel of other diseases of the respiratory apparatus, and some writers contend that phthisis is the termination of chronic disease of the lungs, characterized by the formation of tubercles within the substance of the lungs.

Symptoms. - The most notable symptoms are emaciation, debility, cough, fever, and purulent expectoration. Expectoration, however, is a feature of this disease, more marked in the human subject than among horses and cattle; yet, in the last stages, we 
occasionally observe nasal discharges of a purulent character. In diagnosing this disease, it is necessary to make ourselves acquainted with the history of the case, and the physical conformation of the animal; for, if the patient be the subject of neglected catarrh, bronchitis, or any other pulmonic or pleuritic difficulty, we have the data for an intelligent diagnosis ; provided the physical conformation corresponds to that which physiologists regard as susceptible of phthisis ; namely, a lean, lank organization, associated with an active, nervous temperament:

Referring to Percivalu for evidence on the subject now under consideration, I find that he considers a colt having long legs, overgrowth, narrow chest, flat sides, pot belly, and an appearance of weakness and unthrivingness, a capital subject for phthisis. A cough occurring in such an animal, of a feeble, painful, hoarse, rattling, or gurgling character, shows conclusively that disorganization of the lungs has commenced. The cough will also be accompanied by a sound which gives us an idea that it is deepseated.

Causes.-Aside from the well-known direct hereditary causes which are known to exist in breed, there are others operating insidiously to produce disease and altered structure in the lungs. The climate may be prejudicial. I have known this disease to make its appearance among cows unsuited to our New England climate-the Alderneys, for example. On the other hand, if cows be removed from a warm, comfortable location, or barn, to a region involving a material difference in temperature, a derangement of the respiratory system is very apt to occur. It may appear at first under the guise of a simple bronchial affection, which insidiously steals on until the substance of the lungs is affected. Aninuals shut up in close and hot stables, where they can not obtain sufficient oxygen to vitalize or decarbonize the blood, are apt, after a short time, to die of tuberculated lungs; or, perhaps, a worse form of disease, known as infectious pleuro-pneumonia, soon terminates their wretched existence. Impure air is at all times operative in exciting pulmonary affections. The least deviation from purity may occasion very serious difficulties; therefore, it should be the business of the farmer to see that his cattle have constantly an abundant supply of pure, uncontaminated airthe breath of life.

Treatment.-In the first place, the patient must be removed to a 
comfortably warm and well-ventilated barn. Should the weather be chilly, a blanket may be thrown over the body; and it will be expedient, also, to clothe the limbs, up to the knees and hocks, with strips of flannel. By this means we promote cutaneous and subcutaneous circulation, and every drop of blood invited and maintained at the surface in the extreme vessels tends to prevent internal congestions. Without proper attention to these matters, we might as foolishly attempt to raise a dead cow to life. Next, the patient, whose appetite is almost sure to be impaired, should be fed on that kind of food which contains more carbon and nitrogen than common hay; namely, oatmeal. In case of a complete suspension of rumination (loss of cud), a due proportion per diem of oatmeal gruel, sweetened with some saccharine matter, may be administered from a bottle. Every morning the patient should have four ounces of the best cod-liver oil. This can be continued until its action is made manifest by purging. Every evening, give the patient a dose of the following:

No. 75. Powdered phosphate of lime........ 3 oz.

Powdered bloodroot.............. 1 oz.

Powdered bayberry bark.......... $4 \mathrm{dr}$.

Powdered sassafras.............. 2 oz.

Divide the mixture into sixteen parts. The above is, according to my experience, the most rational method of treating this disease; but the farmer must not feel disappointed if he fails in arresting it, for it frequently baffles the most consummate skill.

\section{Pharyngitis (Sore Throat).}

The term pharyngitis signifies inflammation of the membrane lining in the pharyngial inlet, or funnel-like entrance into the œsophagus, or gullet.

Symptom.-The diagnostic symptom of this affection is as follows: The subject is unable to swallow, and thus the food taken into the mouth is apt to be returned by the nostrils. This happens occasionally, although the passage of the nasal inlet is much smaller than it is in the horse. On exploring the inferior region of the throat, from ear to ear, considerable swelling or tumefaction is encountered, yet the pharyngial muscles appear to be constricted. It generally appears among cattle as a simple local affection, yet it often accompanies other diseases of the respiratory character, 
and whenever it does appear as a local malady, it is apt to merge into something else. It is a very distressing affection, and the animal gets but little relief until suppuration commences; then a free discharge takes place.

Treatment.-Let the animal's throat be rubbed twice daily with

No. 76. Oil of cedar.................1 oz.

Cod-liver oil..................6 6 oz.

Spirits of ammonia............. $2 \mathrm{dr}$.

Mix.

Keep a sloppy bran-mash before the patient, or some flaxseed tea, into which stir a small quantity of powdered niter of liquorice. This will relieve the cough, if any be present, and tend to lessen irritation of the lining membrane of the pharynx. When the patient begins to expectorate, or has the least discharge from the nose, give

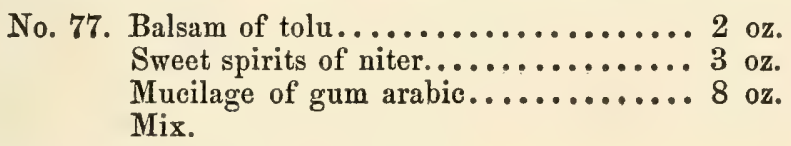

Dose, one wine-glassful, twice daily.

Description of the pharynx.-The pharynx is the commencement of the tube known as the esophagus, or "gullet." It is a funnelshaped cavity, lodged between the mouth, gullet, and windpipe. The pharynx is composed of muscular and membraneous tissues. The most important muscles which enter into the composition of the pharynx are the constrictors. They give the membrane forming the funnel-shaped sac a complete covering, and their function is to force the food beyond the action of the tongue, into the resophagus. The pharynx is divided from the mouth by the soft palate and the epiglottis ; therefore, except in the act of swallowing or coughing, there is no direct communication. The interior of the pharynx is lined by a membrane having within its structure a vast number of minute glands, with excretory ducts, from which a viscid or lubricating fluid issues. This lubricates the pellets of food, so that, by this process, their passage into the œsophagus is insured without the casuality of friction.

\section{Pulmonary Apoplexy.}

This disease occasionally appears among cattle in the Western States. It attacks animals irrespective of age, sex, or condition. 
It is generally sudden in its attacks, and death frequently ensues in the course of a few hours.

Symptoms.-The pulse and respirations are very much accelerated, the former sometimes running as high as 110 ; an augmented salivary secretion is observed to run from the mouth; the tongue is much swollen; so, also, are the eyelids, and tears run down each side of the face; various parts of the body are swollen and congested; the stomach is distended with gas, and the evacuations are profuse and watery. Post mortem examinations reveal a highlycongested state of the lungs ; in fact, they are engorged with blood, and the muscles in the region of the tumefied parts are in a similar engorged condition.

Treatment.-No time should be lost in administering the following drench, for it will preserve the tissues against decomposition, and, perhaps, save the animal:

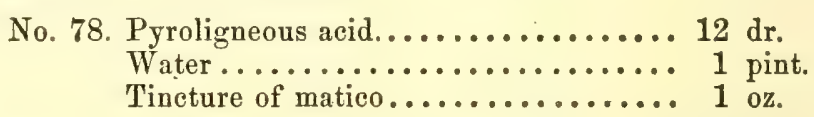

Repeat the dose after a lapse of six hours, and rub the tumefaction occasionally with

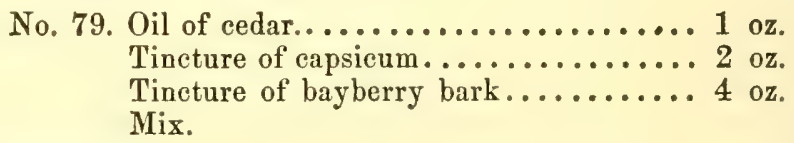

It appears that in this disease there is a morbid impulse directed to various parts, which results in local accumulations, rendering the parts turgid. Then the blood is thrown out of the capillary vessels, and sometimes they suffer a rupture, which accounts for the engorgement and extravasation.

As there is generally some effusion present in this malady, some good may be accomplished in acting slightly on the kidneys; yet, as the pulse and respirations are both accelerated, the ordinary diuretic (sweet spirits of niter) is not admissible, for the simple reason that it is too stimulating; therefore I recommend the use of common niter-half an ounce, every four hours, to be given in a little water. The tumefied tongue should be rubbed often with table salt. The animal does not require any kind of food until amendment takes place. The best drink for the patient is cold water, to which a little table salt may be added. 


\section{Pifeurisy and Description of the Pleeura.}

The delicate, transparent membrane which lines the cavity of the thorax, or chest, is duplicated as an external tunic on the lungs, and forms a partition called mediastinum, which divides the cavity of the chest into two equal parts, termed right and left cavities of the thorax. It is, therefore, a reflected membrane. That portion which gives a lining to the chest is termed pleura costalis, and that which invests the lungs is called pleura pulmonalis, although, at all points, they are precisely similar in structure and function. The pleura is called a serous membrane. It is dense, shining, and transparent; its texture is penetrated by bloodvessels, nerves, absorbents, and exhalents. The minute ramifications of the arteries give origin to a vast number of exhalents; and these furnish a serous or watery vapor, which is distributed over every part of the pleura, and thus all friction and irritation is prevented. In the disease known as hydrothorax (dropsy of the chest), the effused fluid found in the cavity of the thorax comes from the exhalents. The absorbents play a very different part. Their function is to absorb or drink up any superabundant serum or blood that may be found in the chest. The functions of these vessels, however, are limited ; for when, in consequence of disease, augmented serous secretion takes place, the absorbents are unequal to the task imposed on them, consequently the subject of hydrothorax often dies with his chest loaded with water.

Symptoms.-This disease is generally ushered in like other febrile affections, by fever and trembling of the fore extremities. The cough accompanying pleurisy is painful, and the animal tries to suppress it as much as possible. The breathing is not laborious, but short, the intercostal muscles not admitting of their usual extension and contraction without intense pain. The diaphragm has to perform the respiratory movement, without the aid of the former muscles. If slight pressure be made on the intercostal spaces, between the ribs, the animal will evince symptoms of intense pain. If a person attempts to back the patient, the latter will moan or grunt, and be very unwilling to move. The patient stands with his fore-legs wide apart, and seldom, if ever, lies down. On applying the ear to the sides of the chest, a slight sound, resembling that of friction, is discernible. This is probably occasioned by the presence of effused lymph on the pleural surfaces. The pulse is 
generally quick, tense, and small. These are the principal symptoms of pleurisy in its early stage. They will vary as the disease progresses, or become complicated. The minor symptoms, such as loss of cud, etc., are not worth noticing, as they are present in various other forms of disease.

Treatment.-Give two drachms of fluid extract of gelseminum every four hours. This acts as a powerful sedative, and will soon afford much relief. In the mean time, let a preparation of mustard and vinegar be applied to both sides of the chest and breast. The patient should now have one ounce of powdered chlorate of potass, dissolved in flaxseed tea or thin gruel. This may be given morning and evening. Should the animal refuse to drink it, give it as a drench, by adding to it one pint of water. In view of mitigating the cough which may be present, and of relieving pain, the patient may be made to inhale an ounce or so of sulphuric ether from a sponge, the latter being merely covered with a towel, except that part which comes in contact with the nostrils. It would not be proper to completely etherize the animal, but merely to stupefy him for a time. This will have an antispasmodic effect, and may be repeated, or not, at intervals of four hours, according to the nature of the effects produced.

It will be proper to administer an occasional enema, and this should be of an antispasmodic character; therefore an infusion of lobelia is recommended. The very moment the animal appears to be relieved of urgent symptoms, the treatment should not proceed on the same principles. The intention should be to guard against debility and hydrothorax, two mortal enemies to the bovine species. Golden seal and buchu are the best remedies to prevent the one and guard against the consequences of the latter. They may be given in the following proportions:

No. 80. Powdered golden seal............ $2 \mathrm{dr}$.

Tincture of buchu............ 4 fluid $d r$.

Hyposulphite of soda.............. $3 \mathrm{dr}$.

Water.................... 1 pint.

This quantity is sufficient for a period of twenty-four hours. The animal appearing much better will be the signal to discontinue all medical treatment. 


\section{SECTION XVI.}

\section{DISEASES OF THE DIGESTIVE ORGANS.}

Descriptron of the Esophagus-Foreign Bodies in the Gullet-GesophagotomX-Laceration of the Esophagus-Aphth e-Description of StomachRumination-Bloat-Distension of the Rumen with Food-Gastro-IntestiNat Inflamation-Dentition of Cattle-Synopsis OF Dentition-Suspended Rumination, or Loss of Cud-Inflamition of the Bowels-Invagination of Intestine-Diarrhea-Cironic Diarrmea-Gut Tre-Internal Ropture of Oxen-Flatulent Colic-Spasm of the Bowelg-Constipation of Bowels.

\section{Description of the CEsophagus.}

TTHE cesophagus, or gullet, extends from the mouth to the 1 stomach, and passes down the left side of the neck, outside of the windpipe, between the two first ribs, and then runs backward, along the upper part of the thorax, until it reaches the diaphragm. Having passed through the latter, it enters into the mechanism of the digestive canal. It is composed of three coats. The outer one is made up of cellular substance, and admits of much distension. The middle one is muscular, and composed of two layers, arranged spirally, and running in opposite directions; that is to say, the fibers wind round the gullet, in contrary directions. This allows or aids the food which has been masticated to pass down the tube into the first compartment of the stomach, viz., the paunch, and, by a reverse action of the other set of spiral muscular fibers, the pellet (cud) ascends into the mouth for remastication. The inner or third coat is similar in structure to the pharyngeal membrane, or that which lines the pharynx, yet it is so arranged as to admit of considerable distension.

\section{Foreign Bodies in the Gullet.}

When an animal attempts to swallow a substance too large for the caliber of the œsophagus, it becomes impacted in the same, and 
the creature is said to be choked. On examining the left side of the neck, the foreign body can be both seen and felt. When an accident of this character oceurs, half a pint of olive oil should immediately be administered. This will lubricate the internal surface of the œsophagus, and aid us when attempting to force the impacted material toward the stomach. Before any attempts are made to introduce the probang, some efforts should be made to manipulate the obstruction, and thus force it downward. If it can be felt, yet can not be moved by external manipulation, there is very little chance for the animal; yet we are not without resource. The probang is our remedy. This is to be introduced through the mouth-piece into the œsophagus. Some slight degree of force may be used to push the obstruction downward. If that fail, no time should be lost in futile efforts, and an operation must be performed termed œesophagotomy.

\section{CEsophagotomy.}

This operation is performed as follows:-Place the animal in the trevis, or cast, and etherize him. Then make an incision over the region of the obstruction, through the skin and subcellular tissues. The resophagus will then be seen. The only vessels in this location that are of any consequence are the jugular vein and common carotid artery ; but there is no danger of wounding them when the osophagus is distended. The obstruction having been found, an incision is to be made through the coats of the gullet sufficiently large to admit of the extraction of the incarcerated body. So soon as this desirable object is effected, the edges of the gullet may be closed by sutures or stitches, one end of which should be cut off close to the knot, and the other left long enough to hang out of the external wound. The integuments are then to be brought together by another set of stitches, taking care to leave a small orifice at the lower part of the neck, for the escape of any morbid matter. The wound, with the exception of this orifice, should be well coated with collodion or liquid cuticle. For a few days after the operation, the patient should be kept on a light, sloppy diet, sufficiently seasoned with common salt to prevent fermentation. In the course of a fortnight the wound will be quite healed. 


\section{Laceration of the Cesophagus.}

This accident very frequently occurs from the objectionable practice of attempting to force a foreign body, such as an apple, potato, or turnip, that the animal has greedily attempted to swallow, down the œesophagus into a more dilated part of the digestive apparatus, by means of such rude instruments as a whip-stock or common stick, in lieu of a proper instrument, known as a probang. The operator sometimes succeeds in removing the obstruction, but soon a swelling is observed in the region of the neck. The animal evinces signs of pain, and symptoms of suffocation ensue. In such cases it is evident that the osophagus is ruptured. The following, from the author's case-book, is introduced in view of showing the folly of forcibly removing such obstructions:

October 10, 1855.--Saw a cow, at Feltonville, the property of $\mathrm{Mr}$. Welch and others. The animal had calved about ten days previous, and done well. Two days after parturition she was purchased by another man for the above party, and driven to her new home. On the way thither she picked up an apple and got choked. The apple was discovered in the upper half of the resophagus. The attendant tried to manipulate it either one way or the other, but, failing in this, he procured a whip-stock, and forced the foreign body toward the

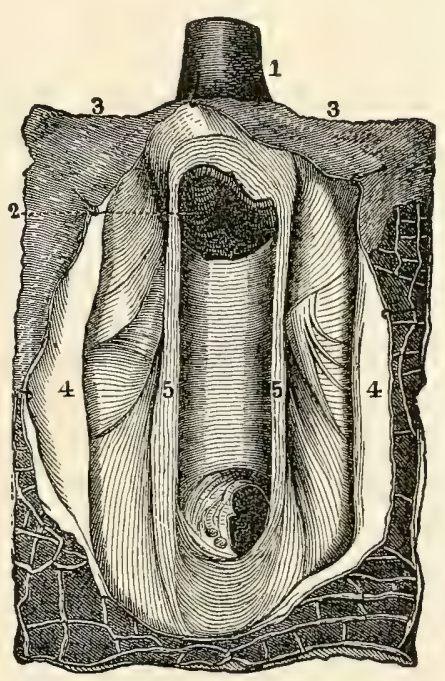

SECTION OF A COW'S STOMACH.

Explanation.-1, A portion of the œsopha. gus, or gullet; 2 , The terminating portion of the gullet; 33 , Lining membrane of the first compartment of the stomach; 44 , Portions of the lining membrane of the second compartment of the stomach, dis. sected and raised, so as to show the mus* cular mechanism beneath it; 55 , The lips of the demi-canal, which are the boundaries of the groove. At the lower part is the entrance into the third compartment of the stomach, termed "many-plies." rumen. This induced convulsions, and the subject threw herself violently down, with the whip-stock in her throat. This feat she repeated several times, to the imminent danger of limb and life. She shortly, however, recovered, and appeared to do well, and the next day, I believe, was received by the above firm. After a brief space, she appeared to be "ail- 
ing," and the owners, thinking the apple was the cause of the same, gave her a full dose of oil. Soon afterward inverted peristaltic action took place, and, whenever she attempted to swallow fluids or solids, vomiting or regurgitation took place. This was her condition at the same time I saw her-ten days after the first attack. I prescribed alkalies and counter-irritation. Next day she appeared better; that is, the vomiting had ceased. She was then drenched with Glauber salts and spearmint, and during the next day received several alkaline injections, and such other treatment as the urgency of the case seemed to require. Notwithstanding this, she died on the third day from my first visit. The owner, in accordance with my request, notified me of the death, and I made an autopsy. The heart, lungs, liver, spleen, pancreas, kidneys, stomach, and bowels all appeared in a healthy state. They presented, however, evidences of debility, in the condensation and pale aspect of their tissues. The omentum-caul was exceedingly dense, scarcely thicker than a piece of writing paper. Tracing the osophagus internally from the mouth to its termination, or base, there were no symptoms of laceration or inflammation; but in the dilated portion of the same, which is contiguous, and receives food after primary mastication, I found a mass of juvenile corn-stalks, about the size of a man's fist, and twice the length of the same-seven inches. This part being considered as the termination of the osophagus, and commencing link of the stomach, was distended beyond its ordinary capacity, and in a high state of gangrene (mortification), and particles of corn-stalk were protruding through its disorganized and lacerated tissues. This accounts for the death; but the reader will probably want to know something about the cause, and may, possibly, say that the whip-stock was the exciting one. This would appear, on first thought, as a rational conclusion, because many valuable animals, both in this and the mother country (as records show) have been destroyed by lacerating the œsophagus with the above or some such instrument; and we might reasonably assign the cause of death to the same, and thus terminate this article. But my readers, I opine, desire the truth, and nothing but the truth. I am satisfied that the whip-stock, however injurious it may have been in other cases (and it is in most cases an objectionable remedy), was inoperative in this ; that is, so far as the vitality of the animal was concerned. Now for the proof. The seat of the disease proved to be, by care- 
ful measurement, forty-three inches from the tip of the lips. The whip-stock was three feet six inches in length, and, according to the testimony of the operator and others, six or eight inches of the same never entered the mouth. Computing the medium at seven inches, the reader will perceive that the diseased location was just seven inches beyond the reach of the instrument.

How, then, are we to account for the death of the animal? I shall try to satisfy the reader on this point. The animal, at the time of purchase, had not recovered from the pain, labor, and excitement of parturition. She was an invalid; in the same condition, yet less well provided for than her lordly mistress, who has the advantages of science, skill, and sympathy to alleviate her woes and mitigate her pains. The poor brute was compelled to perform a journey when she ought to have been kept at rest, and kindly cared for. The common sympathies of our race ought to have been extended to her. She ought to have been dosed with groaning cordial, instead of corn-stalks; and, instead of performing a journey through apple orchards, she ought to have been kept at home until health and strength reappeared.

Changes in food, location, and barn management, are, at times, and under certain circumstances, operative in inducing disease, especially when the subject be in a weak or delicate condition. Corn-stalks, therefore, being very indigestible, tend to overtax and irritate the stomach, and the oil, probably, induced nausea. In efforts at vomiting, the rough particles of food found a lodgment at the point indicated, where they accumulated, produced irritation, inflammation, and, finally, mortification. The direct cause of death, therefore, was obstruction within the gullet; the morbid appearances were the consequence of the same.

The best instrument I know of for the accident of choking, or obstruction within the gullet, is a flexible probang; but, as that instrument is not always to be obtained, every farmer should be provided with the next best instrument, which consists of a piece of smooth, flexible rattan, about five feet in length, armed at one end with an oblong ivory ball, which must be securely fastened. If much force be used, laceration of the osophagus is apt to occur; therefore, if the foreign body will not yield to gentle pressure, I should pour down a little olive oil, wait awhile, and then try the probang again. If, however, the foreign body can be detected, and the probang fails to remove it, we should immediately perform 
the operation of osophagotomy, which consists of making an opening into the gullet large enough to remove the former, and then bringing the divided edges together again by means of sutures (stitches).

Cases like the above (when the medical man is not called on until after the lapse of several days from the time of the accident) do not admit of the introduction of a probang.

The following case, which occurred in the practice of W. Sanders, student Royal Veterinary College, goes to show that an animal should never be consigned to death until the skill of a qualified person proves unavailing: "In the month of March, a young Alderney cow became choked with a Swede turnip, which was found to be impacted about the termination of the third of the cervical portion of the esophagus. The owner immediately sent for a person whom he had been in the habit of employing, but who, not having a proper probang, substituted a common ash stick. After some difficulty, the obstruction in this way was removed. In the course of a fortnight the neck was found much swollen, and the animal not only evinced a great deal of pain, but sometimes, as I was informed, appeared as though threatened with suffocation. It was supposed that the oesophagus must be ruptured, in consequence of, as it appeared, a considerable portion of food having protruded between the muscles of the neck, just anterior to the sternum. A small orifice was made through the skin, and a portion of the food (for food it proved to be) removed. The cow continued to get worse for several days, and putrefaction had apparently commenced. The attendant now advised the owner to have the cow slaughtered; but, as her condition precluded the prospect of selling her advantageously, it was deemed expedient to risk the chances of recovery, especially as she was young and in calf. Under these circumstances, my brother, who resides in that locality, was consulted. On his arrival he gave but little hopes of recovery, seeing she was to so great a degree debilitated. A stimulant was administered, and an incision, about seven inches in length, was made in the most depending part of the swelling, through which more than a quarter of a peck of food was removed from between the muscles. The wound being now held open, and a candle placed in front, the rupture of the esophagus became quite apparent. It proved full three inches in length. The wound was cleaned. A common side-saddle was placed upon her 
back, and fastened on with a girth in the usual manner ; a smallsized piece of ash-wood was shaved so as to render it pliable at one end. The large end was then tied to the crupper-loop of the saddle, and passed then between the pummels, which kept it in its place, and elevated it half way along the neck. The œsophagus at this part being rather deep-seated within the loose fleshy part of the dewlap, it was not convenient to pass sutures through the edges of the laceration. A pledget of tow, dipped in some digestive, was put into the wound, and pressed against the ruptured part. A pad of flannel was then placed over the outside, and a broad bandage, with some thin but flat pieces of wood sewn to it, to prevent its becoming too narrow. This was carried round and over the stick above the neck, which, being pliable, acted as a spring, that kept a moderate but equal pressure as she moved her head up and down. She was mainly supported upon gruel made. of barley and bean and wheat flour; but in addition, occasionally, was allowed a little green food. Tonics and stimulants were administered. Any food that accumulated in the wound was removed, and it was regularly dressed twice a day. Three weeks after she calved a live calf; but, from the weakness before parturition, she became so reduced as to require assistance to get up for a short time. I went home from college on the 6th of May. On the 8 th I saw her myself. There was then a small circular opening, leading into the csophagus, sufficiently large to admit the finger, which was prevented healing by the frequent escape of food. The edges of the wound were thickened, and apparently healed over. They were occasionally scarified, with the view of promoting cicatrization; for it was my opinion that, should the wound heal, it would leave a stricture in that part of the tube, and consequently render the animal susceptible of becoming again choked. The food continued to pass, more or less, through the opening until the beginning of June, when the wound became entirely closed. Since then she has become again in calf, and from that has continued to go on well; nor has she ever, that I am aware, again shown any symptoms of choking, although she has taken her chance as to the nature of her food. She is still in the same person's possession; cousequently I know, from the information $I$ have received, that she continues, up to the present time, apparently as well as ever she was; and it is now eight months since the wound healed over." 


\section{АРнтнж.}

This disease is commonly known as "thrush, or sore mouth. It appears as a vesicular eruption on the tongue, gums, and on the buccal and palatine membrane. When the disease is mild, and confined to the above parts, it is easily cured by daily applications of a portion of the following:

No. 7. Powdered golden seal.............. 2 oz. Tincture of matico.............. $1 \mathrm{oz}$ Honey................... 4 oz. Mix.

Apply by means of a swab or sponge.

When the disease is violent and of long standing, it is apt to extend through the whole course of the alimentary canal, from the mouth to the anus. If this be the case, the animal will purge, as if a powerful cathartic had been administered, and will be otherwise unwell. The method of cure, in this event, is to prescribe tonics and astringents. The remedies are tincture of matico, golden seal, and sulphur, in the following proportions:

No. 8. Tincture of matico.............. $4 \mathrm{dr}$.

Powdered golden seal............. $2 \mathrm{dr}$.

Sulphur..................... $2 \mathrm{dr}$

Water....................... 1 pint.

Mix.

The patient's diet should consist of oatmeal gruel, slightly alkalized with hyposulphite of soda.

The following article upon epizoötic aphthæ is from the "Veterinarian:"

"Epizoötic aphthæ, commonly known as foot and mouth complaint, is a febrile, very contagious, and panzoötic affection; that is, readily communicable from one species to another-met with in the horse, goat, pig, fowl, hare, etc., but most commonly in the ox and sheep, in which two last-mentioned animals it consists of vesicles breaking out on the mouth, gums, lips, teats, and around the coronary surface of the foot, between the skin and the hoof. It is an epizoötic affection, spreading over large tracts of country, interfering very seriously with the capability of the ox to put on fat 'while preparing for the butcher,' and in the milch cow, rendering the supply of milk small, if not altogether suspending it. In this disease the ox exhibits the following symptoms: Suspen- 
sion of rumination ; constipation ; sometimes, though rarely, diarrhea; great flow of saliva from the mouth, and severe lameness.

Milk taken from cows affected with this disease should never be drank, as it will most readily produce aphthæ in man. To prove this fact, Professor Hertwig, together with two medical men, Mann and Vilion, drank the warm milk of an aphthous cow, and the result was that each became the subjects of severe inflammation of the throat, associated withe the vesicular eruptions mentioned above as indicative of this malady. Professor Simonds also gave the warm milk of an aphthous cow to pigs, with a similar result."

\section{Description of the Stomach.}

The stomach of a ruminating animal presents a very complex arrangement, of which the purpose seems to be to favor the mechanical reduction of the food, and its impregnation by the salivial and gastric fluids, before it is subjected to the action of the biliary and pancreatic juices.

The stomach of an ox is divided into four parts or cavities, viz.: rumen, or paunch ; reticulum, manyplus, abomasum.

The rumen, like the cosophagus, is composed of three coats. The internal one is studded with numerous papillæ, which incline in the direction that the food takes. They are erectile, and by inclining in different directions, they can, to a certain extent, favor or oppose the passage of food.

The openings into the rumen are two-one at the base of the cesophagus, through which the food and fluids pass; the other is below this. It is much larger, and communicates with the second stomach. The paunch is not so highly organized as some other parts of the stomach. This compartment is capable of extraordinary distension, and, in some cases of tympanites, becomes so distended as to produce suffocation by pressure on the diaphragm and lungs.

The reticulum, or second stomach, sometimes called the honeycomb, presents a very irregular surface, consisting of open cells, varying in size and depth, looking very much like a piece of honeycomb on a large scale. There are two openings into this stomach-one through the floor of the gullet, the other into the paunch. The pellet of food to be returned for remastication is thrown into the œsophagean canal by the reticulum. 
The manyplus, sometimes called manyplies or manyfolds, is made up internally of laminæ, or leaves, which hang down from every part of it. By this arrangement an immense digestive surface is crowded into a small space. The cuticular covering of these leaves or laminæ is very singular. It is thickly studded with eminences, varying in size and form. In some places they resemble little hooks, others look like papillæ; some are soft, others are hard and durable, like horn. The function of the laminæ and papillæ is to comminute and properly prepare the food ere it enters the fourth compartment.

The abomasum is the true digestive stomach. Its function is to secrete the gastric juice; at least the gastric juice is secreted by small glandular bodies, having ducts which terminate on the villous membrane, which lines the abomasum. The lower orifice of this part of the stomach is termed pylorus. It is guarded by a projecting body, which answers the purpose of a sphincter muscle, to guard against regurgitation from the duodenum.

\section{Rumination, or Remastication and Ingestion of Food.}

The ox, a member of the group ruminantia, has four compartments in the stomach, yet two of them are nothing more than dilatations of the œsophagus.

The food having been gathered by the lips, tongue, and teeth within the mouth, it undergoes a grinding process between the molars, and receives the admixture of salivial fluids secreted by the submaxillary, parotid, thyroid, and sublingual glands. It then passes down the œsophagus into the paunch. The character of the food, however, regulates its passage into the various compartments. If the pellet of food be solid, the paunch receives it; if it be semi-fluids, it goes beyond the paunch to the second and perhaps third compartment. This is the case with a sucking calf. The milk, which forms its nutriment, requires no remastication, and, therefore, passes directly into the true digestive cavity-the fourth compartment.

It appears, therefore, that the functions of digestion and remas. tication are involuntary, and are governed by the same sort of power which causes the heart to pulsate, expands the lungs, secretes the bile, pancreatic juice, etc., without the aid or consent of the animal. We may, however, to a certain extent, increase 
or decrease these functions, by artificial means; but their primary operations are uncontrollable, simply because they are involuntary. Some persons have doubted the fact of rumination, and if any of my readers be skeptical on this subject, let them satisfy themselves by experiment. The best subjects for demonstrating the acts of rumination are animals with lean necks. For example, let a person stand on the left side of the animal, in the region of the neck (supposing the latter to be in the ruminating mood). He perceives the cud re-ascend through the gullet, and re-descend again into the stomach. At the period of re-ascension, place the ear in the region of the gullet, and a gurgling sound will be heard, different from that accompanying re-descension. The action has been described as undulating, alternate, coming and going, like the motion of a ship; but this is regulated by the respiratory movements and different attitudes of the body. We can, however, at the moment of the reascent, perceive a flank movement, deep inspiration, succeeded by a rapid expiration, showing conclusively that a powerful nervous concurrent force (involuntary) controls the action of rumination.

Finally, the cud can be made to ascend or descend, in the following manner: We perceive the cud descend; now grasp the gullet firmly, and it re-ascends into the mouth. We next perceive the cud ascending; arrest it by compressing the gullet, and it rapidly descends again into the stomach; hence the phenomenon of remastication can readily be demonstrated.

The solid food, when once in the paunch, receives the admixture of fluid secreted from its walls. After maceration for a short time, the more solid parts are returned to the mouth, where they undergo another mastication, and are again saturated with the salivial fluids and swallowed. If properly masticated it reaches the third stomach (manyplus or omasum). Here it undergoes a further reduction, becomes quite pulpy, after which it enters the fourth stomach.

CARPENTER thus describes the phenomena of rumination: "The direction of the food into one or the other of the digestive cavities, appears to be affected without any voluntary effort on the part of the animal itself, but to result simply from the very peculiar endowments of the lower part of the cesophagus. This does not entirely terminate at its opening into the first stomach or paunch, but it is continued onward as a deep groove with two 
lips. By the closure of these lips it is made to form a tube, which serves to convey the food onward into the third stomach; but when they separate, the food is allowed to pass either into the first or second stomach. When the food is first swallowed, it has undergone but very little mastication; it is, consequently, firm in consistence, and is brought down to the termination of the œesophagus in dry bulky masses. These separate the lips of the groove or demi-canal, and pass into the first and second stomachs. After they have been macerated in the fluids of these cavities, they are returned to the mouth by a reverse peristaltic action of the cesophagus. This return takes place in a very regular manner, the food being shaped into globular pellets by compression within a sort of mold formed by the ends of the demi-canal, drawn together, and these being conveyed to the mouth at regular intervals, apparently by a rhythmical movement of the œesophagus. After its second mastication, it is again swallowed in a pulpy semi-fluid state. It now passes along the groove which forms the continuation of the esophagus, without opening its lips, and is thus conveyed into the third stomach, whence it passes to the fourth."

\section{Bloat, Hoven, or Tympanites.}

The term "bloat" has long been discarded by veterinarians as an indefinite term, signifying a state of turgescence, dilation, inflation, or puffiness, which is merely indicative of changes in the form and condition of parts, without regard to the actual seat or nature of the difficulty. For example, a horse is bloated when he becomes the subject of subcellular emphysema (distension of the cellular membrane beneath the skin with gas), or odema (dropsical tumefaction), etc. However, as every farmer appears to be somewhat conversant with the condition of the animal known as "bloat," or "hoven," we shall not offer any remarks calculated to mystify him, but merely suggest that the term tympanites be substituted for "bloat." Tympanites intestinalis signifies a distension of the intestines with wind or gas, accompanied by an elastic distension of the abdomen. The latter, when struck or sounded by a blow, sounds like a drum, and indicates a windy distension of the abdominal viscera (a bowel or organ within the body), commonly known as flatulent colic. Tympanites rumenites 
signifies distension of the rumen in the bovine species-the ox and cow-and, in the phraseology of the grazier, is known as bloat or hoven.

Causes.-The direct cause of flatulency and windy distension is imperfect digestion. In such cases the food, instead of undergoing the normal process of digestion, whereby it is converted into chyme and chyle, ferments and evolves gases, either carbonic acid, or sulphureted hydrogen, and, as "a little leaven leavens the whole loaf," so the fermentation, once commenced in the stomach, goes on until the food is in a state of putrefaction, or up to the period when all its gaseous material has been extracted. Before this takes place, it frequently happens that the animal dies, either by rupture of the rumen or some portion of the abdominal viscera. In some cases, unrelieved, the distension is so great that the animal dies in a state of suffocation, occasioned by the pressure on the diaphragm, and other important parts and organs.

Imperfect indigestion may be occasioned by a deranged condition of the digestive organs, induced by various causes, such as give rise to the same phenomena in man, namely, errors in diet, or sudden changes of the same. Thus, if stall-fed animals be turned into a field of elover, or into a luxuriant pasture, they not only eat greedily, and create an undue distension of the stomach, but they partake of food containing a large amount of aqueous matter, which, every one knows, is more indigestible than dry food; and such a sudden change of diet is not always to be tolerated. There can be no dispute about the causes of bloat, hoven, or tympanites. It evidently is occasioned by imperfect digestion. As a general proposition, therefore, we may contend that all indigestible matter may, directly or indirectly, produce a tympany of the abdominal viscera ; and we may also contend that an animal may occasionally become tympanitic, under the most intelligent management, owing to some inherent idiosyncrasy in the local organs, honestly inherited from sire or dam, or their ancestors. Hence, the reader will infer that this dyspepsia, or indigestion, is, like various other diseases which seem to appear without any direct cause, transmissible, not always directly, but by predisposition to this and other maladies, which is said " to lurk in breed and conformation," over which we have but little control other than palliative.

Treatment.-Supposing the abdomen to be distended to its utmost capacity by the extricated gas, and the animal is oppressed 
and distressed in the act of breathing, there is no time to be lost. It is useless to resort to drug medication. The case is imminent. The gas must be evacuated immediately, and we therefore puncture the flank on the left side, in its most salient region, by means of the trocar and canula (an instrument somewhat similar to that used for tapping the chest). Immediate escape of the gas is the result, and the patient is soon relieved. Now we may resort to medication, and that medicine is the best which is calculated to arouse the action of the stomach and arrest fermentation. With these objects in view, I recommend the following:

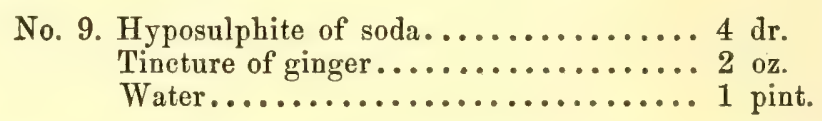

Dissolve the hyposulphite in the water, and then add the tincture of ginger. Drench the animal with the same. If the tincture of ginger can not be obtained, then substitute four drachms of the pulverized root. If the case be curable, the above treatment is almost sure to afford relief. The medicine, however, may be repeated at the end of four hours, if necessary.

Remarks on the introduction of the Trocar.-Having ascertained that the animal is in a dangerous condition, owing to the great quantity of gas present within the rumen, the most prominent point of the left flank should then be selected. Here make an incision through the integument, sufficiently large to admit the instrument. Then draw the skin upward, and puncture the abdomen; in this way we make an indirect opening, so that, when the trocar is withdrawn, the integument covers the orifice made last. The trocar must be kept very sharp or keen, so that it may, without using much force, penetrate the peritoneum, and, lastly, the rumen. Once within the latter, all resistance ceases. The trocar is now withdrawn, and the canula remains, for the passage of the gas. In bad eases, the moment the cutting instrument is withdrawn from its sheath, the gas will escape, with a noise resembling a steam-whistle, which conveys to us the idea that we are in the presence of a living locomotive, issuing a blast of warning to keep out of smelling distance, for ofttimes the odor is intolerable.

It is best to let the tube remain in the stomach or paunch until the abdomen is reduced to about its natural size. The instrument must occasionally be drawn forth a little, or pushed forward, as 
the case requires; and when it becomes obstructed with any portion of the contents of the stomach, a quill or straw may be used to clear the obstruction, and, as the gas escapes and the paunch or bowels recede, the canula, which is about six inches in length, must be pushed forward as far as it will go.

Relief may sometimes be obtained by passing the probang into the stomach, and I should advise its use in such cases as those attended by eructation of wind by the mouth from the stomach.

\section{Distension of the Rumen with Food.}

It occasionally happens that the function of the stomach, as a whole or a part of the same, become impaired. The food is then very apt to accumulate in the rumen. Or it may happen that an animal has partaken voraciously of meal or corn, which, becoming saturated with the fluid found in the paunch, swells to such an extent that there is danger of its bursting.

Symptoms.-At first the animal is noticed to be uneasy, and frequently shifts its position; occasionally moans; the left flank is swollen and hard. This swelling may be determined by a person taking a position directly behind the animal ; he will immediately perceive the enlargement. On striking the part with the hand, it has no drum-like (tympanitic) sound, as in hoven or bloat, but has a solid sound, showing that the distention is owing to the presence of a quantity of solid food. Should the medicinal preparations fiil to relieve the animal, rumination then ceases, the symptoms become aggravated, the brain sympathizes, unconsciousness and convulsions occur, which soon end in death.

Treatment.-In cases of extreme distension, it is all folly to waste time in administering medicine. An incision, about five inches in length, should be made through the left flank into the stomach. Then, by means of the hand, the indigestible mass is to be removed. This should be done carefully, so as to prevent the food falling into the abdominal cavity. The incision made into the rumen is then to be stitched, or sutured; and, lastly, the integuments are brought together in the same way. It may be proper to apply a little tincture of matico or tincture of aloes to the wound. Having finished the operation, the next object is to arouse the action of the stomach, for which purpose I recommend the following: 
No. 10. Powdered golden seal.............. $3 \mathrm{dr}$. Hyposulphite of soda.............4 dr. Powdered ginger................. $2 \mathrm{dr}$. Warm water.................. 1 pint. Mix.

Drench from a bottle. The patient had better be kept hungry the first twenty-four hours after the operation; then an occasional bran-mash, well seasoned with table salt, may be allowed.

Many cases of very considerable severity have been relieved by the above treatment. There is very little danger attending an operation of this kind, for the rumen is not very highly organized with either blood-vessels or nerves. I have known several instances in which this operation has been performed in the rudest possible manner, with a common jackknife, and yet the animals operated on recovered. The intelligent husbandman, however, will, if possible, secure the services of a surgeon for the performance of all operations requiring skill and good judgment.

Removal of the Sutures, or Stitches.-The incision into the rumen having been secured by very fine yet.strong thread, and the ends cut off, needs none of our attention. The external sutures, however, will have to be removed in the course of a week or ten days. We merely cut the knot and withdraw the suture.

\section{Gastro-intestinal Inflammation.}

Gastro-intestinal inflammation of the stomach and bowels is frequently occasioned by the presence of concretions and hair-balls, or some other foreign bodies. There are many plants, such as hemlock, crowfoot, henbane, wild poppy, ete., which act as poisons, and induce an inflammatory condition of the stomach and intestines. An animal may feed on substances which are too dry and fibrous. They accumulate and distend the stomach beyond its normal capacity. Or the manyplus may contract spasmodically and imprison the food. In either case an inflammatory condition is the result. The treatment of a disease of this character is very unsatisfactory, and it is very difficult to write out any directions that shall meet the emergencies in cases of the above character.

The following case, communicated for the "Veterinarian" by Surgeon REDwood, will give the reader a better idea of this malady than the author can furnish, his experience being rather limited in treatment of this affection: 
"I have forwarded to you a jar containing portions of the abomasum of a cow that I have had slaughtered, seeing that further treatment was altogether useless. You will perceive $a^{a}$ large gangrenous spot on the inner coat of the above viscus, which I consider was the cause of the symptoms presented. I have also sent you portions of the ilium and jejunum, which will afford you some idea of the intensity of the spasmodic action with which these intestines were affected. The whole of the small intestines presented the most marked spasmodic condition; in fact they were like so many muscular cylindrical cords, and impervious to any body larger than that of an ordinary-sized goose-quill.

Now, all the diseased conditions are enumerated in the above brief description. Every other organ-except the liver, which had a little deposit of earthy matter in a few of its larger biliary tubes, a very common condition of the gland-both in the thorax and the abdomen, presented the most healthy aspect. The animal was ill but thirty-six hours.

The following were the symptoms observed and the treatment adopted: The patient was a dairy cow, five years old, in excellent condition, living on straw alone for the last fourteen days, five months advanced in pregnancy, and never had been ill before, being bred on the farm. When I first visited her she evinced all the symptoms of hoven, and that, to a great extent, so much so, that at one time I was about to introduce the trocar, for the purpose of affording relief; yet, knowing the animal could not have had access to succulent diet, I came to the conclusion that this was the effect of the chemical laws acting on vegetable matter, over which the stomachs had, in some degree, lost their vital influence. The poor animal was in great agony, shown by loud groaning, though rarely lying down; eyes, sunk in their orbits; pulse, quick and irritable, but not such as to indicate or warrant depletion. Occasionally a quantity of the fluid contents of the rumen were regurgitated through the nose and mouth, almost, it would appear, involuntarily. She also, at intervals, voided a small quantity of commingled aqueous and mucous fluid per anum, although, as before remarked, from the moment she was observed to be ill, not the smallest portion of ingesta passed the abomasum, which, together with all the other stomachs, was partially filled with food in a pultaceous state. It appeared to me evident that the obstruction to the passage was caused by spas- 
modic action of the pyloric orifice, the action extending throughout the greater portion or all of the small intestines.

Will you favor me with your opinion as to the cause of the circumscribed and intense inflammation of the villous coat of the true stomach, and say if you think it arose from any chemical irritant?

The treatment consisted in the exhibition of spirits of ammonia aromatic with the carbonate; aperients, and at last the chlorides; but all proved inert, no benefit whatever accruing therefrom. Had I suspected spasm to such a marked extent, I should certainly have given large doses of extract belladonna and hyosciami, both in the form of enema and by the mouth. As it was, my treatment was directed solely to the symptoms evinced; and when I found that all the usual remedies were of no avail, as the animal was in high condition, I thought it best to have her destroyed.

[The lining membrane of that portion of the alimentary canal forwarded by Mr. Redwood was, throughout, in a state of congestion, presenting here and there depressed or cup-like patches, which apparently resulted from ulceration, commeneing in the submucous tissue. The ulceration had also penetrated, in spots, the substance of the mucous membrane. This condition of parts is somewhat singular, and might have had its origin in the existence of some local irritant.]"

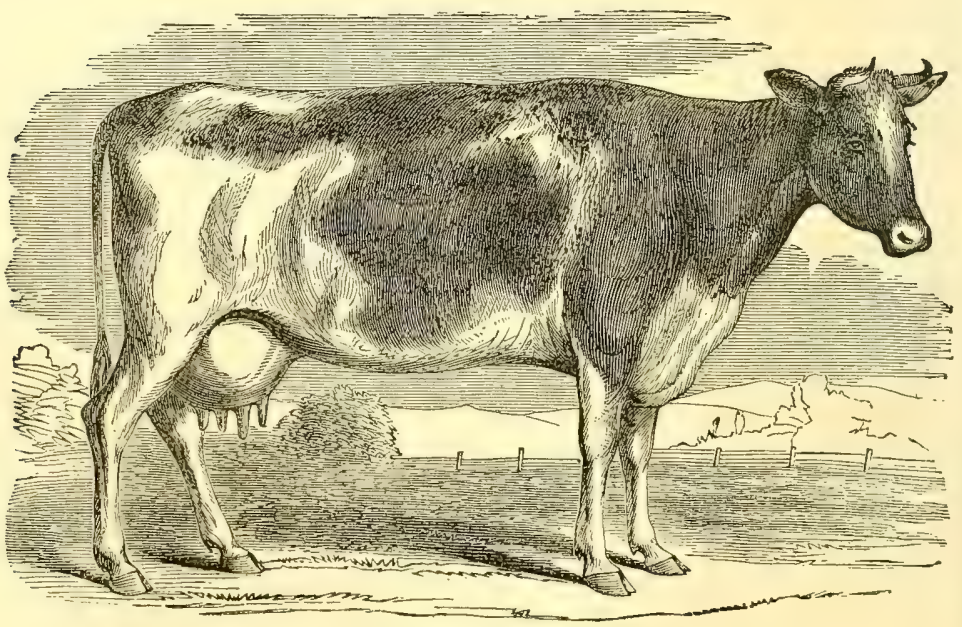

IMPORTED DUTCI COW PURMER.

[Imported by W. W. Chenery, of Belmont, Mass.] 


\section{Dentition of Cattle.}

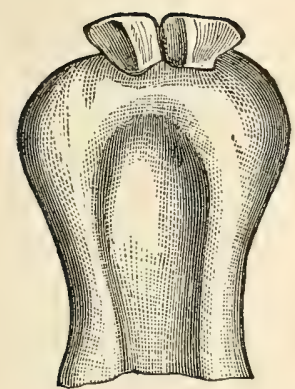

APPEARANCE OF THE TEMPORARY INCISORS $\triangle T$ BIRTH.

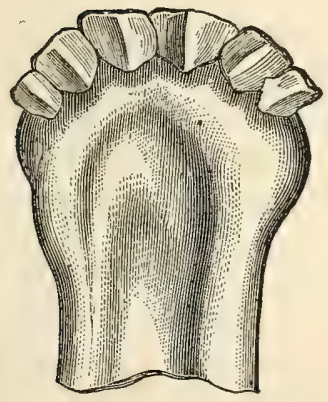

APPEARANCE OF THE TEMPORART INCISORS ABOUT TWO WEEKS AFTER BIRTH.

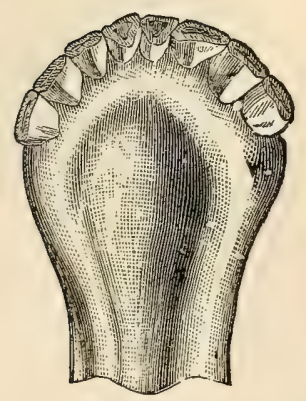

APPEARANCE OF THE TEMPORART INCISORS AT THE AGE OF EIGHT OB NINE MONTHS.

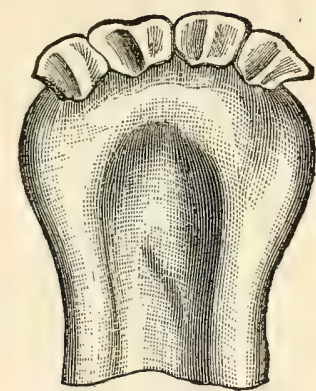

APPEARANCE OF THE TEMPORARY INCISORS ABOUT ONE WEEK AFTER BIRTH.

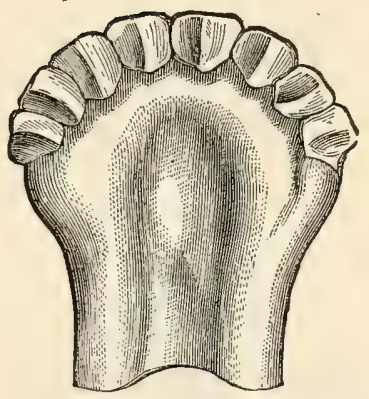

APPEARANCE OF THE TEMPORARY INCISORS ABOUT THEEE WEEKS OB $\triangle$ MONTH AFTER BIRTH.

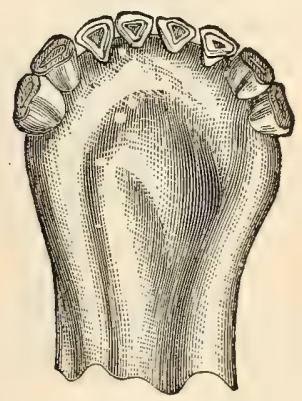

APPEARANCE OF THE TEMPORART INCISORS AT THE AGE OF TEN OR ELEVEN MONTHS. 


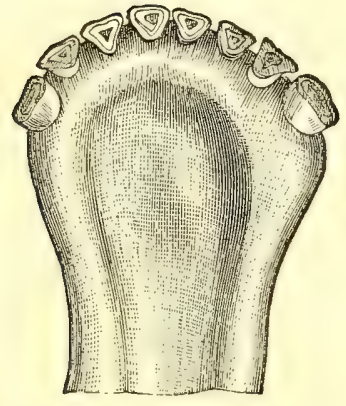

APPEARANCE OF THE TEMPORART INCISORS AT THE AGE OF FIFTEEN OR SIXTEEN MONTUS.

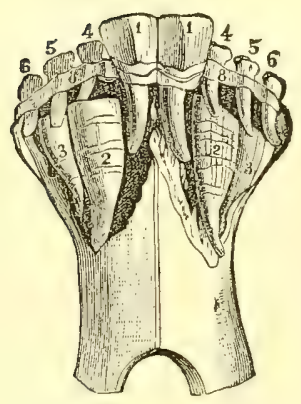

APPEARANCE OF THE TEMPORARY AND PERMANENT INCISORS AT THE AGE OF ABOUT TWO YEARS.

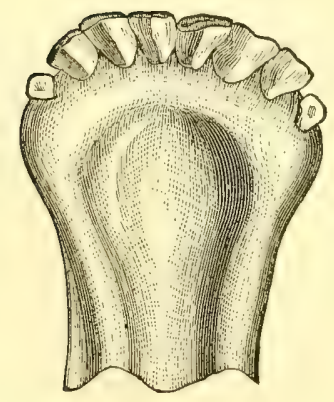

APPEARANCE OF SIX PERMANENT INCISORS AND TWO TEMPORARY AT THE $\triangle G E$ OF FOUR.

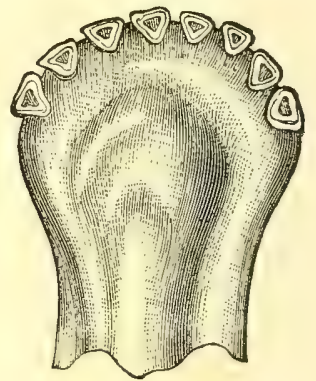

APPEARANCE OF THE TEMPORART INCrSORS AT THE AGE OF EIGHTEEN OB NINETEEN MONTHS.

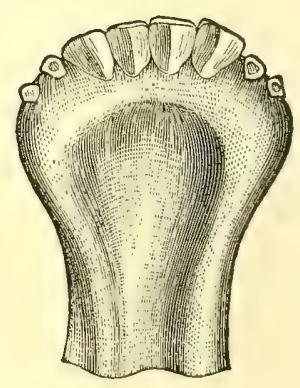

APPEARANCE OF THE PERMANENT AND TEMPORARY TEETH AT THE AGE OF THREE YEARS.

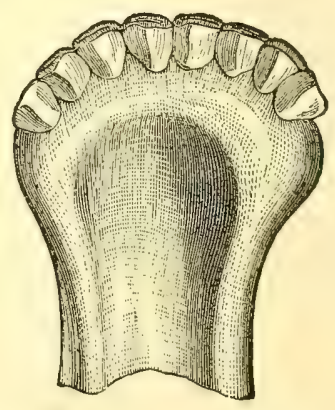

APPEARANCE OF ALL THE PERMANENT TEETII AT THE AGE OF FIVE. 


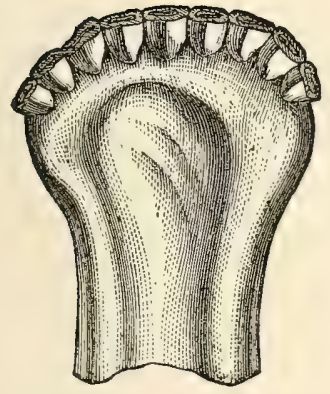

APPEARANCE OF THE PERMANENP TEETI AT THE AGE OF ABOUT BIX YEABS.

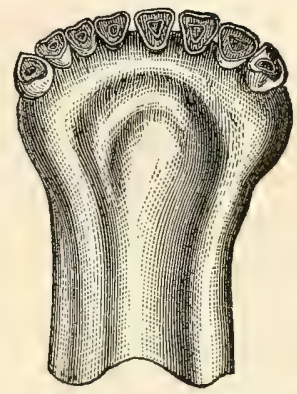

APPEARANCE OF THE PERMANENT TEETH AT THE AGE OF ABOUT TEN YEABS.

\section{Synopsis of Dentition.}

INCISORS.

Temporary incisors all prominent at the end of one month.

The two central permanent incisors appear in from twenty to twenty-four months.

The two inner middle permanent incisors appear at about the age of three years.

The two outer middle permanent incisors appear at about the age of four years.

The corner permanent incisors appear between the ages of five and six years.

\section{MOLARS, OR GRINDERS.}

Temporary molars, three in each jaw, above and below, on both sides, are prominent at birth, or within four weeks of that period.

Fourth temporary molars are cut at the age of six months.

Fifth temporary molars are cut at the age of fifteen months.

Sixth temporary molars are cut at the age of from two years to thirty months.

The first and second permanent molars are cut at the age of about two years.

The third molar is cut at about the age of three; the fourth appears at the age of four; the fifth at the age of five; and the sixth at the age of six. 


\section{Suspended Rumination, or Loss of Cud.}

Some people, who are in the habit of prescribing for loss of eud, more properly termed "cessation of rumination," suppose that if they can only restore the lost function the trouble will end. This is certainly very desirable, for an improvement in the appetite of sick animals is a sure sign of rapid recovery; yet a restoration of the cud alone will not always insure a cure, neither are the remedies prescribed by some calculated to accomplish this object. One man reccommends a red herring to be thrust down the throat; another a portion of the quid, or cud, of a healthy cow. Others recommend raw beef, pork, pepper, etc. This results from mistaking symptoms for disease; for loss of cud is nothing more than a symptom of deranged digestive function, or that of other organs sympathetically associated with it. Those who have the care of cattle, and prescribe for them when sick, are not supposed to be able to trace loss of cud to derangement of one or a class of organs, unless they shall have had the advantages of a medical education, which is not often the case. They are not acquainted with the various sympathetic relations that exist in the animal economy, neither can they understand why an abnormal condition of one organ produces a corresponding effect in one or a class of organs remotely situated; nevertheless, such are the facts. Many a poor cow has been thus forced to swallow down a red herring (bones and all) or "another cow's quid" (not of tobacco, for none other than two-legged animals chew quids of this description), and we can readily conceive that such articles may, for the time being, arouse the digestive organs, and create an unnatural appetite in animals of such refined taste and nice discrimination as the cow. A red herring, either whole or comminuted by the grinders, and then swallowed, only creates irritation on the mucous surfaces of the various compartments of the stomach; an unusual abundance of blood flows into the walls of that organ, the circulation of that fluid is quickened, the gastric juice flows more readily, and the digestive function is exalted. A repetition of the practice enfeebles the power of the stomach; its action is quickened at expenditure of power, for all unnatural excitement of a natural function is followed by a corresponding depression. A stimulant-and red herring may be classed as such-creates a desire for food; but then the animal may be laboring under an acute disease of some organ, 
when food would be inadmissible; or the stomach may be overburdened and unable to digest what is already there, and, therefore, requires rest, as any other organ would after long-continued action.

The stomach is one of the most important organs, and performs some of the most delicate operations in the animal economy. Its functions may be suspended from various causes, and it is the province of the practitioner to learn and understand the why and wherefore of its derangement. In many cases the stomach craves no food, because it can not digest it; and merely creating an appetite by stimulants is worse than useless, for whatever is then eaten can not be converted into chyme, nor can the lacteals take it up and apply it to the purposes of nutrition. Whenever an animal is suffering from disease, pain, or excitement, there will often be absence of appetite (loss of cud), and this function is suspended because the animal is drawn from it by his sufferings.

The real seat of sensation of hunger is in the brain, not in the stomach alone, as some suppose. The latter may first communicate some such sensation to the brain, yet if that organ be in a deranged condition, unable to recognize the want, then we must impart healthy action to it, and establish an equilibrium between the nervous and general system, in order to produce a natural appetite. Do you wish to know how to do this? If so, study the veterinary art. That the appetite is affected by the state of health, both of the body and mind, is certain. Human practitioners realize that. "In fever, pain, and in certain dyspeptic states, the stomach craves little or no food. So in mental distress, in times of great fear, or sorrow, or extreme anxiety, the appetite fails. Even in a single moment the appetite may be suspended by any sudden mental affection or emotion." Animals are known to manifest mental emotion when separated from their offspring, or from those with whom they have been accustomed to work or associate; and many cases are on record showing that animals, when deprived of the society of their fellows, refuse their food, and die of grief, so that, in this particular, the brute does not differ from his master. Is it not a matter of importance, then, to ascertain the cause of loss of cud (appetite) before we prescribe red herrings, etc.?

"Loss of cud," says Mr. YouATT, "is more a symptom of disease than a disease of itself. It accompanies most inflammatory complaints, and is often connected with those of debility. It will 
be the duty of the practitioner to ascertain the cause of this suspension of second mastication, and to adapt his mode of treatment to the nature of that cause. A dose of physic, with a small portion of aromatic medicine, will be indicated if any fever ean be detected. More than the usual quantity of aromatic should be added in the absence of fever, and still more, with tonic and alterative medicine, if general debility is indicated. The caraway and ginger powder are the best aromaties, and will supersede every other. The gentian and ginger, with Epsom salts, will prove a very useful tonic and alterative in cases of loss of cud that can not be traced to any particular diseased state of the animal."

A word to the farmer, and I have done. If you have permitted your animals to subsist on innutritious diet, so that their digestive powers have been overtaxed, and that function is paralyzed, then the blame rests with you. On the other hand, have you been preparing them for market-piling on the fat, at the same time depriving them of pure air and exercise? If so, you are equally blamable. Is your barn and dairy management consistent with the received opinion of those who are the best judges in these matters? If not, make them so, and, depend upon it, your cattle will thrive and be blessed with a natural appetite; for disease, in nine cases out of ten, results from violating the laws of Nature.

\section{INFLammation of the Bowels (ENTERITIS).}

Inflammation of the bowels, occurring in any of the divisions of the intestines, is one of frequent occurrence; yet among cattle it is not liable to become so suddenly fatal as among horses.

Symptoms.-This disease bears some analogy to colic, in the suddenness of its attack. A healthy animal is all at once attacked with abdominal pain; gets down, and moans from incessant or persistent pain ; The pulse generally ranges from 60 to 70 ; rumination has ceased, and the feces is hard, covered with slime or streaked with blood; the flanks heave (as the saying is); the limbs are tremulous, and the animal has a staggering gait; the visible surfaces of the eye, mouth, and nostrils are reddened; pressure upon the abdominal region (which is generally tucked up), elicits symptoms of pain. As the disease proceeds, these symptoms are aggravated, and intestinal hemorrhage or mortification 
terminates the sufferings of the animal. The post mortem examination generally reveals engorgement of the blood-vessels of the intestines, with highly-carbonized and coagulated blood; the lining membrane is of a dark velvet color, and the large intestines are, more or less, inflamed or gangrenous.

Treatment.-The principal objects in the treatment of this disease are, to lubricate the interior of the stomach and intestines with mucilage of slippery elm, and to equalize the circulation and nervous action over the general system. The remedies are as follows:

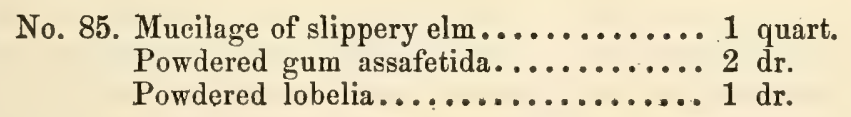

The above dose may be repeated at the expiration of eight hours. In the mean time, apply a mustard poultice to a part of the abdomen, and excite the circulation on the surface of the body and extremities by friction with wisps of straw. An occasional antispasmodic injection should be thrown into the rectum. Two quarts of hot water to one ounce of powdered lobelia are about the proper proportions. Should the patient appear to suffer from distension of the intestines with gas, then give one ounce of hyposulphite of soda, two drachms of powdered golden seal, and one pint of water.

Animals in the above condition can not bear cathartics, yet cases now and then occur which require a gentle aperient, merely to aid in the removal of fecal accumulations. When such remedy is indicated, I recommend the following, to be used as a drench :

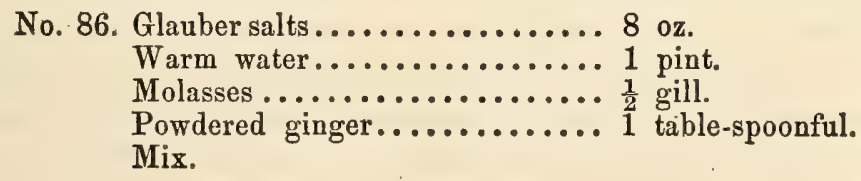

\section{Invagination of INTESTiNes.}

Invagination of intestine signifies a portion of intestine drawn within another, there becoming strangulated. This difficulty does occasionally occur, and the symptoms do not differ materially from those attending strangulation occasioned by the cord, or vas deferens. 
Symptoms.-The animal passes nothing from the rectum but a small quantity of blood; suffers incessant pain; is very uneasy, and rumination is suspended; he will occasionally anxiously regard the flanks and moan. This trouble is generally preceded by flatulency.

Treatment.-The best plan of relieving the animal is to etherize him, for it often happens that, during etherization, a relaxation of the muscular tissues takes place, and such condition is favorable to the return of the bowel. It may be proper, while the animal is down, and under the influence of ether, to introduce a hand into the rectum and try what can be done by manipulation and traction. - The small intestines, in which the difficulty occurs, can be seized through the wall of the rectum, and, perhaps, by some slight traction or movement, the difficulty may be remedied. It is impossible to tell what is the result of our efforts until the animal is relieved, and has had time to recover from the effects of the ether. This having taken place, without improvement, I should immediately perform the right-flank operation, search for the seat of the difficulty, and reduce the invagination.

This operation may appear to some as a very formidable affairvery dangerous and painful; but a skillful surgeon, armed with the great Godsend, ether, can perform the same without the least danger. I have opened the abdominal cavity several times, in cases of sterility, merely to explore the uterus and ovaries, and never met with any accident; and to encourage those who have never had any experience in the performance of this operation, I relate the following incident: I was requested, a short time ago, to visit a very valuable Durham cow, the property of Mr. G. Clarke, of Hyde Hall, Otsego County, N. Y., which had been purchased of Lord Ducie, accompanied by a warranty of pregnancy by one of the best bulls in England. Time revealed the fact that she was not pregnant. She had afterward several connections with the opposite sex, yet could not be impregnated. The object in securing my services was to solve the problem of her sterility. Now comes the pith of the matter. I cast her and explored the vagina. It appeared that there was a sort of hour-glass contraction in the vagina, so that the hand could not be introduced more than six inches. At this point there appeared to exist a mechanical obstruction to further penetration. This was sufficient evidence of incapacity of the sexual congress, yet, having traveled a long 
distance, at great expense to the owner, and there being some prospect of litigation in the matter, I was determined to remove all doubts. I therefore performed the flank operation, and explored the uterus and ovaries. The whole were in a state of atrophy, except the right ovary, which was as large as the kidney of an ox. Being desirous of ascertaining what the character of the enlargement was, I put my thumb and finger into it. By so doing I ruptured a vessel, which bled very profusely. My immediate impressions were that the animal must die of internal hemorrhage. It was a matter of impossibility to secure the bleeding vessel, so I sutured the incision, expecting that the animal would die in the course of a few hours; but, contrary to my expectations she got well.

\section{Diarrhea.}

Diarrhea consists of an increased number of lower-bowel discharges, augmented peristaltic motion of the bowels, irritability of the same, and a too rapid propulsion of the secretions. Diarrhea is sometimes salutary-an effort of Nature to rid the system of morbific matter. It frequently occurs at the commencement of various forms of disease, and is scarcely, if ever, an isolated affection. GALLUP, in his "Institutes of Medicine," contends that the muco-intestinal discharges are portions of the centrifugal circulations, which probably have their functions accelerated by the force of the heart and arteries, above that of the centripetal series. A fluid, quite similar to the cold sweat of the skin, and by a similar process, is forced out in abundance, and produces the diarrhea.

A moderate diarrhea, occurring in a plethoric animal, is often salutary ; for it relieves the relative plethora of the vascular system, which occurs at the onset of acute disease. When the discharges are of a yellow or greenish color, there is, generally, functional derangement of the liver. When an abundance of mucous material is observed, it goes to show that the mucous membrane is congested. Sometimes an excess of serous fluid is observed. This occurs more particularly in dropsical affections. There is also an alvinous variety of diarrhea, caused by an abnormal secretion or exudation of coagulable lymph. It frequently comes away in shreds, or detached pieces, from various portions of the intestinal canal. 
Treatment.-Almost any form of this affection, except the chronic kind, can be checked, and perhaps cured, by administering a few doses of charcoal and lime-water. I use these agents in the following proportions:

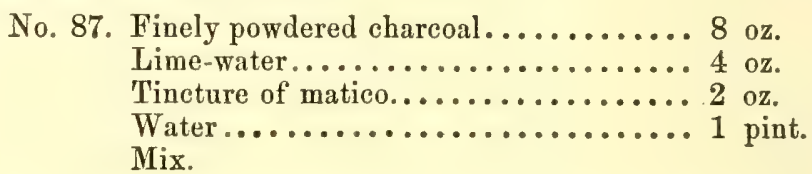

One-fourth of the above is a dose for an ox or cow, which may be repeated every four hours. The same remedy answers for calves, only they require a smaller quantity. The astringent properties of the above mixture can be augmented by increasing the quantity of matico, or by adding a small quantity of powdered bayberry bark.

In all cases of diarrhea, I usually order a change of diet; for, in some cases, the food is operative in producing the difficulty. If it occur while at grass, the animal should be taken to the barn for a short time, and allowed a few moderate meals of ground oatmeal. When sucking calves are under treatment for diarrhea, the mother should also have a few doses of the above remedy; for it often happens that she is ailing at the time, and her milk has a morbid tendency on the calf. In the advanced stages, when the fecal discharges emit a bad odor, and rumination is suspended, the patient should have a few quarts of milk porridge per day. Scalded milk alone will sustain and benefit the calf.

\section{Chronic Diarrhea.}

Chronic diarrhea is usually accompanied by loss of condition ; the coat stares, and, although the animal is allowed the very best kind of food, still it thrives not; the skin and ribs seem to have entered into permanent relationship. In a case of this character, I recommend the following:

No. 88. Phosphate of lime.............6 oz.

Powdered ginger................ 3 oz.

Powdered charcoal.............. 4 oz.

Powdered golden seal............. 2 oz.

Powdered bayberry bark.......... 1 oz.

Mix.

Divide the mass into eight equal parts, and mix one of them 
in the food night and morning. This will generally have the desired effect. Should it fail, I would increase the quantity of bayberry bark to three ounces.

\section{GUT-TIE.}

It would seem that an apology is due the reader from the author for introducing such an apparently vulgar term as the above; but the fact is, this term has a meaning-perhaps better understood than any other that can be introduced at the present time. It would appear that "gut-tie" is often occasioned by a faulty method of castration, or "altering." This is the testimony of experts; but it is my opinion that gut-tie often occurs more accidentally than otherwise, for animals have been known to die of gut-tie after being castrated in the best possible manner. I once operated on an animal for this mechanical trouble, and am satisfied that the castrator was as well qualified to perform the operation as the best of the craft; yet the cord had retracted into the abdomen, formed a false tissue, and had also a large bulbous extremity, which no skill or foresight could have possibly guarded against.

Symptoms.-The animal operated on presented the following symptoms: Pulse, small and quick ; respirations, hurried ; animal very uneasy (all the time), down and up again very often; rectum, empty, yet coated with slime; the patient refused both food and water, and had not ruminated nor passed any feces for several hours.

Treatment.-I proposed an operation, with the intention of exploring the abdominal cavity, to which the owner consented. The patient was cast on the near or left side, and etherized. An incision, five inches in length, was then made through the integuments of the right flank, midway between the last rib and anterior part of the ilium, about three inches below the transverse spines of the lumbar vertebra. The muscles and peritoneum were also divided in the same manner as when spaying a cow. After introducing my hand, I found a portion of intestine incarcerated by the remains of the spermatic cord, which seemed, together with sôme false membrane, to have entangled the intestine. After liberating the latter, the divided muscles were closed by suture, one end of which was left long enough to hang out of the wound. The integuments were then sutured, and dressed with tincture of aloes. 
The patient staggered a little when he got up, but soon commenced eating. I did not see him afterward, but, a fortnight from the time of the operation, was informed that the animal experienced immediate relief from the difficulty, and was then well.

Up to the present time there appears to be no information afloat in this country regarding the cause and remedy of this singular difficulty; hence, I presume that some reliable account from other sources may be acceptable to the reader of this work. The following account of "gut-tie" is from the pen of Surgeon CRowHURST, published in the "London Veterinarian :"

"I was requested on the 15 th of last month, to attend a twoyear old steer, the property of W. W. Daws, Esq., of Ewhurst, which was taken ill the day previously; but, it being late when the illness was observed, and my residence nine miles distant, some aperient medicine was administered, and orders given to send for me the next morning. When I arrived, the steer was lying down in a shed, but soon got up and walked across the yard. His back was arched, and the abdomen tucked up. He was somewhat excited at first, but soon began to tremble, and to step in a backward direction, by putting one hind leg directly behind the other. Occasionally he turned his head toward his side, and would kick at his belly, and, now and then, stretch himself out, and curve his loins to a considerable extent. After being in the yard for a short time, he returned into the shed and lay down again, doing this in a very careful manner. He soon got up a second time, and stepped backward as before, seemingly for the purpose of getting his hind-quarters against some resisting body. The man in charge of the animal explained that the symptons had undergone but very little change since he was taken ill, and that he had not eaten any thing nor voided any feces. There were, however, several evacuations of mucus lying about the yard, which had been expelled from the bowels.

Having procured a wagon-rope, and obtained the assistance of two or three men, I had the animal cast, and examined him per rectum. There were no feces present, but, on passing my hand onward, I readily detected a band, which was drawn tightly round the bowel. It appeared to pass from behind forward, and undêr the rectum. By pulling at this the animal struggled from pain. Finding this state of things, I at once decided upon operating, as I could not see the patient any more that day, having many press- 
ing professional engagements. I first cut off the hair from the right side, which I prefer to do while the animal is standing, that I may know better where to commence my incision when he is cast. I will here describe my plan of casting the animal, that, should any person have a preferable one, I may put it in requisition in my after-proceedings. I first fastened one end of a rope to the off fore-leg, then passed it in front and around the near fore-leg, and afterward under the part of the rope between the legs, so as to pull them close together. Next, it was carried between the hind legs and round the near one, and then under that part which passed from the fore to the hind legs. By then bringing the end of the rope over the bullock's back, I was enabled to throw him on his near side, and prevent his rising by drawing the near hind leg forward, and fixing it between the fore-legs. As the off hind leg was still at liberty, it was secured by drawing it backward, and fastening it by another rope to a crowbar fixed in the ground. A man was then placed on the animal's neck, to keep his head down, and prevent his rising. Every thing being ready, I commenced the operation by cutting through the common integument and abdominal muscles, midway between the antero-inferior spinous process of the ilium and the last rib, a short distance below the transverse processes of the lumbar vertebra, so as to avoid the arteries of the part. The peritoneum was then broken through with the fingers, and afterward the left hand was introduced into the abdomen, in a direction toward the pelvis. I experienced no difficulty in finding the band, which consisted of the vas deferens, and which formed a semicircular projection in the abdomen from the intestines passing behind it, or from below upward, as the steer lay on his side. It was drawn quite tight, and had produced strangulation of the intestines. I was soon enabled to liberate the intestines, after which I drew the vas deferens toward the opening in the muscles and divided it, excising a portion to the extent of about three or four inches. The part cut through retracted, and was left floating free in the abdomen. The operation was completed by passing some interrupted sutures of wide tape through the common integument only. After the animal was released, I gave him some aperient medicine, and ordered gruel to be administered at regular intervals.

Before I was a student at the college, I attended a post mortem 
examination of an old Welch runt, which my father ordered to be killed, as the proprietor neglected sending soon enough for him to be of any service. In this case there were about six inches of the small intestines strangulated, and quite black in color. The question arises, Is this affection produced from any particular mode of castration? My father has castrated animals in the same way as taught him by his father for forty-five years, and has never had one case among those he has operated upon, although he has been called to several which had been operated upon by other persons.

The calf which I have alluded to was not castrated by him, nor was the two-year old steer, (the subject of this communication,) by either of us, which tends to prove that castration has to do with its production, these cases having occurred in our district. I had, until recently, supposed that the spermatic artery, on being drawn at until it ruptures, in the operation of castration, might recede into the abdomen, and, hanging loose, afterward strangulate the intestines by getting around them, but I feel convinced this is not the case."

The following, which lately appeared in the Edinburgh "Veterinary Review," is offered for the reader's instruction. It is a translation from GIERER, by Mr. GAMGEE:

\section{"ON the Internal Ruptures in Oxen.}

"Gierer alludes to the fact that all authors agree as to the nature of the internal or peritoneal ruptures termed 'ueber-wurf,' by the Germans-' gut-tie' by the English. There is a separation of the atrophied spermatic cord from the sides of the pelvis, and, under peculiar circumstances, the peritoneum is lacerated; a portion of intestine slips downward and backward, and the cord is entwined round it, so as to constrict it, obstruct the passage of excrement, and inflammation, with other consequences, result. Gierer especially describes his method of discovering the seat of the constriction, and his plan of operating. He says that it is not always so easy to find the spermatic cord, and to discover precisely which intestine is incarcerated. Most cases occur in oxen from a year and a half to four years old. When symptoms of colic, and no discharge of feces induce him to suspect 'gut-tie,' he examines per rectum, and, by careful manipulation, can always 
detect the spermatic cord and imprisoned intestine. Gierer has never seen this strangulation relieved spontaneously; and there are two methods of cure to adopt-the one palliative, and the other radical. The first consists in the return of the intestine through the fissure in the spermatic cord, by quickly driving an ox or raising his hind-quarters in a stall. This should be tried soon after the first appearance of the disease. When this does not answer, the normal condition of the parts must be restored by the taxis effected through the rectum.

Very often there may be a relapse, from the loose spermatic cord and fissure of the peritoneum remaining in statu quo. There are two methods of radical cure-one by the introduction of the hand through an incision through the abdominal walls, and the other by the rectum. Gierer always operates by the rectum, and his method consists in the simple return of the spermatic cord in contact with its natural point of attachment against the abdominal wall. To effeet this a limited, successive, and slightly outdrawing traction forward, or from behind forward, of the intestine is effected ; and, having accomplished this, the soft part of the thumb of the right hand, which is used from the beginning of the operation, is applied through the coats of the rectum in the lower and posterior part of the cord, and, with a jerk, or forward movement, the operation is completed.

Gierer says that the introduction of the hand in the abdomen is only needed when, from the amount of intestine imprisoned, it is difficult to feel the rudimentary spermatic cord; but, under these circumstances, inflammation and gangrene would have ensued already, and the operation would, therefore, be useless, and it is many years since he had occasion to perform it."

\section{Flatulent Colid.}

This disease is generally occasioned by some derangement of the digestive organs, whereby the food, instead of being properly digested, undergoes fermentation, and thus carbonic acid gas, or sulphureted hydrogen is evolved.

Symptoms.-It is attended by considerable pain; the animal will be very restless, continually lying down and getting up again; discharges gas from the anus; strikes the belly occasionally with the hind feet, and the abdomen is enlarged. 
Treatment.-This species of colic can generally be relieved as follows: Take one ounce of hyposulphite of soda, dissolve the same in a quart of water; then add tincture of ginger and tincture of golden seal, of each, one ounce. Drench the animal with the same. Clysters of soap-suds, to which a little salt may be added, should be thrown into the rectum occasionally. The belly should be well rubbed with coarse straw, and, in severe cases, I should rub some mustard, moistened with vinegar, on the lower part of the abdomen. After a lapse of two hours, should the patient appear unrelieved, a second dose of the colic drench may be given. Generally, however, one dose is sufficient.

\section{SPASM OF THE Bowels.}

Spasm of the bowels, generally termed spasmodic colic, is occasioned by contraction of the longitudinal and circular fibers of the muscular tunic of the intestines. In this disease nearly the same symptoms are observed as those alluded to in flatulent colic, with the exception of flatulency. The patient, however, is more irritable and dangerous to handle than in flatulent colic, and he has periods of relaxation from pain which return at intervals with increased violence. It is supposed that this affection is caused by the presence of irritating matters in the intestinal canal, either in the form of bad food, poisonous plants, or water impregnated with lead. As the muscles of the intestines belong to that class known as involuntary, it follows that the state of spasm is the result of some excitability or deranged condition of the nerves of involuntary motion; hence antispasmodies are indicated. In view of relieving the spasm, I recommend the following:

No. 89. Powdered assafetida.............. $1 \mathrm{dr}$.

Sulphuric ether................ $2 \mathrm{dr}$.

Thin gruel.................... 1 pint.

Mix.

Use as a drench ; then administer, occasionally, an antispasmodic clyster, composed of warm water and a small quantity of powdered lobelia.

Failing to relieve the animal by the above means, I should get him under the influence of sulphuric ether, to be applied to the nostrils by means of a sponge. There is no necessity for fully etherizing the animal, for, if kept in a partial state of stupefaction 
for about twenty minutes, the spasm will relax; after which, let the patient be kept on bran-mashes for a day or more.

\section{Constipation of the Bowels.}

Constipation is generally the result of impaired digestion, yet, now and then, it is the precursor of some definite malady. The old method of resorting to powerful cathartics generally aggravates the symptoms, and the animal dies unrelieved of the constipation.

The best method of treating this affection is to administer, with an aperient, stimulants and bitters; in this way we arouse the action of the digestive organs, and create a lax state of the bowels. Let the following drench be given:

No. 90. Glauber salts................. 12 oz.

Powdered golden seal............. $3 \mathrm{dr}$.

Powdered ginger................ $2 \mathrm{dr}$.

Dissolve the Glauber salts in one quart of tepid water; then add the other ingredients. After a lapse of eight hours, the animal should be walked about, have its belly rubbed, and"an occasional clyster of warm soap-suds may be thrown into the rectum. Should the medicine not operate at the expected time, administer two drachms of powdered mandrake, the same quantity of golden seal, and half an ounce of hyposulphite of soda, in about a quart of water.

To prevent a recurrence of the constipation, let the animal have half an ounce of equal parts of golden seal and carbonate of soda daily, which may be mixed in the food. It is important, also, in view of guarding against constipation, to make some change in the diet. Thus, if the animal has been long fed on meal, some coarser material (shorts) should be substituted, for a brief period at least.

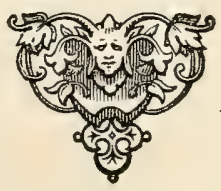




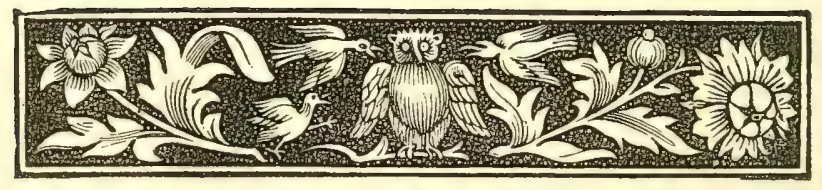

\title{
SECTION XVII.
}

\author{
PARTURITION.
}

Sygns of Labor, or Parturition-Natural Labor-Unnatoral LaborFore-tegs Presenting - One Fore-leg Presenting- Head Presenting without the Legs-Extraction of a Calf on its Back--Hind Legs Presenting-Breech Presentation-Cleansing, or Removal of the Afterbirth-Back of the Calf Presenting at the Brim of the Pelvis-Uterine Hemorrhage-Birth of Twins-Triplets.

\section{Signs of Labor, or Parturition.}

A $\mathrm{T}$ the end of two hundred and seventy days from the period Al of a cow's impregnation, some enlargement of the udder will be perceived, and the labii pudendi (external parts of the genital organs) are relaxed, and appear tumefied, and a sort of glistening discharge issues from the same. The animal is also restless, and appears desirous of avoiding the society of other cows. Her respirations are somewhat quickened; she becomes nervous and irritable, and labor pains set in, occurring at stated periods, until, at last, the neck of the uterus dilates, the foetal membranes present themselves in the form of a watery tumor, and the parts admit of the delivery of the foetus.

\section{NATURAL Labor.}

Natural labor consists of the presentation of the placental membranes, inclosed fluid, with the head and two fore-feet of the foetus. In the act of natural expulsion the membranes become ruptured, and the liquor amnii (water) escapes. This lubricates the parts, and greatly facilitates the birth of the foetus. After delivery a few after-pains occur, by which means the placenta, or after-birth, is expelled. This completes the painful routine of natural labor. 


\section{UnNatural Labor.}

A cow failing to give birth after the fashion described in the preceding article, and being in a state of parturition, having regular uterine pains, increasing in severity as they successively occur, yet no appearance of the foetus, is probably the subject of false presentation. The character of this presentation must be ascertained, and our efforts then directed to the replacement of the part to its natural position. The person who intends to render assistance to the parturient cow should be clad in suitable garments. His arms must be bared to the shoulders, and, in view of guarding against the absorption of morbid virus, the person's arms should be lubricated with glycerine or olive oil. The instruments required are, embryotomy knife, embryotomy hooks, and slip-nooses. (See cut of instruments.)

\section{Fore-Legs Presenting.}

The most common false presentation is that when the two forelegs are advanced into the vagina, sometimes beyond it, and the head turned upon the fotal body. This is occasioned by the muzzle having caught at the brim of the pelvis. The delivery can not be effected until the position is changed, without danger to the mother and certain destruction to the calf. The best plan is to attach a cord, or the slip-nooses, to each fore-leg, which are then to be forced back into the uterus. The head must then be sought for, and constant pressure exerted on the same until it is sent forward far enough to enable the operator to release it from the brim of the pelvis, and guide it into the vaginal outlet. A noose may then be slipped over the lower jaw; then traction on it and those of the fore-legs will accomplish the delivery.

If the calf is dead I should use the embryotomy hook in preference to the noose; but, in view of saving the calf, the latter is the safest. Some care, however, is necessary in drawing out the fore-feet, lest the points of the hoofs lacerate the vagina. While the assistants are drawing steadily on the cords, the operator should give them a lateral action, from side to side, and upward and downward. This is far better than pulling persistently in one direction, for it tends to loosen and alter the position of impacted parts. 


\section{One Fore-Leg Presenting.}

This is also a common occurrence, and, if seen early, the delivery may be safely effected by attaching the noose to the protruded leg. This is to be pushed back, the other sought for and secured in the same manner, and again to be returned. The head must then be properly placed, the legs drawn outward, and the delivery may be accomplished with every prospect of bringing forth a live calf.

\section{Head Presenting without the Legs.}

In a case of this character, it is evident that the legs are doubled up within the vagina and uterus, and, unless attended to early, the calf will be dead; therefore, in order to save time and trouble, I should decapitate the calf, which is done as follows: Make a circular incision around the neck through the integuments; then cut down in a region between the first and second cervical vertebræ, and sever the capsular ligaments and spinal marrow. A person not expert in these matters will probably succeed better in detaching the head at this point than at the base of the cranium. Before the neck is returned, the embryotomy hooks should be inserted into it, and the blades secured by tying the cord which passes through them. Having pushed back the neck, I run my hand along one limb at a time, and find the hoofs. These are brought forward and noosed. Traction now being made on the three cords, the delivery is secured.

Supposing the calf to be alive, I proceed as follows: A noose is affixed to the lower jaw; the head is then pushed back as far as it ean be got; the fore-legs are then to be brought into position as above described, after which the calf is readily brought away. Some difficulty may be experienced in extracting the hind parts. If they require much force in extraction, the probabilities are that the pelvis of the fœetus is impacted in that of the cow. The long diameter of the pelvis is crosswise of the body, so that, if the calf be in a position that opposes its long pelvic diameter to the short one of the mother, it must be pushed back a few inches, and turned, so that its feet shall be downward, in a line with the cow's limbs.

It is very important that the calf should be in the right posi- 
tion as regards the diameter of the pelvis, for many valuable cows are ruined by the violent means used in the extraction of the hind parts when in a faulty position. A little tact in securing a right position for the exit of the calf would save a vast amount of unnecessary and cruel traction, which in our rural districts, where veterinary surgeons are not to be found, is too often employed.

\section{Extraction of a Calf on its Back, Hind Legs Presenting.}

Some persons have an idea that when a wrong presentation of this kind takes place the calf may be turned. This is an impossibility, and it is only a waste of time and a feat of ignorance to even attempt it. The calf must be extracted in the manner of presentation. The traction, however, should be made in a direction toward the bones of the coccygis, or tail. In the early stages of this kind of parturition, the back rests on the belly of the mother, and the feet come in contact with her spine. If my services were sought at this early period, I should endeavor to bring the feet down, one at a time, and noose them, and proceed to deliver without making any futile attempts to change the position of the calf. In a case of this character, which occurred in my practice a short time ago, I found it impossible (the cow being down) to dislodge the feet from the spinal region. I therefore procured a double and single block tackle, and fastened it to a beam which ran across the barn; the hind extremities were then attached to the single block by means of straps, and in this way the posterior parts were elevated. The consequence was, that the whole foetal apparatus receded into the abdominal cavity, the feet were dislodged from the spine, and I had the satisfaction of delivering the animal of a live calf.

The following case is related by Surgeon CARTwRIGHT, in the "Veterinarian :"

"On the 30th of April, 1850, Mr. _ came for me to see a cow, four years old, that could not calve, as, in a former instance, another celebrated man at such work had been in attendance on her, but, from the state of the os uteri, was fairly frightened from making an attempt to remove it, as, he said, 'an operation' must be performed on it. I found her well off at the hips, and about the vulva well relaxed. On introducing my hand into the 
vagina, I ascertained that the os uteri was dilated to about five inches in diameter, in an apparent rigid state. On passing my hand through the os uteri, I found that the calf lay on its back. The hind feet could be felt, but they were doubled up at the fetlocks, and pressed against the rectum and inside of the upper portion of the os uteri. In consequence of the calf lying on its back, and its feet being doubled up, the latter was not forced into the os uteri, from which cause the os uteri could not be dilated for the cow to calve. I immediately got one of the legs straight, brought it forward into the vagina, and passed a cord around it, and then served the other the same. Afterward we used gradual traction to the feet, until the os uteri was fully dilated, and, in the course of an hour, we removed a live calf, which, together with the cow, did well. I fear such cases as these may induce persons to divide the os uteri, thinking it in a scirrhous state. In a fortnight after, this person had an exactly similar case; but, from using gross force, they burst open the pelvis somewhere, and the consequence was, the cow was obliged to be destroyed."

\section{Breech Presentation.}

A presentation of this kind is generally attended with difficulty and danger. The difficulties depend, however, somewhat on the length of time which has elapsed since the commencement of the labor. If it be recent, there is some hope for both mother and calf, but in a protracted case there is little hope for the mother; and one reason is, the vagina or uterus is often injured or ruptured by the struggles of the foetus in trying to free itself from its uncomfortable position. Then, again, the uterus has contracted upon the foetus so as almost to imprison it, at least forcing and impacting it within the cavity of the pelvis, so that the little animal is almost immovable. Let a person unacquainted with bovine midwifery introduce his hand and arm in a case of this character, and he will be astonished at the amount of force it will require to thread his hand between the foetus and pelvis; and after accomplishing his object, the hand and arm become so benumbed by the pressure that he can not accomplish much, if any thing, until he can succeed in foreing the fœetus forward, which, in some cases, when uterine action is strong, can not be effected without elevating the posterior parts of the cow by means of a 
hoisting tackle. It requires a person with a long arm to be of much service in a case of this kind, for the limbs are extended a long distance into the abdomen. A breach presentation is very readily detected by the presence of the calf's tail, which occasionally hangs out of the vagina.

The mode of extracting the foetus when the breech presents is as follows: Pressure must be made upon the buttocks of the calf in the interim of labor pains. Having succeeded in pushing the calf forward, the hocks may possibly be reached; afterward the feet. These are to be brought into the vaginal passage; then, by traction, and altering the position of the calf, if necessary, the delivery is completed. Should it be found impossible to push the foetus forward, I should lose no time in raising the hind-quarters of the cow by means of hoisting apparatus, which most farmers have on hand. In order to avoid hurting or injuring the cow's limbs when hoisting the hind parts from the ground, I encircle the legs, just above the fetlock, with some old gunnybag, or something of the sort; then affix a strap to each leg, into which the tackle must be hooked. The cow is, of course, raised from the floor, belly upward.

\section{Cleansing, or Removal of the After-birth.}

After the delivery, the after-birth should be detached, if possible; for, if it be allowed to occupy the uterus, the latter contracts upon it, and there it may remain for a week or more, and, at last, come away a mass of putridity-not, however, before the cow has suffered some derangement of health. So soon as the calf is born, I introduce my hand, and pull, in various directions, on the umbilical cord. Failing to bring the placenta away, I introduce my whole arm, and carefully detach the now foreign body from the cotyledons of the uterus. The uterus shortly afterward contracts, and thus effectually prevents uterine hemorrhage.

It has been my universal custom, of late, to remove the placenta immediately after the birth of the calf, and I do so because I consider it unwise to allow it to remain; for the labor is not then completed. No practitioner of midwifery in human medicine would ever deem it proper to leave his patient until the "afterbirth" was removed, for it would, most undoubtedly, endanger the mother's life if it were left to rot away, as is too often the case 
with the poor uncomplaining cow. In the removal of the placenta, I am careful not to pull too hard on the umbilical cord, lest inversion of the uterus ensue. A little tact and patience will often accomplish wonders. The placenta may be detached from the cotyledons, and yet can not be drawn away. The probability is that some irregular contraction of the uterus retains it. Now we st exercise a little patience, and then introduce the arm and el for the seat of contraction. This can probably be dilated by the fingers, and then the placenta may easily be removed.

In cases of protracted labor, when the eow is much exhausted, the placenta may remain in the uncontracted uterus for want of muscular power in the same to expel it. In such a case I should give the cow about a quart of ginger tea, and if any tympany of the intestines exist, I should add a small quantity of carbonate of soda.

The absurd practice of attaching a weight to the membranes, or rolling them on a stick, as heretofore recommended, is not in accordance with my views of an enlightened system of practice. To say the least, it is unscientific, and presents an unsightly appearance: The odor which arises from the putrid mass, in the course of a few days, is enough to sicken a dog, and no doubt it does sicken pregnant cows, and may induce abortion. The membranes must be removed.

The abominable practice of allowing the cow to devour the after-birth is much to be deplored. I know the custom has the sanction of long usage, but that, in my opinion, is no argument in its favor. The cow is not a cannibal nor a carniverous animal, yet, for the sake of getting rid of an unsightly and filthy mass of carrion, which ought to have been removed from her sight, she is tempted, and finally does devour it. She may, however, sometimes be led to devour the placenta and foetal membranes through the promptings of a morbid appetite. The best and safest way to dispose of the after-birth is to burn it, for the odor arising from it under the process of decomposition has a bad effect on pregnant cows of a highly imaginative and nervous temperament, and the odoriferous morbid germ is more active in warm than cold weather. 
Back of the Calf presenting at the Brim of the Pelvis.

This unfortunate presentation is one of rare occurrence. I never saw but one case, and that I now propose to introduce for the instruction of my readers. I was called, a short time ago, to visit a cow, the property of Mr. R—, of Winchester. The animal had been in labor, with strong parturient pains, for twelve hours. In the mean time several persons had tried their skill on the poor brute, without doing the least good. At the time of my visit, she was in a deplorable condition. Her ears, horns, and extremities were icy cold; she was delirious, throwing her head about in a reckless manner, as if in convulsions; the vaginal lips were very much tumefied, their lining membrane being highly inflamed. I immediately gave her a good drench of stimulating medicine, which appeared to have a good effect in restoring warmth on the external surface and in the extremities. On making an examination, I discovered that the back or spinal column of the foetus was firmly impacted within the brim of the pelvis; consequently every uterine effort to expel the same was only making matters worse. I employed all the usual means to change the position of the calf to no purpose. Finally, I proposed an operation, to which the owner consented. I now etherized the cow, turned her on her left side, and made an incision through the right flank, beginning at a point two inches beneath the transverse processes of the lumbar vertebra, mid-distance of the last rib and anterior spine of the pelvis. The length of the incision was about ten inches. I then divided the muscles in this region known as the transversalis, external and internal oblique. Having thus exposed the peritoneum, I punctured it, and, by means of a probe-pointed bistoury, dilated it to the extent of the external incision. I then made an incision through the uterus, disemboweled the calf, so as to reduce its bulk, and, finally, removed the heart and lungs; yet I could not extract the carcass (which was of extraordinary size). I therefore made a section of the spinal column, and removed the foetus in halves; then took away the placenta, and removed all fluids by means of a sponge. The incision was properly sutured, and, after a short period, the cow got up and partook of a bran-mash. Twenty-four hours after the operation the cow died. This probably occurred from the exhausted condition of the animal at the 
period of operating. When nothing except an operation of this kind can save the mother, I recommend that it be performed early, so that there shall be vitality enough in the system to bear up against it.

\section{Uterine Hemorrhage.}

Uterine hemorrhage is known, in common parlance, as flooding from the womb. Occasionally it does occur as a sequel of forcible extraction of the calf, followed by an unwarrantable harshness in extracting the placenta and its membranes from the uterine cotyledans. The best plan of arresting this kind of hemorrhage is to drench the cow with two ounces of tincture of matico, and then encircle the body, in the region of the small of the back, with a cold-water bandage. The object, in a case of this kind, is to induce contraction of the uterus, for, when once contracted, the flooding will soon cease.

\section{Birth of Twins.}

There are many cases on record of cows giving birth to twins, and even triplets, without manual assistance; yet occasionally, in consequence of two presenting in the passage at once, some assistance is needed. When called to a case of this character, the object should be to ascertain if the parts presenting belong to one or two calves. If the latter be the case, one must be pushed back and the other advanced; for they can not both be born at once without periling the life of the mother.

\section{TRIPLETS.}

Three years ago I visited Keene, N. H., on the occasion of the State Fair, and saw a cow of native breed, the property of $\mathrm{Mr}$. Aldrich. I learned that at two previous births she had brought forth twins, and now was the mother of three at one birth. These were milk-white, and a perfect counterpart of each other. I understood $\mathrm{Mr}$. Aldrich to say that the cow had never been bred to the same bull, so that the plural and triple births were the result of a peculiarity of constitution on the part of the cow. 'This remarkable peculiarity seems to favor the hypotheses of Pythagoras and Aristotle, who maintained that the female parent affords all 
the materials necessary for the formation of the offspring, the office of the male being merely to awaken the dormant formative powers residing in the female ovaducts. The "ovists" further assume that the foetal germs already exist, with all their organs, in some part of the female organs of generation, and that the action of the male is merely that of exciting and endowing the fotus with vitality. These theories, however, appear irreconcilable with the phenomena of the offspring inheriting the faults and defects of the male. The cow alluded to never required any assistance in labor. This may be accounted for from the fact that the mother had a large pelvis, and her offspring at the time of birth were all small.

But here are two other remarkable cases, as related by $\mathrm{Mr}$. GAMGEE, in his translations:

"The subject of this notice had been delivered of her first calf in January, 1857, when two years old-a fine heifer of the Brittany breed, and of medium size. Soon after she was served (only once) - by a bull of the district, and became impregnated. During gestation the animal was healthy, walked easily, and her belly presented no uncommon appearance as regards size. On December 26, her term of gestation having closed with little pain or expulsive efforts, she gave birth, in a very few minutes, to two calves (male and female), the first being in the normal position, the last having the posterior limbs presented first. At the same time the fotal envelopes of the calves came away.

During delivery the cow showed no suffering, and would eat and drink as usual. Shortly after, the attendants perceived, between the lips of the vulva, two white, soft tumors, which they took for inverted uterus, and at once hastened for the cow-doctor. Meanwhile, a neighbor, thinking he recognized in them the waterbags, pricked them, and, on the escape of the water, another calf was seen, with a natural presentation, which. was soon delivered by the efforts of the mother. This was immediately followed by a fourth, which was also easily expelled. These two last-the first a female, the last a male-died in a few seconds, though, doubtless, had the liquor amnii been at once evacuated, both might have survived, as they were even stronger than the two first. They were very fat, and weighed, when given to the butcher, 25 kilogrammes each.

"The cow which has shown such remarkable fecundity (five 
calyes in one year) has all along preserved the most perfect health."

Remarkable Fecundation in a Cow.-Mr. M. B. Forbes sent the London "Veterinarian" the following particulars relating to the birth of five calves at one time: "A cow of the short-horned breed, six years old, the property of Mr. Richard Knight, farmer, Santon, about a mile from Ryegate, was safely delivered on Monday morning, the 21st of February, three weeks before her time, of five calves-four bulls and one cow. Three of the calves died a few hours after birth, but the fourth survived until Tuesday, and the fifth until the following day, Wednesday." Mr. Fonbes saw the cow on the $23 \mathrm{~d}$, and found her going on well. It was her third calving.

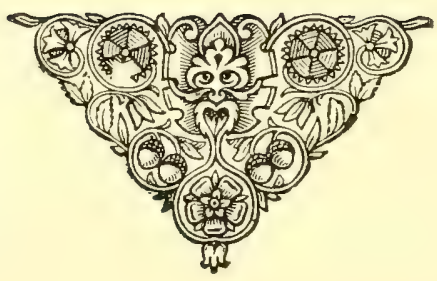




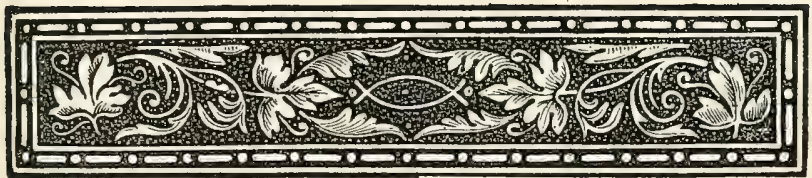

\section{SECTION XVIII.}

\section{DISEASES OF THE GENERATIVE ORGANS.}

Constriction of the Neck of the Uterus-Embryotomy-Treatment of Cows during Pregnancy - Symptoms of Pregnancy-Dropsy of the Womb-Puerperal Feter-Faliting of the Womb.

\section{Constriction at the Neck OF the Uterus.}

O WING to some abnormal condition of the neck of the uterus, it is occasionally the seat of constriction. The difficulty can only be determined by introducing the hand into the vagina; then, by bringing the fingers in contact with the mouth of the womb, it is found in an undilated and indurated or hardened state. Should the labor pains be quite strong, and no signs of relaxation appearing, I should then introduce a probe-pointed bistoury or the embryotomy knife into the neck of the uterus, and dilate the stricture, by making incisions into the inner border of the strictured or hardened mass. This will allow of some slight dilatation, large enough for a bladder of fœtal membrane to gain entrance. After this takes place, the labor will gradually progress, and, if every thing goes right, as the saying is, a live calf may soon be expected to make its appearance, and the cow may also be expected to survive the operation.

\section{EMBRYotomy.}

The operation known among medical men as embryotomy, signifies dismemberment of the calf within the vagina and uterus. I presume no man, except he be acquainted with the anatomy of the parts, would dare to attempt the dismemberment and disembowelment of the foetus. It is an operation, however, which has to be performed very often, and it has saved the lives of very many valuable cows; therefore I shall try to "post" the reader on the 
subject. The instrument used for this purpose is called an embryotomy knife (see cut of instruments), and is introduced into the uterine cavity, concealed in the hand, so that its cutting edge shall not injure the genital organs of the cow.

Mode of Operation.-Having introduced the knife within the uterine cavity, I run my hand along the foetal limb to the top of the shoulder, if possible, and there turn the knife and send its beak point through the integument, and slit the same to the region of the knee. Here I make a circular incision of the integument around the knee. A slip-noose is affixed to the fetlock, and, while an assistant is making steady traction on the same, I loosen the integument from the limb; then, by a little dexterity in the use of the knife, at the top of the shoulder and elsewhere, the whole leg is drawn away. After amputating the shoulder, I make an incision through the cartilages of the ribs. This exposes the whole of the thoracic viscera, which I remove. If the bulk of the calf appears to be sufficiently reduced to insure its extraction, I affix a noose to the remaining fore-leg, and, by traction, remove the carcass. The only difficulty in the way of a prompt extraction occurs, oftentimes, in consequence of the foetal head being bent round on the opposite side of the chest; but, under ordinary circumstances, the calf can be extracted without proceeding to disembowel or decapitate it. If, however, either of the latter operations will facilitate the birth of the calf, I should certainly perform one or the other, or both. Having extracted the whole of the fœetus, I next remove the placenta and membranes, and then drench the cow with the following:

No. 91. Fluid extract of ginger........... $\frac{1}{2}$ oz.

Tincture of matico............. 1 oz.

Warm water................... 1 quart.

This will improve the condition of the prostrate animal, and insure contraction of the uterus. The uterine expulsive power, so favorable to the liberation of the calf, if dormant, can readily be aroused by administering a stimulating drench.

\section{Treatment of Cows During Pregnancy.}

The cow is the only animal with which I am acquainted that yields milk regularly during the trying and prostrating probation of pregnancy. She has not only to yield milk for the profit of 
her owner, but she must also furnish an identical preparation for the nourishment of the foetus in utero. Then, again, the integrity of her own system requires that her digestive organs shall furnish enough of chyme and chyle for the manufacture of good, rich blood, to repair incidental and extraordinary wear and tear of her system; therefore she should be well fed and cared for. Any stinting or miserly economy in the feeding of pregnant cows is very poor policy, and is a sort of starvation procedure, which can never be expected to pay. It is impossible for the cow to discharge these treble duties unless she be generously fed on nutrimental agents, containing the necessary equivalents. I am aware that most cows are too well fed, hence milk fever and other inflammatory affections; yet the food given to some cows in this region, such as swill, brewer's grains, corn-stalks, coarse, damaged hay, etc., is not suitable for pregnant cows. They should have a mess of roots occasionally, and about a quart of meal night and morning, which may be stirred in a bucket of water, to which add a tea-spoonful of salt. A reasonable quantity of good, sweet hay should be allowed daily. This will not only keep the stomach distended to a healthy capacity, but will also furnish matter for remastication, by which process a large amount of saliva is secreted, and passes into the stomach, where it operates on the food therein contained as a powerful digester.

A pregnant cow should never be confined to the cow-house, for want of proper exercise induces plethora, and a plethoric condition of the system retards the development of the foetus; hence the cow is very apt to go over her time. The pregnant animal needs, and must have, exercise. It aids in the circulation of blood through her system: it brings the blood oftener in contact with aerating surfaces, and thus invigorates it; in short, the whole animal economy is benefited by exercise in the open air. If the season of the year admits, the cow should be sent to pasture. Here, under the advantages arising from pure air, natural food, etc., she will get in fine condition, so that when the period of calving arrives she will be strong and vigorous, and can bring forth a healthy calf. It must be borne in mind, however, that the petted, stall-fed cow is not a safe subject to leave in the pasture at night. The cold, damp ground and air are apt to derange her health; therefore she should be housed at night and on stormy days.

As regards milking the animal during pregnancy, it must de- 
pend on circumstances. A cow in good condition may be milked longer than a poor one, and the poor, overmilked animal must "go dry," as the saying is, for a couple of months prior to calving. Should a pregnant animal yield milk up to within a fortnight of calving, she should have a few bran-mashes, and the daily quantity of food and drink must be diminished. She should also have free access to salt, and occasionally a table-spoonful or so of phosphate of lime may be sprinkled over her fodder.

\section{Symptoms of Pregnancy.}

A cow in healthy condition will be in heat (a state of menstruation) about once a month. This lasts for a period of four days, more or less. About three or four months after conception has taken place, the belly is enlarged, and, on making pressure on the right flank, the motions of a live fotus can be distinctly felt. Pregnancy may be determined earlier than this by auscultation (the art of diagnosis by listening to the sounds of the heart). The beating of the fotal heart can be distinctly heard. The ear should be applied to the right flank.

\section{Dropsy oF the WoMrB.}

This affection generally prevails among aged cows, in the latter period of pregnancy; The causes of it are, perhaps obscure; yet it may be attributable, like other dropsies, to a debilitated condition of the system, and an impoverished state of the blood.

Symptoms.-The symptoms noticed in this affection are as follows: An unhealthy and debilitated state of the animal; visible membranes, pale and watery ; a pendulous and much enlarged condition of the abdomen; spinal column, curved in a downward direction; and the animal, when down, is observed to rise with difficulty.

Treatment.-It often happens that in dropsy of the uterus, the walls of the abdomen are ruptured, and the fluid escapes into the cellular tissue beneath the common integuments. This is readily detected by an unnatural tumefaction, or swelling, in some part of the abdominal region. My usual practice in a case of this character is to puncture the integument, and allow the fluid to escape. I then direct that the animal have a few doses of the following: 
No. 92. Fluid extract of buchur............ 1 oz.

Powdered chlorate of potass......... $4 \mathrm{dr}$.

Water....................... 1 pint.

Mix.

Give a dose of the above once or twice daily.

Dropsy confined to the uterus is a formidable affection, because it requires the services of an experienced surgeon, who will, probably, tap the dropsical membranes through the vagina; yet the operation is not always advisable, for it frequently results in premature parturition.

\section{Puerperal Fever.}

During the past few years, very many valuable cows have died of " milk fever" (puerperal fever), puerperal convulsions, etc. It is a remarkable fact that this disease almost always attacks cows in high condition; hence, in view of prevention, we should endeavor to guard against the accumulation of fat; and this can be done by withholding meal and substituting shorts, and also by allowing considerable quantities of roots and grass, when they can be had.

Puerperal fever is a disease of an inflammatory type. The state of plethora, which is observed among fine cows owned by wealthy individuals, who dispense provender liberally, because they hate to see a "poor" cow, is more likely to occur in a cow when pregnant and stall-fed (from the fat of the crib) than otherwise, because, under such management, she does not get exercise enough to make away with the carbonaceous material, and, therefore, it increases from day to day, and is harvested into the cell reservoirs, proving in its bulk detrimental to full and free circulation, respiration, and intestinal peristaltic action.

The warm and impure atmosphere which the stall-fed animal is compelled to respire is decidedly operative in bringing about that condition known as plethora; therefore our object should be to secure a current of cool and pure air throughout the cow-stable. Pure and cool air is a very potent physiological, sedative, contrastimulant, and, as the fat animal is in a morbid state of excitation from the stimulus of the food, as well as artificial atmosphere, the suffering creature will surely be benefited by inhaling the requisite amount of an uncontaminated atmosphere.

The principal causes of puerperal fever in women are said to 
be misplaced benevolence, which benevolently(!) furnishes her with choice viands, rich caudles, and stimulants, in view of obviating debility and insuring hilarity of mind. Now, the condition of the pregnant female being one of increased susceptibility, it fully demonstrates the incompatibility of using even what may be considered as the ordinary stimulations of health. Therefore, in the case of the cow, stimulating food, and an unnecessary amount of the same, given daily after the cow has "come to her growth," as the saying is, may be termed misplaced benevolence; whereas, if the cow has not attained her full growth, the practice of feeding liberally is not objectionable, for she requires a large amount of nutriment to develop the various parts of her organism, and promote the integrity of the same, and also to nourish the fœus in utero, and, lastly, for the purpose of furnishing the necessary material for the lacteal secretion. It has been noticed that great milkers, as.well as fat animals, are often the subjects of puerperal fever; and probably the suppression of the milk secretion, in the advanced stage of pregnancy, may be an exciting cause of the difficulty, yet not in all cases, for we are pretty certain that some cows, owing to a peculiarity of constitution, are predisposed to puerperal hysteritis. In view, therefore, of guarding against the consequences which may occur when the animal is suddenly "dried up," we should be careful to diminish the daily allowance of food, and also feed lightly from this period up to that of parturition.

Milking the cow before calving, in view of preventing puerperal fever, is very mischievous, for it is apt to excite premature parturition. Many of the cows I have attended for the above complaint had been so treated. Occasionally the udder becomes distended to a painful degree, so that it is necessary to practice moderate milking, otherwise the practice is injurious.

It is generally supposed that this disease first manifests itself in some parts of the tissues entering into the composition of the reproductive organs; or, in other language, primary congestion and irritation of the womb, ending in cerebral congestion and convulsions. The cerebral congestion, however, is not always the cause of convulsions, for we have many cases on record in which phlebotomy was practiced on the heroic plan (an incredible amount of blood having been abstracted) without arresting the convulsions; hence, in such cases, congestion is the result, not the cause, 
of convulsions. A very eminent physician (Dr. LogAN) has stated, as the result of observation, that puerperal convulsions were generally found in females of highly organized nervous systems, and who were peculiarly susceptible to irritation of that system; yet our experience in this country, in cattle practice, confirms what we have written in the preceding part of this article; namely, that puerperal convulsions are more frequent among fat animals than in those of lean condition. The theory of Dr. LoGAN may, in the main, be correct; for a lean animal (nervous temperament) can, by artificial means, be brought into the condition known as plethora.

YodatT contends that "cows in high condition are most subject to an attack of puerperal fever. Their excess of condition, or state of plethora, disposes them to affections of an inflammatory character, at all times and under all circumstances." If it shall prove to be the case that puerperal fever is the result of uterine inflammation, we should diagnose the case as puerperal hysteritis, and treat accordingly. Puerperal hysteritis may, however, occur at any period of pregnancy.

Symptoms. - The early symptoms attending a disease of this character are such as are found to prevail in a disease of a febrile character; namely, loss of appetite, accelerated pulse and respiration (the latter attended with strong but quick abdominal flank movements); tongue, slightly coated; mouth, hot and clammy; muzzle, dry. There is also a sort of wildness about the eyes; the animal is nervous, and some unusual spasmodic twitchings of the muscles will generally be observed. Soon the animal grates her teeth, foams at the mouth, dashes her head recklessly on the floor (for she is now on the floor). Examine the eye at this stage, and the pupil will be found in an amaurotic state. The udder becomes swollen, hot, and tender, and the lacteal secretion is partly suspended.

The disease generally appears within a fortnight after calving, and it may occur within a very few hours. Its duration is very brief, both the curable and incurable cases terminating either one way or the other in a short time; and if violent convulsions set in, we generally find that the patient has but little time to live. Especially is this the case when paraplegia be present, or paralysis in any form. The principal symptoms in which the diagnosis must be founded are as follows: We first ascertain whether or not 
the animal has, within the period of a fortnight, given birth to a calf. If so, and she manifests the usual symptoms of convulsions, (refuses to notice her calf, has lost the use, more or less, of her limbs, and the eyes present a wild stare, the pupils being dilated, and the udder swollen,) we may then safely conclude that we have a case of milk fever to deal with.

Treatment.-The professional man treats the disease according to its indications, and the non-professional, if he attempt to treat a case of this character, must endeavor to do likewise. I can not possibly commit to paper all the necessary information, but shall merely lay down a few simple rules for the management of such cases. At the same time, I would advise the owner of the sick cow to secure, if possible, the services of some competent veterinarian. Some practitioners recommend the abstraction of blood from the jugular vein, but my practice is to bleed from the bowels, by administering purgative medicine. In this way the animal can be depleted with greater safety than by blood-letting. The cathartic is as follows:

No. 93. Glauber salts................ 12 oz.

Powdered ginger.............. $\frac{1}{2}$ oz.

Warm water.................. 1 qt.

First dissolve the salts in the above amount of water, then add the ginger. Drench the animal by means of a quart bottle. Take time to pour it down the osophagus, and the more speedily will it act. If I have no faith in blood-letting, I may be said to have great faith in purgation; for, as Pencivall very truly observes, in regard to the treatment of staggers in horses, "Purge a horse and you cure him," so I say in regard to the treatment of puerperal fever, Purge a cow and she shall be cured. The bowels must be made to liberate their contents. After having administered the medicine, the patient must be watched, and, when, she lies down, let her have a comfortable bed of hay ; and the attendant should occasionally sponge her head with cold water. When down, a good stimulating alkaline enema may be thrown into the rectum, composed of

No. 94. Warm water.................. 2 qts.

Ginger...................

Fine salt.................. 2 oz.

Supposing that, after a lapse of six or eight hours, the bowels 
fail to respond to the purgative, half the quantity just recommended may be given; and perhaps it may be proper, in view of creating a vacuum in the rectum, to repeat the enema; for purgation once established our patient is safe; yet, in view of producing this very desirable result, it is not a rational procedure to convert the stomach into an apothecary's shop, and gorge it with useless drugs, as is, unfortunately, too often the case; for, by this means, a medicinal disease is created, which is generally more uncontrollable than the original one.

In curable cases it is very rare for the above medicine to fail in exciting eatharsis; however, such remedy is not always at hand (I mean the salts). The next best remedy is common table salt, to be substituted for the Glauber or Epsom salts.

Other indications to be fulfilled in the Treatment of this Disease.-The palsied limbs require attention. Let them be diligently rubbed with tincture of capsicum (hot drops), in view of producing reaction. By this means we equalize the circulation, and thus relieve internal congestion. Should the bowels be tympanitic, or distended with gas, a quart of ginger tea may be given; and it may do more good if a small quantity of carbonate of soda be added to the same. Should the animal be thirsty, a small quantity of powdered niter may be dissolved in water or thin gruel, and offered to her. After the bowels have operated, the danger passed, a few doses of alterative medicine may be given. This is composed of

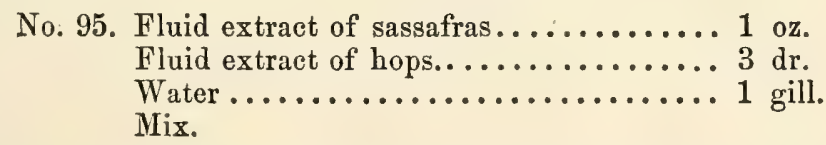

The following case, from the author's note-book, is here introduced to illustrate a new mode of treatment. The case occurred in a five-year old cow, the property of Mr. G—, of Malden, Mass. The animal gave birth, without assistance, to a healthy male calf. The birth took place during the night-time, at pasture, the weather being rather tempestuous. Three days after parturition, the cow showed symptoms of failing health, and the owner, like a sensible man, instead of boring the animal's horns, at the suggestion of the neighbors, preferred to seek advice; hence my services were secured.

The patient was down on the left side; pupils, amaurotic; 
pulse, scarcely perceptible; respiration, of a stertorous character, and accelerated; surface of the body, comfortably warm; visible surfaces, of a leaden hue; abdomen, slightly tympanitic. The animal occasionally turned its head toward the costal region, and returned it recklessly to the floor ; mamma, tumefied and hot; internal surface of the labia pudendi, inflamed. The patient takes no notice of its offspring. Applied counter-irritants to the spinal and pectoral regions, and then applied sulphuric ether to the nostrils. The object in administering sulphuric ether was to diminish the reflex excitability of the nervous system, and so cut short the convulsive paroxysms. The ether appeared to prove invaluable; for, soon after its administration, the animal appeared calm, tranquil, and rational, and soon began to take notice of its offspring. I then administered table salt, eight ounces; water, one pint. Flannels, saturated with infusion of hops, were now applied to the mamma, and renewed occasionally. In the course of thirty-six hours after the administration of the ether, the patient was standing up, nursing her off'spring, and, at the same time, partook of a bran-mash. The following three days, the patient got two drachms of fluid extract of chamomile flowers per diem, and was then turned out to grass, and did very well.

Remarks on the Preceding Case.-The treatment of puerperal fever, in bygone days, is a disgrace to what has been considered as a "learned profession." It is a very unfortunate circumstance that such learned men as Ransbotham, Churchild, Meigs, and other equally distinguished midwifarians, should contend that "the lancet is our sheet-anchor in puerperal convulsions." They inform the world of medical non-thinkers that the daring use of "the lancet is demanded;" that "the lancet is our sheet-anchor;" that "blood must be taken largely;" yet a great proportion of their patients thus treated prematurely pay the debt of Nature secundum artem. What a libel on a "learned profession" is this state of affairs! And, unfortunately, the distinguished French surgeon Cazeaux has indorsed the reckless tactics of England's universally wretehed practice; namely, "sanguine emissions." Now, I contend that the routine practice of the acknowledged authorities will not stand the test of modern medical logic; that it is contrary to all demonstrative experience, and, therefore, should receive the seal of oblivious antiquity, never to be reacted. or countenanced by sensible men. The old school heretics are 
great sticklers for the support of their tottering fabric-namely, "that fever and inflammation must be subdued"-and they commit outrages on the physiological laws of animality by instituting a series of manipulations, redolent of the aroma, acceptable to the god of pathology, whom they worship with the spontaneous faith of a new convert.

\section{Falimag (or Inversion) of the Womb.}

This sad accident is generally occasioned by extra uterine expulsive action, at the moment of the birth of the calf, aided by adhesions of the placenta to the cotyledons, by which means, in the process of delivery, the uterus is actually turned inside out. The relaxation of the ligaments, which ordinarily confine the

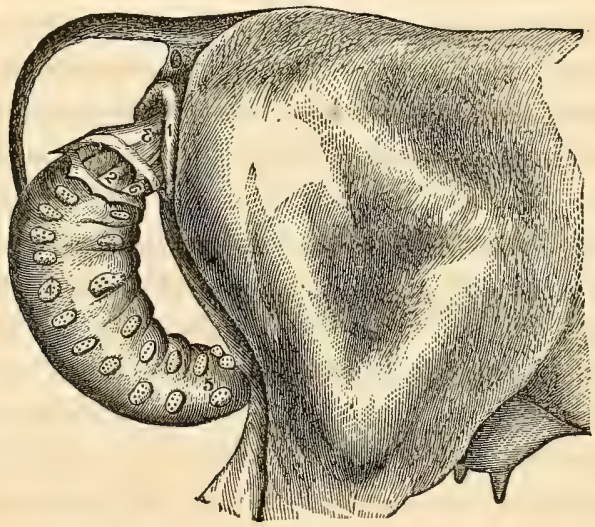

TALLING OF THE WOMB.

Explanation.-Fig. 1, Pudendum; 2 , Region of the neck of the womb; 3, Vagina; 4, The cotyledons of glands of the uterus; 5 , Body of the womb turned inside out.

uterus to the pelvic cavity, may be one among the various causes which tend to induce inversion. The accident, so far as my experience goes, is apt to occur in animals of an inferior class, coarse and flabbily organized, and among others in a debilitated condition. The only way to prevent a recurrence of inversion is to spay the cow.

Treatment. - The old method of introducing the arm, in order to force back the protruded parts, is now superseded by a bulbous rod of iron, which must be applied to the fundus or base of the 
womb. A due amount of pressure being steadily made, an assistant manipulates the sides, and the womb is re-inverted. Sometimes it is very difficult to get the womb back, in consequence of latent nterine action, which convulsively resists our efforts, and violently sends it out again at the moment of return. Should the convulsive action of the uterus continue any great length of time, I should etherize the animal, and, when fully under its influence, the reduction may again be attempted, and probably consummated. The instrument should be kept in the womb for some time after reduction, so as to give the muscular fibers of its walls time enough to contract; and it may be necessary to have a man in attendance for a whole day or more, in view of accomplishing this result.

So soon as the reduction is complete, I should sponge the loins often with cold water; or a better plan, perhaps, would be to pack the loins, after the fashion of hydropathy. The loins and abdomen will, by this method, receive considerable support, which will be favorable to induce contractile power in the uterus.

Sometimes the presence of the bulbous instrument in the uterus, after reluction, tends to keep up the after-pains, or expulsive action, and if so, it must be removed, previous to which, however, some stout tape should be sent through the thick skin in the region of the prominences found on each side of the vaginal outlet, known as the tuberosity of the ischium. This is a much better plan than that of stitching the vaginal lips. After removing the instrument, a pad may be placed on the lips of the vagina; then tie the tape sutures moderately tight. Two sutures will answer, and they can be allowed to remain until all danger of re-inversion has passed. The animal should be placed in a situation where the hind parts can be elevated a few inches.

The following cases appear interesting enough to obtain a space in this work, and I recommend them to the reader's perusal. They occurred in the practice of Surgeon Younghusband:

"On the morning of the $3 \mathrm{~d}$ of January, 1850, I was sent for, in great haste, to attend a cow that three days before had calved, and, apparently, up to this time, had done well; but on that morning, on the cow-keeper attending as usual, he found the cow down, and the whole of the uterus protruding through the vagina. On my arrival, I found her as described, with the uterus in a most loathsome state, from being suffered to remain unprotected among 
the dung and urine of the cow-house, and also the placental membranes adherent. Plenty of assistance being at hand, we had her up, well-raised her hind parts, and in that position secured her, so that she could not well slip down again. Having carefully detached the placenta, I next proceeded to cleanse the parts by washing them with a mixture of weak spirit and water. Having accomplished this, I proceeded to return the part; but, on minutely examining that viscus, before attempting its replacement, I discovered in it a large rent, through which I easily introduced my hand; and, for the better satisfaction of the owner, I caused one of the bystanders to do the same, to show them that it was not through any mismanagement of mine that this untoward accident had taken place; for, in my belief, another cow had trod upon it while she was down, and thus done the mischief. After this, I effected its return without much difficulty. Judging that I had got all the part into a right position, ete., I made an attempt to withdraw my arm; but, in doing this, the cow immediately began to strain with such violence that it was not without the greatest difficulty that I could retain the part in statu quo. But, by a fortunate slip, her anterior parts were brought so near the ground that I now easily accomplished that which, for a length of time, I had found the greatest difficulty in attempting to do; namely, getting the part into a right position, retaining it there, and withdrawing my arm without difficulty. The cow now straining very little or none, I applied the usual means of prevention, gave an anodyne, had her set up from the awkward position into which she had got, and waited to see the result. I retreated for a short time, leaving a watch, in case any thing untoward should again take place. On my return, I found her still up, attended with no bad symptoms, very little straining, and appearing to be more comfortable than her situation would warrant. She was now offered a little food, of which she seemed to partake freely. Still, I warned the owner of the danger, and told him I had not the slightest hope of her recovery. To be brief, from that time she had a little fever medicine occasionally, and I paid her a few visits, still finding her apparently improving, and on my last visit, on the 10th, found her in so favorable a situation that I told the owner that, being at a great distance, unless I heard more from him, I would discontinue my attendance; nor had I more occasion to repeat them. The cow did well, 
and has since had two more calves, without needing any of my assistance.

The second case was that of a cow belonging to Mr. T. Monkhouse, of Moredale, in my neighborhood, which calved apparently with the greatest ease, showing no signs of particular uneasiness; but, on paying her a visit, he found her with the uterus protruded and the placenta attached. They immediately secured the part, so as it might receive little or no injury from the contact of foreign bodies. In the mean time, a messenger was dispatched for me. Being at home, I was not long in being at my post. After having her put in a position which I considered favorable, I proceeded to detach the placenta, which was easily done, and the part being free from dirt, was soon ready for returning, which, from the dilated state of the parts of generation, was, of all cases I ever had, the most easily accomplished. But mark the sequel. When I imagined I had made all right, the cow, appearing to suffer very little from the effects of the operation, was let up from her situation, and I had withdrawn my arm, when, behold, a portion of the small intestine made its appearance through the vulva! Judge of my consternation now, having no cause to fear such an untoward act. I told the owner how the case stood, and frankly confessed my ignorance of its cause. I now proceeded to find out the rent in the uterus, which I soon did, it being in its posterior part, and, without much difficulty, got the intestine returned. My next aim was to cause as much contraction of the uterus as I could, so as to bring the divided edges of the organ together. This I did by the application of camphorated tincture of opium, and which, I am proud to say, soon gave me the required satisfiction-so much so, indeed, that before I withdrew my arm the laceration was scarcely distinguishable to the touch. 'The cow, in this case, appearing in so easy a state, no truss was applied the first night, but a person stayed with her, if possible to prevent future ills. Now, I must say, of all cows, this one has gone on most favorably. To give a description of her treatment would be a waste of time and paper, since as to the medical treatment it amounted almost to nothing. Careful nursing, with a few solitary doses of medicine, constituted the whole." 


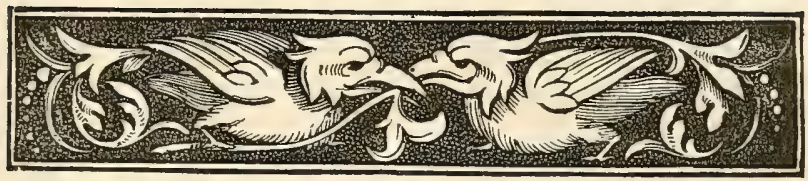

\title{
SECTION XIX.
}

\author{
DISEASES OF THE UDDER, TEATS, ETC.
}

Mammitis-Stricture in Cows' Teats-Obstruction at the end of the TeatsObstruction in the Teats-Tumors in the Teats-Injuries to the TeatsSore Teats-Chapped Teats and Chafed Udder-Inversion of the Vagina -Laceration of the Vagina-Abortion-Inflammatory Affections of the Hind Limbs after Calving.

\section{MAMMITIS.}

$\mathrm{M}^{1}$ AMMITIS signifies inflammation of the udder. It usually consists of tumefaction, attended with heat and pain, and generally sets in shortly after calving. The treatment of this affection should be antiphlogistic. Let the animal have a full dose of Glauber salts, and apply a refrigerating lotion to the udder (cold water will answer), in view of reducing the temperature of the same. In this state of the udder scarcely any milk flows, and what comes is often bloody. Soon an entire obstruction takes place, or nothing but a watery secretion can be got away. Next, the udder hardens in places, abscesses form, and then the secretory function of one or more quarters is destroyed. The animal now has the "garget," and ten chances to one if the part or parts are ever restored. The only way to prevent garget is to let the calf suck immediately after it is born, or else introduce a tube into one or more of the teats, and thus evacuate the milk ere it coagulates.

Symptoms.-The disease, at its commencement, invariably consists of an inflamed condition of the mamma, or "bag," characterized by pain, heat, swelling, and more or less febrile symptoms. It is precisely the same disease which many nursing women are prone to and suffer from, and its terminations, when not arrested in the early stage, are exactly the same ; namely, suppuration, formation of an abscess, induration, or hardening of the walls of the 
bag. In the human female the suppurative stage is known to nurses as "broken breast;" and the state of induration or hardening which follows, or may exist independent of an abscess, is commonly called "caked" breast; hence the term caked udder. Such is the character of this disease as it occurs among cows in the United States. In Europe it oceasionally assumes a more malignant form, and it often becomes necessary to extirpate the whole gland.

Treatment.-The disease should be attended to in its early stage, and the milk must be evacuated, so that it shall not accumulate nor coagulate. In order to do this, a metallic tube may be inserted into the teat, and allowed to remain there, so that the milk shall flow as fast as it is secreted. The inflamed part must be bathed with cold water several times during the day, to which add a few drops of tincture of arnica, and, if the part be very painful, an infusion of hops may be used. Afterward anoint the parts with a small quantity of glycerine. The animal must be kept on a very light diet (scalded shorts are good); and if she be fat, or the least constipation of bowels exist, I should give one pound of Epsom salts, dissolved in warm water, to which may be added a small quantity of molasses and a tea-spoonful of ginger.

Supposing the case to be in the suppurative stage, and it is evident that pus or "matter" is forming within the "bag," or its walls, it may be poulticed with flaxseed, or rubbed twice daily with some stimulating liniment-say linseed oil, two ounces; spirits of hartshorn, one drachm. So soon as the matter burrows to the surface, and a soft spot can be detected, it should have a free opening made into it, by means of a thumb-lancet. The matter must then be squeezed out, and into the cavity syringe some salt and water, or a little tincture of aloes. In the suppurative stage I generally order a generous diet.

In the indurated stage, the treatment consists in exciting absorption of the parts; and in this view I recommend iodide of potassium, known as "hydriodate of potassa." It is one of the most efficient remedies for the absorption of abnormal growths that I have ever used, and it is highly recommended in our textbooks by men well acquainted with its modus operandi. The dose of hydriodate of potassa is twenty grains per day, to be pulverized and dissolved in water. Being inodorous and almost tasteless, there is no trouble about the patient drinking it. The medicine 
may be continued until the enlargement disappears, when the dose may be gradually lessened. If I were called upon to treat a chronic case of long standing, I should, in addition to the above, besmear the parts daily with a portion of the following:

No. 96. Iodide of potassium............. $1 \mathrm{dr}$.

Glycerine ...........,.,.,., $7 \mathrm{dr}$.

Mix.

\section{Stricture in Cows' Teats.}

I was lately consulted in reference to the case of a very valuable imported cow, that had obstruction in the off posterior teat. She had given birth, about a week previous, to twin calves. The obstruction appeared to be located about half way up the teat. I fomented the parts with an infusion of lobelia, after which the tube was easily introduced.

\section{Obstruction at the Ends of the Teats.}

It occasionally happens that a fungous or warty excrescence makes its appearance at the end and center of the teat, which obstructs the flow of milk, and is very annoying and painful to the animal. This should be removed by the scalpel, taking eare to dissect away every portion of the morbid growth. The part is then to be sprinkled with powdered bloodroot, in order to prevent union of the edges of the outlet of the teat. The milk-tube, welloiled, must now and then be introduced.

\section{Obstruction in the Teats.}

A simple obstruction in the teats is frequently occasioned by imperfect union in the lining membrane.

Treatment.-This is easily remedied by introducing a tube constructed for the purpose, which should be well lubricated with olive oil, and allowed to remain in the lactiferous channel for several hours daily, or until all danger of readhesion has passed away. The lactiferous outlet is sometimes obstructed by false membranes running across its channel. These must be annihilated by the introduction of the tube. 


\section{TUMors in the Teats.}

Tumors are occasionally found in the teats. Their presence is determined by bulbous enlargement, which, on manipulation, appear very evident.

Treatment.-The method of operation in such cases is to introduce a tube, well smeared with iodine ointment, and repeat the operation, two or three times daily, until the milk passes freely.

\section{INJURIES TO THE TEATS.}

I have met with several cases of injury to the teats in the form of an incision, which occurred accidentally on the animal rising from the ground, cutting or lacerating the same with its own hoofs.

Treatment.-When the accident is discovered shortly after it happens, the parts may be brought together by uninterrupted suture. The seam is then coated with collodion, and the milk must be evacuated wholly by the tube until the parts have united. Sometimes the union is not complete, but a small fistulous opening is left, through which the milk is constantly dribbling. The only way to remedy this is to convert the fistula into a simple fleshwound. This is done by means of a sharp-pointed knife, which removes the thin callus forming the interior of the fistula. The raw edges are then to be brought together by suture, and collodion and the tube used, as before.

\section{SORE Teats.}

Treatment.-First, wash with warm water and castile soap; then lubricate the parts with equal portions of lime-water and linseed oil.

\section{Chapped Teats and Chafed Udder.}

Treatment.-Foment the parts daily with an infusion of chamomile flowers for at least fifteen minutes at a time; then wipe dry, and use the lime liniment. These temporary, or what might, with more propriety, be termed local maladies, will, if the system be free from morbid matter, generally yield to local remedies. If, however, no change for the better can be observed, the following aperient should be given: 
No. 97. Fluid extract of golden seal..... $3 \mathrm{dr}$.

Powdered mandrake.......... $\frac{1}{2}$ oz.

Powdered ginger............ 1 tea-spoonful.

Dissolve in warm water, one quart, and drench the animal with the same. In the event of the above remedies failing to give relief, anoint the parts twice daily with a compound composed of glycerine, four ounces, and fine elm flour enough to form a thin paste.

\section{INVERSION OF THE VAGINA.}

Inversion of the vagina generally occurs in the latter months of pregnancy, at a time when the digestive organs are somewhat impaired, either actually or sympathetically. In either condition the food is very apt to be imperfectly remasticated, and ferments. This gives rise to the formation of gas within the alimentary canal, and occasions tumefaction of the compartments of the stomach and large intestines. In this state they take up more room than can be spared for their occupancy; hence the pressure in a posterior direction, which forces the vagina out of its location.

Treatment.-When this difficulty occurs in a cow on the eve of parturition, there will be some danger of inversion of the uterus, unless the reduction is effected before delivery; therefore I should endeavor to make room in the abdominal cavity, by putting a stop to the fermentation which is going on in the stomach. In this view, I recommend that the animal have the following drench:

No. 98. Hyposulphite of soda............ 1 oz.

Powdered golden seal............ $4 \mathrm{dr}$.

Water....................... 1 qt.

Mix.

Having administered this drench, procure a soft sponge, and foment the part (if it be much inflamed and painful) with a tepid infusion of hops. If the inversion be of recent origin, and not much congested, cold water may be used instead. After awhile pressure and manipulation may accomplish the reduction; yet it is apt to reappear, and continue to do so until the animal has got rid of her burden. The reduction, however, must be effected, if possible, every time the protrusion makes its appearance, or the cow will suffer from retention of urine.

It is very evident to me that very many cases of inversion of the vagina are caused by indigestion; therefore great care should 
be exercised in the feeding of cows during the last months of pregnancy.

\section{Laceration of the Vagina.}

This is an accident of very frequent occurrence among cows, yet seldom proves fatal. It often occurs from the rough manner, sometimes practiced, of bringing the feet forward; therefore great care is necessary in conducting an artificial labor to a successful termination.

The following case of extensive laceration of the vagina of the cow, given by the "London Veterinarian," goes to show the potency of Nature in the cure of disease and injuries:

"Mr. J. W. Maw, veterinary student, has sent us the following particulars relating to a case of willful laceration of the vagina, and contiguous parts of a cow: 'Mr. Crosby, who is in practice at Thornton Pickering, Yorkshire, as a veterinary surgeon, was called, a short time ago, to attend a milch cow which presented symptoms of colic. On his visiting her, he observed a piece of stick, about three inches long, protruding from the vagina, and, on removing it, he found that it was about a yard in length, and had been thrust through the vagina, and likewise the rectum, into the abdomen. Previous to its removal the cow did not evince such symptoms as one would have been led to expect in an animal suffering from such a severe injury. There was but little external hemorrhage, the symptoms, upon the whole, rather indicating that internal hemorrhage was going on. Anodynes were administered, and quietude enjoined. No inflammatory symptoms supervened, and, in the course of ten days or a fortnight, the animal had recovered. The atrocious act is supposed to have been perpetrated by some gypsies, who were located near the place at the time, from their going, in the following morning, to beg the carcass, thinking, probably, that the poor animal was dead."

\section{Abortion in Cows.}

The cow is the most liable of all domestic animals to abortion, and those that have once been the subjects of this mishap are liable to a recurrence of the same. Mr. Youat, in his work on cattle, furnishes some very useful information on this subject; still, the direct causes of abortion are, like many other enzoötic and epi- 
zoötic affections, involved in obscurity. That it occasionally rages as an enzoötic pest, is clearly shown by the records of the past, and by what occurred, a short time ago, among the dairy cows of a milking establishment at Concord, N. H., without mentioning other cases, which of late have been chronicled in our agricultural periodicals.

It occasionally appears as an insolated evil. An Alderney cow, the property of Mr. Burnet, of Southboro, has, in the course of three years, aborted four times. At the time of writing this article my attention was called to her. I recommended that she should be spayed; the owner consented, and, of course, she thereafter became a more useful animal in supporting other offspring than her own. I call this an isolated case, because Mr. Burnet informed me that his other cows had not aborted. She inherits a tendency to abort.

A theory has been broached by some writer that severe winters, succeeded by warm springs, hilly pasturage, the practice of allowing young stock and one and two-year old bulls to run with the breeding cows is likely to end in abortion. This is sheer nonsense, and is not entitled to the least consideration; and, relying on the intelligence of my readers, I refrain from offering any argument in view of controverting evident absurdities. The fact is, some cows will abort, no matter what may be the nature of the pasturage or condition of the atmosphere ; and so will women miscarry occasionally, in spite of their own precautions and the advice of their physicians to prevent it. It is evident, therefore, that there exists in the animal economy of some subjects peculiarities of constitution termed idiocyncrasies, which, under certain circumstances, and on the application of the cause, (indirect,) develop the latent pathological fire, and thus they abort.

When abortion prevails among a whole herd of cows on one man's farm, I should consider it as an enzoötic, arising spontaneously, and afterward propagated by infection or by sympathetic influence. YouatT gives a quotation which favors these views: "In the Leipsic 'Agricultural Gazette,' it is stated that, 'by an unheard-of fatality, the abortion of cows in that district was almost general, and that, after the most anxious search, no assignable cause for it could be discovered, nor would any medicine or medical treatment arrest the plague,",

I shall now, in a brief manner, throw out a few hints for the 
consideration of dairymen and breeders, in view of the adoption of preventive measures; for, since neither "medicine nor medical treatment" can arrest the plague, our only hopes of stopping abortions, which in this country are alarmingly on the increase, lies in the practice of preventive measures.

A very objectionable, and I may add, ruinous, practice prevails at some milking establishments, of keeping the cows impregnated all the time. The mother no sooner gets through the pains and perils of parturition than she is again, ofttimes, compelled to submit to a re-impregnation. This is what I call an excessive use of the reproductive organs, which must eventually impair their integrity. It is well known to all physiologists, and I presume that the reader must have some knowledge of the facts, that the uterine organs, like those of digestion and respiration, can be overtaxed; and disease, in some form or other, is very apt to occur in overworked organs. If actual disease does not set in, debility of function or organ must eventually ensue. The uterus, like various other organs of the animal economy, must have periods of rest, or the day of reckoning will surely arrive. An excessive use of the reproductive organs generally, if not always, impairs the integrity of the nutritive organs, and vice versta; hence, if a cow is kept pregnant all the time for the unwise purpose of making her yield a constant supply of milk, it should not appear strange if she fall off in the quantity of milk, appear unthrifty, be off her feed, and have a glairy discharge from the vagina. These are the symptoms which usually precede abortion.

I lately visited an imported cow, the property of a gentleman in Illinois, who informed me that she had aborted three times at about the seventh month of her pregnancy, and she was generally put to the bull shortly after the mishap. I told him that this bad practice was the sole predisposing cause of the trouble; for the short space of time which occurred between the premature expulsion of the foetus and re-impregnation was not sufficient for the entire recovery of tone in the sexual organs; hence, so long as this practice continued, his cow would never go her full time. I strongly urge the necessity of spaying such an animal. She ought, at least, to be separated from the breeding cows, and her foetal calf, membranes, and placenta should be burned in the open air, for there is no safety in burying them a few inches under the earth's surface. 
It is very probable that many cases of abortion occur through a deranged condition of the digestive organs; therefore the means most likely to prove effectual in keeping the stomach in a healthy state should be adopted. What an immense amount of labor the digestive organs have to perform! They have to be almost constantly engaged in converting the elements of good and bad food into chyme and chyle; and they must not slacken much, else where are sixteen quarts, more or less, per day of milk to come from? How is the foetus in utero to be nourished, and the wear and tear of the cow's organism to be provided for, when the stomach is not in working order?

The practice of milking cows to within a short period of parturition is highly injurious, yet some persons with whom I have had conversations on this subject contend that, in some cases, it is impossible to "dry the cow." I advised them to reduce the animal's food to less than one-half, or even one-fourth, if necessary; in fact, there would be no harm in withholding food altogether for a short time, and in substituting for food a dose or two of aperient medicine. This course must necessarily soon lessen the amount of blood in the system, and as the milk is concocted from the blood, it will decrease in the same ratio, or, rather, be essentially lessened; and in view of reducing the quantity of milk, I also urge the necessity of keeping the cow on a small quantity of water. This practice may not, on all occasions succeed in arresting the lacteal secretion, still it is the only rational way of accomplishing the object.

Abortion is sometimes attributed to a debilitated state of the animal. This also arises from a derangement of the stomach. Then an opposite mode of treatment must be pursued, such as a change of food to that of a more nutritious character, and the frequent administration of small doses of ginger and golden seal.

Breeding cows require a great deal of care and some watching. Careful selection of the right kind of food for the necessary wants of the animal and her footus in utero is indispensable. Neither the one nor the other can be expected to enjoy good health on a diet of slops, swill, brewer's grains, corn-stalks, rotten potatoes, frozen turnips, damaged meal, musty hay, or sour apples, etc. Care is also requisite in providing for the animal's wants in regard to "watering." This is a subject of great importance, yet very few husbandmen give it that attention which its importance 
demands. The horrible stagnated stuff found in some pastures and water-troughs, which the thirsty creatures are compelled, from sheer necessity, to imbibe, is surely operative in producing very many unnecessary diseases, derangements, and abortions.

Some watching is necessary. A cow likely to abort is generally "off her feed," does not ruminate according to her accustomed activity; she fails to yield the daily quantum of milk; her condition is noticed to be unthrifty, and, on inspecting the vagina, it is observed to be the seat of a glairy discharge. Such an animal should be immediately isolated from the rest of the herd, and proceeded with as above directed. It is said that a very common cause of abortion is sympathetic influence.

There is another matter of some importance to be considered in view of prevention? I allude to breeding. It is my opinion that thousands of the abortions which occur among our domesticated animals of this country are for the benefit of the several races present and to follow; for if the offspring of cows whose systems have been debilitated by a too early use of the sexual organs, and those of other parents, laboring under organic disease, malformation, and stunted growth, together with the apology of offspring of cows doomed to drag out a miserable existence in some horrible "swill-milk establishment," where death runs riot, or runs into the milk-pail, to kill off unnursed infants, these being permitted to come into the world with their inherited imperfections, and being suffered to grow up and become parents, issuing a worse edition than the original copy in turn-these bad specimens, not of Nature's handiwork, but of man's ignorance and folly, or willful transgression of the law of Nature, being permitted to live, they would be so many libels on creative power; and, in order to remove them from the face of the earth, and put a check on the monstrous evil, a friendly pestilence must eventually ensue. This would be a mournful event; for many valuable animals might, by infection or contagion, be involved in the general ruin ; therefore Nature adopts the lesser evil, and applies the law of destruction prior to the birth of monstrosity.

The laws of reproduction and destruction are nicely and wisely balanced. "Thus far shalt thou go, and no further." The offspring of weak, emaciated, ill-formed, scrofulous, consumptive, and otherwise defective animals, should never be used for breeding purposes; in fact, they are not even fit for the butcher. 
Finally, I would advise breeders to let the female get her growth ere she is forced into copulation; for prior to maturity all the energies of her system are concentrated upon the perfection and integrity of her organism, and, until that important period arrives, the reproductive system can not be exercised without running great risk of violating one of the fundamental laws of Nature.

I have now directed the reader's attention to most of the causes likely to influeatce or induce abortion, and I leave the rest, if any there be, to be discovered by their own experience. It is impossible for me to furnish definite instructions as regards the treatment of individual cases, either prior or subsequent to abortion, as each require a certain course, according to the condition and prevailing symptoms; yet, if I have succeeded in pointing out only a few of the errors which prevail in our present faulty system of breeding and management, and have been practical enough to secure the reader's attention in a perusal of this article, I have accomplished more than I expected at the time of its composition.

\section{Inflamatory Affection of the Hind Limbs after Calving.}

Some cows, after calving, suffer from an inflammatory affection of the hind limbs, which renders them lame, or they are unable to use the parts with that promptitude which characterizes the physiological condition or state of health. The limbs are generally hot, and, in certain localities, are swollen, and the general health of the animal seems to be impaired, yet there is no great danger attending the condition. The tumefaction appears to consist of a local effusion (dropsy) of serum into the cellular tissue, which readily disappears through the local application of cold water, and the action of the calf on the teats.

The attention of veterinarians has been directed to this affection by LECOUTURIER, in the following paragraph, translated by Mr. Gamgee :

"A few days after parturition, and always when it has been normal, a difficulty in the movements of the hind-quarters is to be observed. The hocks become sensitive; the anterior surface swells up; the synovial capsule is disturbed; great pain is felt at the slightest touch, accompanied with heat. Then the appetite 
is lost; the secretion of milk diminished; pulse quick and strong; and a certain amount of fever sets in. The patient can not lie down, and still is not safe on its legs. The writer never saw the affection but on recently-calved cows; never after the ninth day, and almost always from the fourth to the seventh. This disease affects generally the good mother, and is of a most benignant nature. A friction of turpentine and alcohol is sufficient to restore to health in most cases; otherwise, brandy and soap. When the fever is strong, a dose of niter, with digitalis, is useful. This disturbance is always of a benignant nature, and is coincident with parturition, as the parturient fever, and a serous diarrhea, which occurs from the second to the third day after calving, easily checked, and never lasting more than twenty-four hours."

Treatment.-My treatment is as follows: Give the patient a drachm, three times per day, of fluid extract of gelseminum, and bathe the limb with a cold infusion of hops, just so long as inflammation and fever exist. Then prepare the following:

No. 99. Fluid extract of buchu........... 8 oz. Chlorate of potass.............. 2 oz. Water...................... 1 pint. Mix.

Dose, a wine-glassful night and morning. Rub the limbs with a portion of the following:

No. 100. Fluid extract of wormwood.......... 3 oz. Proof spirit................... $1 \mathrm{pt}$.

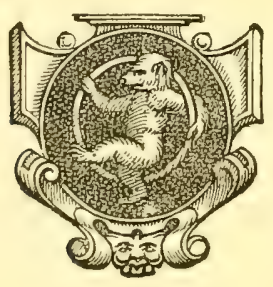




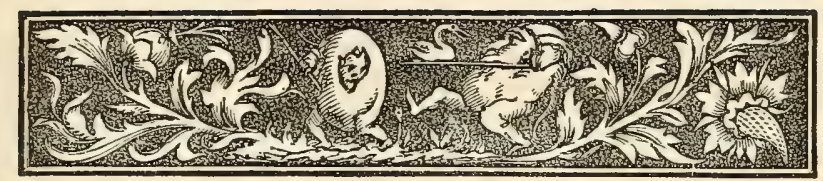

\section{SECTION XX.}

\section{DISEASES OF THE URINARY ORGANS.}

Description of the Urinary Ststejr-The Ureters-The Bladder-The URETHRA-HeMATURIA-INFLAMMATION OF THE KIDNEYS-URINART CALCULi-Calculi in tire Bladder-Black Water-Red Water.

\section{Description of the Urinary System.}

THE primary organs of the urinary system are the kidneys, 1 two ovoid conglomerate bodies, of a purple color, located in the lumbar region, or loins, separated from each other by the inferior part of the spinal column. They are generally imbedded in adipose tissue denominated suet. The kidneys are the great emunctorics of the body, are constantly engaged in secreting waste and morbid fluids from the blood, and we find them much larger in cattle than in horses. When the function of any other excretory organ is impaired, an extra amount of work is in store for them to perform. In health their function never ceases-a constant secretion of urine is going on all the time. Whether the animal be awake or asleep it matters not, there is no rest for the kidneys. On cutting through the kidneys, it is found to be composed of two substances, termed cortical and medullary.

The kidneys are supplied with blood by the emulgent arteries. They are of large caliber, and furnish a large quantity of blood from which the urine is secreted. Within the center of each kidney is a receptacle known as the pelvis, which terminates in a funnel-shaped outlet, the commencement of the ureter. The emulgent arteries, which supply the kidneys with arterial blood, are derived from the posterior aorta. They subdivide and ramify into the substance of the gland, and end in a very complex network. The emulgent veins are much larger than the arteries. They accompany the latter, and, finally, converge into one trunk, 
and terminate in the posterior vena cava. The nerves of the kidneys are derived from the renal plexus.

\section{The Ureters.}

Each kidney is connected with the bladder by a common duct or tube, known as the ureter. These ducts are much stronger and larger than those found in the horse. They are composed of two tunics. The external one is both fibrous and muscular in a longitudinal direction, which permits it to contract and extend in exact ratio as the bladder contracts when empty, and expands when filled. The internal membrane is of mucous texture, and is constantly lubricated with a mucous secretion, which defends it against the irritating qualities of the urine.

\section{The Bladder.}

The bladder of an ox is much larger than that of the horse. It is a musculo-membranous sac, or bag, and is located within the pelvis, bounded above by the rectum, and below by the internal surface of the pubic bones. In the cow we find the bladder located between the rectum and the uterine organs. The bladder has a transverse ligament inserted into the lateral parts of the pelvis. It has three coats. One is derived from the peritoneum, or lining membrane of the abdominal cavity, which gives the bladder an external covering. The middle cont of the bladder is composed of muscular fibers, which run in longitudinal and circular directions. The internal membrane, or coat, is more highly organized than the others. It is furnished with excretory outlets, from which issues a mucous secretion for its own protection.

The bladder is divided into fundus, body, and cervix. The fundus is that prominent part which faces anteriorly, or toward the intestines, and when the bladder is full of urine, it protrudes into the abdominal cavity. The body is the bulky or capacious part, generally more rounded, when full, than in the horse's bladder. The cervix is the neck, or contracted part of the bladder. It is surrounded by muscular fibers that involuntarily contract, so as to close the passage, except in the act of urinating. Some anatomists consider this muscle as a distinct one in the horse, and have given to it the name of sphincter ; but in oxen it 
does not seem to be a distinct muscle, neither does it contract with that force or persistency which is known to occur in the case of a horse.

\section{The Urethra.}

The urethra arises from the neck of the bladder, and extends from it to the end of the penis. It is much smaller in caliber than in the horse. It affords a passage for the urine and seminal fluid. Interiorly it is composed of mucous membrane, which is a prolongation of that found within the bladder.

\section{Hematuria.}

Hæmaturia signifies voiding of blood with the urine. In cattle-raising districts, this disease, or rather the symptoms of one, is generally known by the term "red water," although a very marked difference exists between hæmaturia and mere discoloration or reddened appearance of the urine; for in the former case blood globules are invariably present, and their presence can be determined by procuring a sample of the urine. After letting it stand for a short time, the blood coagulæ may be detected by the naked eye, at the bottom of the vessel in which the urine is caught.

Causes-Its Character and Symptoms.-The voiding of blood with the urine is generally supposed to be occasioned by some violence, such as local injury in the lumbar region, calculi within the ureters or the bladder; from local hemorrhage, occasioned by congestion, or inflammation of some portion of the urinary organs. Congestion is apt to occur in overfed animals, and, perhaps, is an effort of Nature to phlebotomize the subject and reduce the amount of circulating fluid, and thus lessen the liability to aceumulate adipose tissue. If such be the case (the patient being in a state of plethora), no immediate danger is to be apprehended. Especially is this the ease when the hemorrhage is merely passive, unaccompanied by symptoms of pain either at the time or between the periods of urinating. A case of this character might very properly be termed congestive hæmaturia, and may be treated on the same principles which prevail in the practice of intelligent physicians in the management of other local congestions. Nephritis (inflammation of the kidneys) may occasion hæmaturia, 
and is almost always accompanied by sure and unmistakable symptoms; namely, those of pain and irritation, either in the act or else before or after urinating. It may be difficult to determine what is the exciting cause of this inflammatory condition. It may be purely idiopathic, or may arise from the irritation consequent on the passage of urinary calculi through the parts in volved; and, notwithstanding there are certain diagnostic symptoms attending both varieties, still the medical attendant (who has just seen the case for the first time,) may find it hazardous to venture an opinion on its exact pathology; yet he shall not be at fault in treating the case as a local inflammatory affection. If it can be shown that the animal has been dosed with strong diureties, and no hemorrhage had existed prior to their administration, but is now quite profuse, we may safely conclude that the hemorrhage is occasioned by the local stimulus. It may, therefore, be laid down, as a general rule, that all cases of hæmaturia, attended with symptoms of pain, are oceasioned by the irritation of some foreign body acting on some part of the secretory surface of the urinary apparatus, or else is the result of some external violence; and it is the business of the person who prescribes to carefully consider these matters, in view of forming a correct diagnosis.

Hrmaturia does occasionally occur in nursing women of a plethoric diathesis. At the period of weaning the infant, the woman notices, on urinating, that the fluid is tinged with blood; but, as the act is unaccompanied by pain, and nothing of the kind being felt in the region of the kidneys, she feels no alarm about it, and searcely if ever consults a medical man on the subject. In the course of a few days, all things being favorable, the urine assumes its natural appearance; therefore, if hæmaturia shall appear in a cow at the period of "drying her up," as the saying is, or if a cow shall be observed to pass blood in the urine at the time of weaning the calf, and neither one nor the other manifest any discernible symptoms of pain, nor any perceivable manifestations of derangement in the vital functions, there will be no necessity to resort to any very active mode of medication, and an intelligent physician would merely recommend a mild laxative, light diet, and a little nursing. A few doses of liquor acetate ammonia might be indicated as a febrifuge. This may be given from a bottle.

Treatment.- Should the owner of the animal be unable to obtain 
the services of a professional man, let him prepare some flaxseed tea (a quart); then add half an ounce of powdered bloodroot; after which let the animal have six drachms of tincture of matico, diluted in a small quantity of water, night and morning. Should the disease be traced to the presence of urinary calculi, a lithontriptic will be indicated, which is prepared as follows:

No. 101. Muriate acid.................. 1 oz.

Water....................... 1 pt.

Fluid extract of hops............ 1 oz.

Dose, half the above quantity, night and morning.

\section{Inflamition of the KidNeys.}

Symptoms. - Cattle affected with inflammation of the kidneys will be observed to have some slight roaching of the back; that is to say, instead of the back or spinal column being straight or slightly concave, as it ought to be, it now presents a convexity, or, in other words, is arched in an upward or superior direction. The arching of the back is oceasioned by tonic spasms of the psoas and iliac muscles, located above the kidneys in the lumbar region. These muscles are known to butchers as "tenderloin." On making pressure over the region of the kidneys, the animal will be observed to flinch, the parts appearing very tender and hot. The animal is generally dull ; the muzzle, dry; a chilliness of the horns and external surface is observed, showing very clearly that there is an unequal circulation of the blood. There is, generally, some difficulty in passing the urine; the animal strains in the act of passing it, and it is redder than usual. The symptoms vary as the disease progresses, but the above are the principal ones on - which the diagnosis must be founded.

Treatment.-Drench the animal with twelve ounces of Glauber salts, dissolved in a quart of warm water; then apply warm-water bandages to the region of the loins, which may be retained in their place by encircling the body with a bandage. An emollient clyster of slippery elm should occasionally be thrown into the rectum, and the patient should be drenched with four ounces of the liquor acetate of ammonia every six hours, until an improvement takes place. Then give tincture of matico, in half-ounce doses, night and morning, until the patient is better. 


\section{Urinary Calculi.}

Cattle are occasionally the subjects of urinary calculi, yet there are not many cases of the kind on record; and, during a practice of twenty-five years, the author has never been called upon to prescribe for nor treat a case of this character. This testimony is diametrically opposed to that of YouatT and others. Youaxt contends that "concretions are oftener found in the urinary passages of eattle than of the horse." This is not in accordance with the experience of American husbandmen. In England, however, cattle may inherit some peculiarity of constitution. which causes a tendency to the formation of concretion; or perhaps the water they drink may be impregnated with alkalies, so as to induce an alkaline diathesis. Urinary calculi are generally composed of carbonate and phosphate of lime, carbonate of magnesia, and some animal matter.

Treatment.- Should calculi be present in either the kindeys or ureters they are sure to occasion some degree of pain. The urine will be reddened, and sometimes blood corpuscles can be detected in the same. After awhile some muco-purulent fluid will be discharged with the urine. In this stage, it might be advisable to give the animal one ounce of acetic acid, in sixteen ounces of. water, per day, to be continued a week or so. Should the general health be impaired, let the animal have a few doses of the following:

No. 102. Fluid extract of hops........... 2 oz.

Hyposulphite of soda........... $\frac{1}{2}$ oz.

Water..................... 1 pint.

Mix.

\section{Calculi in the Bladder.}

A stone once having formed in the bladder will defy all our attempts to dissolve or decompose it; yet if any one wishes to try the experiment, they can resort to the use of muriatic acid, sufficiently diluted with water to render it harmless to the animal tissues with which it may come in contact. The stone can, however, be removed by the operation of lithotomy, which will require the services of a skillful surgeon. In such a case the owner may possibly decide upon slaughtering the animal. Perhaps this is the best thing he can do. 
Symptoms.-There are various symptoms of deranged health which often attend stone in the bladder; yet, as they frequently accompany other diseases, and throw no special light on this, I shall take no notice of them, but offer something brief, yet more reliable. The presence of a stone in the bladder will generally occasion some interruption to that free and uniform flow of urine which takes place in a healthy animal without any symptoms of stone in the bladder ; consequently if, in the act of urination, the flow of fluid is suddenly stopped, to commence again a few moments afterward, at the same moment a slight trembling of the hind limbs is observed, and the animal strains ineffectually to urinate, only passing a few drops, and this state of things has existed for some time, it may be inferred that a stone is present in the bladder, and if so, the animal had better be slaughtered.

\section{BLACK WATER.}

A discharge from the urethral outlet of fluid quite black in color very frequently indicates a grave form of disease existing in the liver. Should black-colored matter be detected in the urine, the probabilities are that the animal will die; for the disease which gives rise to the abnormal discharge is probably organic.

Symptoms.-In some cases black water is occasioned by engorgement of the liver, spleen, or kidneys, and when an animal dies of these engorgements, we find the capillary veins much distended; and these engorgements frequently occur, also, in many of the diseases of an epizoötic or malignant character. Such condition or congestion is owing to a loss of equilibrium in the circulation. The arteries are very impressible to excitation, and hold out their physiological actions the longest. The veins being more distensible than arteries, the blood is forced into the venous radicles faster than they can absorb it; hence the free egress of blood is retaraed. The result is an accumulation of blood, distending the organs or veins, producing engorgements and a darkened color of the blood and tissues. The blood assumes a darkened color partly from delay and partly from deficient pulmonary absorption and aeration.

The causes of these local congestions are not always to be determined, yet they almost always receive their chief force from a morbid habit pervading the general system; at least this is the case when no local disease exists to account for them. 
Treatment.-The principal object in the treatment of black water is to equalize the circulation and decarbonize the blood. Diffusible stimulants and nauseants fulfill the first indication, and ammonia the latter; therefore $I$ use the following:

No. 103. Fluid extract of ginger........... 1 oz.

Chlorate of potass............... 1 oz.

Spirits of ammonia............. $2 \mathrm{dr}$.

Water........................ 1 pint.

Give the above quantity once daily.

\section{RED WATER.}

The changes which occur in the color of the urine are the results of various causes. In a preceding article the reader is informed that hæmaturia (voiding of blood with urine) is traceable to certain direct causes, hence the difficulty is of an acute character. Now, suppose we take a well animal as a subject for experiment. We administer repeated doses of sweet spirits of niter or tincture of juniper. The first thing we notice is, the urine is augmented, or rather more profuse than usual, and of a pale color. Continue the medicaments over a given period, and the urine assumes a darker color-first, yellow ; next, brown, or coffee color, purple, or even black. This shows conclusively that the color of the urine can be altered by the use of medicinal agents; therefore, if a reddened appearance of the urine follow the exhibition of strong diureties, the cause is plain enough, and the cure is effected by discontinuing the diuretic medicine, and in restoring the equilibrium of action which should exist among the various excretory orgaris. Various kinds of food are also operative in producing changes in the color and quantity of the urinary secretion. Beets and earrots, when fed in quantity for a sufficient length of time, alter essentially the color of the fluids of the body, urine included. As regards quantity, musty oats and an excess of stimulating food are notorious causes in explanation of augmented and discolored urinary secretion.

Discoloration of the urine, which some of our Western farmers term "red water," is usually occasioned by derangement of the liver and other parts of the digestive apparatus. The urine, however, is more likely to have a tinge of brown or yellow than red, and, therefore, several writers, Mr. Youatr included, have termed 
such cases "chronic red water;" and they all agree that such an affection is not primarily one of the kidneys, but of the liver, and I presume that the "red-water" which prevails among cattle in the West owes its origin to the same causes as above. For example: A friend informs me that he lost a valuable cow, "which died of red water," and that the urine was of a "yellow-brown color," indicating, very clearly, that the animal labored under an affection of the liver; and, in view of dispelling all doubt on the subject, I here introduce a short paragraph from my correspondent's letter: "On skinning the animal, I found that the parts beneath (subcellular tissues) had a yellow appearance. On cutting open the abdomen, I noticed that the liver was of a very dark color, and appeared to be filled with black blood. The gall-bladder was very large, and appeared darker than usual; the manyplies (manyplus) was full of caked food, and there were no appearances of disease in any other parts."

Of course, it will be perceived that my correspondent was far from being an adept in the art of autopsy; however, the appearances which he has described lead us to the necessary conclusion that the so-called red water (which he contends caused the death of his cow) was the result of functional, perhaps organic, disease of the liver.

Mr. Youatr, when discussing the theory of the pathology of chronic red water, offers the following:

"Chronic red water is more prevalent than that which is acute, and in its first stage is far more a disease of the digestive organs, and especially of the liver than of the kidney. The urine is observed to be of a brown color, or brown tinged with yellow. The beast feeds nearly as well as before, but ruminates rather more lazily. In a few days a natural diarrhea comes on, and the animal is well at once; or a purgative drench is administered, and a cure is presently affected. This occurs frequently in cows and calves of weak constitution. At other times there is manifest indisposition. The animal is dull, heavy, languid; the ears droop, the back is bowed; she separates from the herd; she refuses her food, and ceases to ruminate. Presently she gets better; she rejoins her companions, but this is only for a little while. The urine, which at first was brown, with a tinge of yellow, has now red mingled with brown, or it is of the color of porter. It is increased in quantity; is discharged sometimes with ease, at other times with considerable straining- 
in little jets, and with additional bowing of the back. The milk diminishes; it acquires a slight tinge of yellow or brown; the taste becomes unpleasant, and it spoils all that it is mingled with. The pulse is accelerated; it reaches to 60 or 70 . If the blood is drawn, the serum which separates from it is brown. The skin is yellow, but of a darker yellow than in jaundice; it has a tinge of brown. The conjunctiva is also yellow, inclining to brown. The urine becomes of a darker hue, and is almost black. The animal usually shrinks when the loins are pressed upon. Occasionally there is much tenderness, but oftener the beast scarcely shrinks more than he is accustomed to do when laboring under almost every disease. The belly is not so much tucked up as drawn together at the sides. There is considerable loss of condition; the legs and ears get cold; the animal is less inclined to move; there is evident general debility. In every stage there is costiveness, and that exceedingly difficult to overcome, but, on close inquiry, it is ascertained that there was diarrhea at the beginning, and which was violent and fetid, and which suddenly stopped."

Testimony of this description, emanating from reliable authority, is entitled to our consideration; and it should teach us to seek for the cause of discolored urine beyond the region of the kidneys, except in those cases which are evidently the results of the action of diuretics or stimulants in the form of improper food. It will generally be found that discolorations in the urine are the symptoms of a disease located elsewhere; hence the disease should be treated instead of the symptoms.

Treatment.-If the discase appears to be located in the liver, indicated by a yellow tinge of the visible surfaces, dark-colored feces, and yellow tinge of the urine, the animal being dull and sleepy, showing symptoms of febrile action, it may be proper to administer a dose of purgative medicine, combined with some agent calculated to restore the physiological action of the liver, and I therefore recommend the following:

No. 104. Epsom salts................... 12 oz. Podophyllum peltatum (mandrake)... $2 \mathrm{dr}$.

Dissolve the salts in tepid water, one pint; then add the podophylium, and administer the same by means of a "drenchinghorn," or bottle.

In administering medicine to cattle, our object is to get it beyond 
the rumen or paunch into the digestive compartment of their complex stomach, and, in aiming to do this, we pour the medicine down the œsophagus slowly. After the bowels have responded to the action of the medicine, it may be proper to administer alteratives which are known to change morbid action. Powdered sulphur and mandrake may be given, in small doses-not sufficient of either to keep up the cathartic action of the salts, but merely to keep the bowels in a soluble condition for a day or two; and if there be any danger of superpurgation, neither mandrake nor sulphur are indicated. In such case I should give an occasional dose of powdered golden seal, two drachms, and the same quantity of carbonate of soda. The patient should be fed sparingly; and if the season permit, a run in the pasture will prove beneficial.

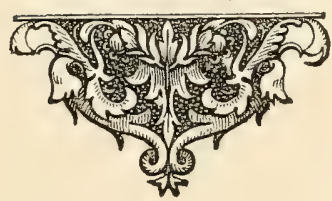




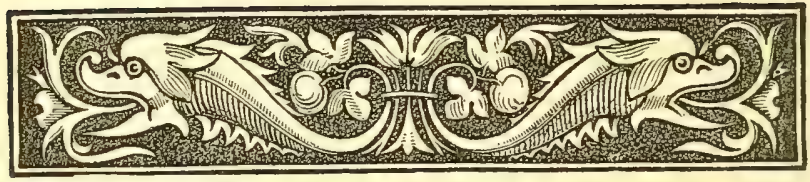

SECTION XXI.

THE HEART-ITS FUNCTION AND DISEASES.

Description of the Heart-The Heart's Function-Diseases of the HeartDilatation of the Heart-Pericarditis-Hypertrophy-Endocarditis.

\section{Description of the Heart.}

THE heart is a wonderful and powerful piece of muscular 1 mechanism. Its function is of the involuntary order, so that regular contractions and expansions, or beatings, occur in the normal state, without the knowledge and consent of the animal. These contractions and expansions, however, can be modified by means of various medicinal agents which act upon the nervous system, thus producing a sort of mixed action (voluntary and involuntary). For example, all medicines known as sedatives operate so as to depress the heart's action, and lessen, for a given period, the number of its pulsations; while, on the other hand, stimulants augment the action of the heart, and increase the number of its pulsations. The average weight of the heart of an ox is eight pounds, yet it is frequently the seat of fatty degeneration, when its weight and bulk is then materially increased.

The heart is anatomically divided into four cavities, two of which being in a superior and anterior direction, and, in consequence of bearing some resemblance to the ears of a dog, are termed auricles. These cavities, known as right and left (or rather anterior and posterior), are divided by a wall, or septum, known as the septum auriculorum. The right auricle is the receptacle for venous blood, and three venous trunks terminate in it; namely, the anterior vena eava, which returns the venous blood from the anterior extremities, head and neck; next, the vena cava posterior, which returns the venous blood from the posterior parts; and, lastly, the coronary vein. The latter returns (540) 
blood which has circulated through the heart itself for its own nourishment. A considerable quantity of dark, venous blood is generally found in this auricle after death, and it opens into the right or anterior ventricle by an aperture denominated the auriculo-ventricular opening; yet, in consequence of a valvular contrivance within the ventricle, the blood can not recede into the auricle.

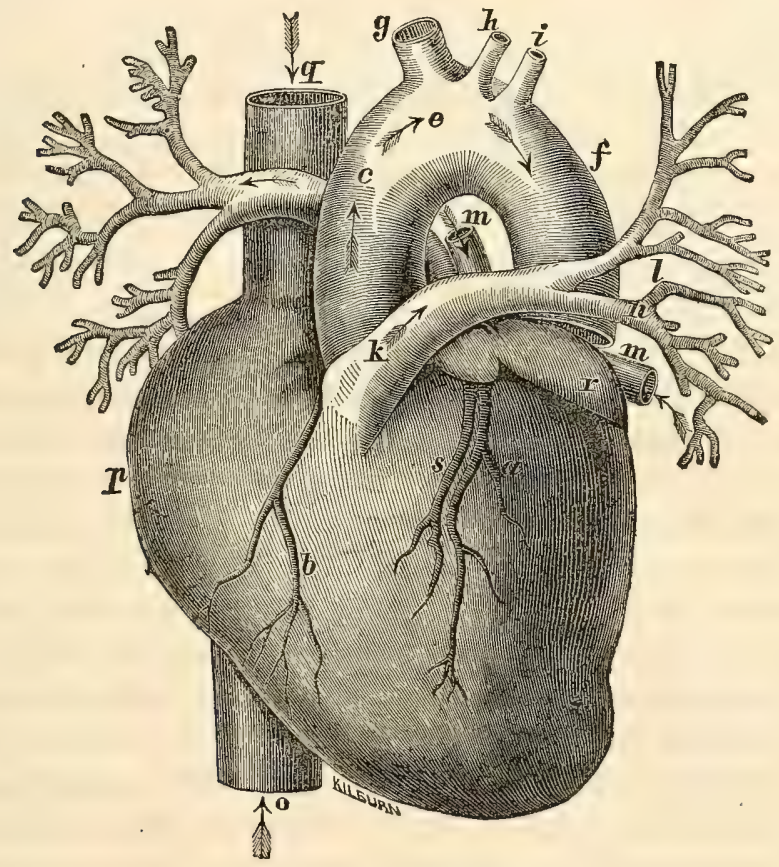

THE HEART; ITS CIRCULATION.

Explanation. $-a$, The left ventricle; $b$, The right ventricle; $c \in f$, The aorta; $g h i$, The carotid and other arteries springing from the aorta; $k$, The pulmonary artery; $l$, Branches of the pulmonary artery in the lungs; $m m$, The pulmonary veins emptying into the left auricle; $n$, The right auricle; $o$, The ascending vena cava; $q$, The descending vena cava; $r$, The left auricle; $s$, The coronary vein and artery.

Internally, the right auricle is lined by a glistening vascular membrane, having, on various parts of its surface, small muscular eminences, termed musculi pectinati. The small cavities which occur, in consequence of this arrangement, are termed cul-de-sacs. The right or venous ventricle is also lined by a nicely-organized membrane, and has beneath it several muscular prominences, named carnoe columnoe, which give origin to as many tendinous 
slips. These are known as cordee tendinoe. They are inserted into a fibrous membrane in the region of the auriculo-ventricular opening, and then get the name (membrane included) valvula tricuspis. The lateral contractions of this ventricle are aided by small tendinous cords, having muscular origins from the wall and septum. The venous blood passes from this cavity into the pulmonary tissues of oxygenation, through the pulmonary artery, which emerges from the superior part of the ventricle. At the commencement of the pulmonary artery are found three valves termed semilunar. Their function is to guard against a retrograde movement of the blood, so that it has no other channel than that which leads to the lungs.

The left ventricle has scarcely any anatomical or structural differences from those observed in the right, although its cavity is smaller, and its walls are somewhat thicker, than those found on the right. It receives the blood from the lungs after purification, by means of the pulmonary veins, which have four openings into this cavity-two proceeding from the right and two from the left lobes. The left or arterial ventricle is the reservoir for arterial blood, which is destined to reanimate, replenish, and perpetuate the vital economy. Having a vastly more important function to perform (which requires augmented muscular mechanism) than its duplicate found on the right side, the thickness of its walls must, therefore, necessarily exceed those of the right. This is found to be the case, so that the outer wall of this is about three times as thick as that found on the other side; and this guide is useful to us in determining at sight, after the heart is detached from the body, which is the left ventricle, and vice vers $\hat{a}$.

The channel of communication between the left auricle and ventricle is named, as is the case on the opposite side, auriculoventricular opening. It is furnished, however, with only two instead of three valvular openings, termed valvula bicuspis, or mitralis. 'This ventricle is one of importance for our consideration, from the fact of the great aorta (the plastic hose, which seldom if ever requires cobbling or repair) here originates. Its margin, or outlet, is guarded by a complete set of valves, three in number, termed semilunar, similar to those found at the origin of the pulmonary artery. This ventricle is divided from the one on the opposite side by a muscular and tendinous vartition termed septum ventriculorum. 
Form, Situation, and Attachment of the Heart.-Its form describes that simulating a cone having a body, base, and apex. Its base being in a superior direction, it follows, as a matter of course, that its apex has an inferior insertion downward and backward. The heart lies in the region occupied by the fourth, fifth, and sixth dorsal vertebra, right in the central region known as the cavity of the chest. Its apex is inclined to the left side. It appears that in the ox the heart differs in construction from that of the horse in the following peculiarities: There is a bone found in the heart termed os cordis, the function of which has never been explained; yet it is evidently intended for some useful purposeperhaps to give strength and durability to the sanguinous forcepump. Next, we notice some difference in the internal mechanism of the right ventricle. There are several fleshy bands, running across from side to side, which prevents abnormal dilatation; for, taken as a whole, the venous system of the bovine species is more developed than in the horse-the veins are larger and more numerous, and more blood is constantly accumulating in the right auricle and ventricle; hence the need of additional muscular mechanism.

\section{The Heart's Function.}

The blood having gone the rounds of the circulation, enters the anterior or right cavity (auricle). It then passes into the venous or right ventricle. By the contraction of this ventricle the blood is forced through the pulmonary arteries into the lungs; there having undergone certain changes (which have been alluded to in another part of this work), it returns by the pulmonary veins to the left auricle; from thence it descends into the left ventricle. The contractions of the left ventricle force the blood into the great aorta (anterior and posterior), which gives rise to a numerous set of arteries, through which channels the blood reaches the extreme parts of the system.

\section{Diseases of the Heart.}

Diseases of the heart in cattle are very common, yet they frequently elude detection, simply because the symptoms are very obscure. Mr. GasigeE contends that the most common cause of diseases of the heart in cattle is the passage of needles, and other 
foreign bodies, from the reticulum through the diaphragm. At the same time, he alludes to many drawings in his possession of polypi in the heart, growing from the auriculo-ventricular valves; also to drawings of deposits beneath the endocardium (lining of the heart's eavity) ; of tumors, also, outside the heart, consisting in masses of cysts, and due to the development of hydatids of the echinacoccus veterinorum.

\section{Dilatation of the Heart.}

The heart is often the seat of dilatation, or amplifications of one or more cavities, with attenuation of the walls of the same. This dilatation is supposed to arise from the mechanical effect of over-distension.

Cause.-The cause of dilatation is deficient power in the heart in proportion to that of the system. This may be acquired or it may be congenital. All obstructions, also, to the circulation, whether situated in the orifices of the heart or in the aortic or pulmonary systems, may induce this dilatation.

Symptoms.-The pulsations are feeble and oppressed, and, on moving the animal about, he becomes distressed; the pulse is soft and feeble; languor in the arterial circulation, as shown by the congested state of the mucous surfaces. The respiration is somewhat affected, not primarily, but through the intervention of irregular nervous action. The jugulars are engorged, and various parts and organs of the body are congested, and the surface and extremities are below the natural temperature.

Treatment.-The principal object should be to improve the general health, by alteratives and tonics. The surface of the body should be kept warm, and often rubbed, so as to excite capillary action. This will also have the effect of relieving the venous congestions. Any hygienic measures, regarding both diet and management, will certainly do good. Pure air, for example, is absolutely needed, to vitalize the blood as it tardily courses the veins. Without such there is very little chance of restoring the animal. The alterative and tonic may be thus prepared:

No. 105. Iodide of potass............... $\frac{1}{2}$ oz.

Fluid extract of sassafras........... $3 \mathrm{oz}$.

Fluid extract of hops............. $1 \mathrm{oz}$.

Water....................... 1 pint. 
Mix, and dissolve the potass in the water; then add the other ingredients. Dose, one-fourth of this quantity night and morning.

\section{The Pericardium and its Diseases.}

The pericardium is a membranous bag that surrounds the heart. It is known to the butchers as the "heart-bag," and is attached to the sternum, diaphragm, and to the roots of the large bloodvessels at the base of the heart. It is composed of two layers, united by cellular tissues. The external one is composed of fibrous tissue; the internal one is a smooth, serous membrane, similar to that which lines the cavity of the chest. This serous membrane is studded by numerous vessels called exhalents. From these issue a vapor of fluid, the purpose of which is to guard against friction. The insertions of the pericardium are such that it confines the heart in its proper situation, and aids in sustaining a reciprocal action between the heart and lungs. It is often the seat of a disease known as pericarditis.

\section{PertCarditis.}

This disease consists of inflammation of the pericardium, the causes of which are supposed to be nearly the same as those of pleurisy, or inflammation of other serous membranes; namely, sudden impressions of cold on the external surface, violence, etc. A special cause has also been assigned for this disease; namely, the penetration of the pericardium by foreign bodies, such as nails, needles, wire, etc., coming from the interior of the stomach, and working their way through it into the former.

Symptoms.-There is considerable increase in the force of the heart's pulsation; the jugular veins are engorged (larger than usual), and an undulating movement is observed in them. There will be acute inflammatory fever, generally preceded by rigors, shivering, and coldness; the visible surfaces of the nostrils and eyes are highly reddened in the early stages, and rumination has ceased, with loss of appetite. The animal occasionally directs its head near the region of the heart, and if pressure be made on the sternum (breast-bone), it elicits distressing symptoms. As the disease progresses, the jugulars increase in volume, the pulse becomes feeble, and an odematous or dropsical state of the lower 
parts of the thorax sets in. This latter feature indicates that a considerable quantity of fluid is present, both in the pericardium and thorax. In this stage the case is incurable.

Treatment.-In the early stage of pericarditis, the treatment should be about the same as that recommended for acute pulmonic and pleuritic affections. Iodide of potassium, in doses of twentyfive grains per day, may prove serviceable; and some form of diuretic medicine may also be exhibited, to increase the action of the kidneys. So soon as the patient shows signs of amendment, tincture of matico, in ounce doses, may be given, twice daily, until the patient has fairly recovered.

The London "Veterinarian" publishes the following case, furnished by Surgeon LEPPER:

"I herewith send you what $I$ think is a rare specimen of a diseased heart, with its investment, which was taken from a cow of the short-horn breed, about seven years old, the property of Mr. Bliss, of Windmill Hill, Waddesdon. On the fourth day after calving she was noticed to fail in her appetite, and her milk also became greatly diminished in quantity. In walking across the yard she staggered and fell. She laid for some considerable time, when, with difficulty, she got up and walked to a shed, where she soon laid down again. It was in this condition that I first saw her. The expression of her countenance did not materially differ from that of a healthy animal. The bowels were regular in their action; the pulse did not exceed fifty beats in the minute, and was soft and full. On the left side of the chest there was an emphysematous swelling, extending over four or five of the middle ribs, and about eight or ten inches wide. On auscultating the chest, I could not discover any unusual sound in the respiratory action; the breathing, also, was regular. The jugular veins were filled to repletion, so as to be observable when standing by the side of the animal - a state of things which $I$ have invariably found to exist in effusion into the pericardium, and, especially, if much fluid is present. I felt satisfied that this was a case of this description, and, consequently, formed an unfavorable opinion as to the issue. To relieve the distended vessels, I took away eight or ten pounds of blood, and gave a slight purgative, which appeared to afford temporary relief. For two or three days the appetite improved, and the cow appeared to move with greater freedom and less pain. On the sixth day after my first visit she 
suddenly relapsed, and died on the seventh. This animal had, for some time past, wasted in flesh, but, as her general appearance was healthy, little notice was taken of it. It is surprising that she should have gone on so well as she did with so extensive disease of the heart. As to the nature of the affection I shall offer no remarks, but leave this to be explained by you."

The following remarks are added by the editor of the abovenamed journal:

"This was but another of several specimens which we have received, from time to time, in which the external surface of the heart was covered with layers of effused and partially-organized fibrine. The pericardium was thickened to three times its natural substance, and within it was contained several ounces of colorless serous fluid. The effused fibrine hung in grapelike appendages from the base of the heart, each of which had a flocculent condition. These bodies were devoid of color, and had altogether such an appearance as would lead us to doubt whether the effusions resulted from active inflammation. The muscular substance of the organ, and its internal lining membrane, were alike free from organic change."

The following case, which occurred in the practice of Mr. WrLliams, may also prove interesting and instructive to the reader:

"On the 16th of June, I was requested by Mr. Thomas Tatum, Gwernafield, to attend a cow of his. Her history he gave as follows: 'She has been unwell since she calved, three weeks ago. The fotal membranes were not expelled for a fortnight after the event, and they came away piecemeal, in a state of putrescence. Her appetite has been very capricious all along, and her bowels rather costive. A discharge of white matter has taken place from the vagina for the last week or so. She gives but little milk. The breathing is tranquil, and she does not cough. She has a peculiar stare in the countenance, and takes a great deal of notice of every thing.'

On inspection, I found her as the owner had stated, the symptoms, to a casual observer, being any thing but indicative of extensive organic changes, but more of general debility after parturition. She was rather emaciated. On closer examination, I found the mucous membranes to be rather pallid; the mouth, cool and moist; the temperature of the body but slightly below the 
natural standard, and equal over all parts of the body; the pulse, very weak, beating 96 in the minute, and regular; the breathing only very slightly hurried.

After advancing thus far in my examination, I must confess that the case fairly puzzled me, and I was inclined to look on it as one of general debility and leucorrhea; but as the discharge from the vagina was very slight, I thought it could not be the sole cause of the loss of appetite and other symptoms present. I now auscultated the chest, and, on applying my ear, the respiratory murmur was heard distinct and natural, but I thought a little increased at the anterior portion of both sides. On auscultating the region of the heart, I failed to detect the sounds of that organ, and on still further examination, both the sounds and the impulse were indetectible. This struck me as being curious, but, on account of the regularity of the pulse, I did not consider either the heart or its membranes to be the seat of disease, and treated the case as one of general debility, loss of appetite, and leucorrhea. The animal lingered for about four weeks more, the symptoms remaining nearly the same as at the time when I first examined her, with the exception of increased emaciation.

The post mortem examination revealed anæmia, the tissues being exceedingly pale and sodden, the blood in the vessels scanty and pale, and scarcely staining the fingers. The abdominal viscera were healthy, with the exception of the liver, which was congested. The gall-bladder was distended with dirty, lemon-colored bile, of thin consistence, and seemingly containing a large amount of mucus. On opening the cavity of the thorax, the pericardium was seen distended to an enormous extent, filling the floor of the cavity, and adherent to the sternum and pleura costalis by a thick layer of exuded lymph, this having no appearance of organization, but seemingly consisting of a shapeless basis-substance, easily separated from the parts it was in contact with. The cavity of the pericardium contained in its inferior part about two pints of a fetid, whey-like fluid. With the exception of that part which contained this fluid, the cavity was obliterated by exudation, matter about two inches in thickness surrounding and gluing together the visceral and parietal surfaces of the pericardium. The heart itself was flabby, pale, and its cavities contained a small amount of blood, of the same thin consistence as that in other parts of the body. The endocardium was healthy. The lungs 
presented no appearance of disease; they were pale, excepting in the parts lying undermost after death. The pluræ, excepting where they were involved in the pericardiac disease, presented no abnormal appearance. The thoracic and abdominal cavities contained a little fluid.

Reflecting on the appearance presented by the pericardium, by its being adherent through the accompanying pleuritis to the walls of the thorax and sternum, and by the heart itself being thus in a manner bound down and fastened by a thick layer of plaster, and elastic material existing between it and the ribs, I was satisfied that the absence of the sounds of impulse was due to these pathological changes, and not, as I had foolishly supposed, to general debility. But, before giving publicity to this opinion, I thought it better to wait for more proof.

September 4, 1857, I was called to attend a cow, the property of W. Gregg, Esq., Syntroll, Mold. I had attended her in May previous for 'retention of the foetal membrane.' She seemed to be very languid and depressed for some days before and after the removal of these; but, as she fed pretty well, I was not requested to prescribe for her. She had given milk freely all the summer, but had not appeared so lively as she ought to be. She seemed as well as usual on the evening of the $3 d$ of September. On the morning of the 4th her quantity of milk was diminished. On examination, I found her wet and cold from the rain of the previous night, standing 'all of a heap ;' head depressed, and ears pendulous; mouth and nose, cold; breathing, tranquil ; bowels, costive; pulse, 100, very feeble, but regular; the mucous membranes slightly tinged yellow. Auscultation failed to detect the sounds of the heart, and the palpitation was absent. I pronounced the case to be one of pericarditis, and that exudation had taken place to some extent; also that congestion of the liver existed, this being secondary, and owing to the feebleness of the circulation.

The treatment consisted in the administration of purgatives and stimulants. Counter-irritants were also applied to the sides, and as the case advanced, tonics were given. But nothing seemed to affect the pulse; it remained feeble throughout, and it was only a few days before death that irregularity was detected.

On Friday, September 18, diarrhea set in, and on the 21st œdematous swellings appeared in different parts of the body, but more 
especially on the dewlap and fore-parts. On the $23 \mathrm{~d}$ she dropped down and died without a struggle.

All throughout, this animal exhibited the same watchful state as observed in the first case, and the appetite was very capricious indeed. One day she would feed pretty well; then, again, she would not touch any thing for days. The bowels were very irregular, and, on account of rumination being suspended, the food passed through her in a half-masticated state.

The post mortem, four hours after death, revealed general anæmia of the subcutaneous and muscular tissues, these being seemingly in a broken-down condition. The blood in the veins was pale and thin. The abomasum, small intestines, and liver were congested; but even here the blood was of the same watery character as in other parts. The gall-bladder was distended with thin, lemoncolored bile. The thoracic eavity contained rather a large amount of clear serum. The pericardium was distended by a layer of lymph, about two inches in thickness, gluing together its two surfaces, and containing, near the apex of the heart, nearly a pint of dirty, whey-colored fluid, of a fetid odor. The lymph was easily removed from the heart, and between it and the muscular tissue of the organ was a layer of cartilaginous substance, about a quarter of an inch in thickness, covered by a serous glistening membrane. Toward the apex of the heart this had formed bands of connection between the two surfaces of the pericardium, but in all other parts it was confined to the visceral pericardium only. The lungs were slightly congested. The pluræ were as described in the former case."

\section{Hypertrophy of the Heart.}

Hypertrophy signifies augmentation-thickening of the muscular substance of an organ, resulting from increased nutrition. It generally pccurs in animals of the sanguine temperament, of vigorous health, their muscles being much harder and firmer than usual. In such animals the blood is rich, and nutrition is very active. I have often visited market for the purpose of inspecting the hearts of slaughtered oxen, and I have observed that hypertrophy generally occurs in the left ventricle. Sometimes its walls are thickened, the cavity retaining its natural dimensions. Occasionally the cavity is dilated and the wall thickened, but, in the 
majority of cases, the walls are thickened and the cavity diminished. This constitutes the disease known as true or concentric hypertrophy (enlargement).

This disease, when moderate and uncomplicated, is not productive of much inconvenience to the bovine species, whose motions are slow; yet occurring in a horse, it would produce much inconvenience, and render the animal almost useless for speed.

Symptoms.-A person unacquainted with the heart's sounds will find it impossible to diagnose a disease of this character; in fact, such a disease will sometimes defy the scrutiny of experts. A deadened sound is always heard when the ear of a person is applied to the region of the heart, yet this depends somewhat on the form of the disease, its complications, the nature and intensity of the exciting causes, and the condition of the patient. On applying the finger to the pulse at the angle of the jaw, or on the temporal artery, it will appear that the circulation is embarrassed.

Treatment.-No rules can be laid down for the treatment of this affection, and the only agent of any value is iodine, or iodide of potassium. It is possible, however, that in a case of this character the butcher may prove to be the best doctor.

\section{ENDOCARDITIS.}

Endocarditis is an inflammatory affection, located in the membrane within the heart. It is a rare disease among cattle, yet very frequently occurs among horses.

Symptoms.-Like all inflammatory conditions, this is attended with heat about the base of the horns; hot and feverish mouth; vitiation of the secretions; cessation of rumination; some degree of suffering, of a peculiar character, not noticed in any other affection. The pulse is violent, abrupt, strong, full, and may range up to eighty; every motion the animal makes accelerates the respiration; yet the lungs show no signs of disease. On examining the heart after death, its internal membrane is thickened, and appears of a dark purple color; clots of extravasated blood are also present on its internal surface.

Treatment.-Administer a cathartic drench, say about sixteen ounces of Glauber salts in a couple of quarts of tepid water; then give half an ounce of powdered nitrate of potassa in a little water, every four hours, until the force of the pulse is essentially lowered. 
In the mean time, administer an occasional clyster of soap and warm water.

This disease has occasionally a rheumatic origin; so that if the animal be taken suddenly with inflammatory muscular lameness assuming the features of rheumatism, shifting from one limb to another, and then leaving the region of the first attack and going to the heart, I should prescribe two fluid drachms of wine of colchicum, in a gill of water, every morning, and at night, twentyfive grains of iodide of potass, in half a gill of water.

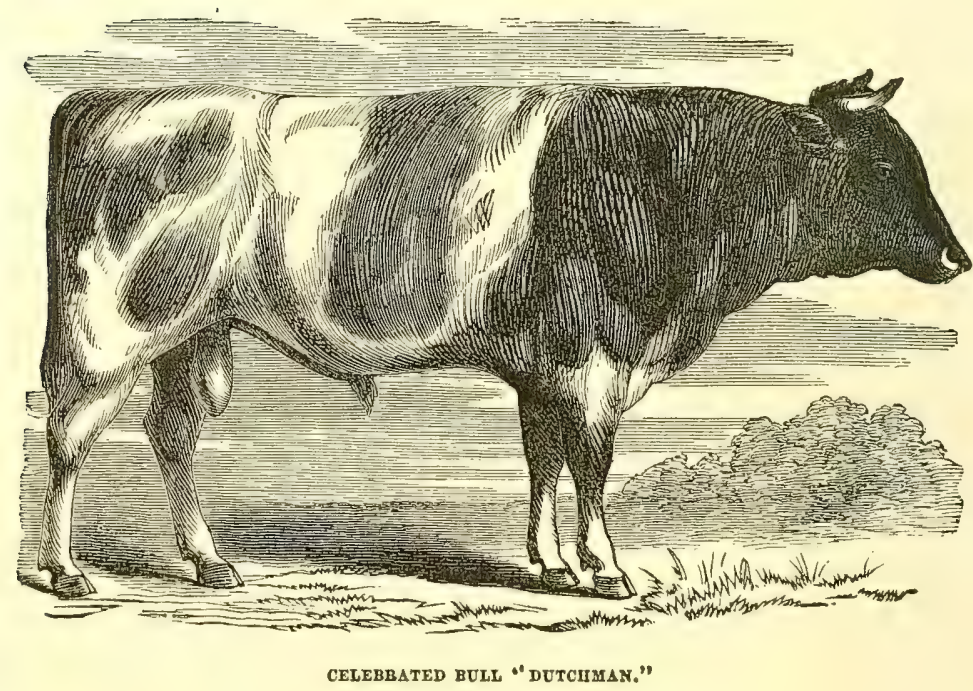




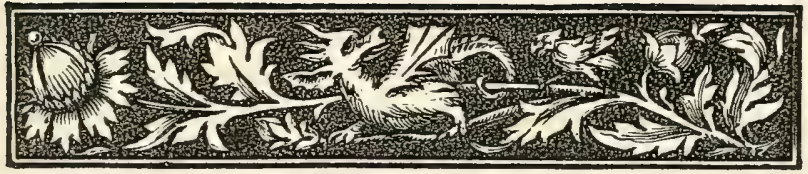

\section{SECTION XXII.}

\section{DISEASES OF THE EYE AND ITS MEMBRANES.}

Description of the Eye-Humors of the Exe-Coats of the Eye-Muscles of the Exe and Eyelids-Ophthalmia-Foreign Bodies in the Eyes-Specific or Periodical Ophthalmia-Cancer in Cattle.

\section{Description of the Eye.}

7 THE eyes of oxen are frequently the seat of various forms of 1 disease, hence it is necessary that the farmer should know something of their wonderful mechanism; I therefore propose to give a description of the same. The eye is protected by a bony casing known as the orbit, which appears to be much stronger than that found in the horse. In the interior part we find a large quantity of fatty matter which acts as a soft cushion for the eye to repose on, and, at the same time, as the fatty matter accumulates, it gives prominence to the eye and increases the range of vision.

\section{Humors of the Eye.}

The humors of the eye are named aqueous, crystalline, and vitreous-three in number. The aqueous humor fills the interval between the cornea and crystalline lens, and is surrounded by a capsule of its own, on the inner surface of which is secreted the aqueous humor. The uses of this limpid fluid appear to be to transmit the rays of light and permit free motions of the iris. The fluid is frequently evacuated and reproduced.

Crystalline Lens.-This is situated in a concavity behind the aqueous humor, and anterior to the vitreous, opposite to the pupil. In appearance it resembles a crystal or lens of magnifying glass, and is composed of concentric lamellæ, and these of radii. Its form is spherical, yet the posterior surface is more convex than 
the anterior. Like the other humors of the eye, it is enveloped in a capsule, and the disease known as cataract consists of altered structure in the lens or its capsule; sometimes both are involved. Acids, alcohol, and boiling water convert it into an opaque, solid body, resembling the white of an egg when boiled. The function of the erystalline lens is to concentrate the rays of light so as to form a distinct image on the interior of the eye.

Vitreous Humor.-This jellylike substance derives its name from its glassy appearance. It occupies the posterior concavity of the globe of the eye, known as the "dark chamber." It fills the membranes, aids to maintain the form of the eye, and keeps the crystalline lens at a proper and physiological distance from the retina. Anatomists have hitherto failed in detecting any signs of vascularity in the vitreous humor.

\section{COATS OF THE Eye.}

1. Tunica Conjunctiva.-This membrane is very highly organized, lines the eyelids, and is reflected over the anterior part of the eyeball. It covers the region known as the pupil, yet is pervious to the rays of light. Previous, however, to being reflected over the globe, it gives a covering to the membran beneath, carunculæ lacrymale, and puncta lachrymalia.

2. Tunica Sclerotica.-This tunic is made up of fibers, which are very dense, yet elastic. It constitutes what is commonly known as the "white of the eye," extends from the junction of the optic nerve with the globe to the cornea, and, more than any other tunic of the eye, tends to preserve its form. It also affords attachment for most of the muscles of the eye-the retractor and four recti.

3. Cornea.-This is a dense and compact membrane, yet composed of laminated structure, which accounts for the opacity or "filmy" obscurity so often encountered in veterinary practice; for, in a physiological condition of the eye, the laminæ are compact, like a house newly shingled; but, in consequence of untoward circumstances, a leak occurs; a watery exudation then sets in. The cornea may be considered as the shield that protects the eye, yet, in consequence of its transparency, it is also operative in the production of vision.

4. Iris.-This is the movable curtain of the eye, adapts the eye 
to vision, possesses the property of contraction and expansion. In its center is a perforation commonly known as the pupil. The dimension of the pupil depends entirely on its contraction and dilatation. In a very dark barn (provided the eye be in a healthy condition), the pupil will be very much dilated; in a barn well lighted, the pupil will be quite small. The iris is a fibro-muscular membrane, abundantly supplied with blood-vessels and nerves, and coated with a substance known as uvea, which is derived from the pigment. The arteries of the iris come from the ciliary; its nerves are derived from the ciliary.

5. Choroid Coat.-This coat, or membrane, lies beneath the sclerotica. It extends from the optic foramen to the ciliary edge of the cornea. Both of its surfaces are coated with pigment, which absorb the rays of light. The outer coating is entirely black; the inner one is a mixture of black and green, which lines the posterior half of the dark chamber.

6. The Retina.-This is the third or inner coat of the eye. It is a delicate, transparent membrane, highly vascular. The principal part of it, probably, consists of an expansion of the optic nerve; it is the part on which the object of vision is depicted. It is supplied with blood by branches from the central artery of the retina.

\section{Muscles of the Eye and Eyelids.}

The first muscle which attracts our attention, on removing the palpebral integument, is the orbicularis palpebrarum, so named from the spherical or circular arrangement of its muscular fibers. It is inserted into the orbital portion of the ungis and os frontis to the palpebral ligament, and to the skin of the lids. Its use is to shut the eyelids.

Levator Palpebrce Superioris.-The action of this muscle is to corrugate and draw the lid upward. It is located above the orbit, and is attached to the forehead by means of an aponeurotic expansion, and is inserted into the upper eyelid, its muscular fibers being blended with those of the preceding muscle. Its action is to raise the upper eyelid, in which action it is aided by the levator palpebræ internus.

Next, we find four muscles known as recti; namely, levator oculi, depressor oculi, abductor oculi, adductor oculi. They are inserted into the cavity of the orbit and external part of the scler- 
otica, at points equidistant from each other. The action of these four muscles are as follows: The levator raises the eyeball in a superior direction; the depressor, as its name indicates, depresses the eyeball; the abductor turns the eyeball from the axis of the body outward; and the adductor rotates the eyeball inward, or toward the axis of the body.

The three remaining muscles, making seven proper to the eye, are named obliquus superiora, et inferiora, and retractor oculi. The obliquus superiora is found in the upper part of the inner cavity of the orbit, and is attached to the margin of the optic faramen. At the inner canthus of the eye it passes through a fibrocartilaginous pulley-like arrangement, by which means the eyeball is rotated in an oblique direction upward and outward.

Obliquus Superiora.-This muscle is found beneath the eyeball, in the front and lower part of the orbit. It is attached to the os unguis, and to the lower and outer part of the sclerotica, at its junction with the transparent part of the globe. Its action is antagonistic to that of the preceding muscle.

The seventh and last muscle to be described is the retractor. It is one of the most admirably-arranged muscles in the whole animal economy. In figure it resembles a hollow cone-its apex turned backward, its base pointing forward, the inner edge surrounding the optic foramen, and, at the same time, offering protection to the optic nerve. Around its sides, equidistant, are found the four recti muscles, making a very complex and wonderful piece of mechanism. The action of this muscle is to draw the eye backward within the orbit.

I shall now make some brief yet instructive allusions in relation to the appendages of the eye. The appendages comprise the eyelids, eyelashes, tarsal cartilages, meibomian glands, lachrymal glands, caruncula lachrymalis, punctu lachrymalia, lachrymal sac, and ductus ad nasum.

Eyelids.-The upper and lower eyelids may be considered as the movable blinds of the eye. 'The upper is the most capable of corrugation, and borders the greatest surface of the eyeball. It is readily resolved into wrinkles. At the junctures of the aperture which separates the eyelids are the canthi, or angles of the eye. The loose portion of integument entering into the composition of the upper lid is derived from the skin covering the frontal region, and that of the lower lid is derived from the face. The lids inter- 
nally form two cavities, thus adapting themselves to the convexity of the globe. Their internal surfaces are lined with the tunica conjunctiva. The borders of the eyelids have two margins. On the outer one we find eyelashes; between the latter and the internal margin we find the orifices of the ciliary glands, through which issue a secretion for the lubrication of the surrounding parts. The edge, or surface between the two margins just alluded to, conduct the tears into the puncta lachrymalis.

Eyelashes.-The eyelashes (cilia) are so familiar to the reader, and their function is so apparent, that I need not trouble him with any remarks about them, only to observe that, by their advantageous arrangement, the rays of light, come from whatever direction they may, are somewhat intercepted; and they also operate as feeders to the seeing as well as the sightless animal, and warn him of his proximity with bodies that he can not immediately perceive.

Tarsal Cartilages.-These fibro-cartilaginous substances are found at the ciliary margins of the upper and lower lids. They possess firm and thick ciliary margins, yet grow thin as they recede from the cilia. In consequence of their close connection with the rim of the orbit, they are called, by some anatomists, "tarsal ligaments." Their function is to give some degree of firmness to the eyelids, or the soft parts of the same, and they also preserve the physiological curvature of the eyelash.

Ciliary or Meibomian Glands.-On inverting the eyelids of a horse, and carefully running the eye over the borders of the lids, within the grooves, occurring in the concave part of the tarsal cartilages, we perceive, through a very fine tunic or membrane, a number of opaque sacs, having a miniature canal, capable of penetration by a small body equal in caliber to the point of a common needle. They are evidently glandular bodies, and, therefore, have excretory ducts, which pour out the necessary lubricating material to prevent the agglutination of the lids.

Lachrymal Gland.-This gland lies underneath the process of bone known as the orbital arch. It is covered by the lining membrane of the orbit. It is a gland of the conglomerate class, being composed of many lobules. These lobules are composed of granules, from which spring the excretory ducts, and they, by interunion, form a set of tubes which terminate on the conjunctiva of the upper lid. The office of this gland is to secrete the 
tears, which run into the ducts, and are then poured on the conjunctiva.

Caruncula Lachrymalis. - At the inferior canthus of the eye, between the eyeball and eyelids, is seen a small, black tubercle, called the caruncula lachrymalis. It is not a secretory organ, but appears to be placed there for the mechanical purpose of directing the tears into the puncta lachrymalis.

Lachrymal Puncta and Conduits.-The puncta are two small orifices, seen in the inner margins of the lids, at the root of the caruncle. They are the openings of two canals found within the substance of the lids, termed lachrymal conduits; they terminate in the lachrymal sac.

Lachrymal Sac.-The lachrymal sac is a funnel-shaped membranous bag, lodged in close proximity with the lachrymal foramen. This sac serves as a reservoir, into which the tears flow from the lachrymal conduits. The lachrymal sac terminates in the ductus ad nasum.

Ductus ad Nasum.-The office of this duct is to convey away the tears as fast as they are collected within the lachrymal sac. The duct terminates at the inner and inferior part of the nostril. It is formed by a prolongation of the membrane which enters into the composition of the lachrymal sac.

\section{OPHTHALMIA.}

Symptoms.-Ophthalmia, or inflammation of the eye, is quite a common affection among neat stock. It frequently occurs without any assignable cause, except the concentration of morbid action or morbid material in the tissual membranes. It is always attended with more or less pain, tenderness, and tumefaction, and the tears being secreted faster than they can be carried through the ductus ad nasum, flow over the lower lid. This simple diseased condition differs from what is known as specific or periodical ophthalmia. There is no constitutional disturbance to be noticed, nor symptomatic fever, and the affection appears to be confined to the conjunctivial membrane only.

Treatment.-The treatment should commence by giving the animal a dose of Glauber salts, which consists of twelve ounces of salts dissolved in one quart of tepid water. The action of this medicine will be to remove morbid matter from the system. The 
eye should be carefully fomented with a warm infusion of hops, after which lay a piece of wetted rag over the eye and fasten it to the horns. I could name many favorite local applications (lotions) for the disease in this stage, but, in my opinion, cold water surpasses them all. The food should consist of scalded shorts, the animal to be kept quiet in comfortable quarters.

\section{Foreign Bodies in the Eye.}

If an animal should suffer in consequence of irritation and tumefaction of the lids of one eye, and a little pus or matter can be detected, the probability is that some foreign body, such as a piece of hay, straw, or dirt, has insinuated itself between the ball and eyelid, and has become lodged there. In a case of this character, the lids must be carefully inverted, one after the other, and examined. The removal of the foreign body is equivalent to a cure; for very little, if any, after-treatment is needed, with the exception of cleansing the eye with cold water.

\section{Specific or Periodical Ophthalmia.}

This dangerous form of disease is termed periodical, because, having once made its appearance, there is a liability of a recurrence. It is not, however, so prevalent among cattle as among horses. It is a disease which, finally, is sure and certain to end in cataract, or disorganization of the eye. In this stage perhaps the butcher would be the best doctor.

It may be proper, however, to give the reader some idea of the nature and symptoms of this malady, so that common ophthalmia may not be confounded with nor mistaken for it. It has been observed, in the preceding article, that common ophthalmia was confined to the membranes of the eye. The disease now under consideration makes its ravages on the inner structures of the eye, the external covering being only sympathetically involved. On parting the swollen lids, and if the cornea be not too opaque, we shall find that the aqueous humor is thick and muddy; the iris, lens, and other internal parts are altered in texture and structure; in fact, the eye has all the appearance of being ruined, which is probably the case. The remote causes of this disease are constitutional predisposition. 
- There are several other affections of the eyes of oxen and cows, but, as they are incurable, the subjects must -be referred to the butcher.

\section{Cancer in Cattrle.}

Cancer is a peculiar malignant growth, affecting one or more parts of the body. It probably owes its origin to some peculiarity of constitution. It is usually divided into two stages. The first is that of scirrhous, or hardening of the soft parts; the second is that of open cancer, or ulceration. In this stage, a foul fungus sore is observed, having an irregular surface of varied hue, with elevated, everted, and rough margins.

Treatment.-The popular method of treating this dreadful malady is to dissect out the diseased parts; but this rarely succeeds, for the disease generally has an origin beyond the reach of our scalpel. The only remedies of any value, in curable cases, are bloodroot and iodide of potassium. The parts should be sprinkled often with powdered bloodroot, and the patient should have twenty grains per day of iodide of potassium.

A writer in the "Eclectic Journal" offers the following as the pathology of cancer :

"According to the researches of the most eminent physiologists, malignant growths are composed of two parts, granules or cells, with cell germs, and granules within them, nourished with fat cells and globules, and of fibrous tissue or stroma, in which the former parts are embedded. Malignant growths are composed chiefly of albumen, supplied with blood-vessels, but differ in their essential elements-namely, in their chemical and microscopic-from healthy tissue. The development of this disease is evidently a perversion of the ordinary process of nutrition. The lymph, or blastema, which exudes through the capillaries, either in the ordinary course of nutrition or through some accidental inflammation, appears to have its vitality perverted; so that, instead of forming itself into one of the proper tissues of the body, it forms the irregular abnormal cells, which constitute this species of growth. These abnormal cells are deposited in distinct masses, or else spread through the tissue of the organ or part, which it ultimately supersedes. When once formed, they increase in size by the constant formation of new cells, which are supplied with fresh material from the blood. In the course of 
time, the older portions of cancerous growth lose their vitality, soften down, their outer surface ulcerates, and a sanious discharge follows."

The following case appeared in the "Veterinarian," and is selected for the instruction of the reader:

"About two years since, we were called in to attend a cow, the property of a gentleman residing in our neighborhood. On examination, we perceived a cauliflower excrescence growing from the membrana nictitans, about the size of a strawberry, from which issued an ichorous discharge that excoriated the adjacent parts, and which bled on the slightest touch. We at once decided upon taking it out, and this was accordingly done, the parts being subsequently touched with lunar caustic. After this the cow appeared to go on well for about twelve months, without any reappearance of the cancerous growth. At the conclusion of that time, we were again sent for, and found the cornea had become of a bottle-green color, and that the sight of the eye was completely gone. About three months after this a fungoid growth sprouted from the cornea, which increased in size very rapidly, but was repressed by the application of a little burnt alum. Shortly after this the eye receded considerably into the socket, and eventually it sloughed entirely away. Some weeks afterward she became partially paralyzed, and was unable to masticate. The lower lip was pendulous; the ear hung down by the side of the neck on the affected side; difficulty of deglutition was experienced, and the saliva flowed from the mouth, mingled with the partially-masticated food. Attenuation of the paralyzed muscles quickly followed, and much general emaciation of the frame. The owner being desirous of obtaining another calf from her, she was kept alive with gruel; but, as it was soon seen she could not live so as to give birth to a calf, she was destroyed. This being a fortnight before her time, the calf was immediately taken out, and it seemed likely to live and do well. It however lived only three days, the immediate cause of death being injudicious feeding." 


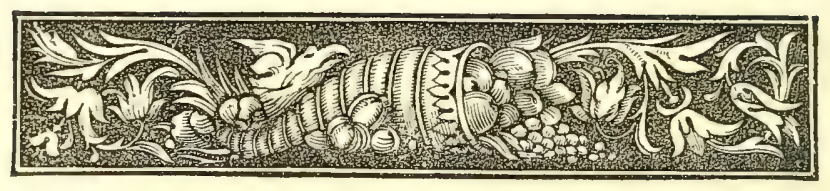

\section{SECTION XXIII.}

HERNIA, OR RUPTURES.

Forms of Rupture-Inguinal Hernia-Strangulated Hernia-Ventrat Hernia.

\section{Hernia.}

TERNIA signifies rupture, or unnatural protrusion of a porI tion of the intestines or the omentum. In consequence of the rough encounters which horned creatures frequently engage in, they are liable to suffer from the consequences of external violence, and a pregnant cow, with a distended abdomen, is more apt to be injured in this way than the male. Still, an accident of this kind will occasionally occur without the intervention of external injuries; for the cow is often the subject of dropsy of the womb, and in such condition the abdomen is distended to an enormous capacity. The only thing that can be done in a case of this character, is to rupture the fœtal membranes by means of a blunt instrument, introduced through the neck of the uterus. This will not only evacuate the fluid, but also bring on premature labor, and thus the lives of both mother and calf may be saved; provided, however, the calf be full grown.

\section{Forms of Rupture.}

There are various forms of rupture. A calf is occasionally born with a tumor or enlargement in the region of the navel. This is known as congenital umbilical hernia. It is termed congenital in consequence of being present at birth, and umbilical because it is found in the umbilical region.

Treatment.-This form of hernia is not a very serious matter, (562) 
and very rarely requires an operation. My usual course is to apply some cotton-batting, wet with an astringent (infusion of bayberry bark), and over the same a bandage, which must be passed twice round the body; yet, if the part is bathed occasionally with infusion of bayberry, the bandage and batting can be dispensed with. The reason why a case of this kind is not a serious affair, is because there is no rupture of the walls of the abdomen, but a mere purse, or dilated state of the common integuments, exists, which, if necessary, can be sloughed off by the application of a common pair of clams.

\section{Inguinat Hernia.}

When rupture in the groin appears at birth, it is congenital, and consists of a portion of intestine, which has found its way through the inguinal canal (the channel through which the testicle descends into the scrotum).

Treatment.-This can readily be reduced by raising the animal by its hind legs, and giving it a few jerks upward. If the intestine slips down again, and the case requires it, I should castrate the animal, and put a stitch or two in the external ring, or else castrate by the clam method, which will produce considerable swelling, and block up the passage so that the bowel can not descend. The clams may be removed at the end of twelve hours.

\section{Strangulated Hernia.}

The intestine will occasionally descend into the scrotum, and become strangulated, so that it can not be returned.

Treatment.-In a case of this character, the operator must dilate the ring (through which the intestine descended), by means of a probe-pointed bistoury. Having returned the bowel, let the animal be castrated, and then apply the clams. Strangulated hernia occurring in any other part of the body must be treated as follows: If the protruded bowel is tumefied in consequence of gas within, it may be punctured, in one or two places, by means of a suture-needle. This will allow the gas to escape. Then efforts should be made to return the protrusion. Failing to accomplish this object, in consequence of stricture or thickening of the walls of the abdomen, I should introduce the bistoury, and dilate the 
wound. The reader will infer, and rightly so, that I am alluding to a case in which there is an accidental external wound; otherwise it would constitute what is called ventral hernia.

\section{Ventral Hernia.}

This form of hernia consists of an escape of a portion of the intestines through a rent in the abdominal muscles, creating a tumor beneath the common integument. This tumor may exist for some time without affecting the animal's health, but when it increases in size, so as to be bulky, or becomes strangulated, an operation must immediately be performed. In performing the operation, however, it must be borne in mind that the protruded bowel has pushed before it a portion of the peritoneum (the lining membrane of the cavity of the abdomen); therefore the intestine is contained in a sac, which should also be returned, along with the intestine, into the abdominal cavity.

Treatment.-The mode of operation in cases of ventral hernia is to cast and etherize the animal. A semilunar incision is then made over the tumor, the convex border of the flap being downward. The panniculus carnosus (subcutaneous muscle), or superficial fascia, is also divided. A probe-pointed bistoury is then introduced between the bowel and edge of the wound. This must be dilated or made sufficiently large to allow the return of the intestine. The rent in the muscles is then to be strongly sutured, and one end of the suture should be left long enough to hang ont of the wound; the integuments are then to be brought together in the same manner, after which dress the wound with tincture of aloes. In the course of eight or ten days a pull may be made on the ends of the deep-seated sutures, to see if they are ready to come away, yet it is best not to remove them until they are quite loose.

It is impossible in a work of this character to furnish the reader with all the information necessary to meet the emergencies which may occur in the various forms of hernia ; for their management and treatment require more than ordinary skill, and many persons, who consider themselves competent to prescribe for the ordinary diseases of cattle, are willing to acknowledge themselves at fault when consulted on the subject of rupture; and, lest my own remarks on this subject may prove of little avail in furnishing the 
husbandman, in the hour of need, the necessary instruction, I introduce the two following cases, selected from the "London Veterinarian." Mr. James Stowar furnishes the following case of strangulated hernia:

"On the 17th of September, 1850, I was called to the farm of Ashogle, to see a cow which had been injured by the horns of another; but, as I had left home that afternoon for a distance, and did not return until late next day, I did not see the cow until the 19th. I found her with a hernial tumor protruding from the right iliac, which remained covered within the skin, so large that a bushel measure would not have contained it. She was evidently in much pain while standing, and would stand only a very short time. I was told that she had eaten a good deal since the accident, and, as a stoppage of the dung was observed, they had given her a pound of Epsom salts. Her abdomen was enormously distended, notwithstanding the largeness of the hernial tumor. The non-escape of the feces was proof positive, under the circumstances, of the hernia being strangulated.

I had her laid on the left side, as carefully as possible, and, after every attempt to return the hernia by external manipulation had failed, I cut into the sac, and attempted to knead in, inch by inch, the gorged intestines; but in this, also, I failed. The hernial opening was large enough to admit three of my fingers, so that it was not want of room in that, but in the abdominal cavity, which was so fully distended by the intestines forming the hernia, that caused the difficulty. I had her laid upon her back, with her hind legs drawn up, so as to throw the contents of the abdomen as much as possible upon the diaphragm. Even then, however, I could not succeed. I therefore, with a sharp-pointed scalpel, cut into the intestine, and emptied it entirely of its semifluid contents, stitched up the wound, and washed it carefully, and so, ultimately, accomplished the reduction. With a strong cord I closed the hernial opening, as well as the one I had made through the skin, nine inches further up; I then turned her on her left side, and in a few minutes the feces began to escape by their proper channel. I gave linseed oil, twenty ounces, and tincture of opium, one ounce. Next morning I found her up, stepping through the shed, inclined to eat, her bowels acting freely. I had her tightly bandaged, to support the abdomen, and made an opening to allow the escape of discharge at the bottom of the sac. I 
saw her daily for a week, and prescribed according to the state in which I found her. On the whole, her health proved good, and her wounds healed pleasantly. She was fourteen years old, and, like most cows, had a large belly. She fattened well, and was sold for the shambles in April. The butcher told the farmer that he found a large abscess in her side when he cut her up."

The next case is that of ventral hernia, furnished by Mr. G. LEWIS:

"In September last, I was requested to examine a heifer, which was said to have a 'swelling on her right side.' I found her with a pendulous bag, hanging very low on the right flank, and of an immense size. Upon a close examination of the parts, I ascertained that the enlargement was produced by an escape of the intestines through a rent in the abdominal muscles. I informed the owner that nothing short of an operation could be productive of benefit to the animal, and I also pointed out to him the nature of the case, and its probable results.

According to the arrangement, the heifer was sent to me on September 6, when, after due preparation by medicine, etc., I had her cast and secured, and then carefully made an opening through the skin and panniculus muscle, immediately over the laceration in the other muscles, but a little longer than it. I thus exposed the rent which existed in the external and also the internal oblique. The edges of the aperture were very uneven and much thickened; and I may state in this place that the enlargement had been observed a month prior to this date. A large portion of the intestines were found to have eseaped into the sac formed by the panniculus and common integument; besides which, a considerable quantity of serous fluid existed benenth the skin, and externally to the panniculus, which had doubtless been thrown out by the injured vessels.

The intestines being returned into the abdominal cavity, the edges of the laceration were brought together with strong metallic sutures, and properly secured. The external wound was then closed; and, before allowing the animal to rise, I passed a seton through the most depending portion of the integumental sac, with a view to give a gradual and continuous exit to the serous effusion. A compress was also placed upon the part to remove as much of the pressure of the viscera as possible, after which the animal was allowed to rise. 
For several days the bowels remained obstinately constipated, and some symptomatic fever was also present; but, by proper measures, these unfavorable symptoms were soon removed. The animal was allowed only linseed tea and thin gruel for a few days. Subsequently a large amount of serous fluid was discharged through the openings made for the introduction of the seton; it however, soon ceased. The sutures also came away in due course, leaving but little appearance of the original injury."

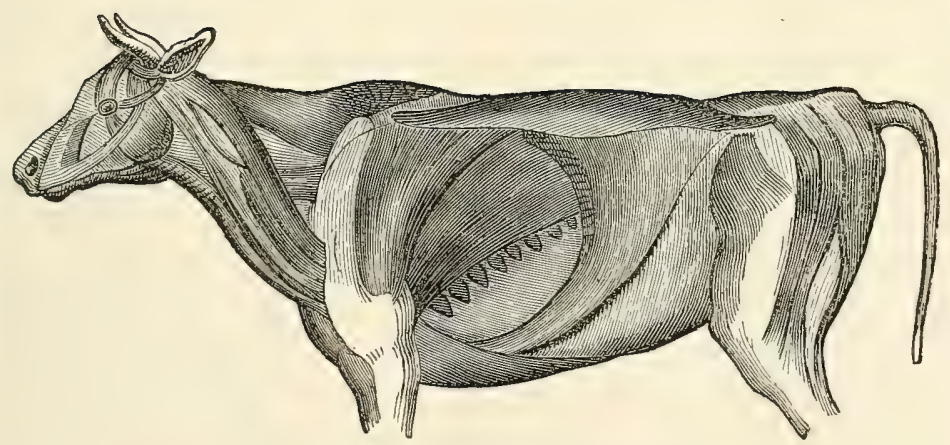

VIEW OF SOME OF THE SUPEREIOTAL MUSCLES. 


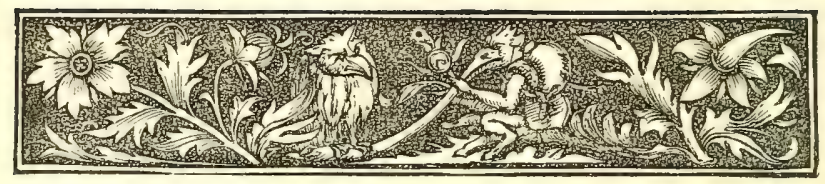

\title{
SECTION XXIV.
}

\author{
DISEASES OF THE BONES.
}

Cachexia Ossafraga-Bone Disorders-Mechanism and Structure of BonesCompozition of Bones-Ultmate Constituents of Bones-Dilatation of the Jat Bones-Abscess beneath the Periosteuni, at the Angle of the JawExostosis, or Deposit of the Calcareous Matter on the Surface of Bones -Caries, ob Ulgeration of Bones-Prelminary to Horn-Ail-On Srmpathy and the Sympathetic Refations which exist in the Animal EconomyHORN-AIL-TAIL-AIL.

\section{Cachexta Ossafraga (the "Cripple").*}

" LTHOUGH using this term as the title of my subject, I do A not consider it to give an idea of the exact character of the disease I am about to describe, but merely to convey an impression of a very prominent symptom of the disease. As many other maladies existing in different localities, this one is very differently treated of in our veterinary works. The inefficiency of our works on cattle medicine is sufficiently apparent to the practitioner, who, after a nominal term spent at college, enters into the world of medicine a sufficiently-educated person, as his diploma gives him to understand; but how frequently is he wofully disappointed when called to any of the several enzoötics in cattle which he finds have not been treated of, perhaps not mentioned either at college or in the books!

The present subject is one, among others, which is not diseussed in British works on cattle medicine. It is a disease very prevalent in many districts I am personally acquainted with, and in all of which it passes under the very expressive term of 'cripple,' the appearance of the animal fully confirming the term. It seldom happens that a solitary case occurs on a farm which is subject to it; 
for all the milch cows, and perhaps some of the younger stock, are more or less affected by it. Such farms are considered to be of much less value than other places on which it does not occur, not only from the unthrifty condition of the stock, but also as regards fertility, as they are never found to be very productive, and among farmers they pass under the cognomen of 'crippled places.' The disease will never be found to exist on farms that are fertile and rich; in fact, a certain guide to the locale of this affection is to look out for farms that are situated on high lands, possessing stunted hedges and trees, a barren, or, at least, a poor soil, producing a dry, short, and innutritious herb. On passing over the farm, the limestone rock will, most generally, be seen to project out of the soil, proving that it is situated on the limestone; or by examining the rivulets, if any, that flow through the farm, the water will appear to be beautifully transparent, so that the smallest pebble may be discerned on its bed. There is, also, in the generality of instances, the limestone rock forming its bed. When this is present, any old farmer will tell you that the water which seems to be so limpid and pure is 'hard,' and that the cattle confined to it are certain to be crippled. I have, however, noted farms where the water which supplied them was charged with a carbonate of lime, and still no cripple exists on them. This is, undoubtedly, owing to the character of the soil, which, on examination, is found loamy and free, and lying on the red sandstone rock. " I have also noticed 'cripple' prevailing on farms supplied with water running over mosses, etc., which, from the experience of intelligent farmers, has been said to be antagonistic to this affection. This, in my opinion, is caused from the character of the soil proving to be of an undoubted calcareous character, intermixed with clay. Although it can not be doubted but that the water supplying farms impregnated with any principle of an injurious character will have an influence in the production of disease, yet, in the instances quoted, it appears that the character of the herbage produced on such farms either counteracted the injurious influence of the water, or, on the other hand, destroyed or overbalanced the influence of the mossy water.

From what has been stated, it will be seen that the 'cripple' will and does exist on those farms where the soil is calcareous and clayey, and that it is evident that such soils are incapable of producing a succulent and healthy plant; in fact, will the herb not be 
surcharged with calcareous and silicious elements? and is there not a deficiency of azote-producing principles? As it is well known that where nitrogen abounds a luxuriant herbage is the result, we need go no further in support of this than a farm on which an abundant supply of its manure is spread. How much more abundant and luxuriant is the character of the vegetation from the use of decomposed vegetable matter and animal excretæ than from any thing else. If, therefore, the herb. grown on these 'crippled farms' be unhealthy, as there is little doubt, the animals partaking of such will, without doubt, in the course of time, have their systems seriously deranged, and the character of derangement will depend either upon an insufficient supply of nitrogenous material, inducing, consequently, emaciation and pure debility; or it may be that, where this deficiency of azotized material exists, the earthy salts may not exist in an abnormal degree, but still, from a want of other nutritious material to counteract their ill effect, the system may become charged with them. When the herbage and other produce of the soil is charged with earthy salts, whether carbonates or silicates, they would, in all probability, become assimilated, or, at least, there would be a tendency to the deposition of these salts into the various textures, such as the cartilages, ligaments, and cardiac valves, etc., all of which lesions are apparent, to a greater or less degree, in all the animals which have died of this disease that I have examined. Some are of opinion that it is owing altogether to an excess of carbonate of lime in the soil and waters; but if this were the case, the character of the farm could not be altered, nor the disease be removed, as has been done, and which will be noticed hereafter. It is most probable, at least, as far as my humble opinion goes, that it is induced from the combined influence of poverty in the soil, and an excess of astringent salts, silicates, etc. When I refer to the poverty of the soil, it must not be understood that I mean an exhausted state of the soil, because many of those places have not been broken up with the plow to any extent. Many farmers are of opinion that the labor and expense required in the cultivation of such lands would not be compensated by their produce. If it were turned up by the plow, the advantages it would derive from exposure to the atmosphere and rain-water would be considerable.

There are many farms which are termed crippled farms, and many cattle called crippled, which are not really so. The mystery 
of this is easily explained. On strict inquiry, you will find that the reason the stock display so many of their bones, and walk off so stiff, is owing altogether to imperfect feeding. It answers as a good excuse for the farmer who overstocks his farm, and is thus compelled to serve out short rations, to say that it is the cripple (or any other name it may possess) that is the cause. The climate will, without doubt, have a great influence in modifying this disease, as is shown by the greater severity in form on those lands that lie the highest, where the protection is scanty from the inclemencies of the seasons. A fact connected with this affection is that when cattle are taken from those farms which are subject to it, even when suffering severely from it, to farms existing on the eastern banks of the river Eden (which flows through the vale of Westmoreland), they recover completely from its effects, and are perfectly free from a recurrence of the attack if they remain in that district. It is a common saying among farmers that 'there is no cripple on that side of Eden.' The character of these farms that are free from the affection is essentially different from those afflicted with it. They have a free, sandy, and rich soil, producing excellent oats and rich meadow-grass, but their climate is colder, owing to their being situated near a range of mountains; but if the cattle are brought on to those farms situated on the western bank of Eden, where the cripple prevails, they soon begin to show the symptoms of an attack. I do not include all the farms on the western bank, as some are perfectly free from it.

From this instance, we may again be led to infer that the herb and water to which they had been previously subject were not sufficiently nutritious, or else impregnated with some injurious matter, because, on removal to farms producing a superior herbage, they have recovered, sometimes in an incredible short time, for the sad state they were in. On all these crippled places cows that yield a great quantity of milk are the soonest attacked, and the farmer knowing this will not take such to his farm, because he would soon have to 'dry' them, or they would rapidly fail ; but animals that have been reared on these places are less liable to its attack, and are seldom so severely afflicted by it.

Many have confounded this affection with rheumatism, but it is, without doubt, a distinct disease in character, as in no case of cripple will be seen the acute symptoms and fever which exist in both the acute and chronic form of rheumatism. In those cases 
where suppuration exists in the joints, and the limbs even rot off, it is, in my opinion, produced from the friction of the joint from ossified cartilage, and an evident deficiency of synovia, these inducing a local inflammation altogether differing from the peculiar characteristics of rheumatism, and, unlike it, the osseous system seems to be the principal seat of disease.

Some cattle evince a greater predisposition than others to an attack, as the elderly cow and the cow that yields a great quantity of milk. I have never noticed young calves become affected by it. Some have stated them to have been so, but, on inquiry, it has been found to be pure arthritis, or joint-felon, from cold and damp houses. While one of the stock of calves has been so afflicted, the others were perhaps perfectly healthy. The symptoms vary in degree of virulence on different farms. Some farms have a very slight attack, while others are very severely afflicted with it; but the symptoms will vary frequently on one farm, as, on entering a cow-house with a collection of ' crippled' cattle, it will be seen that some are slightly affected, while others are evidently suffering greatly. The disease being of a subacute character, the period from its first appearance in an animal to its last stage occupies frequently some years, according to the vigor of their constitutions, and the mild or severe form of the disease. There is one farm I am acquainted with where the owner is compelled to change his stock every two years, to prevent heavy losses occurring.

Symptoms.-In the primary stage of the disease the animal presents a lean, emaciated condition. The bones in every part of the body are prominent; the skin is tight, hard, and void of all suppleness; the hair presents a coarse appearance, standing almost straight up, having none of its naturally smooth character about it; but if we see our patient walk, it will strike us that there are no joints in her limbs, as she is peculiarly stiff, knocking her hock-joints up against each other without any regard to the unstateliness of the gait. She will, also, while walking, make an extaordinary cracking noise, showing an insufficiency of synovia to lubricate the joints; the pulse, on pressure, feels flaceid, but the caliber is rather increased than diminished; rumination is performed rather slow and sluggishly; appetite is not indifferent, eating her hay, straw, or turnips with a degree of zest. If we allow her to come in the neighborhood of the hedge where the farmer's wife has displayed her linen, she will indicate the phe- 
nomenon of bulimia or depraved appetite. She will seize with avidity the first article she reaches, and chew it with wonderful pleasure; she will also hunt with avidity any sour or filthy liquids, even human urine, and drink them greedily; and when in the pasture will pick up a bone, if such ean be found, and chew it for an hour at a time, seeming to enjoy the occupation. Stones, iron, sticks, clothes, etc., are all alike objects of attraction to her. This peculiar habit is attributable, I should think, to acidity of the stomach. The farmer imagines that a sod, cut from a field and placed before her, will palliate her affection, and the cow, to appreciate his kindness, commences to lick it, and even to devour the soil that is on it. I have noticed them chewing these different articles, and never saw one display any anxiety to swallow the object, but merely a desire of chewing. When such articles are swallowed, it will, I think, be more accidental than intentional on the part of the animal, but I can not be positive on this point. They show a particular desire to chew articles of clothing, as is testified by a person going to a stall-head between two of them. They will immediately seize his dress and chew it. The bowels are always more or less constipated, and the secretion of milk is partly checked, although but slightly. They may continue in this manner for a longer or shorter period, according to constitution and external circumstances, before they display any of the worst symptoms concomitant on the termination of the disease. It is a gradual declining from the primary symptoms to those of a more fatal character. These may be marked by the appearance of tumors over the hips or stifle-joints, or, perhaps, a tumefaction extending from the stifle to the hock-joint, which, in the course of time, suppurates. In the mean time, the system, it is evident, is sinking. The pulse is flaccid, but still retains its caliber, although accelerated; appetite is impaired, and rumination is performed very lazily; constipation, if a milch cow ; the milk is now wholly suspended, or nearly so. If, perchance, the animal has swallowed any irritating or indigestible substance, which is almost always the case, the symptoms of indigestion will be more severe, with perhaps an intermittent pulse, great lameness, increasing to such an extent that the animal is either unwilling or incapable of rising; or, perhaps, on closer examination, some bone will be found to have become fractured, either the scapula, femur, or the pelvis, or it may have occurred in any other bone, as there is the 
same tendency in all the bones to fracture, although the animal is fastened up in his stall, and, therefore, not subject to any of the ordinary causes of fracture.

I recollect a case in which the animal could not stand. On examination, it was found that the radius and ulna were completely fractured; next day the scapula and femur were broken; in fact, one after another, all the bones of the limbs became fractured, until the animal died from the extent of irritative fever set up, consequent on the fractures. Another instance of this brittleness of bone: A milch cow was purchased as perfectly sound. She was poor, and the excuse was that she was rather crippled. A fair price was paid for her. She walked to her new home extremely stiff and lame. Next morning I saw her, and found the os ilium fractured; afterward the femur broke, and she died.

In the greater part of cases the brittleness of bone is present, and in such I never noticed any suppurative tumors, excepting some cases where the joints were affected, and opened from local inflammation. On examining some cases after death, I found attenuation of muscles, the bones polished at their extremities, and brittle. In some rare cases exostosis may be noticed on the body of the bone, but such are scarce. On the epiphyses of the bones, however, they will frequently be found, more especially at the lower extremity of the tibia. Sometimes they have existed to such an extent as to anchylose the hock-joint. In all the articulations there is evident deficiency of synovia. The different articulatory cartilages displayed an undoubted tendency during life to the deposition of osseous matter in them, as all of them possessed a degree of hardness inconsistent with nature.

In regard to prevention, it will be evident enough that the removal of the animal from the innutritious farm to one of a richer and more luxuriant character will be attended with the greatest benefit. Many cases have been recruited even when the animal has been compelled to lie and eat. An important fact I wish to mention here is, that there are some two or three large farms in this neighborhood which were formerly severely afflicted with this disease; but now, since they have become possessed by enterprising agriculturists, the disease is much abated, in fuct, proving a very rare visitor, although these farms possess the characteristics previously described as 'crippled places,' and were, in fact, situations where this disease prevailed to a serious extent. The means which 
have been resorted to by these men were irrigation and extensive use of artificial and other manures on the farms, thus altering the character of the herbage, as it is not only more luxuriant, but evidently of a more succulent and nutritious character. They also cultivate a good quantity of the mangel-wurzel and linseed for consumption by the stock. These articles were never known on the farms while suffering from ' cripple.' These means have evidently produced a wonderful effect, as they are seldom if ever afflicted with it. As the disease is found to be worse during the autumn and winter seasons, when the cattle are brought in to dry food, the necessity of a succulent diet is obvious; but still I have seen many bad cases during summer, on farms where the soils were calcareous or clayey. Where there is a scanty supply of turnips, and where oil-cake or other of these useful articles of diet are scarce, the 'cripple' will be the most severe. In a word, to prevent it, soft nutritious diet, as turnips, mangel-wurzel, oil-cake, etc., must be resorted to; and the hay or straw with which the animals are supplied should not be the stunted and dry material which is produced by those lands on which the 'cripple' is known to exist, but such as is produced by the plentiful use of artificial and other manures, to counteract the tendency of the soil to produce a dry and stunted herb. The use of crushed oats and barley, along with other nutritious food, will, in the course of time, have a very beneficial effect also.

When the practitioner is sought to a case of confirmed 'cripple,' where the preventive means have not been resorted to, it will be necessary to employ more active measures. Many remedies have been tried, with more or less benefit, according to the advancement of the disease, where the cachexia ossifraga is so predominant, and one bone becoming rapidly fractured after another, the case may be looked upon as altogether hopeless; but in those cases where the tendency to fracture is not so great, the animal may be recruited most generally. In recommending remedies for a disease possessing different phases, it is necessary to apply those means which seem most applicable to the one particular case in hand. Many disappointments have originated from the reported success of a particular remedy from parties who, no doubt, did succeed with it, while others, without taking into consideration the modifications of the disease, ruled by circumstances, climate, etc., have unmistakably failed, although the same remedy may 
have been employed, thus showing that one remedy may succeed admirably in one district, while in another it is sometimes nearly useless.

Treatment.-The means which I have seen applied with success, according to the modification of the disease, were, in the cases where the attack is primary, the use of malt ale, administered in pint doses every night for a length of time; water acidulated with sulphuric acid, diluted, or with nitric acid, allowing the animal no other water to drink excepting this; also administering the diluted sulphuric acid, twice daily; and where constipation is threatened by its use, it must be obviated by a laxative diet. Hydrochloric acid and nitric acid have been used, but the preference is given to the sulphuric. Sulphate of soda, in quarterpound doses, combined with vegetable tonics, will sometimes be found useful. Camphor, in large doses, combined with tonics, given in cold ale instead of gruel, have been attended with good success; also, the use of a decoction of the willow or poplar barks, given in pint doses, will be found an admirable tonic for advanced cases. As to the use of sedatives, the animal rapidly sinks under their influence. The only class of medicines that have been the most successful were tonics. There are many cases of chronic rheumatism and arthrites that have been confounded with cripple, but any one acquainted with these diseases will readily recognize an essential difference between them. But, in fact, every remedy will be comparatively useless unless the animal be put upon a more nutritious diet-a diet free from astringent qualities."

\section{BONE DisORder.}

During the past few years I have noticed a number of articles in our agricultural periodicals referring to a disease named by the writers "Bone Disorder." The name, as the reader will perceive, does not throw the least ray of light on the pathology of the malady; in fact, it savors strongly of the so-called "horn-ail" and "tail-ail" - terms which apply to symptoms rather than to any real disorder. One writer informs us that when an animal becomes the subject of bone disorder, "the bones threaten to cave in-have wasted away." If they do threaten to cave in, the best method I am acquainted with to insure an opposite tendency, is to promote the healthy functions of the body, and thus keep dis- 
ease and the cattle separate. If the bones waste away, and then assume their original shape by merely feeding bone meal, as the writers contend, then the bones of cattle must possess some peculiarities that I have never, in my professional capacity, been able to discover.

If the animals recover (as we are told they do), then it might be inferred that the bones possess properties analogous to that of the soft tissues-an inference which the reader knows is not correct. I would observe, however, that all parts of the animal organism undergo a very perceptible augmentation, and decrease or waste. Thus, up to the period of adult life, all parts of the animal frame increase in bulk, and give to the animal that rotund and symmetrical appearance which attracts the eye of all those who love to admire the works of Nature; but, at last, old age comes on, which is attended by a gradual waste or shrinkage. This occurs, however, more in the muscles than in the bones; yet the latter undergo some condensation, which may possibly lessen their volume. This, however, is a physiological result, the work of uncompromising Nature, over which human agency (bone meal included) has not the least control.

Whenever the bones do become diseased, it is the result of hereditary predisposition, of local injury, or of impaired digestion. So far as my inquiries have extended in relation to the character of the so-called bone disorder, I infer that it is a disease of a debilitating character, originating, in most cases, in the digestive and nutritive organs affecting not only the bones but various other tissues of the body, and, therefore, the only rational plan of treatment consists in improving the general health of the animal. The ways and means of accomplishing this very desirable result are as various as the causes which occasion the derangements. If it be evident (as the bone disorder theorists contend) that the animal organism, in such cases, is deficient in phosphate of lime, I have no objection to offer against the popular custom of giving the patient a few doses of bone meal ; for, whenever there shall be a deficiency of carbon in the system, bone meal may be useful in supporting pulmonary combustion, and thus insure healthy action of the organs and functions of the animal economy; yet I would suggest to the intelligent reader that as bruised oats, ground corn, and linseed meal contain a large amount of phosphates, they should be selected in preference to bone meal, which I think is 
more valuable as a remedial agent for a carnivorous than for a herbivorous animal.

As I understand the complex theory of digestion, the value of food or remedial agents is in exact ratio to their adaptation for assimilation; so that if their elements are only held in union by an uncomplex chemical affinity, as is the case with all vegetable productions known as food, their solution is easily and promptly effected by the ordinary process of insalivation, mastication, and remastication; but bone meal (a popular remedy) is actually an animal production, (vegetable matter animalized,) and, in order to convert the same into the component parts of the cow's structure, it will perhaps require more chemico-vital force to effect the solution than when oatmeal, etc., are used, the chemical affinity

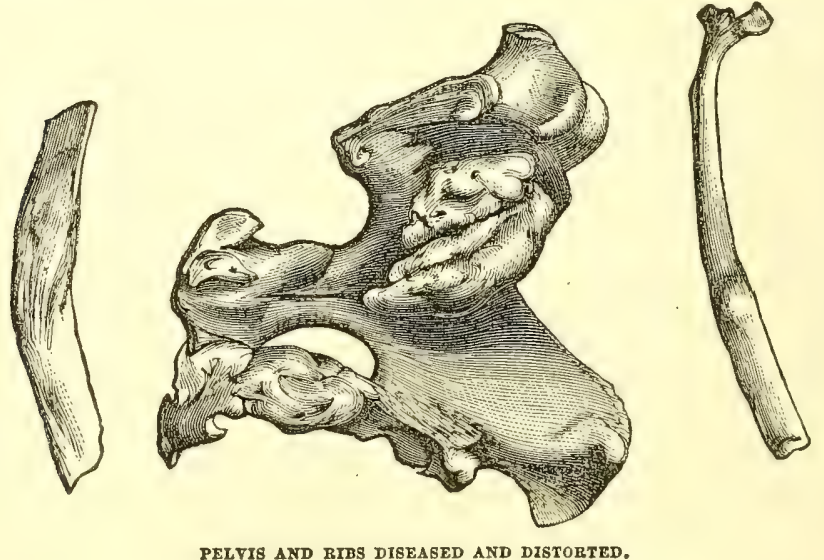

here being weaker than is the case with bones. In the treatment of any and every disease, it is usual to endeavor to ascertain the direct eauses, and, if possible, effect their removal, and whatever seems to be indicated we are justified in supplying; so that if phosphates are indicated, they may be prescribed; yet in cases of emaciation, when weakness and debility preside, I should prefer to use nutritious food, tonics, and stimulants, instead of bone meal. Why not add the bone meal to the barnyard manure? In this way the animal would get the benefit of it in the form of fodder.

In reference to a deficiency of phosphate of lime in the mill, which the writers alluded to have noticed, I remark that it may, 
in part, arise from functional derangement in the digestive organs (in such cases a large quantity of phosphate, etc., is expelled from the system in the excrement); or the fodder itself may be deficient in its usual yield of phosphate of lime. We then have an evidence of vegetable disease, for I believe that the phosphate of lime is as necessary for the growth and integrity of the plant as it seems to be for the same purposes in the animal kingdom. But I believe that the plant can not lack phosphates without there being a corresponding deficiency in several other constituents which go to form the healthy vegetable; so that, in such cases the disease is general, not local. The plant is not fiber-sick nor the cow bonesick, but, in both cases, (the food of each being innutritious,) debility is the disease.

It is well known that successive cultivation exhausts the soil. and uses up the constituents necessary for the growth and maturity of grains and fodder; yet the pastures and plowed land might be made to yield good crops and rich harvests by depositing in the soil (in the form of animal excrement, straw, wood, ashes, lime, charcoal, etc.,) as much as we take out of it. The soil can not create any thing of itself; therefore an increase in crops can only be obtained by adding more of certain agents to the soil than we take out of it.

"In Flanders the yearly loss of the necessary matters in the soil is completely restored by covering the fields with ashes of wood or bones, which may or may not have been lixiviated. The great importance of manuring with ashes has been long recognized by agriculturists as the result of experience. So great a value, indeed, is attached to this material, in the vicinity of Marburg and in the Wetterau, (two well-known agricultural districts,) that it is transported, as a manure, from the distance of eighteen or twenty-four miles. Its use will be at once perceived, when it is considered that the ashes, after being washed with water, contain silicate of potash exactly in the same proportion as in the straw, and that their only other constituents are salts of phosphoric acid."

It is a fact well-known to husbandmen, that some breeding cows do not come up to the standard of health or fair condition, although they are fed from the cream of the crib, on the best kind of fodder. The inference is that the digestive organs are not in working condition; therefore, in such cases, the food operates as 
an exciting cause of disease. The effects of cheap and damaged food are too well known. Poor food is dear at any price which may be asked for it. It may not furnish sufficient carbon; if so, the animal is deprived of the power of reproducing itself, and must, eventually, suffer.

\section{Mechanisur and Structure of Bones.}

Bones have many things in common with the soft tissues and organs; for example, arteries, veins, nerves, lymphatics, and a connecting cellular web. Their structure, in the embryotic state, is highly vascular, yielding, and gelatinous. They have externally a fibrous investment, known as periosteum, which is well supplied with arteries, veins, nerves, and absorbents; and it is through the intervention of this fibrous tunic that the vessels proper to bones reach their ultimate destination. On the interior surface of hollow bones we find a membrane of similar structure, only more delicately organized. The cavity of the shaft-bones is usually occupied by a quantity of adipose matter, known as marrow. This is inclosed in laminated cells, and is supposed to be a sort of aliment in reserve, to provide against accidental emergencies of non-nutrition.

\section{Composition of Bones.}

Bones consist of two constituents; namely, animal basis and calcareous matter. In the healthy adult the proportions are as follows:

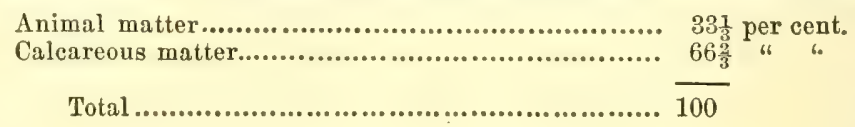

\section{Ultimate Constituents of Bones.}

The ultimate constituents of bones are gelatine, animal matter, carbonate, phosphate, and fluate of lime, phosphates of soda, and magnesia. Their growth, like that of shells, is effected by the addition of new tissues to that already formed. Bones which have a central cavity are protected internally by cartilaginous fibers or unions; hence they expand or burst so soon as their cartilaginous braces are decomposed by a diseased condition of the parts. 


\section{Dilatation of the Jaw Bones.}

This disease seems to be more prevalent among horses than cattle. In equine practice it is termed "big head;" in bovine practice we call it spina ventosa (a bony tumor, in which the interior of the bone is absorbed, leaving a mere shell of bone, divided into cells, containing purulent, cheesy matter) ; but perhaps "dilatation of bone" will give the reader a better idea of the character of the disease than any other term. On inspecting the jaw bones of cattle, the subjects of dilatation, we find the greatest enlargement at the angle of the lower jaw. On cutting into the same, its cavity is occupied by a preparation resembling soft cheese. On removing this, it is discovered that the cartilaginous braces which hold the sides of the bone together are decomposed; hence the dilatation. This foreign material often degenerates into true pus, and burrows through the bones toward the surface. In this condition a fluctuating tumor can be felt at the angle of the jaw.

Sometimes the bones of the jaw appear to have lost their cohesive firmness and vitality; they then border on a state known as necrosis. Now, if a portion of bone in this condition be macerated, for only a short time, in a weak solution of muriatic acid, it can be rolled up like a piece of paper, showing that it is deficient in calcareous matter; but if it takes a day or more to put the bone in this condition, the experiment would not be of much value, as all bones can be more or less softened by means of weak acids.

Treatment.-Having ascertained that the osseous structure is deficient of earthy matter, and that the animal matter preponderates, we are then in possession of facts which can be used to great advantage in preventing the malady, and perhaps treating it in its early stage. The remedies are phosphate of lime, vegetable tonics, and stimulants. I use them in the following form:

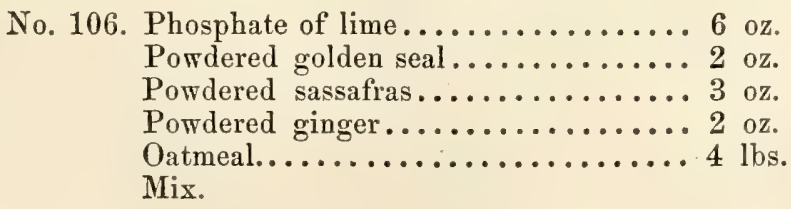

This is to be divided into sixteen parts, one of which may be incorporated with the food every night. I recommend the medi- 
cine in this form because it is calculated to give tone to the function of nutrition.

It is well known that the maintenance of the functions of animal life are almost entirely dependent on the due performance of the nutritive operations, and, therefore, the integrity and properties of all the hard as well as the soft tissues depend on their regular nutrition by a due supply of perfectly elaborated blood. This can not be effected unless the functions of circulation, respiration, and secretion are performed with regularity. Circulation is necessary to convey a supply of nutritious fluid, and respiration and secretion separate the blood from its impurities. Therefore, in cases of this character, I advise stock-owners to endeavor to improve the general health of the patient by means just suggested, and also that they should see that the animal gets that kind of food which is rich in phosphates.

It is very difficult to define the causes of this disease. It may originate in a peculiar morbid habit of body, or it may be the sequence of faulty nutrition or hereditary predisposition. When an animal labors under any morbid habit of body he is in a state far removed from that of health, and various parts of the body become affected by the change; and even should the power of forming good healthy blood remain, the organic force by which the constituents of blood are transformed into osseous structure must necessarily be enfeebled by the morbid habit, so that the power to produce metamorphoses is necessarily diminished. It is my opinion that big head usually commences in the fibrous tissues which are found in the internal surface of bones. A very peculiar feature of these fibrous tunics is, that when they once become diseased they run rapidly to purulency; and this accounts for the large amount of purulent matter often found in the cavities of jaw bones when buried or exposed to a drying process. It is then, however, in a spongy state.

A very distinguished French writer contends that "fibrous tunies or tissues hardly ever contribute to the formation of pus." This is evidently an error; for when the periosteum (covering of bones), which covers the fang of a tooth, and gives a lining to the cavity into which it is inserted, becomes inflamed, it suppurates, and the tooth has to be removed. I contend that it is the most common tissue that excites the flow of those exudations from arterial capillaries, which becomes converted into pus; hence, in this 
way I account for the collections of pus often found on and under fibrous ligamentary tissues and coverings of muscles; also upon and beneath the peritoseum, and in the vicinity of fibrous tissues in other parts of the system.

The surgical treatment of a case of this kind is to liberate the pent-up pus or matter. With this object in view, I cast the subject, and, after having brought him under the influence of ether, I make an incision through the integuments, etc., and expose the bone; then, by means of a pair of common bone forceps, I make an opening into the same, liberate the imprisoned matter, and inject the cavity with pyroligneous acid. Having removed as much as possible of the morbid matter, I then cram the cavity with equal parts of powdered bloodroot and bayberry bark. The external wound must not be closed by suture, but left open, so as to allow of a free discharge from the parts ; for the healing process must begin at the upper part of the cavity, and the integument should be the last to heal. I continue to throw into the cavity, by means of a glass syringe, a small quantity of pyroligneous acid daily, until the discharge ceases. Then the parts are to be dressed with common tincture of aloes. After an operation of this character there will remain some enlargement of the tissual structures about the parts. This can gradually be reduced by a few applications of a portion of the following:

No. 107. Iodide of potassium............. $1 \mathrm{dr}$.

Glycerine.................. 1 oz.

Mix, and keep the preparation in a glass vial, well corked.

\section{Abscess beneath the Periosteum, at the angle of THE JAW.}

This is a very frequent and formidable disease among cattle, and is ofttimes occasioned by blows inflicted purposely or accidentally on a region known as the angle of the jaw. It is a formidable disease, because it frequently ends in caries or ulceration of the jaw-bone.

Symptoms.-It originates in an inflammatory condition of the periosteum, accompanied by a gradual enlargement at the angle of the jaw. It is very painful, the animal being very unwilling to have the part handled. The pain is occasioned by distension 
of the periosteum, and this is sometimes found to be indurated or thickened. The animal does not eat as well as usual, in consequence of the pain occasioned by the act of mastication, and he generally labors under some degree, more or less severe, of febrile excitement. Ordinarily it is a very easy matter to detect the presence of pus in any of the superficial regions; but in this, located directly on the bone, and covered by a fibrous membrane which only admits of a limited amount of distension, it is very difficult for an unskillful person to discover it; yet, if the above symptoms are observed, I should decide that there is pus beneath the periosteum.

Treatment.-No treatment is of any advantage except that of making a free opening through the skin, muscle, and periosteum, and thus insuring a complete discharge of the morbid matter; and the sooner this is done the better, for it will relieve the animal of much suffering and prevent ulceration of the jaw-bone. Very little after-treatment is required. It is only necessary to cleanse the part daily, and dress with tincture of matico.

\section{Exostosis, or deposit of Calcareous Matter on the SURFACE OF BONES.}

Exostosis signifies an unnatural growth of bone, or deposit of calcareous matter on the surface of bones. Among horses this disease occurs in the form of splent, spavin, and ring-bone. It is very seldom that cattle are treated for this affection; for the deformity is so slight, and the lameness so obscure, in consequence of the slow motions of the animal, that very little notice is taken of it.

Treatment.-The proper mode of treatment is to apply, daily, a small quantity of iodide of glycerine, prepared as follows :

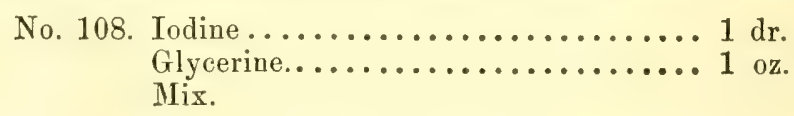

Apply by means of a small piece of sponge. This is to be applied daily for a period of two weeks, after which use acetic acid, one part; water, six parts. With a portion of this sponge the enlargement until pain and lameness disappear. 


\section{Caries, or Ulceration of Bones.}

This is a very common disease among cattle, and is preceded by inflammation and suppuration. It sometimes proceeds from a blow received at the angle of the jaw ; generally, however, it is a constitutional disease of a malignant character.

Treatment.-In the first stages of this disease, the part being hot, painful, and tender, I should foment with warm vinegar, which will tend to hasten suppuration; then, having ascertained that there is some pus or matter locally imprisoned, the part must be freely punctured, the pus evacuated, and the cavity injected with pure pyroligneous acid. Supposing, or rather suspecting, the disease to be constitutional, I should administer the following:

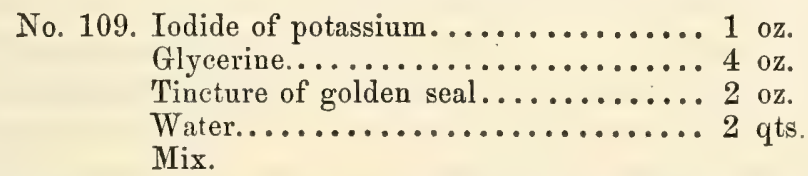

The dose is one-sixteenth part of the above quantity, to be administered daily.

\section{Prelminary to Horn-Ail.}

Many very valuable animals die prematurely, under treatment, in consequence of mistaking symptoms for the disease. Before I discuss horn-ail, I propose to offer a few brief remarks on the subject of sympathy, so that the non-professional reader may be prepared to comprehend the why and wherefore of sympathetic diseases, as they occur in neat stock during the progress of primary affections.

\section{On Sympathy and the Sympathetic Relations which} Exist in the Animal Economy.

The animal structures are so sympathetically related to each other, and so dependent are various organs and functions on an equilibrium of vital action, based on the law of sympathy, that the animal economy, as a whole, may be said to compose a vast machine, the integrity of which depends on the free and full play of all parts composing its intricate mechanism. The media by 
which sympathy is aroused and maintained are the nerves. These originate from the brain and medulla spinalis, or spinal marrow. Ten pairs of nerves radiate directly from the brain, termed cerebral; thirty-nine pairs from the spinal marrow, termed spinal. They are named after that of the region in which they are found; hence we have seven pairs of nerves issuing from the cervical region (neck), eighteen from the dorsal (back), five from the lumbar region, and five sacral, within the pelvis, four coccygealmaking, in all, forty-nine pairs cerebro-spinal nerves. They are distributed from each side of the spine to various parts of the body, and their terminations are called "sentient," this being the seat of sensation.

The most important nerve, however, for our consideration, is the sympathetic. It is supposed by some writers to be in itself a complete nervous system. It originates in a branch issuing from the base of the brain, and communicates with every other nervous filament of the body. The connection takes place in the following manner: The sympathetic nerve has, at various points, a number of gangliform plexuses. From these thread-like filaments arise, which radiate and connect with similar ganglions found on the cerebral and spinal nerves. On these divergent filaments we have other ganglionic structures, which send branches to the liver, spleen, kidneys, etc. The sympathetic nerves of one side amalgamate with those of the opposite, and this is their mode of communication. The nerves of the sympathetic system possess a certain degree of power in exciting muscular contractions, as well as sympathetic actions, in the various parts to which they are distributed. CARPenter has observed that, by "irritating a branch of the sympathetic nerve, immediately after the death of an animal, contractions may be excited in any part of the alimentary canal from the pharynx to the rectum, according to the trunks which are irritated; in the heart, after its ordinary movements have ceased; in the aorta, vena cava, and thoracic duct; in the ductus choledochus, uterus, fallopian tubes, vas deferens, and vesiculæ seminales, etc. It is probable that the sympathetic system not only thus brings the organic functions into relation with the animal, but that it also tends to harmonize the former with each other, so as to bring the various acts of secretion, nutrition, etc., into mutual conformity."

There are several orders of sympathetic nerves. Some pass 
into the parenchyma of the organic viscera, and others are distributed on the outer coats of arteries, continuing, throughout their minute capillary structures, into the papillæ. By this arrangement the harmony between the internal, external, and remote parts is perpetuated.

Correct knowledge regarding the sympathetic relations peculiar to animal organization seems absolutely necessary, in order to correct the erroneous impressions that some persons have formed of the nature and seat of disease incidental to horses and cattle, and, in this view, I offer these preliminary remarks as introductory to the subjects of "horn and tail-ails" (imaginary diseases, which oftentimes, perhaps always, are the result of a fertile imagination, or, rather, a sequence of that faulty mode of reasoning which confounds effects with causes). Any person conversant with the sympathetic relations existing in the animal economy, can readily discover the difference between a pathological condition and the local or general symptoms which usually accompany it. An animal, for example, is attacked with acute disease of the liver. He evinces signs of pain from pressure on the right or off side, in the region of the liver, and, possibly, the lameness is of so grave a character as to mislead the non-medical observer, and he necessarily concludes that the subject is lame, "and nothing more." He prescribes an external remedy, accordingly, which is neither calculated to cure nor palliate the liver difficulty. Thus, for want of the necessary knowledge, the symptoms are mistaken for the disease.

In derangement of the digestive organs, more particularly of the stomach, the brain is usually sympathetically affected. The symptoms of disturbance in that organ, or its functions, may escape the attention of the "cow-leach," yet they are always present, and range from what has been observed as "dullness" up to somnolency, accompanied by other morbid phenomena well understood by the physician. A knowledge of these and other sympathetic relations existing throughout the animal economy, enables us to understand what occasions vomiting in a man when a blow of sufficient force is received on the skull. The blow arouses a certain set of involuntary operations which the subject is unable to control, as in the cases of vomiting, etc. It explains, also, why giddiness or vertiginous symptoms usually follow when a blow is received on the region of the stomach; how the impreg- 
nated uterus influences the mammæ and stomach, causing increase of function and volume in the former, and morning nausea in the latter; how a diseased condition of the internal mucous membrane reacts on the common integument, and vice versad. Even in the osseous structures the law of sympathy prevails. A disease in the upper extremity of the bone gives rise to sympathetic pain at the opposite extremity. And as regards the muscles and tendons, there exists a very marked sympathy, although in the mind of pathologists this is not strange, for, anatomically considered, the tendons are neither more nor less than tendinous terminations of muscles. Puncture of a tendon is often followed by great derangement of the nervous system, and other pathological conditions, not unfrequently ending in trismus (lock-jaw.) This is owing to the same law of sympathetic association just alluded to. There is, therefore, a tendency in certain organs to become deranged or diseased in consequence of a malady locating in others, although they may not always be identical in function.

\section{HORN-AIL.}

Persons who are in the habit of prescribing for sick brutes, and have never made themselves acquainted with the sympathetic relations existing in the animal economy, to which I have referred, are liable to commit errors in diagnosing disease, and, when questioned regarding the seat of the same, their opinion generally is that the suffering animal has either the horn-ail or tail-ail. The idea, in almost all cases, is so supremely absurd, that, if any thing other than a living animal were the subject of the barbarities which, according to mistaken notions of cure, are sure to follow, I should feel disposed to burlesque the whole procedure regarding both horn-ail and its treatment. In my opinion, horn-ail, in ninety-nine cases out of one hundred, exists only in the imagination of those persons who allow error to overcome their better judgment, or else they have not given the subject a passing thought; therefore, they are incompetent to even guess at the true nature of the malady with any chances of correctness. No allusion, that I am aware of, has ever been made by the authors of standard works or text-books on veterinary science, to horn-ail; and if educated veterinary surgeons were as numerous here as in England or France, and they had the same means to 
reach the ears and the understandings of our husbandmen, the latter would soon be convinced of the absurdity which is here described, and, consequently, be induced to protect their animals from that species of cruel quackery or ignorance which would refer all their aches and diseases to horns or tails, and which sanctions the boring of the former, and curtailing or docking the latter.

The pathological conditions on which the absurd theory of horn-ail seems to be founded are heat or coldness of the horns. These are the principal, and, in fact, only symptoms which the unlearned expounders of a popular malady have given us; but every one ought to be aware that variations in the temperature of a part so inferiorly organized as the horns are is no criterion as regards the nature of the disease which occasions, in this vicinity, merely an increase or decrease of temperature. The actual disease which occasions a loss or increase of temperature of the external surface of the body, horns included, may be, and often is, located in either the brain, stomach, or bowels, and at other times is the result of local congestions of the lungs and other parts-mere effects-the results of preëxisting disease; therefore, I contend that the term horn-ail, when used to express the condition of parts sympathetically affected or aroused, throws no light on the true nature of the disease under which the animal labors.

I shall contend that neither the augmentation nor decrease in the temperature of the horns constitutes actual disease of the same, but may indicate a loss of equilibrium in the circulation of blood. If the horns are at fever-heat, and the surface of the body be cool, we know that the brain or its membranes may be actively or passively congested. On the other hand, should the horns and the extremities be cold, it goes to prove that the animal is the subject of internal congestion or disease. But why locate it in the horns, when in the animal economy are found so many hundred parts, sections, and divisions of parts, more important, sensitive, and of vastly more consequence to the preservation of vital integrity, than the horns? The variations in the temperature of the horns and other parts of the body, which the horn-ail theorists neglect to notice, enter into a class of symptoms from and by means of which an educated surgeon makes up an intelligent and correct diagnosis, and, consequently, are only of value, in point of fact, in 
so far as they go to show the actual state of the whole animal mechanism. The proposition admits of the following appropriate and convincing illustration: A man is attacked with acute disease of the liver, and almost always experiences a pain in the right shoulder, a purely sympathetic affection. Now, it would be very ridiculous for a physician to overlook the diseased condition of the liver, and merely prescribe for the sympathetic shoulder-lameness, and call it "shoulder-ail." Such ignorance is calamitous, but does not often occur when the educated physician is employed; otherwise it does, and many such eases are quite fresh in my memory. Now, instead of prescribing local agents, as some have done, in view of mitigating sympathetic local pain, would it not be more in accordance with reason and science to administer medicinal agents, such as are calculated to restore the liver to a natural physiological condition? A man who thus ignorantly prescribes falls into the same error with him who would refer all diseases of neat stock to their horns or tails, merely because the parts are not in their natural physiological condition, owing, as I have said, to actual disease seated elsewhere. Thus the symptoms are mistaken for the disease, and the treatment, in so far as boring, sawing off horns, and curtailing the caudal appendage is concerned, is highly injurious, barbarous, and, in these enlightened times, deplorable.

It requires no argument to convince many men that horn-ail is a very prevalent disease, for the false doctrine has been promulgated, and has received attention from men whose domains extend from Maine to California. The error has been sown broadcast, and has acquired such hold on the minds of some that it will take many years to root out the evil. One writer on this subject, believing that horn-ail is a sort of national disease, recommends the barn-yard faculty (for no regular physician will heed his advice) to carry gimlets in their pockets, so that they may be armed and equipped to encounter and subdue that which is more imaginary than real.

I have made examinations of the bodies of cattle, subsequent to death, said to have died of horn-ail. Among them were evident traces of softening of the brain; and this is a feature of disease very often present, as I shall attempt to show, in many of the socalled cases of horn-ail. Softening of the brain is a disease of so grave a character, that any morbid symptoms attending the same, as local heat or coldness of horns, might compare in the ratio of a 
mole-hill placed beside a mountain. Softening of the brain is the ultimatum of a grave disease occurring in that organ; and if the owners of live stock are disposed to believe that horn difficulty is the most preponderant and alarming, and they can sleep soundly in the belief that no danger threatens, then, "If ignorance is bliss, 't is folly to be wise." I can not indorse this sentiment, however; for, in this day of intellectual maturity, no one has a right to be a dunce in his chosen profession, nor a fool, to thwart the intentions of those who would substitute light for darkness.

Softening of the brain is always preceded by acute, and, subsequently, chronic, inflammation of the same or its investing tunics, and constitutes the last stage of disease; so that the days of such a subject are numbered, and the man of gimlet and ignorance, armed with his munitions of warfare, treats the disease at long odds. He pockets the fees but loses the case. His patient, on which a too confiding owner has foolishly permitted him to practice his cruelty and legerdemain, dies, and he knows not the why nor the wherefore.

But, in order to convince the reader of the follies of the above practice, $\mathrm{I}$ offer the following illustration, from a reliable source. The case was diagnosed and treated as horn-ail. I quote from the "Southern Planter:"

"After death, examined the head. Crest between horns perfectly hollow. All the little divisions and offshoots of bone which are usually found in the cavity were removed, in a greater or less degree, and there were only the white of egg matter and pus. The horns, also, entirely hollow; one of them filled with nearly a pint of lymphy and purulent matter. The cavity extending to the orbit of the eye, thence communicating with the nostrils, especially on one side. The brain, which lies in very near contact with this cavity of the crest (which we may as well call the frontal sinuses), was softened and fallen, in one hemisphere, into a thick mush. A small part only of this half was of healthy consistency, preserving its form or vessels entire. The other half (hemisphere) was not softened, but the vessels were very full of blood, and the membranes exhibited signs of intense inflammation. No other region or organs examined."

In regard to this quotation I remark: It is very natural that the frontal sinuses should be hollow; for, if they were solid, the 
weight of the head would be enormous; and I can readily perceive the wisdom of the Divine Artist in thus adapting the parts for the convenience of the animal, and relieving him of what, had it been otherwise, must have been a burden, and would have required powerful muscles to support the head. The bones about the head are divided into two plates, separated by numerous vacuities or cells; but, unlike those of the horse, they extend through the whole of the bone-nay, penetrate even through the parietal and occipital bones. Hence it happens that the frontal sinuses (so these cavities are called in cattle as well as the horse) extend from the angle of the eye to the very foramen through which the brain escapes from the skull-nay, to the very tip of the horn. Hence the parts may be said to be hollow; and it is well that every farmer should know this, for some suppose them solid, and are very much surprised to find them hollow. On making a section of the horn, from tip to base, it will be found partly hollow, "having sinuses that extend almost to its tip." The lymphy and purulent matter found within the frontal sinuses and those of the horn, together with the softening of one hemisphere of the brain, proved that the parts were all involved in disease; but then the disease never had its origin in the horn. The brain, or perhaps its membranes, were the primary seat of the affection, and, after softening, (decomposing,) involved the surrounding parts in ruin. "The other half of the brain was not softened, but the vessels were full of blood, and the membranes exhibited signs of intense inflammation." Probably this was the state of affairs in the diseased hemisphere in its early stage, and some physical impediments to the return of blood from the brain had induced cerebral appolexy. The animal was in a plethoric state, "very fat." There was too great redundancy of blood-just the subject for such disease. Softening of the brain, I think, would be a better designation of the disease than "horn-ail."

The editor of the "Planter," in a subsequent number of his journal, remarks: "The notion that this disease originates in the horn itself seems to us to be an error, resulting from that backward mode of reasoning which confounds symptoms with disease. The disorder in that organ, 'the horn,' should rather seem to be secondary. No less erroneous do we deem the opinion, held by some, that the horn becomes frost-bitten, and then putrifies. Though its whole inner surface is exceedingly vascular, yet it is 
perfectly protected by the almost insensible horny covering in which it is cased throughout, except only a very minute ring at its base, whose pulsations are the most accurate index of the pulse; and that ring, hardly wider than a thread, can not be affected by cold. When it is considered that the horn is a substance of lower vitality than the hoof, which never becomes frost-bitten, even though chilled by an iron shoe, reason should teach us that it can not be injured by cold."

A similar case occurred in the experience of the editor of the "Planter," which "ran to a speedy and fatal termination." Upon dissection of the head, the left hemisphere of the brain was found completely "broken down," or disorganized; the base of the left horn was slightly implicated. The introduction of a gimlet, therefore, into the horns of cattle thus affected, and afterward cramming in pepper, turpentine, and other foreign bodies, can not possibly reach the disease nor benefit the animal, but is apt to do much harm; for puncture of the lining membrane of the frontal sinuses, which generally takes place when the instrument is introduced near the base of the horn, is attended with danger, both as regards inflammation and hemorrhage; and it is just as unwise to fill the parts with pepper, turpentine, etc., in view of cure, as it would be if one of our own race were concerned.

I now have an impression that the reader, like myself, has come to the conclusion that, in the generality of cases occurring among neat stock, the disease christened horn and tail-ails, is located elsewhere. Yet some persons contend that the practice, which I denominate as unscientific and barbarous, saves some of the afflicted animals. I have doubts about the treatment saving them. They may survive it; for it is well known that both men and animals often recover after an unfavorable prognosis is made, and they will also survive very severe injuries, fractures, punctures, and formidable wounds, etc.; so that health returns, in such cases, in spite of the violence opposed to it. Hence, if an animal should be restored to health, after having been compelled to submit to the fashionable barbarities of horn-boring, that is no proof of the utility of the means used.

If we could only collect all the facts in the case of an animal said to be the subject of horn-ail, we should probably discover that, in four cases out of five, the animal's stomach was the seat of the original difficulty, for the stomach is more frequently deranged 
than any other organ of the body; and it often occurs in this way: A man has a cow, an ox, or a lot of cattle, which he intends to bring to market, in view of exchanging them for dollars and cents. He puts them through (as the moderns have it) the fattening process, furnishing them with a superabundance of carbon, in the form of meal and other nitrogenous equivalents. The result is an accumulation of adipose tissue; the animal becomes fat, and, consequently, plethoric. The accumulation of fat offers an impediment to the free and full play of the heart, lungs, and diaphragm; and, should the subject be a pregnant cow, she is liable, a few hours after the period of parturition, to be attacked with milk or puerperal fever, or convulsions; therefore the liability to disease in more important structures than the horns is a matter that I seriously urge our itinerant cattle doctors to consider.

Neat stook are often the subjects of catarrh, (or "hoose," as it is termed,) nasal gleet, etc. In either case, a profuse discharge occurs from the surface of the nasal membrane, extending to the frontal sinuses, up to the very tip of some horns. This is often called horn-ail, and the gimlet is brought into requisition, and, on withdrawing it, some of the "matter" may possibly escape from the orifice; hence the gimlet is said to do good. This I deny; for the more rational way of favoring the discharge of the matter would be to steam the nostrils, and adopt such other means as the nature of the case may seem to require.

Even granting what some contend for-namely, that an abscess occasionally forms in one of the nasal cavities - then it would not be proper to bore the horns, for the pus must necessarily be inclosed within a sac, which the gimlet may rupture. Then the morbid matter escapes into the frontal sinuses, and is apt to set up diseased action on the tissue with which it comes into contact. If it be ever necessary to puncture an abscess of this character, it should be done by way of the nostrils. Even should the animal labor under any disease located within the horns, the introduction of a gimlet would be a very unsafe remedy, as all veterinary surgeons are ready to testify. It is a dangerous business to puncture a membrane so highly organized as that found within the frontal sinuses,* especially when so rude an instrument as a gimlet is

* The gimlet, before it enters the lining membrane of the parts (termed schneiderian), must necessarily puncture a highly vascular membrane (termed 
used. 'The instrument must lacerate the parts, and induce severe inflammation and suppuration; consequently, it is better calculated to produce disease in this region than to cure one existing there.

I have examined many animals after their horns have been bored, and have always found an undue degree of heat in the cranial region; throbbing pulsations about the base of the horn, and a general disturbance of the functions; sometimes an accumulation of pus within the cavity. Then, again, internal hemorrhage, effusion, etc., of blood, are apt to occur after boring the horns; and every intelligent physician would be unwilling to incur the risk attending it. Many a valuable animal has been lost by hemorrhage, following fracture, puncture, and sawing off the horns.

Horn-ail is said to be so prevalent among neat stock, that the farmer (in the imaginative mood) fears it as he would the plague. And the only remedy for this lamentable state of ignorance is for farmers to use their reason, godlike and rational, and avail themselves of the experience of those competent to diagnose disease by its concomitant and persistent symptoms. We are now in possession of facts showing that the introduction of a foreign body (a gimlet) may induce a formidable disease; therefore, it should never be used.

Among the diseases mistaken for horn-ail I name indigestion; and this disease is almost as prevalent among neat stock as it proves to be among the members of the human family in this country. Loss of cud, or a cessation of rumination, is one of the principal symptoms of acute indigestion. I made an autopsy, in a case of this character, only a short time ago, and found the abomasum, or fourth compartment of the stomach, enormously distended with food, and, on removing the same, the mucous membrane readily peeled off. I particularly pointed out to the owner of the animal the pathological appearances as they occurred; but, as he had made up his mind, and the neighbors confirmed his opinion that the animal was the subject of horn-ail, he still believes that the trouble first commenced in the horns, which had been bored in several places. 
I am not contending that the vascular membrane within and without the osseous structure of the horns and contiguous parts enjoys immunity from disease. It is subject to it, as are other parts, precisely identical in function and structure. But I am exposing a false theory, which confounds functional and organic diseases with their symptoms or manifestations.

A local disease of the horns is not unusual. For example, a blow in the region of the base of the horn often occasions a local affection; and this is not to be wondered at, when we know that the parts in this region are merely defended by a thin cuticular envelope, and, therefore, offers but little protection to the subtissues. This region is denominated by brutal men the "tender spot;" and, in view of subduing a restive animal, they aim their bludgeons in this direction, with terrible effect. Local injuries of this character, and those occurring in the savage encounters which horned animals frequently engage in, the boring of their horns, and sawing them off, etc., are accidents and processes more or less operative in exciting a local affection..

A local disease of this character admits of the following demonstration; namely, percussion. Percussion is the art of striking various parts of the body, with a view of ascertaining the seat of disease. Every substance, when thrown into sonorous vibration, produces a sound the tone of which is peculiar to itself, and every change which takes place in the composition of that substance, or in the arrangement of its component parts, is accompanied by a corresponding change in its tone. If we strike a solid body, it emits a solid sound; strike an empty one, and it emits a hollow sound. In like manner, if we strike a healthy horn, we get a sound of hollowness. When the horn and frontal sinuses are occupied by matter, we elicit a dull sound, without vibration. The difference between the sound elicited from an empty barrel and that from a full one, or the sound from a solid post and that from a hollow one, when struck with a hammer, is not more remarkable than in the cases of healthy and diseased horns. By the same means we diagnose diseases of the chest. We are, therefore enabled, with absolute certainty, to determine the presence of pus within the horns; and, keeping in view the history of the case, which it is our business to inquire into, we can generally tell whether or not such abscesses result from local injury or disease within the parts, or if it be the sequence of some other disease. 
Treatment.-In regard to the treatment of an abscess located in the region of the base of the horns, the proper method is to trephine the skull, which operation can only be performed by a veterinary surgeon.

\section{TAIL-AIL.}

In view of sustaining the respectability of veterinary science and my own professional reputation, I would inform the reader that tail-ail is an imaginary disease, appearing only in localities which the apostles of true veterinary science have never visited. The supposed affection is said to induce partial or complete paralysis of the hind extremities, and, contrary to the principles of science and the testimony of several veterinary writers, this condition is often termed tail-ail. The faulty theory is, a soft spot is found at the end of the tail, (this is a feature of every healthy cow's tail, when it has not been docked,) which takes the strength out of her back, and produces paralysis. The remedy is either to amputate the caudal appendage, or slit open the soft spot and stuff in tar, pepper, salt, or whatever remedy Neighbor So-and-so recommends. It seems a pity that the poor animals I am now writing about can not, like their masters, receive the benefits resulting from the investigations of scientific men in improved methods of treating disease. However, it is gratifying to know that the errors of the past are fast "dying out," and that the days of ignorance are numbered.

The tail is sometimes the seat of a local affection arising from blows, etc., or it may be the seat of a cutaneous disease; but neither one nor the other can be of so grave a character as to produce paralysis of the posterior limbs. The soft extremity of the tail may also occasionally become congested, or else odematous (dropsical). But these states of the part would not justify a man in cutting off the tail ; for the limbs are often found in the same condition, and no one would ever think of lopping them off, for the remedy would be worse than the disease; and, so far as the tail is concerned in being the seat of local congestion, or œdema, there is no disease at all, and, therefore, does not require local treatment. But I am not discussing the probabilities of diseases of the tail. This is not my purpose. I only aim to show the folly of making the cow's tail the indicator of the various diseases of her body, and, also, that of confounding a disease of the nervous system 
with a slight congestion of the tail ; and, further, the folly of arguing that the cow's strength is taken out of her back, etc., because the end of her tail is soft. If the tails of neat stock, or those of any other animals, become diseased, in the name of humanity let them be prescribed for; but I do hope that those who read this article will never be caught prying into the end of the above useful appendage for the purpose of demonstrating that which never existed.

Almost all animals said to have the tail-ail are laboring under various forms of disease remote from the tail, and, however diligent men may be in performing their barbarous operations on the same, the disease under which the animal suffers, perhaps located either in the brain, spinal marrow, liver, or pancreas, entirely unsuspected, may progress to a fatal termination, while an uneducated cow-leech is amusing himself by taking unwarrantable liberties with the uncomplaining animal's tail. I am often told that animals, after being thus operated on, get well. Very likely. Some animals will endure the most cruel torture, and I once saw a cow, in apparent health, who a fortnight previous drank a pint of white paint. Such cases I look upon as nothing more nor less than lucky escapes.

I shall close this article by introducing a selection from "Youatt on Cattle." Mr. Youatr, when treating of palsy, thus alludes to "tail-ill," or "tail-slip":

"In many parts of the kingdom palsy is traced to a most ridiculous cause. The original evil is said to be in the tail, and all maladies of this kind, involving the partial or total loss of motion in the hind limbs of the animal, are classed under the name of tail-ill, or tail-slip. Our friend, Mr. Dick, of Edinburgh, has taken up this subject, in a very interesting point of view, in the fourteenth number of the 'Journal of Agriculture,' and the public are much indebted to him for dispelling a false, injurious, and cruel superstition. The farmer and the cow-leech believe that the mischief passes along the cow's tail to the back, and that it is on account of something wrong in the tail that she loses the use of her legs; and then some set to work and cut the cow's tail off, while others, less cruel or more scientific, make an incision into the under surface, and allow the wound to bleed freely, and then fill it up with a mixture of tar and salt, and we know not what. *** $*$ * Mr. Dick, with a kind consideration for which he deserves much 
credit, condescends to reason the case with these foolish people; and what he says is so much to the purpose, that we can not refrain from introducing it here: 'The disease, in ordinary cases, is said to consist in a softening about the extremity of the tail, and is to be distinguished by the point of the tail being easily doubled back upon itself, and having, at this doubling, a soft and rather crepitating kind of feel. But what is the real state of the case? The tail is lengthened out to the extent of about three feet, and is formed like a common whip. Toward the extremity the bones terminate gradually, becoming insensibly smaller as they proceed downward. At this part is said to be found a soft place (the tailslip). Beyond this, again, a firm cartilaginous portion is found, covered with hair, to brush off the flies within its reach. Now, why have we the long columns of bones-the termination with a soft space of a few inches-this thickened, hard, cartilaginous part at the very extremity, and that extremity covered with hair, but with a view to form a whip, to drive off, with the greatest possible effect, the insects which wound and torment the animal?"

After such testimony as the above, I firmly believe that the readers of this work will never be caught in the foolish act of locating paralysis at the extremity of the caudal appendage.

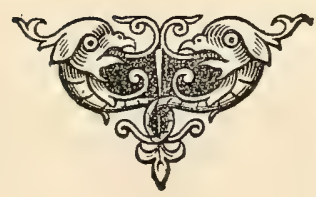




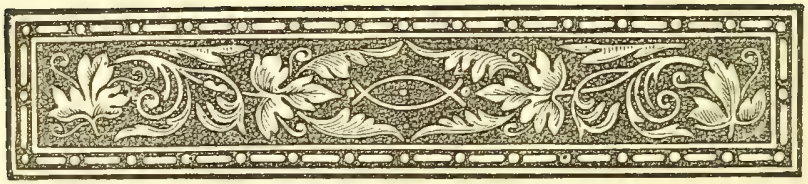

SECTION XXV.

DISEASES OF THE BRAIN.

Inflammation of the Brain and its Membranes-Sturdx in Cattle-Shaking PaLsY-Hydrophobia.

\section{Inflamiation of the Brain and its Mejrbranes.}

TT makes but little difference, so far as our method of treatment 1 is concerned, whether the brain or its membranes be the seat of inflammation; for the treatment, in both cases, must be the same, and the difference, in a pathological point of view, can not be very great between a disease of the brain and its investing membranes, although each may have peculiarities of appearance. The affection is generally sudden in its attack, and it is often accompanied by symptoms of frenzy, and the animal sometimes becomes frantic, and decidedly mischievous. Soon, however, alterations in the structure of the parts take place, as softening, effusion, etc., and then the animal dies.

In regard to the treatment, I must confess that it is much easier to write about it than accomplish it. In cases when delirious fits occur, accompanied by convulsions, which make it dangerous to approach the animal, I have no remedies to offer; the case is beyond the reach of art. I may, however, add that the terminations of this disease are different. They depend on the intensity of the malady and the structural susceptibility. The disease is generally treated on the antiphlogistic plan : cold water to the head, active cathartics, and counter-irritation on the region of the spine.

Causes.-The causes of a disease of this character are often obscure, yet I have known it to occur as a symptomatic affection. I examined the carcass of an ox, a short time ago, that died of $(600)$ 
what the owner termed " mad staggers." I found the brain highly congested, and several adhesions between it and its membranes. There were also large patches, intensely red, on the lining membrane of the third and fourth apartments of the stomach. I was informed by the owner that the animal died twenty-four hours from the time of its first attack. The disease probably originated on the digestive surfaces, in consequence of the irritating nature of the food-moldy hay and tough corn-stalks, with a sprinkling of damaged meal and brewer's grains.

Symptoms. - The symptoms of symptomatic disease of the brain are as follows: Dullness, loss of appetite, staring of the coat; and, if the animal be a milch cow, diminution in the quantity of milk is observed; the extremities are cold, and the animal grates its teeth; the respiration is at first tranquil, and the pulse slow but full; the patient will frequently be seized with a kind of epileptic fit, which lasts but a few minutes, during which time some of them will exhibit the most violent symptoms, such as bellowing hideously, pawing the earth, and running at any thing within their reach; they will also break out into profuse perspiration, and press their heads forcibly against the wall, even to such an extent as to break off their horns. Many are seized with violent tremblings and twitchings, and, toward the latter period of the disease, the respiration becomes extremely laborious, the jaws are firmly closed, convulsions succeed each other rapidly, and death shortly closes the scene.

Treatment.-Give the animal two drachms of fluid extract of gelseminum in a gill of water, twice daily, until the pulse and respirations become more natural. Give occasional enemas of soap-suds, and keep the head and spine sponged often with cold water. So soon as the inflammatory symptoms subside, discontinue the gelseminum, and administer, daily, doses of

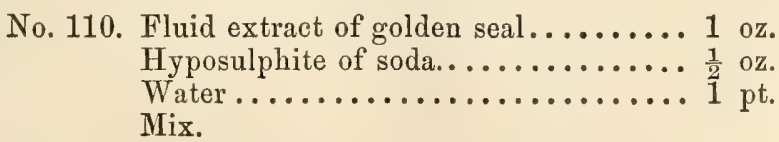

\section{Sturdy, or Cerebral Parasttes.}

The brain, and its investing membranes, are often infested with a species of entozoa, termed cœnurus. They consist of a parent 
sac, or membranous tunic, from which, externally, germination takes place. This mode of multiplication of this group of parasites differs from that which is observed in the hydatid (fluke), in which it occurs internally.

Symptoms. - The symptoms will depend altogether upon the number and size of the parasites. In their early state they take up but little room, and do not occasion any very marked symptoms; yet, if the animal could only speak, we might be informed that he was the subject of headache. As the parasite or parasites increase in size, they produce pressure on the brain, which makes the animal appear giddy, confused, nervous, and desirous of separating itself from the herd; and it is in consequence of these peculiar symptoms making their appearance, when no other form of disease is present, that the term "sturdy" is applied, which is simply used to denote the presence of cerebral parasites.

Treatment.-When once these parasites have fairly taken up their abode in the cranial cavity of an ox, I fear there is very little help. An operation, such as that alluded to by the writer of the appended article, may, once in awhile, prove successful, yet, in my opinion, the remedy is about as bad as the disease; therefore I recommend prevention rather than attempts at cure. The preventive remedies are salt, sulphur, and charcoal, equal parts. This is a specific for all parasites. About a table-spoonful of the mixture, given occasionally in the food, will prevent the germination of many forms of parasites.

The following interesting translations are by Mr. GAugeE:

"Sturdy in Cattle.-No less than seven bladders of the coenurus were found in the left hemisphere of the cerebrum, in an old cow affected with the sturdy. Between the dura mater and the cranial parieties there were several transparent vesicles, about the size of a pea, embedded in the substance of the bones. The left hemisphere of the cerebrum only weighed three drachms and a scruple less than the right, although the former contained all the bladders. Hering could not find any heads of the cœnurus on the interior of the sacs, and he held them to be yet undeveloped hydatids.-Repertorium fiir Thoerheil, p. 21. 1855: Stuttgart.

"In the second volume of the Milan 'Veterinary Journal,' at page 52, is a case of sturdy, recorded by Patellani. It occurred in a two-year old animal, that had shown, for several days, attacks of madness, and in one of these it had broken a horn off. 
Patellani found her lying senseless on the ground; the head, bent on one side; the forehead, hot, and painful on percussion; the sound produced, hollow. " On examination after death, the membrane of the brain was found injected, and in the right ventricle of the cerebrum were hydatids with several heads.

"The trephine has, of late years, been much recommended in cases of cerebral hydatids in cattle; and in Bavaria and Wurtemberg it has frequently been employed, and often with good results. At the Clinique of the Munich Veterinary School, in the month of November, 1854, a year-old heifer was presented, with expansion of the right frontal bone. There were symptoms of giddiness, with turning toward the right side, dullness, etc. Ramoser found, on percussing the seat of the disease, that the sound was most hollow to the left. The case was observed for forty-three days, during which time the symptoms became more severe; the animal was trephined, and about two ounces of serum passed out, followed by the bag of the parasite. "The wound would have been closed with a clay plaster, but the animal had to be slaughtered the following day. The membranes of the brain were inflamed, especially to the right, and blood was extravasated on its surface. The expansion, thinning, and even perforation of the upper part of the right lateral ventricle, showed that the bladder was lodged in the ventricle itself, as had been seen the previous year, in another case that had been operated upon. Death was then attributable to the far-advanced stage of the malady, and to the abrupt collapse of the parietes of the ventricle, after contraction of the bladder."-Miinchen Jahresber, fer 1854-5, p. 13.

The following is from the pen of Mr. JoHn GamgeE, (Edinburgh,) on sturdy in sheep, in which much useful information relative to parasites is given:

"The more inquiries made, the more accurate will the conclusion arrived at by scientific men appear, that dogs and sheep must live together for certain parasites to extend their ravages with effect. On all the sheep-farms I visited last summer, sturdy was complained of, and on all such farms there were dogs. Such was the case at Cairnton, Mill of Kincarnardine, on Glendye, and in other localities. Mr. Falconer, of Balnakettle, a gentleman of great experience, and other farmers, assured me that there has been more giddiness in sheep within the last two or three years than they had ever witnessed before, and the losses, in consequence, 
are sometimes greater than by that fatal malady "braxy." This clearly does not depend on a large number of dogs being kept; but there are many conditions affecting the propagation of parasites, and if destructive agencies were not as universal as the productive, much more effectually would vermin and parasites multiply and spread their baneful influence, to the detriment of mankind. It is accidental circumstances that affect the development of diseases of animals. If every germ produced by a single tape-worm, in a limited period of time, were to take effect, it would be quite sufficient to exterminate the flocks of Great Britain; but, I repeat, the laws established to procure the multiplication of any animal are counteracted by an infinity of uncontrolled, but, perhaps, not uncontrollable, agencies. The latent vitality of the eggs of such parasites is extremely difficult to destroy, and, to use the words of one of Kuchenmelster's reviewers, after months of exposure to warmth and moisture, the pulpy and putrid debris of segments of the tænia solium yield ova which show no sign of any approach of degeneration or decay. And the writer of this review has been struck by the remarkable way in which the size and structure of these ova allow them to elude all precautions that may be taken against their mechanical dispersion. In spite of every attempt to insure their destruction, by steeping the specimen glasses he may have used in strong acids, and by afterward bathing them in the flame of a spirit-lamp, he has once or twice found the characteristic ova appear most unaccountably in healthy and diseased tissues of secretions of the human body, which he has subsequently examined with these glasses. The dissolution of the parent tissues ultimately sets fiee the eggs contained in their interior, to be carried by the winds and waves wherever accident may determine. How vast a number of them miscarry is evident when we attempt to take the census of a single tape-worm! Or imagine the million of eggs such a parent foists upon society during the years it may inhabit a given animal. What becomes of these abortive germs, how long they retain any vitality, and what are the circumstances that may rob them of it are questions we can not answer, save by the conjecture that their albuminous and fatty materials are either applied to the soil in a decomposed form, or are consumed as food by various of the minute intervertebra that throng the surface of the earth and the waters. But the more fortunate minority of these eggs, the des- 
tiny of which is to eat instead of being eaten, after many and long wanderings of this passive nature, are, at length, engulfed by some unconscious animal in company with its food, and, through its alimentary canal, attain the locality of their second form of existence. During this passive emigration, the worm has retained its previous size (1-700th of an inch) and shape. But its thick wall bursts and sets free the inclosed embryo, which is an ovoid body, of nearly equal size, armed with six hooklets at one extremity. Impelled by instinct to begin its active migration, the embryo pierces the first portion of its path by bringing together the anterior pair of hooks, so as to form with them a kind of wedgeshaped stiletto, and now drags itself forward in the same direction by means of the succeeding pairs of hooks, which it uses like a person who, in attempting to get out of a bow-window, thrusts himself forward by his elbows. In this way the minute embryo penetrates the body it inhabits, and only increases its efforts on reaching the place its instinct recognizes as suitable for its abode, prior to the next series of changes it has to undergo. Streaks of reactive inflammation and exudation generally indicate the minute channel by which the embryo thus traverses the wall of the digestive canal, in its course to the liver or other organs. The migration of a tænia is probably a passive process. Various facts suggest it to be so- " a true locomotion, effected under the impulse of an instinct, and by means of certain special organs. The germs of parasites are evidently carried through the system in the stream of circulating blood, and they do not always travel themselves through the interstices of tissues.

Sturdy was long considered as dependent on a simple accumulation of water on the brain, generally affecting one side. Lœcke had observed, in 1780, that the water-bladders on the brain of giddy sheep were animals; and Fabricus (Harvey's master) was the first to assert the same respecting the cysticercus of the pig. Albildguard, the founder of the Copenhagen Veterinary School, observed that a tape-worm (the bothriocephalus latus) which existed in the abdominal cavity of the stickle-back, and in the intestinal canal of certain water birds, never had eggs in the former but only in the latter situation; and that from the firstmentioned creature it passed into the second, he ascertained by direct experiments with ducks, which he fed on banstickles. Gotze, in 1782, had perceived the great resemblance between the 
head of the hydatid of the liver of mice and rats, the cysticercus fasciolaris, and of the tape-worm of the cat (tænia crassicollis.) The cercariæ was first studied by Müller, and lastly by Bejanus, in 1818, who recognized them as parasitic in certain snails, inclosed in bags. M. Wagner and Von Siebold and Steenstrup fancied they had discovered the change cereariæ underwent to become true fluke-worms. Ehrenberg, in 1852, disputed Steenstrup's accepted metamorphoses, and only gives the resemblance of the tailless cercariœ, with a trematode worm. Dr. Kuchenmeister, of Sittau, instituted ingenious experiments to settle these hypotheses. He made dogs and cats swallow hydatids, which developed into tape-worms in the intestines. From the hydatids of the liver of cats and mice the tænia crassicollis in the intestines of the cat arose, and from the cysticercus of the hare and rabbit the tænia serrata in the intestine of the dog; so that if the waterbladder is lost, the head of the worm attaches itself to the head of the mucous membrane, the rings constituting the body of the tape-worms, including the organs of reproduction, and they are thus formed. By repeated experiments, made by several eminent zoölogists, the deductions were confirmed."

\section{Shaking Palsy.}

Shaking palsy is a condition of the animal economy known to medical men as irregular or abnormal nervous action. It generally occurs in cows of the nervous temperament, whose digestive organs are deranged; and, so far as my experience goes, the disease is confined to imported stock, of the Alderney breed. I lately attended an Alderney cow, the property of Mr. Chenery, at the "Highland Stock Farm," Belmont, Mass.

Symptoms.-The patient was suddenly attacked with symptoms of irregular nervous action of the muscles of the chest and forelegs, simulating shaking palsy. The membranes of the eyes were highly injected; pulse, jerking in unison with the irregular muscular action; external surface, extremities, and horns, quite chilly; respirations, normal; pulse small and languid.

Treatment.-I gave the patient three drachms of fluid extract of golden-seal, and the same quantity of fluid extract of camomile flowers. The spine and fore extremities were then irritated by the application of tincture of capsicum. On visiting the animal, the 
next day, I found her in better condition. The tremulous motion had somewhat subsided, and I pronounced her out of danger. She finally recovered without much subsequent treatment.

It is my opinion that this case had its origin in derangement of the digestive organs and constipation; for, after the exhibition of the tonics ("bitters,") which aroused the action of the stomach, the animal passed an immense quantity of black, fetid excrement. The quantity was so great that it occasioned remarks on the subject from all who visited her.

\section{HYDROPHOBIA.}

There are generally some circumstances connected with a case of hydrophobia which afford a clue to its real nature. It often happens that evidence conclusive is furnished that the animal has been bitten by a rabid dog. If this be the case, all doubts are at an end.

Symptoms.-In the early stages of the disease, there may be nothing in the symptoms to excite suspicion as regards the true character of the affection; they may merely denote approaching illness; but if the animal has been bitten by a rabid dog, or any other animal, and has become inoculated with the virus, which is usually communicated through the medium of the saliva, then any slight deviation from health, as shown by suspension of rumination, or by any other abnormal condition which may attract the attention of the farmer, is the precursor of the dreadful malady which is to follow. The most marked symptoms of hydrophobia are, protrusion of the eyeballs; the conjunctivial membrane is very much reddened, and, in fact, all the visible surfaces of the eyes, nostrils, and mouth are much inflamed. The animal is ripe for mischief, bellows occasionally, will paw and tear up the ground with its horns, and, on the least excitement, will become more dangerous than a rabid dog, trying, however, to do injury, to friend or foe, with its horns rather than with its teeth. The rabid cow or ox will drink water, if it can swallow; in fact, it generally suffers from intense thirst; but the fact is, the poor creature can not swallow a drop. The least attempt at deglutition induces spasms of the larynx, and puts the animal into the most distressing agony. It is not the sight of water that puts a rabid animal into convulsions, as some persons suppose; for in the early stage 
of the disease, when the membrane of the larynx is not much affected, they will drink freely.

Trectment.-It is well known that the poison of all rabid animals resides in the saliva, consequently they can not be handled and drenched without fear of danger; for if a small quantity of the saliva comes in contact with an abraded surface or sore, the unfortunate individual is just as much in danger as if he had been bitten. The only remedies that seem likely to be of any service are the plantain leaf (plantago major) and lobelia. Take four ounces of each of these herbs, and infuse them in two quarts of boiling water; when cool, strain through a fine sieve, and administer at once. If at the end of a few hours the patient has not improved, it will be advisable to destroy him.

The following interesting case occurred in the practice of $\mathrm{Mr}$. G. LEwIS, of Monmouth, England:

"I was requested to see a cow, the property of an extensive farmer near this town. Upon my arrival he gave me the following history of the case: 'On the 15th ult., the calf from this cow, tied in an out-house, was severely bitten in the nose and mouth by a dog, it was believed, although none was seen. But the cattle which were in the same meadow were in a very excited state, lowing and bellowing, as also the ealf; and, upon the arrival of the shepherd, who hastened to the spot, he found the calf much torn, and the cow with blood upon her nose. The calf, from this period until the 27 th, could not take its milk in the natural manner, and was obliged to be drenched. But from the above date, the wounds having healed, and the animal apparently recovered from the injuries it had received, it was turned to the cow, and took its milk in the usual way, which it continued to do up to the 31st, at which period the teats of the cow were bitten by the calf. From this date the calf became very ill, appeared to have sore throat, made a very peculiar noise, a kind of half bellow and roar, continued to get worse, and died on the $3 d$ inst.' I did not see him, but such is the description given to me by Mr. Jand I know that it is a faithful one. My attention was now directed to the cow. She was observed yesterday to separate herself from the others, and to bellow occasionally; but this morning she was seen to foam greatly at the mouth, and appeared much excited. The other cattle, also, would not associate with her, but kept at a respectful distance, with their heads and tails 
erect. She was now brought to the house, at which time I first saw her. She was standing; the eyes were half-closed; she appeared to be in a kind of stupor, or half comatose state; extremities, natural temperature; respiration, natural. She was looking rather thin; she was always a remarkably quiet creature, but now the least noise appears greatly to agitate her. The human voice, or the slightest movement, is sufficient to cause her eyes to glare, and set her bellowing, which ends in something between a growl and a roar. A person whistling, or the bark of a dog, produces fearful excitement. These paroxysms also appear to come on spontaneously. At their termination, the eyes again become halfclosed, the abdominal muscles tremble, the respiration is slightly quickened, and the pulse is quick and tremulous. I remarked that some water might be brought. Upon presenting the same she plunged her nose into the bucket, but could not swallow, and the effect was fearful to behold.

I informed Mr. J_ as to the nature of the case, stating that I had no hesitation in pronouncing it to be a case of hydrophobia; but, as he did not wish to have her destroyed, and was very anxious that I should give her something, and watch the case, I merely ordered a purgative, combined with a little febrifuge medicine, at the same time pointing out the propriety of being cautious while administering the medicine, and the probable impracticability of it, which was verified.

September 14.-All the symptoms of yesterday are aggravated intensively. The eyes have still a heavy appearance, when suddenly they appear like two brilliants. I observe that the spasm is more severe, and that she bites the wood-work of her stall.

September 15.-Much worse; the cornea of the left eye, in its center, is become opaque, and appears as though nitrate of silver had been applied to it. She sometimes lies down, but gets up again and roars. Upon a person present putting his foot near her mouth, she made an effort to seize it, uttering a growl. She died this evening.

"Post mortem examination, seventeen hours after death.-The brain-its substance appeared healthy. The pia mater showed intense inflammation in small patches; its vessels generally were very fully congested. The larynx showed traces of intense inflammation, as also did the membrane lining of the trachea, throughout its entire length, and was most beautifully spotted, 
as was also the lungs, pleura pulmonalis, pericardium, and heart. The coats of the first and third stomachs parted upon the slightest touch, in patches. The abdominal viscera, also, was similarly spotted, as was the diaphragm, etc., although not so thickly as the respiratory organs."

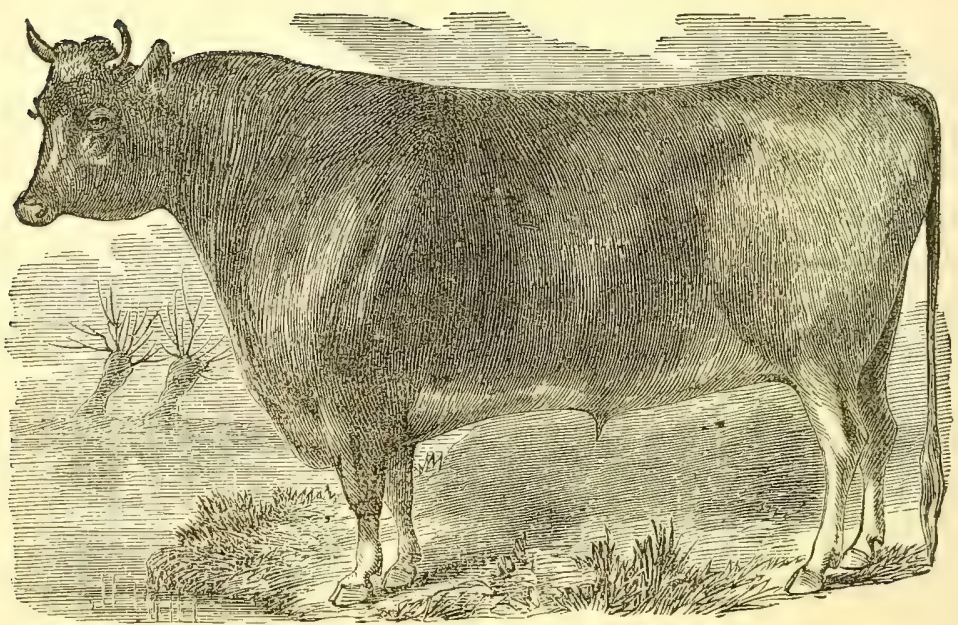

SPECIBEN OF AN ALDEIRNEY BULL. 


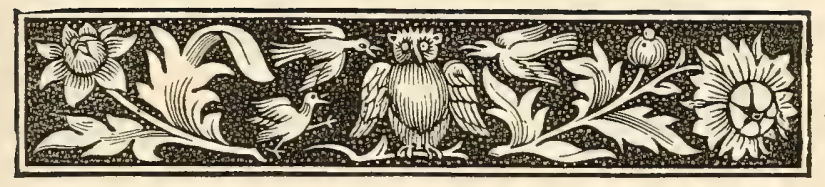

\section{SECTION XXVI.}

THE LIVER AND ITS DISEASES.

Description of the Liver-Secretion of Bile; tts Uses, etc.-Gall-bladderInflammation of the Liver-Hydatids-Javndice, or Yellows.

\section{Description OF THE Liver.}

TTHE liver is the largest gland in the body. Unlike the liver 1 of a horse, it has a large reservoir for the reception of the bile, called the gall-bladder. In form it is irregular, being convex anteriorly, or toward the diaphragm, with which it is in contact, and concave on its posterior surface, or toward and in the vicinity of a part of the stomach. It is composed of two lobes. That on the right side is the larger. The central portion of the gland is the thickest, and it gradually becomes thin toward its borders. The mass consists of a vast number of minute lobules, varying in size and form, containing a net-work of biliary ducts connected with their main trunks, and a large number of biliary cells; and each is connected, in like manner, with three blood-vessels ; namely, the hepatic or nutrient artery of the liver; the vena porta, which returns the venous blood after it has circulated through the intestines; the hepatic vein, which carries back the blood received from both the other sources. It will be seen, therefore, that the venous blood, which is brought to the liver by the vena portæ, is intended for the elimination of bile; therefore this vessel seems to act in the double capacity of vein and artery; for, as a vein, it receives blood from the abdominal viscera; as an artery it ramifies through the liver, forms a capillary net-work, and then secretes the bile. From this capillary net-work, which can be traced to the center of the minute lobules, the hepatic vein takes its origin, collecting the blood from the capillary net-work. It then unites with other 
radicles to form the main trunk, by which it is delivered into the vena cava.

The branches of the hepatic artery are principally distributed upon the walls of the hepatic ducts, and upon the trunks and branches of the portal and hepatic veins, supplying these and the contiguous parts with the necessary amount of arterial blood for their nourishment, as well as that of the whole gland.

The investing membrane of the liver, from which prolongations extend into its substance, is termed Glisson's capsule.

The liver derives its nerves and nervo-vital power from the great sympathetic and eighth pair.

The liver is confined to its situation by ligaments, which get the name of broad, lateral, coronary, and round. The round ligament is the remains of the umbilical vein of the fetus. The others are continuations or duplicatures of the peritoneum.

\section{Secretion of Bile; its Uses, etc.}

The bile is secreted from the capillaries of the hepatic artery, by minute glands found on the surface of the biliary ducts. It then passes through the biliary pores and branches of the hepatic duct. By this duct it is conveyed to the ductus communis choledochus (union of the cystic and hepatic ducts), from whence, in part, it passes by the cystic duct to the gall-bladder. When needed in the duodenum, it returns by the cystic duct, and mixes in the ductus communis choledochus with fresh bile from the hepatic duct, and then passes into the duodenum. The bile having entered the intestine, mixes with the aliment and pancreatic juice. The pancreatic juice changes the digested aliment into a brown mass, termed chyme, and then emulsifies it. After being emulsified, a portion of the chyle is taken up by the lacteals and enters the receptaculum chyli. In a healthy state of the system, should any bile escape with the chyle, it is absorbed by the mesenteric glands, and returns to the liver by the vena portæ.

\section{Galim-Bladder.}

The gall-bladder is a pear-shaped bag connected with the concave and posterior surface of the liver, by the above vessels and cellular membrane. It has four coats, termed peritoneal, cellular, 
musclar, and villous. The villous coat is the internal one, and is thrown into numerous minute folds, arranged in a reticular form, filled with small ducts, or follicles, and glands. The latter secrete a fluid for the protection of the internal surface.

\section{INFLAMMATION OF THE LIVER.}

Cattle and young stock, when fed too high, or when allowed to luxuriate in a rich meadow, are often attacked with an acute disease of the liver.

Symptoms.-The principal symptoms of this malady are yellowness of the membrane which lines the eyelids, and covers that part known as the "white of the eye." The visible surfaces of the mouth are also of a yellow tinge. In addition to the above symptoms, the animal is feverish, thirsty ; mouth and base of the horns, hot; pulse, accelerated; breathing, rather laborious ; rumination is suspended, and the animal is said to have "lost its cud." Some fullness will also generally be observed on the right side, in the region of the liver, and the animal will occasionally turn its head in that direction, as if it were the seat of pain, which is probably the case ; yet the most reliable symptoms, in a disease of this character, are a yellow tinge of the visible surfaces, accompanied by febrile symptoms.

Treatment.- The most rational method of treating this disease is to endeavor to mitigate the inflammatory diathesis, and restore the normal function of the liver. In view of accomplishing these desirable results, I recommend the following prescription :

No. 111. Glauber salts.................. 16 oz.
Powdered mandrake.......... $2 \mathrm{dr}$.

The salts should be dissolved in one quart of tepid water; then add the mandrake, and drench the animal by means of a common porter-bottle. This drench should be poured down the œsophagus in a gradual manner, so as to prevent its being received into the rumen, or paunch.

The patient should, if possible, be dieted on green fodder. If such can not be procured, some sliced cabbage, turnips, or carrots may be substituted. A tea-spoonful of mandrake should be given daily in the food, until the visible surfaces assume their natural color. A curable case will generally yield under the above treatment. 
This disease sometimes runs into a chronic type, and is known by the yellow color of visible surfaces, dull, sleepy appearance of the subject, and absence of those acute symptoms which are invariably present in the inflammatory stage. A chronic disease of this character may exist for months and even years without interfering very essentially with the general health. Finally, however, the liver undergoes alterations in structure, becomes hardened or indurated, or else it becomes tuberculous, or is the seat of hydatids, and the fluke-worm is often found in the ducts. The treatment for chronic disease of the liver is as follows:

\section{No. 112. Powdered iodide of potassium......2 dr. Powdered golden seal.......... 4 oz. Mix.}

Divide the mass into six equal parts, and give one in a little water, every morning, on an empty stomach. The medicine may be continued for some length of time without the least danger. If this treatment does not benefit the animal, the case is probably incurable.

\section{HYDATIDS,}

Hydatids, commonly known as "flukes," consist of a sac or vesicle filled with fluid. To the naked eye it appears as a simple enveloping cyst; but on examining it more closely, by means of the microscope, it shows many tunics or coats, and these are the rudimentary cells in various stages of growth. According to CARPENTER, these rudimentary developments project more and more into the parent cell, and, at last, become detached from its wall, and lie loosely within it. Shortly before this separation, however, the young hydatid is seen to contain smaller cells, which increase in size along with it. This increase continues until the new brood thus formed entirely fills the cavity of the parent, and a further increase causes the rupture of the sac and the escape of the progeny; and these, in their turn, undergo the same evolution, becoming parent hydatids in distinct cysts, and setting free their contained cells as a subsequent generation. These cystic entozoa are never found in the alimentary canal, but are always embedded in the liver, brain, or glands of organs. They obtain their food by absorption from the inner surface of the investing membrane. $\mathrm{Hy}$ datids infest the various organs of the bodies of all mammalia, and, although they are said to possess an independent existence 
while residents of the organ or organs which they inhabit, they die immediately when removed from their chosen habitation.

"The principal genera of cystic entozoa are, cysticercus, cœnurus, echinococcus, to which may be added acephalocystis. Several species of cysticercus are enumerated, but the most common are cysticercus tenicollis and cysticercus cellulosus. The former (tænia hydatagenia, hydatis globosa) is met with frequently in the peritoneum and pleura of ruminating animals and pigs. It is often generated in the disease called rot, where another entozoön, the distoma, or fluke-worm, is met with in the biliary ducts (liver). The cysticercus cellulosis is found generally lodged in the tissues of the muscles. It occurs sometimes in man, but more frequently in animals, particularly in the hog, where it causes the disease denominated measles. Of the genus cœnurus (hydatis polycephalus), the species cerebralis is found in the brain of sheep, oxen, and other ruminating animals. These hydatids on the brain of sheep cause the disease called sturdy, or giddiness. The hydatids belonging to the genus echinococcus are considered, by some, as varieties of the acephalocyst. They are commonly called granular hydatids, from the presence of numerous granules which float in the fluid of the cyst, or adhere to its walls. There are two species of echinococcus. One, echinococcus hominis, has been met with in the brain and abdomen of man, in a few instances; the other, echinococcus veterinorum, occurs in the hog and other animals.

Hydatids occur much less frequently in the brains of cattle in Great Britain than in other countries, as Youatt has correctly said, in his treatise on the 'Diseases of the Ox.' It occurs only in the young animals. Indeed, it obeys the same laws that guide the development of the conurus in the sheep; and it is a well-established fact that it is only when animals are growing that the germs for the propagation of the bladder-worms will be taken up, and carried to parts probably through the blood.

The success attending the trephine, in cases of hydatids in the brain of the ox, is very great and encouraging; and not only have I read interesting and convincing records on this subject, but, in conversation with skillful and experienced veterinarians, I have learned that trephining is an operation often to be relied upon." *

The liver is sometimes enormously enlarged, in consequence of 
the presence of a numerous progeny of the fluke-worm. The following ease, contributed to the "Veterinarian" by Surgeon J. B. Gregory, will serve to illustrate this matter. The morbid parts referred to were sent by Mr. Gregony to the editor of the "Veterinarian," who makes the remarks inclosed in brackets:

"The morbid parts I send were taken from a well-bred, shorthorned eow, six years old, estimated to weigh, when fat, one hundred and twenty-five stones of eight pounds. She was purchased in August last, with a warranty to calve in November following. On September $2 d$ her owner called on me, and wished me to give her some medicine, as he thought she was not quite recovered from the effects of being driven sixteen miles. The medicine I sent had a beneficial effect, as the cow's appetite returned, and she appeared to be quite well again. After this I heard no more of her until the 6 th of January last, when I was requested to go and give my opinion as to her being in calf, it being two months beyond the time she was expected to calve. I found that her appetite and rumination were natural, the pulse regular, and the kidneys and bowels acting well. There was, however, a peculiar expression of her countenance, that told of continuous pain. Her eyes, also, were sunk in their orbits, and she had a frequent and troublesome cough. Her skin, likewise, had lost its pliancy, and she was sadly out of condition, but no yellowness of the mouth or eyes was present. I first examined her abdomen externally, by percussing the right side, with a view of determining her pregnancy; but the body my hand came in contact with was too large for, and had not the feel of, a fotus. As the cow had occasionally been observed to strain, I was now induced to examine her per vaginum, when I found the os uteri to be indurated and unyielding. I could also distinctly feel a round and hard substance, about the size of a large cricket-ball, and which I supposed to be the right ovary; the other one, however, I could not find. I told the owner that the cow was not in calf, unless it were extra uterine, and that, in all probability, the mass we could feel from the outside was a tumor within the abdomen. I also added that medicine could do no good in such a case. He at once decided on having her killed, which afforded me the opportunity of making a post mortem examination. All the viscera were healthy, with the exception of those I send. The liver, as you will see, is the organ principally affected. It weighed, when first removed, one hundred and forty-six pounds. Its great 
size led to its encroaching on the space occupied by the other viscera, all of which were more or less compressed. It did not adhere to the side of the abdomen, but was firmly attached to the diaphragm, and also, in places, to the intestines. In my examination I had the assistance of Mr. Hearn, M. R. C. V. S., who was recently your pupil, and who has, I believe, also written to you on the subject. The cause of the enlargement of the liver is evident enough, being produced by hydatids, but I leave to you to describe the variety to which they belong. I have attended post mortem examinations of many cattle since 1828 , but never met with any thing like this before.

[The description given by Mr. Gregory leaves us but little to say respecting this extraordinary enlargement of the liver, as stated by him. The sole cause of its increase in size was due to the presence of an immense number of hydatids in the substance of the organ. These entozoa belonged to the variety designated the acephalocystis endogena, and each cyst contained within it a considerable quantity of the so-called echinococci. In the 'Transactions of the Veterinary Medical Association,' for 1842-3, a similar case is recorded as occurring in a pig, which is illustrated by a colored plate, and which may be said to depict equally as well the condition of the liver of the animal in question.]"

\section{JAUNDICE, OR YELLOWS.}

This disease is of very common occurrence among horned creatures. The stall-fed animal is, probably, more subject to it than those otherwise fed. From this the reader will infer that it is more likely to occur in the winter or spring than at any other season, which is the case. In order to prove this, let any one visit our markets, and note the color of the fat of beef, and he will notice the yellow appearance of almost all the beef offered for sale. There may be some exceptions, yet this feature of fat is a sure sign that the animals, while living, were not entirely free from functional derangement of the liver. This peculiar color, however, is very rarely, if ever, found to pervade the brain, humors of the eye, or the milk during lactation.

Occasionally a yellow tinge of the visible surfaces (membranes of the mouth, nose, and eyes) appears very suddenly, and is supposed to originate from sympathetic action of the brain upon the 
liver; yet it may arise in the same sudden manner, in consequence of gastro-intestinal irritation, produced by the introduction of poisonous plants and rough food, such as corn-stalks and sugarcane, into the stomach. Jaundice, in a very protracted form, often owes its origin to the presence of biliary calculi. So say the authorities; but I have examined many gall-bladders after death, yet have never succeded in finding any; and I have inquired of many slaughterers, and am informed that they never observed any stones in the gall-bladder; yet, in various alkaline districts of this country, I presume biliary calculi may be as common as in the chalky regions of the old world. According to concurrent testimony, the presence of biliary calculi in the gall-bladder of an ox does not interfere much with the general health; but they occasion a jaundiced appearance, a general eye-sore, which renders yellow beef unacceptable to the palate of an epicure, and the shrewd butcher declines to deal in any thing yellow, excêpt pure "dust." I know of no symptoms by which the presence of biliary calculi can be detected prior to death.

The following paragraph occurs in "Youatr on Cattle," and may throw some light on the subject:

"Sometimes, they (calculi) enter the duct (the cyst) which conveys the bile to the intestines. They are likely to do this on account of their swimming on the surface of the fluid which the bladder contains. The cystic duct is large at its union with the bladder. It is a continuation of the neck of the bladder, and the gall-stone may be easily pressed into the commencement of the tube; but it has scarcely entered it before its passage is obstructed by the folds of the inner coat of the duct. These assume a semilunar form, with the edges projecting toward the bladder, and they act as partial valves, retarding the progress of the bile, so that it may not all be pressed out at once, but gradually escape as the process of digestion may require. The gall-stone being thus impacted, violent spasmodic action takes place in the muscles of the duct, occasioned by the irritation of its continued pressure. It is fortunate, however, that, although the muscles of these ducts act with some power, the obstruction is usually, with no great difficulty, overcome. The duct distends; as it distends these valvular folds lie closer to the sides, and no longer oppose the passage of the calculus, which is pressed on until it reaches the common duct. The caliber of this tube is larger, and, unless the calculus is of con- 
siderable bulk, no further difficulty occurs until it reaches the opening into the duodenum, which, being situated in the center of a muscular prominence, acting as a valve, and preventing the passage of all matters, whether fluid or solid, from the intestine into the ducts, a new difficulty is opposed to the progress of the gallstones, and there is some return of pain, and, in a few cases, the pain is evidently more intense than in the early stage. At length, this sphincter muscle of the duodenum dilates, the calculus enters the intestinal canal, the pain ceases, and the natural color of the skin returns. In this species of jaundice, we have, in addition to the yellow skin, the heaving of the flanks, the hard, concentrated pulse, the diminished appetite, the insatiable thirst, and the other symptoms of fever. Then, too, we have the alternate cold and heat of the ears, the roughness of the coat, the urine becoming first of a transparent yellow, and then opaque red, saffron-colored, or brown, and the sediment brown. The bowels are constipated, the feces seldom evacuated, and, when appearing, are hard and black."

Treatment.-When occasioned by the presence of gall-stones, the animal should have a daily drench, composed of

No. 113. Hydrochloric acid.............. 2 dr.

Water........................ 1 quart.

Every other day, give three drachms of fluid extract of mandrake. This will arouse the liver to healthy and vigorous action and will remove from the intestinal canal all irritating substances.

When jaundice is occasioned by either functional or organic disease of the liver, I generally give the following:

No. 114. Fluid extract of mandrake......... 1 oz.

Powdered hyposulphite of soda...... $\frac{1}{2}$ oz.

Water...................... 1 pint.

Mix.

Let this dose be given every morning, before feeding, for five or six days, or until the visible surface of the mouth becomes of a more natural color. In the day time the animal should be turned out to grass, if the season permits. 


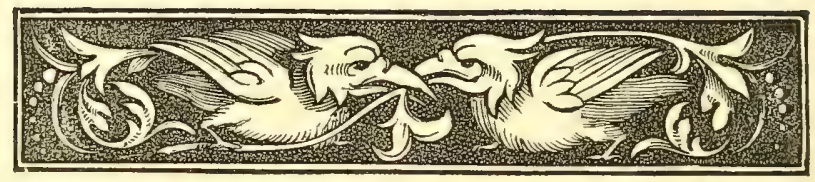

\section{SECTION XXVII.}

\section{DISEASES OF THE SKIN.}

Exanthemata, or Eruptive Fever-Mange-Four in, the Foot-WartsGadflies-Yoke Galls-The Hair of Cattle an Epidermic AppendagePemphygis or Vesicular Eruption-Cracks in the Heeis of Cattle.

Exanthemata, or Eruptive Fever.

TXANTHEMATA is the name given to a contagious (someIld times epizoötic) or eruptive fever which occurs among cattle. The following cases will probably give the reader a good idea of the cause and nature of the malady now under consideration :

An unusual and alarming disease having made its appearance on the premises of Mr. Waterman, at Warsaw, I was requested to visit the place, in view of ascertaining, if possible, the nature of the disease, its cause, and treatment. On arriving at the place designated, I learned that disease and death had run riot, making terrible ravages among a herd of cattle which, only a few weeks ago, numbered eight hundred, but are now reduced to six hundred. The loss of two hundred fine animals in the course of a few weeks was a calamity calculated to alarm the husbandmen in the whole neighborhood.

From information obtained on the premises, I learned that no disease of the kind had ever prevailed there, nor in the regious where the cattle had been purchased, but the most positive evidence seemed to show that the malady had a spontaneous origin on the premises of the proprietor. Some of the animals were purchased in Missouri, and, when they arrived at Warsaw, were as poor as Pharaoh's "lean kine," splendid specimens of skin and bone. The dietary arrangements of the establishment were not calculated to benefit their condition, their principal food being "slop" from the distillery. It was not the intention of the owner 
to feed this large herd of cattle exclusively on "swill," but his stock of hay having become exhausted, and the roads being in very bad condition, it was impossible to obtain a supply of the same. In consequence the function of rumination, or remastication, was suspended. The hot "swill" from the distillery failed to distend the complex stomach of the animal to a healthy capacity; it operated so as to produce a lax state of the bowels, amounting, in a large number of cases, to actual diarrhea, or liquid stools having an offensive odor. The constant discharge from the bowels of liquid feces augmented the debility of the "lean kine." They became afflicted with a mortal malady, or enzoötic affection, which ran like wild fire through the whole herd. It manifested itself, first, in a condition of anemia; then depilation of the hair took place; the external surface of the body, in many cases, presented a raw surface, and the animals seemed to be crazy to rub themselves against any fulcrum that would mitigate their intolerable itchiness. Many of them got down on the floor, and, by contact, abraded the external surface of the body in many places, so that the raw flesh was exposed, and, in some cases, the hip bones protruded. It was noticed that whenever an animal thus affected got down on the floor, death invariably closed the scene. Some of the creatures thus having the finger of Death upon them were charitably put out of their misery, by killing them outright.

It appeared to be of an exanthematous character, and contagious. The proof of its contagiousness seemed to be evident, from the fact that the whole herd were affected with precisely the same pathological symptoms ; namely, depilation of hair, debility, eruptions on the surface of the body, anemia, diarrhea, etc. I might be mistaken about the contagious element of the disease, but give my readers the facts, and leave them to form their own opinions. It may be that the evils to which the animals were subjected, and which was operative in implanting the malady in the system of the first victim, was operative, and produced the same in all the rest of the herd; but I have no proof of a positive character to offer in favor of either theory.

I had supposed that the skin disease was, like itch, dependent on the presence of parasites located under the scarfskin; but in the use of a good magnifying power, the supposed parasites were not discernible, but beneath the scarfskin, in the integuments and 
subtissues, were observed a multitude of minute local beds of lymph, from which sprang the superficial local malady on the skin. Incrustations, or scales, solitary and in clusters, formed wherever the deeper-seated integuments were affected; and in some cases, when the scabs were rubbed off, the parts looked red and inflamed, and some persons contended that the animals had the red or mad itch. Almost every person who saw the animals declared that itch was the disease; but, knowing that itch is due to the presence of parasites, and failing to detect any, I could not so name the malady.

I examined very carefully the external surface of the body, yet could not detect either lice or parasites to account for the intolerable itchiness which was present, more or less, in the whole herd. From all the evidence I was enabled to obtain during a brief visit, I was forced to the conclusion that the cutaneous affection was of an exanthematous character, and resembled eczema. Nearly all the animals were in a state of debility; all had daily been losing flesh, and those which I had examined after death were anemiac (bloodless). Their muscles were pale, like those of a calf when bled to death. Their stomachs and intestines were void of food; the hot swill seemed to have found no resting-place in the digestive cavities, but ran through them like a dose of cathartic medicine. The internal organs of those animals I examined presented no signs of disease, except that they were pale and bloodless.

Causes.-The causes of the disease may be summed up as follows: Debility, from insufficient nutritive food; the filthy condition of the cattle-pens and surroundings; herding of too many animals together in a small space; want of pure air, exercise, and muscle-making nitrogenous fodder.

- Treatment.-I informed the proprietor of the establishment that no medicinal remedies would be of any avail unless the exciting causes were removed, and suggested that the afflicted animals be allowed the use of their limbs and lungs on dry ground, in the open air, within an extensive inclosure, and that they should be allowed a bounteous supply of hay, corn, and corn-stalks; then medicinal remedies of a tonic, stimulant, and alterative charácter, which I prescribed, might prove beneficial. For the local affection, I prescribed linseed oil, petroleum, lime-water, and sulphur. Several applications of this compound were made, and the subjects 
were much benefited thereby. Many of the affected animals, however, were in such a deplorable condition that I urged the owner to have them destroyed, as a deed of charity.

\section{MANGE.}

This disease is too well known to need any particular description from me. It is due to the presence of parasites; hence it can be communicated by contact or touch, and is, therefore, contagious. This latter fact suggests the propriety of removing the diseased animals from the healthy ones.

Treatment.-Let the animal have a table-spoonful of sulphur in the food, for three or four days in succession; in the mean time, anoint the affected parts daily with a portion of the following:

No. 115. Cod-liver oil................. 6 oz.

Sublimated sulphur............. 2 oz.

Mix.

Apply by means of a sponge.

In the course of four or five days, wash the surface of the body with warm water and soap, and then give the body a thorough sponging with the following:

No. 116. Lime-water................. 1 qt.

Sublimated sulphur.............. 2 oz.

The above treatment generally cures the most inveterate cases.

\section{Foul IN the Foot.}

In cases of this character the animal is dead lame, and often an intolerable stench arises from the parts between the claws. This is followed by the discharge of sanious and, finally, purulent matter. In this stage astringents and antiseptics are indicated; therefore I recommend the following:

No. 117. Tincture of matico.............. 2 oz.

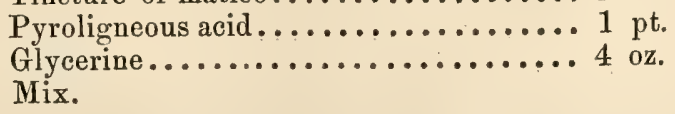

Saturate a small piece of sponge with a portion of the above, and introduce it between the "cleft" of the foot; the hoof and contiguous parts are then to be bathed with the preparation; and, 
finally, in view of keeping the sponge in place, and to produce a good effect on the external parts, a narrow bandage must be applied, so as to encircle the hoof. If any heat or tenderness exist, the bandage should be kept moist with cold water.

\section{WARTS.}

Warts, as they occur on cattle, are of three kinds. One makes its appearance upon the skin, and consists, in part, of an increased development of the epidermis or scarfskin; the next kind originates under the epidermis, and, as it grows, elevates the same; the latter, however, is nothing more than a common encysted fibrous tumor, without organization. This can readily be removed by cutting down upon it, and dissecting out the cyst or sac which enshrouds it. The epidermic wart sometimes has a very broad base, and, in order to remove it, a double-armed ligature must be sent through it, so that it can be tied in two equal halves. Occasionally this kind of wart is pendulous-has a contracted neck at its base. This may be encircled by a single ligature, and, in the course of thirty-six hours, will fall off. The third kind of wart is a sort of fungous excrescence-not horny or hard, like the two former, but possessing great vascularity, and, when rubbed or bruised, they often bleed profusely. I lately removed one from the inferior jaw of an ox, which bled very profusely after the removal, and did not cease until a hemostatic had been repeatedly applied. The hemostatic was powdered matico leaves, which coagulated the albumen of the blood. There is great danger in removing warts of this kind. The following case, occurring in the practice of Surgeon CARTwRIGHT, will illustrate the proposition:

"On the 1st of June, 1846, I removed several warts from the linea alba of a year-old heifer, belonging to Samuel Worthington, Esq. I cast her, and cut them off with the knife, and did not apply any thing to the wounds, as they did not bleed an ounce. I could see one or two large blood-vessels on the surface of the wounds. We loosed her from the hobbles, and then tied her up, and the wounds soon left off bleeding. Gave her an aperient, and left her for the night.

The next morning she was found down, and could scarcely get up from loss of blood, which continued to issue through a large 
band that was passed round her. I was immediately sent for, but was gone to Liverpool. They, in consequence, applied to a druggist, who sent some alum-water, which stopped the blood. In a day or two I saw her, but, as a scab was then forming on the wounds, I did not meddle with it; soon afterward the parts were cicatrized.

There is no doubt but that, after she was left, she commenced licking the wounds, and so brought on the secondary hemorrhage. The person who looked after her believes that, had not something been done at the time, she would have bled to death. From this and other cases that I have seen, I think it absolutely necessary that we should guard against the animals licking themselves, and perhaps it would be quite as well to apply the cautery to the parts, after removing the warts, or subsequently."

When a large surface is occupied by warts which do not possess any definite necks, they must be cut off close to the skin, and the surfaces thus exposed should be touched with strong pyroligneous acid, and then sprinkled with powdered bloodroot.

Warts on the teats are to be removed by ligaturing them at their base, by means of saddler's silk, or horsehair. If securely tied, so as to obstruct circulation, they drop off in the course of thirty-six hours. Any sores which may occur in consequence of the removal of warts, can be healed by covering them once or twice daily with a small quantity of glycerine.

\section{GadFlies.}

The gadfly is known to naturalists as the ostris bovis. It punctures the integument of cattle, and then deposits its ova, or eggs. In this situation the ova mature, until they are capable of enjoying an independent existence. They then make their exit through the external outlet, fall to and burrow into the ground, and remain there until the period of their metamorphosis takes place, when they assume the form of winged insects. In this form they multiply the species by the deposit of their ova. They probably occasion much irritation; and, in view of getting rid of them, -I usually puncture the tumor, by means of a thumb-lancet, and squeeze out the parasite.

The following paragraph is from the pen of GUNTHER; and I urge the reader, if he be the owner of live stock, to give them an 
occasional dose of sulphur; for I contend that no living creature can ever be infested with parasites when its system is saturated with sulphur:

"The gadfly not only persecutes healthy oxen, by its bites, during summer, but also deposits its eggs in their skin, which give rise to tumors on the back and other parts, in which the larvæ become developed. They live there on the succulent fluid which the soft parts secrete, and make their escape thence in the following spring, in order to become metamorphosed. The greater the number of tumors, the more is the strength of the animal diminished by the pain and suppuration. For this reason we should endeavor to free the animal, as soon as possible, from these larvæ pests, by frequently washing these tumor's with camphorated brandy or forcibly compressing them, which either crushes the insect or forces it to make its exit. When they have attained the size of a filbert, an incision must be made into the part, which is then to be covered with a pitch plaster. A few doses of sulphur are to be given internally. We are told that those oxen which have taken sulphur for a long period of time are not infested by gadflies."

\section{YoKe GaLLS.}

The exciting cause is local irritation occasioned by the yoke.

Treatment.-So soon as an abrasion is discovered on the neck, the animal should be excused from duty for a few days. The abraded part should be lubricated two or three times daily, with a small quantity of glycerine. In most cases, however, a few applications of tincture of aloes and myrrh will produce a healthy action, and thus restore the parts to soundness. Should there be no abrasion, yet some tumefaction, heat, and tenderness, a coldwater bandage, renewed as occasion seems to require, will, in most cases, have the desired effect. Occasionally the integuments are so bruised as to induce induration (hardening). Local induration in the neck is a morbid condition of parts, known to the farriers of old as "sit-fast." The treatment consists in smearing the part with a portion of the following:

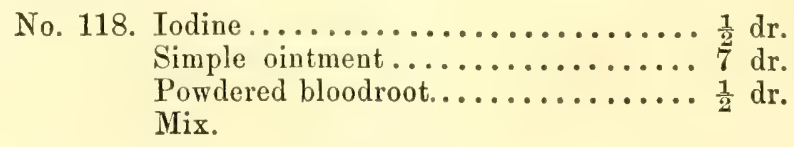


A few applications of a portion of the above will have the effect of removing the sit-fast, or eschar, when a healthy granulating surface will appear.

Some animals, owing to a peculiarity of constitution, will "chafe," as the saying is, in those parts which come in contact with the yoke, and no human foresight or mechanical contrivance can prevent it; therefore, in view of protecting the parts against the local irritation and its consequences, I recommend the following liquid cuticle:

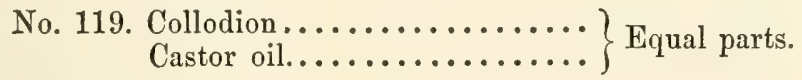

After washing the abrasion with soap and water, wipe dry, and smear it all over with a portion of the above invaluable liquid cuticle.

\section{The Hair of Cattle is an Epidermic Appendage.}

According to CARPenter, hair is an epidermic appendage, although not developed upon the external surface, but in the interior of a follicle (sac or fold) formed by a depression of the true skin. This follicle is lined by a continuation of the epidermis (scarfskin), the cells of which are developed in peculiar abundance from a spot at its deepest portion, the dense exterior of the cluster thus formed being known as the "bulb of the hair," while the softer interior is termed its pulp. Although the hairs of different animals vary in the appearances they present, we may generally distinguish two elementary principles corresponding with those which we meet with in the stem of a feather; namely, a cortical resembling bark, and a medullary resembling marrow. The fullest development of both substances is seen in the spine hairs of the hedgehog, and in the quills of the porcupine, which are but hairs on a magnified scale. The cortical envelope of hairs is a continuation of the outer scales or layers of the epidermis that lines the follicles, while the medullary is derived from the deeper stratum, whose cells are produced in usual abundance at its cœcal (blind) extremity; and it is by the constant development of new cells at this point that the continual growth of the hair is kept up. 


\section{Pemphigus, or Vesicular Eruption.}

In the United States, (at least in the East,) pemphigus is of rare occurrence, yet it has made its appearance in Texas and in the Western States. The following symptoms, which usually accompany this eruptive affection, may enable the reader to recognize it.

Symptoms.-The breathing is generally hurried and sonorous, accompanied by a slight cough ; the animal shivers, which is occasioned by deranged and irregular nervous action ; the integument in the regions of the neck, shoulders, anus, loins, and haunches is elevated by effusion into the cellular tissue; the cuticle, or external tunic of the body, is the seat of watery bladders, varying in size from a Beverly bean to a walnut; in other parts of the skin a serous or watery fluid may be observed to dribble from the hair. The ears are drooping and moist, and their temperature is much elevated; the base of the horns are hot, and the whole external surface of the body is in the same condition; the visible surfacesnamely, those of the eyes, mouth, and nose-are highly injected with a reddened yellow tinge; the tongue is swollen or tumefied, and an abundance of saliva flows from the mouth; the pulse will often rum up to 80 , yet may be imperceptible at the jaw; the spinal column presents a convexity, or is arched upward; the urine is scanty and dark-colored, and the feces are of a lighter color than usual, showing very conclusively that the function of the liver is impaired.. As the disease advances, the serous tumors burst, exposing broad excoriated patches.

Treatment.-Let a portion of the following mixture be applied to the surface of the body, morning and evening:

$$
\begin{aligned}
& \text { No. 120. Glycerine ................. } 2 \text { oz. } \\
& \text { Hyposulphite of soda........... } 2 \text { oz. } \\
& \text { Rain-water.................. } 1 \text { pint. }
\end{aligned}
$$

Mix well together. After this lotion has been applied a few times, a decided improvement in the skin will be apparent.

A few daily doses of the following medicine must also be given:

No. 121. Fluid extract of poke-ropt......... $3 \mathrm{dr}$

Chlorate of potass............. $4 \mathrm{dr}$.

Water...................... $\frac{1}{2}$ pint.

A few years ago a vesicular epizoötic broke out among cattle and milch cows at Stratford-on-Avon, England, the symptoms of which are thus described by J. Tours, V. S.: 
"The symptoms were, increased pulsation; copious discharge of saliva from the mouth; respiration, disturbed. These symptoms continued for two or three days, when a tremendous inflammation of the integuments and cellular tissues set in, around the coronets at the heels and between the hoofs, causing exceeding lameness and excruciating pain. The swelling quickly extended to the fetlocks. The poor beasts lay down the greater portion of their time-panted and perspired profusely, with occasional deep groaning. At this stage of the disease the mouth improved. When made to get up, they could hardly walk, and soon lay down again. The beating of the heart could be distinetly heard several yards off. In some, all four feet were affected; in others, the fore-feet; others, the hind feet, and in some one fore-foot. Suppuration took place in the integumentary and cellular membranes, five or six days subsequently to the original attack, which process afforded great relief. In the interim they became emaciated, and lost their milk. In a day or two after, extensive sloughing supervened of the integuments of the coronets and heels, and between the hoofs, leaving the pedal and coronary bones bare, with deep sinuses. The putrid parts, while sloughing, emitted an unbearable stench. Granulations of new flesh soon formed, which became prominent and luxuriant, forcing the hoofs wide apart. At this period of the complaint the urgent and painful symptoms considerably lessened; the animals could then hobble out of the yard into a grass-field close by, where they were prior to the attack. The milk, flesh, and strength began to return, and the lameness slowly diminished, as the thickening of the integuments and cellular membrane became absorbed. New hoofs formed, which in some grew irregularly, and caused great tenderness for some length of time."

\section{Cracks in the Heels of Catthe.}

Cracked heels are generally more prevalent in the horse than cow; yet as I have had an opportunity of seeing and prescribing for a few cases of this character, I shall give the reader the benefit of my experience, so that, when cases of this character do occur, the farmer may know what to do. Some persons suppose that uncleanliness is the sole cause of cracks. This is not the case; the subjects are predisposed to a humory condition and congestion of the feet. 
Treatment.-The part must first be sponged, or, rather, washed, with tepid water, slightly alkalized with a small quantity of carbonate of soda. I prefer soda instead of soap. The latter is apt to irritate the parts; and there exists no dirt nor morbid matter which can not be removed by an alkaline wash. After cleansing the parts, apply a portion of the following:

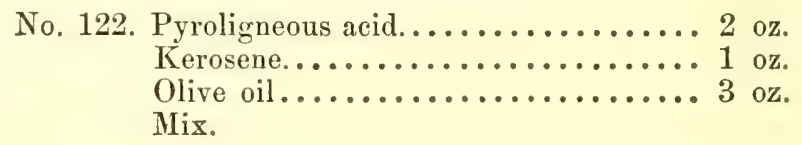

Apply by means of a piece of sponge. The parts should be dressed once per day, and oftener if necessary. The edges of the crack sometimes become inverted. In this case procure a small piece of linen, form it into a pad, and, after smearing its surface with a portion of the above preparation, apply it so as to press the lips of the gap together, and then bind it on.

If the disease be constitutional, which may be inferred from the fact that the animal is said to be "subject to it" in the winter, then a few doses of alterative medicine may be given. The following forms a very excellent stimulating alterative:

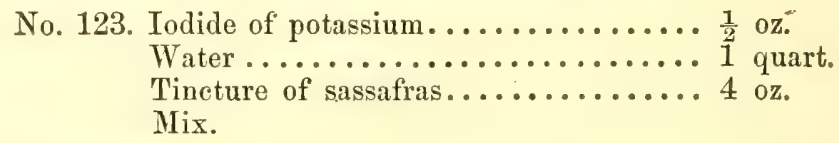

Give a wine-glassful every morning, before feeding-time. 


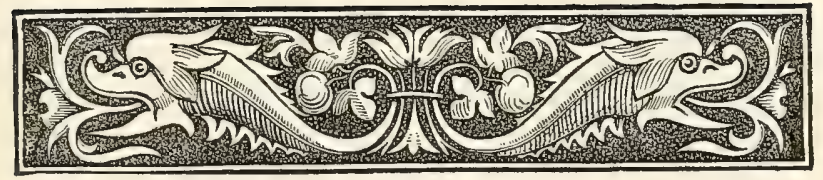

\title{
SECTION XXVIII.
}

\author{
VARIOUS OPERATIONS AND DISEASES.
}

Castration-Enzoötic Milk Sickness, or Trembles-Improved Method of Milking-Diseased Thymus Gland-Cords in Young Calves-Steaned and Cooked Food for Stock-Cheap Fodder for Cows-Black Tongue-FraCtured Bones-Fractured Radius of a Calf-Light in Barns-Diarrhea in Calves-Sprains or Strains-Spaying Cows.

\section{Castration.}

IiABOR under an impression that the husbandmen of this I country commit some awful mistakes in the theory and practice of castration. The usual practice is to castrate the calf from one to three months after birth. This is evidently done to the manifest injury of form, size, and muscular development. The operation may tend toward fattening the animal, and improving the epicurean quality of its meat; and it may render the animal docile, and thus increase his usefulness; but his strength, stamina, and endurance are compromised by early castration. If animals are needed as working oxen, they should not be castrated until they have attained the age of three or four years. At these pericids the muscles of the neck and chest have undergone remarkable clevelopments, and the animals have strength and endurance to make really valuable oxen for work; whereas, if castrated at too early a period, the muscles of the forward parts are always defective, lank, and lean. I am aware that there are difficulties in the way of keeping bulls up to the age here indicated; yet the experiment has been tried, in this country and elsewhere, to the entire satisfaction of the parties that made the experiments; therefore, I recommend farmers not to castrate calves intended for work-oxen until their muscular system is well developed; and even in view of obtaining good beef, and developing the propensity to fatten, 
I should let the calves remain uncastrated for six months or more. It has been urged, by some writers, that the danger of castration is less in young than adult animals. This may be correct, yet I apprehend but little danger in castrating either a horse or bull at any age, provided the creature is in the enjoyment of health, and the operation be properly performed.

In many parts of France the bull-calf is castrated by means of a curious species of torsion, termed bistournage. The animal is thrown and secured. The operator places himself behind the animal, and opposite to the tail; he seizes the testicles with both his hands, and pushes them violently upward and downward several times, in order to destroy their adhesion to their coverings. $\mathrm{He}$ continnes this manipulation until he thinks that he has produced sufficient lengthening of the cords, and dilatation of the bag itself. He then pushes up the left testicle as nearly as possible to the ring, leaving the right one low in the bag; he seizes the cord of the right testicle between the finger and thumb of the left hand, about an inch above the testicle, and, grasping the bottom of the scrotum with his right hand, he turns the testicle, and pushes it forcibly upward, until he has reversed it, and its inferior extremity is uppermost. Some little practice is required in order readily to effect this. Then, the right hand holding the testicle while the left hand raises the cord, the testicle is turned round from right to left four or five or six times, until there is a degree of tension and difficulty in the turning, which indicates that the spermatic vessels are so far compressed or obliterated as to be deprived of the power of secreting or conveying the seminal fluid. The testicle is by this means brought up nearly to the abdominal ring, where it is retained by turning the serotum over it, while the left testicle is brought down, reversed, and turned in the same manner. Last of all, in order to prevent the untwisting of the cords and the descent of the testicles, the operator grasps the bottom of the scrotum in his left hand, and, holding one end of a piece of cord, eighteen inches in length, and about as large as a quill, between his teeth, and having the other end in his right hand, he makes with it several turns round the scrotum with considerable firmness below and close to the testicles, yet not so tightly as quite to stop the circulation of blood through the bag. This is taken away at the end of the second day, after which the testicles will remain fixed against the abdomen, and will gradually wither 
away. The animal is usually bled after the operation, and half of its allowance of food taken away.

I lately castrated two horses, at the respective ages of twelve and seventeen years, and they have both done well; yet, if they had been "prepared," as the books recommend, I might have lost both animals; and I verily believe that the once popular method of physicking (prostrating) animals before castration has been the cause of many unnecessary deaths.

In castrating bullocks, I apply a ligature around the whole cord, for it is not always safe to merely ligature the spermatic artery, as the reader will perceive by reading the following paragraph :

Herring has observed that, after tying the spermatic artery, without difficulty, in two places, and cutting between them, the spermatic cord being then cut across two inches below, arterial hemorrhage sometimes ensued. It is easy to account for blood flowing through the spermatic veins, after this operation, by a retrograde circulation in the wide vessels; but, as regards the arterial hemorrhage, Herring was in doubt, until, after several injections of the spermatic cord, he found that the spermatic artery often divides into two nearly equal branches; if but one be tied, on removing the testicles, the other division bleeds. HERRING afterward injected several testicles and spermatic cords of bulls, and found an extraordinarily rich net-work of veins, which differs in many points from the pampiniform plexus of the spermatic cord in man, horse, dog, etc. In the spermatic cord of the horse, we observe the artery making a large number of curves on itself until it reaches the testicle; and from the latter organ arise numerous veins, which coil upward, but now join in several branches which pass up with the artery, anastomosing at intervals, and forming a net-work, the meshes of which are in the shape of parallelograms. In the bull, on the contrary, the veins are exceedingly numerous, and spin round the artery like the tendrils of a climbing plant round a wire; and this is seen high up in the abdomen, so that, in successful injections, the spermatic artery is completely hidden.

Some persons may object to the plan of applying a ligature around the whole cord, and YoUATT speaks of it as a cruel operation. I can not conceive how there can be any more cruelty in it than when the "clams" are used. The pressure on the cord 
is the same; and all the difference is, I use saddler's silk instead of wooden clams. I have castrated a great number of animals, at all ages, by means of a ligature around the cord, and have never met with loss or accident.

Method of Castration.-There is very little danger in castrating a young calf, and it is very rare that a surgeon is ever called upon to perform the operation; yet I would advise persons who are in the habit of castrating these young animals to make free openings into the scrotum and inner covering of the testicles. This inner covering is called tunica vaginalis. If too small an opening be made, the swollen cord will be imprisoned by the divided edges of the tunica vaginalis, which will untimately end in hardening or scirrhosity of the end of the cord, or else there will be scrotal abseess. A large opening into the serotum and tunic is necessary, in order to postpone union of the external parts, until the tissues above and within have healed.

When castrating bullocks, I either secure them in the trevis and partly etherize them, or else cast them with the hobbles (see cut of instruments), and render them completely insensible by ether. I then grasp the scrotum, between the belly and testicle, and make an incision on one side and at the lower part of the scrotum sufficiently large to allow the testicle to escape. The testicle then hangs by the cord. A ligature is then passed around the latter, which must be tied tight enough to compress the bloodvessels and prevent after-bleeding. The cord is then divided, and one end of the string may be cut very close to the knot, so that a slight pull will untie it. The other testicle is to be proceeded with in the same manner, and the operation is complete. It is proper, however, to return the cord within the inner tunic, or covering of the testicle, and one end of the ligature is left long enough to hang out of the wound. In the course of a week, if the ligatures do not come away, a slight pull will detach them.

I never make use of any dressing, except when a bad odor arises from the parts. The best remedy, under such circumstances, is diluted pyroligneous acid, in the following proportions:

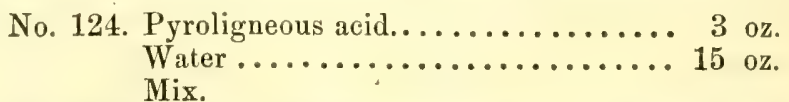

Mix.

Let the parts be well cleansed, night and morning, with a por tion of the above. Should any undue amount of swelling ensue, 
the parts are to be rubbed occasionally with an ounce or two of sweet spirits of niter.

I find that exercise operates favorably in preventing scrotal swelling, which is a condition of the parts known to surgeons as local effusion (dropsy); and, as exercise at pasture always tends to reduce these kinds of swellings, I generally, if the weather permits, place the castrated animal under the care of "Doctor Grecn," (a good pasture,) where the patient can vitalize its blood with pure air, promote the integrity of the whole system by voluntary exercise of the muscles, and partake of herbage calculated to benefit the whole animal economy.

\section{Enzootic Milk Sickness, or Trembles.}

The cause or origin of enzoötic milk sickness (commonly denominated "trembles") is a subject of much speculation. Various are the opinions and conclusions of medical and non-medical men. It has been asserted that a creeping vine, known to luxuriate in forest regions, occasions the disease. The theory is that this vine, or vegetable parasite, is matured in the latter months of summer, or the first autumnal, at which season of the year the grass becomes dry and tough, when the cattle resort to the timbered land for sustenance, feeding upon the (supposed) vine; and as the animal is (without satisfactory evidence) susceptible to its (imaginary) influence, it often sickens and dies; yet, strange to relate, many animals located in the same regions escape the pest. And, in order to sustain this faulty theory, we are informed that the lucky creatures who live and die at a respectable bovine age know enough to give the poisonous plant the go-by. This is nothing more than mere speculation, for the disease very frequently occurs when the ground is covered with snow.

Dr. GRAFF informs us that the general appearance of the infected districts is somewhat peculiar. "The quality of the soil is, in general, of an inferior description. The growth of timber is not observed to be so luxuriant as in situations otherwise similar, but is scrubby, and stunted in its perfect development, in many instances, simulating what, in the West, is denominated 'barrens.'"

Now, it is possible that these barrens do not furnish a sufficient amount of carbon, in the form of food, for the metamorphoses of 
the tissues; and if we take into consideration that the animal receives, during the day, while in search of this food, a large supply of oxygen, and at the same time the waste of the body is increased by the extra labor required to select sufficient nutriment-it being scanty in such situations-then it follows that this disproportion between the quantity of carbon in the food and that of oxygen absorbed by the skin and lungs must induce a diseased or abnormal condition. The animal is sometimes fat, at others lean. Some of the cows attacked with this disease were fat, and in apparent health, and nothing peculiar was observed until immediately preceding the outbreak of the fatal symptoms. The presence of fat is generally proof positive of an abnormal state; and, in such cases, the liver or spleen is often diseased. The blood then becomes loaded with fat and oil, and is finally deposited in the cellular tissues. The reader will now understand how an animal accumulates fat, notwithstanding it be furnished with insufficient diet. All that I wish to contend for is, that in such cases vital resistance is compromised. We are told that, in the situation alluded to, vegetation was stunted, etc.; and knowing that vegetables are composed of nearly the same materials which constitute animal organization-the carbon or fat of the former being deposited in the seeds and fruits, and that of the latter in the cellular structurethen we can arrive at but one conclusion; namely, that any location unfavorable to vegetation is likewise ill-adapted to preserve the integrity of animal life.

In connection with this, it must be remembered that, during the night, the soil emits excrementitious vapors, which are taken into the animal system by the process of respiration. In the act of rumination, vapor is also inclosed in the globules of saliva, and thus reaches the stomach. Many plants which, during the day, may be eaten with impunity by cattle, actually become poisonous during the night! This, I am aware, will meet with some opposition, to combat which I quote from LIEBIG:

"How powerful, indeed, must the resistance appear which the vital force supplies to leaves charged with oil of turpentine or tannic acid, when we consider the affinity of oxygen for these compounds! This intensity of action, or of resistance, the plant obtains by means of the sun's light, the effect of which, in chemical actions, may be, and is, compared to that of a very high temperature (moderate red heat). During the night an opposite 
process goes on in the plant. We see, then, that the constituents of the leaves and green parts combine with the oxygen of the air-a property which in daylight they did not possess. From these facts we can draw no other conclusion but this: that the intensity of the vital force diminishes with the abstraction of light; that with the approach of night a state of equilibrium is established, and that in complete darkness all those constituents of plants which, during the day, possessed the power of separating oxygen from chemical combinations, and of resisting its action, lose their power completely.

A precisely similar phenomenon is abserved in animals. The living animal body exhibits its peculiar manifestations of vitality only at certain temperatures. When exposed to a certain degree of cold, these vital phenomena entirely cease. The abstraction of heat must, therefore, be viewed as quite equivalent to a diminution of the vital energy. The resistance opposed by the vital force to external causes of disturbance must diminish, in certain temperatures, in the same ratio in which the tendency of the elements of the body to combine with the oxygen of the air increases.

It is obvious that the cause of the generation of force-namely, the change of matter-is diminished, because, with the abstraction of heat, as in the plant by abstraction of light, the intensity of the vital force diminishes. It is also obvious that the momentum of force in a living part depends on its proper temperature, exactly as the effect of a falling body stands in a fixed relation to certain other conditions; for example, to the velocity attained in falling. When the temperature sinks, the vital energy diminishes; when it again rises, the momentum of force in the living parts appears once more in all its original intensity. The production of force for mechanical purposes, and the temperature of the body, must, consequently, bear a fixed relation to the amount of oxygen which can be absorbed in a given time by the animal body.

The quantities of oxygen which a whale and a carrier's horse can inspire in a given time are very unequal. The temperature as well as the quantity of oxygen is much greater in the horse. The force exerted by a whale, when struck with the harpoon, his body being supported by the surrounding medium, and the force exerted by a carrier's horse, which carries its own weight and a heavy burden for eight or ten hours, must both bear the same ratio to the oxygen consumed. If we take into consideration the 
time during which the force is manifested, it is obvious that the amount of force developed by the horse is far greater than in the case of the whale.

In climbing high mountains, where, in consequence of the respiration of a highly rarefied atmosphere, much less oxygen is conveyed to the blood, in equal times, than in valleys or at the level of the sea, the change of matter diminishes in the same ratio, and with it the amount of force available for mechanical purposes. For the most part, drowsiness and want of force for mechanical exertions come on; after twenty or thirty steps, fatigue compels us to a fresh accumulation of force by means of rest (absorption of oxygen without waste of force in voluntary motions.)"

In the situations alluded to we generally find poisonous and noxious plants, with an abundance of decayed vegetable matter. An English writer has said: "The farmers of England might advantageously employ a million, at least, of additional laborers in clearing their wild domains of noxious plants, * which would amply repay them in the superior quality of their produce. They would then feel the truth of that axiom in philosophy, 'that he who can contrive to make two blades of grass, or wholesome grain, grow where one poisonous plant grew before, is a greater benefactor to the human race than all the conquerors or heroes who have ever lived." "

The noxious plants found in such abundance in the Western States are among the principal causes, either directly or indirectly, of the great mortality among men, horses, cattle, and sheep. The hay would be just as destructive as when in its green state, were it not that, in the process of drying, the volatile and poisonous properties of the buttercup, dandelion, poppy, and hundreds of similar destructive plants found in the hay, evaporate. It is evident that

* The American farmers are just beginning to wake up on this subject, and before long I hope to see our pasture lands free from all poisonous plants. Dr. Whitlaw says: "A friend of mine had two fields cleared of buttercups, dandelion, ox-eye, daisy, sorrel, hawk-weed, thistles, mullein, and a variety of other poisonous or noxious plants. They were dried, burnt, and their ashes strewed over the fields. He had them sown as usual, and found that the crops of hay and pasturage were more than double what they had been before. I was furnished with butter for two summers, during the months of July and August. The butter kept for thirty days, and proved, at the end of that time, better than that fresh churned and brought to the Brighton or Margate markets. It would bear salting at that season of the year." 
if animals have partaken of such plants, although death in all cases do not immediately follow, there must be a deficiency of vital resistance, or loss of equilibrium, and the animal is in a negative state. It is, consequently, obvious that when in such a state it is more liable to receive impressions from external agents ; in short, is more subject to disease, and this disease may assume a definite form, regulated by location. It has been observed, also, that in the infected districts the water is not of the best kind, neither is it very abundant; hence, in consequence of its insufficiency or unwholesome character, the equilibrium of health may become disturbed.

A loss of vital resistance may also be the result of exposure. It has been observed that cattle which have been housed regularly have escaped the attacks of this malady, and that, when suffered to run at large, they were frequently seized with it. Therefore we may conclude that the indirect causes of milk sickness, or trembles, are any thing that disturbs the general health.

Now, let us suppose that one or a combination of the preceding causes has operated so as to produce an abnormal state in the system of a cow. She is then suffered to remain in the unhealthy district during the night. While there, exposed to the emanations from the soil, she requires the whole force of her vital energies to ward off chemical decompositions, and prevent encroachment on the various functions. A contest commences between the vital force and chemical action, and, after a hard conflict, in their incessant endeavors to overcome each other, the chemical agency obtains the ascendency, and disease of a putrid type (milk fever) is the result. The disease may not immediately be recognized, for the process of decomposition may be insidious; yet the milk and flesh of such an animal may communicate the disease to man and other animals. It is well known that almost any part of animal bodies in a state of putrefaction, such as milk, cheese, muscle, pus, etc., communicate their own state of decomposition to other bodies. Many eminent medical men have lost their lives while dissecting, simply by putrefactive matter coming in contact with a slight wound or puncture. Dr. GRAFF made numerous experiments on dogs, with the flesh, etc., of animals which died of milk sickness. He says: "My trials with the poisoned flesh were, for the most part, made on dogs, which I confined; and I often watched the effect of the poison when administered at regular intervals. In the space of forty-eight hours from the commence- 
ment of the administration of either the butter, cheese, or flesh, I have observed unequivocal appearances of their peculiar action, while the appetite remains unimpaired until the expiration of the fourth or fifth day." From the foregoing remarks, the reader will agree with me that the disease is of a putrid type, and has a definite character. What is the reason of this definite character? All diseases are under the control of the immutable laws of $\mathrm{Na}$ ture. They preserve their identity in the same manner that races of men preserve theirs. Milk sickness of the malignant type luxuriates $i^{*}$ the locations referred to, for the same reasons that yellow fever is peculiar to warm climates and consumptions to cold ones, and that different localities have distinct diseases; for example, ship fever, jail fever, etc.

Before disease can attack and develop itself in the bodies of men or animals, the existing equilibrium of the vital powers must be disturbed; and the most common causes of this disturbance I have already alluded to. In reference to the milk, butter, cheese, etc., of infected animals, and their adaptation to develop disease in man, and in other locations than those referred to, I observe that when a quantity, however small, of contagious matter is introduced into the stomach, if its antiseptic properties are the least deranged, the original disease (milk sickness) is produced, just as a small quantity of yeast will ferment a whole loaf. The transformation takes place through the medium of the blood, and produces a body identical with, or similar to, the exciting or contagious matter. The quantity of the latter must constantly augment; for the state of change or decomposition which affects one particle of the blood is imparted to others. The time necessary to accomplish it, however, depends on the amount of vital resistance, and, of course, varies in different animals. In process of time the whole body becomes affected, and, in like manner, it is communicated to other individuals; and this may take place by simply respiring the carbonic acid gas or morbific materials from the lungs of diseased animals in the infected districts.

My principal object is to show what are the causes of this malady, so that the farmer can prevent its occurrence, for the treatment is very unsatisfactory. A writer in the "Atlanta Medical Journal" informs us:

"Where stock cattle, for instance, are kept pent up until after the morning's dew, they are never affected, though they are pas- 
tured where it is known to abound. Again : if food, in the form of bundles of hay, or fodder, or sheaves of oats, has been cast on the surface of the earth where it was suspected to exist, fed to calves or a calf, during the morning, while wet with dew, the result is the death of the animal. Facts like these are, to my mind, evidence conclusive of its origin in the form of vapor. But let it originate from whence it may, it is only known in timbered land, and there disappears, after being once cleared, cultivated, and seeded with tame grass, which shows, again, if of a telluric source, that the toxical agent lies near the surface, and is destroyed by being shifted from its lurking-place."

Symptoms.- The principal symptoms are irregular, nervous action; trembling, trémors, spasms, and, lastly, convulsions. The other symptoms are such as are noticed in affections of a low typhoid type. The pulse is quickened, yet small; the tongue slightly swollen, and coated with a brown fur; the urine is highcolored, and the bowels are constipated; the membranes of the eyes are reddened, and the breath has a bad odor.

Treatment.-If the bowels are constipated, I should give an aperient, composed or

No. 125. Glauber salts................ 10 oz.

Powdered ginger................ 1 dr.

Golden seal.................... $1 \mathrm{dr}$.

Tepid water.................. 1 quart.

Then let the whole length of the spine be well rubbed with two two or three ounces of oil of cedar. Should the breath or excrements have a very bad odor, the following must be given :

No. 126. Pyroligneous acid.............. 2 oz.

Glycerine.................. 4 oz.

Water...................... 1 quart.

Mix.

Dose, a wine-glassful three or four times daily, until an improvement takes place.

To relieve the nervous irritation and trembling, I give two drachms of tincture of Indian hemp, in a little water, twice daily. The patient is to be kept on oatmeal gruel, the quantity to be regulated according to actual necessity. A curable case will be very likely to improve under the above treatment; if it fail, the owner will have the satisfaction of knowing that the patient was not destroyed by meddlesome medication. 


\section{Improved Method of Miliking,}

The first process in the operation of milking is to make the cow's acquaintance, and give her to understand that the milker approaches her with none other than friendly intentions; for if he swears, scolds, or kicks her, she is likely to prove refractory, and may, possibly, give the uncouth and unfeeling milker the benefit of her heels, which, in my opinion, he is justly entitled to.

Before commencing to milk the amimal she should be fed, or have some kind of fodder. In the enjoyment of the mastication of the same, her attention is withdrawn from the milker's operations, and the milk is not "held up," as the saying is, but is yielded freely: The milker should not sit off at a distance, like a coward, but his left arm should be in close contact with the leg of the cow, so that she can not kick. If she make the attempt when the milker is in close proximity with the cow's body, the former merely gets a push instead of a blow. Before commencing to milk, the teats are to be washed with cold water in warm weather, and warm water in winter. The object is to remove accumulated dirt, which otherwise would fall into the milk-pail, to the disgust of persons who love pure milk and hate uncleanliness. Here is a chance for improvement.

The best milker is a merciful man. The udder and teats are highly organized and very sensitive, and these facts should be taken into consideration, especially when milking a young animal, for the parts are sometimes excessively tender, and the hard tugging and squeezing which many poor sensitive ereatures have to endure at the hands of some thoughtless, hard-fisted man, are really distressing to witness. A better milker than even a merciful man is a woman. The principal part of the milking in private establishments in foreign countries, is done by women; and in these United States there are thousands of capable women out of employment that might be advantageously employed, in private and dairy establishments, as milkmaids. Therefore, in view of improvement in the art of milking, I advise farmers to learn their wives, daughters, and female domestics how to strip the cows. An indolent person (slow coach) should never be suffered to touch a cow's teats. The process, to say the least of it, is painful ; therefore, the best milker is the one that can abstract the milk in the quickest time. Finally, milk the cow dry. The last of the milk is the most val- 
uable, yet Mr. Hurry-up can not spare time to attend to this matter ; consequently he loses the best of the strippings, and actually ruins the cow as a milker.

\section{Diseased Thymus Gland.}

The thymus gland, commonly known as the sweetbread, is usually found, after adult life, in the region of the thoracic duct, just within the anterior or front part of the chest; yet the greatest activity and development of this gland is during fotal life. This is one of the glands which, according to Goonsin, is an involuted portion of the germinal membrane, acting as the first assimilating organ possessed by the fotus; hence, as soon as the animal is borm, and the legitimate organs of digestion are called into operation, the thymus dwindles away, and, in some cases, almost disappears. Occasionally, however, it becomes enlarged, and has to be removed by a surgical operation. The following instructive case occurred in the practice of W. LyoN, V. S., and appeared in the "London Veterinarian:"

"I beg to forward for your inspection a tumor, being the largest of two which were removed on the 15 th instant from under the cervial vertebræ of a yearling quey, and which, although now considerably shrunk and dry, weighs forty-nine ounces, and measures twenty-three inches in its greatest circumference. It rested upon the œsophagus, trachea, blood-vessels, and sternal muscles, excepting when the animal's head was depressed; so much so, that had the same degree of pressure been made on the gullet, by a tumor existing elsewhere, as within the chest, permanent hove, etc., would have been produced.

The operation consisted in an incision through the integuments of the off-side of the neck, over and parallel to the course of the vessels, which exposed part of the levator humeri ; the next incision being made right through that muscle, in the direction of its fibers, which brought to view part of the great tumor, exposing also the sub-scapulo-hyoid muscle, which was considerably but favorably displaced by the pressure of the lesser tumor, which inclined to the off-side. The remaining incisions were made, with care, through numerous ligamentous-like bands and cellular membrane, by which the tumor was attached to the inter-vertebral and other muscles. The lesser tumor, which was more superficial, was 
then removed in the same manner, after having been very useful in keeping the said sub-scalpulo-hyoid muscle out of the way. Both tumors seemed to be indifferently supplied with blood. Excepting the cutaneous veins, which yielded less than an ounce of blood, there were no blood-vessels divided; hence no artery nor vein required to be taken up. A few interrupted stitches were put in, and as the quey had necessarily to stand during the operation, there being only one man and a boy present at its commencement, orders were given to let go the under-jaw, when the animal immediately sprang orer a four-foot wall. I have not seen her since, but have learned that she is doing well.

I need make no remark on the origin, progress, structure, locality, and treatment of such tumors, such being already well-known to veterinary practitioners. Farmers, however, would do well (now that the disease is very common) to feed off cattle so affected or predisposed, when it is possible to do so, and not to breed from such stock, as it is generally difficult and sometimes impossible to remove the tumors when once formed. It would also be for their interest to pay less attention to the direct treatment, by intrusting that in the hands of a practitioner, and to attend more to the negative treatment, such as blood-letting, avoiding refrigerants, such as nitrate of potash, and all other medicines that tend to diminish the fibrine of the blood, particularly all mercurial preparations, and, in general, to avoid every thing formerly used in the treatment of serofula in the human subject. But this they will not always attend to when any thing is wrong with any of their cattle. They must not merely get direct treatment, but, without due discrimination, they must share and share alike."

\section{Cords in Young Calves.}

A disease to which the above name is given occasionally appears among young calves. It is an inflammatory affection, and particularly manifests itself in contractions of the sinews (tendinous structures); hence the name, "cords." WHITE contends that calves are most liable to be affected by this disorder during the first days or weeks after they are dropped. If they outlive five or six weeks, they are seldom in any danger. Calves that suck their mothers are not so liable to the disease as those which are reared by hand. The greatest number of calves that fall a sacrifice to 
this disease, if not the whole of them, are those which are closely confined to the house from their birth, without ever being exposed to the free, open air. It is a well-known fact that ealves which are dropped and remain in the fields are in little or no danger. Mr. Lawrence, in his "Treatise on Cattle," observes, that "a complaint called the cords has recently destroyed a number of young calves in Scotland, both such as have been calved abroad and under shelter. Those which are brought up by hand are most liable, and the most dangerous period is the first week or two after birth."

Treatment.-As a preventive I should give the new-born calf (provided we have no discharge from the bowels) a wine-glassful of castor-oil; yct if the mother yield milk, and the calf immediately after birth imbibes the same, there is no need of giving any medicine. The object in giving castor-oil is to purge off the meconium-first excrement. The first flow of milk, however, will meet the emergency, and it is only in cases of suspended lacteal secretion, or in cases of acute garget, when the cow can not yield milk, that I recommend the castor-oil. Should the oil purge the calf too actively, twenty drops of the oil of anise-seed or peppermint, may be given in half a pint of flour gruel. Should the animal be dangerously attacked, and the contractions of the tendinous structures violent, let the little creature be placed in a warm bath for a few minutes; then give him a good rubbing with coarse towels, after which he will probably do well.

\section{Steamed ANd Cooked Food for Stock.}

In view of fattening neat stock, and augmenting the quantity of milk in dairy cows, I recommend the use of steamed and cooked food. It has been satisfactorily proved that fat cattle of the best quality, may be produced by feeding them on boiled food.

Dr. Whithaw says: "On one occasion, a number of cows were selected from a large stock, for the express purpose of making the trial; they were such as appeared to be of the best kind, and those that gave the richest milk. In order to ascertain what particular food would produce the best milk, different species of grass and clover were tried separately, and the quality and flavor of the butter were found to vary very much. But what was of the most importance, many of the grasses were found to be coated with 
silicia, or decomposed sand, too hard and insoluble for the stomachs of cattle. In consequence of this the grass was cut and well steamed, and was found to be readily digested; and the butter that was made from the milk much firmer, better flavored, and would keep longer without salt than any other kind. Another circumstance that attended the experiment was that, in all the various grasses and grains that were intended by our Creator as food for man or beast, the various oils that enter into their composition were so powerfully assimilated or combined with the other properties of the farinaceous plants, that the oil partook of the character of essential oil, and was not so easily evaporated as that of poisonous vegetables; and experience has proved that the same quantity of grass, steamed and given to the cattle, will produce more butter than when given in its dry state. This fact being established from numerous experiments, then, there must be a great saving and superiority in this mode of feeding. The meat of such cattle is more wholesome, tender, and better flavored than when fed on the ordinary food.

"A mixed diet (boiled) is supposed to be the most economical for fattening cattle. 'A Scotchman, who fattens one hundred and fifty head of Galloway cattle annually, finds it most profitable to feed with bruised flaxseed, boiled with meal or barley, oats or Indian corn, at the rate of one part flaxseed to three parts meal, by weight-the cooked compound to be afterward mixed with cut straw or hay. From four to twelve pounds of the compound are given to each beast per day.' The editor of the 'Albany Cultivator' adds: 'Would it not be well for some of our farmers who stall-feed cattle to try this or a similar mode? We are by no means certain that the ordinary food (meaning, probably, bad hay and corn-stalks) would pay the expense of cooking; but flaxseed is known to be highly nutritious, and the cooking would not only facilitate its digestion, but it would serve, by mixing, to render the other food palatable, and, by promoting the appetite and health of the animal, would be likely to hasten its thrift." "

An article on steamed food for stock lately appeared in the columns of the "Mark Lane Express." It does not sustain my theory exactly, yet the closing paragraph proves all I contend for at the commencement of this article. It is my opinion that, in order to test the real value of steamed over raw food, the experiments must be conducted with great care, and be continued for 
some time, during which it must not be expected that the animal will thrive in health and flesh, unless it have an occasional meal of its more natural food in its raw state. What I mean by the "real value" of steamed food, is its usefulness in the animal economy, as a co-agent with the unsteamed articles of fodder; and, considerable discretion will be needed in making selections among articles of food, some of which might be improved, or, rather, rendered more acceptable to the palate and convenient for mastication, while others might be deteriorated by the same process. I select the following from the above source:

"As to steaming food for cattle, there is considerable difference of opinion among theoretical writers. Among practical men there is little difference of opinion, especially where experiments have been individually undertaken. The late Mr. Howden, of Lawhead, East Lothian, undertook a series of experiments on feeding cattle with steamed food. Lots of cattle of similar age and breeding were selected and divided. Those cattle fed on turnips and potatoes, given raw, made rather greater progress than those fed on the prepared food, equal quantities being given. The difference was slight; still it was perceptible, the rate of progress being tested by girthing the animals, and the condition generally, by handling. The experiments were carried on for more than one season, with nearly the same result. A lot of young cattle, a year and a half old, fed on boiled beans, made very rapid progress, and left a profit beyond payment for their food consumed. They were cheap when purchased, and the value of beef was at the time relatively high with that of lean cattle. Of course, the straw was not prepared, either by steaming or cutting into chaff. Other experiments with steamed food have been, from time to time, undertaken in that county, and with nearly the same result. In every case that came under our observation, the preparing of food by steaming and boiling was, after a time, given up. In feeding dairy stock when in milk, steaming and boiling food is known to be profitable; but the health of the animal sometimes suffers, compared with that of cows kept partly on raw and partly on prepared food." 


\section{Cheap Fodder for Cows.}

Straw contains much farinaceous aliment. The attention of agriculturists in France has recently been directed to the discovery of a method of converting straw into a kind of bran. The discovery has been claimed by two individuals. The first is a miller, near Dijon, who, it is said, on trying the mill-stone of a new mill, discovered the possibility of converting straw into a nourishing food; the second, M. Joseph Maitre, of Villotte, near Chatillon. This distinguished agriculturist, known for the purity and perfection of his breeds of sheep, conceived the idea of converting into farina not only the straw of wheat and other grains, but of hay, trefoil, lucern, sanfoin, ete. His efforts have been perfectly successful, and his discovery arrived at, not by chance, but by long experiment and research. The aliment which he has produced is said to be a complete substitute for bran. It is given to sheep and lambs, who consume it with avidity, and may be given to all other graminivorous animals as a grateful and substantial food. We know, in this country, that the mere chopping of straw adds greatly to its powers, by facilitating mastication and digestion. We may believe that a more perfect comminution of its parts will produce a corresponding effect, and extend very widely the uses of straw and other fodder as a means of feeding our domestic animals. This sort of aliment is very excellent, when combined with a sufficient amount of nutrimental matter, for animals whose systems lack the requisite amount of phosphates and phosphoric acids. A milch cow, for example, whose lacteal vessels yield, in the form of milk, the above equivalents, may be benefited by an occasional feed of straw meal.

\section{Black Leg, or Anthrax.}

Various are the names given to an affection of the above kind. Some persons call it inflammatory fever, gangrene, etc. Then again it gets its name from the region in which it first appeared. So, if it first appears in the region of the thigh, it is called quarter ill, quarter evil, black quarter, joint murrain, ete. ; hence it appears to have as many names as there are locations for it.

The Nature of Black Leg.-YoustT, and other orthodox veterinary writers contend that this affection is a pure inflammatory 
fever, yet, at the same time, they admit that cattle of all descriptions, ages, and conditions are subject to it. Now, I think it will be very difficult to prove that inflammatory fever can attuin a very high grade in the system of an animal in poor condition. The very reverse is the case; for disease of a low typhoid type, and those which are known to run a rapid course, are apt to pounce upon animals having but little vital resistance-being out of condition, as the saying is.

In my opinion, this is an epizoötic affection, and, like most epizoötics, its pathology must necessarily be obscure. In applying the terms black leg, black quarter, etc., to this affection (epizoötic or enzoötic) leads us into error in supposing that it is merely a local affection, limited to a certain part, when, in fact, it is a general affection, showing itself in various parts of the economy at the same time. For example, in the early stage, when a limb or part is swollen and distended with gas, the various organs and functions of the body are more or less disturbed. Even in the early stage the respirations are short and quick; pulse, accelerated (a mere fluttering action, scarcely perceptible); the coat stares; rumination is suspended; the bowels are constipated, and the nervous system is affected; for the beast staggers, and soon becomes comatose; death frequently takes place in the course of from twelve to twenty-four hours. It will be perceived, therefore, that the local affection is not proportionate, in degree or extent, to the severity and magnitude of the general symptoms; therefore it is a general disease.

The autopsy clearly indicates the ravages which this epizoötic or enzoötic makes on the general economy. Youatr informs us that "the chief appearances after death will be venous congestion every-where. It affects both of the pleura, the whole substance of the lungs, brain, and peritoneum; the intestines and stomach are also affected. Therefore what we perceive externally, in the form of tumors, emphysema, ulcers, sloughing, and mortification are only the symptoms of a general malady. Mr. ERnes informs us, through the pages of the "Veterinarian," that "black quarter" (which is the same as 'black leg') is neither more nor less than that dreadful malady of horses and cattle called by the French charbon. Anthrax would, perhaps, be the better appellation, seeing its characters are a hard, circumscribed tumor, exceedingly painful, with tension and burning heat in the subcutaneous cel- 
lular tissue. This is often depressed in its center, and sometimes preceded by a small opening.

According to authorities, "the tumor of black leg, though small at first, suldenly increases in size, sometimes to that of a child's head. Gangrene soon supervenes, beginning in the center and extending to the circumference, which rapidly converts the whole into an eschar of a black color, similar to a piece of charcoal; hence its name-charbon. The eschar is sometimes several inches in diameter, and is almost always either preceded or accompanied by bladders, which form a sort of areola around it. There is also always an œdematous swelling, more or less considerable, owing to an emphysematous state, and an infiltration of serum or serogelatine in to the cellular tissue, which crackles on pressure, caused by the presence of gases. The danger and rapidity of the disease are such that, when an animal is attacked, after a violent access of fever, it falls a sacrifice to it in a few hours, rarely exceeding twenty-four or thirty-six. All animals are subject to it, but the herbivorous more so than others. It is either epizoötic or enzoötic ; the first is the most destructive."

I now consider the point settled regarding the nature of the disease; namely, it is an epizoötic or enzoötic affection, and, of course, is subject to the same laws which govern diseases of this type. This will explain the otherwise unaccountable variations which are observed in the symptoms of the affection when prevailing in different localities, and it also enables us to account for the great losses which Messrs. Shortfeed and Overfeed are continually encountering. The fact is, all overfed animals may be ranked as gluttons, and all half-starved animals furnish a savory morsel for the great epizoötic pathological glutton, which, like the epidemic one that, hovering around the city of New Orleans, a few years ago, destroyed several thousands of its inhabitants ere it touched a single sober citizen. When this disease ranges in a single locality, it is supposed to have a spontaneous origin. Then the term enzoötic is applied to it; and if it prevail among the cattle of an extensive region, then it is called epizoötic.

Causes.-The causes of this affection are as obscure as those of cholera, influenza, potato rot, ete. They seem to appear independent of local causes, occur at uncertain intervals, prevail for indefinite periods, and run their course in a short space of time. It is supposed by some persons that a disease of this character and 
nature is propagated by contagion or infection. How far it is engendered in these ways I am not prepared to decide. There must, however, have been a time when the disease did not exist, but must have arisen from a concurrence of natural causes; and if these were adequate to its production at an anterior period, they must be so at the present time. I shall, therefore, abandon all further speculation in this direction as unprofitable, for there is evidently no direct cause, but various are the predisposing, exciting, indirect, and morbid causes. The only way that I know of to prevent this malady is to keep the cattle in a physiological condition, by paying proper attention to breeding, feeding, rearing, and housing; for animals in a perfectly healthy state are certain to enjoy immunity from this and other diseases.

I have noticed, at various times, articles in agricultural papers on the prevention of black leg, which, in the name of common humanity I am compelled to notice. The one I have thought proper to select reads as follows:

"Preventive.-Take spring calves in the month of October; cut a small incision in the hollow above the foot. On the top of the flesh a small blue vein appears; take a crooked instrument, in the shape of an awl, and put the point under the vein, raise it up so that it can be cut, and take about an eighth of an inch out of the vein. Don't sew up the incision. It must be done on all the four feet. I have cut many hundreds, and have known of thousands being cut, and never knew of one dying with the above disease after being cut."

Now, I am not disposed to scold or find fault, nor question the intentions of men who recommend or practice such outrageous barbarities under the guise of doctoring sick animals, but I wish to remind the intelligent reader that cattle have nerves to feel, and are as keenly sensible to pain as we are; therefore all unnecessary operations, even should they have received the seal of antiquity, ought to be avoided. This is the age of progression. The lamp of veterinary science is illuminating the mystified halo which has hitherto surrounded our barn-yard practice; and before the barbarities of bygone days are practiced on our domestic animals, let us be satisfied that we are using rational means for the recovery of the sick, such as science and common sense confirms. Just as rational would it be, if it were at all rational, to take an infant and divide one of the posterior veins of both feet, in view 
of preventing disease common to adult life, which, after all, might never occur, the little creature not being predisposed thereto.

Let any one just study. the anatomical structure of the foot of an $o x$, and he will learn that the vein which we are recommended to sever and amputate from is called the coronary, and is engaged in returning blood from the vast venous plexuses of the foot, which requires to come in contact with the lungs for purification and oxygenation. Any impediments, such as severing a vein, which interrupts the free circulation or return of blood to the heart and lungs, can not be beneficial, but otherwise. It will be seen that the vein is nothing more than an elastic hollow tube, a mere vehicle, through which the blood courses; therefore it can not be supposed to have any specific power over other parts of the organization in warding off disease.

I shall not trouble the reader with any further remarks on the subject of irrational and barbarous prevention; for I presume that the introduction of the foregoing paragraph will answer all the purposes intended. Among physicians the disease is known as hœmatosepsis; and our experience is that it usually occurs among young stock, and generally in well-bred animals in high condition, or in native stock in low condition; yet it may occur in the system of any bovine, as the result of sudden change in the quality of the food.

Treatment.-It is an unfortunate occurrence that this disease should ever have been classed as an inflammatory affection, for the error has led to a system of practice disastrous as the cattle plague. The following paragraph from YouATT will serve to show the orthodox method of treating inflammatory fever, and will also explain the reason why a great proportion of the animals treated die:

"The very name of the disease (inflammatory fever) indicates the mode of treatment. In a case of excessive vascular action, the first and most important step is copious depletion. As much blood must be taken as the animal will bear to lose; and the stream must flow on until the animal staggers or threatens to fall. Here, more than in any other cliscise, there must be no foolish directions about quantities. As much blood must be taken away as can be got; for it is only by the bold and persevering use of depletory measures that a malady can be subdued that runs its course so rapidly. Purging must immediately follow. The Ep- 
som salts are here, as in most inflammatory diseases, the hest purgative. A pound and a half, dissolved in water or gruel, and poured down the throat as gently as possible, should be our first dose; and no aromatic should accompany it. If this does not operate in the course of six hours, another pound should be given; and after that, half-pound doses, every six hours, until the effect is produced."

It is a wonder to me how any creature can survive such barbarous treatment as this; yet, even at the present day, just such doctrines are taught in the schools and practiced on cattle, to the disgrace of science and shame on those men who perpetrate the wickedness. Blood-letting and purging can have no good effect on a disease like this, so prostrating, and which runs its course to mortification so rapidly. Such a wretched system of practice has always failed, and ever will fail.

That form of treatment which reason and experience suggests is the best. For example, it would naturally occur, to the mind free from the prejudice of veterinary orthodoxy, that a disease which runs into sloughing and mortification in a few short hours must require life-sustaining agents; hence I recommend a very different mode of treatment from that heretofore practiced. I commence the treatment thus:

No. 127. Spirits of hartshorn........... $4 \mathrm{dr}$. Tar-water.................... 1 qt. Tineture of bloodroot........... $1 \mathrm{oz}$.

Mix the hartshorn and tar-water first; then add the bloodroot. Drench (dose No. 1). The object in giving the hartshorn is to decarbonize the blood, and impart healthy stimulus to the nutrient system of blood-vessels and nerves; and this agent will do it.

Should the animal show any lameness in the back or hind quarters, apply the following:

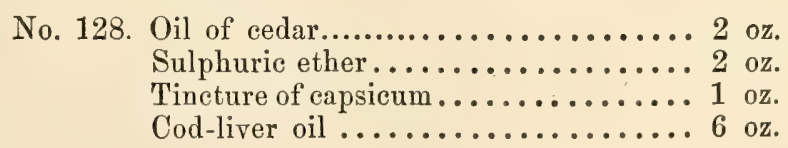

First, mix the two oils; then add the ether, and shake them thoroughly; lastly, add the tincture. The object in applying this preparation is to arouse capillary action, and thus prevent the engorgements, which, according to the best authorities, are everywhere found. 
Four hours after administering "dose No. 1," drench the patient with the following:

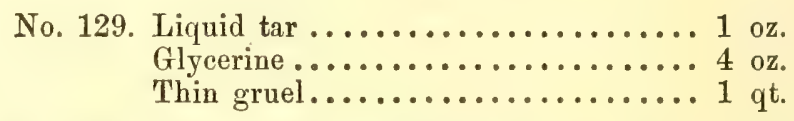

If the urgency of the case demands it, this dose may be repeated, at intervals of four hours, until symptoms of improvement are observed. The tar is a powerful antiseptic, and prevents decomposition, emphysema, and mortification.

All sores and ulcers must be dressed with pyroligneous acid, and kept constantly sprinkled with powdered bloodroot. All abscesses must be punctured with a thumb-lancet, their contents evacuated by pressure, and syringing them with soap-suds; and their cavities must be crammed with fine flour. Should any sores or ulcers be observed about the muzzle, mouth, or throat, tincture of matico is the remedy, or liquid tar. One ounce of the same may be introduced into the mouth of the patient; it will surely do good. If the patient be weak and debilitated, tonics are indicated; golden-seal, in two-drachm doses, may be incorporated in the food, in direct ratio to the urgency of the case.

\section{Black Tongue.}

This epizoötic has raged very extensively, at different times, in North and South Carolina, Georgia, and Florida. Several persons have died from drinking milk from cows thus diseased. It appears that domestic cattle are not alone the sufferers, but that in Florida, particularly, the deer perish from the same disease in large numbers; and, according to a letter in the Savannah "Republican," dogs, and the buzzards in Burke County, Georgia, that have eaten the flesh of cattle that have died of the black tongue, have perished from the effects of such poisonous diet. The cause of this malignant disease does not seem to have been yet ascertained, though by many it is attributed to the rust, which, in varirious parts of the Southern States, as elsewhere, has affected the grain crops, and, it is said, in some places, the grass also. The cattle are attacked by stiffness, and walk as though foundered, while froth is discharged in large quantities from the mouth; they can eat nothing, fall away rapidly, and the tongue and gums 
become dreadfully swollen, and turn black, and death speedily releases them from their agony.

Treatment.-The rapid progress which usually attends this dreadful epizoötic calls for prompt and energetic treatment. It is evidently a congestive disease, and very apt to run into the typhoid stage and end in sudden death. The moment an animal is suspected to be the subject of this malady, he should be drenched with

No. 130. Table salt................... 12 oz.

Warm water................. 1 qt.

Tincture of capsicum............ 2 oz.

This medicine will act as a powerful antiseptic and stimulating tonic, thus preserving the animal tissues against putrescence; and at the same time it relieves the venous congestion. If, on applying the hand to any part of the body, a crackling sound is elicited, the animal is then said to be emphysematous, which signifies an accumulation of gas beneath the skin. The patient should then be immediately drenched with

No. 131. Pyroligneous acid ............. 2 oz.

Pure oil of sassafras.............. 28 drops.

Linseed tea.................. 1 qt.

Mix the oil with the latter, then add the acid. After having drenched the animal, apply a portion of the following to the tumefactions, or emphysematous region:

No. 132. Soft soap ................. 4 oz.

Oil of sassafras............... $\frac{1}{2} \mathrm{oz}$.

Dissolve the sassafras in two ounces of alcohol.

Tincture of capsicum ........... $2 \mathrm{oz}$.

Tineture of Peruvian bark......... 1 pt.

Mix, and rub the external surface with a portion of the same.

The swollen tongue should be frequently covered with fine salt; and the moment there appears any improvement, tonic medicines should be given. One ounce of the fluid extract of camomile flowers may be given, twice daily. This remedy will give tone to the system and restore the appetite.

\section{Fractured Bones.}

Fracture of bones, occurring among neat stock, is generally considered as a justifiable cause for their destruction; but I object 
to this summary mode of disposing of unfortunate yet valuable animals; for the truth is many are killed that might be saved. The trouble of managing, and the expense of treating cases of fracture, often deter husbandmen from performing a duty incumbent on them in view of protecting their property ; but the trouble and expense are mere trifles when the usefulness of a valuable animal is involved.

The remedy in case of a simple fracture of bones, under the improved system of practice, is neither tedious nor expensive. The bones unite very readily, if kept in contact, and the unity is secured by means of starched bandages. Where there is any laceration of the soft parts, and the bone is broken into. several pieces, the better way is to put an end to the sufferings of the creature, for recovery is impossible. The following case will give some idea of the method of treating simple fractures. An animal under treatment for fracture, may be placed in the trevis, if necessary; but I prefer to let the patient have its liberty in a box stall. The limb opposite to the fractured one will have to sustain more weight than usual; therefore I try to prevent swelling and stiffness by occasional hand-rubbing, or by bathing it once or twice daily, with a portion of the following:

No. 133. Oil of wormwood.............. 1 oz.

Alcohol..................... 2 oz.

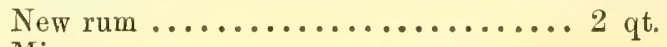

Mix.

The following case of fracture of the radius I extract from my note-book:

This was a case of simple fracture of the radius (bone above the knee) of a calf about six months old, the property of a gentleman residing in Brookline. The accident was oceasioned in consequence of the animal getting his leg entangled in a fold of chain, the latter being used for the purpose of confining him, in the day time, to a grazing spot. The fracture was crosswise of the long diameter of the bone in the central region; namely, midway between its superior and inferior extremities. No laceration of the soft tissues, nor comminution of bone. The diagnostic symptom was crepitis (crackling noise).

Treatment.-The bones were brought in apposition, and secured by means of slips of pasteboard and starched bandages; and, in order to keep them from slipping downward, they were sewed to 
a broad belt, which passed over the inferior cervial region, in the form of a figure 8 . The animal did not appear to like this contrivance, or else he liked to lick the starch, for he soon commenced to nibble the wick-yarn with which it was marled on, and so loosened the bandage that, in the course of a few hours, it slipped below the carpus. So soon as this occurred I was again summoned to visit the patient. I now procured some thick tar, and with it smeared the limb to the extent which was to be covered by bandage. The bandage was composed of common sheeting, three inches wide, three yards long, and this was also well tarred, and, after being neatly applied, was secured by means of the manytailed, tarred bandage. This had the desired effect; for, however well the animal might have liked the taste of starch, he apparently had no relish for tar. The bandage remained undisturbed.

The accident happened on the 18th day of August, 1857; on the 4th day of October the osseous union was complete. The bandage had been removed some time prior to the latter date, yet the condensed tar was allowed to remain on the skin for some time. In a few months his limb was just as symmetrical as the other, and no one but a skillful surgeon would ever discover that the bone had been fractured.

\section{LiGHT IN BARNS.}

Barns should be so constructed, by the insertion of windows in various parts of the building, that they shall be as "light as day." A "dark" barn is only a suitable black-hole for some vicious animal; it is the very worst location for any thing that breathes. Sir A. Nylie (who was long at the head of the medical staff in the Russian army) states that the cases of disease on the dark side of an extensive barrack, at St. Petersburg, have been uniformly, for many years, in the proportion of three to one to those on the side exposed to a strong and uniform light. HumвоцDт has also remarked that among bipeds the residents of South America, who wear very little clothing, thus allowing the cutaneous, as well as the orbital surfaces to receive a free ray of light, enjoyed immunity from various diseases which prevailed extensively among the inhabitants of dark rooms and underground locations; and so excellent an authority as LiNNeus contends that the constant exposure to solar light is one of the causes which render a summer 
journey through high northern latitudes so peculiarly healthful and invigorating. Dr. EDwaros has also remarked that persons who live in caves or cellars, or in very dark or narrow streets, are apt to produce deformed children; and that men who work in mines are liable to disease and deformity. Light, therefore, is a condition of vital activity; and, in view only of preserving the sight of animals, it is absolutely necessary that, while they are in the barn, their optics shall have free access to the sun's rays.

If a cow were in the same condition as a polype, with no organs of vision, who shuns light, a dark barn might prove to be its earthly paradise; but as the cow has special organs of yision, evidently susceptible to the influence of light, and the integrity of its organism, or a part of the same, depending entirely on the admission of light, it is absolutely necessary that barns should be constructed accordingly.

\section{Diarrhea in Calves.}

Diarrhea is a very prevalent disease among calves. The sucking calf is liable to be the subject of this affection whenever the general health of the parent is impaired. In such cases the mother is to be treated instead of the calf. She, probably, is the subject of a deranged condition of the digestive organs, which can easily be remedied by the administration of a few doses of the following:

No. 134. Pulverized chareoal.......... )

Carbonate of soda..........

Pulverized ginger...........

Dose, two ounces daily, to be incorporated with the food; or it can be given as a drench, by adding a pint of scalded milk.

The disease occasionally occurs in consequence of weaning the calf (in view of husbanding the cow's milk), and feeding the juvenile on improper food. This kind of diarrhea must be treated as follows:

No. 135. Phosphate of lime.............. 1 oz.

Carbonate of soda.............. $2 \mathrm{dr}$.

Scalded milk................. 1 pt.

Mix the same, and administer by means of a drenching-horn or bottle. It may be divided into "broken" doses, or may be given at once, as a single dose.

If the above remedies fail in arresting the diarrhea, I should 
give three drachms of tincture of matico, every four hours, until the patient showed some signs of improvement.

\section{SPratns or Strains.}

Sprains or strains are generally occasioned by unnatural distension of parts, or rupture of cellular structure, which connects muscles or tendons. They are generally accompanied by heat, tenderness, and lameness. Working oxen are more liable to sprain than cows, because, when used in the yoke for draught, they are as liable to strain or rupture parts as horses, by sudden exertions, or violent efforts of the muscles to guard against a sudden slip and fall. The slightest strain will sometimes occasion lameness, because the parts concerned are composed of minute fibers and cells, delicately organized; and a portion or the whole of such minute tissues are easily injured. In bad cases of strain, considerable tumefaction or swelling is observed. This is occasioned by the presence of serum, or water, in the cellular tissues beneath the skin. If proper means are adopted, this fluid can be taken up by the absorbent vessels, so as to leave no enlargement; but if the case be badly treated, the enlargement becomes organized into a permanent, hard mass, which is known to medical men as induration.

Treatment.-The very best and cheapest remedies in the early stages of strain are rest and cold water. This very soon lessens the vascular excitement; and, if there is no laceration, the animal will soon get well. It will be necessary to shower the sprained spot two or three times per day, until the acute symptoms have subsided. Then a common bandage may be applied. This should be wet with vinegar nceasionally. Should the strained part be very painful, I would foment with infusion of hops-a handful of hops to a quart of boiling water, to be applied when cool. If this does not relieve the pain, let the part be occasionally sponged with a small quantity of sulphuric ether or chloroform.

\section{Bronchocele.}

Bronchocele is a disease known in common parlance as swelling in the throats of cattle. When it occurs among members of the human family it is denominated goitre; yet it is my opinion that 
bronchocele occurring among cattle, and goitre in man, are very different affections. For example: Bronchocele occurring in cattle is curable, and, so far as I have been able to ascertain, is confined to the thyroid glands, although in a protracted state of the malady, the surrounding tissues may become so involved as to render the case incurable. Goitre, as I understand it, is a diffuse tumor occupying the anterior part of the neck, occurring principally among the inhabitants of the Alps, and is considered incurable. Medicine and external applications seem to have little if any effect on it, and its removal by operation is generally fatal. The following cases are offered in support of my argument as to curability:

A couple of cows, the property of Mr. Humphrey, of Brookline, Mass., were observed to be the subjects of an enlargement in the thyroid region, which gradually increased up to the size of a man's fist. At this period I was requested to see them. The animals were natives; their ages, seven and eight; in fair condition, and yielding the usual quantity of milk. The only fault the owner had to complain of was that their appetites were not so good as usual, and he merely consulted me for the purpose of ascertaining what could be done for the thyroid tumors. On making careful examination of both animals, there was very little of abnormal action to be perceived, either internally or externally. The pulse was regular; respirations, normal ; the surface of the body, comfortably warm; coat, glossy; nothing unusual about the feces nor urine; and all I could say about the cases was, that they were mild forms of hypertrophy of the thyroid glands (enlargement withont change of structure). The tumor in one animal occupied the right side of the thyroid region; in the other it was found in the left; both immovable, yet having no morbid adhesions to skin nor subtissue.

Treatment.--The owner being unwilling to incur the expense of professional attention, and desiring to treat them himself, under advice, I accordingly prescribed as follows : Each animal to have daily ten grains of iodide of potassium in half a gill of water. The tumor to be anointed daily with a portion of the following:

No. 136. Simple ointment................ 2 oz.

Iodide of potassium............ $2 \mathrm{dr}$.

Mix.

During a period of seven days each animal got seventy grains of iodide of potassium, and by inunction received one drachm each. 
According to the testimony of Mr. Humphrey, the remedly was effective; for at the end of two weeks the tumors had disippeared, and the animals were on the high road to health.

\section{Spaying Cows.}

Before I commence to point out the method of spaying, it may be proper to allude briefly to the female organs of generation.

Uterus, or Womb.-This is a hollow, musculo-membranous organ, united to the front part of the vagina, and terminating beneath the anus, in what is known as the lips of the pudendum. The womb is destined for the reception of the foetus. It is situated within the cavity of the pelvis, between the region of the bladder and that of the rectum, and is an organ capable of extraordinary distension.

The womb is divided into body, horns, neck, and mouth. The body is the oblong, cylindrical part, growing out of the front part of the vagina, in the center of which it terminates, internally, by the mouth of the womb, termed by physicians the os uteri. The front part of the body of the womb, by branching into two divisions, forms the horns. They diverge laterally (sideways) toward the front part of the pelvis, and, finally, terminate in oval extremities, (fallopian tubes,) and, lastly, the testes, or ovaries. The part termed the neck protrudes backward into the vagina, and is only perceptible when the parts are unimpregnated.

The uterus and ovaries are partly covered, and confined to the sides of the pelvis by a portion of the peritoneum, called broad or lateral ligiments, which also inclose within its folds the fallopian tubes.

The ovaries are two soft bodies, about the size of a walnut, and resembling somewhat in form the testicles of the male. They are filled with little vesicles, or bladders, which can be seen through the surface texture, containing a small quantity of a whitish yellow fluid. These yellow bodies, or spots, termed corpora lutea, are supposed to contain the ova. The cicatrices, or marks left in the region where the vesicles have burst, denote the number of times the animal has been impregnated. The contents of the ovarian vesicles, from one or both testicles, are discharged into the uterus through the fallopian tubes, and the same is vitalized by the male semen in the act of copulation; so it will be perceived 
that the uterus has two inlets, coming from the ovaries, and one outlet, which is the lips of the vagina.

The arteries which supply the uterine organs with blood are named vaginal, uterine, and spermatic. The vaginal is derived from the internal iliac; the uterine, from the external iliac; and the spermatic comes directly from the great aorta.

This brief description of the form, function, and location of the uterine organs may possibly prove of some value to the husbandmen of this country, who are not expected to be posted on the subject, and can not spare the time to familiarize themselves with the technicalities of the schools, yet have intelligence enough to comprehend plain matters of fact.

On the Selection of Animals for Spaying.-If the animal be intended for milking purposes, a good milker must be selected, for the operation will not transform a poor milker into a good one. She must necessarily be in possession of those constitutional peculiarities which have been observed to prevail in animals renowned as first-class milkers. The operation will, without doubt, improve the quality of the milk, yet may not, under all circumstances, increase the quantity, although the French dairymen contend that " the corw will be found to give as much milk after eighteen months as immediately after the operation ; and there was found, in quantity, in favor of the spayed cows, a great difference." About three years ago $I$ spayed a cow, the property of a gentleman residing in Southboro'. She continued during this period to yield her usual quantity of milk, yet gradually accumulated fat, so that she was known in the neighborhood as the "fat cow." When in this condition, the daily yield of milk gradually decreased, and the owner at last sold her to the butcher. I learned from parties who bought the meat that it was of superior quality even to that of an ox or steer, and commanded a higher price. Among other animals that I have spayed several have run to fat, as the saying is, and at the end of from eighteen months to three years, fell into the hands of the butcher as first-rate beef. I am, therefore, inclined to think that if animals are predisposed to fatten easily, they will not remain uniform milkers beyond the above period; yet, from a report made by the Rheims Academy, I learn that this liability to fatten was not observed in the environs of Paris, where, in some milking establishments, one hundred and fifty cows are kept, all spayed except fifteen or twenty. It may happen, however, that in conse- 
quence of their not being permitted to go to pasture, and their artificial food not of the best quality, and perhaps scanty, they do not have enough adipose matter to spare and store away in the fat-cells, as is the case with stall-fed animals in private establishments, where only one or two are kept.

In view, therefore, of securing a permanent milker, I should select a cow of compact muscular organization (native breed), having little, if any, predisposition to accumulate fat. She must be a good milker, and the mother of at least three calves. The best period for spaying is in the spring, when the unimpregnated animal is in her full flow of milk. If the object of spaying be to furnish the market with fat, tender, juicy meat, then I should select animals, barren or otherwise, that keep in good condition on a small quantity of food. The operation may be performed at any period, from the age of three months to nine or more years.

Mode of Operation.-The principal authority on the modus operandi of spaying is M. MoRIs, a celebrated French veterinary surgeon. I am not in the habit of performing the operation according to his directions, yet the reader may desire to know how it has been performed in a country where the results have been so remarkably successful ; therefore I introduce the following quotations :

"Having covered the head of the cow to be operated on, we place her against a wall provided with five rings, firmly fastened and placed as follows: The first corresponds to the top of the withers; the second to the lower anterior part of the breast; the third is placed a little distance from the angle of the shoulder; the fourth is opposite to the anterior and superior part of the lower region, and the fifth, which is behind, answers to the under part of the buttocks. We place a strong assistant between the wall and the head of the animal, who firmly holds the horn in the left hand, and with his right the muzzle, which he elevates a little. This done, we pass through and fasten the end of a long, strong, platted cord in the ring to the lower part of the breast; we bring the free end of the cord along the left flank, and pass it through the ring which is below and in front of the withers; we bring it down along the breast, behind the shoulders and the angle of the foreleg, to pass it through the third ring; from there we pass it through the ring which is at the top of the back; then it must be passed around against the outer angle of the left hip, and we fasten 
it, after having drawn it tightly to the posterior ring, by a simple bow-knot. The cow being firmly fixed to the wall, we place a cord, fastened by a slip-noose, around the hocks, to keep them together in such a manner that the animal can not kick the operator. The free end of the cord and the tail are held by an assistant. The cow, thus secured, ean not, during the operation, move forward nor lie down; and the veterinary surgeon has all the ease desirable, and is protected from accident.

M. Leorant advises that an assistant should hold a plank or bar of wood obliquely under the teats and before its limbs, to ward off the kicks; but this method is not always without danger, both to the operator and the animal, because, at the commencementthat is, when the surgeon makes the incision through the hide and the muscles-the cow makes such sudden movements, and tries so frequently to strike with its left hind foot, that it may happen that, upon every movement, the plank or the bar may be struck against the operator's legs. On the other hand, although the defense may be firmly held by the assistant, yet it may happen that, in spite of his exertions, he sometimes may be thrown against the operator, by the movements she may attempt, and there may be an uncontrollable displacement of the plank or bar; and then it may happen that she becomes wounded, and, at the same time, prevents the operation; while, by the mode we point out, there is no fear of accident, either to the operator or the beast. In case of the want of well-provided rings, we may use a strong palisade, a solid fence, or two trees, at suitable distances apart. Across we fix two strong boards of wood, separated from each other, according to the size of the cow.

There is another means of confining them that we have employed for some time past, where the cows were very strong and irritable, more simple than the preceding, less fatiguing to the animal, less troublesome to the operator, and which answers perfectly. It consists in leaving the cow almost free, covering her eyes, holding her head by two strong assistants, one of whom seizes the nose with his hand, and strongly pinches the nostrils whenever the animal makes any strong movement during the operation; in causing another assistant to hold the two hind legs, kept together by means of a cord passed above and beneath the hocks. This assistant also holds the tail, and pulls it whenever the animal seeks to change its place. 
The cow being conveniently disposed of, and the instruments and appliances, (such as curved scissors, upon a table, a convexedged bistoury, a straight one, and one buttoned at the point, suture-needle filled with double thread of desired length, pledgets of lint of appropriate size and length, a mass of tow in pledgets, being collected in a shallow basket held by an assistant,) we place ourselves opposite to the left flank, our back turned a little toward the head of the animal; we cut off the hair which covers the hide in the middle of the flanks, at an equal distance between the back and hip, for the space of thirteen or fourteen centimeters in circumference. This done, we take the convex bistoury, and place it open between our teeth, the edge out, the point to the left ; then, with both hands, we seize the hide in the middle of the flank, and form of it a wrinkle of the requisite elevation, and running lengthwise of the body.

We then direct an assistant to seize, with his right hand, the right side of this wrinkle. We then take the bistoury, and cut the wrinkle at one stroke through the middle. The wrinkle having been suffered to go down, a separation of the hide is presented, of sufficient length to enable us to introduce the hand. Thereupon we separate the edges of the hide with the thumb and fore-finger of the left hand, and, in like manner, we cut through the abdominal muscles, the iliac (rather obliquely) and the lumbar (cross), for a distance of a centimeter from the lower extremity of the incision made in the hide. This done, armed with the straight bistoury, we make a puncture of the peritoneum, at the upper extremity of the wound; we then introduce the buttoned bistoury, and move it obliquely from above to the lower part, up to the termination of the incision made in the abdominal muscles. The flank being opened, we introduce the right hand into the abdomen, and direct it along the right side of the cavity of the pelvis, behind the paunch and underneath the rectum, where we find the horns of the uterus. After we have ascertained the position of these viscera, we search for the ovaries, which are at the extremity of the cornua, or horns (fallopian tubes); and when we have found them, we seize them between the thumb and fore-finger, detach them completely from the ligaments that keep them in their place, pull lightly, separating the cord and the vessels (uterine or fallopian tubes) at their place of union with the ovarium, by means of the nails of the thumb and fore-finger, which presents 
itself at the point of touch; in fact, we break the cord, and bring away the ovarium.

We then introduce the hand again in the abdominal cavity, and proceed in the same manner to extract the other ovarium. This operation terminated, by the assistance of a needle we place a suture of three or four double threads, waxed, at an equal distance, and at two centimeter's or a little less from the lips of the wound. Passing it through the divided tissues, we move from the left hand with the pice of thread; having reached that point, we fasten with a double knot. We place the seam in the intervals of the thread from the right, and, as we approach the lips of the wound, we fasten by a simple knot, being careful not to close too tightly the lower part of the seam, so that the suppuration, which may be established in the wound, may be able to escape. The operation effected, we cover up the wound with a pledget of lint, kept in its place by three or four threads passed through the stitches, and all is completed. It happens sometimes that, in cutting the muscles of which we have before spoken, we cut one or two of the arteries, which bleed so much that there is no necessity for a ligature before opening the peritoneal sac, because if this precaution be omitted, blood will escape into the abdomen, and may occasion the most serious consequences."

Improved Method of Spaying.-I cast the cow, by means of the hobbles (see cut of instruments), on her right side. I then apply to the nostrils a sponge, saturated with concentrated sulphuric ether. When the animal is completely unconscious, I slacken the casting-rope, so as to free the limbs and prevent any pressure on the walls of the abdomen. By so doing, I secure room enough to introduce a hand and arm, for the purpose of searching for the ovaries.

The first stage of the operation consists in pinching up a fold of the skin on the left side, midway between the prominent bone of the haunch or pelvis, and the last or posterior rib, about four inches below the transverse processes of the lumbar (back) vertebræ. Having divided the integuments to the extent of about five or six inches, I make a similar incision through the abdominal muscles, until the peritoneum (lining membrane of the abdominal cavity) is exposed. This membrane is then punctured by means of a beak-pointed bistoury, into which puncture I insinuate a knife having a probe point, and then divide the peritoneum to the extent of the external incision. 
The second stage of the operation commences with the introduction of the operator's right arm, he kneeling down in close contact with the cow's back. The hand is then passed within the brim or cavity of the pelvis. Having found the deep-seated or right ovary, it must be removed by laceration. I find that the best and most expeditious way is to slip the other hand into the abdominal cavity; then, with the right hand, I seize the broad ligament at the base of the ovary; my left then clasps the ovary, and in this way, by using trifling force, the ovary is detached or torn away. The left ovary is then to be sought for, and an assistant depresses the edges of the incision. At the same time the operator, having a firm hold on the ovary, brings it into view, so that it can be removed by means of a pair of blunt-pointed scissors.

The third stage of the operation is the process of uniting the abdominal muscles, by means of stitches or sutures. A curved needle, armed with four or five threads of shoemaker's twine, well beeswaxed, is to be passed through the abdominal muscles, without penetrating the peritoneum, (lining membrane of the abdominal cavity,) at interrupted distances of one inch, more or less. Each suture is to be securely tied; one end of the same is to be cut close to the knot, the other is left long enough to protrude through the integumental incision. The skin, or integument, is then to be closed by means of ligature or metallic wire, leaving a small orifice at the inferior or lower region of the external incision, for the escape of morbid matter. This completes the operation. In the course of a week or ten days the deep-seated ligatures may be pulled away, and when the integument is well united, the external stitches may also be removed. The wound is healed by Nature, and scarcely, if ever, requires any sort of dressing. 


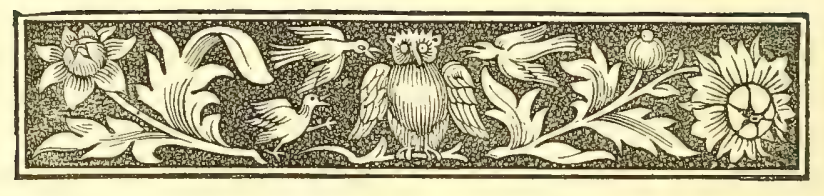

\section{SECTION XXIX.}

\section{REMARKS ON THE CATTLE PLAGUE**}

$\mathrm{D}$

ISEASES of all kinds are attributable to predisposing and exciting causes. The majority of maladies require a combination of agencies to induce them; but there are some which can only be developed by one unchangeable and specific means, which we designate contagion. A sheep can only become scabby by the scab-insect creeping on its body, and there multiplying. A dog only becomes rabid by the virus of another rabid animal entering its system; and scientific men are agreed that an animal ean only be seized with rinderpest as the result of direct or indirect communication of the rinderpest virus from a diseased to a healthy animal. These are accepted truths which somewhat shock the minds of people unacquainted with the mysterious operations of Nature. The question occurs to most persons whether, under extraordinary circumstances, these contagious maladies are not generated de novo. All we can say is, that as yet no one has demonstrated that in the steppes (Russian plains) cattle can be placed under such circumstances, apart from being subjected to the influence of contagion, as can induce the disorder. The disease is always there, roving to and fro, winter and summer, among esttle of all kinds, sometimes killing many, and at other times attacking few. My belief is, after searching in vain for evidence of the spontaneous development of the rinderpest in Asiatic or European Russia, that the malady (just like human small-pox) is never developed, per se, out of nothing, but is propagated in obedience to immutable laws, such as those which operate in perpetuating small-pox in man. There are periods of recrudescence which are,

* Condensed from Mr. John Gamaen's late great work on the Cattle Plague. (668) 
to a great extent, accounted for by periodic and extraordinary movements of stock, as in times of war, or by an activated cattle trade; but neither cold nor heat, rain nor drought, storms nor severe frosts, affect the propagation of the malady, except in so far as they affect the movements of people and the traffic in cattle.

The predisposition said to exist in the cattle of the steppes (the special idiosyncrasy which has been spoken of) is observed, during outbreaks in Western Europe, to manifest itself in rendering the disorder less severe, far less malignant, in the cattle of Russia than in any other known breeds. JESSEN tells us that foreign stock in Russia, acclimatized and bred there, succumbs from attacks of the disease as rapidly as in its native country. Thus Devon cattle imported into Russia, bred there, for years continue to indicate a terrible susceptibility, and are rapidly exterminated when the disease is communicated to them by accidental contact or artificial inoculation. It is difficult to understand that these animals, manifesting an extraordinary susceptibility, should not, as it is proved they do not, suffer from spontaneous manifestations of the disease, which have been regarded as likely among the less susceptible herds of the steppes. I do not believe that the flesh and blood of the Russian cattle are impregnated with this malady, nor that they inherit it as human beings do scrofula; and I am strongly disposed to regard the specific poison of the cattle plague as obeying the same laws of reproduction as those which we know regulate the development of the virus of variola.

We are asked again, How did the first case occur? We must give an Irishman's answer, and ask, How did the first case of human small-pox or hydrophobia occur? We can prove absolutely nothing as to their primary origin, and all we can say is, the most careful observations show that the transmission and procreation of certain animal poisons, including that of the cattle plague, obey laws similar to those which govern the transmission and procreation of living organisms. Some day we may know more of the vitality of animal poisons. We now know that their destructive operations are not more extraordinary, and the reasons for their existence not more unfathomable, than those of many parasitic plants and animals which seem to live and to multiply by undeviating processes of generation, only to shorten the existence of the higher orders of the animal or vegetable kingdoms.

It is strange, but true, that the poison of the steppe murrain 
may be compared to any living organism which only requires a. favorable habitat for its indefinite increase. Just as the scabinsect lives and breeds almost exclusively on the skin of a sheep, so does the cattle-plague virus grow in quantity only when it meets with conditions favorable to its fructification in the systems of bovine animals. This statement is not affected by the occasional appearance of the disease among goats, sheep, and other animals; for we well know, after the experience of centuries, that whatever animals may be in a country, whenever the horned stock has succumbed the disease vanishes. It does, indeed, inflict some damage on the flocks of sheep; but its slow and imperfect propagation among them indicates that it needs a more favorable nidus for its perfect and complete development.

If all known facts seem to favor the view that the Russian murrain owes its origin to contagion alone (even in Russia), no one having a knowledge of the subject believes that any other cause can induce it beyond the confines of that country. Numberless outbreaks, traced without doubt to contagion, and the certainty with which the early slaughter of diseased animals extinguishes any manifestation of the malady, even in countries where it appears often, have tended to discard from our minds the possibility of any spontaneous development of the plague over the European continent, in the new world, which it has never visited, or in the islands of the globe. It is singular, and it is very instructive, that the disease should alone be kept up in a country such as Russia. The conditions there are all in favor of the perpetuation of contagious maladies; and it is important to mention that even small-pox in sheep is preserved in Europe by its constant presence and frequent recrudeseence in Russia.

The view of the purely contagious nature of rinderpest is materially strengthened by the definite knowledge of the origin of all diseases, which, undoubtedly, originate spontaneously in various parts of the world. The Siberian boil plague, which has been confounded with the steppe murrain, is one of those enzoötic disorders due to excessive heat in the broad plains of Siberia, and especially in certain spots where an elevated temperature operating on retentive and ill-drained soils, produces a malady which certainly acquires contagious properties, but which ceases with the advent of cold and the washing of miasmata into the earth. The Siberian boil plague springs suddenly into existence, is prop- 
agated a certain distance, but can not spread far, after the manner of purely contagious disorders, which are not influenced by seasons or weather. I could mention many similar instances, were it necessary, and there is none better than that virulent blood disease which has gone on increasing of late in this country, in districts where farmers grow the largest crops by liberally distributing manure on fertile soils. That disease is splenic apoplexy, which has been studied on the Northumberland hills, in the fens of Lincolnshire, and the Somersetshire pastures. Professor Voelcker traced, in his laboratory, one of the potent causes inducing this malady in the west of England, and proved how wrong it was to give animals water charged with the products of organic waste, such as nitrates and other salts. The Russian cattle plague never did and never can appear here but as an imported pestilence. All who have seen this disease must, at all events, admit its highly infectious and contagious character. The air surrounding a diseased animal is impregnated with volatile poison, and every part of the animal's system is charged with the same principle, but in a tangible form capable of being carried on the point of an inoculating needle, and of being plunged with effect into the tissues of a healthy ox. Admitting, therefore, that contagion is the great and all-potent exciting cause, it may be well to enter into details on two points. We must first discuss whether certain conditions directly affecting an animal render its system more than ordinarily susceptible to the disease; and, secondly, what external conditions favor the propagation of the virus.

Causes.-Individual susceptibility or idiosyncrasy affects the communication of the disorder to some, though to a very limited extent. We no doubt have the striking illustration of the Russian $0 x$, which is often attacked in a mild manner; and so we find in this country that a herd is killed out in five days, and another, of a very similar kind, is not killed out in a month, and several animals do not suffer at all. This fact is partially explained by the poison sometimes growing weaker as it passes from herd to herd. We witness this with all poisons; and hence the great importance of guarding against fresh importations of virus, even during the prevalence of the steppe murrain in a country. The broader the area over which the poison can meet with favorable conditions for its development, the greater the tendency to severe recrudescence during an outbreak. 
There are some unaccountable instances of constitutional immunity, and animals in this country have appeared to withstand the disease with effect, or it has attacked them so mildly as scarcely to be observed. This, it is true, is extremely rare, but has been observed sufficiently often to indicate that, whatever may be the cause, one animal is very susceptible, and another less so, to attacks. We can not ascertain the special predisposition until the effects of contagion on an animal have been witnessed.

Age exerts no influence on the disease. Kersting has, however, said that, as the result of inoculation, young calves and cows suffer most severely. Lean or fat oxen are also badly affected; but animals in moderate condition, and at maturity, seem to bear up best against the disease. As to the influence of sex, it would appear that, as with other contagious maladies, cows yielding milk are very susceptible. Pregnant animals readily catch the disease, but probably not so readily during gestation as at the period of calving.

The conditions under which animals are kept do not seem to have much influence on the character and rapid spread of the disorder. We believe that, as a rule, the disease will be most speedily propagated where animals are congregated in a shed under one roof. Indeed, numerous instances have occurred of animals, by being separated, escaping the disease; and, although it is virulent enough in the open air, the cases do not follow each other quite so rapidly as where the cattle are housed. A proof of the aggravation of the disease among housed stock is its general manifestation where sheep are constantly penned with cattle, and the common escape of these animals when they only mix with oxen or cows in the open air. Dr. Maresch, who first deseribed the cattle plague in sheep with accuracy, showed that the malady was only rife in flocks housed with bovine animals. The question then arises, Does the housing affect the constitution of animals, or does it simply concentrate the poison? It is not improbable that it acts in both ways. Every one agrees that the depressing effects of bad ventilation, of breathing an impure atmosphere, tend to aggravate and accelerate the malady, though there are not wanting cases to prove the exact contrary. The same is observed in other contagious diseases; and I have known two herds of heifers, belonging to the same owner, one housed and the other in the fields, seized with pleuro-pneumonia about the salse time. The boused 
herd, by no means kept eleanly, suffered little, while few survived in the open fields. I am a great believer in fresh air, wholesome food, and pure water; but there is absolutely nothing to prove that animals die more readily from the cattle plague where the ventilation is imperfect, and the food and water far from being of the best quality.

It is understood that the poison of the cattle plague comes from abroad, and there are those who think that it may be the result of confining cattle for days and nights together in crowded ships, surrounded with dirt, ill-fed, and supplied with an insufficient quantity of water. Vivid descriptions have been written of the heat, fetor, and steaming sweat which rises from the holds of ships engaged in the cattle traffic. We are asked if it be possible that animals should be thus ill-used without suffering from any or all diseases; and the foot and mouth disease, pleuro-pneumonia, and the cattle plague, which differ in intensity, but not in character, have each been ascribed to these general causes. It is certain that maltreatment is not invigorating. It may lead to suffocation or severe constitutional disturbance; but, in spite of all the mismanagement in bringing cattle across the sea, no case of specific disease has, to my knowledge, ever thereby been induced. However much the animals may suffer, they can only die of the cattle plague with varying rapidity when that plague is brought among them by a diseased beast or infected materials. The same remarks. apply to markets, farm-yards, and town cow-sheds. The filthier these places are, the greater, undoubtedly, the facilities for infection ; but no amount of filth ever produced a specific outbreak of lung disease or rinderpest. We concentrate the poison in foul places, but we do not create it.

We can, with some show of reason, attribute to steam-power the outbreak of contagious diseases in this and other countries during the last quarter of a century. But steam has operated in facilitating locomotion, and in placing distant parts in regular and rapid communication with each other. Before countries were intersected, as they now are, by railways, it took several days to transport animals a distance of one hundred miles. It is, of course, better to move an animal in a pure van or truck than to walk it along roads where its feet may plunge in excrement, or its lungs inhale the breath of any sick animal passing, and which, by some strange fatality, is sure to be approached. Cattle are inquisitive, and 
sheep flock toward sheep; so that if they meet animals of their kind, and especially sick ones, they are sure to run up to them and smell about, and thus endanger their own lives.

The excrement which drops from animals affected with rinderpest, and which are driven or placed in trucks or vans, is highly charged with poison. It readily communicates the disease, as has been proved by myself and others, by inoculation; and it is well known that if a cow places its foot in a mass of this excrement, the chances are that it will be inoculated. The intestinal gases have been collected and then discharged by the diseased animal, so that, if healthy cattle inhale these gases, the probability is that they will contract the disease. The discharge from sick animals generally, and their manure in particular, are very apt to secure an indirect contagion. The greatest care should, therefore, be exercised not to disseminate the poison by such means. Food which has been mouthed or breathed on by sick eattle is capable of communicating disease, and this should always be kept in mind. It is not easy to determine if water, under the same circumstances, has a contagious effect. Some persons have supposed that if animals with the Russian plague drink from a river whose waters run through other farms or districts, the disease may be carried by the stream to great distances. This I do not believe, inasmuch as water is a great purifying and diluting agent, and even all the poison that a large herd of cattle may drop into a river can have no effect, as it passes on in an enormous mass of water, which effectually weakens and destroys it. The case is different if animals are made to drink out of a common pail or trough; for, in such a. case, it is not so much the water that carries the virus as the sides of the vessel containing that water. It may be noted, however, that cattle of different farms often stand for hours together in a stream at only a few yards apart; and here, as in the drinking-trough, the virus contained in the discharges may reach healthy animals unchanged, and thus lead to their contamination.

In countries or districts where contagious diseases prevail among cattle and sheep, we find that slaughter-houses are favorable for their dissemination. This is due to the fact that plague-stricken animals are taken there for slaughter, are sometimes kept alive for hours, or even days, and, when killed, much that is charged with virus escapes in surface-drains, or on an open causeway, so that, within a certain radius, there is a chance of contaminating healthy 
stock. One way in which the cattle plague may be carried from diseased cattle or from a slaughter-house is unquestionably by flies, which, after resting on the carcass or offal of sick animals, fly about, rest again on the animal, especially on any wounded parts, and thus produce a direct inoculation. Any place in which many animals affected with rinderpest are slaughtered, must charge the surrounding neighborhood with enough poison to kill large numbers of cattle. The hides and meat of diseased animals carry the infection. In Hungary I am told that one common cause of rinderpest outbreak on a farm is the hawking about of the flesh of animals slaughtered during an attack of the disease. If the water in which such meat is washed be thrown into a yard to which cattle have access, an outbreak is almost sure to follow. JESSEN speaks of cattle becoming infected by drinking the water used in soaking or washing salt meat. Slaughter-houses and the traffic in hides and meat are probably not so active in favoring the spread of the rinderpest as cattle-dealers' farms, and the changes of stock which necessarily occur there. The grazing of cattle in neighboring fields, feeding them on the road-sides, driving them along paths through fields where there is a right of way, are all potent circumstances in the dissemination of rinderpest.

No one doubts that dogs which feast on the carcasses of diseased animals are very liable to carry infection. I have been consulted more than once as to the probability of a pack of fox-hounds carrying infection over a country by passing through fields containing sick cattle, and afterward crossing healthy farms. It is possible that a pack of hounds may carry much excrement charged with organic poison from one field to another; therefore, in an infected district hunting should, in my opinion, be discontinued. Human beings, as well as quadrupeds, are accused of harboring the poison and distributing it, and no doubt they do carry it, more or less, if great care be not exercised. I have been assured that in Russia one common cause of wide-spread outbreaks was the practice of calling priests and people together to pray in the cattlesheds, that the plague might be stayed, and the assembled people moving thence from farm to farm. Vicq d'Azyr demonstrated, last century, that if clothes worn by attendants on diseased cattle were placed on sound stock, three animals out of six would be seized with the disease.

Without entering into further details, I may state that the 
causes here enumerated as affecting the transmission of the cattle plague are not imaginary but real. Enough to mention that farmers and others, who have any thing to do with rinderpest, must regard it as a purely contagious and specific disease, incapable of spontaneous development, but most readily and certainly communicable from diseased to healthy cattle, and sometimes from cattle to sheep, or vice versâ.

Symptoms. - The recognition of this disease is greatly facilitated, especially when it first appears in a herd, by a knowledge of its prevalence in any district or country. I mention this, as in many diseases we have premonitory signs similar to those of the cattle plague; and it is especially when we know of its existence in or near a country, district, or farm that the earlier symptoms are of value. Indeed, when an outbreak is studied, great importance should be attached to the period which elapses from the introduction of a diseased animal, or other source of infection, to the first appearance of sickness in a stock. Every specific fever has its period of incubation-that period during which the poison is insidiously attacking the system of a man 'or animal-and there is no more distinct feature of the eattle plague than its latent stage. From four to seven days is the usual period of incubation. It often extends , to a week, but very rarely, indeed, beyond the eighth day. Some say it may be as short as twenty-four hours, and others that it occasionally extends to twenty-eight days. There are no reliable facts in proof of these extremes, but there is a large amount of evidence to show that an animal may be regarded as absolutely free if it does not show signs of disorder within ten days after having been exposed to the contagion. Indeed, recent inquiries and observations would lead one to regard the incubative stage as not often exceeding six days, though the earlier or premonitory signs of an attack are not apparent to nonprofessional observers. I consider this point of the greatest importance in relation to the subject of veterinary inspection, and one affording scope for many experiments as to the facilities offered for the prevention of the disease, by recognizing sickness in animals before they become dangerous from the discharge of that poison which is the cause of the propagation of the malady. Whenever medical treatment may have to be tried, it is at this early period that good results may be anticipated.

A delicate thermometer indicates an elevation of temperature in 
the earliest stage of the disease varying from one to four degrees. The elevation precedes the acceleration of the pulse and every other symptom. It is not uncommon to find it in healthy animals varying one or two degrees at different periods of the day, so that reliable observations can only be made on a number of cattle at the same time, obeying in all the same conditions as to the instrument used, the part in which the observation is made, whether it be the rectum or vagina, and the length of time the instrument is inserted, etc. Sometimes, when animals are excited on a hot day, and are hurried into a shed from a field, the thermometer may rise one or two-tenths more than usual; but if a whole stock be examined, any animals suffering, however slightly, from the disease, indicate an elevation amounting even to five or six degrees. Such an exaltation of temperature is generally incompatible with health, and the only exception yet known to this rule is that observed during cestrum or sexual excitement; the temperature then rises three or four degrees, and the same may be seen just after parturition. A number of experiments prove:

First. That the temperature is much exalted when the pulse indicates slight or no variation from the normal standard.

Secondly. That there are variations in the frequency of the pulse and the temperature during the course of the disease.

Thirdly. A sudden lowering of temperature usually, if not always, precedes death. In the cases quoted above, where the temperature last taken is marked as high as $105^{\circ}$ and $104^{\circ}$, death did not take place until ten or twelve hours after the last observation.

Fourthly. With the lowering of temperature before death there is a greatly increased frequency of pulse, varying from 120 beats per minute to such a rate as to render observations almost impossible.

When animals recover, the temperature decreases gradually till it reaches its normal standard. There is an absence of very marked and sudden change. The transition from sickness to convalescence occurs steadily and with regularity.

I consider it impossible to over-estimate the importance of thermometric observations such as those referred to, and, although similar results may be obtained in the investigation of other diseases, it is evident that the thermometer affords unerring as well as early evidence of an animal sickening, recovering, or about to die. No other indication is so unmistakable and satisfactory; 
and though there are other febrile diseases associated with an early elevation in temperature, it must be admitted that, taken with the history and symptoms of the case, the accuracy afforded by thermometric observations is of the highest moment.

The visible premonitory signs consist in shivering, muscular twitchings, and uneasiness. In some cases there is dullness, and in others excitement amounting even to delirium and associated with remarkable sensitiveness. There is often a short, husky cough ; the appetite is irregular, capricious, and then entirely lost; rumination ceases; the animal grinds its teeth, yawns, arches its back, and draws its legs together under its body; the eyes, nose, and mouth are dry, red, and hot; the extremities are cold, though the internal heat is high; constipation, as a rule, exists; and secretion is generally arrested, as indicated in milch cows, by the milk at once ceasing to flow. The respirations are often, but not invariably, increased in frequency; expirations succeed the inspirations tardily, and with each there is a low moan; the temperature continues to rise, though the animal's skin becomes rigid, and indicates functional derangement by a staring coat, dryness, and eruptions.

The redness of the visible mucous membranes, especially of the gums, lips, papillæ on the inside of the cheeks, is partial, pale, and patehy at first. Dr. WEBER has spoken of the aphthous eruption of the mouth, the aphthæ being of circular or indefinite form, covered with whitish-yellow granular exudation, which adheres very slightly and is easily removed. He also speaks of the redness of the papillæ of the cheeks as always beginning at the apex. JESSEN has published a pamphlet on the appearance of the buccal membranes, which he describes as sometimes consisting in small, round nodules (seldom larger than a millet-seed), still covered with epithelium when discovered, through which a yellowish or yellowish-gray material can be distinguished. Within twentyfour hours the epithelium gives way and the contents become visible. The result is a superficial lesion, which soon heals. In other cases the nodules become confluent, and form a considerable enervated ulcer, with irregular margins. The so-called aphthæ are described by JESSEN as small vesicles due to raising of the epithelium, and either contains a clear watery or a turbid fluid, and leave behind round, flattened excoriations, with even edges. My observations, in a considerable number of cases, have shown 
that on the inner surface of the lips, on the inside of the nostrils, and other parts of the mucous membrane, there are at first scarcely visible whitish opaque specks, about the size of a small pin's head. These are the starting-points for the softening and desquamation of epithelium, which results in the dirtyish-yellow, flaky appearance in some of the worst cases.

When a number of milch cows has to be examined, one of the first symptoms to look for is redness, and a mottled appearance of the lining membrane of the vulva and vagina. Animals in apparently perfect health, eating well, ruminating, yielding a full quantity of milk, are seen to have a reddened condition of the mucous membrane of the external organs of generation. The redness of the vagina also occurs in animals that have recently calved, and it is important to guard against this source of fallacy. In many cases the continuous rigors, and singular muscular twitchings of the face, ears, and neck, may be regarded as characteristic. They are not, however, so typical as the discharge from the eyes and nose, which soon appears, and which, from being glary and watery, changes shortly to a turbid secretion. No symptoms can better illustrate the care required in diagnosis than the discharge both from the eyes and nose. A marked symptom is restlessness, lying down and rising again; sometimes looking round to the flank, and by drawing the hind legs forward, denoting more or less colic or abdominal pain. Animals often lie on the left side, with the head stretched across the right flank. Severe diarrhea sets in, and the animal becomes very thirsty. Emphysematous (windy) swellings are apt to form at this period, and there are exacerbations (violent) of all the symptoms toward night-time. The discharges are all fetid, especially in severe cases. The urine is rather scanty, and generally, if not always, albuminous. This stage lasts about three days. The symptoms increase in severity. The dysentery is aggravated, and the animal becomes extremely weak; it stands and walks with difficulty, and lies much. The pulse becomes feeble and indistinct at the jaw; it beats from 90 to 130 per minute. The discharge from the eyes, nose, and vagina increases; the cough becomes less audible and soft. On the buccal and schneiderian membranes, as well as in the clefts of the feet, there is a deep redness, with flaky discharge of epithelium. The muzzle, angles of the mouth, and membrane round the nasal orifices are sometimes ulcerated, with a greenish- 
yellow and somewhat dense granular and epithelial deposit. On opening the mouth, a similar change about the base of the tongue and on the inside of the lips is often found. The coldness of the extremities, or of the body generally, the stupor or drowsiness, quick breathing, and fetor of the exhalations, with spasmodic action of the alæ nasi, jerking respiration, and moaning, are among the most unfavorable symptoms. The feces, at first dark, become slimy, charged with masses of detached epithelium, are very fetid, and are more or less tinged with blood. The urine acquires a dark color, due to the coloring principles of bile. Cows abort, and all symptoms of sensibility or consciousness gradually disappear.

I have seen many cases which presented, from the earliest moment, great disturbance of the organs of respiration. A hacking cough, depressed and protruded head, spasmodic action of the nostrils and flanks, indicate serious pulmonary lesions. Emphysema takes place, and usually begins in the anterior lobes. Dr. WEBER remarks that the anterior intercostal spaces become somewhat fixed, whereas the posterior true ribs are raised with an effort, and sink rapidly. On percussion, the thorax is found to be very resonant, and this resonance becomes greater as the emphysema increases. On auscultation, râles of various pitches are heard, either accompanying the vesicular murmur or superseding it. The heart's sounds become inaudible, and impulse imperceptible on the left side. As death approaches, the mucous membranes often acquire a leaden hue; the erosions (ulcerations) are marked, and blood-spots, or ecchymoses, occur. The partially open and dark red or otherwise discolored aspect of the inner surface of the lips or the vulva can not fail to be noticed at this stage. The involuntary evacuation of excrement, extreme fetor of all discharges, tendency to tympanitis, muscular twitching, lowering of temperature, and increasing listlessness betoken the approach of death.

In some cases there are signs of improvement about the third day, and then a relapse occurs. Animals may become quite convalescent; but still the gastric or intestinal lesions advance, and when least expected, a fortnight or three weeks after marked improvement, alarming symptoms supervene, severe diarrhea occurs with the return of other discharges, and the animal soon sinks and dies.

In favorable cases we find a cutaneous eruption on various parts 
of the skin, especially on the neck, back, and teats, not unlike cow-pox. There are instances of severe illness and death with this eruption, and, indeed, in bad cases, we sometimes find a dirty yellow appearance of the skin of the back, and a desquamation of epidermis, which indicates a morbid process of the skin, similar to that affecting the mucous membranes. The surface of the skin over the neck and withers is often moist or greasy from an abundant sebaceous secretion. There are no vesicles, and an entire absence, as a rule, of pustules. Convalescence is indicated by a certain vivacity, return of appetite, equable temperature of the body and extremities, restored secretion of milk, moist muzzle, and other well-known signs of health.

As with other fevers, we find in the rinderpest a marked periodicity in its manifestations. Improvement in the morning, violence of symptoms at night; a distinct subdivision of an attack into stages, and, from the date of the crisis, either sudden aggravation or gradual abatement of alarming symptoms. There are, at times, chronic cases, as in lung disease, and animals get into a hectic state, out of which they can not be rallied. It is, therefore, evident that the duration of the malady varies. I have seen animals dead in the evening which had only indicated active signs of the eomplaint for the first time during the early part of the same day. As a rule, death occurs from the third to the sixth day.

Symptoms in the Sheep.-The cattle plague among sheep is characterized by comparatively mild symptoms, and frequently speedy restoration to health. In 1857, Dr. Kreutzer first described the symptoms of the cattle plague as observed on a sheep which had been inoculated on the 1st of October. The period of incubation lasted till the $9 \mathrm{th}$, and was followed by general disturbance, discharge from eyes and nose, prostration, moaning and diarrhea. The animal died on the 13th. Dr. Maresch observed the disease more carefully from 1860 to 1863 , and since then much information has been obtained. The period of incubation extends usually to seven or eight days; languor and dullness appear, with redness and prominence of the conjunctiva at the inner angle of the eye. There is a yellow discharge which trickles down the face, and a viscid phlegm flows from the nostrils; the head droops, and there is grinding of teeth; the appetite is diminished and capricious; rumination, suspended; feces, thinnish, and partly adhering to the hind legs and tail; there is an occasional cough, 
with frequent pulse and labored breathing; the pulse rises from 120 to 160 beats per minute. Ewes not unfrequently abort, or bring forth weakly lambs, which afterward rally. It is not at all unusual for the disease to cease at this period, and the animals to recover rapidly. In other cases the diarrhea increases; there is painful straining, or tenesmus, panting respiration, very feeble pulse, and the animal sinks. Greatt weakness, awkward gait, and somewhat severe nervous or convulsive symptoms mark fatal cases, when death occurs about the fourth, fifth, or sixth day from the commencement of the disease. When a case takes the more usual and favorable turn, there is more liveliness, improved appetite, restored rumination, less discharge from the eyes and nose, and diminished frequency of pulse and respirations. The animals are quite convalescent in from ten to fourteen days. In some cases, such as those observed by Dr. Leicht in 1860, the disease is more rapid. The animals stagger, lie down much, shake their heads, have a dense discharge from the eyes, are subject to diarrhea, and die, sometimes, within twenty-four hours of the first appearance of premonitory signs.

There are as great variations in the symptoms in sheep as there are among cattle. Some cases differ from those described above, as the animals stand, are dull, and keep apart from the flock; there is no appetite, no rumination; constipation at first, followed by diarrhea; arched back; elevated temperature of the skin; eyes sunken and discharging; from the nose a quantity of dense, grayish-yellow, flaky matter drops, and the mucous membranes of the mouth and nose are reddened, showing erosions and desquamations of epithelium; the breathing and pulse are accelerated; there is pain on pressing the loins, and, at last, general prostration.

Special Symptoms in cases of Cattle Plague coupled with Pleuropneumonia.-The animals seized with the two diseases at once are observed to suffer from greater prostration and more labored breathing at the outset. The short grunt of lung disease begins early; there is a spasmodic action of the nostrils, and, on auscultation, the impervious condition of the portion of the diseased lung is ascertained. Any one acquainted with the two diseases can readily recognize such cases.

Special Symptoms in cases of Cattle Plague coupled with the Foot and Mouth Disease.-It is more difficult to diagnose rinderpest in its earliest stages when epizoötic aphtha has attacked a 
herd. Smacking of the lips, eruption, and salivation exist; but the greatest reliance is to be placed in the usual lameness and morbid condition of the feet in foot and mouth disease, as also the usual eruption on the teats, and tendency to congestion and inflammation of the udder. When the cattle plague advances, there is the shivering, discharge from the eyes and nose, the diarrhea and prostration not usually seen in epizoötic aphtha.

The Nature of the Cattle Plague.-From all that has been said in the preceding pages, it is evident that the murrain of the steppes is not typhus, nor is it the typhoid or enteric fever which we observe in man. Not only is it distinct in its origin, progrêss, and essential nature from any known contagious disease of the human subject, but it is, undoubtedly, a specific bovine fever, manifesting all its characteristic features in horned cattle alone, though experience has proved that there are circumstances under which the plague may be communicated to the buffalo, goat, sheep, deer, gazelle, zebu, yak, auroch, ibex, and other wild ruminants. One experiment, performed by myself, proves the communication of the disease to the deer ; but it is remarkable that until the recent outbreak of rinderpest in the gardens of the Paris Acclimatization Society, in the Bois de Boulogne, it was not known that the majority of wild ruminants could catch the disease and suffer from it in a virulent form. It never attacks men, horses, dogs, and, indeed, the great majority of warm-blooded animals; and it is worthy of special remark that the virulent animal poison, which is the active agent in the development and propagation of the malady, originates in the system of the $o x$, is perpetuated in countries where herds of eattle ahound, and is not to be found where bovine animals are wanting. The history of the cattle plague clearly indicates that the disease has been always recognized as attacking horned stock almost exclusively, and that it spreads from country to country through the trade in cattle or the transport of oxen in the rear of armies. The poison does pass through the system of some ruminants besides those of the bovine race, but it appears to be deprived of much of its force until it returns to members of the ox tribe.

The cattle plague is not a local disorder; it is not an affection of any special organ or group of organs. It is a systemic diseasea fever in which the mucous membranes and skin are specially implicated. There are important local and characteristic mani- 
festations, usually most marked in the mouth, fourth stomach, and intestines, in the organs of generation, and frequently in the respiratory passages. It is, however, something different, and something more than an inflammation of the breathing, or digestive, or generative systems. The cell growth, fatty and molecular disintegrations, desquamation, and discharge of the epithelial and epidermic cells, are typical of this disease. In this way it can only be classified among general diseases, with fevers of a specific kind, and which originate from specific causes, run a definite course, manifest a singular periodicity in their progress, and have a marked tendency to destroy life.

The pathological process, observed from first to last, indicates an early development of severe febrile disturbance. All the functions are modified. The temperature of the body fast rises; general functional disturbance soon follows, and the blood loses its watery parts and soluble albumen, indicating, also, a large increase in the proportion of blood-corpuscles and fibrine to serum. The process of assimilation is checked; the large reservoirs in which the food is prepared for true gastric and intestinal digestion become torpid; the rumen, reticulum, and ornasum retain a large quantity of solid food, and their movements are stopped; the fourth stomach ceases to secrete gastric juice, its epithelium is thrown off, a morbid cellular deposit clogs the gastric glands, and the proper preparation of aliment for intestinal digestion can no longer occur. The intestine is the seat of inflammation and extravasation; its contents indicate the drain of the blood of its soluble albumen and other products; the intestinal epithelium is rapidly ejected, and, in some cases, many of the glands become clogged with a deposit similar to that which is found in the follicles of the gastric mucous membrane. A free suppuration is nowhere witnessed, but discharges flow from the mucous membranes, and are highly charged with epithelial cells and the specific virus of the disease. The rapidity and completeness of the general functional disturbance is indicated not only by the checked process of assimilation, the nature of the intestinal contents, and the general implication of the mucous membranes, but it is recognized on an examination of the albuminous and dark-colored urine, the disposition to early putrefaction, fetor of the secretions, etc. All shows that the cattle plague poison speedily induces in the system of a susceptible animal changes which defy the successful use of medi- 
cine, and which must inevitably result in death. Like most animal poisons, the rinderpest virus is reproduced with marvelous rapidity in, and discharged abundantly from, the bodies of sick animals. The breath of a sick ox inspired by a healthy animal, and the solid products of the disease, seem to be alike capable of inducing the malady; and antidotes are applied too late when an attempt is made to reach the poison in the animal's system. I know of no antidote to be used internally. A Agents are employed which, when directly mixed with the virus, kill it; and caustic alkalies, mineral acids, chlorine, iodine, carbolic acid, creosote, the alkaline permanganates, and other oxidizing or decomposing agents, render it perfectly inert. I have no faith in our ever reaching the virus with effect in the living animal. We must not even expect too much from the system of treating certain symptoms during the progress of the diseuse, which is frequently confounded by the unskilled with the effectual treatment of the disease itself. A large proportion of animals seized must always die. A certain percentage will always recover, and this depends on the severity of the attack, the constitutional resistance of the animals seized, and the consequent extent and rapidity of the morbid changes which occur.

Treatment.-The steppe murrain is essentially one of those diseases for which prevention is better than attempted cure. Urgently enforcing this doctrine has cost me the good opinion of some, who, without duly considering the importance of slaughtering the diseased and infected animals for the protection of many untainted herds, argue that it is not scientific to exterminate the malady by destroying diseased animals. The eattle plague can be prevented and can be extinguished, at comparatively small cost and with the greatest certainty, in countries such as England. Whatever tentative trials are made as to treatment, this fact must ultimately reveal itself, that it is impossible to deal with outbreaks of rinderpest by administering drugs. I hold it to be eminently scientific to act on our knowledge of the cause of any disease; and, knowing, from lengthened experience, how easily disseminated and malignant cattle plague virus is, it has been my duty to insist on its early and complete destruction, so that it should never reach the systems of herds of animals, in which, when it has once entered, it can not be counteracted by any known antidote.

The cattle plague is not so deadly in its effects as equine glan- 
ders, cancer, or well-developed human phthisis; but no known remedy restores an animal once severely attacked, and the administration of medicine is, as a rule, not required to save the small percentage which may recover. As, however, the malady has been suffered to spread, veterinarians are called upon to adopt means of cure. With a view, therefore, to indicate what has been suggested and what may be attempted, I shall draw attention to the following points:

Precautions to be observed so as to prevent the SPREAD OF DISEASE FROM A HERD UNDER TREATMENT.

Hygienic management.

Medicinal agents used.

METHODS OF TREATMENT SUGGESTED AT VARIOUS TIMES IN THIS COUNTRY AND ABROAD.

Precautions to be observed so as to Prevent the Spread of Disease from a herd under Treatment.-Diseased cattle should be placed for treatment in warm and detached buildings where proper ventilation, drainage, and facilities for feeding can be obtained. They should not be left in the fields, and are best kept entirely in the house until perfectly recovered. The sheds should be kept clean, frequently disinfected, and every particle of urine and excrement must be disinfected by means of chlorinated lime. People attending the stock must be kept there for that purpose, and not allowed to rove about and go on other farms, or to fairs and markets. All dogs should be tied up, and every attention paid to cleanliness with other stock and in farm-yards generally. Dirt and negligence are great propagators of the cattle plague. It is impossible to be too clean or too careful in avoiding the carriage of tainted objects from an infected to a healthy farm or district.

Hygienic Management.-This is of far greater importance than any medicine or system of medication hitherto recommended; and I have to direct the attention of my professional brethren to several points of incalculable importance. It is not desirable to have many animals in the same shed when under treatment, and several attendants are essential, so that proper care be taken of the cattle. When a large stock has to be treated, the labor and trouble is enormous; it will not do, therefore, to trust to a few attendants, who get tired and faint-hearted over their work, especially when the number of animals recovering is not large. If possible, it is undoubtedly best to have the animals loose; but this can only be 
secured where isolated cases are under treatment. With forty or fifty beasts sick at one time, loose boxes can not usually be obtained; and it is not desirable to keep many sick animals loose in a yard. As a rule, in winter all the animals should be clothed, lightly, but sufficiently, so as to favor cutaneous exhalation. The shed is best kept at a proper and never-varying temperature, not exceeding $58^{\circ}$ Fahrenheit. Proper ventilation is indispensable to recovery, and heat must not be purchased at the expense of atmospheric purity. Clothe the animals rather than close the aperatures made for the purposes of ventilation. In order to secure purity of the atmosphere, every attention should be paid to sweeping away excrement and washing the stalls thoroughly by means of common soda and water. The use of chlorinated lime is not to be advocated where stock is under treatment, as the chlorine gas induces great disturbance, irritation of the respiratory passages, and a troublesome cough. I prefer cleanliness and abundance of fresh air where the cattle are actually diseased, to any system of disinfectants, and the chlorinated lime is best used to disinfect the manure-heap and other objects outside the shed containing the sick. The food allowed to animals varies much according to circumstances. During the various stages of acute disease it should be given sparingly. At first linseed tea or linseed gruel, wèll boiled, may serve as a laxative, with or without medicine. Linseed is not to be continued when purging begins; well-boiled oatmeal gruel should then be substituted for it. The oatmeal gruel, which is perhaps the best nutritive material to be given from first to last, is made by taking a peck of meal for every three or four animals, and adding some cold water. Boiling water is then mixed and stirred with the whole, until it acquires a proper consistency to be poured through a towel or sieve; and thus all the coarser and more indigestible portions of the meal are removed. The gruel is boiled for at least ten minutes or a quarter of an hour, and is used warm. About half a gallon of it may be given three or four times daily; and if the cattle drink it voluntarily, so much the better. Badly-made oatmeal gruel irritates the stomach and intestines, and aggravates the diarrhea. When animals are severely affected they do not eat; but if they have any appetite, well-boiled mashes should be made for them. Of these we can suggest a great variety, such as steeping barley for a time, throwing off the water, adding fresh, and boiling the barley; the whole is then allowed to rest for 
awhile, and bran is mixed with it, as well as a certain quantity of well-boiled turnips. The mash must be moderately salted. The barley may be replaced by brewer's grains, and a moderate quantity of bean or peasemeal-say half a pound of the latter morning and night. One of the best managers of cows $I$ ever knew made, for every sick animal that would eat, a mash of four handfuls of bran, four of brewer's draff, one pound of peasemeal, and two pounds of well-boiled mashed turnips. This was given thrice daily to each animal. Cattle have been fed on milk, soups, etc., but not with the same benefit as with vegetable food which was properly cooked, and not allowed to get sour. Great moderation in diet is essential. The sick cattle must have cold water to drink in small quantities, and at least every two hours. Their mouths are parched, and great benefit is derived from frequent allowances of fresh water, which may be acidulated with a little vinegar, or given alone. All animals under treatment must be kept scrupulously clean, and are benefited by brushing, washing, hand-rubbing, bandaging the legs, etc. As a means of insuring cleanliness and action of the skin, I think it is very probable that the hot-air bath, when at hand, may be of great service, as also the vapor bath or the wet packing, used occasionally.

Medicines.-The plan principally adopted in the treatment of cattle suffering from steppe murrian has consisted in giving solutions, mixtures, and powders, either alone or in food. There are various ways, however, which might be resorted to, and perhaps with benefit, and which would effectually set aside some of the uncertain and often injurious administration of draughts. In order to explain myself fully on this subject, I shall consider the various methods by which remedies may be given internally.

Administration by the Mouth.-It is often possible, in the early stage of disease, and in favorable cases when appetite returns in the later stages, to give animals tasteless or palatable drugs in food, and this is by far the best plan. The frequent, troublesome, and somewhat dangerous drenching to which sick cattle are subjected, should, if possible, be dispensed with. I have seen so many animals tormented and injured by the administration of medicines, treacle, gruel, and other liquids, that I venture to make a few remarks on the operation of giving a draught. In the first place, glass bottles are dangerous ; and nothing is so useful as a horn of proper shape, or a tin instrument made in the 
shape of a horn, with its mouth well rounded off. The operator should go up to the right side of the animal, pass his hand over the face into the angle of the mouth on the left side. The head is bent round, not elevated, except to a very slight extent; and if the person giving the draught plants his feet well on the ground, with his back against the animal's shoulder, he can steady himself well, and, holding the horn or other instrument charged with the draught in his right hand, he can pour it by degrees into the animal's mouth. It has often pained me to see sick cattle seized by the nose, and, with their heads drawn up and necks stretched out, required to gulp down a quantity of liquid which can not be conveniently swallowed in such a position. Often have I seen an animal get worse after such treatment, and the passage of medicine into the windpipe and lungs was not unfrequently the cause of aggravated symptoms. One great objection to the administration of draughts when an animal is suffering from the cattle plague is, that they accumulate in the rumen, do not reach the true stomach until the diarrhea, inseparable from the disease, sets in, and then the accumulated drugs exert a most prejudicial influence. It is incredible how long a vast amount of medicine, and very acrid, irritating medicine, too, may remain unchanged, unabsorbed, and inactive in the paunch of an ox, which is usually full of vegetable food throughout the progress of an attack of the plague.

Administration by the Rectum.-Injections, given carefully with an enema funnel, have the benefit of creating little disturbance, and the power of solution and absorption of remedies is probably as great, if not greater, in the rectum during this disease as in the stomach and small intestine. Simple warm-water injections, cathartic, stimulating, sedative, and even nutritive enemata, deserve a fair trial, and as much good may be anticipated from them as from the administration of draughts.

Subcutaneous Injections.-Various medicinal agents have been effectually introduced into the system by being injected into the open tissue beneath the skin. This plan can be adopted if such remedies as tincture of aconite, solutions of belladonna, of various alkaloids, and other agents which do not irritate and inflame the tissue, are used.

Injection into Veins.-The principal object in view in resorting to this operation would be to try the effect of injecting about a 
pint or a quart of water, at $100^{\circ}$ Fahrenheit, after abstracting a similar quantity of blood from one of the jugular veins. The operation should be tried in the early stage of the disease, and the result usually observed is purgation, action of the kidneys, and free exhalation from the skin. The system might thus be prepared for any further course of treatment. The operation is simple, and unattended with bad results.

External Applications.-The method of applying heat, cold rubefacients, and blisters to the surface of the skin is too well known to deserve any lengthened description. Dashing cold water over the body, and then using towels and wisps of hay to dry and warm the animal, has been often recommended. The vapor bath, by covering the animal, head excepted, with woolen rugs, fixed out by sticks, etc., and then heating the air between the rugs and the skin by a spirit or gas-lamp, has been suggested. If mustard poultices are used, they should be large, warm, and applied with a rug to the body, so as to be kept on for three or four hours. Rugs dipped in boiling water, wrung out thoroughly, and applied to the abdomen, have been resorted to.

Internal Remedies.-A close observotion of the treatment adopted by empirics has led me to the conclusion that no remedy, or class of remedies, can be regarded as absolutely efficacious in any real case of rinderpest. Some animals recover despite the mode of treatment, but the majority die. I shall here notice some of the principal remedial agents used by myself and others under various heads.

Most veterinarians consider that the over-distended stomachs and the marked constipation should be relieved. From the fact that the omasum is loaded with dry food, even in the later stages of cases attended by great diarrhea, it is believed by some that smart and early purging is desirable. Full quantities of Epsom salts, linseed oil, and even croton oil, have been given, and a very common result of drastic eathartic doses is to induce an early and very fatal diarrhea. There are more advocates for mild laxatives, such as treacle, small and repeated doses of sulphate of soda, sulphur, and oil. I have treated many cases successfully without attempting to relax the bowels, and have certainly had as much success then as when either mild or active purgatives have been prescribed. A purgative which may be used advantageously is the following: 
No. 137. Sulphate of magnesia .......... $12 \mathrm{oz}$.

Sulphur.................. 4 oz.

Spirits of nitric ether.......... 1 oz.

Niter..................... $\frac{1}{2}$ oz.

Water ...................... 1 quart.

As this is not apt to induce much purgation, a bottle of linseed oil may be given after it. Oil of turpentine, to the extent of two or four ounces, has been given with a quart bottle of linseed oil, and with good effect when no other purgative has been administered.

I think that injections have been too much overlooked in the treatment of this disease. They may be given at first to move the bowels, and various forms of medicated or nutritive injections might prove of service. When the diarrhea comes on, and there is some tenesmus, an injection may be given of one pint of starch emulsion, containing one ounce of laudanum, to be repeated, if necessary. It is desirable to guard against irritation of the rectum as a result of frequent enemata.

Niter has been extensively used during the present outbreak, and in considerable doses, with a view to aid in the elimination of the virus. It is apt to weaken, and must be prescribed in moderation. It has no specific action of value. Oil of turpentine, which is a stimulant as well as diuretic, has been strongly recommended by some, in ounce and two ounce doses.

Warm clothing, the application of heat to the skin by means of heating the air, confined with woolen covers arranged round the animal, and the internal use of liquor ammoniac acetatis, oil of turpentine, and other agents calculated to excite perspiration, have been most strongly advocated. I undoubtedly prefer to excite the action of the skin by raising the temperature of the air rather than by the employment of internal remedies, which often act feebly and very imperfectly on the lower animals.

Stimulants have had their strong advocates. I have given whisky, brandy, spirits of wine, in two ounce doses, every two or three hours, and some animals have recovered while others have died. Carbonate of ammonia, in half ounce doses, has been largely employed, and seems to agree as well as any thing with the sick animals. Strong ale, porter, port wine, and other more or less active beverages containing alcohol, have been principally used when animals have been convalescent.

I have not resorted to sedatives to any great extent in this 
malady. Tincture of aconite, in thirty-drop doses, has been administered at frequent intervals. Extract of belladonna and considerable doses of opium have been chosen from among narcotic remedies as affording the best chance of regulating, at different times, the condition of the bowels.

Nitrate of potash, chlorate of potash, and acetate of ammonia, have been freely administered, and, in some cases, with apparently good effect. These agents are almost exclusively relied on in certain febrile disorders from their action on the blood, and the favorable effects they induce in activating the secretion of the skin, kidneys, and mucous membranes generally. They are administered singly or combined, in moderate and repeated doses, with or without carbonate of ammonia, and dissolved in a considerable quantity of water.

Mineral and vegetable tonics are used with benefit in the convalescent state, and, if not given in large doses, so as to induce derangement of the stomach and bowels, they accelerate the return to health in a very decided manner. Vegetable tonics, such as chinchona bark in decoction, or infusion of gentian, are given about an hour before feeding-time, and serve to stimulate the appetite. Of all the mineral tonics, sulphate of iron is the best, and should be given in drachm doses in food. Preparations of iron have been supposed to act powerfully as prophylactics, and for this purpose I recommended them when I first recognized the existence of the disease in the country.

Mineral acids have claimed a great share of attention, and I have been in the habit of using them freely. Hydrochloric acid, largely diluted, has lately been vaunted as a specific. Sulphuric acid has been more frequently used. Nitromuriatic acid is serviceable in the convalescent stage.

Methods of treatment may be classified under two heads-Preventive and Curative 'Treatment.

Prevertive Treatment.-A somewhat rational plan, recommended from the commencement, was the daily administration of tonics, in addition to proper feeding, ventilation, and use of disinfectants. Iron has proved so useful in my own practice during outbreaks of contagious diseases, that, in a circular entitled "Advice to Owners of Cattle," dated the 3d of August, I said: "The administration of tonics in moderation, and especially preparations of iron, may be recommended for all cattle that have been acci- 
dentally subjected to the contact of infected animals. All should be done to support the animal's strength, that it may withstand the disorder." Shortly afterward, the British Consul at Warsaw brought to the notice of the British Government, in the month of August, a remedy for the rinderpest, adopted with much success in Poland, in 1857: "The putting of old iron into the troughs from which the cattle drink, so as to produce a highly chalybeate water. The efficacy of this mode of treatment is said to have been discovered by the almost entire immunity from the disease, in 1857, of the cattle on a farm where there was a chalybeate spring." An observer recommends a plentiful supply of bruised oak bark as a great preserver, and fresh-powdered carbonate of ammonia, dissolved in about the proportion of a tea-spoonful to a quart of the water supplied to the cattle, to keep their blood pure and in a state to resist infection. Sulphurous acid and its salts have been strongly advocated, and Dr. Druitr, Dr. Sirart, Dr. Wilkinson, and others, have recommended daily doses of hyposulphite of soda to healthy cattle in infected districts. The use of vinegar has also been suggested. Two calves were placed among a number of diseased cows, and had a wine-glassful of vinegar in a pint of water twice a day; they also had their eyes and nostrils sponged with the same mixture, with complete success. Tar-water, made of the best Barbadoes tar and capsicum, was also suggested. Hot tar was to be painted on the cribs and mangers, and a little tar was to be smeared on each nostril, and upon every foot, and between each hoof.

Curative Treatment.-It is difficult to classify the many extraordinary methods of treatment suggested since the 1st of August last for the treatment of steppe murrain. Advocates of hot-air and vapor baths have been very positive in their statements, some, like Mr. R. Monterte, insisting on the value of the first, and others asserting their firm belief in the second, especially in combination with other remedies. The vapor bath is an old method of treatment, said to originate with the celebrated THAER, the father of scientific agriculture in Germany, who is reported to have applied it with signal success during the murrain year of 1828. A large copper kettle is sunk in the earth and filled with water. A strong fire is then kindled underneath the kettle, over which perforated boards have been laid. When the water boils, the sick animal is placed on the boards and covered with a woolen 
cloth, and a linen one above it. The animal is kept there from half to one hour, then rubbed dry, kept warm, and allowed hay and gruel. Great attention was paid to this method of treatment by a Russian farmer, who explained it, in a letter to our Ambassador at the Court of the Czar, Sir A. Buchanan, which was forwarded in a dispatch to Earl Russelt, on the $3 \mathrm{~d}$ of October, 1865. Mr. Feuling, the gentleman in question, spoke confidently of the value of the vapor bath; and the faith in the remedy increased when Mr. Grahas, of Capellie, near Glasgow, detailed recoveries under the following system. Mr. Graham administered

No. 138. Sulphur.............. 3 table-spoonfuls.

Niter.................. 3 table-spoonfuls.

Ginger............... $\frac{1}{2}$ table-spoonful.

Treacle............... 1 pound.

In warm water.

The animal was to be packed in three heavy horse-rugs, well saturated with cold water, and three other rugs were wrapped over them. In a letter to Sir Fitzroy Kelly, Dr. Druitr enters somewhat fully into the kind of remedies which may be found serviceable in the treatment of the plague, and his observations are condensed in the subjoined note:

"In any zymotic disease, the first question is, Can we stop it? Can we put out the fire? Have we any medicines capable of summarily checking the disease before it comes to the point at which it is naturally spent? We wish we had, but have not. Providence will show us the way some day, if we exert ourselves; but at present the only fevers which we can cut short are the malarious ones-ague, remittent, jungle fevers, ete.-against which we have quinine, arsenic, and other tonics. We have not at present any remedies on which we can rely for cutting short any true zymotic disease, such as small-pox, chicken-pox, measles, searlet fever, typhoid, typhus, whooping-cough, and all that other dreary catalogue; but if we are to make experiments on the rinderpest, there are two or three glimmers of light to guide us. Supposing that we seek for remedies for the early stage. To cut the disease short, deliberate trials should be made-first, into the various alkaloids, amorphous or crystallized, produced from chinchona bark, of which quinine is the chief. But there are many others, such as chinchonine, chinchonidine, and quinidine, which are got 
from the bark, which may now, or will soon be, obtained abundantly in India, and which are cheaper. We have just as much reason, and no more, to expect these substances to cure the cattle plague as the Spanish pilgrims had, three centuries ago, to expect the bark to cure the Countess of Chinchon's ague. It was a fair experiment, which succeeded. Certainly, large doses of these alkaloids ought to be well tried. If we give six doses, of ten grains each, to a man weighing one hundred and sixty pounds, we ought to give in the same proportion per weight to a cow. Secondly, there are various combinations of bitters, astringents, and aromatics, with stimulants. When they could get no Peruvian bark on the continent, during the war with France, combinations of alum, oak bark, gentian, and aromatics were used, with more or less success, for their malarious fevers. Thirdly, there are certain saline matters, such as nitrate of potass and chlorate of potass, antimony, purgatives, etc., which have more or less virtue in allaying some feverish symptoms, but which are not to be relied on in any zymotic or malarious disease. Lastly, there are stimulants which may be tried in the commencenuent of the attack with the hope of enabling the animal to shake off the disease, even as a glass of hot brandy-and-water, Turkish bath, and other excitants, may, as I believe, enable man to shake off the earliest stage of influenza. So far, then, as remedies are concerned which tend to cut the disease short, experience bids us look for specifics among the quinine group. And it is experience only that can teach us. But, confessing that we are unable to deal with the disease in its essence, there is a very great deal we can do in the way of mitigating its local effects - of keeping up the strength, of preventing the patient being poisoned by the poisonous secretions generated within her own system; of checking exhausting discharges; and of rendering those secretions innoxious to other animals. It must, however, be borne in mind that while we speak of the different stages of the disease, and can always separate them in idea, they may be all so crowded together that it may be necessary to begin at the very outset with the treatment adapted for preventing local ravages and intestinal infection. Now, the chief local effects produced by the rinderpest are a softened, congested state of the mucous membrane of the alimentary canal, with copious fetid discharge from the bowels; and the most likely class of remedies are those which will restrain this discharge, and so pre- 
vent it from draining the patient, and which shall deprive it of its poisonous qualities, and thus hinder it from doing mischief to the patient, or, if voided, from contuminating other animals. If putrid excretions be poured out in the bowels of any animal, they poison the animal's blood, cause all fresh food to be tainted, and, when voided, they are the source of contagion to others. Now, then, we seek a remedy which shall not be poisonous in itself, which shall have tonic or nutritive virtues, which shall restrain excessive discharges, and which shall have the power of deodorizing and disinfecting the contents of the alimentary canal. Such a remedy is the tincture of sesquichloride of iron. The mineral acids, well diluted-as the nitric, muriatic, and sulphuric-do in a lesser degree what the tincture of steel does. Their influence in atonic diarrhea is marvelous; and we ought to know their results in the rinderpest. Small quantities of opium may be added. The trisnitrate of bismuth, in ounce doses, would deodorize the alimentary canal, and soothe it and restrain diarrhea. I have published cases showing how, in threatened ulceration of the bowels, the human alimentary canal may be quieted by adequate doses of this excellent drug. Sulphate of potass, and other compounds of sulphurous acid, have great virtue in checking fermentation, and have been proposed by Polli as remedies in zymotic fevers. We want experiments with large doses of these. But I suspect they are of more value as preventives than as curatives - to disinfect unwholesome water, to rinse the mouths of animals exposed to contagion, and the like. There is the class of creosotes-medicines, such as carbolic acid, which check fermentation, act as antiseptics and deodorizers, and are capital in certain cases of dyspepsia and diarrhea. Here, then, we have half-a-dozen remedies, and the example I have given from practice on man shows the precise method in which good results are to be looked for. I reiterate again the necessity of disinfecting the whole tract of bowels, of restraining discharge, and of rendering the fecal matter innoxious to the patient and her neighbors-all this, too, as a means of helping the patient to live through her disease till its fury be overpast. Whisky, sweet spirits of niter, beef-tea, and other nourishment, should be given freely. There are other remedies, too, as yeast, in large quantities, whose modus operandi is probably that of an antiseptic, and others. Enough if I can succeed in inducing any one, who can do so with 
safety, to make a resolute series of experiments on diseased animals." A veterinary chemist recommends :

No. 139. Chlorate of potash............... 1 oz.

Nitrate of potash.............. 1 oz.

Strong muriatic acid............ $1 \mathrm{dr}$.

Powdered opium............... $1 \mathrm{dr}$.

Decoction of linseed............ $1 \frac{1}{2}$ pints.

Mix together for one dose.

This, it is said, "can not fail to alleviate the trouble, if not entirely ward off the disease, if given on the first symptoms, and followed up three times a day."

The following prescription has also been recommended:

No. 140. Chlorate of potash..............

Common salt.................. Each 1 oz.

Dissolve them in a pint of hot water, in which an ounce of dilute hydrochloric acid has been mixed, and administer in a sufficient quantity of their gruel for a dose. Mr. Moffat's prescription consists of

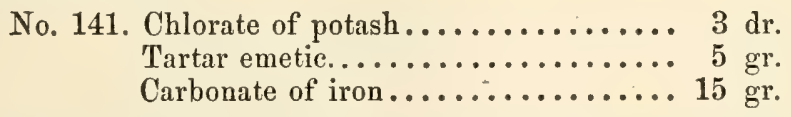

Mr. Crotch, M. A., recommended the injection of various antisceptic and parasiticide salts into the circulation of the veins, and the hyposulphite of soda, the permanganate of potash, or the perchlorate of soda are said to be well adapted to this purpose. Dr. CARR speaks of a dozen cows cured "by removing the diseased cattle from the shed and tethering them in a shaded spot in the field, administering warm drinks of gruel or linseed tea, with aromatic seeds (fenuel or caraway), an astringent, if diarrhea be urgent, and blistering the throat. The mouth is frequently washed with strong vinegar and water; and vinegar is also administered to the extent of a pint or more daily."

Dr. SicarT's method of treatment has been described by himself as follows:

"General Principles of Treatment.-These are based upon a knowledge of the pathology of the disease, and indicate the line of treatment to be adopted in dealing with it.

1. The Animal Temperature is Lowered and Deficient. - This has to be restored and maintained. To do so the affected animal is 
protected from all direct draughts of air, placed in a house or byre with an equable temperature not under $70^{\circ}$ Fahrenheit, and the hide thoroughly cleaned and rubbed down, and a warm covering kept on the animal throughout the progress of the case.

2. The Stomachs are Loaded and Distended with Food.-This condition, by preventing access of medicine and suitable nourishment, presents a very great obstacle to treatment. But it also indicates the line of treatment to be adopted at this stage; namely, to remove the hurtful accumulation as quickly as possible. This must be done by mild purgation, suited to the already irritable condition of the lining membrane of the stomach and bowels. The medicines here indicated are gentle relaxants, combined with diuretic action.

3. Extreme Vital Depression is characteristic of the Disease throughout its entire Progress.-This is conjoined with a very peculiar and rapidly-destructive change of some of the internal structures. Stimulants to support the depressed vital powers, and resist, as far as possible, this tendency to destructive dissolution, are thus clearly indicated from the very commencement. And as it is of importance to make the healthy organs subserve the purpose of removing from the blood the morbid materials that may exist in it and in the general system, stimulant treatment should conjoin with it remedies fitted to excite the functional activity of the two great eliminators of this class; namely, the skin and kidneys. Hence stimulant, diaphoretic, and diuretic action are here indicated. Regular milking of the diseased cow, in order to prevent the retention in the blood of the elements of the milk, is, also, on the same general principles, clearly indicated throughout the entire course of the disease.

4. It almost appears an axiom to say that a properly-regulated and rational system of nursing is in the treatment of disease in cattle, as in man, of very great importance to the comfort of the sick, and as an aid to their recovery. In the present example, no method of combating the malady can be of any use in which careful nursing does not form the basis of every other effort to restore health. It is not idle to repeat this, because, in any system of treatment hitherto made public, the importance of this fact has either been insufficiently recognized or entirely overlooked. Hence arises the necessity of there being kind, skillful, and experienced attendants, and a well-regulated dietary. 
Remedies.-These are few, simple, and selected on the principles above stated. My experience of their suitableness is every day more established by fresh examples of their efficacy. There are yet only three kinds of drugs which I found it requisite to employ. 1. Laxative, with diuretic action. This is principally used in the early, but often required at other periods, in the progress of the disease. It is composed of

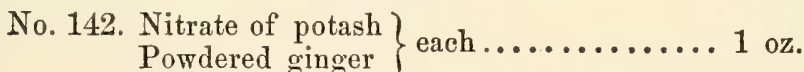

Powder of sublimed sulphur.......... 2 oz.

Treacle...................... $1 \mathrm{lb}$.

Water to make a quart, and well mixed.

This quantity is given night and morning, or, if requisite, oftener, until scouring is produced. Afterward, an oceasional bottle will maintain the free movement of the bowels, without inducing excessive action. As the vital powers sink rapidly, there should be as little delay as possible in administering stimulants. I have found the following mixture possessing stimulant, diuretic, and diaphoretic properties, very efficacious:

No. 143. Carbonate of ammonia ............

$\left.\begin{array}{l}\text { Sweet spirit of niter } \\ \text { Spirit of mindereris }\end{array}\right\}$ each $\ldots \ldots \ldots \ldots 1 \frac{1}{2}$ oz.

Cold water.................. 9 oz.

Mix.

This dose, from the commencement of treatment, is administered thrice a day during the entire course of the disease. When prostration is great, it is sometimes needful to give it from the commencement, and to combine it with any other medicine that may be given. In such cases, the doses may be reduced one-third. When convalescence is fully established, a simple tonic hastens recovery. I find none so good and safe as chinchona bark. The best quality only should be used, and given in doses of one ounce and a half of the powder. This tonic, in the early period of convalescence, is combined with the stimulant, and at a later period with a quart of good, sweet ale, given once daily. It is best administered at night. Two table-spoonfuls of laudanum may be added at any time to any other medicine which the animal is getting, or given in the food when it becomes requisite to control excessive diarrhea, or obviate straining. With this exception, there are at present no other drugs employed.

Diet.-It should be simple, and, until decided convalescence, 
well cooked, and given in small and regulated quantity. I use the following full mash. It is composed of,

No. 144. Bran................ 4 handfuls.

Brewer's draff ...........4 handfuls.

Peasemeal .............. 1 pound.

Mashed turnips (well boiled)... 2 pounds.

Not too thick, and given night and morning. At midday a drink of gruel is given, made with two pounds of oatmeal, well boiled in six quarts of water. In addition to these, some raw turnip (two pounds, for example, of greentops), and one pound of hay, may be allowed in small quantities during the twenty-four hours. To allay thirst, three to four quarts of water, previously boiled and allowed to cool, is given in mouthfuls during the day. This constitutes the full diet of a decided convalescent. Half of this diet is, in most instances, during the acute course of the disease, too much. In all cases, the same kind of food and periods of giving it are followed. There are some animals that, for a time, refuse all food, not excepting gruel. In such cases the gruel is administered by the bottle, thrice daily, along with or after the medicine. The animal should get a little mash so soon as it takes it voluntarily. It is often expedient to miss a meal, especially whenever symptoms of an unfavorable indication appear. These are not of unfrequent occurrence during the course of treatment. Grass is given, and the quantity of hay and turnip increased as there is progress toward more perfect recovery.

Symptoms of Convalescence.-The more obvious indications are, 1. Recovery of appetite; 2. Greater animation; 3. Return of breathing and pulse to their normal condition; 4 . Increase of milk; 5. Chewing the cud. The seventh, fourteenth, and twentyfirst days are critical periods in the progress of the disease.

Summary of Treatment.-1. The animal is at once taken from its ordinary food and separated from the rest. 2. Placed in a well-aired byre, or house, free from draughts, and the temperature of which is maintained at $70^{\circ}$ or $75^{\circ}$ Fahrenheit. 3 . It is to be well rubbed down, and thoroughly cleaned, and covered with a good rug. 4. If there be constipation, begin with laxative, and continue night and morning, or, if required, oftener, until there is free scouring. 5. Let there be no delay in giving the stimulant, and, if needful, combine it with the laxative. 6. Defer giving ale and bark until convalescence appears. 7. To obviate 
straining or excessive purging, two table-spoonfuls of laudanum, night and morning, may be added to other medicine. 8. Be careful to avoid overfeeding, as an error in diet may prove fatal. 9. See that the cow is well milked, night and morning, (even when there is no yield,) during the course of the disease. 10. All the droppings should be at once disinfected by solution of chloride of lime, and quickly removed. 11. The affected animals should be frequently and closely observed, and threatening indications treated as they occur."

The Edinburgh Committee on the Cattle Plague, having been authorized by the Royal Cattle Plague Commission to make observations and experiments in reference to the prevention and treatment of the disease, considered it desirable, in addition to the experiments on treatment which they proposed to institute themselves, to obtain a record of observations and experiments made by as large a number as possible of qualified veterinary practitioners throughout the country. With this view, they drew up the following suggestions for methods of treatment of various kinds, prophylactic and curative, which they were anxious should be tested on an extensive scale:

"The Committee, before specifying the various methods of treatment in detail, would premise a few general remarks, which they consider to be applicable to all cases.

Finst, as to General Sanitary Measures, Disinfection, etc.-The Committee content themselves with referring for full information on these matters to the 'Supplement of the Report of the Royal Cattle Plague Commission,' which is in the hands of all veterinary inspectors.

Seconduy, as to Food.-The Committee deem it desirable to state it as their opinion that, as a general rule, at all stages of the disease, and whatever treatment is used, food should not be pressed on the affected animal. They believe that too much, even of the softest food, is hurtful, the powers of digestion being so greatly impaired by the disease. During the earlier stages, they believe that the safest articles of diet are oatmeal gruel, barleymeal gruel, with linseed tea, hay tea, or bran tea, and that little, if any, addition to these is needed. During convalescence it is also very necessary that the food should be both sparing and of easy digestion. The same diet as during the earlier stages may be continued, with the addition of mashes of well-boiled turnips or 
carrots, but in moderate quantity. When rumination commences to be reëstablished, a handful of sound hay, damped with salt and water, may then be given in addition.

Thirdix, as to the Maintenance of the Heat of the Animal.The tendency to chill of the surface is a marked feature of the disease, and it is very essential that the animal should be guarded against cold. The byre should be kept heated up to a temperature of sixty-five degrees. The animal should be thoroughly rubbed down from time to time, and be kept covered with an ample, clean rug, fastened on with a roller, or band of any kind.

Fourthly, as to the State of the Bowels.-In the early stages they are apt to be constipated. To remove this condition mild laxatives may be required, but strong purgatives of all kinds are both unnecessary and unsafe. The best laxatives are either raw linseed oil, in the dose of a chopin-bottleful (an English quart), or from two to three ounces of flowers of sulphur, mixed up with two pounds by weight of treacle and two chopin-bottlefuls of water. These doses may be repeated cautiously, according to circumstances. Sometimes even in the early stages, but more frequently when the disease has continued for some days, diarrhea or scouring is apt to come on, and to prove irritating and exhausting to the animal. The simplest and best treatment for this symptom is one ounce of laudanum, mixed with a chopin-bottleful of lime-water, repeated twice or even thrice a day if necessary.

Having thus premised these general recommendations, the Committee proceed to state, in detail, particular methods of treatment, classified under the heads of
A. Diaphoretic and Stimulant Treatment.
B. Acid Treatment.
C. Restorative Treaturent without Drugs.
D. Prophylactic Treatment.

A. Diaphoretic and Stimulant.-The Committee are anxious to give a full trial to the method of exciting sweating by means of the vapor bath. The method of using this agent is as follows: The animal is to be placed in a stall inclosed on all sides, the height of the inclosure being a few inches more than that of the animal. Over the top of the box or inclosure thus formed is thrown a tarpaulin, which should cover it completely, with the exception 
that an opening is left in it sufficient for the animal's head to pass through. There is then to be placed on the floor of the inclosure, under the animal, a tub containing boiling water, to the depth of half a foot. A continuous evolution of steam is to be maintained for half an hour by means of red-hot bricks thrown into the tub one after another. Under the use of this steam bath, if properly managed, the animal may be expected to become warm and to perspire profusely. After each vapor bath the animal should be washed with tepid water containing McDougall's disinfecting soap, taking care to dry it well after the washing. It should then be covered with an ample rug, kept, as already stated, closely applied to its body by means of a roller, or band of any kind. The bath may be repeated either on the same day or following days, according to circumstances. During and after the bath the animal should be allowed a draught of cold water, which helps to promote perspiration. The objects chiefly aimed at by the use of the vapor bath are to promote the circulation at the surface, to relieve the congestion of the mucous membranes, and to eliminate the poison from the system. Combined with the vapor bath may be used various other remedies not incompatible with it, but calculated to aid its action.

Several of these remedies the Committee now proceed to mention, it being, however, understood that only one of them is to be used along with the bath in each case where the experiment is made; they are not to be used together in the same case. 1. Oil of turpentine. This may be administered in doses of four tablespoonfuls, well shaken up with a chopin-bottleful of gruel, and may be given twice a day. This remedy may be expected to act beneficially by its powers of stimulating and of exciting perspiration. It may probably, also, in most cases where it is used, supersede the necessity for giving any laxative medicine. 2. Infusion of coffee. The method of preparing this remedy is by infusing two ounces of ground roasted coffee, for a quarter of an hour, in a chopin-bottleful of boiling water. It must, of course, be allowed to cool somewhat before being administered, and may be given in the above quantity every six hours. In addition to its stimulant and nutritive qualities, the coffee may act beneficially in consequence of the empyreumatic oil and caseine which it contains. 3. Carbonate of ammonia. This medicine, which has been found in many cases to act beneficially as a powerful diffusible stimulant, 
may be administered three times a day, in doses of half an ounce, either alone or preferably combined with three drachms of niter, dissolved in a chopin-bottleful of gruel.

B. Acid Treatment.-This treatment is suggested in consequence of the alkaline state of the secretions which is found to exist uniformly in the cattle plague. 1. Diluted muriatic acid is said to have been successful in Holland. It may be given twice a day, in doses of three drachms, mixed with a chopin-bottleful of gruel. 2. Vinegar. This may be used in doses of two ounces, mixed with a chopin-bottleful of gruel, and may be given four times a day.

C. Restorative Treatment without Drugs.-This consists in carrying out in full the sanitary instructions of the Royal Cattle Plague Commission: in regulating the diet according to the instructions already given, in keeping the animal warm, and in administering two chopin-bottlefuls of good Scotch sweet ale three or four times a day. It is desirable that this system should be carried out in a certain proportion of cases, all drugs being rigidly abstained from.

D. Prophylactic Treatment.-The Committee would further desire to draw attention to the importance of experiments being made as to the efficacy of prophylactic (protective) treatment, either in preventing the development of the disease or modifying the intensity of the symptoms when the disease becomes developed in animals which have been exposed to the infection. In such cases, of course, all the sanitary measures of the Cattle Plague Commission should be strictly carried out. There may also be given, at the earliest possible period, prophylactic drugs, of which those most deserving of trial seem to be, 1 . Sulphite of soda, given morning and evening, in doses of one ounce, dissolved in a bucketful of water. 2. McDougall's solution, of which a wine-glassful in a bucketful of water may be given twice a day. 3. A mixture of half an ounce of sulphite of soda and two table-spoonfuls of MeDougall's solution, in a bucketful of water, may be given twice a day.

Prevention of the Cattle Plague.-There is but one rational and sufficient system whereby the Russian plague can be kept in check. That is by preventing direct or indirect contact between animals affected with the disease and those that are sound. All else is valueless. This is clearly established if we consider how and why we are losing the horned stock of Great Britain. 
We should have prohibited direct importation from Russia.

We should have established foreign stock-markets and slaughterhouses.

We should not have trusted to the insufficient system of inspection at our ports and markets.

The disease should have been recognized earlier.

We should have killed the diseased and infected animals with the greatest determination from the very first.

The Government should have acted promptly, without trusting to the tardy development of public opinion and the hesitating action of country magistrates, mayors, and others intrusted with authority.

Greater reliance should have been placed in veterinary surgeons, who, knowing the disease and the desperate measures it required, could have saved the lives of thousands of valuable animals.

I did my best on the 29th and 31st of July, 1865, to rouse the Government to a true sense of our position. I then advocated the formation of a national association for the prevention of cattle diseases, of a national insurance fund, and created some astonishment when I said, on the 1st of August, that "the present calamity will not fall on a class, as in the case of the cotton famine. It must affect the nation throughout its length and breadth. It is too late now to avert such a result, and, with a view to enable us to cope with very extraordinary difficulties, there must be a general and extraordinary coöperation among the people." I afterward advocated the continental system, and said, "I quite agree with the Austrian veterinarians, that to cure is, in this case, to kill, because so long as sick and convalescent animals are kept about, the danger of propagating the malady is enormous. Kill by all means; and if you can compensate, kill all that have been near a contaminated animal." 


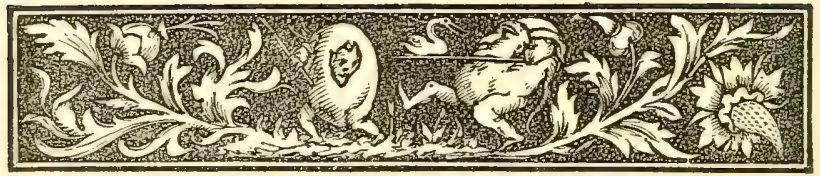

\section{SECTION XXX.}

\section{DISEASES OF SHEEP.}

Improvement in Sheep-Rot, or Hydatids in the Liver-Yeliows-Vertigo, OR Giddiness-Jackets or Blankets for SheEp-Foot-rot-Grubs IN THE Nostrils-Ticks-Inflammation of the Exe-Inflamiation of the LungsCommon Catarri-Diarrhea and Dysentert-Constipation of the Bowels-Tympanites, or Windy Distension of the Intestines and Abdonen.

\section{Improvement in Sheep.}

TO country in the world is better calculated for raising sheep 1 than the United States. The diversity of climate, together with the abundance and variety of the products of the soil, united with the industry and perseverance of agriculturists, render this country highly favorabie for the breeding, maturing, and improving the different varieties of sheep. The American people, as a nation, are stronger intellectually than any other on the face of God's earth; consequently they are all-powerful, " for the mind is mightier than the sword." Should this intelligent nation of husbandmen direct their whole attention to the improvement of sheep, then in a few years America shall outshine her more favored European rivals, and her husbandmen shall feel proud of their improved stock. What the American people have accomplished during the past half century, in the arts and sciences, cultivation of the soil, etc., is an earnest of what they can do in improving the constitution and condition of live stock, provided they take hold of the subject in good earnest. Let any one who is acquainted with the subject of degeneration, its causes and fatal results, not only in reference to the stock itself, but as regards the pocket of the breeder, carefully investigate the subject, and it will be perceived that there still exists a fine field for improvement. Nuch has already been accomplished in improving the different breeds in America, and 
our farmers deserve great credit for their praiseworthy efforts in endeavoring to raise fine stock, and I presume their labors have proved remunerative.

In order to show what a whole community of stock-raisers can accomplish, in view of improvement, when they have an eye single to the object, I here introduce some of the results attending such an enterprise, conducted by a single individual-Mr. BAKEWELL.

"His breeding animals were, in the first place, selected from different breeds. These he crossed with the best to be had. After the cross had been carried to the desired point, he confined his selections to his own herds or flocks. He formed in his mind a standard of perfection for each kind of animals, and to this he constantly endeavored to bring them. That he was eminently successful in the attainment of his object can not be denied. $\mathrm{He}$ began his farming operations about 1750 . In 1760 his rams did not sell for more than two or three guineas per head. From this time he gradually advanced in terms, and in 1760 he let some for twenty-five guineas a head for the season. Marshall states that, in 1786, Bakewell let two-thirds of a ram (reserving a third for himself) to two breeders for a hundred guineas each, the entire services of the ram being rated at three hundred guineas for the season. It is also stated that he made that year, by letting rams, more than one thousand pounds. In 1789 he made twelve hundred guineas by three 'ram brothers,' and two thousand guineas from seven, and, from his whole letting, full three thousand guineas. Six or seven other breeders made from five hundred guineas to a thousand each by the same operation. The whole amount of ramletting of Bakewell's breed is said to have been not less that year than ten thousand pounds (forty-eight thousand dollars).

It is true that still more extraordinary prices were obtained for the use of rams of this breed after Mr. Bakewell's death. Pitt, in his 'Survey of Leicestershire,' mentions that, in 1795, Mr. Astley gave three hundred guineas for the use of a ram of this breed, engaging, at the same time, that he should serve gratis twenty ewes owned by the man of whom the ram was hired, making for the entire use of the ram that season four hundred and twenty guineas. In $1796 \mathrm{Mr}$. Astley gave for the use of the same ram three hundred guineas, and took forty ewes to be served gratis. At the price charged for the service of the ram to each ewe, the whole value for the season was five hundred guineas. He served one hundred ewes. 
In 1797 the same ram was let to another person at three hundred guineas, and twenty ewes sent with him, the serving of which was reckoned at a hundred guineas, and the ram was restricted to sixty more, which brought his value for the season to four hundred guineas. Thus the ram made, in three seasons, the enormous sum of thirteen hundred guineas.

We have nothing to do, at present, with the question whether the value of these animals was not exaggerated. The actual superiority of the breed over the stock of the country must have been obvious, and this point we wish kept in mind. This breed of sheep is continued to the present day, and it has been remarked, by a respected writer, that they will ' remain a lasting monument of Bakewell's skill.' As to their origin, the testimony shows them to have been of mixed breed, though no breed is more distinct in its characters, or transmits its qualities with more certainty; and if we were without any other example of successful crossing, the advocates of the system might still point triumphantly to the Leicester or Bakewell sheep.

But what are the opinions of our best modern breeders in regard to the practicability of producing distinct breeds by crossing? Robert Smith, of Burley, Rutlandshire, an eminent sheep-breeder, in an essay on the 'Breeding and Management of Sheep,' for which he received a prize from the Royal Agricultural Society, (1847,) makes the following remarks: "The crossing of pure breeds has been a subject of great interest among every class of breeders. While all agree that the first cross may be attended with good results, there exists a diversity of opinion upon the future movements, or putting the crosses together. Having tried experiments (and I am now pursuing them for confirmation) in every way possible, I do not hesitate to express my opinion that, by proper and judicious crossing through many generations, a most valuable breed of sheep may be raised and established; in support of which I may mention the career of the celebrated Bakewell, who raised a new variety from other long-wooled breeds, by dint of perseverance and propagation, and which have subsequently corrected all other long-wooled breeds.'" 


\section{Rot, or Hydatids in the Liver.}

This parasite, which affects the liver of sheep, is known as the fluke, (fasciola hepaticum, and is usually found in the biliary ducts. Rot, in its advanced stage, is a disease of a very formidable character, and its associations closely resemble dropsy. A serous fluid accumulates beneath the skin, hence some people call it the water rot.

Causes.-Wet pastures, and exposure to storms and changes of weather, with innutritious diet, are the exciting causes of this malady.

Treatment.-If flukes are present, it is evident that, in order to strike at the root of the malady, we must get rid of them, which can only be effected by bringing about a healthy condition of the system. Nothing that can be done by the application of medicine will act on them to affect their vitality. It is only by strengthening the animal powers that we are enabled to give sufficient tone to the system to throw off the flukes. For that purpose many advocate salt. Salt is an excellent stimulative to the digestive organs, and may also be of service in restoring the biliary secretion, from the soda which it contains. So well is its stimulative action known, that some individuals always keep salt in the troughs containing animals' food. That this is a preventive they have good proof, seeing that it matters not how much the soil might be in salt marshes, no sheep are ever attacked by rot in them, while those sent there infected very often come back free. Salt, therefore, must not be neglected. But then comes the question, can they not do something more? I believe they can. They must throw tonics into the system, especially those that are obtained in the mineral kingdom. I should prefer the sulphate of iron (iron is found in animal matter). It is one of the constituents of the blood, and, used in the form of sulphate, it gives a greater tone and energy to the frame than in any other form. Its use, therefore, ought never to be neglected in the earlier stages of the disease. I have already alluded to the fact that, when the liver does not perform its functions, a greater effort is made by the kidneys to depurate the blood. The kidneys should, therefore, be stimulated. But I must not be supposed to advise the exhibition of diuretics, which would induce debility, but of medicines, which would give strength to the frame, and, at the same time, act on 
the kidneys, for which purpose nitric ether is an agent which ought to be employed. The principles I wish to lay down are, to husband the animals' powers by placing them in a situation where they shall not be exposed to the debilitating effects of cold storms; to supply them with nutritious food, and such as contains but a small quantity of water; and to mix salt with the water; and likewise to administer sulphate of iron, and occasional doses of nitric ether.*

\section{YELLOWS.}

This term is used by shepherds to designate disease of the liver. Symptoms. - The affected animal has a peculiar languid appearance, frequently shaking the head, as if parasites were in the nostrils; the head is pressed against any elevated ground, sometimes against the fence or a post; the animal moves lazily along, with its nose almost touching the ground; the membranes of the eyes and nose have a yellow tinge; the same is true of the skin; a marked yellowness is observed on the insides of the thighs and anus. As the disease advances these morbid appearances are augmented. The patient grates its teeth; stands with the head pendulous, almost touching the ground; then soon falls, and dies without - a struggle.

The autopsy reveals the following: The liver has a mottled appearance, and its structure is easily broken down; the gall-bladder is usually full of dark-colored, tarry-looking bile; kidneys, stomach, and intestines, tolerably healthy. Should any urine be found in the bladder, it will be of a dark color. The heart is pale and flabby; the lungs, healthy, yet the chest usually contains a quart or more of dark-colored serum. On removing the skin, the surface of the body is yellow (jaundiced).

Treatment.-Remove the diseased animals from the flock and put them in a dry shed. Then prepare the following:

No. 137. Fluid extract of leptandra (Culver's root).................... 1 pound.

Powdered hyposulphite of soda......6 oz.

Water....................... 1 quart.

Dissolve the soda in the water, then add the leptandrin. Dose, one ounce (fluid) twice daily. Let the affected animals have salt; grass should also be cut and placed before them.

* Professor Simonds. 


\section{Vertigo, or Giddiness.}

Vertigo, or giddiness, is a disease of the nervous system, oceasioned, most frequently, by a parasite located in the brain. The parasite is named cænarus cerebralis, and belongs to the species known as hydatids. Lambs from the age of two months, or from four to twelve months, are, when predisposed, apt to become the subjects of it. The disease is very apt to end in organic disease of the brain and spinal marrow.

The disease is of hereditary origin, and comes from faults or defects in one or both parents, and also from the too early practice of breeding, which often obtains in some sections of country. In order to guard against the disease, we must put out of the breeding-fold both males and females that have shown any signs of the disorder, and not breed from the ewes under the age of thirty months, nor from rams until they have attained their second year.

Treatment.-In the treatment of the disease, our object is to give tone to the system, and saturate the blood with some agent (sulphur) which is known to be obnoxious to parasites in general. Take of

No. 138. Sulphur................ 1 pound.

Powdered sulphate of iron......6 $6 \mathrm{oz}$.

Powdered poplar bark......... 1 pound.

Let this be thoroughly mixed, place it in a stoppered bottle, and keep it in a dark place. Mix a table-spoonful daily in bran, and place it in the feed-trough, or mix it in a table-spoonful of syrup, and administer it by means of an iron spoon.

Some persons contend that turnips, when fed to sheep, are apt to produce hydatids. On this subject, and on that of protecting sheep from the inclemencies of the weather, the following article is offered :

\section{JACKeTs, or Blankets for Sheep.}

A writer in a late number of the "London Agricultural Gazette" says: "We find, on examining our mortality tables for the last twelve months, that out of six hundred Cheviot and black-faced Evehogs, the number of deaths has been but sixteen. Be it remembered, also, that, with the exception of about a score, none of these ever tasted a turnip, but fared with the ewes on the hill. Since we commenced the use of jackets, (small blankets, we have 
especially noticed an extraordinary diminution of the cases of 'sturdy,' or water in the head. Hydatids in the brain are generally understood to be induced by long-continued heavy rains, cold winds, and general privation. Any one conversant with sheep must have observed the wool along the back parts in such a way as fully to expose the skin. The connection between the spine and the brain is obvious, and it can not be wondered that hydatids (little sacs filled with water) should be formed in the brains of sheep much exposed to severe storms, without due shelter. Hence the advantage of covering their backs with some material which will protect them, in a great measure, from the chilling effects of wind and rain. The material used is woollen, the size being twenty-three inches by fifteen. We lately purchased some coarse blankets that made excellent covers, each jacket costing fourpence. 'The rams were put with the ewes on the $22 \mathrm{~d}$ of November, and we allow forty-five to each male."

The above remarks, from a flockmaster of large experience, in reference to the cause of hydatids, or what we should call water in the brains of sheep, are interesting in a medical and physiological point of view. We know one breeder in Vermont who covers the back of each sheep with a half yard of common sheeting; painted, to shed rain. The practice is founded in reason, and is likely to extend, literally making cotton tributary to the production of wool. The growers of the former staple will not object if every sheep in the United States and Europe has a cotten "jacket;" for one that will answer every intention can be made cheaper of cotton than of wool. The comfort of domestic animals at the South is sadly and most expensively neglected.

\section{Fоот-Rот.}

Causes.-General debility, exposure in wet pastures, contagion, foul habit of body.

Symptoms.-The animal is observed to limp in one or both of the fore or hind legs. Sometimes the whole four are affected. The parts are hot, tender, and swollen, and exude a fetid fluid. The animal is now incapable of walking, and, if not speedily relieved, death ensues. This form of rot is contagious, so that, if the diseased animals are not separated from the healthy, the latter snon become infected. To propagate malignant rot, it is quite 
sufficient that a flock should pass over a place which has a little before been walked over by a diseased sheep.

Treatment.-Endeavor to ascertain the exciting cause, and, if possible, remove it. If the disease has assumed a putrid type, the superfluous horn may be removed. The parts are then to be washed with

No. 139. Pyroligneous acid.............. 4 oz.

Water.................... 3 oz.

Mix.

A piece of lint is afterward to be saturated with the above, and applied as a dressing, and changed as occasion may require.

The local remedy will avail but little unless we sustain the living powers, and thus improve the secretions. Our usual remedies are,

No. 140. Powdered golden seal........... 1 oz.

Powdered sulphur............. $\frac{1}{2}$ oz.

Powdered charcoal............... 1 oz.

Powdered sassafras.............. $1 \mathrm{oz}$

Powdered assafetida............. $2 \mathrm{dr}$.

Flaxseed ................... 2 lbs.

Mix, and give a table-spoonful twice a day in the food.

If I were writing exclusively for my medical brethren, I should recommend them to treat the disease according to its indications. The above agents will, however, generally prove efficacious, (even in the hands of the non-medical,) provided due attention be paid to the wants of the animal, and such additional assistance be rendered as common sense will suggest. Supposing a number of animals to be affected, it would occupy too much time to treat them singly; hence I recommend that they be made to walk slowly, or linger for some time in a wooden trough, the floor of which may be covered, to the depth of one inch, with the following:

No. 141. Linseed oil.................. 2 pt.

Pyroligneous acid............... 4 pt

Kerosene ................... 1 pt

In the above proportions, the required quantity may be prepared.

\section{Grubs in the Nostrils:}

Grubs in the nostrils are occasioned by the gadfly, which deposits her ova in the nostrils of sheep. After a short time the ova bring forth parasites in the larvæal state; the latter migrate 
within the interior of the nostrils, causing the sheep much pain and annoyance. When the larvæ are capable of exercising an independent existence, they undergo the same evolution which obtains in the case of the bot parasite; namely, they burrow into the earth, and finally become metamorphosed into the gadfly.

It seems that the gadfly selects its subjects, and the weakest and most unpromising of the flock are usually its victims; hence close attention to the requirements and condition of a flock may, to a certain extent, act as a preventive. Some farmers, in view of preventing the attacks of the gadfly, smear the noses of their sheep with common tar. Others plow up a piece of land where sheep are pastured, into which they thrust their noses, and then, for the time being, they baffle the gadfly. I do not think it would be good policy to attempt, either by mechanical or medicinal means, to dislodge the parasites; for the remedy might be worse than the disease. When the ova have arrived at maturity, the sheep themselves aid in the dislodgment with acts of snorting, sneezing, and coughing.

\section{Ticks.}

These troublesome parasites may easily be got rid of by dipping the sheep in an infusion of tobacco. A compound tobacco sheep-dip is now manufactured, and can be obtained at the drug stores, with full directions for use.

\section{INFLAMmation OF THE Eye.}

Inflammation of the eye is very readily detected by an acute redness which pervades the lining membranes of the eyelids and that which affords a partial covering to the eyeball. The afflicted animal keeps the eyelids partly closed; an effusion of tears runs over the lower angle of the eye; there is intolerance to light, and the animal appears to suffer considerable pain.

Treatment.-Separate the patient from the flock, and bathe the eye occasionally with the following lotion:

No. 142. Sugar of milk............... 1 oz.

Fluid extract of hops........... $1 \mathrm{dr}$.

Rain-water................... 6 oz.

Mix.

Should the disease run on to cataract, the sight can not be restored. 


\section{Inflammation OF THE LUNGS.}

Inflammation of the lungs is usually the result of exposure; or it may arise in consequence of herding too many sheep together; sometimes it makes its appearance without any perceptible cause. An impure atmosphere, however, may be set down as the ordinary exciting cause of this malady.

Treatment.-Place upon the tongue ten drops of fluid extract of gelseminum, morning and evening. Dissolve one ounce of chlorate of potass in half a pint of flaxseed tea, and give it daily as a drench until the animal improves. Let the patient be placed in a secluded spot, under cover, and if the case is curable, health will soon return.

The most marked symptoms of pneumonia are panting and heaving at the flanks, quickened respiration, discharge from the nose, and cough. There is also a cessation of rumination. In short, the same symptoms prevail in this disease as in pneumonia of cattle.

\section{Common Catarrh.}

This affection prevails most extensively among sheep that have been exposed to rains and unpleasant weather. The disease manifests itself in the form of a defluxion from the nostrils of a mucoserous discharge, accompanied by frequent sneezing, and occasional cough. As soon as the disease is discovered, the affected animals should be placed in comfortable quarters. Then prepare the following drench :

No. 143. Composition powder.............. 2 oz.

Pour the boiling water on the powder; let the mixture stand in a warm place for an hour; pour off the clear liquor, and add two ounces of sugar of milk. Dose: A wine-glassful once or twice daily.

Malignant epizoötic catarrh may be treated in the same manner, with the addition of one ounce of chlorate of potass per day, which can be dissolved in the above drench. 


\section{Diarrhea and Dysentery.}

Curable cases of the above character are brought to a favorable termination by using the following drench:

No. 144. Finely pulverized animal charcoal.... 1 oz.

Scalded cow's milk............. 1 gill.

Hyposulphite of soda............ 1 dr.

Mix.

The above constitutes a dose. It may be repeated as often as the emergency seems to require; but should the subject be a young lamb one-half the above quantity will suffice.

\section{Constipation of the Bowels.}

Constipation is almost always the result of a deranged condition of the digestive organs. A deranged condition of the liver, for example, will result in costiveness, for which I recommend the following drench:

No. 145. Glauber salts.............. 2 oz.

Fluid extract of leptandra....... 1 tea-spoonful.

Thin gruel................ $\frac{1}{2} \mathrm{pt}$.

Dissolve the salts in the gruel, and drench the animal with the same.

\section{Trmpanites, or Windy Distension of the Intestines AND ABdomen.}

This disease is very easily recognized by the bloated appearance of the animal. It is occasioned by the food running into fermentation and generating gas. The following remedy is a sure cure for tympanites, administered as a drench:

No. 146. Hyposulphite of soda ....... $4 \mathrm{dr}$.

Fluid extract of golden seal .... $1 \mathrm{dr}$.

Fluid extract of ginger........ $2 \mathrm{dr}$.

Water.................. 1 wine-glassful. 


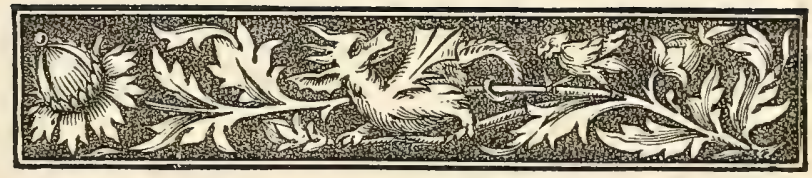

\section{SECTION XXXI.}

\section{DISEASES OF SWINE.}

Erfects of Impure Air on Swine-Measles in Pork-Quinst-Hernia, or Rupture-Intestinal Worms-Hog Cholera-Trichina Spinalis.

\section{The Treatment of the Diseases of Swine.}

THE nature of the most common diseases incidental to swine, 1 and the treatment of the same, are subjects of great importance to those engaged in raising this description of property, and it is very gratifying to know that farmers are anxious to obtain reliable information on such subjects. It is only of late years that any special attention has been given to swine pathology in this country, either by farmers or surgeons, and in former years the treatment of swine diseases was extremely barbarous, and consisted of little else than of splitting their ears, cutting off their tails, and dosing them with poisons. Such treatment must have been any thing but pleasant to the poor brutes, and very unsatisfactory in its results.

Swine, in certain periods in the history of the world, have been denominated "unclean creatures, of gross habits," etc. If they are unclean and of gross habits, their owners gave them their first lesson, and are more to blame than the animal. How can it be expected that they shall exhibit better nabits while confined in miserable quarters and filthy localities, where they are often compelled to wallow knee-deep in mud and excrement, dragging out a woful existence in the midst of a perfect hot-bed of filth, often respiring an atmosphere concocted from the decomposition of rotten excrements within their pest-hole, or local habitation. And the food that such poor creatures are compelled to devour, what shall I say of that? It is too beastly to describe, yet the cravings 
of an appetite, not naturally morbid, induce the animal to convert his stomach into a living cess-pool. Some persons appear to have an idea that any system of management is good enough for hogs; hence how can we expect that animals thus neglected and misused shall improve, either in habits or condition? Hence, if domesticated swine have lost their natural instincts, and acquired gross and filthy habits, the blame should fall where it belongs. Husbandmen know, from experience, that if young pigs are properly provided for and kept in comfortable quarters, and not too numerously herded, they will refuse to partake of the horrid garbage which their city cousins, occupying a down-cellar locality, are, from sheer necessity, compelled to devour, and they will exercise the same sagacity in the selection of nice morsels as tha wild originals have been known to manifest.

The ancients were led to believe that "the use of swine's flesh was an abomination, and its touch pollution." This was, and is, a mere theory, unsustained by facts-the result of prejudice; for the Egyptians, Jews, and Mohammedans, who considered the flesh of swine as the exciting cause of leprosy and tape-worms, were the greatest sufferers, yet they never touched pork. On perusing the records of the Jewish historians, we are informed that the strangers sojourning in the camp of the Egyptians, partook freely of pork, yet no cases of scrofula nor leprosy appeared among them; yet the followers of Moses, who, from prejudice or antiquated religious custom, abstained from pork, were the greatest sufferers from the terrible diseases known as scrofula and leprosy. Pork is the principal article of food among the Romans, Greeks, Chinese, natives of the Archipelago Islands, and the negroes inhabiting the burning regions of Asia; also the more refined and straight-haired inhabitants of Northern Asia partake freely of pork.

The people of England consume an immense amount of pork, as do also the people of this country. Our soldiers in and out of camp, and when on the march, devour pork, both cooked and uncooked, with impunity; hence, if pork is really the cause of leprosy, scrofula, or tape-worm, we might expect to see many lepers in this country, and an equal number of unfortunate individuals infested with tape-worms; but we all know that the converse is the case, and many American physicians inform me that leprosy and tapéworm are very rare afflictions in this country; so that the uncom- 
plaining and much-abused creature that $\mathrm{I}$ am writing about, has been most awfully slandered and ill-used.

I contend that the flesh of swine, when the animal has been judiciously fed, is nutritive, wholesome, and palatable, and in its excellency is considered a great delicacy. Like other substantial food, however, it does not agree with some persons; but the fault is not in the pork, but in the stomach of the invalid, who should not taste that which disagrees with him. Still, it would appear to be in perfect harmony with retributive justice, if the diseases alluded to were the result of man's criminal neglect of a class of animals that should claim his care and protection.

I have alluded to the condition of swine, such as are kept in cities. What shall I say of those which are kept and fed at the slaughter-houses in Chicago and at Cincinnati? We are all conversant with the facts in the case; they are too notorious to need repetition. I only urge those who have the power and will to inaugurate a reform where reforms are most needed. Credit is due, however, to our farmers, who pay much attention to the raising and welfare of swine. The errors I complain of are not so prevalent in the country as in our large cities. I contend that the hog does not thrive so well in the city as in the country. He was never cut out for a citizen; he never chews tobacco, nor does he "smile." He is one of Nature's pets, and thrives best on roots and fruit, in the universal sty. The city air does not vitalize his blood-does not agree with him. Down-cellar locations, and narrow, filthy pens, are abominations. They tend to toughen muscle and to tubercularize, often sending thousands of otherwise valuable animals into a galloping consumption, from which the knife of the butcher rescues them.

\section{EFfects of IMPURe AIR ON SWINe.}

A great proportion of the diseases which afflict swine are, no doubt, caused by bad management, and the crowding of them together on a limited space, where they must necessarily breathe a highly-contaminated atmosphere. It is one of the chief causes of pulmonary apoplexy, and other pneumonic affections which are so notoriously prevalent. I have often seen a lot of swine disembarked from the cars the subjects of congestion of the lungs, the external surface of the bodies of some being as blue as indigo, (quite 
livid,) showing very conclusively that their blood was highly charged with carbon; that either for want of pure air, or from temporary incapacity of the lungs, in consequence of crowding too many animals together, the lungs failed to oxygenize the blood, and thus they suffered. Swine require pure fresh air as much as we do for the vitalization of their blood, and can no more live without it than did the unfortunate individuals that perished in the Black Hole of Calcutta. Under such circumstances, whether the animals be confined in close cars or in filthy pens, it makes but little difference-they are liable to become diseased; and it often happens that when certain forms of disease are engendered spontaneously in this way, other animals exposed to the infection are attacked, and thus we have an epizoötic, which is sure to commit sad havoc ere it dies out or can be arrested. It is probable that bad air, inferior diet, and crowding animals into filthy locations have more to do with the production of the so-called "hog cholera" and the well-known pleuro-pneumonia than some persons are apt to realize. If this be true, and I think it is, then our husbandmen must see the necessity of paying great attention to the hygienic management of swine. The latter are generally the most neglected and despised of all our domestic animals, except when they are of aristocratic breed, and owned by amateurs of means, or when they are designed for "home-fed pork." Now, if it pays the amateur and lover of "home-fed" to give the animal that attention and care which he not only requires, but is entitled to, then a similar course of practice must increase the gains of those who raise their animals for the market.

Pure air, good food, plenty of space, and comfortable quarters are the best preventives of disease that I know of. Much better are they than all the drugs in the country; and when the animal becomes sick, without proper attention to hygiene, medicine is not worth a straw. It is well-known to the scientific farmer that when swine are crammed on a limited portion of land whence they derive their food, without regard to the laws which govern the distribution of living beings throughout creation, disease and death run riot, and the bodies of such animals are almost sure to be infested with various kinds of parasites.

The same facts have been observed in regard to sheep, when they are crowded and the land overstocked. It is the forerunner of parasitic disease. An English writer, having considerable ex- 
perience in the matter, contends that it is the crowding of sheep on the low lands which has engendered "rot" and "scab," and which causes thousands to die, with the respiratory organs filled with strongyli, or the intestines with tape-worms. The Australian farmers can testify to the ravages of the tape-worm in sheep as the result of crowding land to an unnatural extent. On our vast prairies, however, there may be no danger of overcrowding, yet it is right that we should be conversant with the danger, in order to avoid it in the future.

Majendie, the celebrated French physician, contends that very many grave and fatal diseases are introduced into the system through the respiratory organs: "And although we with difficulty arrive at any certain knowledge of the miasms rising in marshy grounds from the decomposition of putrid vegetable and animal substances, there can be no doubt whatever of their entering into the blood through the medium of the air, and so producing many fatal diseases. In some countries, epidemic affections especially rage with extreme intensity. In hot, humid climates, and especially on the sea-coast and borders of certain rivers, as, for instance, the Gulf of Mexico, Vera Cruz, New Orleans, etc. Such causes admit of demonstration, since, by introduction of them into the blood experimentally, may be produced, though not exactly yellow fever, symptoms bearing the greatest resemblance to it, with black vomit and speedy death. Lower Egypt, where the plague formerly prevailed so alarmingly, owing to such causes, has been rendered comparatively rare by the improved condition of the country."

\section{Measles In Pork.}

Measles in the hog is identical with tape-worm in man. It has been discovered that the hydatids of the hog, known as measles, (named in the zoölogical system cysticercus cellulosa,) have exactly the same head as the common tape-worm of man (tænia solium). Many experiments have, from time to time, been made, setting the matter beyond a doubt. Kuckenmeister, who wished to make sure of the fact, made the experiment upon a criminal who was soon to be executed, and, as was to be expected, with perfect success. Measles taken from fresh pork, and put into sausages which the criminal ate raw, at certain intervals, before his death, were found again, in the post mortem examination, as tape-worms in 
his intestines, and in different stages of development, according to the intervals in which the measles had been taken. Thus it was discovered that all measles in swine are tape-worm larvæ, which, when swallowed with a portion of the animal in which they live by another animal, develop the tape-worm. In zoölogy tapeworms are named cestoidea. About two hundred different species are described by naturalists, which vary much in size, the smallest being barely visible to the naked eye, while the largest will sometimes measure one hundred feet in length.

Different species of animals are infested with different species of tape-worms. They are found in the intestines of the horse, ass, sheep, goat, dog, deer, antelope, rat, mouse, and many other animals and insects. All tape-worms are hermaphrodites; the sexes are not only united in the whole of the tape-worm, but each joint of the compound parasite for itself has its own independent male and female sexual organs, testicles, and ovaries.

Treatment.-Put into the food of each hog, daily, two drachms of powdered sulphur, half an ounce of powdered poplar bark, and half a table-spoonful of fine salt. The best method of preventing measles in swine is to keep their lodging-places perfectly elean and dry, and to allow them pure air and exercise. Their food should be of a very nutritious quality. Swine may be suspected to have measles when they do not fatten nor thrive well.

\section{Quinsy.}

This is a very common disease among swine, and affects the lining membrane of the respiratory passages, which become inflamed and tumefied. This condition occasions great difficulty in swallowing either fluids or solids, and the respiration is much embarrassed; the neck appears to be stiff, and the nose is protruded. A distressing cough is usually present; the animal froths at the mouth as if he were choking; the glands in the region of the throat are enlarged, and this adds to the difficulty in breathing.

Treatment.-A mild case of quinsy, unaccompanied with tumefaction of the throat or the thyroid glands, may be cured by transferring the animal to a warm pen where he can burrow in clean straw. Let him have a mess of thin gruel three times daily, into which stir half an ounce of powdered chlorate of potass. It is not safe to drench an animal the subject of quinsy, for he may 
choke to death; therefore, if possible, the medicine must be incorporated with the food. In bad cases the throat must be lubricated with cod-liver oil occasionally. Should the bowels be constipated, a few ounces of Epsom salts or a couple of ounces of castor-oil are indicated; whereas a looseness or temporary diarrhea must be held in check by means of pulverized anthracite coal, or else by means of animal charcoal. These ingredients should be mixed in scalded milk, and placed in a clean trough. It is very evident, however, that, in the absence of pure air, comfortable quarters, and good nursing, medicine will have but little if any effect.

Quinsy mostly owes its origin to exposure, fatigue in traveling long distances, and to bad accommodations; therefore proper attention to the wants and comfort of these animals will act as the best of preventives.

The wretched condition in which some of these animals are compelled to exist, and the vile garbage which is often fed to them, is the exciting cause of most of their maladies ; therefore such matters should command the attention of husbandmen.

\section{Hernia, OR RUPTURE.}

Pigs are very frequently born in a ruptured condition. The disease is then called congenital, and scrotal when within the scrotum. The disease consists of adhesion of a protruded portion of intestine to the testicle after its descent into the scrotum. This adhesion often takes place while the testicle is yet in the abdomen. On the descent of the testicle into the scrotum the intestine descends with it.

Treatment.- Secure the limbs, or engage some persons to hold them; then place the animal on a bench, and cut carefully over the region of the enlargement until the testicle is exposed. Carefully dissect the intestine from the testicle; remove the testicle in the usual way; then return the intestine within the abdomen. Should the intestine be so large that it seems impossible to return it, then enlarge the opening by means of a probe-pointed knife; then raise the hind-quarters of the pig, and the bowel will return into the abdominal cavity, after which take a couple of stitches through the skin and superficial tissues, and the operation is finished. In a few days the stitches may be cut away; there is no need of any dressing. 


\section{INTESTINAL WoRMS.}

When swine are infested with intestinal parasites they generally become unthrifty-will not fatten, yet have a voracious appetite, and will devour greedily every thing that is placed before them.

Treatment.-For solitary cases give the animal an occasional dose of the following:

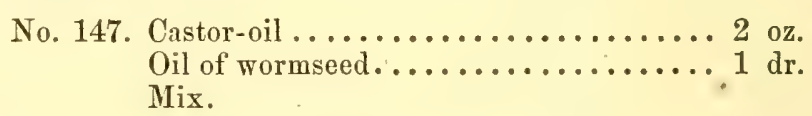

When a large number of animals are infested with intestinal parasites, prepare the following:

No. 148. Pulverized anthracite coal.......... 2 lbs.

Powdered poplar bark............. $1 \mathrm{lb}$.

Powdered sulphur............. $\frac{1}{2} \mathrm{lb}$.

Powdered salt................ 4 oz.

Mix.

Give a table-spoonful with the food twice daily, until the animal takes on a more thrifty condition.

\section{Inflammation of the Lungs (PNeumonia).}

Inflammation of the lungs, or what may very properly be termed apoplexy of the same, is a very fatal malady in swine, and runs its course to a fatal termination very rapidly.

Symptoms.-The early symptoms are accelerated and laborious respiration, shivering fits, cough, loss of appetite, and rapid wasting of fat and flesh.

Treatment.-Dissolve half an ounce of nitrate of potass in about a quart of thin gruel and set it before the patient; if he refuse to drink it, then a drench of the same must be given. In the mean time place on the tongue ten drops (three times daily) of fluid extract of gelseminum until the febrile symptoms have subsided; then give every morning half an ounce of hyposulphite of soda until the animal is convalescent. Bleeding and purging, as recommended in ancient works, is sure death to the animal. 


\section{Hog Cholera.}

The disease to which the name of "hog cholera" has been generally but improperly applied, has prevailed more extensively in this country than most persons are aware of. The first appearance of the disease, of which I have been able to find any notice, was in Indiana, in the summer of 1856 . It has continued, to a greater or less extent, since that time in our Western and Southern States, in nearly all of which it has, at some period, attained the character of a wide-spread and fatal epidemic. Its victims in those States are numbered by hundreds of thousands, if not by millions. During the last five years this disease has been seen, from time to time, in portions of the more eastern States, sometimes, as in Western New York in 1856, proving quite severe and fatal in comparatively limited localities; but in the Eastern States it has, to a great extent, originated with and has generally been confined to, hogs imported from the West. I think that in no State east of Ohio has the disease prevailed extensively, or attained the character of a wide-spread epidemic. In the vicinity of Providence, Rhode Island, it has prevailed to some extent, more particularly among large herds of swine, during each of the last five winters, but has been mostly confined to hogs brought from the West, and has usually disappeared with the approach of warm weather. During the last winter it was more severe than in any preceding, and was not confined to Western hogs. Neither did the disease, as heretofore, cease with the cold weather, but it continued until August, having destroyed more than five hundred hogs in Providence and in the adjoining towns during the first seven months of the year 1861. I have also heard of its prevalence in various towns in Massachusetts during the same period.

It is evident that a subject of so great importance to the agricultural interests of the community deserves attention. It is also interesting to all classes of the community, on account of the relations of this disease to epidemic diseases which afflict the human race, and on account of its effects upon the supply of animal food for cities. For these reasons $\mathrm{I}$ have, in connection with Dir. CotLINS and other physicians of this city, made some investigations upon the subject. We have visited and examined many sick hogs, and have examined the bodies of some of them after death. I have also made inquiries of those who have had the care of the 
hogs in relation to the symptoms, and have obtained information, so far as possible, from those who have seen the disease at the West, where it has prevailed so extensively. The results of these investigations I propose to give briefly as follows:

Symptoms.-The symptoms, as described by persons unaccustomed to such observations, are extremely various. By combining the information obtained from others with the results of my own observations, the symptoms, as seen during the life of the animal, are nearly as follows:

1. Refusal of food.-This is the first symptom usually noticed by those who have the care of the animals, though, as will be seen hercafter, this symptom by no means indicates the beginning of the disease. The refusal of food, after it is first noticed, generally continues through the whole sickness, and food of every description is mostly refused.

2. Great thirst.-This is constant, and large quantities of cold water will be swallowed if it can be obtained. Even after the animal is unable to stand alone it will drink cold water with eagorness.

3. After a time, the length of which varies very much, the animal begins to show signs of weakness; reels, staggers, and, in attempting to walk, often falls down.

4. In most cases there is a diarrhea, with copious fluid discharges of dark, bilious, and very offensive matters. In a few cases there is no diarrhea, but evacuations of hard, black balls; but in some of these cases the fluid offensive matter is found in the intestines after death.

5. In a few cases there is vomiting; but this is not often severe, nor is it continuous for any length of time.

6. The external appearance of the animal is at first paler than usual; but toward the last of the sickness purple spots appear, first on the nose and sides of the head. These extend along the sides and belly, and between the hind legs; after which the animal soon dies.

7. In many cases, perhaps a majority, ulcers are found on the different parts of the borly. These were particularly noticed on the inside of the lips and gums, and on the feet, and were often quite deep and excavated. In some cases these ulcers were seen in the nostrils, and in one case there were extensive ulcerations in the back part of the mouth, on the tonsils. 
8. In some cases the legs are swelled, and the animal is lame; sometimes the ears and sides of the head are swelled and red; sometimes the eyes are sore and inflamed; sometimes swellings, like carbuncles, are seen; and, generaliy, the glands near the surface seem to be enlarged.

9. In most cases the pulse is quickened, the breathing is hurried and difficult, and there is much cough; but in some genuine cases there is no perceptible trouble with the lungs, and no important signs of disease are found in them after death.

10. The duration of the disease in fatal cases, after the first symptoms are noticed, is extremely variable. We have seen some which have died within two or three hours; others have lived many days. It is difficult, however, to fix the time of the appearance of the first symptoms. The first noticed is usually the refusal of food; but it is probable, indeed it is certain, that the sickness is in progress for a considerable period before the animal refuses food. Cases like the following are sometimes seen: A hog refuses to eat; it soon grows weak; staggers in walking; turns purple on the sides and belly, and dies within two or three hours after the first symptom is noticed. But, on examination after death, extensive disease is found in the intestines, or in the lungs, or in both, at a stage of development which must have required many days to reach.

Such are the symptoms as obtained from inquiries and observations in this vicinity. Since the preceding description was written, I have obtained a valuable article upon the disease, written by Dr. George Sutton, of Aurora, Dearborn County, Indiana, and published in the "North American Medico-Chirurgical Review," for May, 1858.

Dr. Sutton made extensive and careful observations of the disease as it prevailed in Indiana, and his artioles comprise the only published information I have been able to obtain in relation to it. His description of the symptoms is as follows: "The hog at first appears weak; his head droops; and sometimes, in a few hours after these symptoms, diarrhea commences. There is frequently vomiting. In some cases the discharges were serous and clay-colored, sometimes dark, also bloody and mucous, resembling those of dysentery. The urine at first was generally small in quantity and high-colored, but as the animal recovered it became abundant and clear. This was one of the symptoms by which 
the men, who were attending the hogs at the distillery, ascertained that they were recovering. In a large number of cases the respiratory organs appeared to be principally affected, and there was coughing, wheezing, and difficult respiration. In some instances the animal lost the power of squealing, and the larynx was diseased. There was frequently swelling of the tongue and bleeding from the nose. In those cases where the respiratory organs were the principal seat of the disease there was gencrally no diarrhea or dysentery. In many instances the disease appeared to be principally confined to the skin; sometimes the nose, the ear, or the side of the head were very much inflamed-the ear swollen to twice its usual thickness. This inflammation would spread along the skin sometimes over the eye, producing complete blindness. Sometimes one or more legs were inflamed and swollen, and the inflammation also extended along the body. The skin, where it was inflamed, was red and swollen. Some had large sores on their flanks or sides, from three to six inches in diameter. In one instance, at the distillery, the inflammation extended along the fore-leg, the foot became ulcerated and sloughed off, and the animal iecovered. Some appeared delirious, as if there was inflammation of the brain. Sudden changes in the weather, particularly from warm to cold, appeared to increase the fatality of the disease."

The symptoms, as described by Dr. Sutron, are similar to those observed in this vicinity, and the descriptions already given are sufficient to enable any one to recognize the disease wherever it prevails. It will be noticed that there is a very great variety in the symptoms described, and that they affect nearly every part of the animal. This would be expected from the nature of the disease itself, as will be seen hereafter.

Post-mortem Appearances.-Having described the symptoms as seen while the animal is living, I will now give, briefly, the appearances found on examination of the bodies after death. In the course of our investigations, during the last winter and spring, the bodies of nine hogs were carefully examined by Drs. G. L. Collins, J. W. C. Ely, and E. 'T. Caswell, of Providence, in the presence of several other physicians. A minute account of each ease was prepared by Dr. Collins, and published in the "Transactions of the Rhode Island Medical Society," for 1861. It will be sufficient for the objects of this paper to give a brief 
synopsis of the diseased appearances which were found in these examinations.

Lungs. - In two cases the lungs were healthy. In the remaining seven cases one or both lungs were more or less inflamed, having a liver-like appearance, called hepatization. In some cases the inflammation was more advanced, and the substance of the lungs was breaking down into a mass of disease. In all cases where the lungs were inflamed there was also pleurisy, and the lungs were adherent to the walls of the chest, the inflammation of the lungs and the pleurisy together constituting true pleuropneumonia. In two cases there were tubercles, or consumption in the lungs; but this is not uncommon in hogs, and is not supposed to have any connection with the special disease we are considering.

Stomach.-The stomach and the small intestines were generally healthy. The stomach was frequently distended with an offensive mixture of food, and in one case the inner surface was ulcerated to some extent. In two cases worms were found in the small intestines; but this was probably a mere accidental occurrence, and had no necessary connection with the disease.

Large Intestines.- The inner coat of the large intestines was generally inflamed and softened, with ulcerations to a greater or less extent, and they were frequently so tender as to be easily torn with the fingers. On account of their diseased condition, their inner coat was frequently discolored. The inflammation and ulceration of these intestines are probably the principal cause of the diarrhea in this disease.

Kidneys.-These organs were, in every case, mǔch more pale and yellow than natural; this condition was well marked.

The liver and bladder were generally healthy. In some eases water was found in the cavity of the belly and of the chest, and in the membrane surrounding the heart (heart-case). In two cases numerous minute purple spots were seen upon the membrane lining the walls of the belly. The urine was often changed from the healthy condition, containing albumen and other diseased products, not, however, noticeable to the eye. Ulcers upon the feet and in the mouth were often found. The brain was not examined, as there were no symptoms observed which seemed to indicate disease of this organ. It may be, at times, affected, but is probably more rarely so than the other organs of the body. 
Such are some of the most important appearances which are found on examination of the bodies of hogs which have died with this disease. It will be noticed that three of the diseased conditions I have described are prominent, important, and such as would be readily recognized by the most ignorant observer. These are, first, the pleurisy and inflammation of the lungs; second, the inflammation, ulceration, and softening of the inner coat of the large intestines; and, third, the pale and yellowish color of the kidneys. One or more of these diseased conditions will be found in every case, and in, perhaps, a majority of cases, they will all be found in the same animal.

Dr. Surton, of Indiana, in the article from which I have already quoted, gives an interesting account of the post mortem appearances in hogs dying from this disease. He examined the bodies of sixty-seven hogs, and it is evident, from this article, that he had the opportunity of observing the disease in a more malignant form than it has presented in this vicinity. He mentions every diseased condition that I have described, and also found unmistakable marks of similar disease in almost every other organ of the body.

With the description already given of symptoms and post mortem appearances, most persons, even without medical knowledge, will be able to recognize the disease. It remains for us to consider, briefly, its nature, causes, prevention, and treatment.

Nature of the Disease.-The first question which arises in the minds of most persons is this: What is the disease? and the question is asked with a special desire to have it answered by a definite name. This can not be done. There is no name which would convey, even to physicians, and certainly not to others, a precise idea of the disease. But though there is no specific name by which it can be called, the facts, as already given, can not fail to suggest, at least to medical men, a correct idea of its nature. Sometimes in the human subject, particularly when portions of the body are undergoing the process of inflammation and suppuration, pus and other diseased products are absorbed, and, entering into the circulation, poison the blood and produce that condition of the system known as pyæmia. In such cases the general poisoning of the system is shown by a low form of fever, great weakness and prostration of the vital powers, frequently accompanied by vomiting and purging, and often resulting in pleurisy, with inflammation 
and deposits of matter in the substance of the lungs, superficial swellings and abscesses, inflammation and suppuration in the joints, and other diseased phenomena. So in severe forms of typhus or ship fever, and in malignant cases of some other diseases, when the whole system seems to be filled with a powerful poison and utterly prostrated, the same or similar effects are seen. So in this disease among hogs there seems to be a general poisoning of the blood, producing local inflammations and ulcerations in various parts of the system, though more frequently in some portions than in others.

This, then, is a general disease of the whole system, resulting from some poisoning of the blood; and the pleurisy, the inflammation of the lungs, the ulceration of the intestines, the superficial ulcers and swellings, and other effects I have described, are only the local effects or results of the general disease. The diarrhea, which exists in a majority of cases, and on account of which the name of "hog cholera" has been given to the disease, is probably caused by the local inflammation and ulceration of the large intestines. The name "hog cholera" is, therefore, entirely improper, as it represents only one of the several prominent symptoms. The discase might as correctly and as incorrectly be called a "pleuropneumonia."

In this connection, it is proper to notice the similarity which exists between this disease in swine and some epidemic diseases which have prevailed among cattle. Some two thousand years since a disease, to which the name of "murrain" was given, was described by Virgil as " a highly inflammatory fever among cattle, accompanied with tumors, carbuncles, ulcers, and purulent deposits throughout the whole body." Any one who reads this and other descriptions of murrain, as given in the "New American Cyclopedia," can not fail to notice the striking similarity between that disease among cattle and the disease among swine which we are now considering. The same is true of the disease which has caused so much excitement in New England, within the last two years, under the name of "pleuro-pneumonia." Its symptoms and post mortem appearances, so far as described in the reports I have seen upon the subject, are very similar to those observed in the disease among hogs which we have examined. The only case of the disease called "pleuro-pneumonia" that I have seen was in the vicinity of Providence, in July, 1861. In that case, in which a 
post mortem examination was made by Dr. Colums, it was the unanimous opinion of the physicians present that there was a remarkable similarity in the symptoms and appearances to those observed in the hogs examined previously.

The conclusion to which I have arrived is, that the "hog cholera" I have described, the "murrain" of Virgil and of all ages since, and the "pleuro-pneumonia" of New England, are similar diseases, having the same general features, producing similar diseased changes in the body, and are the result of similar causes. If a definite name is required, the name of "murrain," which is derived from a Greek word which means to waste or to weaken, is the best. The names "hog cholera" and "pleuro-pneumonia" are both improper, and give very erroneous ideas in relation to the disease.

Causes.-In this and other similar diseases among animals, as well as in epidemic diseases in the human race, the universal tendency of the public mind is to ascribe their propagation to contagion. Dr. Sutton, whom I have already quoted, says: "Although this disease must occasionally have a spontaneous origin, yet, when once produced, it will spread rapidly by contagion." The investigations of the cattle disease in Massachusetts in 1860, and the action of the Legislature in regard to it, seem to have been based upon the theory that the disease was a "pleuro-pneumonia" only, and that it was contagious.

If this be an epidemic or epizoötic* disease, its causes are similar in their nature and operation to those of other epidemics. The following, then, according to my understanding of the subject, are the causes not only of this disease among swine, but also of the disease referred to among eattle, as well as of epidemics in the human race:

1. An epidemic atmospherical poison.

2. The local conditions or circumstances adapted to receive and propagate the poison existing in the atmosphere.

With regard to the first cause, the atmospherical poison, very little is certainly known. It may be an animal or vegetable exist-

"The phrase "epidemic disease among animals" is incorrect, as the word "epidemic" can properly be applied only to diseases of the human race. The word "epizoötic" has the same meaning with reference to diseases among animals that the word "epidemic" has with reference to diseases among men. 
ence, or a chemical or electrical change in the atmosphere. Nor do we know any thing of the differences in the condition of the atmosphere by which such dissimilar epidemic and epizoötic diseases are produced at different times. But, judging from their effects, we conclude that these primary causes of epidemics probably exist in the atmosphere; that they progress over a greater or less extent of country, in accordance with laws with which we are not acquainted, and, lighting upon the earth, produce their effects wherever they find the local conditions adapted to their propagation.

The local conditions, or causes of this disease among swine, are more obvious and better understood. They are, briefly, impure air, arising from the filth with which the animals are surrounded, the location and want of ventilation of the pens in which they are kept, the use of improper and unhealthy food, and the want of pure water. Common sense shows that cleanliness, good food, pure air, and pure water are as important to prevent disease in hogs as in men, though the fact is generally ignored by those who have the care of these animals. Another condition, though not absolutely essential to produce the disease, has a very great influence in increasing its severity and fatality. It is the crowding of large numbers of animals together. It is a well-established fact that the severity and fatality of cholera, and of other epidemics in the human family, is in direct proportion to the density of the population. The coëxistence of both the causes given-namely, the atmospherical poison and the local conditions-is necessary for the extensive development of the disease. When both these causes are present in any locality, and healthy animals are brought into the locality, a portion of them, and sometimes all, will contract the disease. But this important fact is to be remembered, that both these causes may exist in a locality whether any animals are present or not.

Perhaps I have said more than some might think necessary in relation to the causes of the disease ; but the subject is of the utmost importance in its relations to preventive measures. If this and other similar diseases are not contagious, but arise from causes which may, and do, originate and exist wholly independent of the presence of animals, it is manifestly absurd to attempt, as has been done in some cases, to prevent and eradicate them by the wholesale destruction of the animals. 
Prevention.-Keeping in mind the causes of the disease as I have given them, the measures necessary for prevention are obvious, and may be stated in a very few words. They are, to remove, as far as possible, the causes. The general cause existing in the atmosphere ean not be removed, nor can it be avoided, except by the removal of the animals beyond the limits of its existence, and this is not usually practicable. But the local causes can, to a very great extent, be removed, and without these the general cause can not produce any very serions results. The hogs can be removed from their filthy pens and can be kept clean; they can be removed from low ground to more elevated places; they can be separated and isolated as far as possible; they can be allowed an abundance of pure air and of pure cold water; and they can be supplied with nourishing, healthy food. In a word, they can be placed in the conditions which common sense shows to be essential to health, whether of hogs or of other animals. When this is done, every thing is done that is necessary or possible for the prevention of this as well as of other similar diseases.

Treatment.-In the treatment of a disease of this character, we have little to expect from the specific or direct action of medicine of any kind. The most that we can hope to do is to support the system so as to enable Nature to overcome the disease. In a human being, with a disease of a similar character, we should first remove the patient to a healthy locality, and provide for an ample supply of pure air and good nursing. This, faithfully done, would be the most important step toward recovery. We should then give, as the symptoms might require, becf-tea or broths, milk, wine-whey, brandy, quinine, and other similar preparations, always remembering that the object is to sustain the animal powers so as to enable the system to withstand the depressing effects of the poison and free itself from its presence.

The treatment of hogs with this disease must be of a similar character. Stimulants and tonies of some description, with plenty of pure air, pure water, and suitable nourishment must be given. Chlorate of potash, in full doses, from its known effects on the human subject, has been proposed as a remedy, but of its value I am unable to speak. Powdered charcoal is undoubtedly of some value, when scattered freely about the pens and given to some extent in the food. But, in the severer cases, treatment of any kind is generally unsuccessful, and if successful would cost more than 
the animal is worth. We might succeed, by the use of the remedies named, and by careful nursing, in prolonging the life of the diseased hog; but success of this kind would hardly compensate the trouble and expense necessary to secure it. But a large number of the milder cases will recover without treatment of any kind, and in this, as in other epidemics of a similar nature, it will be found that the first cases that occur will be very severe, and will nearly all prove fatal; while, after the epidemic has existed for a time, the disease will seem to become more mild, and a much larger proportion will recover. The important fact to be remembered is, that it is for the interest of the owner of these animals to use every possible means to prevent the disease, as prevention is of infinitely more importance to him than treatment; but if the disease becomes established, it should be treated in a rational manner, not forgetting that Nature, when properly aided, will do infinitely more than medicine for the cure.

Note.-The above valuable article on "Hog Cholera" is from the pen of Dr. E. M. SNow and is the most reliable one ever published on this disease.

DR. DADD'S REMEDY FOR "HOG CHOLERA."

No. 149. Powdered anthracite coal........4 4 pounds. Powdered ginger............. 1 pound. Powdered sulphur............ $\frac{1}{2}$ pound. Salt...................... 7 oz. Phosphate of lime........... 1 pound Mix.

Let each affected animal have one handful of the above mixture every day. Mix it in a pint of dry oatmeal. If the animal refuses to eat it, liquefy the same with cow's milk, and give as a drench. 


\section{TRICHINA SPIRALIS."}

ThE American people have been startled lately by the published accounts of a new and terrible disease in Germany, and especially in Saxony, which brings to mind some of the most dreadful plagues of Egypt. The disease in question, termed Trichiniasis, caused by the ravages on the human muscle of a minute worm, called TRIchinis Spiralis, coming so close upon the cattle disease, did, in-

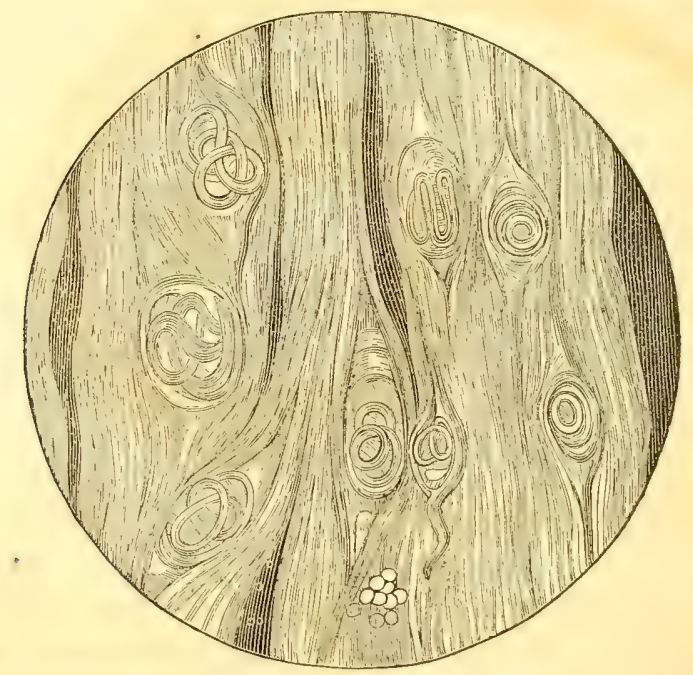

TRICHINA SPIRALIS AND OVA, AS SEEN IN THE MUSCLES OF THE HUMAN GUBJECT, MAGNIFIED ABOUT TWO HUNDRED AND FIFTY TIMES.

deed, to the ignorant, appear to justify some of the recent prophecies of Dr. Cumming, but to the more intelligent, and especially to the medical mind, it came as an old story. Singularly enough, the worm which is now occupying the attention of German anatomists was discovered as long ago as 1835, by Professor Owen. Both Mr. John Hilton, a demonstrator of anatomy at Guy's, and Mr. Thorirald, the demonstrator at St. Bartholomew's, had, two years previously, observed small white bodies interspersed among the muscles of subjects under dissection, and that they were of a gritty 
character was evident from the manner in which they turned the edges of the knives. One of these specimens of affected muscle was, in the year mentioned, given to Professor Owen by Mr. PAGET, then a student, for inspection. These speckles the distinguished anatomist discovered, under the microscope, to be the capsule of a very fine worm, which was seen coiled up closely within it. From its hair-like fineness, its discoverer derived the term Trichina, and from the spiral manner in which it was invariably found coiled up within its envelope, he added the word Spiralis; hence the name by which it is known.

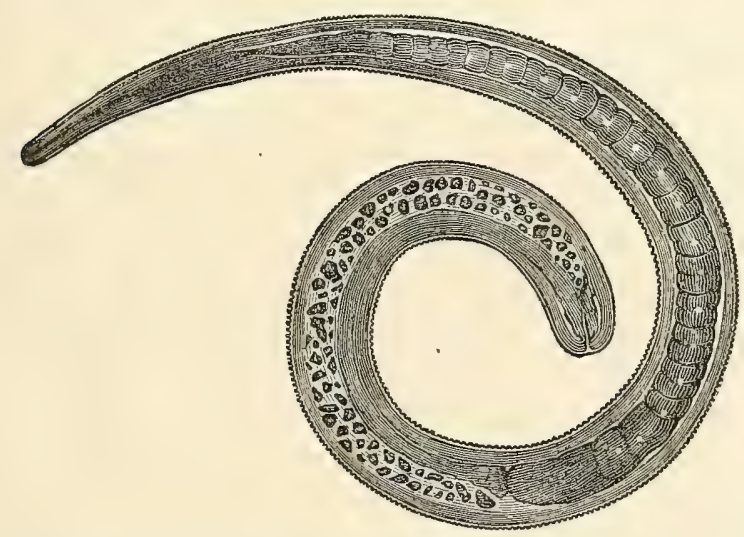

TRICAINA SPIRALIB IN PORK, MAGNIFIED ABOUT TWO HUNDRED DIAUETERS. AFTER AN ORIGINAL DRAWING BY MR. DRAPER.

An account of this newly-discovered parasite was published by Professor Owen, in the "Transactions of the Zoölogical Society," in 18.35, headed, "Description of a Microscopic Entozoön infesting the Muscles of the Human Body." This paper gave a very minute account of the creature, illustrated with drawings, and established his claim to be the discoverer of one of our latest-found inhabitants, which has made such a sensation in the world. The discovery made much noise at the time throughout Europe, and the Professor's paper drew the attention of the anatomists of Europe to the worm. But one or two cases were recorded of the presence of the parasite in the human body, and the matter remained in abeyance for some years, until the German professors again drew attention to it, and completed our knowledge of its method of introduction. Professor Luschka, of Tübingen, car- 
ried our knowledge of the worm, perhaps, up to the highest point anatomically, and in the same year the method of transmission of the worm from one animal to another was made out by a series of experiments instituted by Herbst von NaCHRichten. He gave the flesh of a hedge-hog, which he knew to be infested with trichina, to young dogs, and speedily found that all their voluntary muscles were full of these worms. But, although this important step was made out, little notice was taken of it. His experiments were repeated in Scotland and England, but the peculiar manner in which the worm got into the muscle was yet undiscovered.

KENKER, in 1860, was lucky enough to supply this knowledge. The body of a servant girl, who had died with many of the symptoms of typhus fever, came under the inspection of the anatomist. He found her voluntary muscles to be full of trichinæ; and upon inquiring into her case, he found that she had assisted in the making of sausages about three weeks before she was taken ill, and that she had eaten some of the raw meat a few days before her illness commenced. The butcher who had killed the pig, and several members of the family, had been affected in the same manner as the girl, but had recovered. The sausages and hams were examined, and were found to be full of worms, encapsuled, as it is termed, or surrounded with an envelope; but in the girl the worms were found among the muscles, in a free state.

From this evidence the manner in which the parasite obtained entrance to the human body was fully made out. Pork (uncooked pork) was the vehicle by means of which the parasite was enabled to enter the human body. But, says the reader, why should pork only be the means of conveying the entozoa to the human body? The reason is that the pig is the only animal eaten by man that is partially a carnivorous feeder. It is supposed that the pig obtains them from dead rats, which are often infested with these worms, or other garbage. Birds, although carrion feeders, can not, for some unknown reason, be infested with the worms. In the horse, calf, and the young and old dog, says Dr. Thudichum, the young trichinæ are born, but they can not pierce the intestines, and, therefore, can not immigrate into the flesh. Of course, it is just possible that the worm may be conveyed, like the tape-worm, through the medium of impure water. We are not likely to drink this, but it often happens that fruit and vegetables are watered from impure tanks, into which these creatures may have got. 
It is certainly an objection to the modern system of watering with liquid manure, that in this way the tape-worm, and possibly the trichina, may find their way on to the vegetables which we eat, and in this way we may be receiving noxious intestinal worms into our system. For instance, some people water their strawberries with liquid manure, not thinking of the little serpent that may be hidden in the fruit. It is now known that, after entering the alimentary canal, the parasite finds its breeding-ground, and brings forth immense numbers of young, which immediately begin to make their way through the coats of the intestines and migrate into the muscles.

It is a singular fact that these disagreeable adventurers always select the voluntary muscles, or those which are moved at our will. The heart and kidneys, and those parts of the viscera which act independently of the will, are scarcely ever affected. It is, indeed, a matter of dispute how the worms get distributed so generally over the body, some anatomists asserting that they make their way directly by boring, as the ship-worm bores through a piece of timber; but Dr. Thudichum, who was appointed, in 1864, to investigate the subject, by the medical officer of the Privy Council, asserts that they enter the circulation, and are, in this manner, distributed equally over every part of the body. To use his words: "Arrived in the capillaries (terminal blood-vessels), they penetrate.their two-coated walls, separating the fibers as a man separates the branches of a hedge, when creeping through it, and are now either at once in muscular tissue, their proper feedingground, or get into inhospitable tissues and cavities, and there either perish or escape from them by a renewed effort at locomotion, enter the circulation a second time, and ultimately perish in the lungs, or arrive in some muscle to obtain a late asylum."

This hypothesis, certainly, seems the most reasonable, as it is in agreement with the known means by which other entozoa migrate. Arrived at the muscular tissues, it seems again questionable whether the worm attacks the muscle only, or whether it is not deposited in the intervals which occur between the bundles of muscles. LEUCKHART says they penetrate the sarcolemma, and eat the muscular fiber itself. Dr. Thudichum says that he has never seen but once the worm in the muscle, but always outside of it. It is certainly a strange fact that, in many cases, persons attacked with trichiniasis have not only perfectly recovered from its effects, 
but have become as strong as ever. It could scarcely have happened that the muscles of these patients had been fed upon by vast colonies of worms, which would have inevitably destroyed them beyond repair. The probability is that the worm finds its way into all the tissues. Between the third and fourth week after immigration, the trichina has become full-grown, and now it begins to prepare its capsule. It becomes fixed to the spot in which it is, solid matter is deposited around it, and, curled up, it lies immovable in its plastic capsule, and dies unless received again into the alimentary canal of another animal, which, in this case, of course, it never does. The presence of these encapsuled trichinæ in the muscles may cause irritation, but that speedily subsides; and it is pretty clear that many persons suffer little harm from them while thus curled up, as they have been found in the bodies of subjects that have been dissected, and whose previous history gave no evidence of their existence.

On the other hand, the disease, when severe, puts on many of the characteristic symptoms of well-known diseases. The fever caused by the presence of the parent worms in the intestines may be, as, indeed, it often has been, taken for gastric fever. Then, again, when the young worms are immigrating into the muscles, the most excruciating agony seizes the patient. He can not move a muscle without the utmost pain, and he lies generally upon his back, with his legs a little apart, covered with perspiration. The face and neck become tumid with a dropsical effusion, which generally extends to the legs and abdomen. An attack of acute rheumatic fever appears to have seized the individual, but for the want of the heart symptoms. Again, the disease stimulates cholera and typhus, and, indeed, poisoning, in many of its symptoms; but those who have seen a genuine case of trichiniasis can not be deceived, as the whole symptoms present are consistent with no other disease. In cases of doubt, a piece of the living muscle has been excised from the biceps muscle of the arm; and this test is almost certain to be conclusive, as the worm is distributed, in severe cases, in profusion through every voluntary muscle of the entire body.

Dr. Thudichum, speaking of a child who died of this disease, says, in his report to Mr. SMmon: "One preparation from the biceps muscle of a child, four and a half years of age, which died on the seventy-ninth day, contained the astounding number of 
fifty-eight. Such a preparation was estimated to weigh one-fifth of a grain, and, thèrefore, every grain of muscle contained, on an average, one hundred trichinæ. Now, assuming the weight of the muscles of an adult to be only forty pounds, and assuming him to be a victim of trichiniasis, and the parasites equally distributed throughout his body, he would contain upward of twenty-eight millions of these animals." The agony of this plague of worms attacking the fine fibers of nerves distributed throughout the frame can, from this estimate, be thoroughly understood in the fever and weakness caused by the destruction of fiber, and the irritation is accounted for with equal ease.

The progress of the disease is pretty much as follows: During the first stage, which lasts from a week to ten days, there is great intestinal disturbance, caused by the presence of the parent trichinæ in the intestines, giving rise, in severe cases, to alarming diarrhea, as may be expected. The second stage lasts a fortnight or three weeks, seldom longer. During this time the immigration of the young trichinæ, hatched in the intestinal passage, is taking place; hence the agony throughout the body, the dropsy in the face, the hurried breathing, and the fever. Although the dropsy becomes genuine, it in no manner depends upon kidney discase, as that organ is never affected in any way. In the fourth week the immigration has entirely ceased, and the worm is beginning to be incapsulated. From this time the patient begins to recover, the appetite improves, the pains become less, and, unless complications arise, as in other severe fevers, the patient gradually passes into a state of health. Death may, however, take place at any stage of the disease. At the great outbreak of this disease which took place at Calbe, in Germany, it was observed to happen on the fifth, eighth, fourteenth, twenty-first, and forty-second days of the illness. Death generally is brought about by exhaustion. The exhaustive diarrhea which sometimes occurs, together with the inability to take food, and the terrible agony, easily explains this termination.

The difficulty connected with the treatment of this disease is consequent upon the impossibility of knowing what is really the matter in its early stages, when treatment is alone useful. In regular outbreaks of the disease the physician is led to suspect the evil in the beginning, and then it can be cut short by destroying and expelling the parent worms before they have had time to col- 
onize the intestines with their young. But at the commencement of an outbreak, or in isolated cases, the symptoms are too like those of gastric fever to lead to a suspicion of the real nature of the affection.

A prevention is far better than cure, and, happily, this can be easily accomplished. As pork is the only means by which the parasite can enter the human frame, we have only to take care that we eat it thoroughly cooked. The Englishman has a very strong prejudice in favor of doing his leg of pork well, however much he may like beef and mutton underdone. The Germans are apt to suffer desperate outbreaks of this disease because they are fond of smoked sausages, in which no heat is applied to the meat. The severity of the infection depends, indeed, upon the amount of cooking to which the trichinous meat has been subjected, and the order in which it is affected is as follows: Raw meat, smoked sausages, cervelat sausages, raw smoked ham, raw smoked sausage, fried sausage, fried meat-balls, brawn, pickled pork, blood sausage, boiled pork. As few people are likely to eat raw pork, there seems little danger to be apprehended from the most dangerous item in the list; but it is well to know that boiled pork is, in all cases, the most harmless. The power of the worm to resist heat and cold is very remarkable. They have been frozen to five degrees below centigrade, and been thawed to life again. Ordinary vermifuges are powerless against them. Their vitality is as great as the wheelworm, which seems almost indestructible. Let our friends, then, take care never to touch the smallest portion of underdone pork, and beware of German sausages, bolognas, and things of the same kind, as they would beware of an assassin.

Before the discovery of the new disease, trichiniasis, several epidemies oceurred in Germany, which very much puzzled the physicians. In two or three cases it was supposed that the persons suffering had been poisoned in some mysterious manner, and judicial inquiries were instituted without any result. More generally, however, the outbreaks were ascribed to rheumatic fever, or typhus fever. It was observed, at the time of their occurrence, that the outbreaks were confined to particular families, regiments, or villages. The symptoms, then obscure, are now recognized as those of trichiniasis; indeed, there seems to be little doubt that they were outbreaks of this disorder. They all occurred in the spring of the year, the time of pig-sticking in Germany, and the 
very characteristic swelling of the face, in the absence of any kidney disease, was observed.

The mortality arising from this disease is in direct ratio of the severity of the attack, and this depends upon the number of worms which may chance to be introduced into the body. One pig is sufficient to cause an epidemic far and wide; indeed, many of those which have ravaged Germany within these last three or four years have been traced to one trichinous pig. At the outbreak at Planen one person died out of thirty attacked. At Calbe, where the epidemic was more severe, seven persons died out of thirty-eight infected; at Hettstädt, where one trichinous pig infeeted one hundred and fifty-eight persons, twenty-eight died. From these facts the formidable nature of the infection may be gathered.

If sudden epidemics can be traced to the action of an obscure worm, may we not hope that many of our disorders, now obscure in their origin, and, consequently, unmanageable and incurable, will in time come to light, and be amenable to treatment? Possibly some more subtle power even than the microscope will be discovered, and give us the power of scrutinizing diseased conditions, and finding out the agents so stealthily at work in bringing the human machine to misery and premature death.

The following very interesting and valuable article on the subject of Trichina is translated from the German, expressly for this work, by E. F. Brown, V. S., of Chicago:

The Trichina (Trichina Spiralis) was known to exist as early as 1832, and in 1835 received its name from Mr. Owen. It was considered, until the year 1860 , to be perfectly harmless. Autopsies revealed the parasite as a very small speck, surrounded by a white, chalky capsule. In the Infirmary of Dresden, the servant girl of a butcher died after suffering violent pains in the muscles. The autopsy revealed numerous trichinæ not surrounded by capsules, hence they were not to be seen with the naked eye. After this, closer inquiries were made into the nature and habits of the trichinæ. The result of the investigations proved that the trichinæ are generated in the hog, and introduced into our system, not with the fat or liver, but with the lean pork; and they are sometimes found in capsules, and at others without them, and that the capsules are very soon destroyed, after their introduction into the 
system, and thus the inclosed parasites are set free. The hybernated trichinæ grow very fast, so that they attain, in about three days, twice their original length, and in the same ratio alter their appearance. Nothing of their generative organs was then known, but now the creatures can be plainly distinguished as male and female. They enter ințo very productive marriages, because one

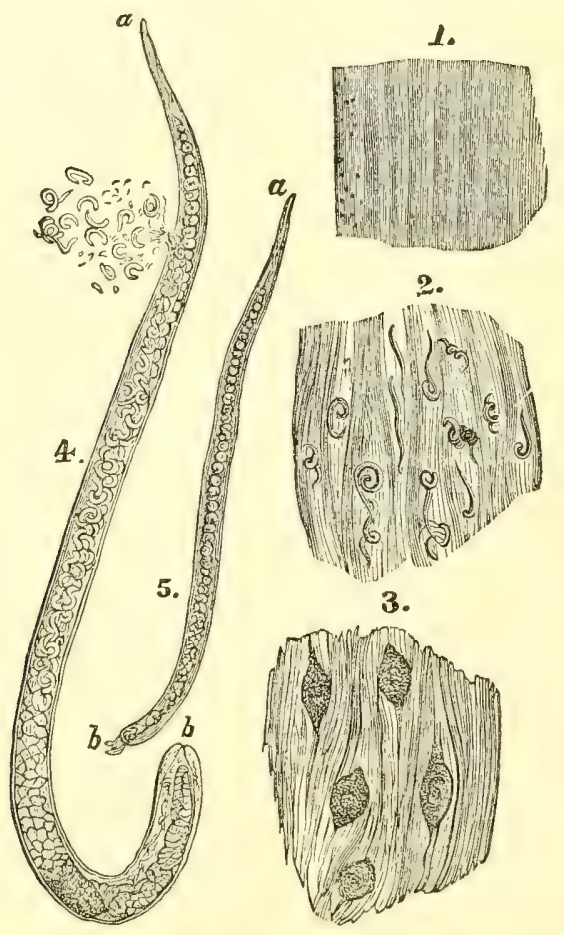

Explanation.-Fig. 1, Trichinous pork, as seen with the nalied eye ; 2, Young minute trichinø roving about, magnified forty diameters; 3 , Trichinæ in capsules, magnified forty diame. ters; 4, Female trichina ; 5, Male trichına, magnified two hundred diameters. $(a)$ Head ; (b) the tail. Near the female trichina is seen the ova and their young ones, just escaped from the genital organs.

female brings forth, in a very short time, hundreds of live young ones. These new-born trichinæ do not resemble their producers, for they are without any generative organs. They are very much like the parasite when first introduced into our system with the pork.

The young trichinæ remain not at home, like the old ones, but 
at once commence to travel. They break through the intestines and enter the muscles known as the voluntary, where they continue their march until they have found, in the finest fibers, a place fit for their encapsulation. On their journey to such locality they can not be seen with the naked eye, but only by means of a microscope. They wander in the shape as presented in fig. 2, straight, or slightly bent. After they arrive at their destination, they curve or bend themselves in various ways, and thus make their way into the flesh fibers, and then they roll themselves up in their spindleformed nest, which is spiral in form, like a watch-spring. The mass around the nest is, in the beginning, soft and transparent, but by degrees it becomes converted into a hard, impenetrable shell. These shells can be seen in the flesh with the naked eye. They are the fine, white spots as seen in fig. 1. The trichina lives now in a perfectly-closed sac, or lemon-formed capsule, and is perfectly harmless. In this state, it seems that the trichina can exist in a latent state, for many years. From some cause or other, the chalk capsules get dissolved, and the hybernating parasites (until now without any genital organs) become males and females, and produce young ones, who live and act just as their predecessors-namely, generate, travel, etc. Accordingly, we find in the human and animal body (mostly in the hog) male and female trichinæ, and those in the stomach either straight or slightly bent. These newborn trichinæ, in the stomach and intestines, very soon become muscle trichinæ, to be encapsuled, like the hermit in his cell.

Every trichina is pointed at the mouth $(a)$ and rounded at the other end $(b)$, the pelvis. Between both openings is the gullet and intestines. The female trichina is about an eighth of an inch long, and has in its hind part (b) a bag containing about sixty or eighty balls (the ovary with eggs). Connected is a pipe conveying the trichinæ, which come from the ova as live parasites, by the vagina. How long the parent can exist and generate can not be told, though it is supposed that it exists for three or four weeks after giving birth to the offspring. The male trichina is about half as large as the female, and on its posterior end $(b)$ is a prominence divided into two flaps. His anterior construction shows the spermatic vessels. The new-born trichinæ without genitals are only to be seen when well magnified. They are very small, and are those which, after penetrating the intestines and the cellular texture of the abdomen and cavity of the chest, enter the muscles, to be encapsuled. 
The traveling trichinæ (fig. 2) grow very fast on their journey. Finding food on their way, they roll themselves up just as they are about becoming encapsuled. In the course of a few weeks the parasite has almost attained its full length, but, not having any generative organs, it can not multiply. It is supposed that the encapsulated trichinæ can live for several years, whereas the parents die in about six or eight weeks.

It is not denied that the trichinæ are dangerous to the human subject, but the danger only occurs when the parasites are very numerous in the intestines and museles. It is certain that a very few small pieces of trichinous pork can introduce a great many males and females into the system, that in a few days may send millions of young parasites into our muscles. The more trichinous food is eaten, and the more those parasites are introduced into the system, and the longer they remain there, the greater must be the suffering and danger. The parts affected or visited by the trichinæ are the stomach, intestines, and muscles; and if this parasite is once introduced in the animal system, it produces an incurable disease, for which there is no remedy. It is well for those who are fond of pork to be very cautious, and cook it thoroughly, because, if well cooked, the death of the parasite is sure.

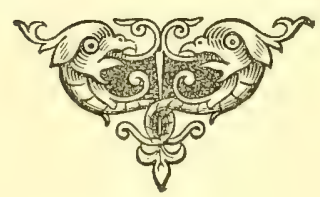




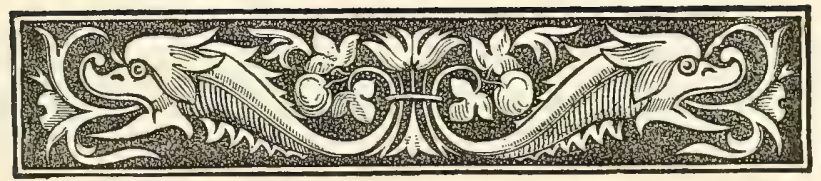

\section{A LIST OF FLUID EXTRACTS,}

RECENTLY INTRODUCED INTO VETERINARY PRACTICE,

By G. H. DADD, V. S.,

WITH INSTRUCTION REGARDING THEIR ACTION, AND

THE QUANTITY TO BE ADMINISTERED.

THE introduction of medicines, in the form of FLUID Ex-

1 TRACTS, for the treatment of diseases incidental to domestic animals is considered a very great improvement over the old method of drenching by the pint or quart, to the great disgust of the patient as well as the practitioner, and the great danger attending the administration of a large quanty of fluid which was necessary when crude materials were used. In the use of fluid extracts all danger is obviated and much labor saved, for the doses are quite small, rarely exceeding one fluid ounce. This can be merely placed on the tongue, the animal's head being slightly elevated, and he swallows it without any difficulty or resistance.

The fluid extracts bear an exact relation to the crude materialsthat is, ounce for ounce-yet, from a variety of circumstances, they may vary slightly from this standard. It is, however, the intention of the manufacturer to completely exhaust the active principle of the crude material and render the medicine uniform in strength. The fluid extracts used by the author are manufactured by Messrs. GARRIson \& Co., manufacturing chemists, of Chicago. They conduct their evaporations at a very low temperature, by means of an improved steam bath, and use only select drugs, thereby preventing the possibility of adulteration. I have used large quantities of medicine manufactured by the above-named firm, and find them 
equal if not superior to any in the market; therefore I do not hesitate to recommend them as efficient and reliable medicines. The doses here recommended apply to both horses and cattle.

\section{Fluid Extract of Chamomile.}

(Anthemis Nobilis.)

This is a valuable tonic, and is used in cases of derangement of the digestive organs. Dose, from two to four drachms.

\section{Fluid Extract of Arnica. (Leopard's-bane.)}

Arnica is chiefly used in veterinary practice as an external application in the treatment of wounds, sprains, and bruises. It is prepared as follows:

Fluid extract of arnica..............4 oz.

Proof spirits (equal parts of water and alcohol)... 1 pint. Mix.

Apply a portion to the affected part two or three times daily.

\section{Fluid Extract of Wormwood.}

(Artemisia Absinthium.)

This is used as an anthelmintic for the expulsion of worms. The dose is one ounce (fluid), to be given every morning for a week, just before feeding-time. It not only has a tendency to expel worms, but gives tone to the digestive organs, and corrects a morbid appetite.

\section{Flum Extract of Pleurisy Root.}

\section{(Asclepias Tuberosa.)}

This is used in pleurisy and irritable sore throat. Dose, four drachms, night and morning. The powdered root enters into the composition of some of the most celebrated cough powders.

\section{Fluid Extract of Male Fern. \\ (Aspidium Filix Mas.)}

This is the celebrated remedy for the treatment of tape-worm, which is sometimes to be found in the intestines of young colts. 
Dose, four drachms, to be given every morning, on an empty stomach, for six or eight successive days, and longer if necessary.

\section{Fluid Extract of Buchu. (Barosma Crenata.)}

This is used exclusively for the treatment of diseases of the urinary organs, retention of the urine, and for the treatment of the various forms of dropsy. Its direct action is diuretic, yet it also gives tone to the parts on which it acts. Dose, from four to eight drachms. Should an animal be suffering from retention of the urine, the diuretic action of the buchu may be augmented by adding to it a small quantity (equal parts) of sweet spirits of niter. For the treatment of spasm at the neck of the bladder, add to the dose of buchu an equal quantity of tincture of assafetida.

\section{Fluid Extract of Indian Hemp (Foreign).}

(Cannabis Indicus Sativus.)

This medicine acts as a narcotic and antispasmodic. Its use is indicated in all diseases attended with pain and restlessness, but is chiefly used for the treatment of tetanus or locked-jaw. Dose, two drachms, to be repeated as occasion may require.

\section{Fluid Extract of Prince's Pine.}

(Pipsissewa.)

Prince's pine is a very excellent tonic and diuretic in chronic diseases of the urinary organs, and has been used with much success in cases of local dropsy of the limbs, known as swelled legs. Dose, from four to six drachms.

\section{Fluid Extract of Black Snakeroot.}

(Cimicifuga Racemosa.)

This extract is considered a valuable agent for the treatment of spasmodic affections, which arise in consequence of derangement of some portion of the nervous system. It is an efficient remedy in hysteria. 


\section{Fluid Extract of Colchicum. \\ (Colchicum Autumnale.)}

This extract is prepared from the roots and seeds of the plant. It is celebrated for the treatment of rheumatism, inflammatory diseases of the joints, and inter-articular lameness. Dose, one drachm, two or three times per day.

\section{Fluid Extract of Yellow Jessamine. \\ (Gelseminum Sempervirens.)}

This agent is used as a sedative in the treatment of pleurisy and pneumonia in the early or acute stage. It is also a powerful febrifuge, hence it is indicated in all acute fevers. It entirely dispenses with use of the fleam. Dose, from one to three drachms.

\section{Fluid Extract of Witch-Hazet.}

(Hamamelis Virginica.)

Witch-hazel is used as a local astringent in the treatment of "bog and blood spavin." It is prepared for use as follows:

Fluid extract of witch-hazel............6 oz.

Proof spirits..................... 1 pint. Mix.

Apply a portion twice daily.

\section{Fluid Extract of Hops.}

(Humulus Lupulus.)

This preparation of hops is used as a tonic in general debility, and as a narcotic in diseases of the nervous system. It is a reliable remedy to induce sleep and relieve pain. Dose, from two to. four drachms, to be repeated as occasion seems to require.

\section{Fluid Extract of Golden Seal. \\ (Hydrastis Canadensis.)}

The golden seal is a pure tonic, and its use is indicated in all cases of debility, loss of appetite, derangement of the digestive organs, and torpidity of the liver. Dose, from two to four drachms. 


\section{Fudid Extract of Sassafras.}

All the preparations of sassafras are more or less alterative. The fluid extract is used in view of purifying the blood in diseases of the skin, and in cases of chronic rheumatism. Dose, from four to six drachms.

\section{Fluid Extract of Culver's Root.}

\section{(Leptandra Virginica.)}

This is employed, in the author's practice, as a substitute for calomel, in the treatment of acute and chronic diseases of the liver. Dose, from two to four drachms.

\section{Fluid Extract of Lobelta.}

Lobelia is a very valuable antispasmodic, and is useful in diseases of a spasmodic character. The author has used it, with great success, in obstinate cases of spasmodic colic. Dose, two drachms.

\section{Fluid Extract of Bayberry.}

\section{(Myrica Cerifera.)}

Bayberry is successfully used as a stimulant and astringent in scours, diarrhea, and super-purgation. Dose, two drachms, to be administered in a small quantity of milk porridge.

\section{Fluid Extract of Poppy-heads.}

\section{(Papaver Somniferium.)}

This is used as a narcotic, in all cases that seem to require the exhibition of opium. It mitigates pain, induces sleep and rest, and is much safer than crude opium. Dose, from two to six drachms.

\section{Flum Extract of Poke-root. \\ (Phytolacca Decandra.)}

This is used as an alterative in a disease occurring among cows, known as "garget," or "caked udder." Dose, from two to four drachms. 


\section{Fluid Extract of Matico.}

(Piper Angustifolium.)

This is used as a styptic to bleeding wounds, internal hemorrhages, and chronic diarrhea. Dose, from four to eight drachms.

\section{Fluid Extract of Cubebs.}

This is a very valuable agent for the treatment of leucorrhea, gleet, nasal gleet, or discharges from mucous surfaces. Dose, from three to six drachms.

\section{Fluid Extract of Black Pepper.}

(Piper Nigrum.)

This is a carminative and stimulant to the digestive organs, and is often used successfully in the treatment of stomach staggers. Dose, from two to four drachms.

\section{Fluid Extract of Mandrake. \\ (Podophyllum Peltatum.)}

Mandrake is now used as a laxative and eathartic, in lieu of aloes. About one ounce will usually purge a horse, if followed up by sloppy bran-mashes.

\section{Flum Extract of Bloodroot. (Sanguinaria Canadensis.)}

This is used as an escharotic in the treatment of foul ulcers, and fungus growths. It is used, also, as a gargle in suppurative laryngitis, croup, putrid sore throat, etc. The gargle is prepared as follows:

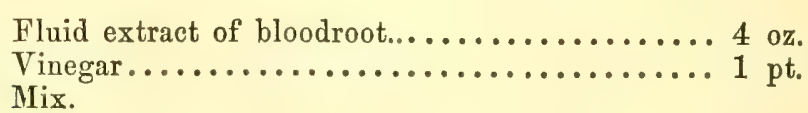

It is applied to the mouth and throat by means of a sponge, affixed to a piece of rattan or whalebone. 


\section{Fluid Extract of Valerian.}

(Valerian Officinalis.)

This is a very useful antispasmodic, and its use is indicated in all cases marked by irregular nervous action and hysterical affections. Dose, four drachms.

\section{Fluid Extract of Ginger.}

\section{(Zinziberis. Officinalis.)}

Fluid extract of ginger is one of the most valuable diffusible stimulants to be found in the whole materia medica. The Jamaica ginger is preferable to any other; it is used in cases of indigestion, flatulency, etc. Dose, from three to six drachms.

Fluid Extract of Ginger enters into the composition of the celebrated colic drench, used by the author of this work and his students, during a period of twenty-five years, with marked success. The following is the formula:

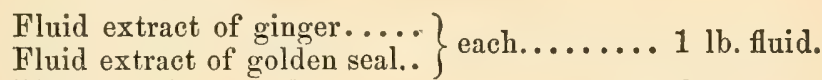
Hyposulphite of soda.............. 6 oz.

Dose, four ounces (fluid) every four hours, until relief is obtained.

\section{REMEDY FOR WOUNDS.}

Apply a compress of several folds of cotton cloth, soaked in a portion of the following:

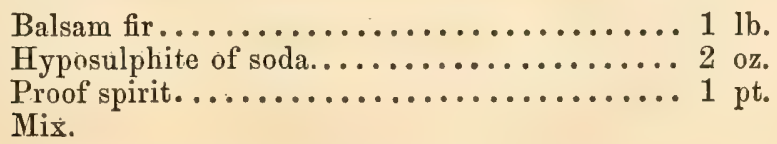

\section{REMEdY FOR TEDIOUS LABOR.}

The natural labor-pains may be excited by administering: fluid extract of ergot (ergota), two drachms, every half hour, until delivery is accomplished. The ergot operates with great energy upon the contractile function of the uterus, of both mares and cows, and to a certain extent prevents inordinate hemorrhage after parturition. 


\section{Remedy for Hemorrhage in Performing Operations.}

Apply, by means of a compress of linen or sponge, a small quantity of persulphite of iron. This is the most valuable styptic known to veterinary science.

\section{Fluid Extract of Aconite.}

\section{(Aconitum Napellus-Monk's-hood.)}

This medicine is used as a very powerful sedative and narcotic, in all cases of active or inflammatory disease of the lungs or brain. It is highly recommended by the professors of our art as a reliable antiphlogistic in the treatment of acute affections. The dose is from ten to twenty drops. This medicine is said to be very efficacious in inflammatory rheumatism, and as a fine topical remedy in localized painful affections, such as laminitis, myalgia, etc.

\section{Hyposulphite of SodA.}

The hyposulphite of soda is useful in all kinds of diseases known as rinderpest, pleuro-pneumonia, typhoid affections, and in enzoötic affections, and in all diseases caused by fermentation in the blood, such as Texas fever, malignant scarlet fever, typhus, etc. The hyposulphite of soda can be used with great success in the treatment of the above and other affections of an enzoötic origin, yet I have found it more efficacious and reliable when prepared as follows:

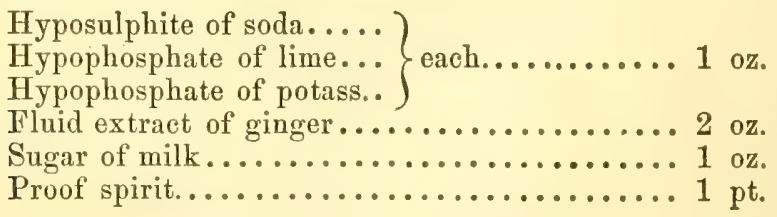

Dose, two ounces, two or three times daily. 


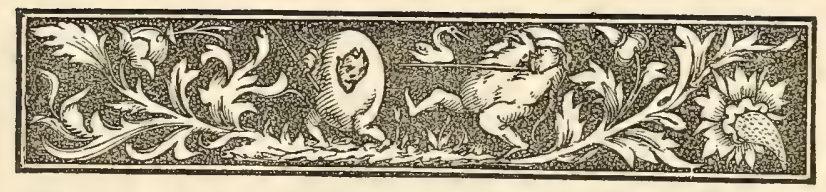

\section{A GUIDE TO DIAGNOSIS;}

OR

\section{COMPLETE INDEX OF SYMPTOMS}

THe following Index of Symptoms is arranged under four heads, viz.: Diseases of Horses; Diseases of Catrle; Diseases of Sheep; Diseases of Swine. Each part is arranged alphabetically, so that ready reference can be made to any symptom.

\section{HOW TO USE THIS INDEX.}

Animals when taken sick, not having the power of speech, can not make known the seat of their disease, except by certain signs or invariable symptoms. It therefore becomes of the highest importance to be able to understand the mute language of the poor dumb brute, and thus to find out what ails him, in order that the proper means of cure may be speedily made use of.

An instance of the use to be made of this Index may be found in the following case: "A horse is noticed to breathe faster than usual, with evident pain; back, arched; legs, straddling; head often turned toward the loins ; urine, reddened and scanty." Now, a reference to these symptoms, as arranged alphabetically, enables us to turn directly to page 223 , where "Inflammation of the Kidneys" is seen to be the disease we are looking for.

The same thing can be done in case a cow, sheep, or hog be the victims of disease.

In observing symptoms, attention should be directed to the 
various parts of the body of the animal which give the earliest warnings of disease. These are, usually,

The Eyes, Tongue, Muzzle, Throat, Pulse, Chest, Heart, Breath, Breathick; Discharges from the Mouth; Discharges from the Nostrils; The Skin, The Coat, The Back, The Attitude, and General Appearance of the WHOLE BODY.

\section{EQUINE DEPARTIENT.}

Abdomen, distended with gas. . . . ........................ 188

do. pressure on, produces pain.............198, 201

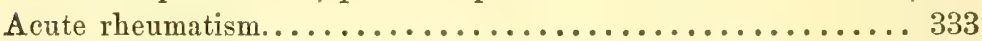

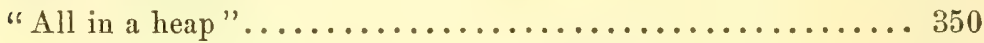

Anus, scurfy, yellow accumulation around margin of........ 220

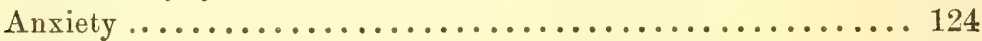

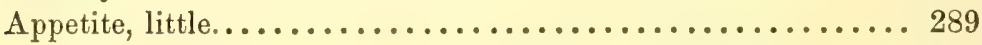

do. loss of...............116, 124, 201, 206, 223

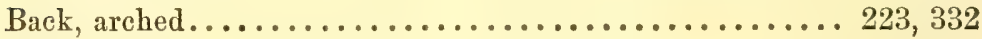

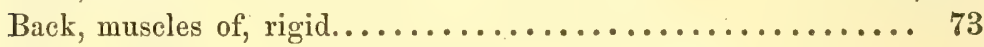

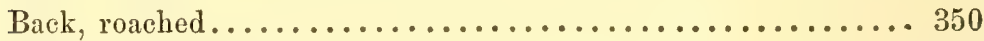

Belching, continually.................... 183, 188

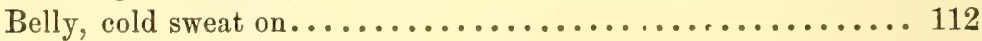

do. dropsical swellings under................ 120,397

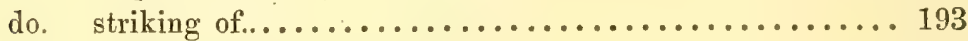

do. suddenly very much bloated................. 187

do. pressure on causes pain.................201, 198

do. "tucked" up........................... 74, 193

Blood from bowels.......................... 297

Body, becomes thin............................ 299

do. cold........................... 94, 112

do. covered with cold sweat................. 187, 188

Bowels, bloody, fetid discharge from................ 112

do. blood from, caused by straining............... 205

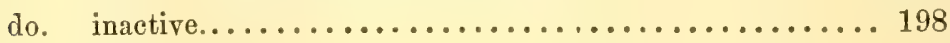

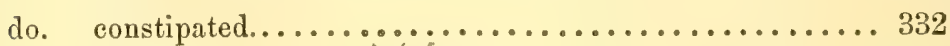

do. irregular........ $5 . \ldots \ldots \ldots \ldots \ldots \ldots \ldots . \ldots . \ldots 220$

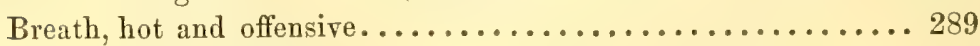

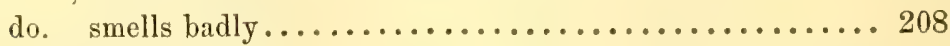

Breathing, grunting.................... 61, 62, 64

do. labored and quick..................... 187 
Breathing, quick and grunting................... 120

do. quick and painful...................... 223

do. oppressed and slow................ 63, 206

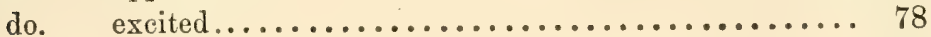

do. oppressed and noisy................. 94, 179

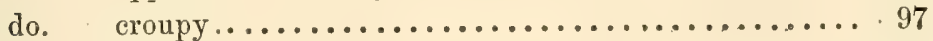

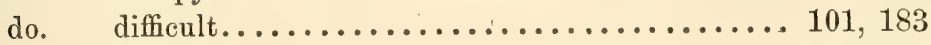

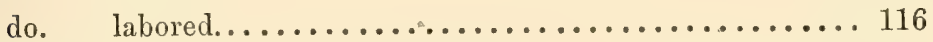

Chest, pressure on side of, gives pain............. 120, 332

do. dropsical swellings under.................... 120

do. tinkling sound in, when ear is held close to......... 124

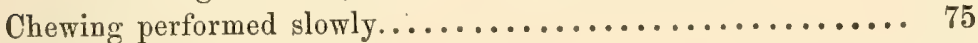

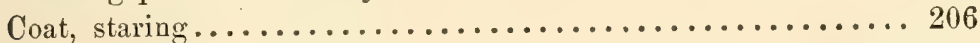

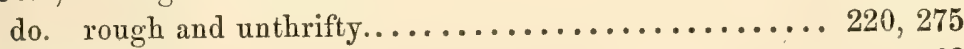

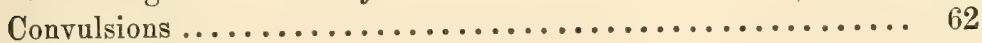

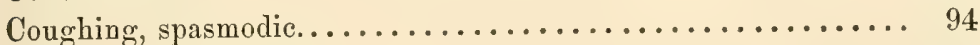

do. and laborious breathing................. 108

do. deep-seated and half suppressed.............. 116

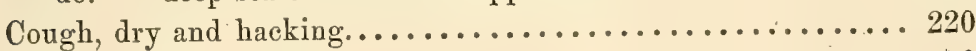

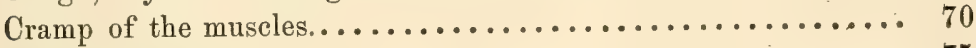

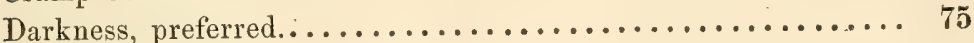

Delirium ............................... 78

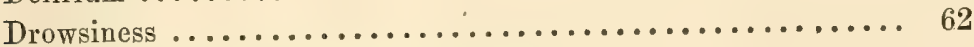

Dung, unusually dark-colored and hard................ 99

do. scanty............................. 120

do. covered with yellow slime................... 220

do. bloody.............................. 297

Ears kept in constant motion, one forward the other backward... 43

do. tips of, cold....................... 97, 208

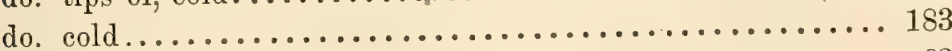

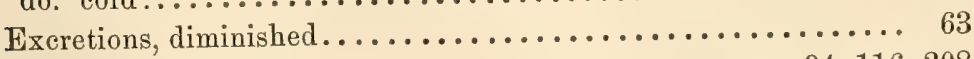

Extremities, cold.................... 94, 116, 208

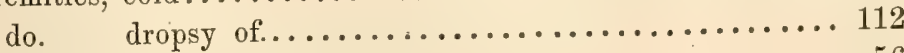

Eye, tenderness in one, with eyelids closed............. 56

do. copious flow of tears from.................... 56

do. white of, slightly red.................... 56

do. anterior chamber of, full of yellow matter........... 56

do. pupil of, dilated................ 59,61, 64, 179

do. staring wildly ....................... 63,196

do. do. as if they would burst from sockets........... 67

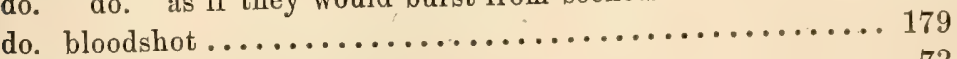

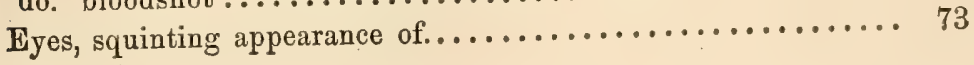


PAGES

Eyes, dull.............................. 75

do. prominent............................. 97

do. glassy and prominent....................... 187

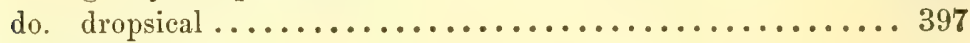

Eyelids kept closed........................... 46

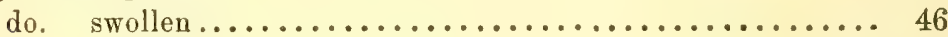

do. internal surface of, red..............46,59, 201

do. profuse discharge of matter from.............. 55

do. closed, with tenderness in one eye............... 56

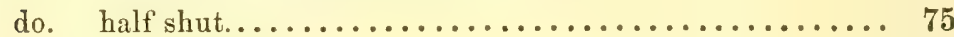

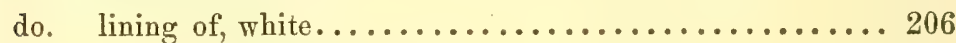

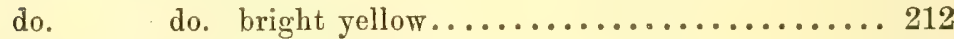

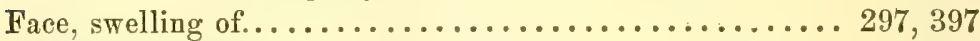

Falling down suddenly on the road................ 64

do. do. with a loud snorting sound.......... 67

Fear and restlessness on approach of heavy vehicle......... 43

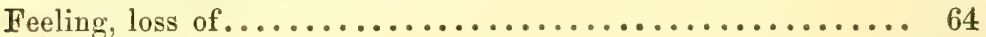

Feed refused........................ 178, 223

Feet, fore ones raised to an unnecessary height in walking..... 43

do. raised very high......................... 75

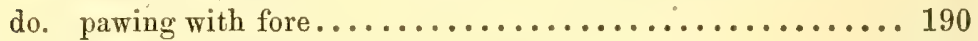

do. fore ones hot and thrown forward............... 332

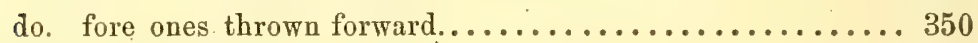

Feverish symptoms...................... 158, 198

Flanks, bedewed with cold perspiration........... 97, 112

do. heaving at the.......................116, 125

do. head turned toward...................... 190

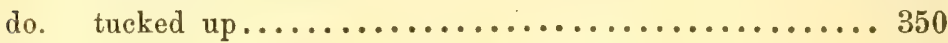

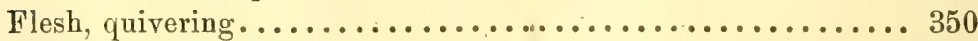

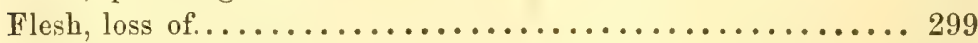

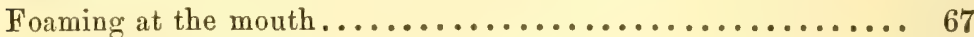

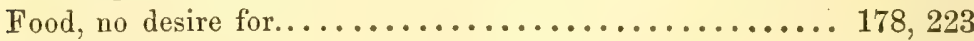

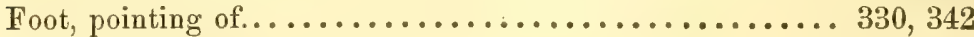

Fore-legs widely separated.................... 116

do. beneath the belly..................... 75

do. in the manger, kicking and tearing............. 78

Gait, heary, slow, and unsteady.............. 75, 289

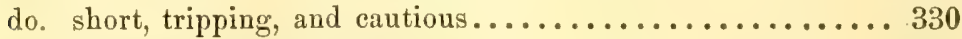

Groaning after passing urine.................... 224

Gums, covered with slime........................ 112

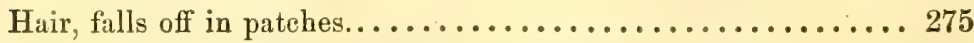

do. do. in circular patches on shoulders and sides....... 291 
PAGES

Hair of neck and legs elevated in blotches.............. 289

do. rough, unthrifty, and not glossy ............ 275, 299

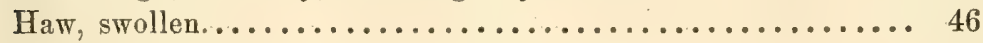

do. shooting above, on raising the jaw.............. 70

Head, depressed and bent to one side.............. 59

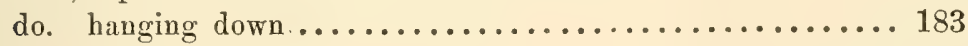

do. held drooping..................... 116, 208

do. kept elevated......................... 43

do. kept near the ground......................... 179

do. reposing on the manger.................... 62

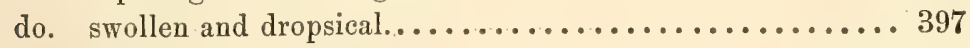

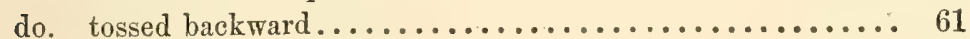

do. turning of to the sides.......................... 120

do. turned to the belly........................... 179

do. turned toward flanks................... 190, 223

do. violently thrust against the rack or wall ........... 62

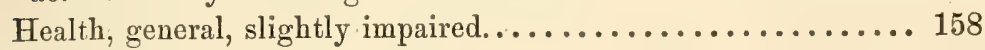

Heart, violent beating of ..................... 206

Heaviness and indolence following suddenly on activity...... 75

Heels, dry, horny scabs on....................... 284

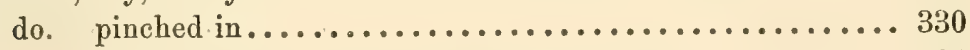

do. ulcers on............................ 284

Hind limbs become suddenly too weak to support the body.... 66

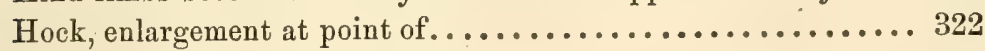

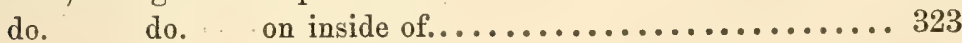

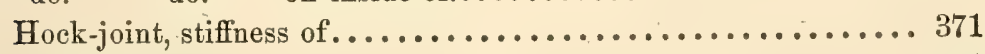

Itching, severe............................ 291

Jaw, swelling under......................... 412

Jugular veins congested........................ 124

do. do. pulsate like an artery................. 124

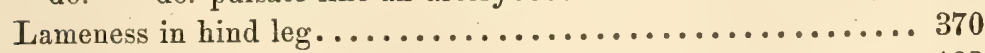

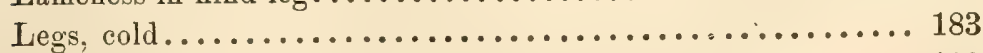

do. hind, dropsical......................... 120

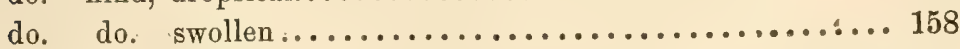

do. do, widely separated .................... 224

do. straddling............................ 223

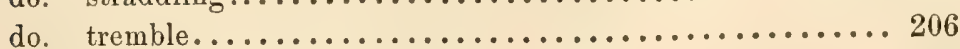

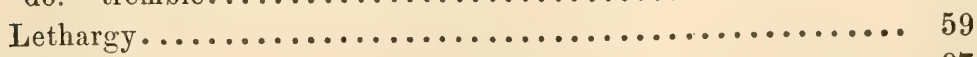

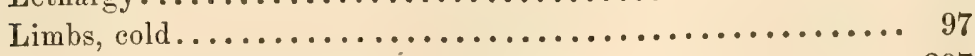

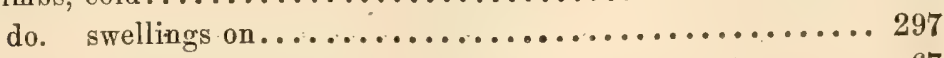

do, violently convulsed................... 67

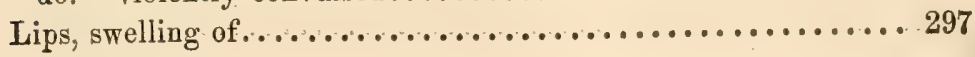


PAGES

Listlessness ............................. 206

Loins, pressure on causes pain and crouching........... 223

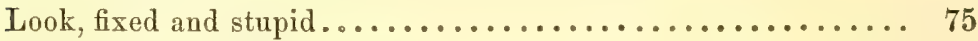

Lying down and soon rising................ 112, 178

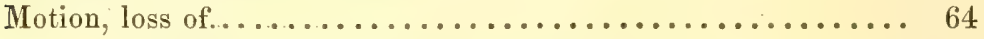

do. tremulous of various parts of the body ........... 64

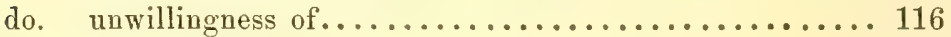

Mouth, bright yellow color of lining of.............. 212

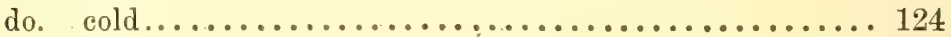

do. dried up............................... 111

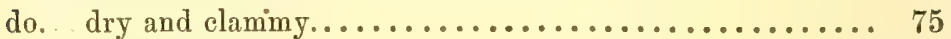

do. foaming at....................... 67,162

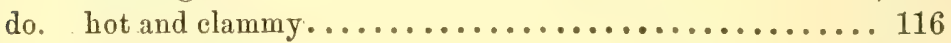

do. hot and dry......................... 201

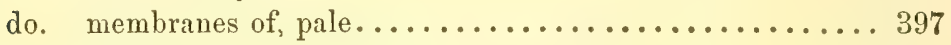

do. yellow tinge on the inside surface of............. 99

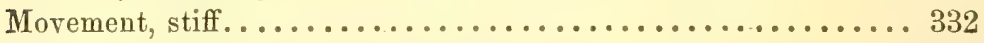

Neck, blotches on, after being driven............... 287

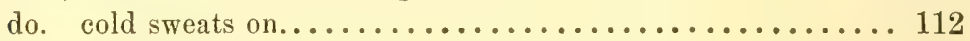

do. muscles of, rigid........................... 73

do. tumor or swelling on nape of................ 293

Nose, bleeding from......................... 112

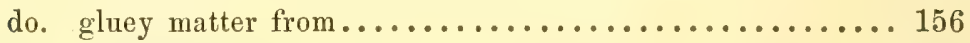

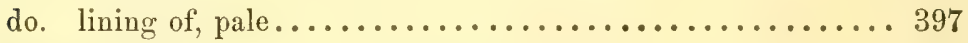

Nostrils, dilated........................... 97

do. discharge from; streaked with blood............ 97

do. mucous discharge from ................... 102

do. redness of lining membranes of.............. 111

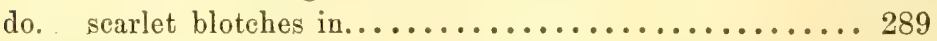

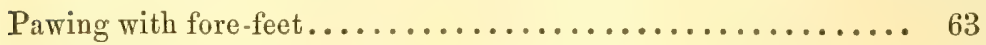

do. do. slightly.................. 198

Perspiration, profuse................... 78,179

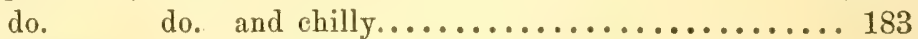

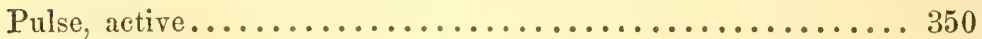

do. feeble............................... 188

do. full, strong, and frequent.............. 111, 183

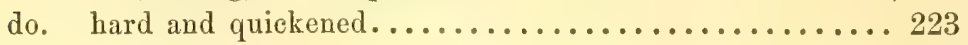

do. nearly gone........................... 94

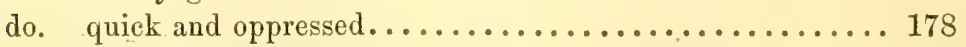

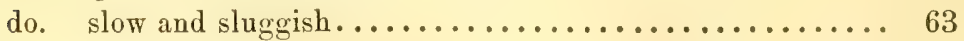

do. small and quick....................... 158

do. small and thready................... 97,187 
Pulse, unnaturally strong........................ 64

do. variable.................................. 116

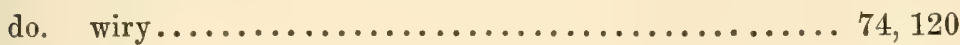

Reeling and falling, on being urged to move........... 59

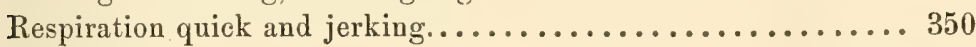

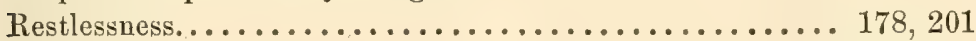

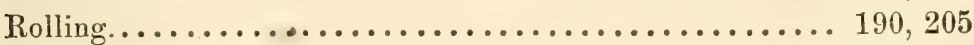

Rolling and tumbling..................... 179, 183

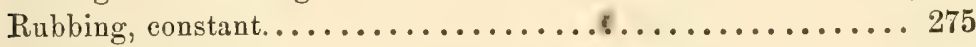

Shivering fit, followed by discharge from nostrils.......... 108

do. do. slight..................... 116, 198

Shoulder, wasting of............................ 324, 330

Sides, blotches on, after being driven................ 287

Skin, coldness of............................ 124

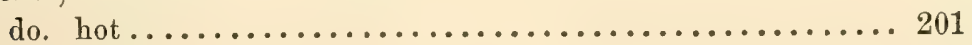

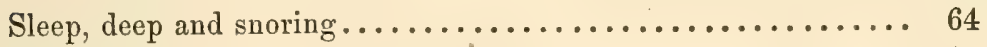

Sleepiness..................... 59, 62, 75, 212

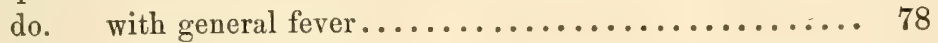

Stamping and pawing continually.............. 193, 205

"Star-gazing"............................. 43

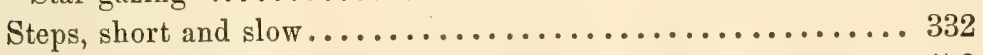

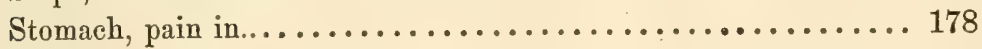

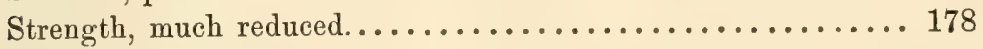

Swallow, loss of power to........................... 64

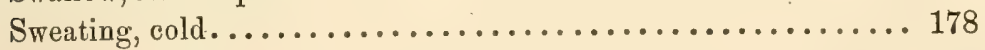

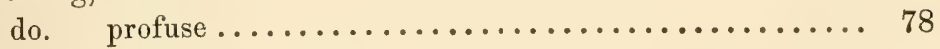

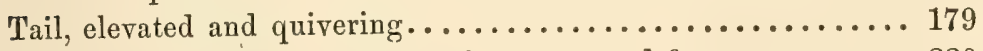

do. great inclination to $\mathrm{rub}$ against posts and fences........ 220

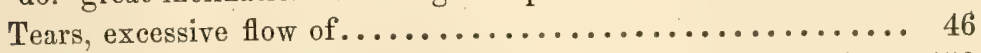

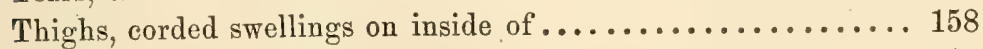

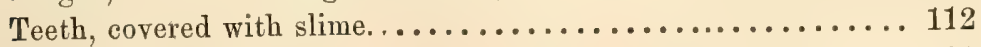

Thirst, great........................ 120, 223, 289

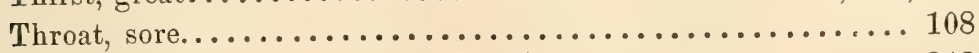

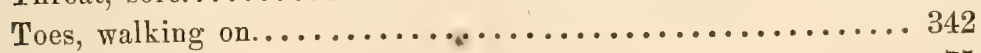

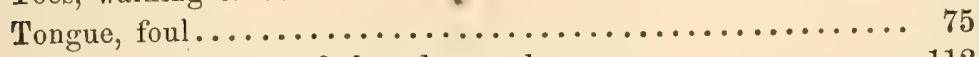

do. has a horny feel, and turns brown............. 112

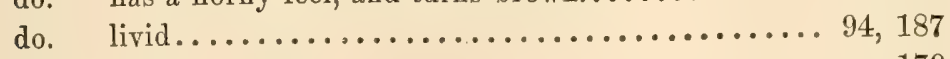

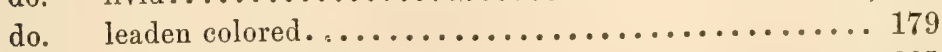

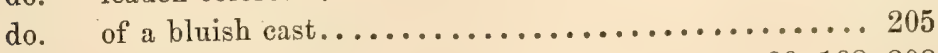

do. slightly coated................. 99, 198, 208

Torpor................................ 61

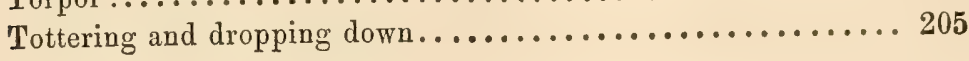


Turning, awkwardness in .......................... 75

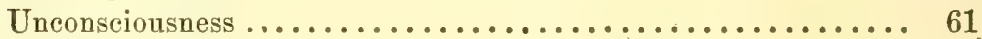

Unsteadiness in moving...................... 208

Urine, of a dark yellow color.................... 99

do. high colored...................... 120, 332

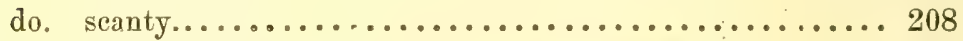

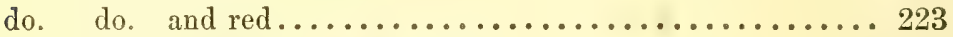

do. frequent and painful discharge of.............. 224

do. light colored, almost transparent..............230

do. has the smell of musty hay ................. 230

do. bloody......................... 237, 297

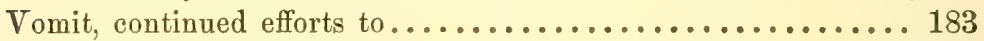

Vomiting, from both nostrils.................... 187

Walking, leaning to one side in...................... 75

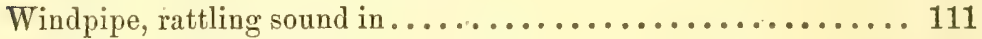

Wind, from the bowels........................... 190

\section{BOVINE DEPARTHENT.}

Abdomen, enlarged and pendulous................. 506 Appetite, loss of ............4 434,444, 447,509, 545, 601 do. morbid, inclining to eat bones, stone, etc........ 572

do. small.............................. 619

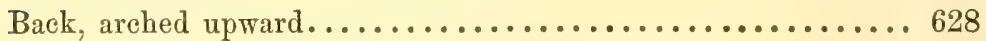

do. slightly roached....................... 533

Bag, inflamed and swollen...................... 517

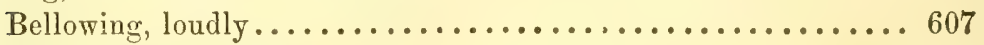

Belly, much enlarged..................... 506

do. enlarged, with great pain in.................. 489

do. pressure upon, produces pain................. 480

do. struck with hind feet.................... 489

do. sudden and severe pain in .................. 480

do. tucked up........................... 480

Blood, from the rectum....................... 482

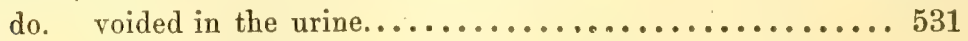

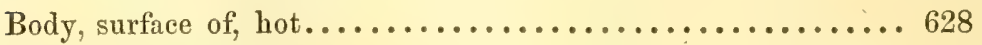

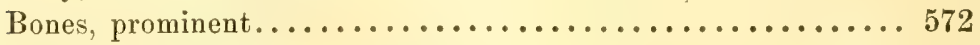

Bowels, constipated..................... 573, 619

do. large, watery discharges from................ 454

do. yellow or greenish do. .............. 483 
Breast-bone, pressure upon, gives great pain............. 545

Chest, sudden and irregular nervous action of the muscles of.... 606

Coat, staring............................ 601, 649

Convulsions................................6. 641

Cough, painful.............................. 455

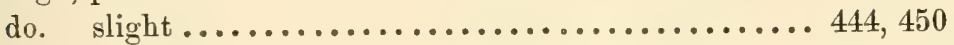

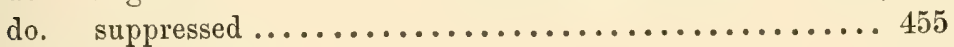

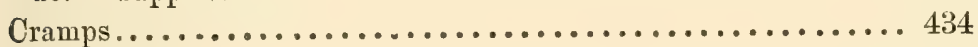

"Cud lost"................................613

Debility............................ 450, 506

Difficulty, in rising........................... 506

Dullness...........................4434, 533, 601

Dung, hard............................... 480

do. do. and black............................6. 619

do. lighter colored than usual................... 628

do. offensive odor from........................ 449

do. streaked with blood....................... 480

Ears, hot, drooping and moist................... 628

Emaciation ............................. 450

Expectoration............................. 450

Eyeballs protrude ("stick out") ...................6 607

Eyelids, much swollen........................ 454

do. of one eye irritated and swollen $\ldots \ldots \ldots \ldots \ldots \ldots \ldots . . . . .559$

Eyes, pain and tenderness of.................... 558 do. visible surfaces of, highly reddened.........545, 607, 641

do. whites of, turned yellow.................... 613

do. wild look from.......................... 509

Feet, cold.............................. 444

Fever.............................. 450, 455

Feverishness ..............................613

Fits, epileptic, accompanied by loud bellowing and pawing the

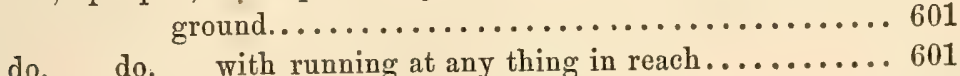

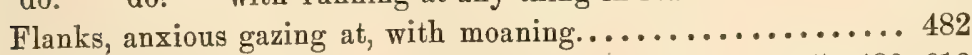

do. heaving at.................... 447, 480, 619

do. left one swollen and hard.................. 471

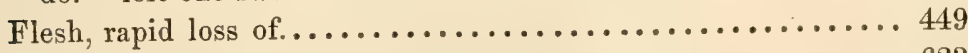

Foot, discharge of bad smelling matter from............. 623

Frantic.....................................6 600

Frenzy................................6 600

Giddiness and nervousness........................6 602

Grunting, or moaning on being backed................ 455 
Gums, eruptions on $\ldots . . . . .464$

do. swollen and black....................... 654

Hair, coarse and standing straight up............... 572

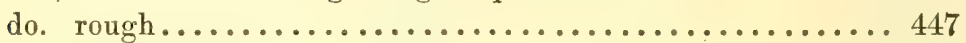

do. watery fluid dribbling from.................. 628

Head, dashed recklessly on the floor................ 509

do. directed toward region of the heart............. 545

do. drooping of......................... 434

do. pressed forcibly against a wall................. 601

do. thrust forward.......................... 444

do. turned toward liver...................... 613

Heart, deadened sound on applying the ear over........... 551

do. increased force of the beating of .............. 515

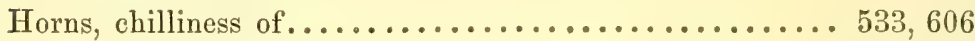

do. heat about the base of............. 551, 613, 628

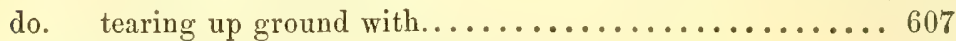

do. trying to injure every thing with............. 607

Jaw, (lower) enlargement at the angle of............... 581

Jaws, angle of, gradual enlargement at.............. 583

do. compressed........................... 434

Jugular veins engorged................... 544, 545

do. do. undulating or "wavy" motion on.......... 545

Kidneys, pressure over region of, causes pain............ 533

Legs, cold.....................444,447,601

do. fore ones, sudden nervous action of muscles..........666 606

do. do. trembling.................... 455

do. do. wide apart................. 444, 455

do. tremulous ............................ 480

Lie down, refusal to................... 444, 455

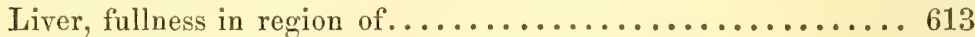

Milk, quantity diminished,.....................661

Motion, gives distress........................ 544

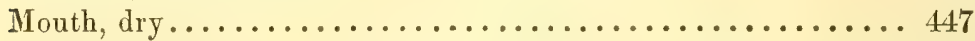

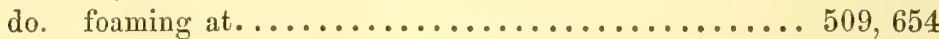

do. hot and clammy.................. 444, 509

do. hot and feverish.................... 551, 613

do. increased flow of saliva from ............ 454,628

do. lining membrane of, pale and watery............ 506

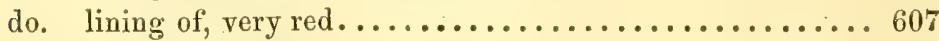

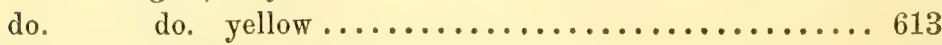

Muscles, nervous and spasmodic twitches of............ 509

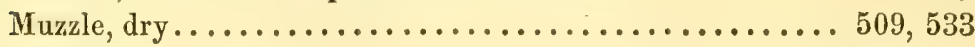


Neck, stiff.................................. 449

Nervous action............................ 641

Nose, glairy discharge from.................... 447

do. lining of, pale and watery................. 506

do. do. reddened.................447, 545, 607

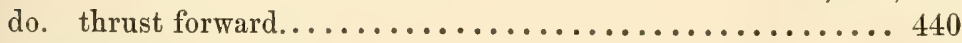

Nostrils, food returned by the................... 452

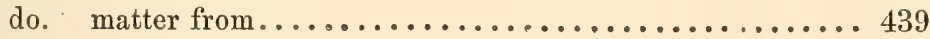

Pain, severe and constant, in the bowels.............. 482

do. do. upon pressing breast-bone.............. 545

Pawing.................................607

Perspiration, profuse, following a fit............... 601

Pulse, feeble and oppressed..................... 544

do. increased force................... 545, 613

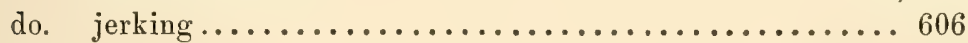

do. quick...................... 444, 447,454

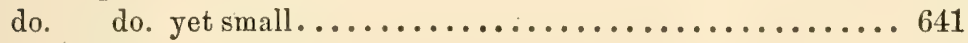

do. violent, abrupt, and strong................. 551

Restlessness............................. 489

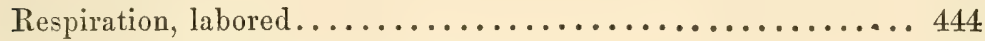

Ribs, pressure between, gives great pain............. 455

Rigors, followed by acute inflammatory fever........... 545

Rumination, ceased.............480, 482, 545, 551, 613, 649

Separation from the herd......................6. 602

Shivering fits.........................444, 628

do. followed by inflammatory fever............. 545

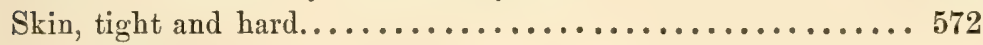

do. yellow..................................619

do. watery bladders formed on ................... 628

"Slobbering".............................. 628

Snorting................................ 447

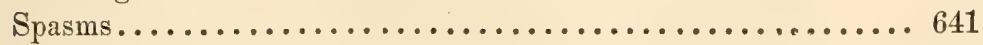

Spinal column curved in downward direction........... 506

Staggering........................4 434, 480,649

Stiffness............................ 434, 654

Stomach, distended with gas................... 454

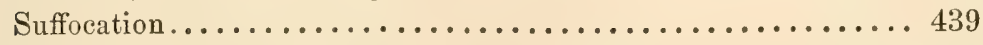

Surface of the body chilly................. 533, 606

Swallowing, loss of power of............... 452, 607

Tears, running down the face................ 454, 558

Teeth, grating of.................... 509, 601

Thinness................................ 450 
Thirst, intense......................... 607,619

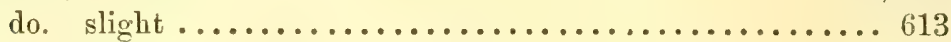

Throat, glands of, enlarged................. 449,659

do. soreness of........................... 447

do. swollen and tender...................... 440

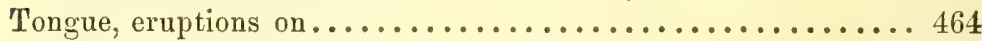

do. much swollen................... 454, 628

do. slightly coated....................... 50 09

do. swollen and coated with brown fur............. 641

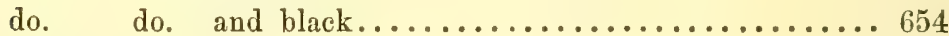

Trembling........................... 434, 641

Tremblings and twitchings, violent...................601

Tumors on various parts of the body, giving, when pressed, a

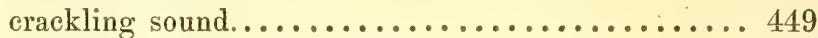

Udder, swollen, hot, and tender............. 509, 517

Urine, black.............................. 535

do. bloody........................ 434, 531

do. difficulty in passing................... 533

do. flow of, suddenly arrested, with trembling of hind legs... 535

do. high-colored ............................ 641

do. reddened ............................. 533

do. scanty and dark-colored..................... 628

do. yellow .............................. 619

Walking, crackling noise heard while................ 572

do. with stiffened joints................... 572

Wasting away............................. 450

Wind, from the anus......................... 489

\section{DISEASES OF SHEEP.}

Anus, inside of, turned yellow................... 710

Bloated appearance of body........................ 716

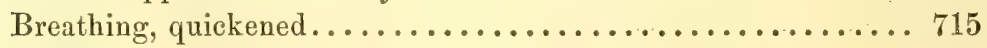

Cough................................... 715

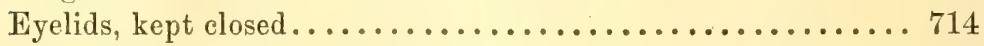

Eyes, tears from lower corner of................... 714

do. whites of, reddened......................... 714

do. do. turned yellow..................... 710

Flanks, heaving at.............................. 715

Head, frequent shaking of...................... 710 
PAGES

Head, pressed against fence or post................. 710

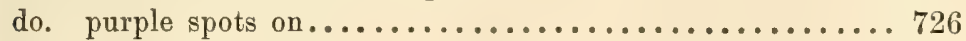

Lameness, in fore or hind legs................... 712

Lazy movements, with nose almost on the ground......... 710

Legs, hot, tender, and swollen, with offensive discharge from ... 712

Light, painful to the eyes..................... 714

Nose, discharge from.......................... 715

do. lining of, turned yellow................... 710

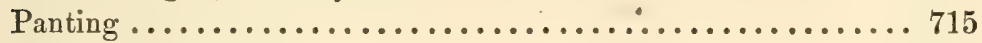

Skin, turned yellow............................ 710

Sneezing................................... 715

Teeth, grating of............................ 710

Thighs, insides of, turned yellow................ 710

\section{DISEASES OF SWINE.}

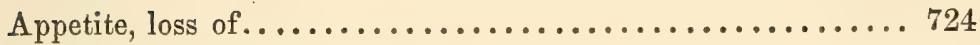

Breathing, quick and difficult..................... 724

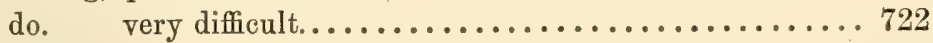

Cough .................................... 724

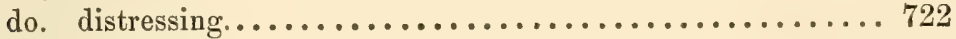

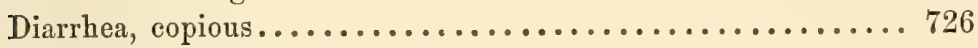

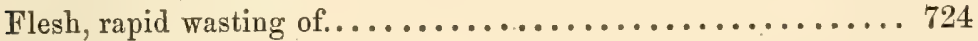

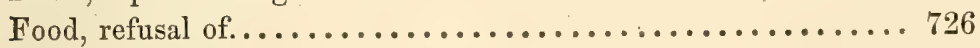

Mouth, frothing at, as if choking................... 722

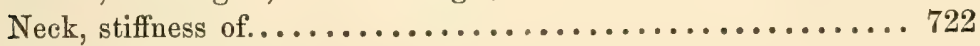

Nose, purple spots on........................... 726

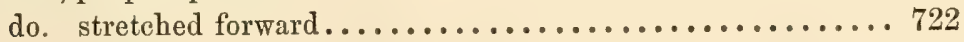

Shivering fits.............................. 724

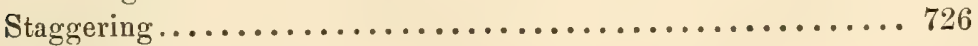

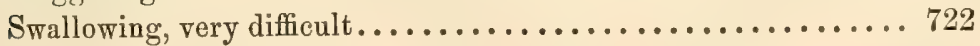

Thirst, great................................. 726

Throat, swollen.............................. 722

Vomiting ................................ 726

Walking, falling down in ...................... 726

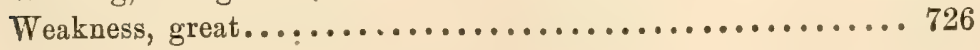




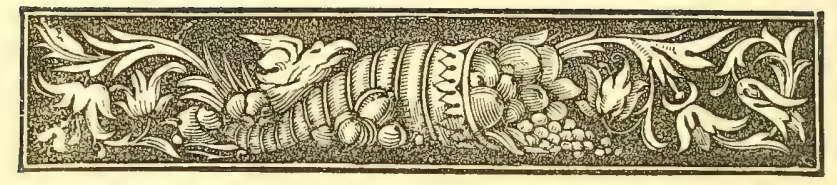

\section{TABLES OF WEIGHTS AND MEASURES.}

\section{APOTHECARIES' WEIGHTS.}

20 grains make one scruple, marked............

3 scruples do. drachm, do. ............ 3j.

8 drachms do. ounce, do. ........... 3 j.

12 ounces do. pound, do. ............. Ibj.

\section{IIQUID MEASURES.}

60 drops make one drachm, marked............ 3j.

8 drachms do. ounce, do. ............ 亏̋j.

20 ounces do. pint, do. ............. Oj.

8 pints do. gallon, do. ............. Cong.

\section{ORDINARY MODES OF MEASURING IIQUIDS,}

A tea-spoonful of liquid equals 60 drops, or one drachm.

A table-spoonful of do. $\frac{1}{2}$ ounce, or four drachms.

A dessert-spoonful of do. 180 drops, or three drachms.

A wine-glassful of do. $1 \frac{1}{2}$ ounces.

The doses vary in quantity in different animals. The amount to be given in any case will be found clearly stated in the treatment of each disease, whether of horses, cattle, sheep, or hogs.

(768) 


\section{GLOSSARY}

OF

\section{MEDICAL AND SCIENTIFIC TERMS.}

ABNORMAL-Irregular.

Absorbents-Medicines used for absorbing; also the vessels of the body which suck up.

ACUTE-Sharp, severe.

Adipose-Fatty.

ADYNAMIC-Debilitated.

AlaE-Wings.

Alteratives-Medicines which change a disease for the better.

Anasarca-Dropsy of cellular membrane.

ANASARCOUS-Dropsical.

Anæmina-Bloodlessness.

Antiseptics-Medicines opposed to putrefaction.

Antispasmodics-Remedies opposed to spasms or convulsions.

Antiphlogistic-Opposed to inflammation.

Aperients-Medicines which open the bowels gently.

Aqueous-Watery.

Ascites-Dropsy of the belly.

AtAXIC-Disordered.

Auscultation-Examination by sounding and listening.

Autopsr-Post-mortem examination.

Bifdrcation-Division into two branches.

Bolus-A large pill.

Buccal Membrane-The lining of the mouth.

Canthus-Corner of the eye.

Capsule-Shell or case. 
Carbonaceots-Containing carbon.

Carminatives-Medicines which relieve pain by expelling wind from the bowels.

Cartilaginous-Composed of cartilage.

Cathartic-Loosening.

Celdular-Cell-like.

Cerebeluum-The brain.

Cerebrum-The brain.

Chancrous-Cancerous.

Clinical-Relating to individual practice.

Coмr-Stupor.

Conatose-Stupefied

Conjunctivial Membrane-The membrane which lines the eyelids and covers the eyeball.

Connea-Transparent coat of the eye.

Cranial-Pertaining to the skull.

CraniUM-Skull.

Crucial--Shaped like a cross.

Dedarbonize-To purify by air.

Diagnosis-The art of telling the nature of diseases.

DiAPHORETICS-Medicines which promote perspiration.

Diathesis-Predisposition to certain diseases.

Dietetics-Regulation of diet.

Diuretics-Medicines which increase the flow of urine.

DucT-C̣anal.

DrNamic-Relating to the vital forces.

Emollients-Substances used to reduce inflammations.

Emphysema-Distention by gas or wind of certain portions of the body.

EMUNCTORIES-Organs which carry off waste matters.

ENCEPHALON-The brain.

EneMa-Injection.

Enzootic-Endemic diseases among animals.

Erizootic-Epidemic among animals.

Equilibrium-Balance.

Equine-Relating to the horse.

EтIOLOGY--The doctrine of the causes of disorders.

Excrementitious-Useless.

EXCRETORY-Relating to vessels which throw off useless matter.

Extravasation-Escape of a fluid of the body from its vessel into surrounding parts. 
ExUdation-Oozing through a membrane.

FAdOES-The throat.

FleAM-Used in bleeding.

Graminivorods-Feeding on grass.

Hematosine-The red coloring matter of the blood.

HEMORRHAGE--Bleeding.

Hippiatric-Relating to diseases of the horse.

HistoLogy-General anatomy.

HYdrocephaLUS-Water in the head.

HyGIENE-Preservation of health.

IcHoRous-Humory.

IDIopathic-Primary affections.

IDIOSYNCRASY-Peculiarity of constitution.

INDURATED-Hardened.

INGUINAL-Belonging to the groin.

INTERCOSTAL-Between the ribs.

INUNCTION-The act of rubbing in.

LACHRymaL Glands-Those which secrete tears.

Lancinating-—"Shooting."

LAXATIVES-Loosening medicines.

LESION-Disorder.

LIGAMENT-The substance which joins bones together.

Mamme-Breasts.

Masseters-Muscles of the jaws.

Morbid-Diseased.

Morbific-Producing disease.

NavicdLAR-One of the bones of the foot.

NEURO-PATHOLOGY-The nervous system in disease.

NodUlous-Like a knot.

NosoLogy-Classification of diseases.

EDematous-Swollen.

OPaque-Not transparent.

Os CALCIS-Bone of the heel.

Osseous-Bony.

Ovold-In form of an egg. 
Palatine-Relating to the palate.

Panzootic-An epidemic affecting animals generally.

Parasite-An animal which lives on another.

Parotid-Largest salivary gland.

Pathologr-The study of the body in disease.

Pedicle-Narrow part of a tumor.

Petechial-Resembling flea-bites.

Phthisis-Wasting away.

Pituitary Membrane-Lining of the nostrils.

Pseudo-membranous-Relating to false membranes.

Pus-Matter.

Pylorus-Entrance into intestines.

RALE-A watery sound heard in sounding the chest in some diseases,

Sanative-Health-giving.

Schneiderian Membrane-The lining of the nostrils.

Sebaceous-Of the nature of suet.

Sedatives-Medicines which produce sleep.

Serous-Watery.

Serum-Watery part of the blood.

- Solvent-That which dissolves.

Sporadic-Scattered.

Submaxillary-Beneath the jaw.

Sudamina-Small eruptions.

SUPra-Renal-Above the kidney.

Thoracic-Relating to the chest.

Thy roID-Shaped like a folding door.

Tonics-Medicines which give tone and strength to the body.

TUbercular-Relating to tumors in the structure of an organ.

Tumefaction-Swelling.

Turbinated Bones-Bones of the nose shaped like a top.

Turgescence-Great amount of humors in any part.

VASCULAR-Full of blood-vessels.

VENTRICLE-Cavity.

Virus-Poison.

VIS A FRONTE-Force from the front.

VIS A TERGO-Force of propulsion.

Viscous-Sticky.

VOICE-BOX-Larynx. 


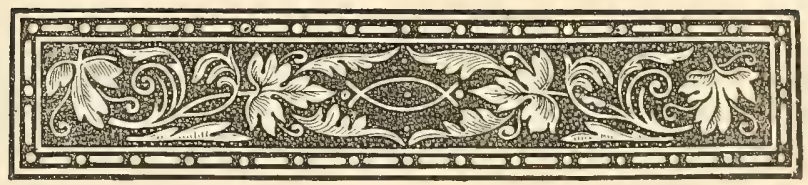

\section{IN DEX.}

\section{EQUINE DEPARTHENT.}

A

Abdominal cavity, wounds of.

Abscess within the substance of the

brain

Absorbents, disease of.

Abstraction of blood.

Acute cough

Acute disease of the laminge of the foot.

Acute disease of the eye.

Acute rheumatism

PAGES

206

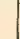

58

156

268

99

350

46 333

Acute and general disease of the brain, known as encephalitis.

Advantages of in-and-in breeding. 315

Affections, typhoid.................... 110

Age of horse, as known by his teeth. 127

Albuminous urine..................... 235

Amaurosis ............................ 40

Amputations .......................... 260

Amputation of the penis............. 261

Anæmia................................ 397

Analysis of the blood in glanders. 25

Anatomy of the lungs............... 90

Anatomy and physiology, importance of studying.............. 315

Anatomy of the tail.................... 394

Animals become parents too early. 312

Apoplexy, cerebral................... 64

Apoplexy of the spine............... 65

Apoplexy of the spleen............... 206

Artereotomy .......................... 268

Ascites, or abdominal dropsy....... 199
PAGES

Atrophy, or wasting of muscles in the region of the shoulderblade. 324

\section{B}

Back, galled......................... 400

Big head and big jaw................ 362

Bladder, inflammation of........... 224

do. stone in..................... 224

do. rupture of................... 232

Bleeding, various modes of......... 268

Blistering............................ 263

Bloody urine........................... 237

Blood, the quantity found in the body........................... 402

Blood, its transfusion................ 404

Bog spavin......................... 323

Bots, stomachic and hemorrhoidal. 164

Bowed legs........................... 381

Brain, abscess within its substance. 58

do. dropsy of..................... 60

do. acute disease of............... 76

Breeding, and the principles of the same ............................ 302

do. in-and-in ............... 307

Brief exposition of the function of the lungs.................... 88

Bronchocele.......................... 103

C

Capped hock......................... 322

Case of laryngitis in Patchen colt.. 94

(773) 


\begin{tabular}{|c|c|}
\hline $\begin{array}{r}\text { PAGES } \\
\text { PAG }\end{array}$ & PAGES \\
\hline Case of croup...................... 97 & Digestive organs, disease of........ 160 \\
\hline Case of pleurisy and dropsy of the & Disease, causes of.................... 19 \\
\hline chest ......................... 120 & do. hereditary causes of........ \\
\hline Case of meteorization................ 186 & do. cases of.......................... \\
\hline Casting, mode of..................... 241 & do. of the urinary organs..... 223 \\
\hline Castration, different methods of.... 243 & Diseases of the skin and sub-tis- \\
\hline Cataract............................... 45 & sues............................ 274 \\
\hline Catarrh, epizoötic................... 106 & Dislocation of the stifle.............. 343 \\
\hline Causes of disease.................... 19 & Division of the flexor tendons...... 259 \\
\hline Cerebral hemorrhage............... 64 & Docking of the tail.................. 395 \\
\hline t on polypus..................... 102 & Dropsy of the brain................... 60 \\
\hline $\log \ldots \ldots \ldots \ldots \ldots \ldots \ldots \ldots \ldots \ldots \ldots \ldots \ldots \ldots \ldots$ & do. of the abdomen............. 199 \\
\hline Chorea, equine........................... & Dropsical limbs....................... 287 \\
\hline Chronic rheumatism................. 336 & Dun, F. on causes of hereditary \\
\hline Circulation of the heart.............. & 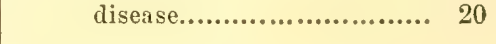 \\
\hline
\end{tabular}

do. experiments on its rapidity...

E

Colic, flatulent........................ 189

do. spasmodic.................... 193

do. treated by inhalation.......... 195

Composition of the blood of a healthy horse................. 25

Composition of the blood glandered 25

Condition, out of..................... 299

Congenital glanders................. 24

Contagiousness of glanders......... 151

do. of strangles........ 413

Contraction of the hoof.............. 344

Copeman on treatment of inflammation

Copeman on meningitis............. 80

Cornea, opacity of.................... 44

Corns ................................ 384

Cough, in its chronic and acute stage ......................... 99

Croup, (cynanche trachealis)....... 95

Curry-comb, use and abuse of...... 397

Curbs............................... 319

Curing diseases, the best method of. 26

Curvation and disease of the spine. 361

Cystitis............................... 224

\section{D}

Death of a horse from ruptured stomach ........................ 179

Dentition ............................ 127

Diabetes ............................... 229

Diet................................... 138

do. changes in, recommended..... 141 Foot, management of, in the stable. 409

Education, importance of.......... 32

Elbow tumor......................... 321

Encephalitis........................... 76

Enteritis ........................... 201

Enlargement of the thyroid glands. 103

Epilepsy............................... $66^{\circ}$

Epiglottis, spasm of................. 90

Epizoötic catarrh................... 106

Equine chorea, or stringhalt........ 81

Experiments by M. Magendie on the brain and nervous system.......................... 83

Experiments on the itch insect.... 277

Eye, acute disease of................ 46

do. worm within.................. 45

do. treatment of inflammation (Copeman on)................ 47

Eyelids, foreign bodies within..... 44

\section{F}

Farey................................. 156

Fever, typhus..................... 110

Films, or specks on the eye......... 44

Firing .............................. 262

Fistulous withers................... 295

Fits, epileptic........................ 66

do. hereditary origin of.......... 67

Flatulent colic....................... 189

Flexor tendons, division of.......... 259

Food, effects of various kinds....... 137

do. quality required............... 138 
PAGES

Foreign bodies within the eyelids.. 44

Founder, bleeding for the same.... 37

Fracture of the pelvis................ 376

Functional disease of the liver..... 211

Function of the pancreas............ 216

Fundament, falling of............... 387

\section{G}

Galled back.

Gamgee on splenic apoplexy........ 206

Gastritis ............................. 178

Giddiness, or vertigo................ 74

Glands, thryoid, enlargement of... 103

Glanders.

do. congenital................. 24

do. analysis of the blood..... 25

do. can be communicated to man..................... 147

do. contagiousness of......... 151

do. why the disease is so called .................... 155

Glass eye............................ 40

Glottis, spasm of.................... 90

Gohier on polypus..................... 103

Goiter .................................. 103

Gorged or over-distended stomach.. 182

Grease................................. 283

do. nature of....................... 23

do. transmission of from horse to $\operatorname{man} . . . . . . . . . . .6 . . . . . . .285$

Gutta serena.

\section{$\mathrm{H}$}

Hæmaturia .......................... 237

Heart, its circulation................. 401

Heaves .............................. 125

Heels, disease of..................... 283

Hemorrhage, cerebral................ 64

do. $\quad$ spinal .................. 65

Hemorrhoidal bots................... 164

Hemorrhoids, or piles................ 391

Hereditary origin of epileptic fits. 66 do. causes of disease....... 20

Herpes............................... 299

Hide-bound............................ 347

Hock, capped....................... 322

Hoof, contraction of.................. 344

Hoof-bound............................ 347

Horses, lice on......................... 282
Horse's foot, management of, in the stable.......................... 409

Hydrocephalus ...................... 60

\section{I}

Importance of ventilating stables, in view of preventing diseases of the lungs............. 86

In-and-in breeding................... 307

Inflammation, nature of............. 38

do. its nature and treatment................ 35

do. treatment of......... 47

do. of the lungs........... 114

do. of the pericardium.. 123

do. of the stomach....... 178

do. . of the peritoneum... 197

do. of the intestines.... 201

do. of the bladder......... 224

do. of the kidneys........ 223

Inflammatory laryngitis............ 92

Influenza, or epizoötic catarrh..... 106

do. followed by acute disease of the brain ....... 108

Inhalation, colic treated by the

same ........................... 195

Intestines, strangulation of........ 205

Intestinal canal, parasites infest-

ing the...................... 217

Iodine, Mörton on.................... 105

Itch.................................. 274

do. insect, experiments on......... 277

K

Kidneys, inflammation of........... 223

Knees, sprung..................... 381

L

Lameness from various causes...... 319

do. navicular ................. 330

do. remarks on............... 385

Laminitis........................... 350

Lampas................................ 160

Laryngitis, suppurative and in-

flammatory .................. 92

Leblane, M., on tracheotomy........ 100

Legs, bowed......................... 381

do. swelled.......................... 287

Lice on horses...................... 282 


$\begin{array}{lr} & \text { PAGES } \\ \text { Limbs, dropsical..................... } & 287 \\ \text { Lithotomy, method of performing... } & 252 \\ \text { Liver, functional disease of........ } & 211 \\ \text { Locked-jaw........................ } & 70 \\ \text { Lungs, anatomy of................. } & 90 \\ \text { do. function of................... } & 88 \\ \text { do. inflammation of............. } & 114\end{array}$

\section{M}

Magendie's experiments on animals

Malignant typhus

Management of the horse's foot in the stable. 409

Mange 274

Meningitis, Copeman on............ 80

Meteorization of the intestines...... 186

Morton on iodine.

105

N
Navicular lameness.................
Navicularthrites....................
Nephrites...........................
Neurotomy, mode of operating.....
Nicking ............................
do. the tail......................
Nitrate of potass, its uses..........
Nostrils, polypus within............

\section{0}

Objections to in-and-in breeding answered...

Eisophagotomy

Estrus equi.

Estrus, hemorrhoidalis.

Opacity of the cornea.

Operations, surgical.

Ophthalmia

do.

$$
\text { purulent. }
$$

do. specific

Osteo porosis, known as big head and big jaw

Out of condition

\section{$\mathrm{P}$}

Pancreas and its function 216 Paraplegia............................. 65 Parasites infesting the intestinal canal.
Patchen colt, laryngitis, case of... 94

Pelvis, fracture of................... 376

Penis, amputation of.................. 261

Peritonitis ........................... 197

Pericarditis ............................ 128

do. Woodger on.............. 124

Peritoneum, inflammation of........ 197

Periosteotomy ....................... 258

Phlebotomy........................... 270

Piles, or hemorrhoids................. 391

Pleurisy ............................... 119

do. and dropsy of the ehest, case of .................... 120

Pleurodynia, painful affection of the pleura and muscles of the chest...................... 332

Poll-evil .............................. 292

Pneumonia ......................... 114

Polypus within the nostrils........ 101

do. Chabert on................... 102

do. Gohier on..................... 103

Pricking the tail....................... 392

Prolapsus ani (falling of the fundament) ...................... 387

Prurigo............................... 291

Pure water needed by horses and cattle .......................... 398

Purulent ophthalmia................ 55

\section{Q}

Quantity of blood in a horse's body. 402 Quarter crack....................... 352

\section{R}

Remarks on veterinary science.... 19

Remedy for tape-worm.............. 221

Retention of urine................... 229

Rheumatism, acute.................. 333

do. chronic................. 336

Ring-bone............................. 337

Ring-worm ........................... 291

Roaring............................... 100

Rowelling............................. 266

Rupture of the bladder............... 232

do. of the stomach............. 178

\section{S}

Saliva, amount of secretion........ 163 217 Salivation, spontaneous slavering.. 162 


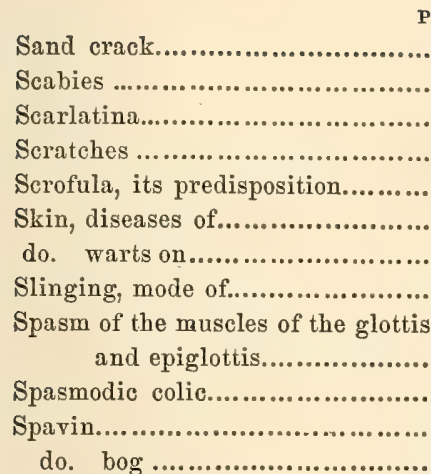

inciples of breeding...

Thorough-pin ......................... 328

Toe sand-crack......................... 354

Trachealis, cynanche................ 95

Tracheotomy ........................ 254

Specific ophthalmia................. 56

Spinal hemorrhage................... 65

Spine, disease and curvature of.... 361

Splent ................................. 378

Splenic apoplexy..................... 206

Sprung knees.......................... 381

Stables, importance of ventilating. 86

Staggers, Coleman on................ 63

Stifle out, dislocation of the stiflebone

Stomachic bots....................... 164

Stomach, gorged..................... 182

do. inflammation of............ 178

do. rupture of................. 178

do. staggers.................. 62

Stone in the bladder................. 224

Stringhalt .......................... 81

St. Vitus's dance.................... 81

Strangles .............................. 411

do. contagiousness of......... 413

Strangulation of the intestines...... 205

Suppurative laryngitis.............. 92

Suppression of urine................ 226

Surgical operations................... 239

Surfeit................................ 286

Sweeny................................ 324

Swelled legs........................ 287

\section{T}

Tail, anatomy of...................... 394

do. docking of....................... 395

do. nicking .......................... 392

Tape-worm, remedy for.............. 221

Temporary teeth, remarks on....... 128

do. Leblanc on............. 100

Transfusion of blood.................. 404

Tumor of the elbow................... 321

Twitch, use of........................ 239

Typhus, or typhoid affections....... 110

do. malignant .................. 113

Tympanitis.......................... 188

\section{U}

Urinary organs, disease of.......... 223

Urination, profuse................... 229

Urine, suppression of................ 226

do. retention of................... 229

do. albuminous................... 235

do. bloody ......................... 237

Use and abuse of the curry-comb.. 397

\section{V}

Vapor bath........................... 114

Varix, or bog spavin............... 323

Ventilation, importance of.......... 86

Vertigo................................ 74

Veterinary science, remarks on.... 19

do. science, the relation it bears to social science .................. 27

do. science, how to inaugurate it................ 30

do. education, importance of.................... 32

W

Warts on the skin.................... 295

Water, necessity of its purity...... 398

Withers, fistulous..................... 295

Woodger on pericarditis............. 124

Worms................................. 217

Worm within the eye............... 45

Wounds penetrating the abdominal cavity .......................... 206 


\section{BOVINE DEPARTMENT.}

A

PAGES

Abortion in cows...................... 522

Abscess at the angle of the jaw.... 583

After-birth, removal of............... 497

Anthrax.............................. 648

Aphthæ................................ 464

Apoplexy, pulmonary................ 458

B

Bile, its secretion and uses......... 612

Bladder, description of.............. 530

do. calculi within............... 534

Black water........................... 535

Black leg...........................6 648

do. tongue........................ 654

Bloat..................................... 468

Bone disorder....................... 576

Bones, their mechanism, structure, and composition............. 580

do. fracture of.................... 655

do. ulceration of................. 585

Bowels, inflammation of............. 480

do. spasm of.................... 490

do. constipation of.............. 491

Brain, inflammation of.............. 600

Bronchitis............................... 442

Bronchial tubes, description of..... 442

Bronchocele......................... 659

\section{C}

Cachexia ossafragia (the cripple).. 568

Calculi in the bladder................ 534

Calves, diarrhea in .................. 658

Cancer of the eye...................... 560

Caries, or ulceration of bones...... 585

Castration............................. 631

Catarrh, common..................... 447

do. epizoötic .................. 449

Cattle, dentition of.................. 475

do. plague, remarks on........... 668

do. do. in Kentueky.......... 433

Cerebral parasites................... 601

Chapped teats and chafed udder... 520

Cheap fodder for cows................ 648

Chronic diarrhea...................... 484

Cleansing, or removal of the afterbirth 497

Coats of the eye.................... 554

Colic, flatulent...................... 489

Constipation of the bowels........... 491

Constriction at the neck of the

uterus......................... 503

Consumption ........................ 450

Contagious pleuro-pneumonia..... 419

Cords in young calves............... 644

Cows, abortion in.................... 522

do. spaying of..................... 661

Cracks in the heels................... 629

Croup ................................. 439

Cud, loss of.......................... 478

$\mathrm{D}$

Dentition of cattle................. 475

do. synopsis................. 477

Description of the larynx........... 441

do. of bronchial tubes..... 442

do. of the pharynx......... 453

do. of the pleura........... 455

do. of oesophagus........... 457

do. of the stomach.......... 465

do. of the urinary sys-

tem................... 529

do. of the heart............. 540

do. of the eye............... 533

do. of the liver.............. 611

Dilatation of the heart.............. 544

do. of the jaw-bones......... 581

Diarrhea.............................. 483

do. chronic.................... 484

do. in calves................... 658

Diseases of the generative organs... 503

do. of the eye and its mem-

branes.................... 553

do. of the bones................ 568

do. of the brain................6 600

do. of the liver................. 611

do. of the skin.................. 620

do. of the udder, teats, etc.... 517

do. of the urinary organs..... 529

Diseased thymus gland............. 643

Disorder of bone..................... 576

Distension of the rumen with food. 471

Dropsy of the womb................. 506 


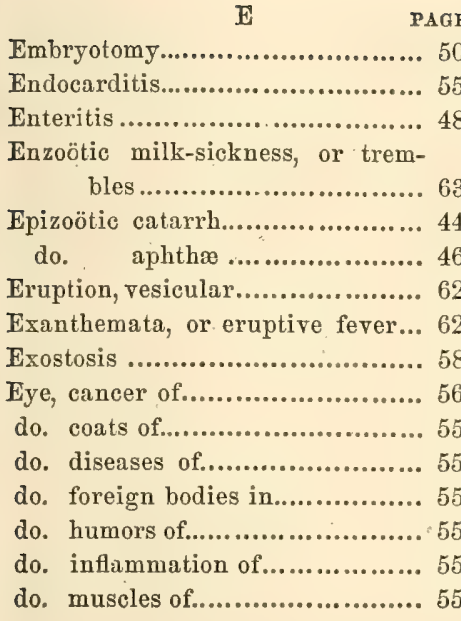

\section{F}

Falling of the womb................ 513

Fever, puerperal..................... 507

do. eruptive ....................... 620

Flatulent colic....................... 489

Food, ingestion of.................... 466

Foreign bodies in the gullet........ 457

Foul in the foot....................... 623

Fractured bones...................... 655

G

Gadflies 625

Gastro-intestinal inflammation..... 472

Generative organs, diseases of...... 503

Gullet, foreign bodies in............ 457

Gut-tie ............................. 485

\section{$\mathrm{H}$}

Hæmaturia 531

Hair of cattle as an epidemic appendage...

Heart, description of................. 540

do. - its function and diseases... 543

do. dilatation of................... 544

do. hypertrophy of............... 550

Heels, cracks in...................... 629

Hemorrhage, uterine................. 500

Hernia, or ruptures.................. 562

do. inguinal.................... 563

do. strangulated ................. 563

do. ventral........................ 564
Horn-ail ............ PAGES

Hoose, or common catarrh........... 447

Hoven................................ 468

Humor of the eye..................... 553

Hydatids .............................. 614

Hydrophobia ....................... 607

I

Improved method of milking....... 642

do. method of spaying........ 666

Inflammation of the lungs............ 443

do. gastro-intestinal..... 472

do. of the bowels........ 480

do. of the eye.............. 558

do. of the brain and its

membranes........6 600

do. of the liver............ 613

do. of the udder.......... 517

Inflammatory affection of the hind

limbs after calv-

ing .................. 527

do. affection of the kid-

neys................. 533

Internal ruptures in oxen........... 488

Invagination of intestines...........4 481

Inversion of the vagina............ 521

\section{J}

Jaundice .............................. 617

Jaw-bones, dilatation of.............. 581

Jaw, abscess at its angle........... 583

\section{K}

Kentucky, eattle plague in......... 433

Kidneys, inflammation of........... 533

\section{I}

Labor, signs of...................... 492

do, natural ........................ 492

do. unnatural...................... 493

Laceration of the vagina........... 522

$\rightarrow$ do of the oesophagus....... 459

Laryngitis ........................... 439

Larynx, description of............... 441

Light in barns, necessity of........ 657

Liver, description of................ 611

do. inflammation of.............. 613

Loss of cud........................... 478

Lungs, inflammation of.............. 443 


M
Mammites ..........................
Mange ..........................
Milking, improved method of.......
Milk siekness......................
Muscles of the eye and eyelids....
N
Natural labor.....................
Esophagus, description of..........
do. laceration of...........
Esophagotomy, operation of........
Ophthalmia ...........................
Origin of pleuro-pneumonia in
Massachusetts................
Oxen, internal ruptures in..........

\section{$\mathrm{P}$}

Palsy, shaking.....

Parasites, cerebral

Parturition

Pemphigus, or vesicular eruption.

Pericarditis

Pericardium and its diseases........

Pharyngitis, or sore throat...........

Pharynx, description of.............. 458

Plague, cattle......................... 668

Pleura, description of................ 455

Pleurisy .............................. 455

Pleuro-pneumonia, contagious...... 419

do. its introduction

into South

Africa........ 427

Pneumonia .............................. 443

Pregnancy, treatment of cows dur-

do. $\begin{array}{r}\text { ing.............................. } 504 \\ \text { symptoms of........... } 506\end{array}$

Puerperal fever....................... 507

Pulmonary apoplexy ................ 453

$\mathrm{R}$

Red water. 536

Rinderpest............................ 419

Rumen, distension of with food.... 471

Rumination, or remastication...... 466 do. suspension of.......... 478

\section{$\mathrm{S}$}

PAGES

Secretion of ile, bits uses, etc...... 612

Shaking palsy......................... 606

Signs of labor........................ 492

Sore throat............................ 452

Spasm of the bowels.................. 490

Spaying cows......................... 661

do. improved method.......... 666

Specific or periodic ophthalmia...... 559

Sprains or strains....................6 659

Steamed and cooked food for stock .......................... 645

Stomach, description of.............. 465

Stricture in cows' teats............. 519

Sturdy, or cerebral parasites........ 601

Sympathy and its relations......... 585

Symptoms of pregnancy.............. 506

Synopsis of dentition................ 477

Suspended rumination.............. 478

T

Tail-ail ................................ 597

Teats, stricture in.................. 519

do. obstruction in................ 519

do. tumors in ...................... 520

do. injuries to...................... 520

do. sore............................. 520

do. chapped......................... 520

Throat, sore.......................... 452

Thrush, or sore mouth................ 464

Thymus gland, disease of.......... 643

Tongue, black....................... 654

Treatment of cows during preg-

Trembles .............................. 635

Triplets, birth of..................... 500

Tympanitis ............................ 468

Twins, birth of....................... 500

U

Udder, inflammation of............. 517

Unnatural labor..................... 493

Ureters .................................. 530

Urinary calculi....................... 534

do. organs, diseases of........ 529

do. system, description of...... 529

Uterine hemorrhage................. 500

Uterus, constriction of.............. 503 
$\nabla \quad$ PAGES

521

Vagina, inversion of

do. laceration of

W

Warts.

624

506
PAGES

Womb, falling of.

513

\section{Y}

Yellows, or jaundice

Yoke-galls

\section{SHEEP AND SWINE.}

A PAGES

Arnica, fluid extract of.............. 748

Aconite, do. do.

B

Bakewell on sheep

Bayberry, fluid extract of

Bully, windy distention of, in sheep 716

Blankets for sheep.................. 711

Black snakeroot, fluid extract of... 749

Black pepper, do. do. ........ 752

Bloodroot, do. do. ........ 752

Bowels, constipation of, in sheep... 716

Breeding of sheep................... 707

Buchu, fluid extract of............... 749

Catarrh, in sheep................... 715

Chamomile, fluid extract of......... 748

Cholera, hog......................... 725

do. do. Sutton on............. 727

do, do. Dadd's remedy for.... 735

Colchicum, fluid extract of.......... 750

Constipation of bowels in sheep... 716

Cubebs, fluid extract of.............. 752

Culver's root, do. .............. 751

Dadd's remedy for hog cholera...... 735

Diagnosis, guide to................... 755

do. Equine Department..... 756

do. Bovine do. ...... 762

Diarrhea in sheep................... 716

Diseases of sheep..................... 706

do. of swine..................... 717

Dysentery in sheep................... 716
E PAGES

Effects of impure air on swine..... 719

Extracts, fluid, list of............... 747

Eye, inflammation of, in sheep..... 714

F

Foot-rot in sheep..................... 712

Fluid extracts, list of............... 747

G

Giddiness in sheep................. 711

Ginger, fluid extract of............. 753

Golden seal, do $\quad . . . . . . . . . .750$

Grubs in the nostrils of sheep..... 713

Guide to diagnosis.................. 755

\section{$\mathrm{H}$}

Hemorrhage, remedy for............. 754

Hernia, or rupture, of swine........ 723

Hog cholera........................... 725

Hops, fluid extract of............... 750

Hyposulphite of soda................. 754

\section{I}

Impure air, effects of on swine..... 719

Indian hemp, fluid extract of....... 749

Inflammation of lungs in swine... 724

Inflammation of the eye in sheep.. 714

do. of lungs do. .. 715

Intestinal worms in swine.......... 724

$$
\mathrm{J}
$$

Jackets for sheep.................... 711

\section{L}

Labor, tedious, remedy for........... 753

Liver of sheep, hydatids in......... 709 


\begin{tabular}{|c|c|}
\hline $\mathbf{A G}$ & PAGT \\
\hline iver of sheep, disease of............ 710 & Sheep, disen'ses of 70 \\
\hline Lobelia, fluid extract of............... 751 & do. improvement of............. 706 \\
\hline ungs of sheep, inflammation of... 715 & do. Bakewell's manner of \\
\hline do of swine, do. $\quad \ldots 724$ & breeding....................... 707 \\
\hline M & $\begin{array}{l}\text { Soda, hyposulphite of.................. } 754 \\
\text { Sutton on hog cholera.............. } 727\end{array}$ \\
\hline Male fern, fluid extract of.......... 748 & Awine, diseases of.................. 717 \\
\hline $\begin{array}{lllll}\text { Matico, do. } & \text { do. } & \text {.......... } 752 \\
\text { Mandrake, do. } & \text { do. } & \ldots . . . . . . & 752\end{array}$ & do. effects of impure air on..... 719 \\
\hline Ieasles in pork........................ 721 & $\mathbf{T}$ \\
\hline $\mathrm{N}$ & Tedious labor, remedy for........... 753 \\
\hline Nostrils of sheep, grubs in.......... 713 & Ticks in sheep........................ 714 \\
\hline & $\begin{array}{c}\text { Trichina spiralis....................... } 736 \\
\text { do. do. Brown on.......... } 743\end{array}$ \\
\hline leurisy root, fluid extract of....... 748 & Tympanites in sheep................. 716 \\
\hline .............. 724 & \\
\hline oot, fluid extract of........... 751 & V \\
\hline oppy-heads, do. do. .......... 751 & Valerian, fluid extract of........... 753 \\
\hline ork, measles in...................... 721 & Vertigo in sheep...................... 711 \\
\hline rince's pine, fluid extract of...... 749 & \\
\hline & W \\
\hline $\begin{array}{lll}7 & 7\end{array}$ & Witch-hazel, fluid extra \\
\hline uinsy in swine.... & ne..................... 724 \\
\hline $\mathbf{R}$ & Wormwood; fluid extract of........ 748 \\
\hline .......... 709 & Wounds, remedy for.................... 753 \\
\hline upture of swine..................... 7 & $\mathbf{Y}$ \\
\hline$\therefore$ & \\
\hline & \\
\hline
\end{tabular}

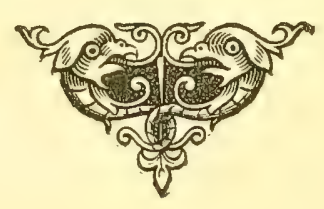







\section{It?}

$0 \%$

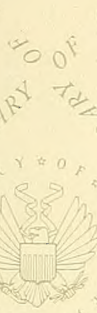

Ell

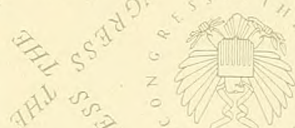

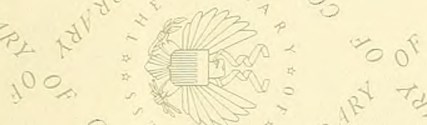

$x_{1 \rightarrow} \rightarrow$

$o^{p}$

"o 0

at +2 -

\section{ent}
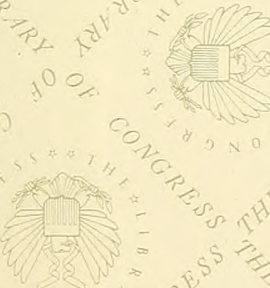

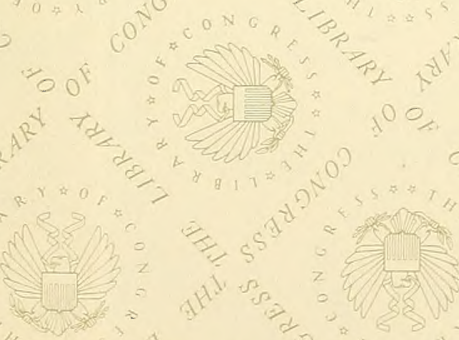

\section{रिक्र}

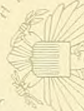

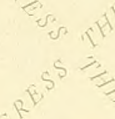

tit?

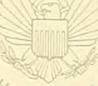

(4)

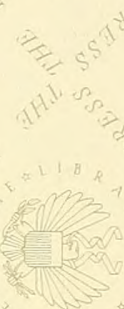

在

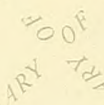

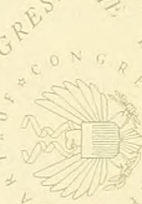

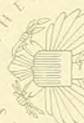

\section{ही?}

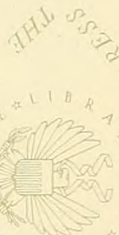

\section{है}
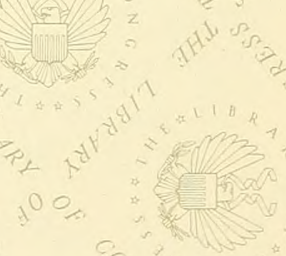

$1^{R}$

\section{=}

$1 \% 00$

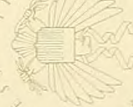

$A^{2}+1 / 2$
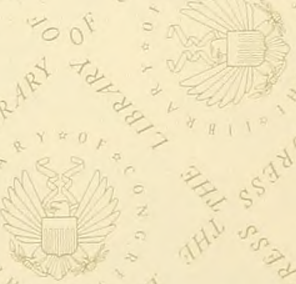

है

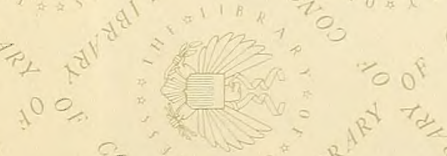

0. 


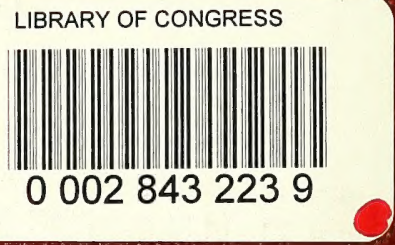

